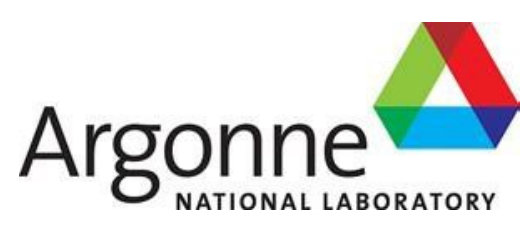

ANL-AFCl-202

(ANL-ABR-4)

\title{
Advanced Burner Reactor 1000MWth Reference Concept
}

Nuclear Engineering Division 


\section{About Argonne National Laboratory}

Argonne is a U.S. Department of Energy laboratory managed by UChicago Argonne, LLC under contract DE-AC02-06CH11357. The Laboratory's main facility is outside Chicago, at 9700 South Cass Avenue, Argonne, Illinois 60439. For information about Argonne and its pioneering science and technology programs, see uww.anl.gov.

DOCUMENT AVAILABILITY

Online Access: U.S. Department of Energy (DOE) reports produced after 1991 and a growing number of pre-1991 documents are available free via DOE's SciTech Connect (http://www.osti.gov/scitech/)

Reports not in digital format may be purchased by the public from the National Technical Information Service (NTIS):

U.S. Department of Commerce

National Technical Information Service

5301 Shawnee $\mathrm{Rd}$

Alexandria, VA 22312

www.ntis.gov

Phone: (800) 553-NTIS (6847) or (703) 605-6000

Fax: (703) 605-6900

Email: orders@ntis.gov

Reports not in digital format are available to DOE and DOE contractors from the Office of Scientific and Technical Information (OSTI):

U.S. Department of Energy

Office of Scientific and Technical Information

P.O. Box 62

Oak Ridge, TN 37831-0062

www.osti.gov

Phone: (865) 576-8401

Fax: (865) 576-5728

Email: reports@osti.gov

\section{Disclaimer}

This report was prepared as an account of work sponsored by an agency of the United States Government. Neither the United States Government nor any agency thereof, nor UChicago Argonne, LLC, nor any of their employees or officers, makes any warranty, express or implied, or assumes any legal liability or responsibility for the accuracy, completeness, or usefulness of any information, apparatus, product, or process disclosed, or represents that its use would not infringe privately owned rights. Reference herein to any specific commercial product, process, or service by trade name, trademark, manufacturer, or otherwise, does not necessarily constitute or imply its endorsement, recommendation, or favoring by the United States Government or any agency thereof. The views and opinions of document authors expressed herein do not necessarily state or reflect those of the United States Government or any agency thereof, Argonne National Laboratory, or UChicago Argonne, LLC. 
ANL-AFCl-202

(ANL-ABR-4)

\section{Advanced Burner Reactor 1000MWth Reference Concept}

Edited by

C. Grandy and R. Seidensticker

Technical Contributors

J. Cahalan, T. Fanning, M. Farmer, C. Grandy, E. Jin, T. Kim, R. Kellogg, L. Krajtl,

S. Lomperski, A. Moisseytsev, Y. Momozaki, Y. Park, C. Reed, F. Salev, R. Seidensticker,

J. Sienicki, Y. Tang, C. Tzanos, T. Wei, W. Yang, and Y. Chikazawa (JAEA Visiting Scientist)

Prepared for the United States Department of Energy

Under Contract No. DE-AC02-06-CH11357

Nuclear Engineering Division

Argonne National Laboratory

September 30, 2007 


\section{Table of Contents}

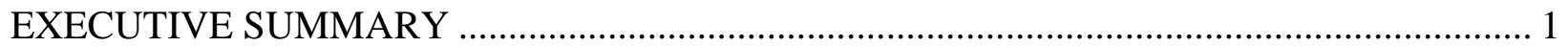

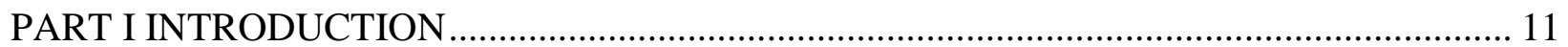

I.1 Overall Objectives .................................................................................................. 11

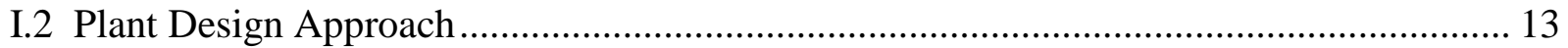

I.3 Safety Design Approach......................................................................................... 16

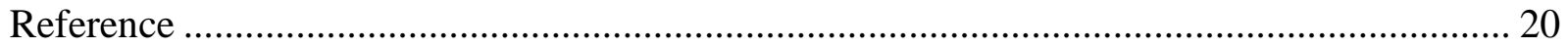

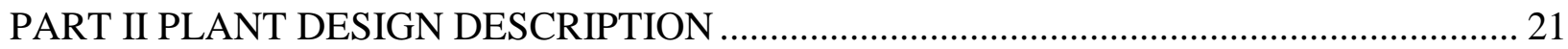

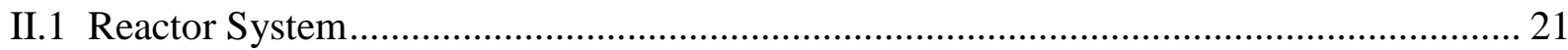

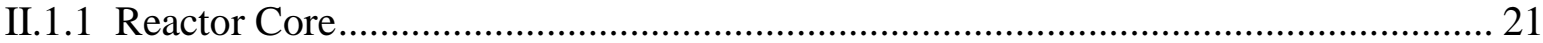

II.1.1.1 Core and Assembly Design Descriptions........................................................... 22

II.1.1.2 Equilibrium Cycle Performance Characteristics ............................................... 26

II.1.1.3 Kinetics Parameters and Reactivity Coefficients................................................ 29

II.1.1.4 Orifice Zoning and Steady-State Thermal-hydraulic Performances ................... 31

II.1.1.5 Spent Fuel Characteristics .............................................................................. 47

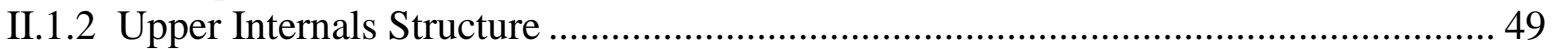

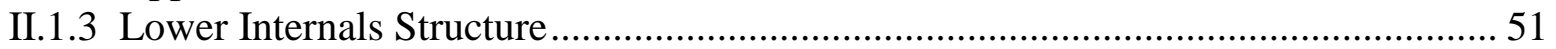

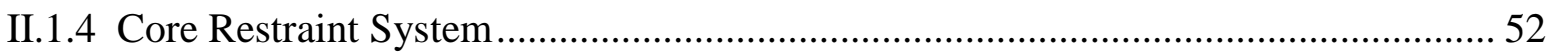

II.1.5 Reactivity Control Requirements and Shutdown Margins.......................................... 54

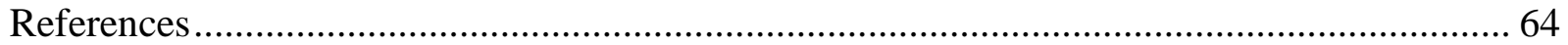

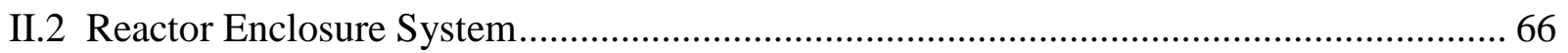

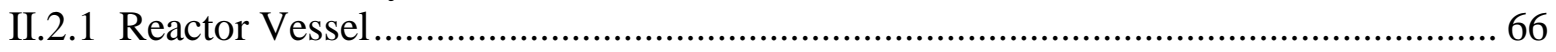

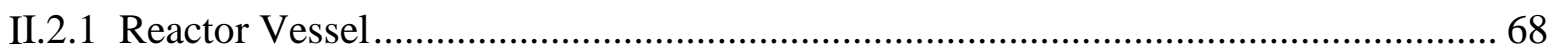

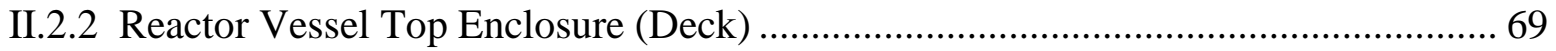

II.2.3 Deck Cooling and Insulation.................................................................................. 73

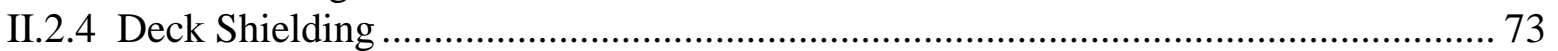

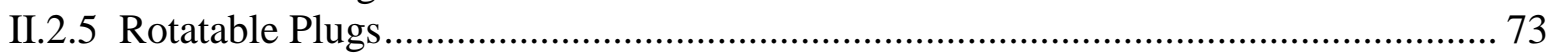

II.2.6 Reactor Vessel and Deck Support Structure .......................................................... 74

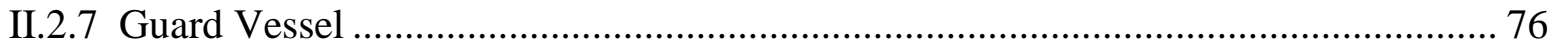

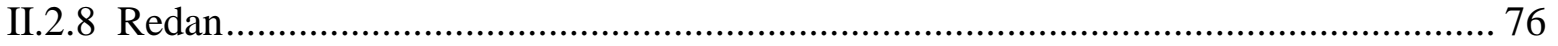

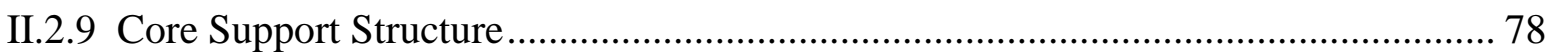

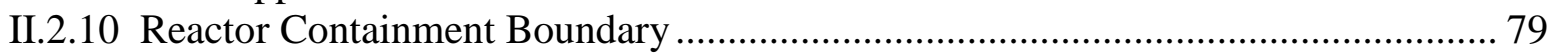

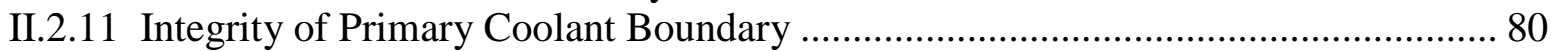

II.3 Primary Heat Transport System .................................................................................... 82

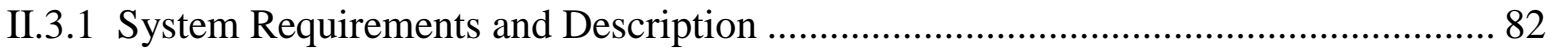

II.3.2 Primary Sodium Mechanical Pump....................................................................... 83

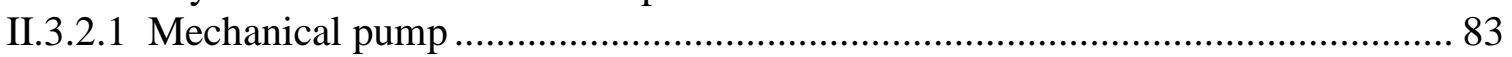

II.3.2.2 Electromagnetic Pump ………………………............................................. 87

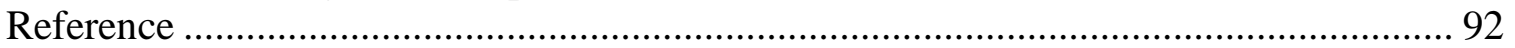

II.3.3 Intermediate Heat Exchanger …………………................................................... 93

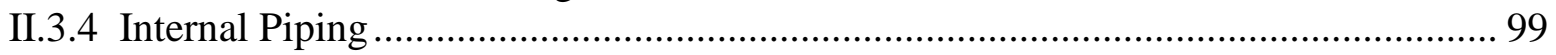

II.4 Intermediate Heat Transport System .............................................................................. 101

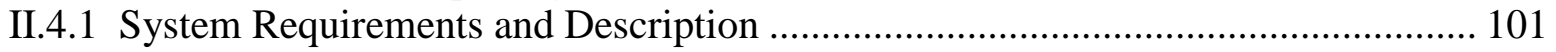

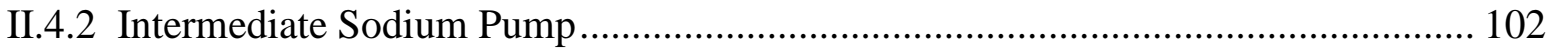




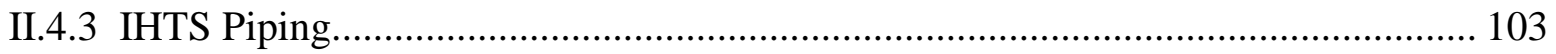

II.4.4 IHTS Sodium Storage Tank, Expansion Tank, and Cleanup System ...................... 105

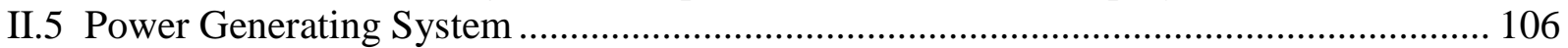

II.5.1 System Requirements and Description .................................................................. 106

II.5.2 Steam Generator System ................................................................................. 107

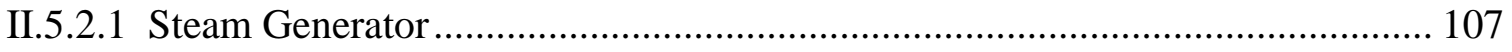

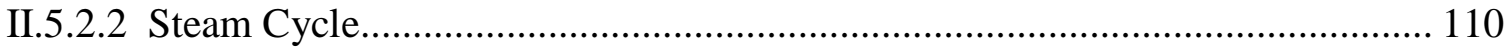

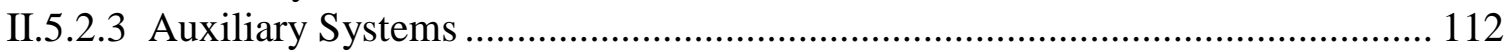

II.5.3 Sodium-Water Reaction Protection System ............................................................... 118

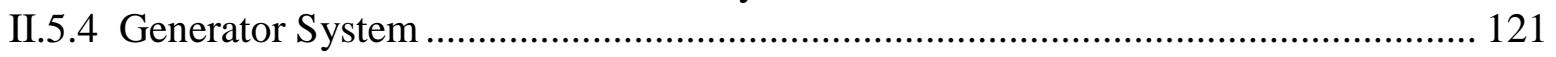

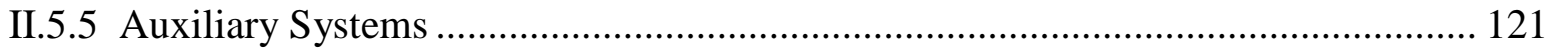

II.6 Shutdown Heat Removal System ............................................................................. 122

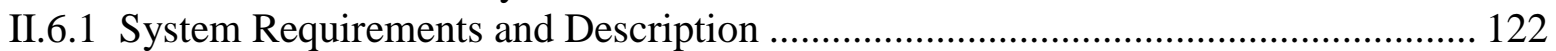

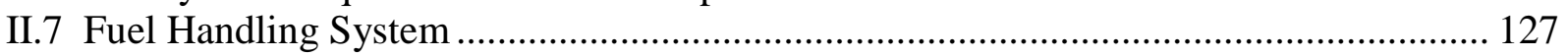

II.7.1 System Functions and Requirements ................................................................... 127

II.7.2 Reference Fuel Handling System Design................................................................. 127

II.7.2.1 Overall System Design .................................................................................. 127

II.7.2.2 Main Components .................................................................................. 128

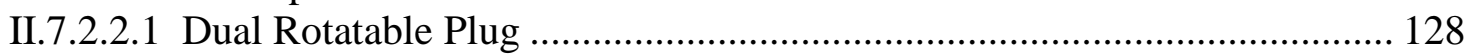

II.7.2.2.2 Fuel Handling Machine......................................................................... 132

II.7.2.2.3 In-vessel Storage ............................................................................ 134

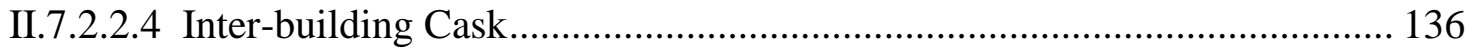

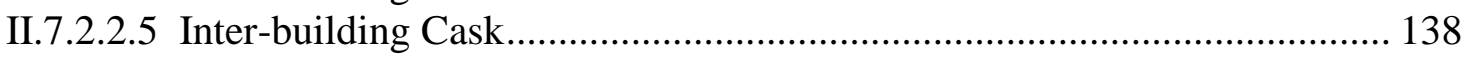

II.8 Instrumentation and Control Systems …………....................................................... 143

II.8.1 Flux Monitoring System.................................................................................. 143

II.8.2 Heat Transport Instrumentation System............................................................... 144

II.8.3 Radiation Monitoring System ....................................................................... 145

II.8.4 Impurity Monitoring and Analysis System ........................................................... 145

II.8.5 Leak Detection System......................................................................................... 146

II.8.6 Data Handling and Signal Transmission System ................................................. 148

II.8.7 Plant Control System................................................................................... 148

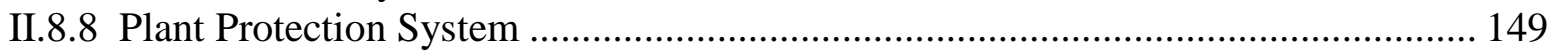

II.8.9 Communications.......................................................................................... 150

II.8.10 Industrial Security and Safeguards System ....................................................... 150

II.9 Primary Plant Auxiliary Systems …………………................................................ 152

II.9.1 Reactor Vessel Heating System ....................................................................... 152

II.9.2 Guard and Thimble Cooling System ...................................................................... 152

II.9.3 Primary Sodium Purification System ........................................................................ 153

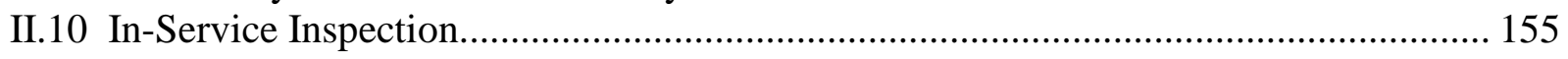

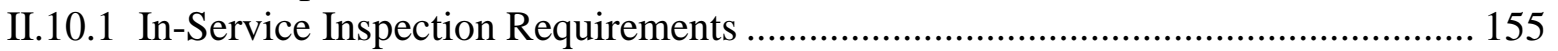

II.10.1.1 Components Subject to Inspection............................................................ 155

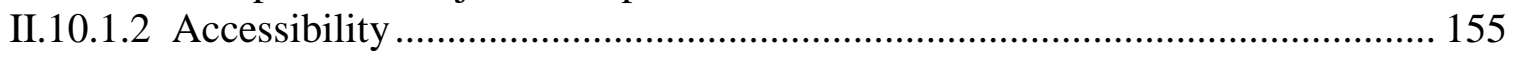

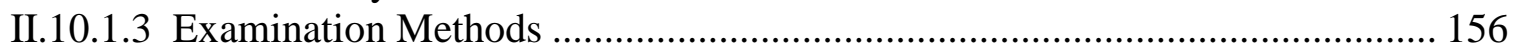

II.10.1.4 Inspection Plans and Schedules................................................................. 156

II.10.2 Examination Techniques ............................................................................... 159

II.10.2.1 Description of Examination Techniques ......................................................... 159 
II.10.2.2 Evaluation of Examination Techniques ......................................................... 160

II.10.3 In-service Inspection Approach for ABR........................................................... 161

II.10.3.1 Approaches for Accessibility ....................................................................... 162

II.10.3.2 Proposed Inspection Methods and Procedures .................................................. 162

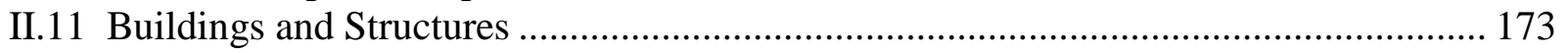

II.11.1 Reactor Building ...................................................................................... 173

II.11.1.1 Containment Design Requirements............................................................. 174

II.11.1.2 Penetrations and Transfer Openings ......................................................... 174

II.11.1.3 Cooling Requirements............................................................................... 177

II.11.2 Balance of Plant Building System........................................................................ 177

II.11.3 Control Room and Personnel Building ............................................................. 177

II.11.4 Radwaste/Maintenance Building....................................................................... 177

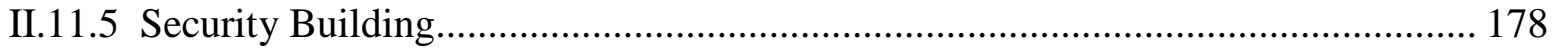

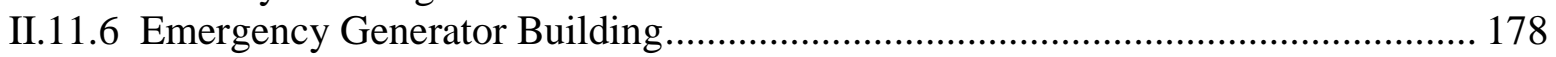

II.11.7 Balance of Plant Services Building System ....................................................... 178

II.11.8 Lift Station and Waste Treatment Plant Building .................................................... 178

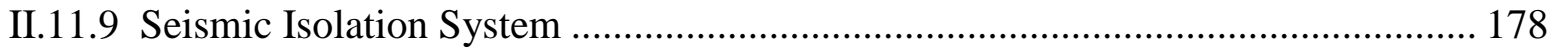

II.12 Balance of Plant Systems ………………………............................................... 179

II.12.1 Compressed Gas System ............................................................................ 179

II.12.2 Chilled Water System........................................................................................ 180

II.12.3 Essential Chilled Water System ........................................................................... 181

II.12.4 Radioactive Waste System ................................................................................ 181

II.12.5 Nuclear Island Heating, Ventilating, and Air Conditioning System....................... 183

II.12.6 Balance of Plant (BOP) Heating, Ventilating, and Air Conditioning System ....... 184

II.12.7 Fire Protection System ......................................................................................... 184

II.12.8 Recirculating Gas Cooling System ……………................................................... 185

II.12.9 Fuel Receiving, Storage, and Shipping System ................................................. 186

II.12.10 Feedwater and Condensate System ..................................................................... 187

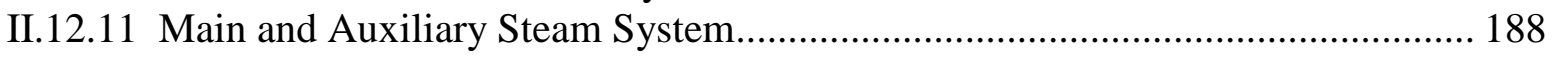

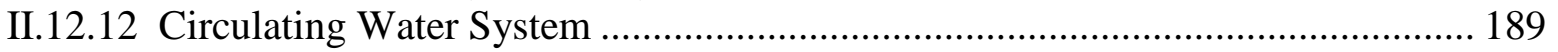

II.12.13 Service Water System ................................................................................. 189

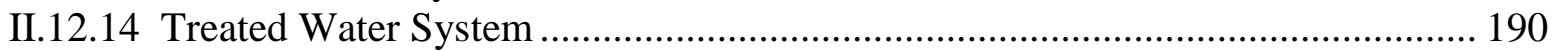

II.12.15 Industrial Waste Water Treatment System..................................................... 190

II.12.16 Liquid Metal Auxiliaries System ........................................................................ 191

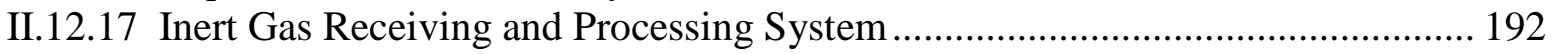

II.13 Electrical Power Systems ……………………................................................... 194

II.13.1 Power Transmission System .................................................................................... 194

II.13.2 Station Power System............................................................................................. 194

II.13.3 Sodium Piping and Equipment Heating and Insulation System ............................. 197

Part III Detailed Design and Engineering Analyses ..................................................................... 198

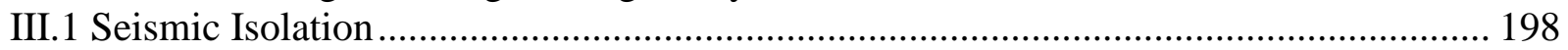

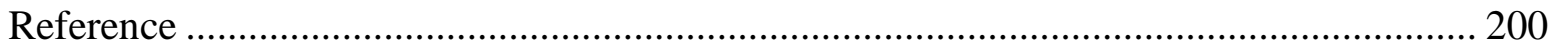

III.2 Structural Analyses ..................................................................................................... 203

III.2.1 Structural Analysis of Reactor Vessel Deck........................................................... 203

III.2.2 Structural Analysis of Reactor Vessel ..................................................................... 207 
III.2.3 Stress Analysis of the Sodium Piping in the Intermediate Heat Transfer System

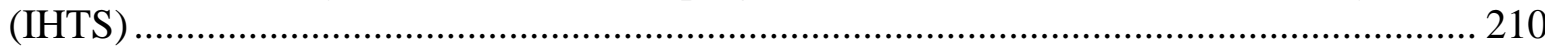

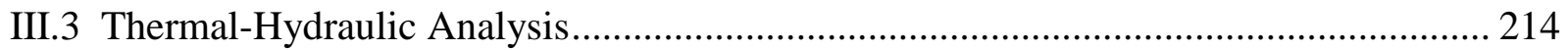

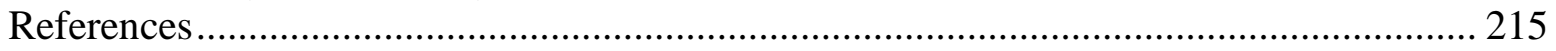

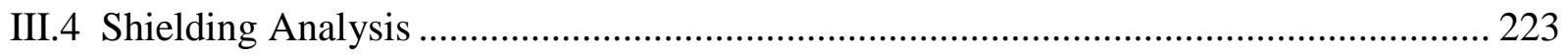

III.4.1 Evaluation of Dose Rate in Reactor Building ................................................. 223

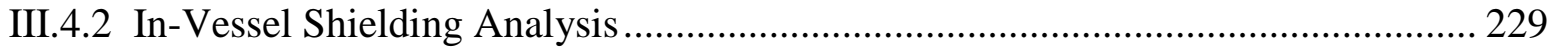

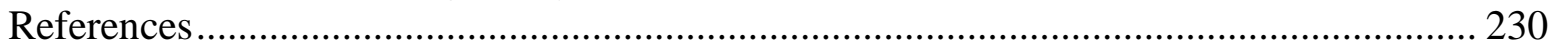

III.5 Response during Natural Circulation Transients ................................................... 232

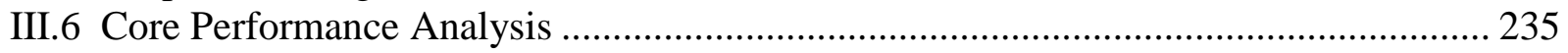

III.6.1 Low Conversion Ratio Core Concepts ........................................................... 236

III.6.1.1 Feasible Design Domain of Low Conversion Ratio Cores............................. 236

III.6.1.2 Low Conversion Ratio Cores........................................................................ 239

III.6.1.3 Impacts of Fixed Absorber........................................................................ 245

III.6.2 High Conversion Ratio Core Concepts .......................................................... 248

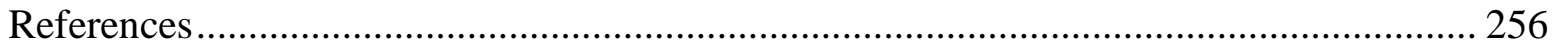

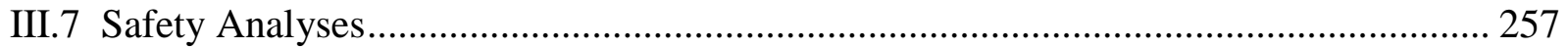

III.7.1 Background and Summary ................................................................... 257

III.7.1.1 Analysis Background .......................................................................... 257

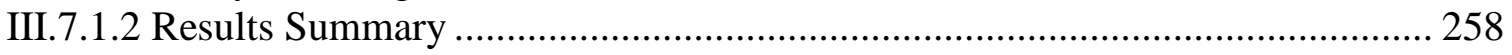

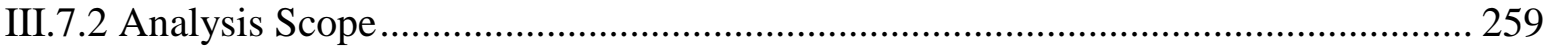

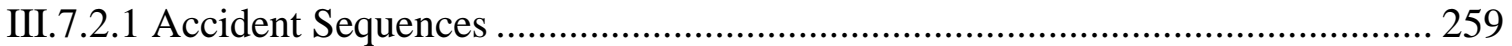

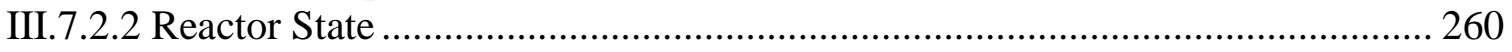

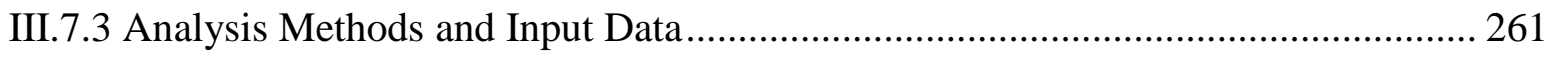

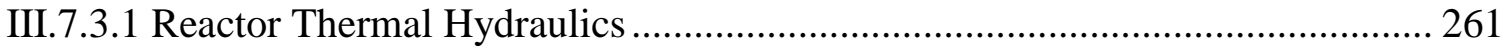

III.7.3.2 Coolant Systems Thermal Hydraulics ...................................................... 272

III.7.3.3 Reactor Kinetics and Reactivity Feedback ............................................... 276

III.7.4 Analysis Results ...................................................................................... 276

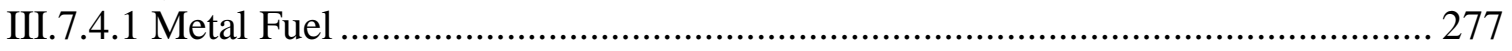

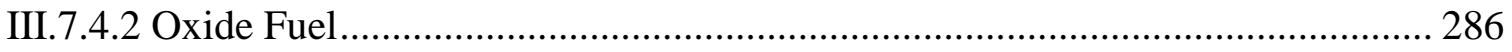

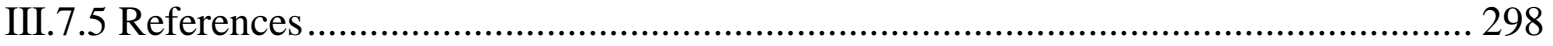

III.7.6 Evaluation Of Safety Design Criteria ............................................................ 299

III.7.6.1 Safety Design Criteria Comparison ........................................................... 300

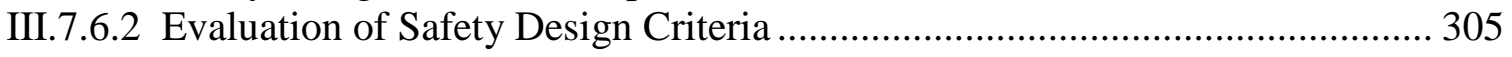

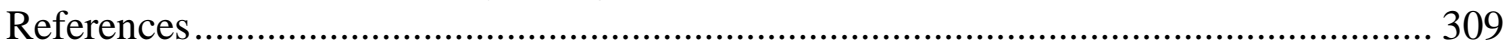

III.8 Supporting Analyses for Heat Transport Systems .................................................... 310

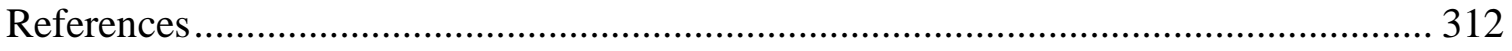

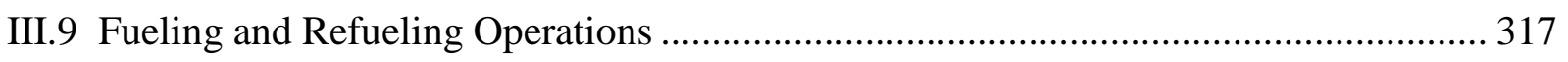

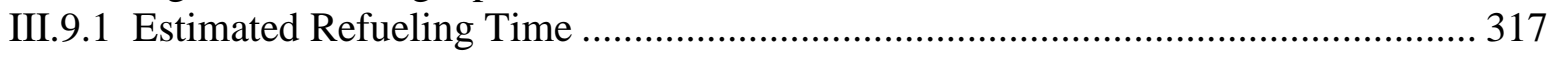

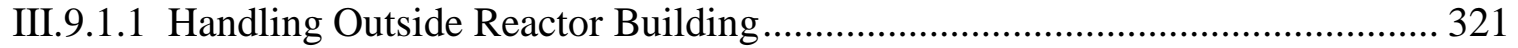

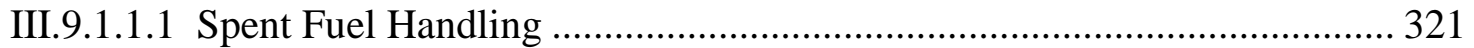

III.9.1.1.2 Fresh Fuel Handling....................................................................... 323

III.9.2 System Design Options ........................................................................... 324

III.9.2.1 Historical Overview of Fuel Handling System ............................................ 324

III.9.2.2 ABR Fuel Handling Design Options ...................................................... 326

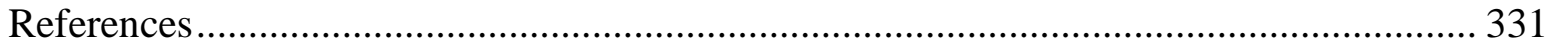




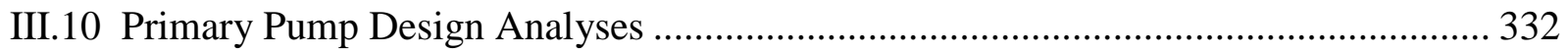

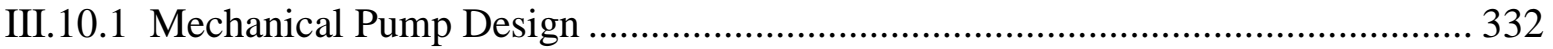

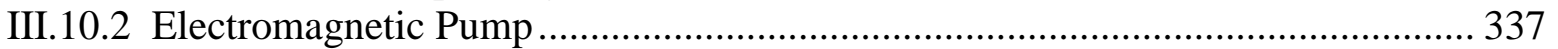

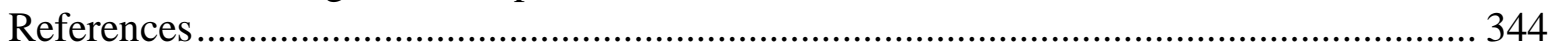

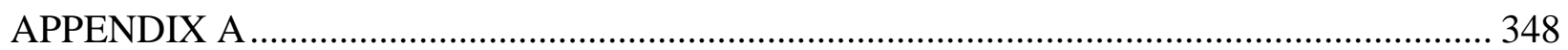

A.1 Requirements for a Standard Commercial Advanced Burner Reactor .......................... 348

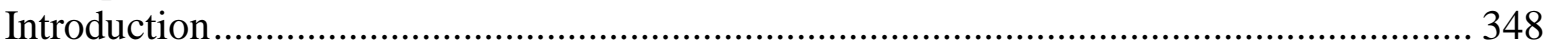

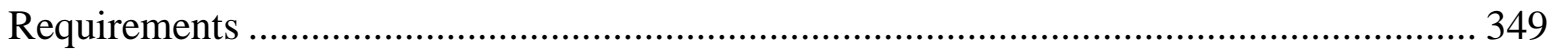

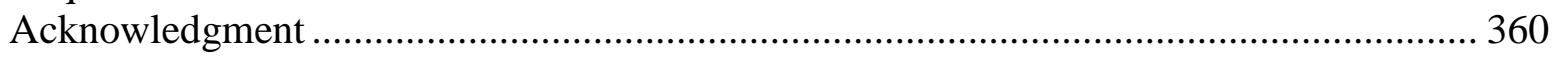

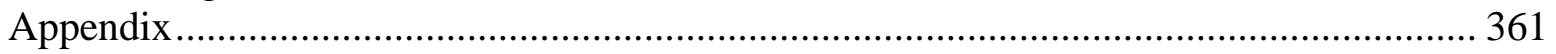




\section{EXECUTIVE SUMMARY}

The goals of the Global Nuclear Energy Partnership (GNEP) are to expand the use of nuclear energy to meet global energy demand, to address nuclear waste management concerns, and to promote non-proliferation. Implementation of the GNEP requires development and demonstration of three major technologies:

- Light water reactor (LWR) spent fuel separations technologies that will recover transuranics to be recycled for fuel but not separate plutonium from other transuranics, thereby providing proliferation-resistance;

- Advanced Burner Reactors (ABRs) based on a fast neutron spectrum that transmute the recycled transuranics to produce energy while also reducing the long term radiotoxicity and decay heat loading in the repository; and

- Fast reactor fuel recycling technologies to recover and refabricate the transuranics for repeated recycling in the fast reactor system.

The primary mission of the ABR Program is to demonstrate the transmutation of transuranics recovered from the LWR spent fuel, and hence, to validate the benefits of the fuel cycle closure to nuclear waste management. The transmutation, or burning of the transuranics is accomplished by fissioning and this is most effectively done in a fast spectrum. In the thermal spectrum of commercial LWRs, some transuranics capture neutrons and become even heavier transuranics rather than being fissioned. Even with repeated recycling, only about $30 \%$ can be transmuted, which is an intrinsic limitation of all thermal spectrum reactors. Only in a fast spectrum can all transuranics be effectively fissioned to eliminate their long-term radiotoxicity and decay heat.

The proposed Advanced Burner Reactor (ABR) Prototype is the first step in demonstrating the transmutation technologies. The Prototype ABR is proposed to have a power range between 250MWth and 2000MWth in the current programmatic environmental impact statement. The ABR reference concept discussed in this report provides a basis for which to compare advanced technologies and concepts going forward and their impact upon site layout, nuclear plant commodities, and overall reactor footprint. The ABR reference concept is $1000 \mathrm{MWth}$ in power level, in the mid-range of the GNEP Programmatic Environmental Impact Study analysis. The ABR reference concept uses essentially proven base technology, and thus is the perfect medium in order to compare future concepts and improvements once developed. The prototype ABR directly supports the development of commercially deployment of ABRs.

The primary objectives of the prototype ABR are:

- To demonstrate reactor-based transmutation of transuranics as part of an advanced fuel cycle;

- To qualify the transuranics-containing fuels and advanced structural materials needed for a full-scale ABR;

- To support the research, development and demonstration required for certification of an ABR standard design by the U.S. Nuclear Regulatory Commission. 
The prototype ABR will also address the following additional objectives:

- To incorporate and demonstrate innovative design concepts and features that may lead to significant improvements in cost, safety, efficiency, reliability, or other favorable characteristics that will promote public acceptance and future private sector investment in ABRs;

- To demonstrate improved technologies for safeguards and security;

- To support development of the U.S. infrastructure for design, fabrication and construction, testing and deployment of systems, structures and components for the ABRs.

To support these objectives, a reference ABR has been developed; it is documented in this report. In addition to meeting the primary and additional objectives listed above, the lessons learned from fast reactor programs in the U.S. and worldwide and the operating experience of more than a dozen fast reactors around the world, in particular the Experimental Breeder Reactor-II, have been incorporated into the design of the reference ABR to the extent possible.

The reactor core design parameters have been selected to be representative of commercial-scale reactors, which results in a moderate conversion ratio of $\sim 0.8$ and a plutonium or transuranics enrichment in the range where extensive irradiation databases exist. However, the core has flexibility to accommodate a wide range of conversion ratios by changing the assembly design parameters appropriately. The reactor core consists of a total of 180 fueled core assemblies 78 assemblies in an inner enrichment zone and 102 assemblies in an outer zone. Reactivity control and neutronic shutdown are provided by 15 primary and 7 secondary control rod assemblies. Fuel or materials specimens could be irradiated in any number of core assembly positions. This core design is the product of extensive trade studies involving power rating, conversion ratio, fuel type (metal, oxide), fissile material (weapons $\mathrm{Pu}$, TRU from LWR spent fuel), control requirements and shutdown margin. The reference design uses weapons-grade plutonium-based ternary metal driver fuel as the initial core and envisions a gradual transition to transuranics-containing driver fuel as it is qualified. It has a TRU conversion ratio of 0.73-0.81 depending upon the fuel.type needed to meet the program goals.

Based on the past trade studies and lessons learned from operating reactors, the pooltype arrangement was selected as the basis for this reference ABR design due to its potential for design simplicity, inherent passive safety and economics.

The key plant design parameters for this reference ABR are summarized in Table 1. The overall plant site arrangement is shown in Figure 1. The major systems - the reactor vessel containing the reactor core and the primary heat transport system, the intermediate heat transport system with the sodium to water stream generators, and the Rankine cycle power conversion system - are shown in an elevation view in Figure 2.

The reactor and the primary and secondary heat transport systems are located essentially below grade. Note that all of the nuclear components of the plant are located on a nuclear island, which is seismically isolated from its base mat foundation, which is also illustrated in Figure 2. 
Table 1 ABR Reference Plant Design Parameters

\begin{tabular}{|l|l|}
\hline Reactor Power & $1000 \mathrm{MWt}, 380 \mathrm{MWe}$ \\
\hline Coolant & Sodium \\
\hline Coolant Temperature, Inlet/Outlet & $355^{\circ} \mathrm{C} / 510^{\circ} \mathrm{C}$ \\
\hline Driver Fuel & $\begin{array}{l}\text { Both metal and oxide startup and recycle cores } \\
\text { were evaluated }\end{array}$ \\
\hline Cladding and Duct Material & HT-9 \\
\hline Cycle Length & 12 months \\
\hline Plant Life & $\begin{array}{l}30 \text { years with the expectation of life extension } \\
\text { to } 60 \text { years }\end{array}$ \\
\hline Reactor Vessel Size & 14.1 m diameter, 14.8 m height \\
\hline Structural and Piping Material & Austenitic Stainless Steel \\
\hline Primary Pump & $\begin{array}{l}\text { Four (4) Mechanical (centrifugal) primary } \\
\text { pumps }\end{array}$ \\
\hline Power Conversion Cycle & Reference: Rankine Steam Cycle \\
\hline Thermal Efficiency & $38 \%$ \\
\hline
\end{tabular}

The primary system is configured in a pool-type arrangement (similar to that used successfully in EBR-II and many other LMR plants), with the reactor core, primary pumps, intermediate heat exchangers, and direct reactor auxiliary cooling system (DRACS) heat exchangers all immersed in a pool of sodium coolant within the reactor vessel. A schematic view of the primary system is shown in Figure 3 and some specific dimensions are given in Figure 4. The pool-type arrangement was selected as the initial reference concept because of its inherent simplicity and safety. All of the primary coolant and associated piping is within the reactor vessel, which greatly reduces the possibility of loss of coolant, and the sodium pool provides a large thermal inertia in the system. In addition, the reactor vessel is a simple shell structure having no penetrations in the shell wall or bottom head; all penetrations are in the top closure head (deck) of the reactor vessel assembly. The hot sodium at core outlet temperature is separated from the cold sodium at core inlet temperature by a structure called the redan. The reactor vessel is exposed only to cold sodium, so it is not subjected to severe thermal transients. A guard vessel is provided as an additional passive safety feature.

Within the reactor vessel, four primary mechanical centrifugal sodium pumps take suction from the lower regions of the cold pool and discharge the sodium into a header that distributes the sodium into multiple feeder pipes, which distribute sodium evenly into the inlet plenum. The inlet plenum distributes the primary sodium to the inlet of the core assemblies, which are individually orificed for proper flow distribution. The sodium is heated as it flows through the core and exits the core assemblies into the outlet plenum. The hot sodium then rises into the redan and then enters the inlet of the intermediate heat exchanger (IHX). After the primary sodium transfers its heat to the intermediate sodium, it exits the IHX into the lower regions of the cold pool. The IHX is round in cross-section 
which is consistent with base sodium heat exchanger technology. The guard vessel that surrounds the reactor vessel will capture and contain any primary sodium coolant in the very unlikely event of a leak in the reactor vessel, and, thus, prevent the IHX inlet, DRACS heat exchangers, and core assemblies from being uncovered.

The baseline power conversion system adopted for this reference concept is the Rankine steam cycle. Four (4) once through helical coil steam generators are used and were sized for the 1000MWth power level. These steam generators generate superheated steam in one pass of the steam generator. A sodium-water reaction protection system was is also included in the design as part of the primary plant auxiliary systems.

The intermediate sodium exits the IHX and flows to the helical coil steam generator located on the nuclear island. The intermediate sodium heats the feedwater which then turns to saturated steam and then to superheated steam before it exits the steam generator. The superheated steam then flows to the turbine-generator performing work and generating electricity. The steam exits the turbine generator and is condensed in the main condenser as condensate. The condensate then goes through a series of heat exchangers and pumps to pressurize and preheat the condensate into feedwater of the right temperature and pressure for introduction into the steam generator. A schematic diagram is given in Figure 5.

Removal of decay heat from the reactor core is a fundamental safety function. In the reference ABR design, normal decay heat removal is through the normal power conversion systems. However, a direct reactor auxiliary cooling system (DRACS) is provided, having both forced flow and natural convection capability. This system removes decay heat from the pool to the atmosphere using heat exchangers located in the cold part of the sodium pool and in the atmosphere above grade. If electrical power is available, forced flow can be used; in an emergency, natural convection flow can remove the decay heat. 


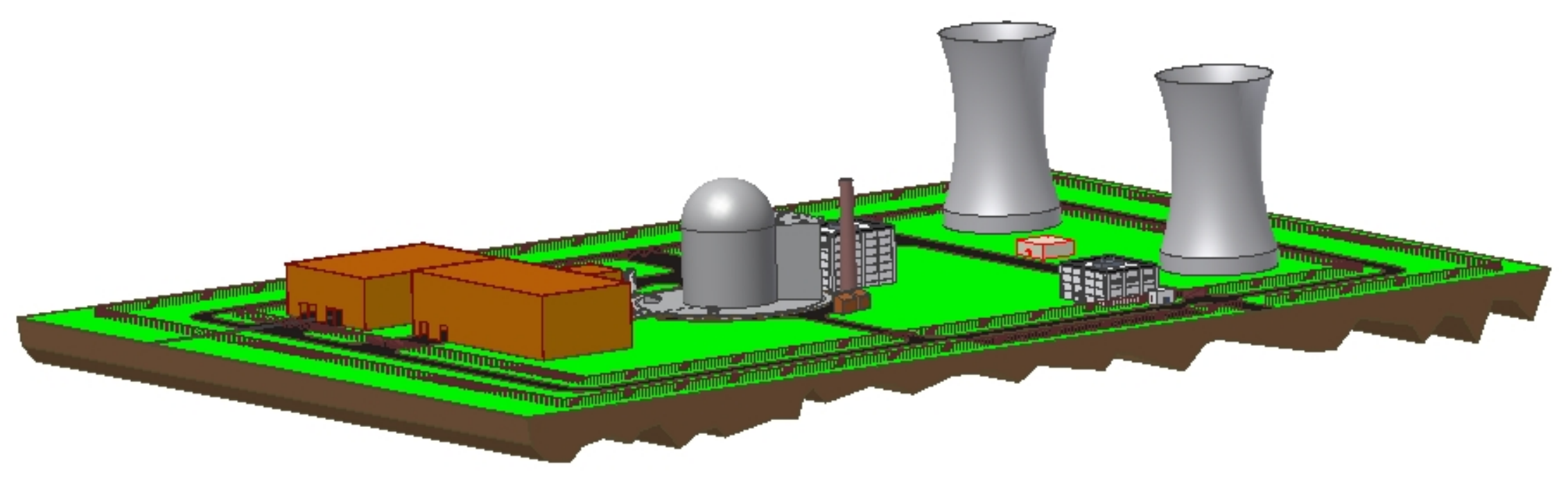

Figure 1 Overall Site View of the ABR Plant 


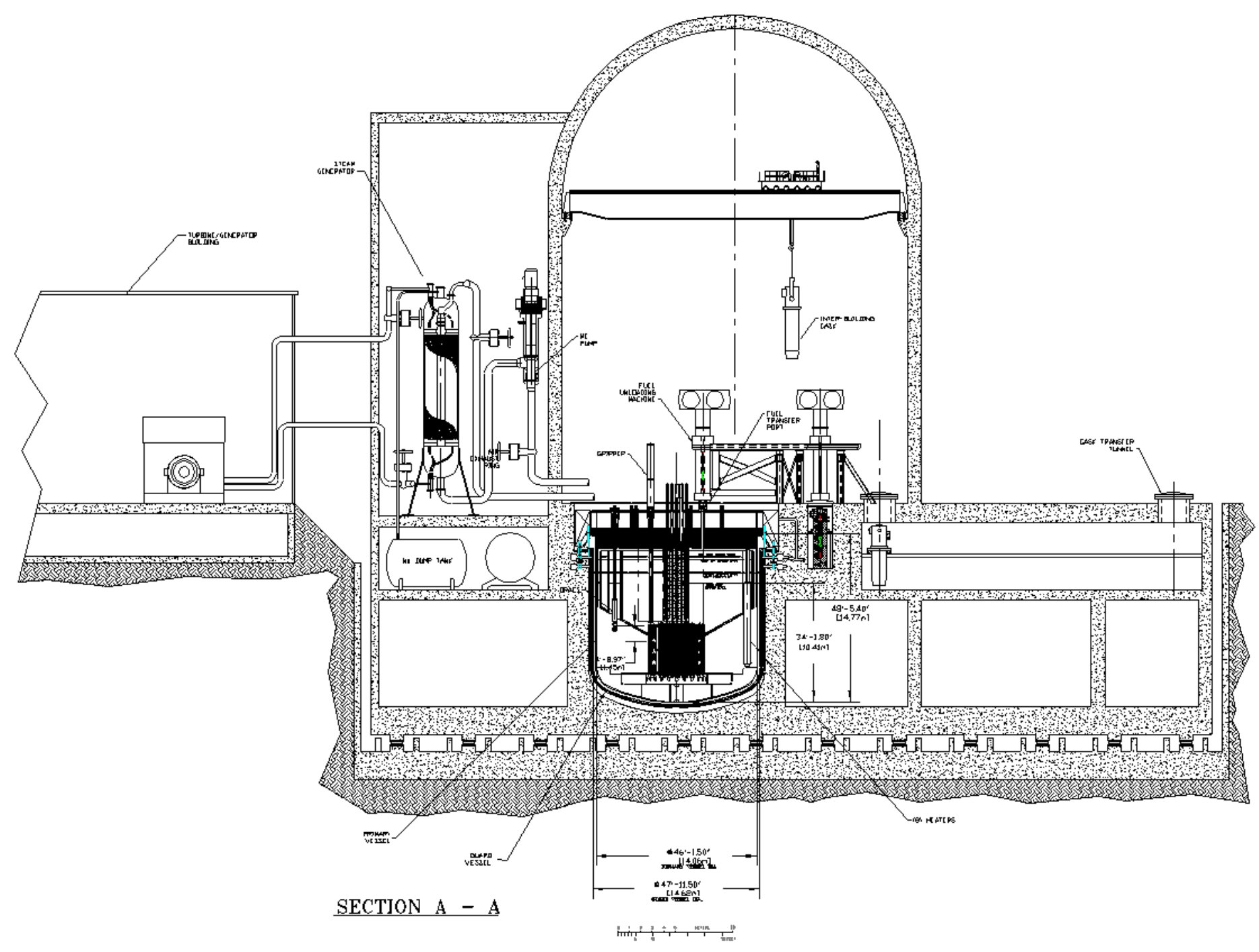

Figure 2 Elevation View of Primary System, Intermediate System, and Steam Generators 


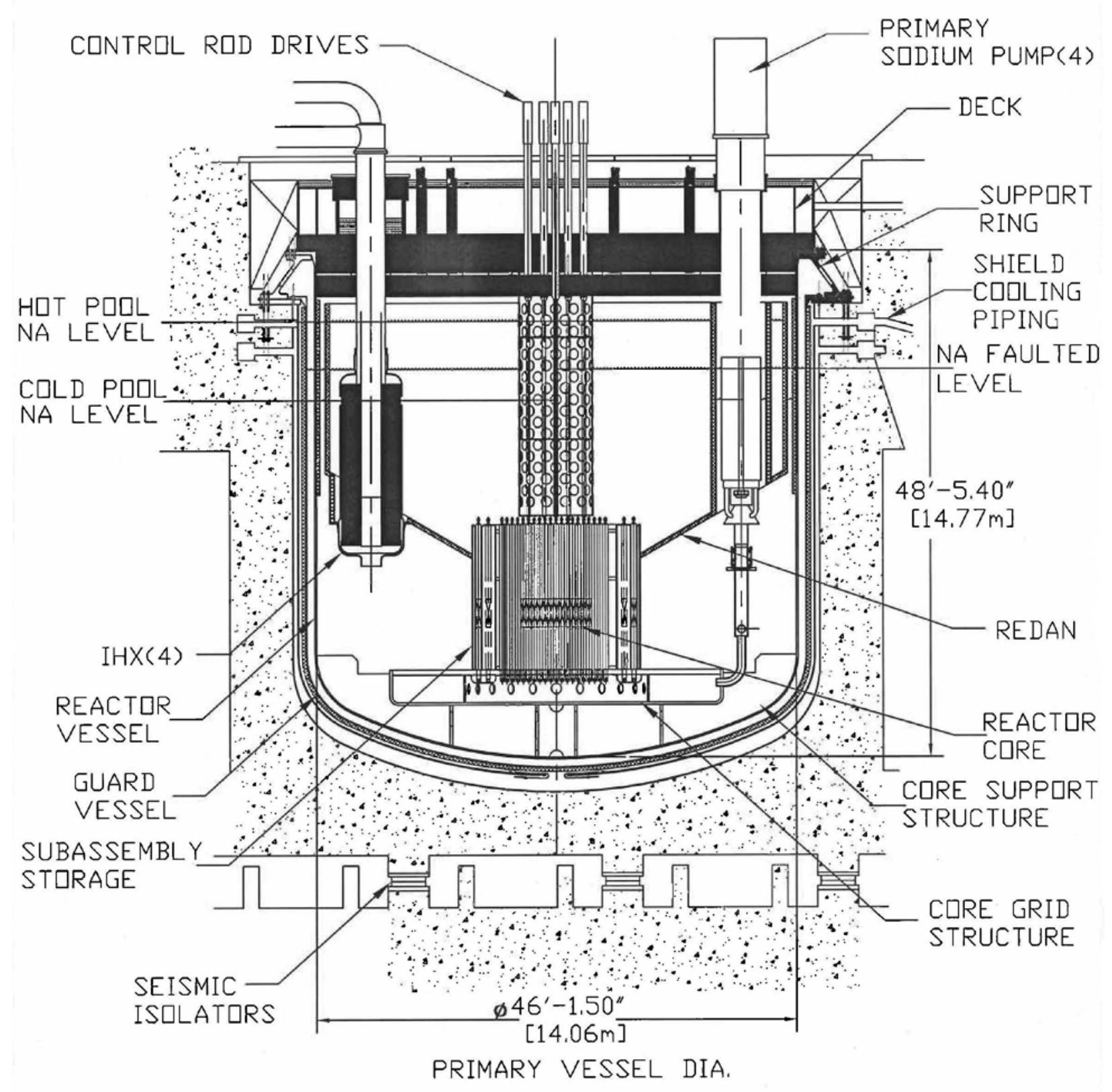

Figure 3 Cross Sectional View of Primary System 


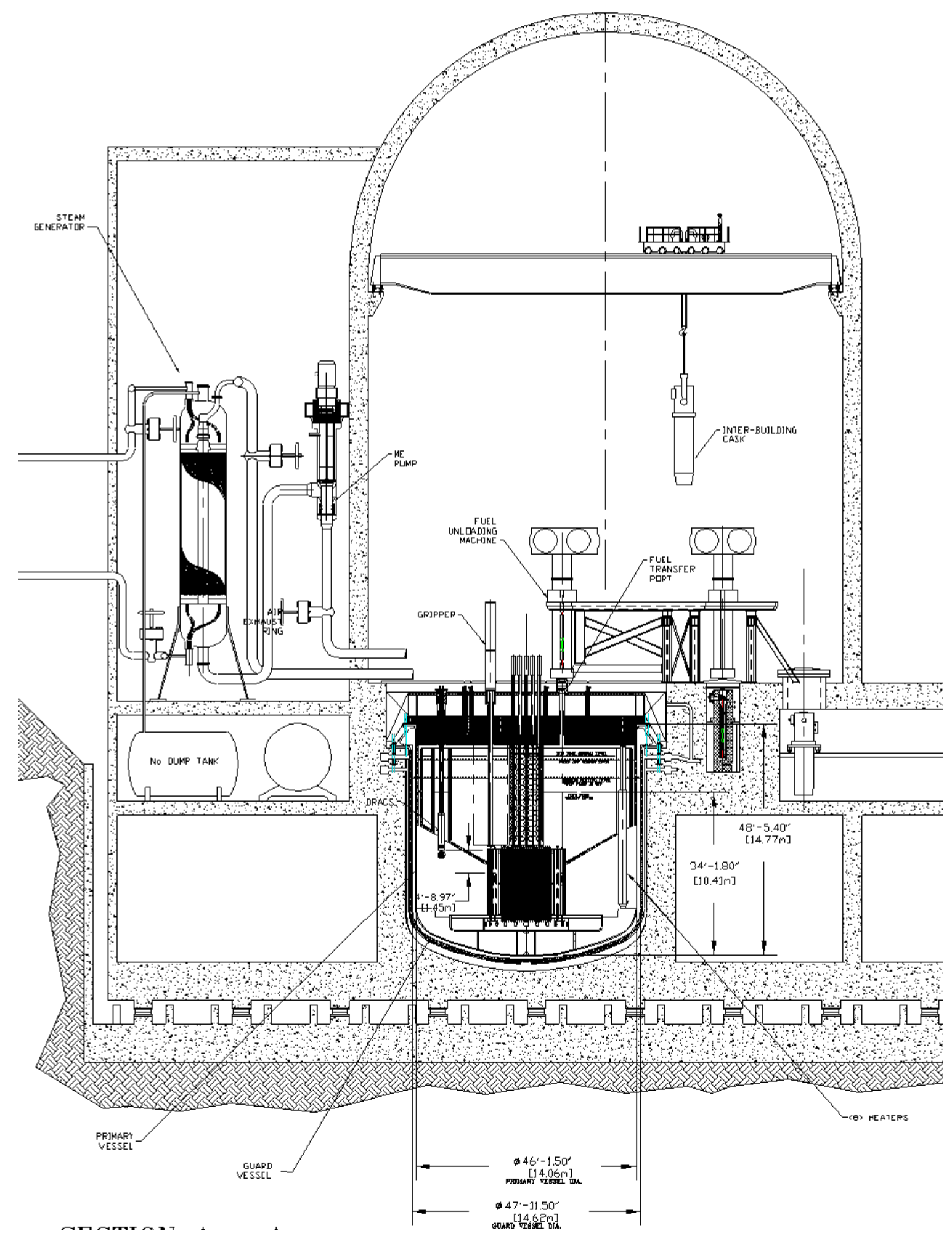

Figure 4 Elevation View of Primary/Intermediate System 
The ABR will have a short refueling interval, so efficient and reliable fuel handling is essential. The reference design provides in-vessel fuel storage cooled by natural convection. Movement of fuel into and out of the core is done using a simple and reliable dual rotatable plug and a straight-pull fuel handling machine. This fuel handling machine, similar to EBR-II, operates be a combination of the dual rotatable plug movement and the fuel handling machine to insert and remove fuel into and from the reactor core. Movement of fuel from the reactor vessel to the fuel handling building is done using a fuel unloading machine and inter-building transfer cask similar to the system used for many years at EBR-II.

The reference design concept presented in this report reflects essentially baseline, proven technology and will be the starting point for extensive and detailed comparison studies of the impacts of advanced technologies on the potential reduction in facility size, reduction in commodities, and economic gain while maintaining the desired attributes of a sodium-fast reactor system. The performance of the reactor and heat transport systems during natural circulation transients and several design basis events was considered. These analyses are documented in Part III of this report. 


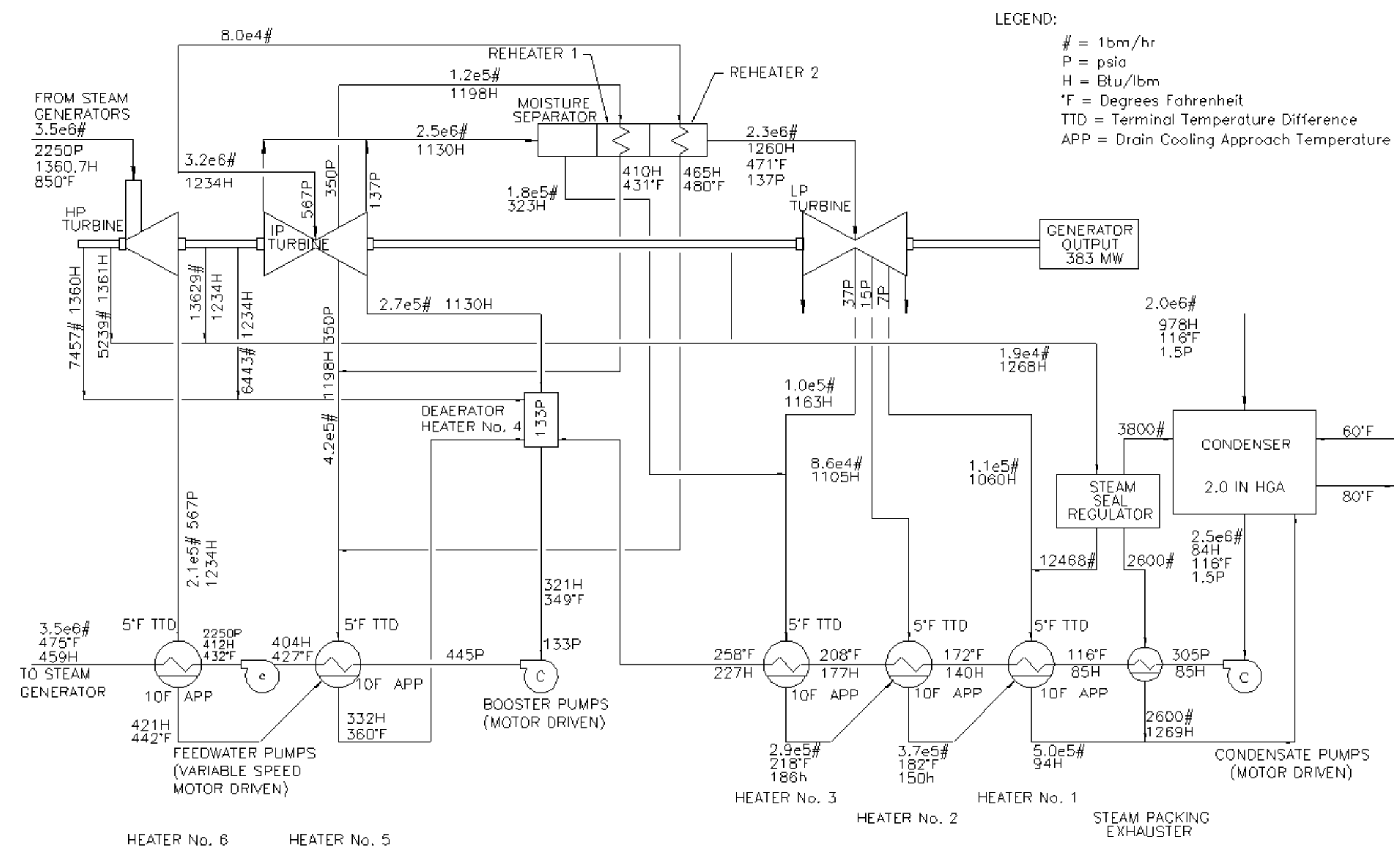

Figure 5 Overall Thermodynamic Cycle 


\section{PART I \\ INTRODUCTION}

\section{I.1 Overall Objectives}

The goals of the Global Nuclear Energy Partnership (GNEP) are to expand the use of nuclear energy to meet increasing global energy demand, to address nuclear waste management concerns and to promote non-proliferation. To enable the expansion of nuclear energy for peaceful purposes and make a major contribution to global development into the 21st century, the United States seeks to pursue and accelerate cooperation to:

- Expand nuclear power to help meet growing energy demand in an environmentally sustainable manner.

- Develop, demonstrate, and deploy advanced technologies for recycling spent nuclear fuel that do not separate plutonium, with the goal over time of ceasing separation of plutonium and eventually eliminating excess stocks of civilian plutonium and drawing down existing stocks of civilian spent fuel. Such advanced fuel cycle technologies would substantially reduce nuclear waste, simplify its disposition, and help to ensure the need for only one geologic repository in the United States through the end of this century.

- Develop, demonstrate, and deploy advanced reactors that consume transuranic elements from recycled spent fuel.

- Establish supply arrangements among nations to provide reliable fuel services worldwide for generating nuclear energy, by providing nuclear fuel and taking back spent fuel for recycling, without spreading enrichment and reprocessing technologies.

- Develop, demonstrate, and deploy advanced proliferation resistant nuclear power reactors appropriate for the power grids of developing countries and regions.

- In cooperation with the IAEA, develop enhanced nuclear safeguards to effectively and efficiently monitor nuclear materials and facilities, to ensure commercial nuclear energy systems are used only for peaceful purposes.

Implementation of the GNEP requires development and demonstration of three major technologies:

- Light water reactor (LWR) spent fuel separations technologies that will recover transuranics to be recycled for fuel but not separate plutonium from other transuranics, thereby providing proliferation-resistance;

- Advanced Burner Reactors (ABRs) based on a fast spectrum that transmute the recycled transuranics to produce energy while also reducing the long term radiotoxicity and decay heat loading in the repository; and

- Fast reactor fuel recycling technologies to recover and refabricate the transuranics for repeated recycling in the fast reactor system. 
The primary mission of the ABR Program is to demonstrate the transmutation of transuranics recovered from the LWR spent fuel, and hence the benefits of the fuel cycle closure to nuclear waste management. The transmutation, or burning of the transuranics is accomplished by fissioning and this is most effectively done in a fast neutron spectrum. In the thermal neutron spectrum of commercial LWRs, some transuranics capture neutrons and become even heavier transuranics rather than being fissioned. Even with repeated recycling, only about $30 \%$ can be transmuted, which is an intrinsic limitation of all thermal spectrum reactors. Only in a fast spectrum can all transuranics be effectively fissioned to eliminate their long-term radiotoxicity and decay heat.

DOE's current vision is to develop an Advanced Burner Reactor (ABR) prototype in the power range of 250MWth to 2000MWth as the first in a series of fast reactor plants that can achieve the mission objectives and as the first step in demonstrating the transmutation technologies. This ABR directly supports development of increasingly larger Advanced Burner Reactor systems, which would result in commercial deployment of ABRs.

The primary objectives of the ABR prototype are:

- To demonstrate reactor-based transmutation of transuranics as part of an advanced fuel cycle;

- To qualify the transuranics-containing fuels and advanced structural materials needed for a full-scale ABR;

- To support the research, development and demonstration required for certification of an ABR standard design by the U.S. Nuclear Regulatory Commission.

The ABR prototype should also address the following additional objectives:

- To incorporate and demonstrate innovative design concepts and features that will lead to significant improvements in cost, safety, efficiency, reliability, or other favorable characteristics that will promote public acceptance and future private sector investment in ABRs;

- To demonstrate improved technologies for safeguards and security;

- To support development of the U.S. infrastructure for design, fabrication and construction, testing and deployment of systems, structures and components for the ABRs.

Based on the overall GNEP and ABR prototype objectives of commercially deploying advanced fast reactors, this 1000MWth ABR has been developed and is documented in this report. This $1000 \mathrm{MWth}$ ABR provides a starting point and a framework to understand the commodity, footprint, and economic impact of potential innovative design features and concepts and how best to include these innovative features in future fast reactor systems to make them more attractive to industry. In addition to meeting the primary and additional objectives listed above, the lessons learned from fast reactor programs in the U.S. and worldwide and the operating experience of more than a dozen fast reactors around the world, in particular the Experimental Breeder Reactor-II have been incorporated into the design of the 1000MWth reference ABR to the extent possible. In addition, the basic goal of the reference concept was to use known and demonstrated technology. 


\section{I.2 Plant Design Approach}

In order to address the ABR objectives, the following major design goals governed the design approach of the 1000MWth reference ABR:

- Demonstrate Transmutation of Transuranics: The reactor core will be designed to demonstrate actinide transmutation in a fast spectrum using the plutonium containing fuel as its initial core driver fuel, gradually transitioning into transuranics containing fuel, recovered from LWR spent fuel. It will accommodate test positions for irradiation testing of transuranic fuels and non-fuel materials within the core, however, there will be no specific accommodation for instrumented subassemblies.

- Demonstrate Fast Reactor Safety: The reactor and system design will incorporate inherent safety features and shall utilize passive safety systems to the maximum extent possible. The design will incorporate the major features that would be expected in a commercial-scale ABR so that safety demonstrations and operating experience is applicable to the future ABRs.

- Economics: The design will emphasize simplicity, reliability and long life of its systems, structures and components.

- Known and Demonstrated Fast Reactor Technology: The design will use known and demonstrated fast reactor technology to support the evaluation of innovative design options in future design studies. The design will emphasize ease of construction and fabrication, operation, inspection and maintenance. The design will be suitable for location on a wide variety of sites in the US.

The reactor core design parameters have been selected to be representative of commercialscale reactors, which results in a moderate conversion ratio of $\sim 0.73-0.81$ and a plutonium or transuranics enrichment in the range where extensive irradiation databases exist. However, the core has flexibility to accommodate a wide range of conversion ratios by changing the assembly design parameters appropriately. This flexibility is discussed in separate reports.

Once the core envelope has been defined, the next key design decision is selection of the overall plant layout option, namely loop-type or pool-type arrangement. Based on the past trade studies and lessons learned from operating reactors, the pool-type arrangement was selected as the reference approach for the 1000MWth ABR. The reasons for this selection are summarized below.

\section{Simplicity}

Assurance of primary system integrity and leak tightness is a major design objective. In the pool concept, the primary system coolant boundary is simple and regular, resulting in low stresses and high structural reliability. There are no penetrations in the reactor vessel wall. Because of the submerged primary piping and other major components, small leakages internal to the primary boundary are permissible. By contrast, in the loop concept, the primary pumps and intermediate heat exchangers are housed external to the reactor vessel in steel-lined, nitrogen-inerted cells. The piping normally penetrates the reactor vessel below the top head closure and runs in inerted pipeways to and from the steel-lined cells. Elimination of these inerted steel-lined primary heat transport system cells and the complex network of piping, 
supports and restraints results in a major simplification of the plant design and reduction of containment building size and associated commodities.

\section{Inherent Passive Safety}

There is a significant difference between the responses of the pool and loop designs to various system transients. Due, in part, to the large inventory of cold sodium coolant, the pool system generally is less sensitive than the loop system to plant upsets associated with loss of heat sink or loss of forced cooling. This reduced sensitivity allows greater opportunity for inherent passive safety features such as natural convective flow and negative reactivity feedback to protect the core. The major design considerations relate to the thermal inertia of the sodium coolant, the rate of pump coastdown, and the reactivity feedback characteristics of the system. An extended coastdown time, combined with the large thermal inertia of the pool system, may well allow reactivity feedback mechanisms to accommodate the unprotected transients. Such mechanisms are inherently provided by the thermal expansion of the support grid and thermal elongation of the control rod drivelines. With these factors properly combined, it may be feasible to design a pool system to survive loss of all electric power (and forced coolant flow) without scram and thus significantly further reduce the probability of a serious accident. (Relevant tests on EBR-II which demonstrated this capability is discussed later in this section.) This is much more difficult to achieve in a loop system because following a scram the primary pumps must coast down rapidly to avoid serious thermal shock to the reactor nozzles, primary piping and downstream components.

Diverse and redundant decay heat removal capacity can be supplied in either a loop or pool system. However, because of the large reactor vessel that contains both primary pumps and intermediate heat exchangers, diversity and redundancy can be achieved more easily and reliably in the pool system. Also, passive protective systems, including decay heat removal, can be provided with simpler engineering solutions. For example, the normal primary heat transport system in a pool design can also be used for in-vessel passive decay heat removal with proper location of dedicated heat removal systems, whereas the loop concept requires an alternative flow path with either check valves or changes in direction of coolant circulation.

\section{Economics}

Past design studies based on use of standard stainless steel materials of construction have concluded that the pool arrangement costs less than the loop arrangement, both in capital cost and in operating costs over the life of the plant. The primary reasons for reduced capital cost lie in the smaller reactor containment building and all of the associated reductions in plant size. Furthermore, the simplicity of the primary system and elimination of the additional inerted steellined cells housing various sodium heat transport system components contribute significantly to cost savings. The simplicity of the pool system can translate into a shortened construction schedule, which also contributes to capital cost savings. The operating and maintenance costs can be reduced because of reduced plant outage times and less radiation exposure to personnel performing inspection, testing, and maintenance work. However, the introduction of advanced technologies could impact the economics of pool and loop plant systems.

\section{Known and Demonstrated Technology}

The goal of this project was to develop a concept that, although it may not be cost competitive with today's advanced light water reactors, contained known and demonstrated technology, for example, dual rotatable plug with a straight pull fuel handling machine, 
mechanical centrifugal primary pumps, steam Rankine balance of plant, etc. Using demonstrated technologies allows for the project to understand the impact of adopting advanced technologies on not only overall plant cost, but also potential impacts on construction duration. As these advanced technologies mature they can be included in a future fast reactor projects with little risk to the overall project success. Thus, the ABR will serve as a reference plant for conducting tradeoffs and for measuring the degree of impact of innovative designs and features.

Even though the pool design described in this report is of a preliminary nature and incomplete, the attractiveness of the design is apparent. In essence, it maximizes the advantages of the pool concept identified in previous design studies by unique design innovations. Some of the known and demonstrated technologies adopted for this reference concept include:

- A vertical redan which reduces the load on the support structure, eliminates complex pump and IHX penetrations, and increases the volume of cold sodium in the primary vessel;

- A passive cooling system to keep the reactor vessel wall at a low and uniform temperature thus reducing costs, enhancing integrity of the primary coolant boundary and simplifying the design;

- Elimination of valves from the primary sodium piping system to improve reliability and reduce cost;

- A reactor support grid incorporating individual inlet modules for reactor assemblies and simple hydraulic hold-down providing flexibility and reducing vertical space requirements;

- Shutdown heat removal system within the reactor vessel which enhances safety and reduces the cost of the secondary system.

- Seismic isolation of the 'nuclear island' so that the need for expensive design features to accommodate large earthquakes is reduced and limitations on the seismicity of candidate sites can be relaxed.

Again, the main purpose for this ABR reference plant is to create a fast reactor concept using essentially known technology that can then be used to evaluate the impact of innovative technology and plant concepts on the overall plant layout, commodities, and performance. This ABR reference plant described in this report is a starting point for understanding the impacts of advanced technologies and plant concepts.

The reference ABR concept was developed based on the key plant parameters summarized in Table I.2-1. Detailed plant design descriptions are presented in Part II and supporting design analyses are presented in Part III. 
Table I.2- 1 Summary of Key Design Parameters

\begin{tabular}{|ll|}
\hline Design Parameter & Value \\
Reactor Power & $1000 \mathrm{MWth}$ \\
Primary Heat Transport System & \\
Reactor Outlet Temperature & $510^{\circ} \mathrm{C} / 950^{\circ} \mathrm{F}$ \\
Reactor Inlet Temperature & $380^{\circ} \mathrm{C} / 716^{\circ} \mathrm{F}$ \\
Reactor $\Delta \mathrm{T}$ & $130^{\circ} \mathrm{C} / 234^{\circ} \mathrm{F}$ \\
Number of Primary Pumps & 4 \\
Number of Intermediate Heat Exchangers & 4 \\
Intermediate Heat Transport System & \\
Hot Leg Temperature & $488^{\circ} \mathrm{C} / 910.4^{\circ} \mathrm{F}$ \\
Cold Leg Temperature & $333^{\circ} \mathrm{C} / 631.4^{\circ} \mathrm{F}$ \\
\hline
\end{tabular}

\section{I.3 Safety Design Approach}

The safety goals in nuclear power reactor design and operation are to assure the health and safety of the public, to protect the plant operating staff from harm, and to prevent plant damage. Traditionally, these goals have been fulfilled by an approach that 1) minimizes risk by maximizing safety margins in design and operation, 2) reduces the likelihood of potentially harmful events by providing safety systems to deal with anticipated events, and 3) provides additional design features to mitigate the harmful consequences of low probability events. This approach is usually identified as "defense- in-depth.”

The basic principle of "defense-in-depth" is to provide multiple levels of protection against an accidental release of radioactive material. One part of defense-in-depth is physical barriers, like the multiple barriers to release of radioactivity provided by the fuel cladding, the primary coolant system boundary and the reactor containment building. Generally, active or passive safety systems are provided to protect the physical barriers. These include the reactor shutdown systems and the reactor cooling systems. Inherent characteristics of the design, such as negative reactivity feedback and long flow coastdown, may provide an additional level of protection. Emergency planning provides an additional layer of defense-in-depth, should the other barriers be threatened. However, in all instances, the "defense-in-depth" strategy depends on the independence of the protective measures, so that no single event can breech more than one protective level.

The ABR safety design approach implements the "defense-in-depth” strategy by adopting the traditional three levels of safety. In addition, the ABR design features have been selected to provide significant safety margin enhancements by inherent passive safety responses to upset conditions and equipment failures.

At the first level, the ABR is designed to operate with a high level of reliability, so that accident initiators are prevented from occurring. The first level of safety is assured in part by selection of fuel, cladding, coolant, and structural materials that are stable and compatible, and by providing large margins between normal operating conditions and limiting failure conditions. Next, the first level of safety is assured by adopting an arrangement of components that allows monitoring, inspection, and testing for performance changes or degradation. Finally, the ABR 
design provides for repair and replacement of components necessary to assure that safety margins are not degraded.

The selection of liquid sodium coolant and a pool-type primary system arrangement provides a highly reliable reactor system with large operational safety margins. The coolant thermophysical properties provide superior heat removal and transport characteristics at low operating pressure with a large temperature margin to boiling. The pool-type primary system confines all significantly radioactive materials within a single vessel, allows for easy removal and replacement of components as well as shutdown heat removal by natural circulation. In addition, the adoption of metal fuel (as one concept) -which operates at a relatively low temperature, well below the coolant boiling point, provides additional safety margin for liquid metal reactor designs due to its high thermal conductivity.

At the second level of safety, the ABR is designed to provide protection in the event of equipment failure or operating error. This level of protection is provided by engineered safety systems for reactor shutdown, reactor heat removal, and emergency power. Each of these safetygrade back-up systems functions in the event of failure in the corresponding operating system, and are subjected to continuous monitoring and periodic testing and inspection.

The ABR design provides an independently powered and instrumented secondary reactor shutdown system that operates automatically to reduce reactor power rapidly in the event that the primary shutdown system fails. For shutdown cooling, the ABR design includes a safety-grade emergency heat removal system, independent from the normal heat removal system and capable of removing residual decay heat by natural circulation. In addition to the normal off-site power supply, the ABR is equipped with a second independent off-site power connection. The two offsite power connections are supplemented by a safety-grade on-site emergency power supply.

The third level of safety provides additional protection of the public health and safety in an extremely unlikely event that is not expected to occur in the life of the plant, or which was not foreseen at the time the plant was designed and constructed.

In the ABR design, the third level 3 protections for cooling assurance and containment of radioactivity is provided by the reactor guard vessel and the reactor containment building. The guard vessel is designed to hold primary coolant in the event of a leak in the reactor vessel. The guard vessel assures that the reactor core remains covered with sodium and cooled by the emergency heat removal system, even if the reactor vessel fails. If primary coolant leaks and oxidizes in the reactor building air atmosphere, or if failures of the cladding and the primary system barriers lead to release of gaseous fission products, the reactor containment building provides a final low-leakage barrier to release of radioactivity to the environment.

The three levels of safety taken together form the safety design basis for ABR. For the purposes of subsequent safety design development, qualification, and documentation, it is customary during the pre-conceptual design phase to identify general design criteria that collectively serve as the basis for safety assessment of the design. A preliminary cross reference and evaluation of existing general design criteria from 10CFR Part 50 Appendix A, from ANSI/ANS Standard 54.1, and from DOE Order 5480.30 are included in Appendix A.

The normal process of safety assessment of a design considers a spectrum of design basis accidents (DBAs) as tests of the various safety systems. These DBAs generally assume single failures. Accidents within the design basis must be accommodated by the design and shown to present risks to the public that are within regulatory standards. Beyond the design basis, there 
exists a class of accidents of such low probability that they have been termed "hypothetical." These events involve multiple failures of safety grade systems, and usually are considered to have a frequency of less than 10-6 per reactor year. Because of the potentially severe consequences of accidents in this class, they have received significant regulatory scrutiny in prior sodium-cooled fast reactor licensing reviews for the purpose of characterizing thermal and structural safety margins beyond the design basis.

Three beyond-design-basis accident (BDBA) sequences, each involving failure of both reactor scram systems, have received attention in past licensing safety assessments. In the unprotected loss-of-flow (ULOF) sequence, it is assumed that power is lost to all primary and secondary coolant pumps and the reactor scram systems fail to activate. In the unprotected transient overpower (UTOP) sequence, it is assumed that one or more inserted control rods are withdrawn, and the reactor scram systems fail to operate. In the unprotected loss-of-heat-sink (LOHS) accident, it is assumed that heat removal through the power conversion system is lost, and the reactor scram systems do not activate. Taken collectively, these three accident initiators encompass all the ways that an operating reactor can be perturbed, i.e. by a change in coolant flow, by a change in reactivity, or by a change in coolant inlet temperature.

The reference ABR design can be made capable of accommodating these beyond design basis accident initiators without producing high temperatures and conditions that might lead to a severe accident, such as coolant boiling, cladding failures, or fuel melting. The inherent neutronic, hydraulic, and thermal performance characteristics of the ABR design provide selfprotection in beyond-design-basis sequences to limit accident consequences without activation of engineered systems or operator actions. This characteristic has been termed 'inherent passive safety.

The efficacy of such passive safety was demonstrated through two landmark tests conducted on the Experimental Breeder Reactor-II (EBR-II), namely loss-of-flow without scram and lossof-heat-sink without scram tests. With the automated safety systems disabled, the two most demanding accident initiating events were deliberately induced with the reactor at full power, first one then the other. Each time the reactor simply coasted to a safe low power state without any damage at all to the fuel or any reactor component. These tests proved conclusively that passive safety design is achievable for metallic fueled fast reactors with sodium cooling.

Within the overall safety framework for ABR, passive safety serves to provide additional margins for public protection in the event of very low probability events whose frequency of occurrence is lower than the normal threshold for deterministic assessment. The ABR passive safety performance characteristic assures that no abnormal radioactivity releases will occur in the event of beyond-design-basis accidents, and that all of the multiple defense-in-depth barriers (fuel cladding, reactor vessel, containment building) for public protection will remain intact, just as for design basis accidents. The passive safety performance of ABR eliminates the potential for severe accident consequences in very low frequency, beyond-design-basis sequences. Consequently, for ABR, beyond-design-basis accidents need to be considered only in the context of probabilistic risk assessments.

Security must now be considered as an integral part of the design. The inherent and passive safety features of the ABR offer a high level of protection against malevolent events, as well as against accidents. Since the inherent and passive features do not rely on operator action, external power or functioning of active components, they remove these potential vulnerabilities. In 
addition, the location of the reactor vessel, the core, and the primary heat transport system below grade within a strong containment structure provides protection against external threats.

\section{Implications on Licensing Approach}

Historically, the safety assessment of reactor designs has been based on a deterministic approach, in which a set of postulated initiating events are defined and the consequences of these events are determined by analysis. The events to be considered are selected to represent challenges to the reactor's control and safety systems, based on judgment, experience and regulatory requirements. The probability of an initiating event occurring is evaluated only qualitatively. USNRC licensing practice using the deterministic approach is highly developed for light-water power reactors and is codified in 10CFR Part 50. The general design criteria found in Appendix A of Part 50 and the related body of regulatory guidance have been applied successfully to licensing of the current fleet of LWRs. They were used with modification in licensing of the Fast Flux Test Facility and the Clinch River Breeder Reactor Project. However, it is recognized that use of criteria designed for LWR technology for new, innovative reactor designs is not efficient, nor does it lead to a stable and predictable regulatory process. In addition, recent trends in safety assessment and licensing have led to an increased emphasis on risk informed decision-making, with a consequent emphasis on the use of probabilistic risk assessment throughout design, safety assessment, licensing and operation. All operating nuclear power plants are now required to have a probabilistic risk analysis (PRA) including both internal and external events, and probabilistic insights are now used in some aspects of operation and regulation.

The USNRC is now developing a risk-informed, performance based alternative to 10CFR Part 50 to be used in licensing of future nuclear power plants [1]. The framework for this alternative approach is based on the NRC's Safety Goals Policy and fundamental safety principles such as defense-in-depth and safety margins. It combines probabilistic and deterministic elements. It is technology-independent, with technology-specific requirements for particular designs.

Under the new regulatory framework, a probabilistic risk assessment would be an integral part of the design process and safety assessment, as well as having a fundamental role in the licensing process. Deterministic criteria and multiple lines of defense against undue radioactive release would continue to be required. Under this approach, a probabilistic analysis would be used to establish the event sequences to be considered in the licensing process and to classify equipment as to its safety significance. The selected events, called Licensing Basis Events (LBEs) would be analyzed deterministically to demonstrate the conservatism of the probabilistic analysis. The allowable consequences of an event would be matched to its frequency quantitatively.

As design work proceeds beyond the pre-conceptual stage, use of probabilistic risk analysis will be central to the design, especially to demonstrating the effectiveness of the inherent and passive safety features of the ABR. It will also allow a quantitative selection of LBEs for deterministic analysis to show compliance with the NRC's Safety Goal Policy. In previous licensing of sodium-cooled fast reactors, a great deal of attention was focused on beyond-designbasis events leading to severe accidents. Probabilistic analysis affords the opportunity to show quantitatively that such events have a frequency below the lower limit for consideration as LBEs (the current proposal is a frequency less than 10-7 per reactor-year); nevertheless, defense-indepth considerations will likely require mitigation features such as low-leakage containment. 
At the present time, the approach to be used for licensing of the ABR is not clear, given the project schedule and the likely time frame for introduction of the risk-informed, performancebased alternative. However, it is clear that an approach to design and safety assessment using both probabilistic and deterministic methods will be needed. In the longer term, such an approach will surely be needed for ABR licensing, and the ABR licensing offers the opportunity to develop the methods and data needed to support the ABR in the future.

\section{Reference}

1. NUREG-1860, Framework for Development of a Risk-Informed, Performance-Based Alternative to 10 CFR Part 50, Working Draft, July 2006. 


\section{PART II \\ PLANT DESIGN DESCRIPTION}

\section{II.1 Reactor System}

\section{II.1.1 Reactor Core}

A reference Advanced Burner Reactor (ABR) core concept of $1000 \mathrm{MWt}$ power rating was developed for the study on the future fast reactor design options. A compact core for a one-year cycle length was developed by trade-off of the TRU conversion ratio and burnup reactivity loss. A ternary metal fuel form of U-TRU-Zr and a mixed oxide form of $\mathrm{UO}_{2}-\mathrm{TRUO}_{2}$ were assumed for the metal and oxide cores, respectively. Low-swelling stainless steel (HT9) cladding was selected as structural material (cladding and duct). In order to allow the interchange of metal and oxide fuel assemblies, it was decided to use the same radial core layout and assembly size for both metal and oxide fuel core concepts.

For both metal and oxide cores, thermal-hydraulic and materials-related design constraints were imposed on selection of design parameters. For the metal fuel, an as-built smeared density of $75 \%$ was assumed to allow free fuel swelling of approximately 33\%, at which point porosity becomes largely interconnected and open to the outside of the fuel, releasing a large fraction of fission gas to the upper fission gas plenum [1]. A smeared fuel density of $85 \%$ theoretical density (TD) was assumed for the oxide fuel for accommodating fuel swelling and to avoid the significant temperature drop from the fuel surface to the cladding [2]. The peak fast fluence limit of HT9 was assumed to be $\sim 4 \times 10^{23} \mathrm{n} / \mathrm{cm}^{2}$, based on the irradiation data obtained in FFTF that showed no HT9 duct elongation or cladding breach after a fast fluence of $3.9 \times 10^{23} \mathrm{n} / \mathrm{cm}^{2}$ [3]. The fuel centerline temperature of both metal and oxide fuels was limited by the fuel melting temperature. For the metal fuel, the peak fuel-cladding interface temperature was limited by $650^{\circ} \mathrm{C}$, which was taken for the maximum interface temperature of EBR-II Mark-V fuel based on the experimental result of the Fuel Behavior Test Apparatus [4]. To minimize the thermal stress damage on the outlet plenum structure, the allowable coolant temperature differential at the outlet of the subassemblies was assumed to be $44{ }^{\circ} \mathrm{C}[5,6]$. Finally, a burnup reactivity loss of $\sim 3.5 \% \Delta \mathrm{k}$ was targeted in order to achieve a sufficient shutdown margin with a reasonable number of primary control assemblies.

Two equilibrium core concepts were developed for both metal and oxide cores: startup and TRU recycled cores. Weapons grade plutonium (WG-Pu) was assumed as the TRU feed for the start-up core without recycling of the ABR spent fuel. For the recycled equilibrium core, the TRU recovered from the ABR spent fuel was used as the primary TRU feed and the TRU recovered from the LWR spent fuel (LWR-SF) was used as the makeup TRU feed. The design descriptions and performance characteristics of the reference core concepts are presented in this section. The impact of alternative core concepts targeting low and high TRU conversion ratios are discussed in Section III.6. 


\section{II.1.1.1 Core and Assembly Design Descriptions}

The 1000 MWt reference ABR core concept was developed using ternary metal alloy (UTRU-10Zr) and mixed oxide $\left(\mathrm{UO}_{2}+\mathrm{TRUO}_{2}\right)$ fuels. Figure II.1-1 shows the radial layout of the reference core, which consists of 180 drivers, 114 reflectors, 66 radial shields, and 15 primary and four secondary control assemblies. The core is divided into inner and outer core zones, which are composed of 78 and 102 fuel assemblies, respectively. Two independent safety-grade reactivity control systems are used. The primary system consists of three control assemblies in the fourth row and 12 control assemblies in the seventh row, and the secondary system contains four control assemblies located at the core center and in the fourth row.

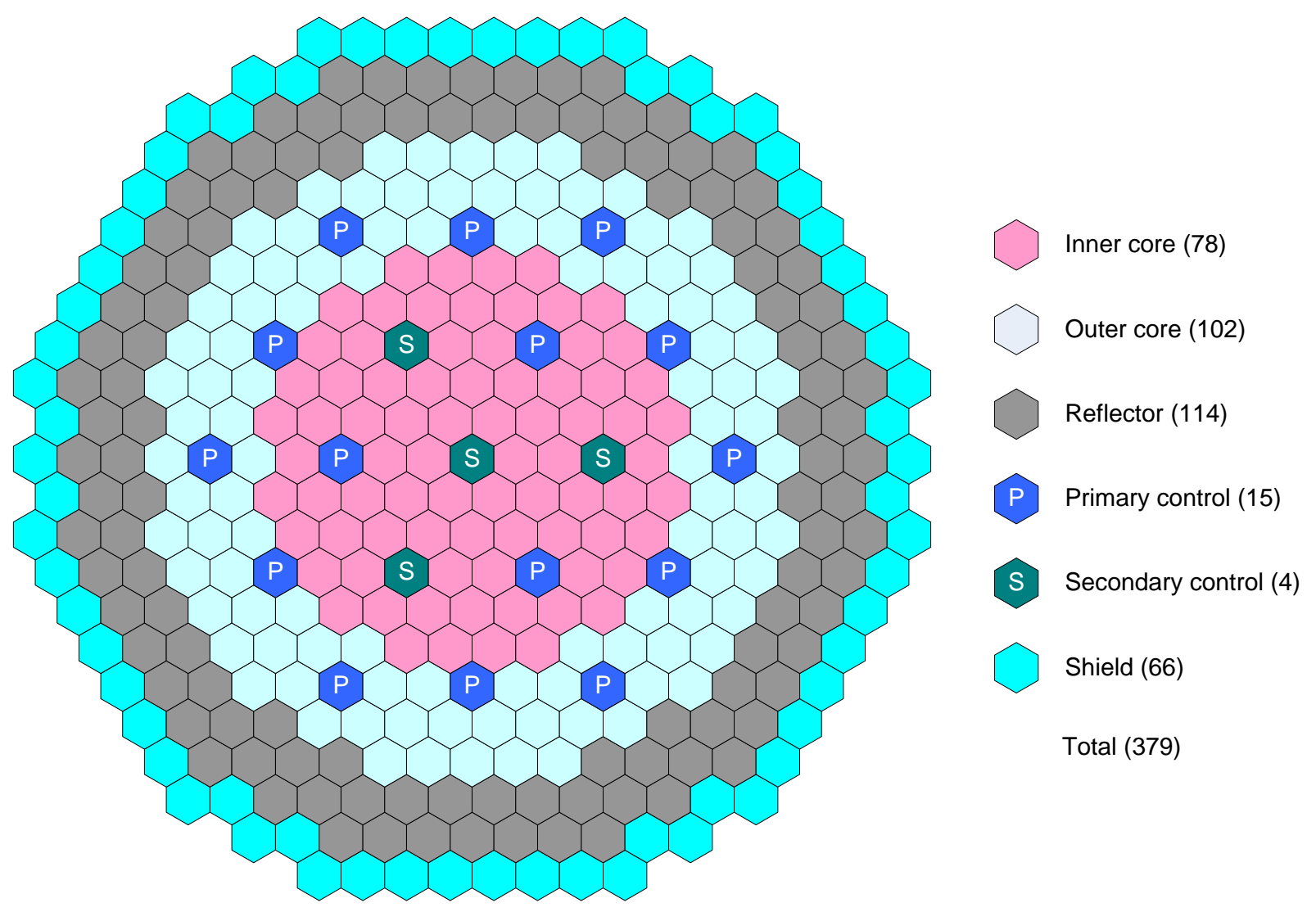

Figure II.1-1 Radial Core Layout of Reference 1000 MWt ABR Concept

Table II.1-1 provides the isotopic fractions of weapons grade plutonium and LWR spent fuel TRU. A $50 \mathrm{MWd} / \mathrm{kg}$ burnup and a 10-year post-irradiation cooling time were assumed for determining the LWR-SF TRU composition. The key assembly design parameters of the metal and oxide fuel assemblies are compared in Table II.1-2, and the schematics of oxide and metal fuel assembly designs are provided in Figure II.1-2. The fuel, reflector and shield assemblies use the same structural components and have an overall length of $477.5 \mathrm{~cm}$ including the nosepiece and handling socket. 
Table II.1-1 Isotopic Fraction of TRU Feeds (\%)

\begin{tabular}{|l|l|l|}
\hline & WG-Pu & LWR-SF \\
\hline Np-237 & & 4.72 \\
\hline Pu-238 & 0.01 & 2.18 \\
Pu-239 & 93.81 & 47.34 \\
Pu-240 & 5.81 & 22.82 \\
Pu-241 & 0.35 & 8.42 \\
Pu-242 & 0.02 & 6.84 \\
\hline Am-241 & & 5.61 \\
Am-242m & & 0.01 \\
Am-243 & & 1.56 \\
\hline Cm-243 & & 0.00 \\
Cm-244 & & 0.46 \\
Cm-245 & & 0.04 \\
\hline
\end{tabular}

For the metal core, the fuel assembly contains 271 fuel pins arranged in a triangular pitch array. The fuel pin diameter and cladding thickness are $7.55 \mathrm{~mm}$ and $0.56 \mathrm{~mm}$, respectively. Fuel pins are made of sealed cladding containing a lower shield, an active core and a gas plenum from the bottom of the pin. The fuel pin is helically wrapped with wire to maintain the pin spacing so that the coolant can flow freely through the pin bundle. In the metal fuel assembly, the active core height is $81.3 \mathrm{~cm}$, and the fission gas plenum height is $124.5 \mathrm{~cm}$. The smeared fuel density is $75 \%$, and sodium is filled as the initial thermal bond between the fuel column and the cladding. The overall length of metal fuel pin is $332.7 \mathrm{~cm}$, including the lower shield of 124.5 $\mathrm{cm}$.

For the oxide core, the oxide fuel assembly design is basically the same as the metal fuel assembly design except for the axial dimensions and the gap size between fuel and cladding. The reflector, shield and control assemblies are identical to those of the metal core. The active core height and fission gas plenum length are increased to $106.7 \mathrm{~cm}$ and $160.0 \mathrm{~cm}$, respectively, and the lower shield length is slightly reduced to $111.8 \mathrm{~cm}$. As a result, the overall pin length is increased to $381.0 \mathrm{~cm}$, which is about $15 \%$ longer than that of the metal core. To keep the overall assembly length of $477.5 \mathrm{~cm}$, the duct standoff length of the oxide fuel assembly is reduced (see Figure II.1-2). The gap between fuel pellet and cladding is $0.008 \mathrm{~cm}$, yielding a fuel smeared density of $85 \%$ TD with the assumed fabrication density of $89.5 \%$ TD. The fuel volume fraction of the oxide fuel is about $7.8 \%$ larger than that of the metal fuel assembly because of reduced gap between fuel and cladding.

The control assemblies consist of an absorber bundle contained in a duct. The absorber bundle is a closely packed array of seven tubes containing compacted boron carbide pellets. To minimize the single assembly reactivity fault, two different B-10 enrichments were used for the primary control assemblies: natural boron (B-10 enrichment of 19.9 atom \%) for the fourth row control assemblies and 60\% enriched boron for the seventh row control assemblies. For the secondary control assemblies, natural boron was used. 
Table II.1-2 Assembly Design Parameters of Reference ABR Cores

Assembly data

- Number of pins

- Assembly pitch, cm

Fuel assembly

Oxide

Metal

Reflector

Shield

Control

- Inter-assembly gap, cm

271

16.142

16.142

91

16.142

19

16.142

7

0.432

0.432

0.432

15.710

15.710

0.432

16.142

0.394

0.394

- Gap between duct and interior duct, cm

-

- Interior duct thickness, cm

- Interior duct inside flat-to-flat distance, cm

$-$

- Overall duct height, cm

477.52

$-$

0.394

15.710

0.432

0.394

15.710

-

Pin data

- Pin material and type

- Bond material

- Overall pin length, cm

- Active core height, $\mathrm{cm}$

- Pellet smeared density, \% TD

- Pellet diameter, $\mathrm{cm}$

- Cladding material

- Clad outer diameter, cm

- Pin pitch-to-diameter ratio

- Cladding thickness, $\mathrm{cm}$

MOX

$\mathrm{He}$

381.0

106.7

85.0

0.627

HT9

0.755

1.180

0.056

0.131

$-$

- Wire wrap diameter, cm

37.0

- Fuel or Absorber

- Structure

477.52

$-$

$-$

$-$

0.394

0.400

$-$

0.394

$-$

13.334

477.52

477.52

477.52

- Coolant

\section{HT9}

$\mathrm{Na}$

332.7

81.3

75.0

0.557

HT9

0.755

1.180

0.056

0.131

29.2

9.8

25.7

35.3 c)

c) $381.0 / 332.7$

-

-

1.54

-

$-$

1.001

-

-

$-$

84.5

15.5 a) $\mathrm{B}_{4} \mathrm{C}$

$\mathrm{He}$

381.0/332.7

81.0

2.553

HT9

3.337

1.001

0.250

-

43.1

10.1

29.7

17.1 b) $\mathrm{B}_{4} \mathrm{C}$

He

112.3/86.3

85.0

4.193

HT9

4.688

1.029

0.070

0.133

42.8

7.6

20.8

28.8

a) Natural boron was used.

b) Natural and $60 \%$ enriched boron was used for $4^{\text {th }}$ and $7^{\text {th }}$ row primary control assemblies, respectively.

c) Data for oxide and metal cores 


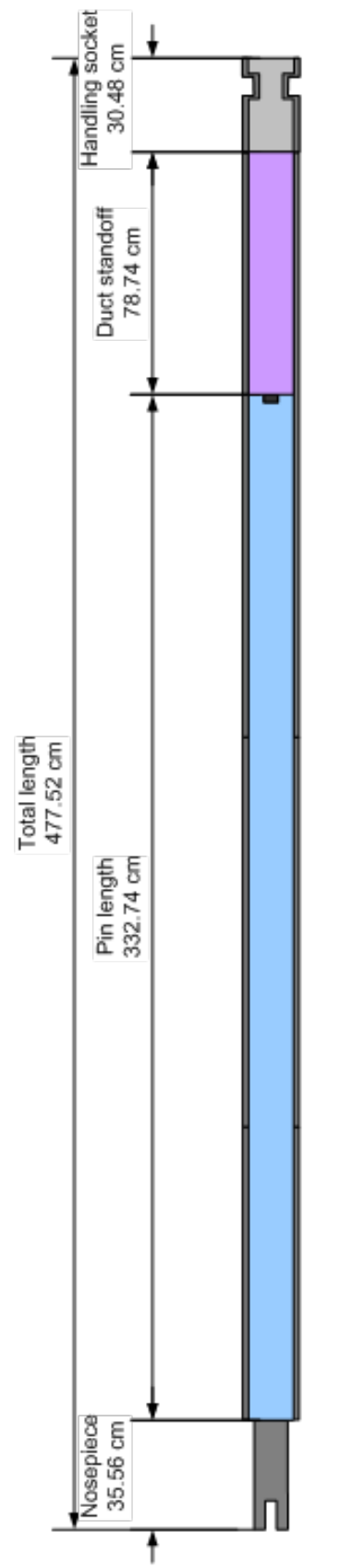

Assembly Duct
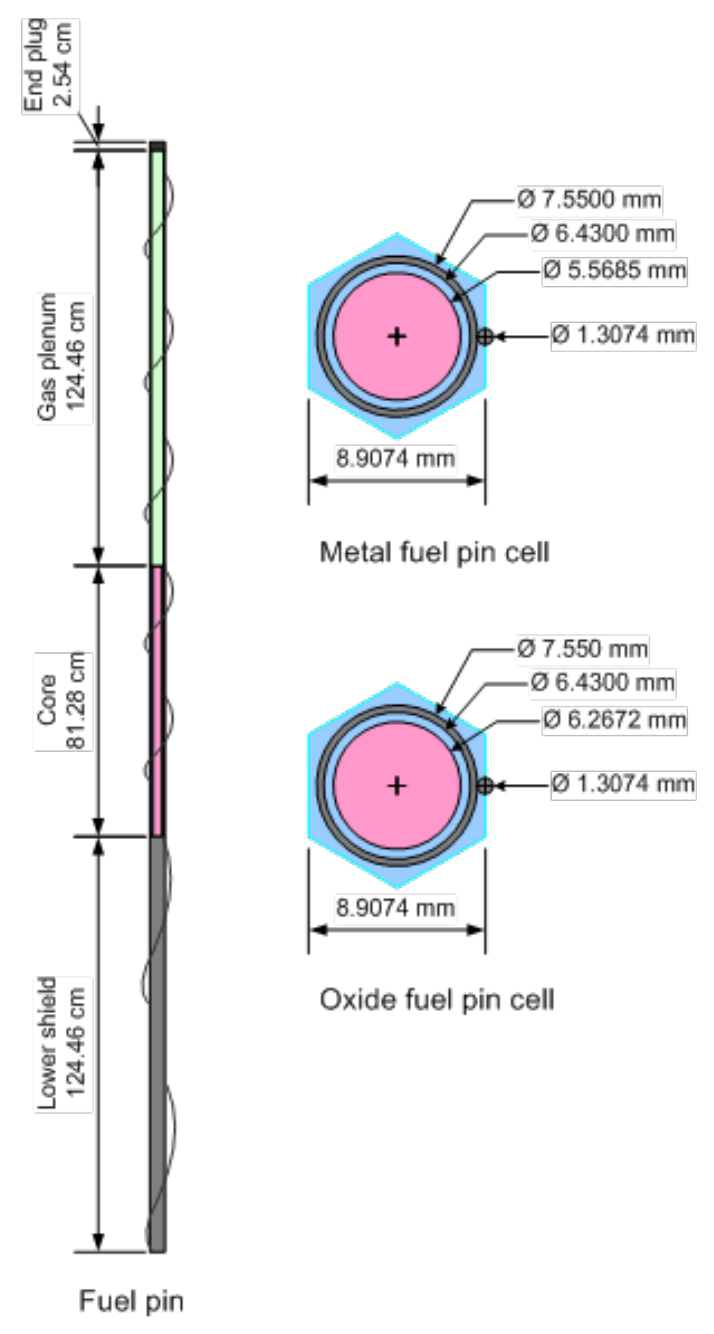

Metal fuel pin cell

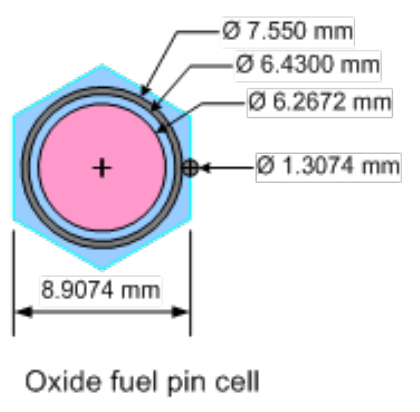

Metal Fuel

แี

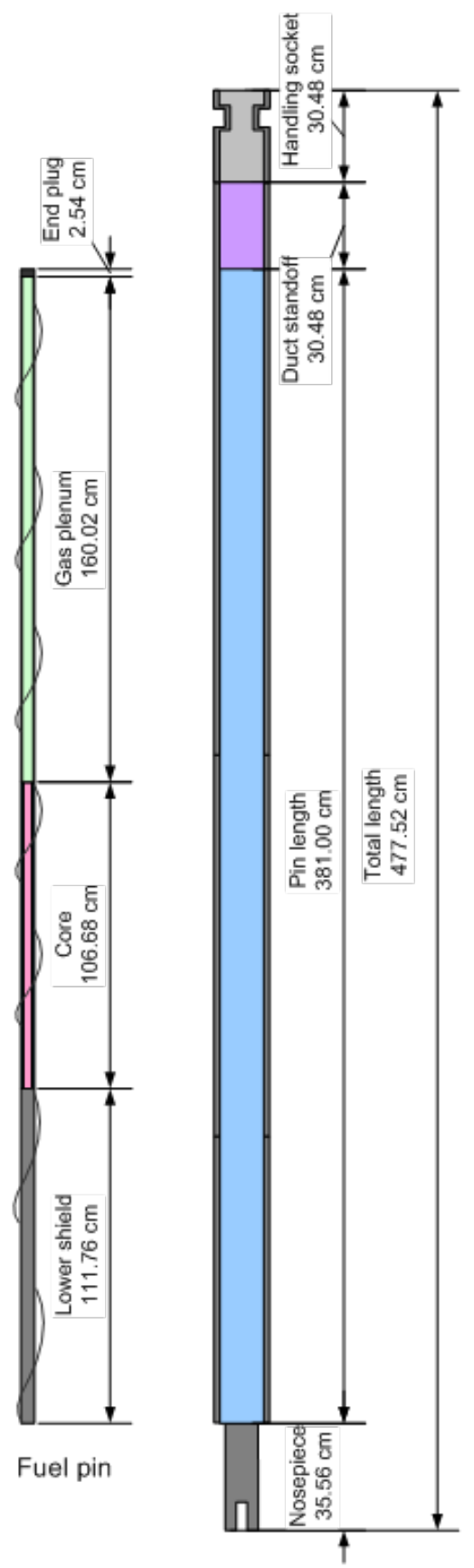

Fuel pin

Assembly Duct

Oxide Fuel

Figure II.1-2 Schematics of Metal and Oxide Fuel Assemblies of Reference Cores 
The reflector assembly contains 91 solid HT9 pins arranged in a triangular pitch array. The HT9 pin volume fraction is $75.3 \%$ and the duct volume fraction is $9.2 \%$, yielding a total HT9 volume fraction of $84.5 \%$. The shield assembly is composed of HT9 cladding and $\mathrm{B}_{4} \mathrm{C}$ absorber. It consists of 19 thick HT9 tubes containing boron carbide pellets. Natural boron is used with a smeared $\mathrm{B}_{4} \mathrm{C}$ pellet density of $81 \%$. The resulting $\mathrm{B}_{4} \mathrm{C}$ and $\mathrm{HT} 9$ volume fractions are $43.1 \%$ and $29.7 \%$, respectively.

\section{II.1.1.2 Equilibrium Cycle Performance Characteristics}

Fuel cycle analyses were performed with the DIF3D/REBUS-3 code system $[7,8]$. Regiondependent 21-group neutron cross section sets generated for metal and oxide fueled cores with the ETOE-2/MC ${ }^{2}-2 / S D X$ code system [9-11] based on ENDF/B-V.2 were used. Using 3dimensional hexagonal-z geometry models, equilibrium cycle analyses were performed with scattered loading. The fuel cycle length was fixed to one year with a capacity factor of $90 \%$. Fuel assembly design parameters were iteratively determined to meet the targeted neutronics performances and the thermal design criteria. Material thermal expansion at operating conditions was modeled by adjusting the hexagonal pitch, axial meshes, and the fuel and structure volume fractions appropriately. Irradiation swelling of metal fuel was considered, and the bond sodium was displaced into the lower part of fission gas plenum. For the oxide fuel, however, the axial expansion caused by irradiation swelling was ignored. Block nuclide depletion was performed by dividing each fuel assembly into five axial depletion zones. For flux calculations, the hexagonalz nodal diffusion theory option of DIF3D [12] was mainly employed. The required TRU enrichment (i.e., TRU fraction in heavy metal) was determined such that the core is critical at the end of equilibrium cycle. An enrichment zoning strategy was employed to flatten the power distribution. The discharge burnup was determined by adjusting the number of batches (i.e., fuel residence time) such that the peak fast neutron fluence is within the fast neutron fluence limit of HT9 cladding. The main core performance parameters of metal and oxide cores are compared in Table II.1-3 and the core heavy metal inventory and mass flow rates are provided in Table II.1-4.

In the metal core, the heavy metal inventory at BOEC is about $13.1 \mathrm{MT}$, which yields a specific power density of $73.2 \mathrm{~kW} / \mathrm{kg}$. The average discharge burnup is $93 \mathrm{MWd} / \mathrm{kg}$ and the peak discharge fast fluence is $4.09 \times 10^{23} \mathrm{n} / \mathrm{cm}^{2}$. The average TRU enrichment is $15.5 \%$ for the startup, and it is increased to $22.1 \%$ for the recycled core because of a significantly lower fissile fraction in the LWR-SF TRU feed relative to the WG-Pu. TRU conversion ratios are 0.81 for the startup core and 0.73 for the recycled core. The resulting TRU consumption rates are $55.5 \mathrm{~kg} / \mathrm{year}$ and $81.6 \mathrm{~kg} /$ year for the startup and recycled cores, respectively. The burnup reactivity loss is 3.6\% $\Delta \mathrm{k}$ for the startup core, and $2.2 \% \Delta \mathrm{k}$ for the recycled core.

Compared to the metal core, the as-fabricated core volume of the oxide core is larger by $31 \%$ because of the increased active core height. Thus, the core average power density is reduced to $231 \mathrm{~W} / \mathrm{cm}^{3}$ from $303 \mathrm{~W} / \mathrm{cm}^{3}$. The heavy metal loading increases slightly to $13.36 \mathrm{MT}$, which decreases the specific power density to $71.2 \mathrm{~kW} / \mathrm{kg}$. The increased number of batches increases the average discharge burnup to $111 \mathrm{MWd} / \mathrm{kg}$. As indicated by the fast flux fraction, the neutron spectrum is significantly softer than that of the metal core. The average flux level of the oxide core is lower by $18 \%$ because of reduced power density. As a result, the number of batches could be increased to five within the fast fluence limit of the HT9 cladding material. The peak discharge fast neutron fluence is less than $3.5 \times 10^{23} \mathrm{n} / \mathrm{cm}^{2}$. 


\section{Table II.1-3 Main Core Performance Parameters}

\begin{tabular}{|c|c|c|c|c|}
\hline & Metal Cor & & Oxide Cor & \\
\hline & Startup & Recycle & Startup & Recycle \\
\hline TRU Feed & WG-Pu & $\begin{array}{l}\text { LWR- } \\
\text { SNF }\end{array}$ & WG-Pu & $\begin{array}{l}\text { LWR- } \\
\text { SNF }\end{array}$ \\
\hline Cycle length, month & 12 & 12 & 12 & 12 \\
\hline Number of batches & 4 & 4 & 5 & 5 \\
\hline Average TRU enrichment, \% & 15.5 & 22.1 & 18.2 & 27.1 \\
\hline Fissile/TRU conversion ratio & $0.71 / 0.81$ & $0.84 / 0.73$ & $0.70 / 0.83$ & $0.85 / 0.71$ \\
\hline HM/TRU inventory at BOEC, MT & $13.1 / 2.0$ & $13.2 / 2.9$ & $13.4 / 2.4$ & $13.4 / 3.6$ \\
\hline Discharge burnup (ave/peak), MWd/kg & $93 / 136$ & $93 / 138$ & $111 / 168$ & $111 / 172$ \\
\hline Specific power density (MW/MT) & 73.2 & 73.2 & 71.2 & 71.2 \\
\hline Average core power density at cold, kW/l & 303 & 303 & 231 & 231 \\
\hline Peak discharge fast fluence, $10^{23} / \mathrm{cm}^{2}$ & 4.07 & 4.09 & 3.42 & 3.45 \\
\hline Burnup reactivity loss, $\% \Delta \mathrm{k}$ & 3.6 & 2.2 & 3.5 & 2.0 \\
\hline Power peaking factor (BOEC/EOEC) & $1.44 / 1.39$ & $1.46 / 1.43$ & $1.52 / 1.48$ & $1.56 / 1.52$ \\
\hline Average linear power, $\mathrm{kW} / \mathrm{m}$ & 23.3 & 23.3 & 18.8 & 18.8 \\
\hline Peak linear power density, $\mathrm{kW} / \mathrm{m}$ & 37.7 & 37.2 & 33.2 & 32.6 \\
\hline Core average flux, $10^{15} / \mathrm{cm}^{2}$-sec & 3.23 & 3.23 & 2.68 & 2.65 \\
\hline Fast flux fraction & 0.67 & 0.68 & 0.58 & 0.58 \\
\hline
\end{tabular}

The softer spectrum of the oxide core decreases the fission neutron yield per neutron absorption ( $\eta$ ) for fissile isotopes and the threshold fission of fertile isotopes, As a result, the required TRU fraction to achieve the targeted one-year cycle length is increased; it is increased to $18.2 \%$ from $15.5 \%$ for the startup core, and to $27.1 \%$ from $22.1 \%$ for the recycled core. The burnup reactivity losses of the oxide cores are comparable to those of the metal cores as targeted. The combined effects of the increased TRU fraction and the softer neutron spectrum result in similar fissile and TRU conversion ratios. Relative to the metal core, TRU conversion ratio is slightly higher in the startup core, but slightly lower in the recycled core. The small difference in TRU conversion ratio yield small variation in the TRU consumption rates. 
Table II.1- 4 Heavy Metal Inventories and Mass Flows

\begin{tabular}{|c|c|c|c|c|c|c|c|c|}
\hline & \multicolumn{4}{|c|}{ Metal Core } & \multicolumn{4}{|c|}{ Oxide Core } \\
\hline \multicolumn{9}{|c|}{ Inventory (kg) } \\
\hline & BOEC & EOEC & BOEC & EOEC & BOEC & EOEC & BOEC & EOEC \\
\hline HM & 13145.1 & 12807.5 & 13150.6 & 12812.6 & 13359.9 & 13027.6 & 13371.9 & 13039.7 \\
\hline TRU & 2025.6 & 1967.6 & 2896.7 & 2815.1 & 2445.7 & 2396.3 & 3629.6 & 3543.1 \\
\hline \multicolumn{9}{|c|}{ Mass Flow (kg/year) } \\
\hline & charge & discharge & charge & discharge & charge & discharge & charge & discharge \\
\hline $\mathrm{U}$ & 2884.7 & 2602.9 & 2660.3 & 2404.1 & 2297.9 & 2015.3 & 2048.3 & 1802.8 \\
\hline $\mathrm{Np}$ & 0.0 & 1.2 & 12.0 & 8.1 & 0.0 & 0.9 & 10.1 & 6.0 \\
\hline Pu-238 & 0.1 & 0.4 & 20.3 & 17.9 & 0.1 & 0.4 & 21.8 & 19.2 \\
\hline Pu-239 & 497.8 & 396.3 & 360.4 & 321.7 & 479.2 & 362.8 & 325.7 & 284.6 \\
\hline Pu-240 & 30.8 & 70.3 & 219.5 & 200.3 & 29.7 & 86.9 & 238.0 & 217.5 \\
\hline Pu-241 & 1.9 & 5.8 & 35.3 & 30.5 & 1.8 & 8.6 & 41.8 & 37.0 \\
\hline Pu-242 & 0.1 & 0.6 & 51.4 & 45.8 & 0.1 & 1.0 & 57.8 & 51.8 \\
\hline Am & 0.0 & 0.5 & 41.8 & 33.9 & 0.0 & 0.9 & 45.9 & 37.2 \\
\hline $\mathrm{Cm}$ & 0.0 & 0.0 & 14.3 & 15.4 & 0.0 & 0.1 & 19.4 & 20.7 \\
\hline HM & 3415.3 & 3078.0 & 3415.3 & 3077.6 & 2808.8 & 2476.7 & 2808.9 & 2476.9 \\
\hline \multirow{2}{*}{ TRU } & 530.6 & 475.1 & 755.1 & 673.5 & 510.9 & 461.4 & 760.6 & 674.1 \\
\hline & -55.5 & & -81.6 & & -49.4 & & -86.5 & \\
\hline
\end{tabular}




\section{II.1.1.3 Kinetics Parameters and Reactivity Coefficients}

Reactivity coefficients and kinetics parameters were calculated for the core configurations at the beginning of equilibrium cycle (BOEC) and the end of equilibrium cycle (EOEC). The coolant, fuel, and structural material density coefficients and the coolant void worth were determined using the VARI3D perturbation code [13]. The first order perturbation theory option was used for density coefficients, while the exact perturbation theory option was employed for the coolant void worth. The effective delayed neutron fraction and prompt neutron lifetime were also calculated using the VARI3D code. The radial and axial expansion coefficients and the control assembly worth were determined by direct eigenvalue differences of the base and perturbed conditions using the DIF3D code.

In the metal core, the BOEC position of the primary control assemblies is $56.8 \mathrm{~cm}$ from the bottom of the active core for the startup core and $64.3 \mathrm{~cm}$ for the recycled core. The EOEC position of primary control assemblies are at the top of the active core. The critical positions of the primary control assemblies are higher in the oxide core than in the metal core, because of the increased active core height. For the oxide core, the BOEC positions of the primary control assemblies are $67.6 \mathrm{~cm}$ and $77.5 \mathrm{~cm}$ for the startup and recycled cores, respectively. The secondary control assemblies were positioned at the top of the active core all the time. Table II.15 compares the calculated kinetics parameters and reactivity coefficients.

In the metal core, the effective delayed neutron fraction $\left(\beta_{\mathrm{eff}}\right)$ is 0.0034 for the startup core and 0.0033 for the recycled core. The prompt neutron lifetime is about $0.37 \mu$ s for the startup core. For the recycled core, it is reduced to $0.33 \mu \mathrm{s}$ because of the increased TRU fraction. For both startup and recycled cores, the prompt neutron lifetime is slightly shorter at BOEC than at EOEC due to the increased neutron absorption in the primary control assemblies. The uniform radial expansion coefficient is around $-0.38 \$ /{ }^{\circ} \mathrm{C}$, and the axial expansion coefficient is -0.05 $\$ /{ }^{\circ} \mathrm{C}$. The sodium void worth and sodium density coefficient are positive because the positive spectrum hardening effect overwhelms the negative neutron leakage effect. The sodium void worth of the recycled core is more positive than that of the startup core because of the higher TRU fraction; i.e., the contribution of $\mathrm{Pu}-240$ and $\mathrm{Pu}-242$ fissions increases significantly.

Compared to the metal core, the effective delayed neutron fraction of the oxide core is slightly smaller ( $\sim 0.0031)$ because of the reduced threshold fission of U-238 due to softer neutron spectrum. The increased slowing down caused by scattering with oxygen increases the prompt neutron lifetime of the oxide core by $30 \%$. The radial expansion coefficient becomes less negative $\left(-0.31\right.$ cents $/{ }^{\circ} \mathrm{C}$ vs. -0.38 cents $/{ }^{\circ} \mathrm{C}$ ) because of increased active core height (i.e., larger core height-to-diameter ratio), which reduces the axial leakage resulting from the radial core expansion. The axial expansion coefficient is -0.05 cents $/{ }^{\circ} \mathrm{C}$, which is comparable to that of the metal core because the same radial core layout was used. The softer spectrum of the oxide core reduces the positive spectral hardening effect, but the increased active core height of the oxide core also reduces the negative axial leakage effect. The combined effects yield slightly less positive sodium void worth and density coefficient compared to the reference metal core. 
Table II.1-5 Kinetics Parameters and Reactivity Coefficients

\begin{tabular}{|c|c|c|c|c|c|c|c|c|}
\hline & \multicolumn{4}{|c|}{ Metal Core } & \multicolumn{4}{|c|}{ Oxide Core } \\
\hline & \multicolumn{2}{|l|}{ Startup } & \multicolumn{2}{|l|}{ Recycle } & \multicolumn{2}{|l|}{ Startup } & \multicolumn{2}{|l|}{ Recycle } \\
\hline & BOEC & EOEC & BOEC & EOEC & BOEC & EOEC & BOEC & EOEC \\
\hline Effective delayed neutron fraction & 0.00335 & 0.00334 & 0.00329 & 0.00326 & 0.00316 & 0.00315 & 0.00312 & 0.00310 \\
\hline Prompt neutron lifetime, $\mu \mathrm{s}$ & 0.36 & 0.38 & 0.32 & 0.33 & 0.48 & 0.50 & 0.41 & 0.42 \\
\hline Radial expansion coefficient, $\Phi /{ }^{\circ} \mathrm{C}$ & -0.39 & -0.38 & -0.38 & 0.38 & -0.32 & -0.31 & -0.31 & -0.30 \\
\hline Axial expansion coefficient, $\mathbb{\$} /{ }^{\circ} \mathrm{C}$ & -0.05 & -0.05 & -0.06 & -0.06 & -0.05 & -0.05 & -0.05 & -0.05 \\
\hline Fuel density coefficient, $\mathbb{\Phi} /{ }^{\circ} \mathrm{C}$ & -0.71 & -0.67 & -0.70 & -0.69 & -0.46 & -0.42 & -0.46 & -0.43 \\
\hline Structure density coefficient, $\mathbb{\Phi} /{ }^{\circ} \mathrm{C}$ & 0.06 & 0.06 & 0.07 & 0.07 & 0.07 & 0.07 & 0.08 & 0.09 \\
\hline Sodium void worth, $\$$ & 4.44 & 4.93 & 6.30 & 6.64 & 4.28 & 4.81 & 6.10 & 6.40 \\
\hline Sodium density coefficient, $\$ /{ }^{\circ} \mathrm{C}$ & 0.11 & 0.13 & 0.15 & 0.16 & 0.10 & 0.12 & 0.15 & 0.16 \\
\hline Doppler constant $^{\text {a) }}$, \$ & -0.70 & -0.71 & -0.56 & -0.57 & -1.54 & -1.55 & -1.14 & -1.15 \\
\hline Doppler coefficient, $\Phi /{ }^{\circ} \mathrm{C}$ & -0.13 & -0.13 & -0.10 & -0.10 & -0.16 & -0.16 & -0.12 & -0.12 \\
\hline Sodium voided Doppler coefficient , $₫ /{ }^{\circ} \mathrm{C}$ & -0.08 & -0.09 & -0.07 & -0.07 & -0.12 & -0.12 & -0.09 & -0.09 \\
\hline
\end{tabular}

a) Reactivity change associated the fuel temperature doubled up. 
Two sets of Doppler coefficients were calculated at flooded and voided sodium conditions. With the approximation that the Doppler coefficients are inversely proportional to the absolute fuel temperature, the "Doppler constant," $\mathrm{T}(\partial \rho / \partial \mathrm{T})$ was calculated by doubling the average fuel temperature, which was assumed to be $850 \mathrm{~K}$ for the metal fuel and $1300 \mathrm{~K}$ for the oxide core. Doppler coefficients in Table II.1-5 were determined by dividing the Doppler constant by the average fuel temperature. The softer neutron spectrum of the oxide core leads to Doppler effects that are about two times more negative than those of the metal core. Because of the higher fuel temperature, however, the Doppler coefficient of the oxide core is only $20 \%$ more negative than that of the metal core. For both the metal and oxide cores, the Doppler coefficient becomes less negative when the flowing sodium is voided due to the spectrum hardening.

\section{II.1.1.4 Orifice Zoning and Steady-State Thermal-hydraulic Performances}

Reactor orifice zoning and flow allocation were determined through detailed steady-state thermal-hydraulic analyses. Heating rates for thermal-hydraulic calculations were determined by coupled neutron and gamma heating calculations. Region-dependent 9-group neutron cross section set and 21-group gamma cross section set were used along with a gamma production matrix generated with NJOY [14] and SDX codes in 9 neutron groups and 21 gamma groups.

Neutron and gamma flux calculations were performed using the triangular-z finite difference option of the DIF3D code. Following the neutron flux calculation, the gamma source distribution was determined with the GAMSOR [15] code. Using this gamma source distribution, the gamma flux calculation was performed as a fixed source problem. The neutron heating distribution was determined by multiplying the neutron flux by the neutron KERMA (Kinetic Energy Release in MAterials) factors, and the gamma heating distribution is determined by multiplying the gamma flux by the gamma KERMA factors. The total heating rate was appropriately normalized to the reactor power. For the power profile calculation, burnup stage factors were also applied to account for the discrete batch effects relative to the batch-averaged equilibrium cycle power. That is, to account for the fact that the real power of each assembly position varies over the fuel residence time depending on the burned stage of fuel, the expected range of assembly power was determined for each fuel assembly position by considering the fuel compositions of individual burned stages.

The sub-channel analysis code SE2-ANL [16] was employed for whole core temperature calculations. SE2-ANL is a modified version of the SUPERENERGY-2 [17] thermal-hydraulic code interfaced with Argonne heating calculations. The SUPERENERGY-2 code is a multiassembly, steady-state sub-channel analysis code designed specifically to perform efficient calculations of the detailed core-wide coolant temperature profiles in sodium cooled fast reactor core geometries. At Argonne, reactor hot spot analysis methods as well as fuel and cladding temperature calculation models have been added to the original version of SUPERENERGY-2 $[18,19]$. Both nominal and two-sigma temperatures are calculated for the fuel and cladding. The nominal fuel and cladding temperatures are obtained by solving a steady-state one-dimensional conduction equation. The two-sigma temperatures are calculated using the semi-statistical method. SE2-ANL calculates the power densities for individual fine mesh intervals and determines the linear heat generating rates and power profiles of individual pins and duct wall faces by interpolating these fine mesh power densities. For the power profile calculation, burnup stage factors are also applied to account for the discrete batch effects relative to the batchaveraged equilibrium cycle power. That is, to account for the fact that the real power of each assembly position varies over the fuel residence time depending on the burned stage of fuel, the 
expected range of assembly power was determined for each fuel assembly position by considering the fuel compositions of individual burned stages.

The batch-averaged and fresh assembly power distributions at BOEC and EOEC are shown in Figures from II.1-5 to II.1-8. The batch-averaged power is the average power of each assembly position by using a homogenized mix of all the burned stages of fuel. The fresh assembly power denotes the power of each assembly position when it is occupied by a fresh assembly. For both metal and oxide cores, about $12 \%$ of total power is generated by gamma heating and the power fraction of non-fueled assemblies is about $2 \%$.

The power shift from BOEC to EOEC is noticeable in the startup core because of the movement of primary control assemblies; the power decreases by $7 \%$ for the metal core and $10 \%$ for the oxide core near the core center and increases by $4 \%$ for the metal core and $12 \%$ for the oxide core around primary control assemblies. This power shift from BOEC to EOEC is smaller for the recycled core because of the reduced stroke of the primary control assemblies. As can be seen, the power of fresh fuel assemblies is significantly higher than the batch-averaged assembly power. In the startup core, the average power increase in fresh fuel assemblies is $6-7 \%$ for the metal core and $8-9 \%$ for the oxide core. This power increase in fresh assemblies is smaller in the recycle core due to reduced fissile content in fresh fuel; $\sim 4 \%$ for the metal core and $\sim 5 \%$ for the oxide core.

The orifice zoning of assemblies and the flow allocation to the assemblies in each orifice zone were iteratively determined with the overall goal of equalizing pin cladding damage accrual and thus pin reliability. The coolant inlet and bulk outlet temperatures were assumed at $355^{\circ} \mathrm{C}$ and $510^{\circ} \mathrm{C}$, respectively. The average flow rate was determined such that the coolant temperature rise across the core is $155^{\circ} \mathrm{C}$. The fuel assemblies were grouped into six orifice zones for both metal and oxide cores. In the metal fuel core, the assembly flow rates were determined such that the peak 2-sigma cladding inner-wall temperatures of individual orifice zones over the one-year cycle are approximately equal to each other. For the oxide core, the $2 \sigma$ cladding mid-wall temperatures corresponding to the peak $2 \sigma$ - fuel centerline temperatures were approximately equalized. The non-fueled assemblies were grouped into eight orifice zones, and the flow rates were determined in proportion to the heating rates in order to obtain uniform coolant outlet temperatures. The same orifice zoning of non-fueled assemblies was used for both the startup and recycled cores, but the fuel assemblies were grouped slightly differently. Temperature calculations were performed using the fresh fuel assembly power (i.e., the maximum power over fuel residence time) for every fuel assembly position. Hot channel factors were also included in temperature predictions to account for core design, analysis, fabrication and operational uncertainties and variations. Figures II-1.9 and II.1-10 show the resulting orifice zones and assembly flow rates of the metal and oxide cores, respectively. 

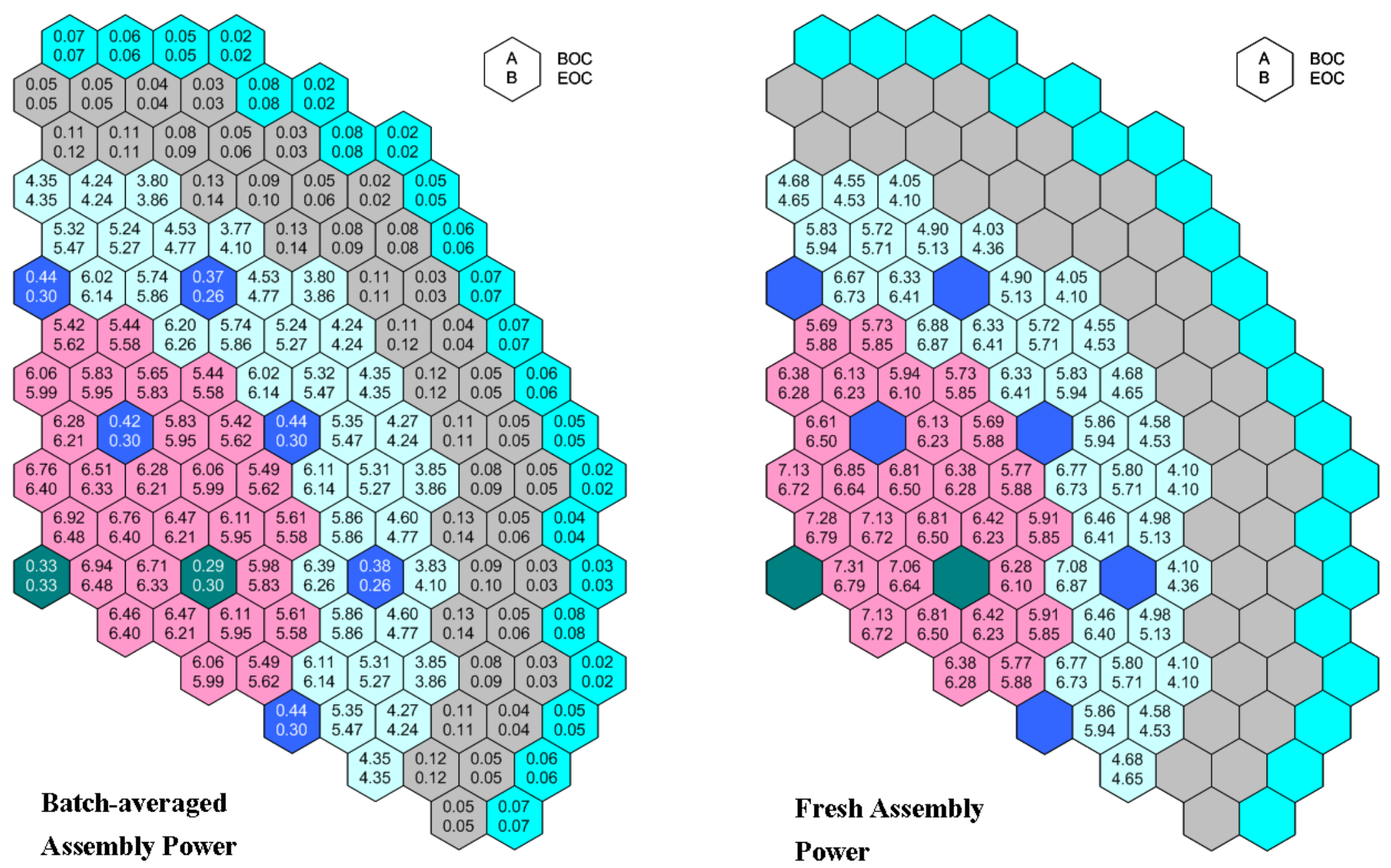

Figure II.1-5 Assembly Power Distributions of Startup Metal Core (MWt) 

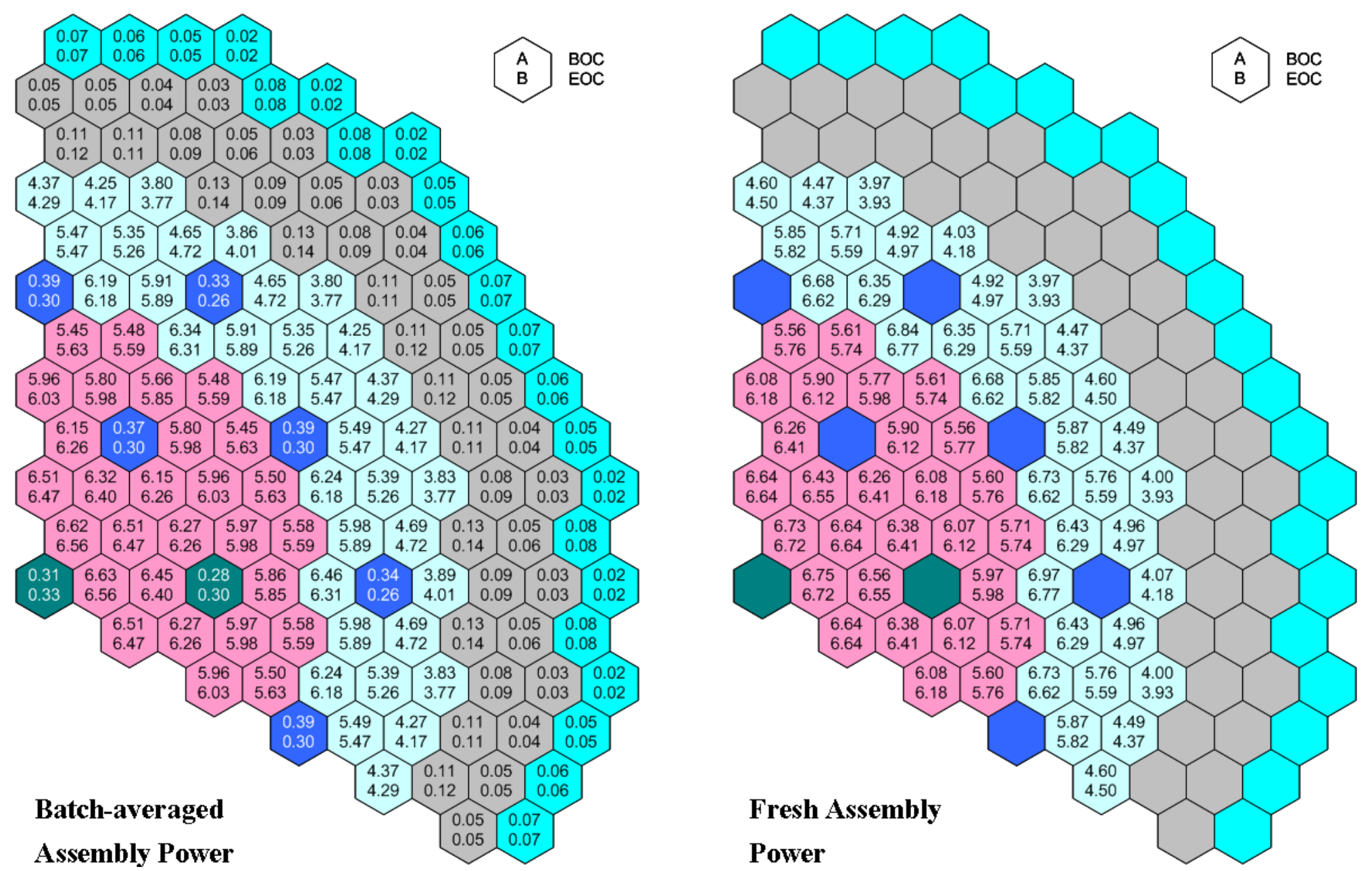

Figure II.1-6 Assembly Power Distributions of Recycled Metal Core (MWt) 

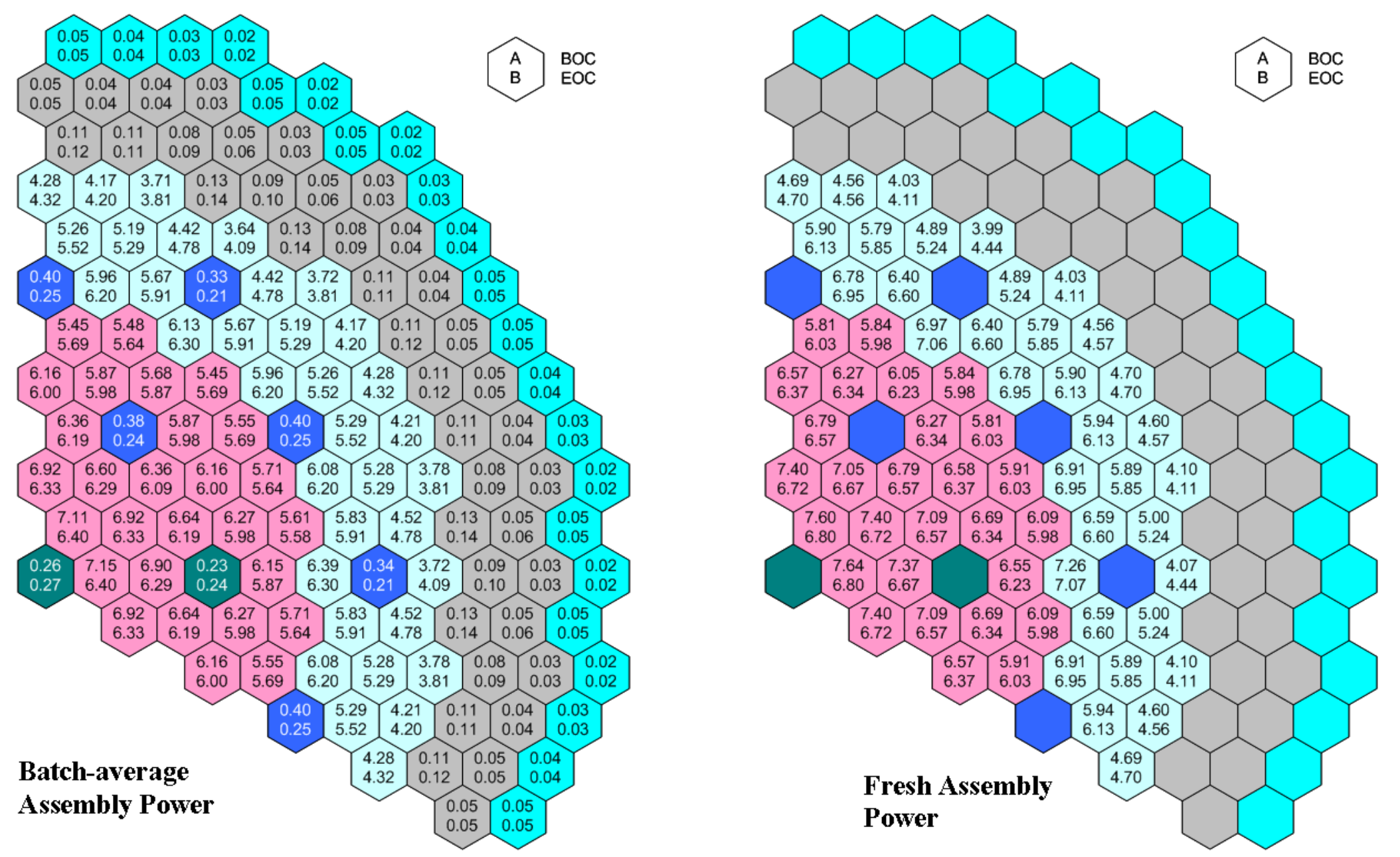

Figure II.1-7 Assembly Power Distributions of Startup Oxide Core (MWt) 

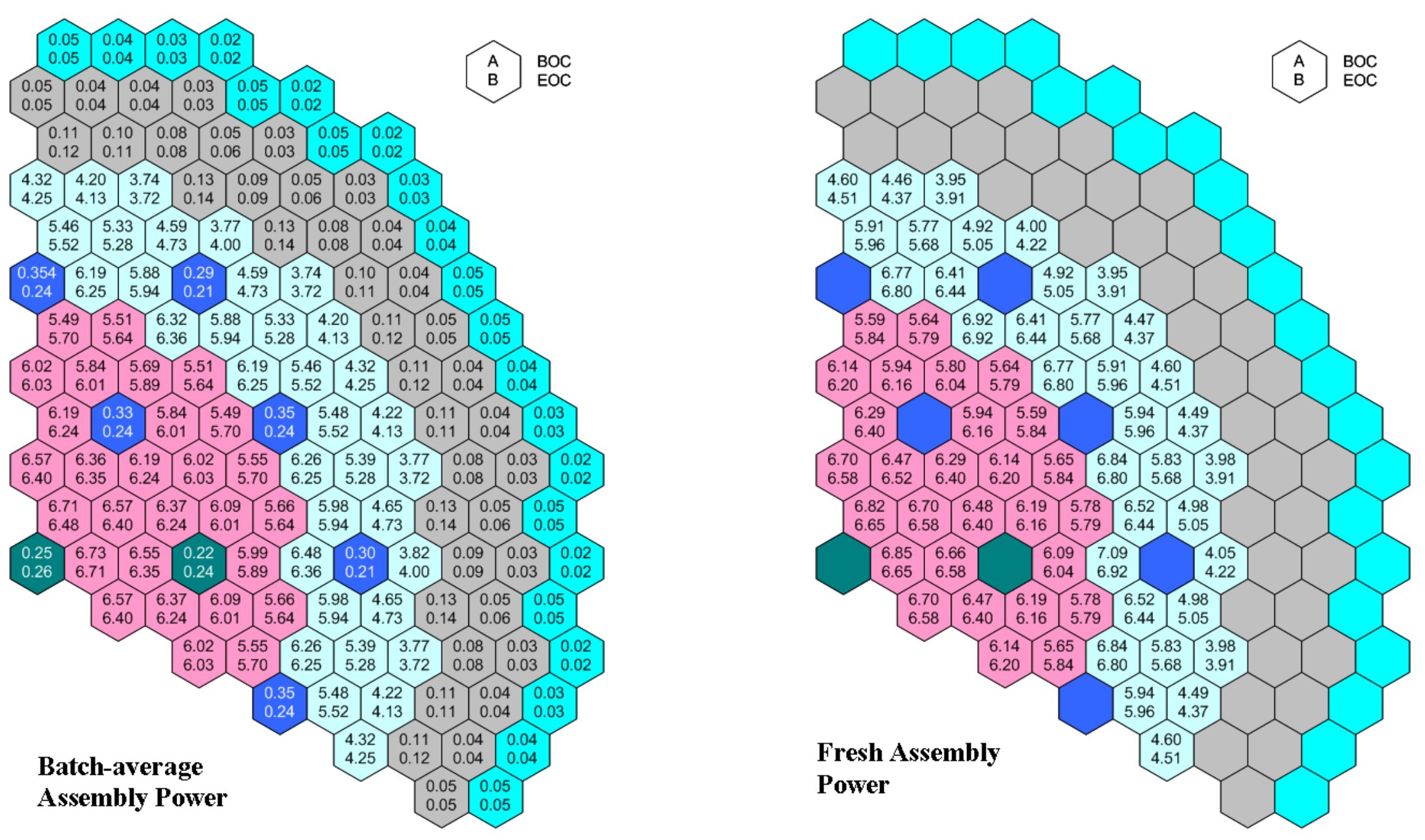

Figure II.1-8 Assembly Power Distributions of Recycled Oxide Core (MWt) 

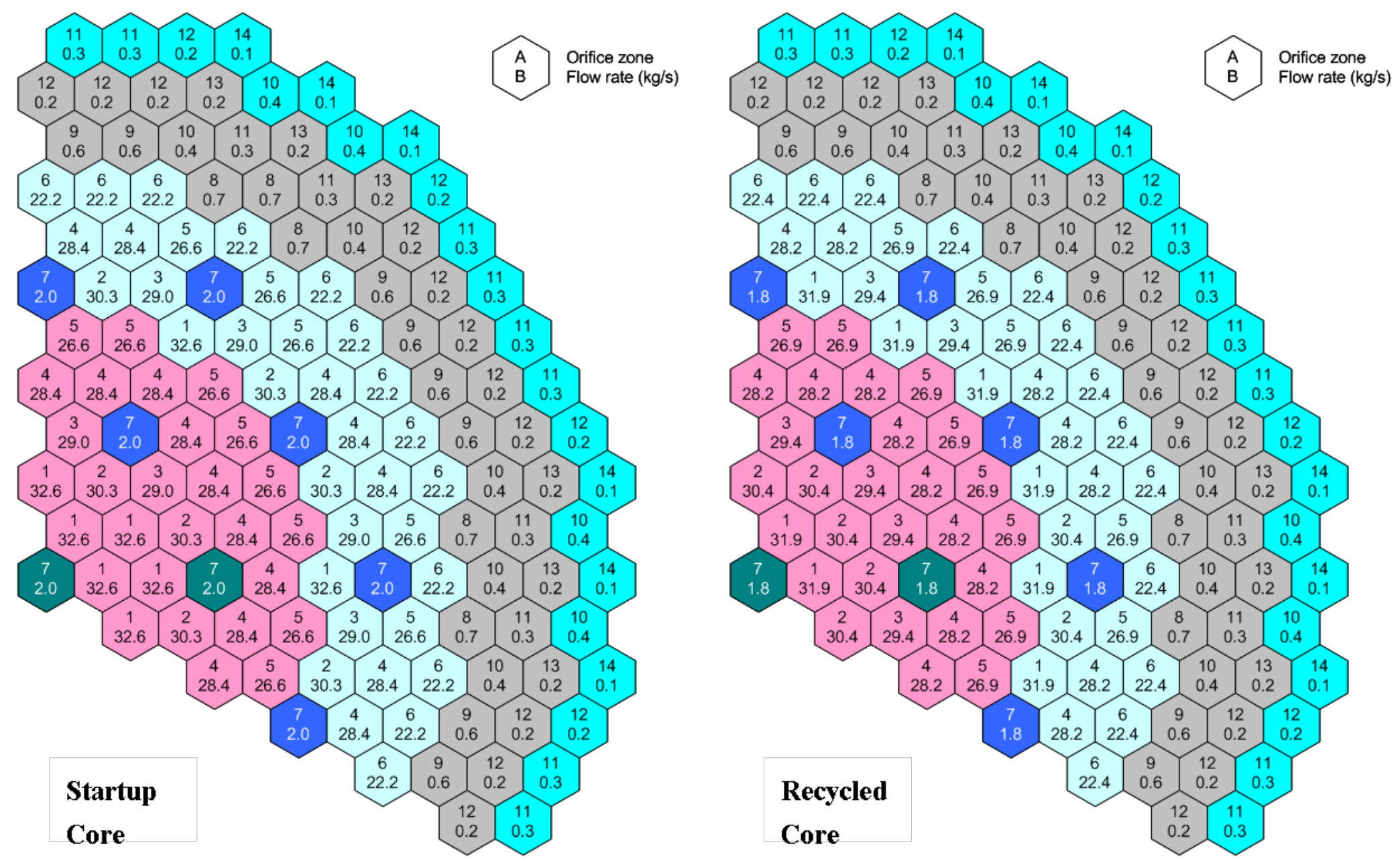

Figure II.1-9 Orifice Zones and Assembly Flow Rates of Metal Core (kg/s) 


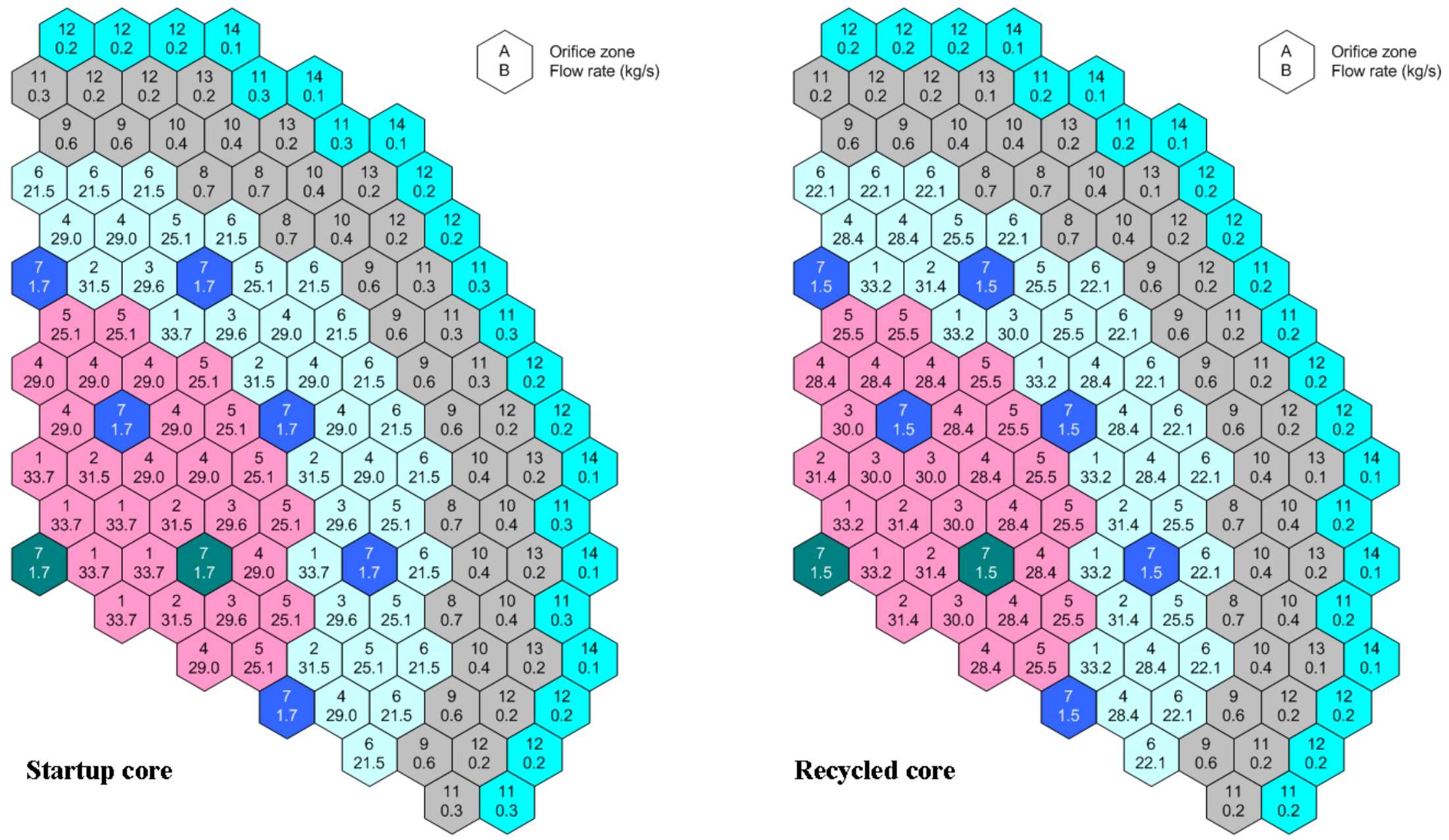

Figure II.1-10 Orifice Zones and Assembly Flow Rates of Oxide Core (kg/s) 
Figure II.1-11 shows the peak 2-sigma cladding inner wall temperatures of the metal core. In the startup core, the maximum of these peak 2-sigma cladding inner wall temperatures occurs in inner core assemblies in the fourth row at BOEC, and moves to outer core assemblies in the seventh row at EOEC. In the recycled core, this maximum occurs in outer core assemblies in the seventh row at BOEC and in inner core assemblies in the third and fifth rows at EOEC. The overall peak 2-sigma cladding inner wall temperature during a cycle is $632.3^{\circ} \mathrm{C}$ in the start-up core and $623.7^{\circ} \mathrm{C}$ in the recycled core. These peak temperatures are lower than the fuel-cladding eutectic temperature of typical ternary metal fuel $\left(650^{\circ} \mathrm{C}\right)$ with a sufficient margin; $18{ }^{\circ} \mathrm{C}$ for the startup core and $26^{\circ} \mathrm{C}$ for the recycled core.

Figures II.1-12 and II.1-13 present the minimum 2-sigma margins to fuel melt of the metal and oxide cores, respectively. The overall minimum 2-sigma margin to fuel melt occurs at an outer core assembly in the seventh row for both metal and oxide cores. The minimum 2-sigma margin of the metal core is $187^{\circ} \mathrm{C}$ for the startup core and $97^{\circ} \mathrm{C}$ for the recycled core. The recycled metal core has a smaller margin since the higher TRU enrichment reduces the solidus temperature of ternary metal fuel. The minimum 2-sigma margin of the oxide core is $694^{\circ} \mathrm{C}$ for the startup core and $727^{\circ} \mathrm{C}$ for the recycled core.

The mixed average coolant outlet temperatures of individual assemblies are shown in Figures II.1-14 and II.1-15. The coolant outlet temperatures of fuel assemblies are higher than the average core outlet temperature of $510^{\circ} \mathrm{C}$, since the fresh assembly heating rates were used for all the fuel assemblies with the nominal flow rates. Considering the differences in power between fresh and burned fuel assemblies, it was estimated for both the startup and recycled cores that the coolant outlet temperature of an assembly would not differ by more than $44^{\circ} \mathrm{C}$ from the average temperature of six surrounding assemblies. Thus, the coolant outlet temperature distribution would not violate the maximum allowable stresses in the reactor upper internal structure resulting from sodium mixing effects.

Figures II.1-16 and II.1-17 show the pin bundle pressure drops of individual fuel assemblies. For the metal core, the maximum pressure drop across the pin bundles is 45.5 psig in the startup core and 43.6 psig in the recycled core. Compared to the metal core, the maximum pressure drop of the oxide core is increased to 55 psig in the startup core and 53 psig in the recycled core because of the increased pin bundle height. 

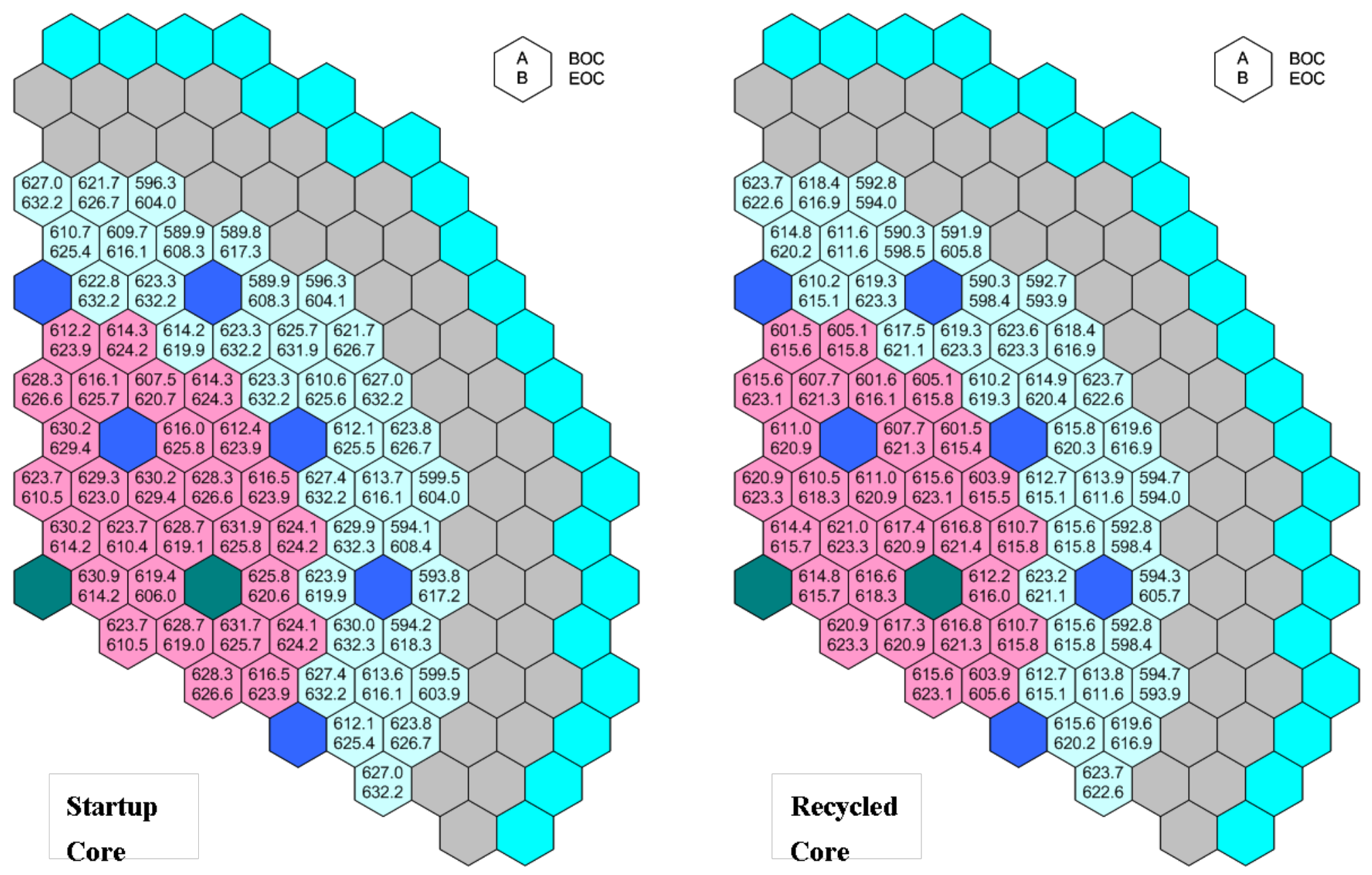

Figure II.1-11 Peak $2 \sigma$ Cladding Inner Wall Temperatures of Metal Core $\left({ }^{\circ} \mathrm{C}\right)$ 

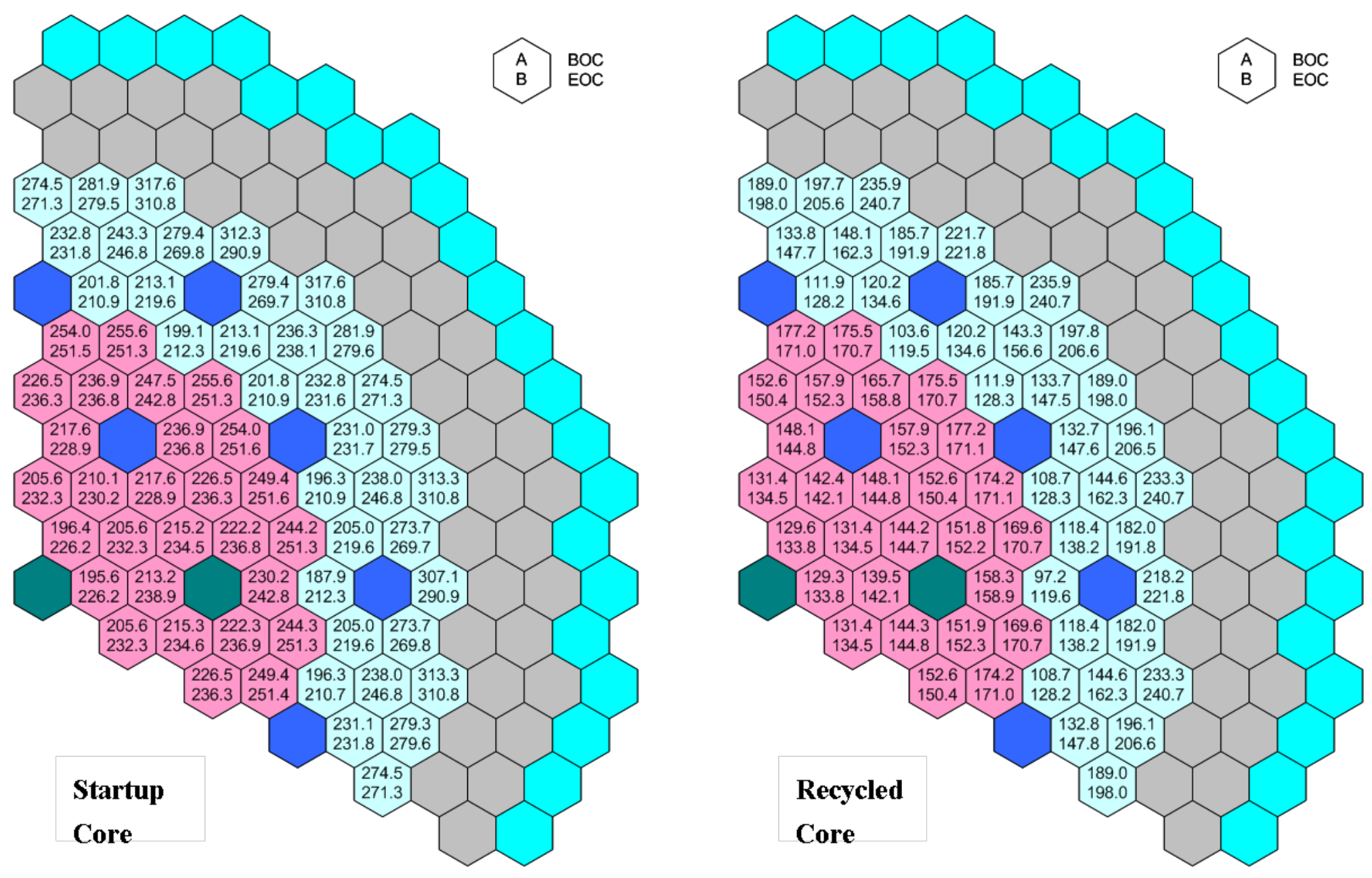

Figure II.1-12 Minimum $2 \sigma$ Margins to Fuel Melt of Metal Core $\left({ }^{\circ} \mathrm{C}\right)$ 

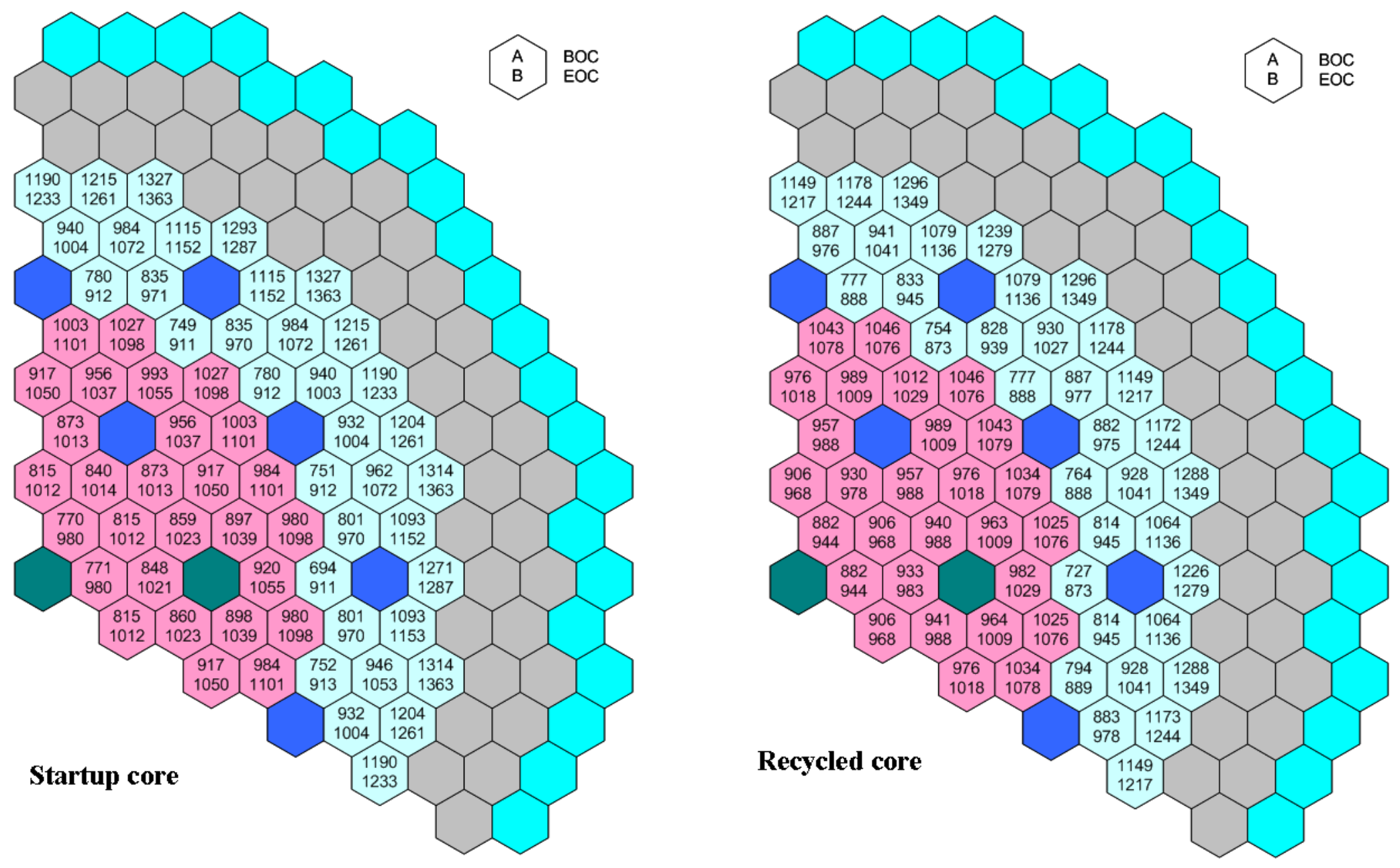

Figure II.1-13 Minimum 2 $\sigma$ Margins to Fuel Melt of Oxide Core $\left({ }^{\circ} \mathrm{C}\right)$ 

\begin{tabular}{|l|l|l|l|}
\hline 494.8 & 486.4 & 488.2 & 158.0 \\
501.7 & 493.5 & 496.6 & 465.0 \\
\hline
\end{tabular}

$\begin{array}{lllllll}517.4 & 516.8 & 501.2 & 507.8 & 485.6 & 463.8\end{array}$

$\begin{array}{llllll}525.5 & 525.6 & 510.2 & 518.8 & 495.9 & 472.8\end{array}$

$\begin{array}{llllllllllll}514.1 & 503.4 & 501.9 & 493.9 & 517.1 & 485.6 & 458.1\end{array}$

$\begin{array}{lllllll}519.4 & 509.3 & 510.5 & 504.8 & 530.7 & 495.9 & 165.0\end{array}$

$\begin{array}{llllllllllll}519.1 & 514.6 & 497.9 & 508.9 & 509.0 & 493.9 & 507.8 & 488.3\end{array}$

$\begin{array}{lllllllll}518.3 & 513.9 & 499.6 & 518.9 & 521.4 & 504.8 & 518.8 & 496.6\end{array}$

$\begin{array}{lllllllllll}516.9 & 513.9 & 500.6 & 496.8 & 508.7 & 501.4 & 501.2 & 486.8\end{array}$

$\begin{array}{llllllllll}519.6 & 513.8 & 506.9 & 507.8 & 518.5 & 510.1 & 510.2 & 493.9\end{array}$

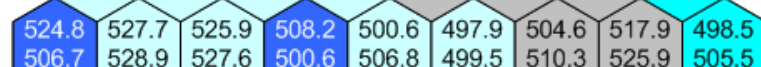

$\begin{array}{llllllllll}523.7 & 524.7 & 520.7 & 525.9 & 523.8 & 515.0 & 514.4 & 523.9 & 498.9\end{array}$

$\begin{array}{lllllllll}528.5 & 528.0 & 520.5 & 527.6 & 523.7 & 514.4 & 519.6 & 532.3 & 505.5\end{array}$

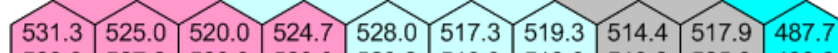

$\begin{array}{llllllllllll}528.8 & 527.2 & 523.8 & 528.0 & 529.2 & 519.9 & 518.6 & 519.2 & 525.9 & 493.9\end{array}$

$\begin{array}{llllllllllllllllll}533.7 & 524.2 & 524.9 & 523.8 & 524.9 & 517.9 & 515.7 & 504.6 & 502.4 & 489.6\end{array}$

$\begin{array}{llllllllllll}530.7 & 511.3 & 527.2 & 528.7 & 512.2 & 519.7 & 513.9 & 509.3 & 510.2 & 496.7\end{array}$

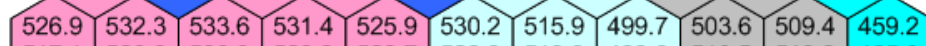
$\begin{array}{llllllllllllll}517.1 & 526.6 & 530.3 & 528.8 & 528.7 & 528.9 & 513.8 & 499.6 & 510.5 & 518.8 & 465.0\end{array}$

$\begin{array}{llllllllllllllllllllll}530.1 & 527.1 & 531.1 & 532.2 & 529.8 & 529.4 & 502.9 & 510.9 & 495.6 & 487.2\end{array}$

$\begin{array}{lllllllllll}518.4 & 517.2 & 523.4 & 527.1 & 528.0 & 527.6 & 506.9 & 518.9 & 504.8 & 495.9\end{array}$

$\begin{array}{lllllllllllllllllllll} & 530.4 & 524.9 & 510.9 & 528.8 & 525.7 & 511.7 & 499.1 & 510.9 & 519.1 & 465.2\end{array}$

$\begin{array}{lllllllllll}518.2 & 514.9 & 508.2 & 523.8 & 520.5 & 500.6 & 507.8 & 521.4 & 530.7 & 472.8\end{array}$

$\begin{array}{lllllllllllllllllllllll}526.8 & 531.1 & 532.1 & 529.8 & 529.3 & 502.7 & 510.7 & 495.6 & 487.2\end{array}$ $\begin{array}{llllllllll}516.8 & 523.3 & 527.1 & 528.0 & 527.4 & 506.7 & 518.4 & 504.8 & 495.8\end{array}$

$\begin{array}{lllllllllllllll}531.7 & 526.1 & 530.3 & 515.9 & 499.8 & 503.2 & 509.4 & 459.2\end{array}$ \begin{tabular}{llllllll}
528.9 & 528.8 & 528.9 & 513.8 & 499.3 & 510.0 & 518.8 & 465.0 \\
\hline
\end{tabular} $\begin{array}{llllllll}528.9 & 518.2 & 515.9 & 505.7 & 502.4 & 489.6 & \end{array}$

\begin{tabular}{llllll}
510.1 & 519.8 & 514.2 & 510.3 & 510.2 & 496.6 \\
\hline
\end{tabular}

\begin{tabular}{llll}
520.3 & 515.4 & 517.8 & 487.7 \\
\hline & 519.9 & 525.8 & 493.9
\end{tabular}

Startup \begin{tabular}{lllll}
519.6 & 515.4 & 517.8 & 487.7 \\
525.8 & 493.9 \\
\hline
\end{tabular}

Core

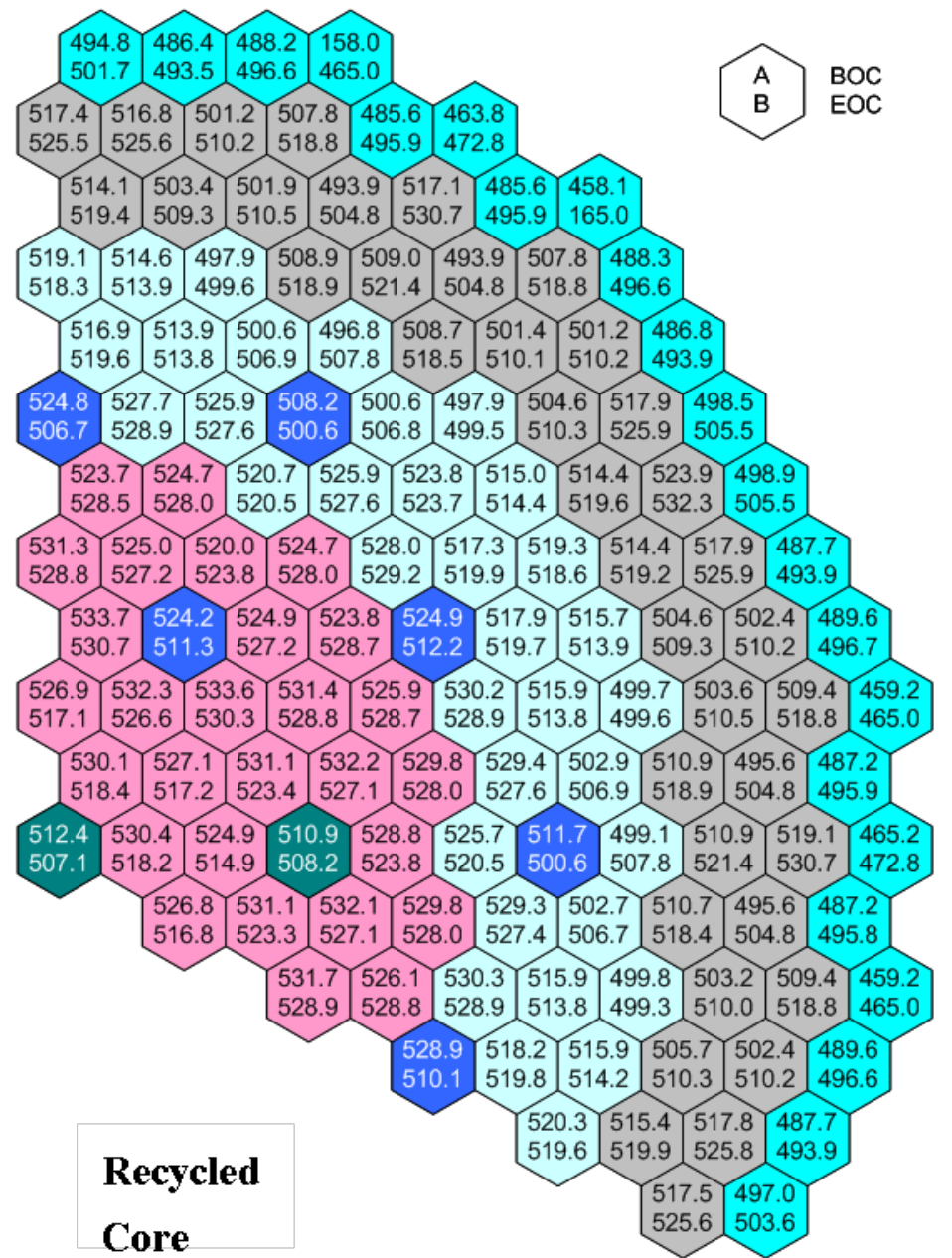

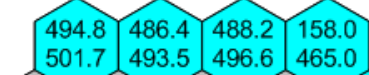

$\begin{array}{lllllll}517.4 & 516.8 & 501.2 & 507.8 & 485.6 & 463.8\end{array}$

525.5 525.6 $510.2 \quad 518.8 \quad 495.9$ 472.8

$\begin{array}{lllllllllllllll}514.1 & 503.4 & 501.9 & 493.9 & 517.1 & 485.6 & 458.1\end{array}$

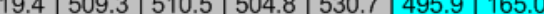

$\begin{array}{llllllll}518.3 & 513.9 & 499.6 & 518.9 & 521.4 & 504.8 & 518.8 & 496.6\end{array}$

$\begin{array}{llllllllllll}516.9 & 513.9 & 500.6 & 496.8 & 508.7 & 501.4 & 501.2 & 486.8\end{array}$

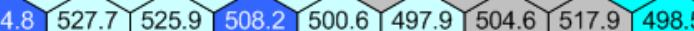

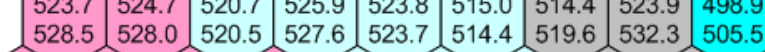

$\begin{array}{lllllllllllllllll}531.3 & 525.0 & 520.0 & 524.7 & 528.0 & 517.3 & 519.3 & 514.4 & 517.9 & 487.7\end{array}$

$\begin{array}{lllllllllll}528.8 & 527.2 & 523.8 & 528.0 & 529.2 & 519.9 & 518.6 & 519.2 & 525.9 & 493.9\end{array}$

$\begin{array}{llllllllllllll}533.7 & 524.2 & 524.9 & 523.8 & 524.9 & 517.9 & 515.7 & 504.6 & 502.4 & 489.6\end{array}$

26.95323533 .6 531.4 525.9 530.2 515.9 499.7 503.6 509.4 459.2

$\begin{array}{lllllllllll} & & & \end{array}$

$\begin{array}{llllllllllllllllllll} & 30.1 & 527.1 & 531.1 & 532.2 & 529.8 & 529.4 & 502.9 & 510.9 & 495.6 & 487.2\end{array}$ $\begin{array}{lllllll} & & \end{array}$

\begin{tabular}{llllllllll|l}
530.4 & 524.9 & 510.9 & 528.8 & 525.7 & 511.7 & 499.1 & 510.9 & 519.1 & 465.2
\end{tabular}

$\begin{array}{lllll} & & \end{array}$

\begin{tabular}{lllllllllll}
5 & 510.8 & 51.1 & 532.1 & 529.8 & 529.3 & 502.7 & 510.7 & 495.6 & 487.2 \\
\hline
\end{tabular}

\begin{tabular}{llllllllll}
531.7 & 526.1 & 530.3 & 515.9 & 499.8 & 503.2 & 509.4 & 459.2 \\
\hline
\end{tabular} $\begin{array}{llllllll}528.9 & 528.8 & 528.9 & 513.8 & 499.3 & 510.0 & 518.8 & 465.0\end{array}$ $\begin{array}{llllllll}528.9 & 518.2 & 515.9 & 505.7 & 502.4 & 489.6\end{array}$

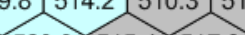

Core

Figure II.1-14 Mixed Mean Coolant Output Temperatures of Metal Core $\left({ }^{\circ} \mathrm{C}\right)$ 


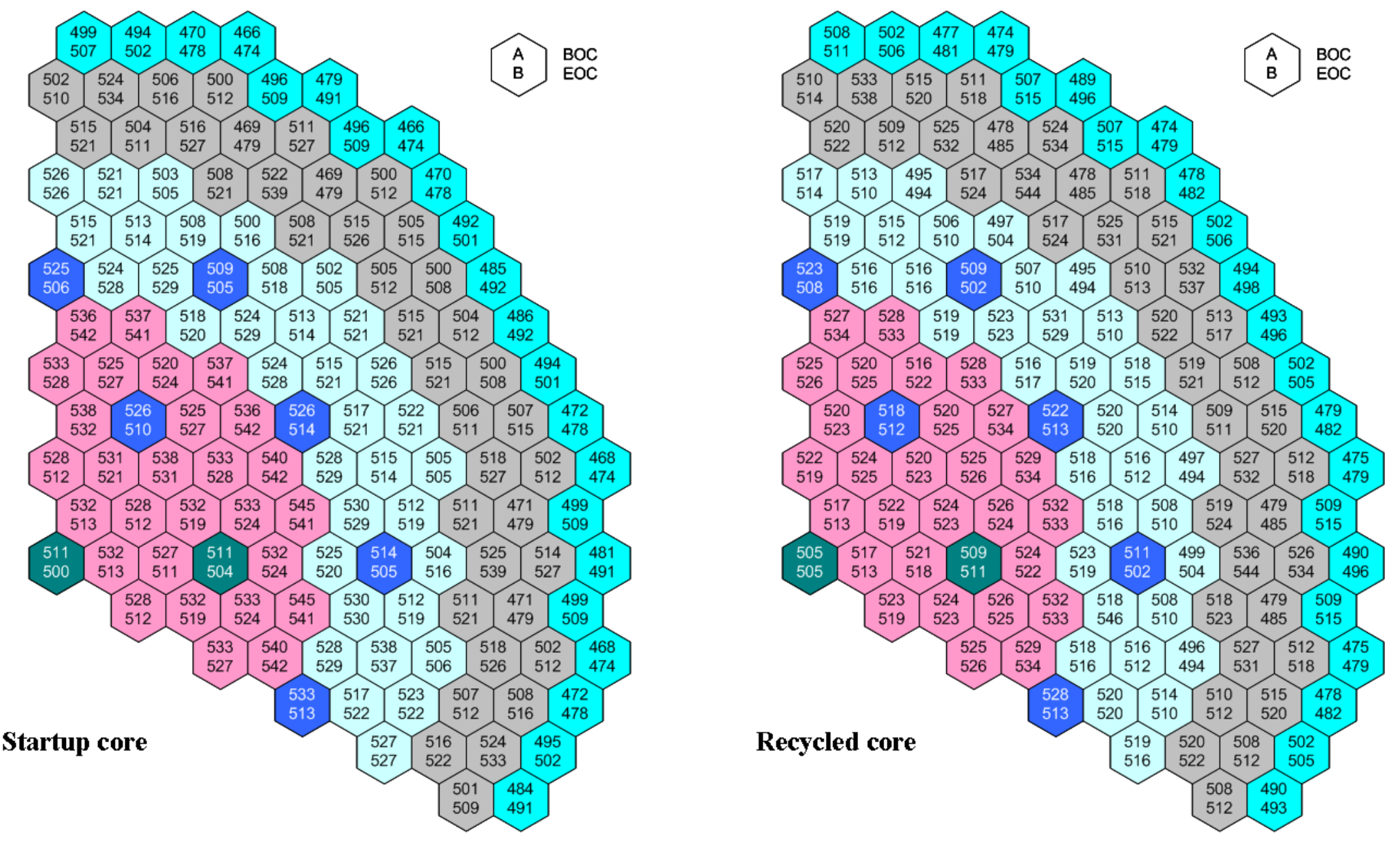

Figure II.1-15 Mixed Mean Coolant Output Temperatures of Oxide Core $\left({ }^{\circ} \mathrm{C}\right)$ 


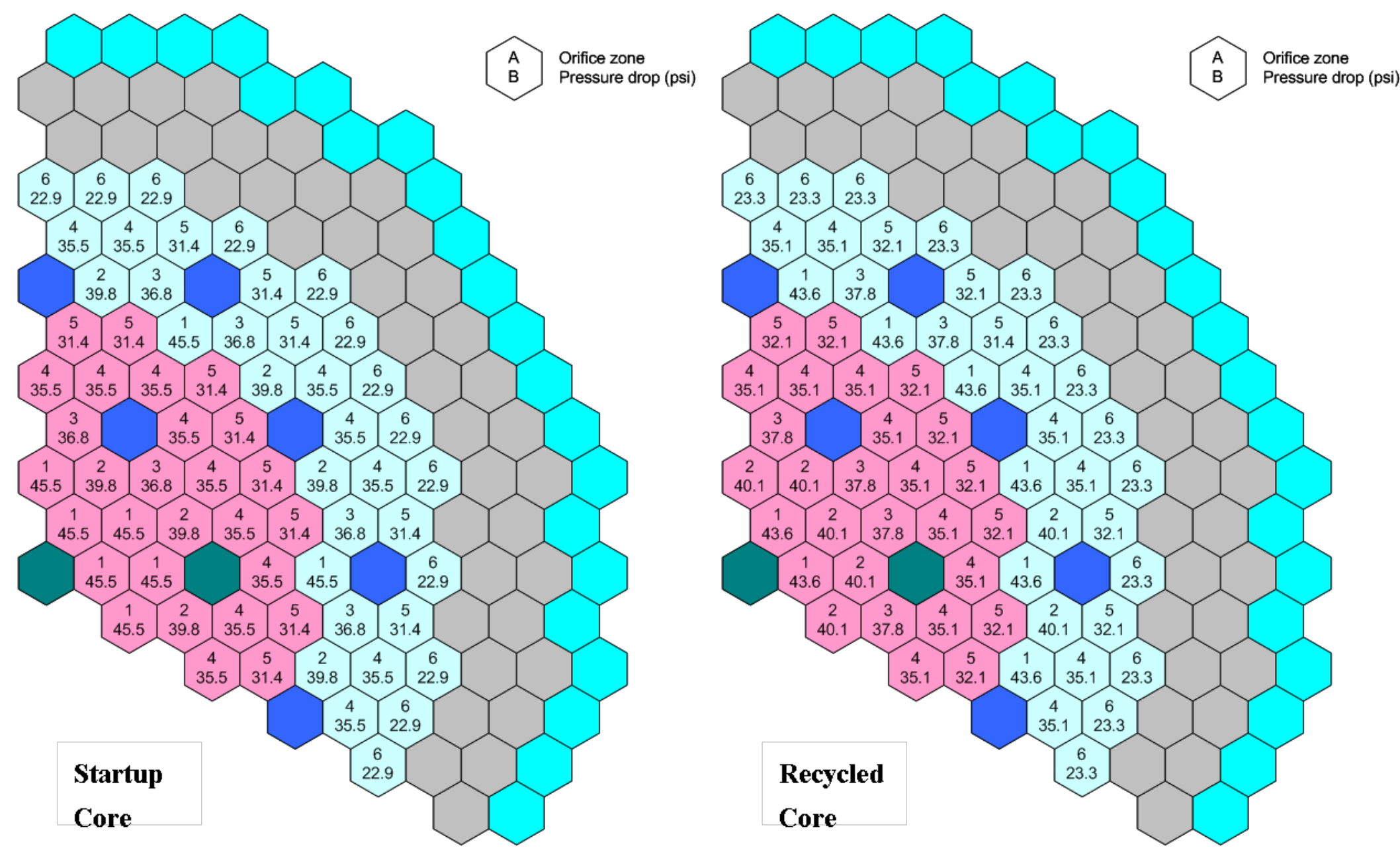

Figure II.1-16 Pin Bundle Pressure Drops of Metal Core (psig) 

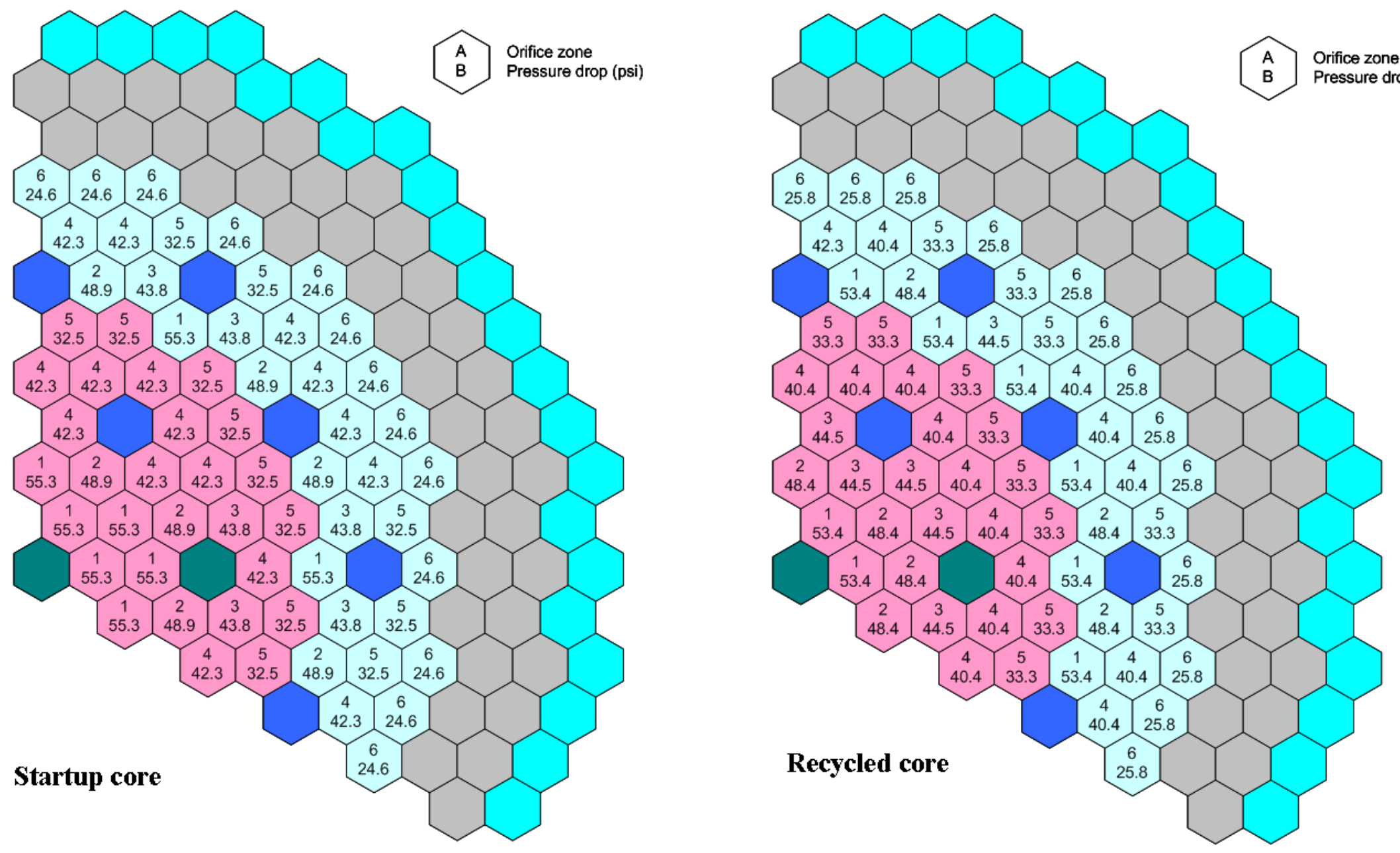

Figure II.1-17 Pin Bundle Pressure Drops of Oxide Core (psig) 


\section{II.1.1.5 Spent Fuel Characteristics}

To provide necessary data for designing the fuel-unloading machine and shielded fuel cask, spent fuel characteristics were estimated. Using the ORIGEN-2 code [20], the decay heat was calculated for a time span from charge to 20 years after discharge. The one-group cross sections determined from REBUS-3 equilibrium cycle calculations were used for actinide isotopes, and the FFTF one-group cross section library of the ORIGEN-2 code package was used for other isotopes. It was confirmed that the use of the actinide cross sections derived from REBUS-3 calculations yields consistent depletion results between ORIGEN-2 and REBUS-3 calculations (the difference in the heavy metal masses at discharge burnup between ORIGEN-2 and REBUS3 calculations was generally less than $1.0 \%$ ).

Table II.1-6 presents the decay heat of ABR spent fuel assemblies depleted up to the average discharge burnup. For metal core, the decay heat per assembly is $0.32-0.33 \mathrm{MW}$, which is equivalent to about $5.7-5.8 \%$ of the average fission power. The decay heat per assembly of the oxide core is slightly higher than that of the metal core because of the higher discharge burnup, and the higher TRU enrichment of the recycled core increases the decay heart compared to the startup core. Figure II.1-18 shows the decay heat of ABR discharged fuels as a function of postirradiation decay time. The decay heat decreases rapidly with post-irradiation decay time, since short-lived fission products are dominant contributors to decay heat.

Table II.1-6 Decay Heat of ABR Spent Fuel Assemblies with Average Discharge Burnup

\begin{tabular}{|c|c|c|c|c|c|c|}
\hline & \multicolumn{2}{|c|}{ Metal Core } & \multicolumn{2}{|c|}{ Oxide Core } \\
\hline & & & Startup & Recycle & Startup & Recycle \\
\hline \multicolumn{3}{|c|}{ Charge TRU enrichment (\%) } & 15.5 & 22.1 & 18.2 & 27.1 \\
\hline \multicolumn{3}{|c|}{ Average discharge burnup (MWd/kg) } & 93 & 93 & 111 & 111 \\
\hline \multicolumn{3}{|c|}{ Initial HM mass per assembly (kg) } & 75.9 & 75.9 & 78.1 & 78.1 \\
\hline \multicolumn{3}{|c|}{ Decay heat fraction of rated power (\%) } & 5.7 & 5.8 & 5.7 & 5.9 \\
\hline \multirow{9}{*}{$\begin{array}{l}\text { Decay heat } \\
\text { per } \\
\text { assembly } \\
(\mathrm{kW})\end{array}$} & \multicolumn{2}{|l|}{ Fresh fuel } & 0.03 & 1.29 & 0.03 & 1.89 \\
\hline & \multirow{8}{*}{$\begin{array}{l}\text { Time after } \\
\text { discharge }\end{array}$} & 0 day & 316.3 & 323.2 & 318.0 & 326.4 \\
\hline & & 10 day & 14.0 & 17.5 & 14.1 & 19.0 \\
\hline & & 1 month & 9.1 & 12.5 & 9.1 & 13.8 \\
\hline & & 3 month & 5.6 & 8.4 & 5.7 & 9.6 \\
\hline & & 1 year & 2.3 & 3.8 & 2.4 & 4.6 \\
\hline & & 3 year & 0.7 & 1.6 & 0.8 & 2.2 \\
\hline & & 5 year & 0.3 & 1.2 & 0.4 & 1.7 \\
\hline & & 20 year & 0.2 & 0.8 & 0.2 & 1.1 \\
\hline
\end{tabular}




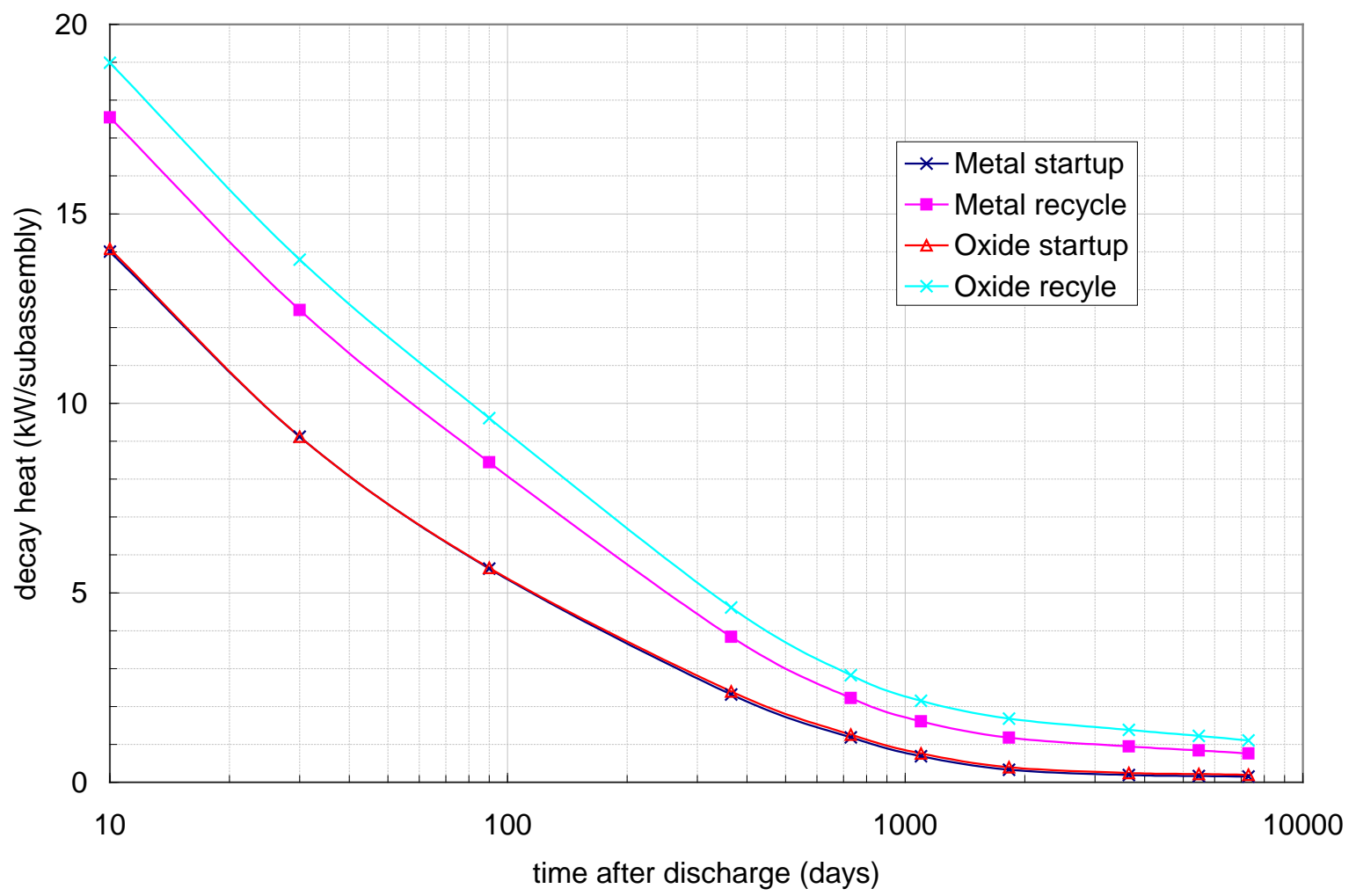

Figure II.1-18 Decay Heat of ABR Spent Fuel Assemblies 


\section{II.1.2 Upper Internals Structure}

The upper internals structure (UIS) is a package of hardware suspended from the rotatable plug to about 3 in. above the core assemblies. The functions of the UIS are to:

1. Support shroud tubes containing the primary and secondary control rod drivelines;

2. Preserve critical alignments between these drivelines and the core lattice, under normal and off-normal (including seismic) conditions;

3. Route and support thermocouples for temperature surveillance of core assemblies;

4. Route and support other instrumentation such as loose-parts monitors and delayedneutron sampling stations;

5. Produce sufficient coolant mixing to mitigate thermal transients to downstream components;

In the present design, outlet thermocouples cover most of the fuel assemblies, and some of the reflector assemblies, and no shield assemblies. Instrument posts of several different geometric configurations are needed when the requirement exists to monitor all heat generating core assemblies. When this requirement is relaxed, however, instrument posts can be dispensed with. In addition to the obvious cost reduction, this is an important design change because thermal striping studies on the CRBR UIS have shown the instrument posts to be the components most vulnerable to thermal fatigue under steady state operating conditions. Additional thermal striping tests are needed for confirmation, but at this time it is believed that the elimination of instrument posts could allow the entire UIS to be constructed of austenitic stainless steel.

Figure II.1-6 illustrates the present reference UIS design. The outer boundary is a 2.2 meter diameter shroud (essentially the outside diameter of the core assemblies) with large-hole penetrations over part of its length. The penetrations release the core effluent horizontally to the hot pool from which it travels upward and then to the inlets of the two IHXs. Within the shroud are 14 - 4 in. outer diameter control rod shroud tubes. Three inches is the nominal gap between the core assemblies and the bottom of the shroud tubes and UIS shroud. The shroud tubes are perforated for the release of coolant to the hot pool at an elevation close to the free surface. The radial position of the shroud tubes is fixed by at least three horizontal guide plates, welded to the UIS shroud. The lower guide plate is close to the core assemblies and is perforated to permit about $85 \%$ of the core effluent to reach the region between guide plates. The balance either goes up the shroud tubes or leaks through the 3 in. peripheral gap. In the region between guide plates are located five loose-parts monitors (high-temperature submersible microphones attached to the outside of selected shroud tubes) and delayed neutron sampling stations attached to all shroud tubes. The latter are part of the failed element detection and location (FEDAL) system. With minimal horizontal mixing of core effluent upstream of the sampling stations, it is expected that the location of a badly breached fuel element can be coarsely identified by the relative signal strengths from these sampling stations. Flow tests are necessary to better establish the expected performance of this part of the FEDAL system.

The upper guide plate is not perforated except for clearances at the 10 shroud penetrations to permit sliding due to differential thermal expansion. The upper guide plate is located high within the upper internal structure. Thus, almost all of the core effluent is forced to enter the hot pool at least $4 \mathrm{ft}$ below the nominal hot pool operating level. This should meet the objective of maintaining a quiet entrainment-free surface. If future flow tests reveal an 
undesirable degree of surface agitation, the upper guide plate can be dished, concave downward, to further suppress any upward flow vectors of sodium leaving the UIS.

Core assembly outlet sodium temperature is monitored by chromel-alumel, stainless steel sheathed, ungrounded thermocouples. These thermocouples are replaceable and installed in helium-filled wells. The thermocouple wells are positioned 3 in. above the centerline of adjacent fuel assemblies. The outside surface of the control rod shroud tubes serves also to support the delayed neutron sampling lines and the leads and oxygen vent lines for the loose parts-monitoring microphones. In the stagnant zone above the upper guide plate, all of these instrument lines are gathered into six instrument conduits which penetrate the rotatable plug.

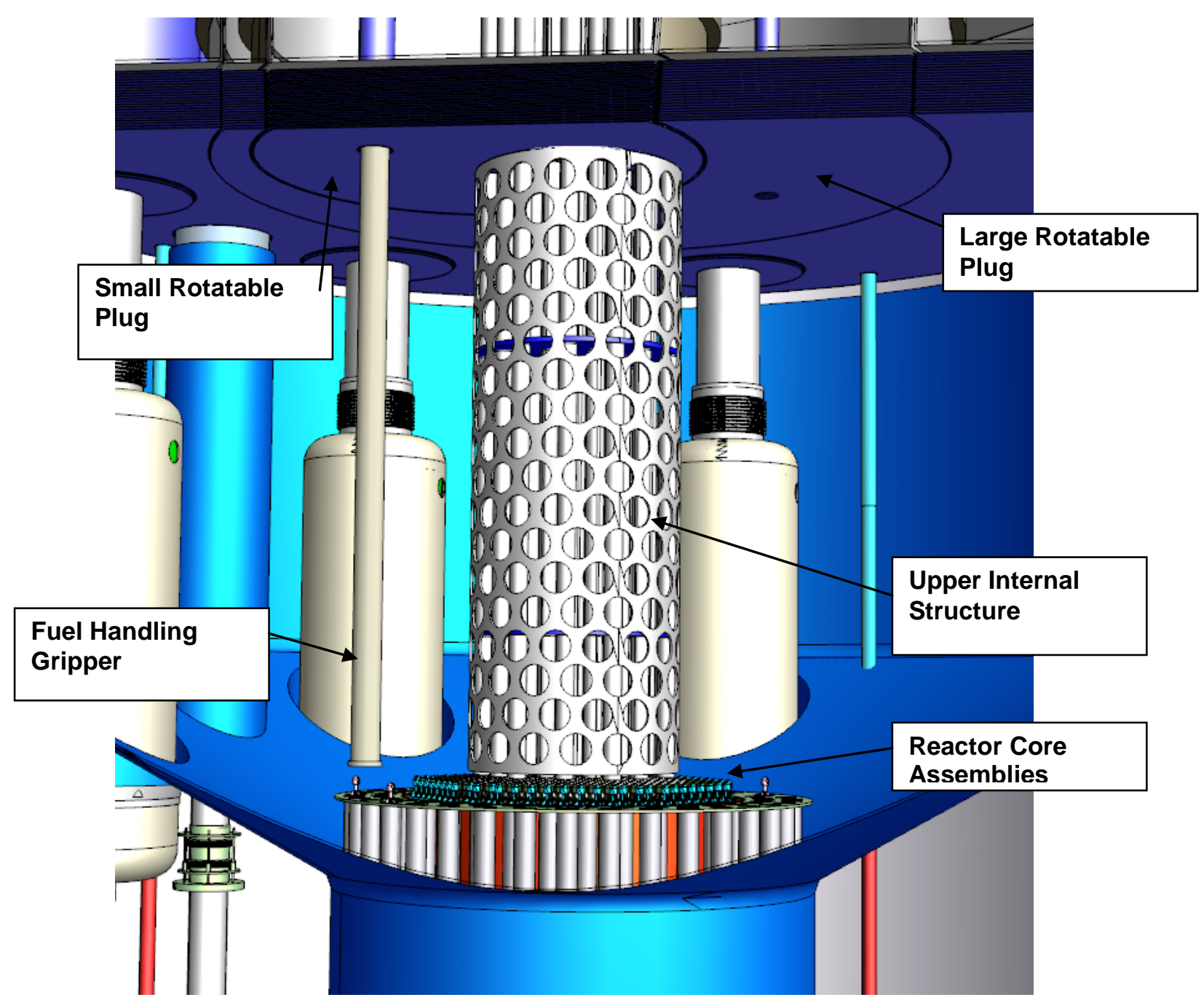

Figure II.1-6 Schematic of Upper Internal Structure 
The UIS design provides backup mechanical hold-down protection, even though this is not considered a design requirement. In the event of a hydraulic liftoff of a fuel or internal blanket assembly, due to a non-mechanistic failure of the hydraulic hold-down system, the assembly would be stopped in 3 in. by the bottom of a shroud or dummy shroud tube if the assembly in question is one of the assemblies that are instrumented. Any of the others would be stopped by the middle guide plate. Radial reflectors and radial shield assemblies have a sufficiently low pressure drop that their liftoff is precluded by dead weight.

Aside from being a convenient shape to support surveillance thermocouples, the funnelshaped lower ends of the control rod shroud tubes capture part of the coolant from the adjacent assemblies. The drivelines are exposed to this effluent for most of their length, before the coolant is released to the hot pool through holes near the top of the shroud tubes. In a hypothetical unprotected loss-of-flow transient, it is expected that this hot effluent will cause some beneficial insertion of absorber material into the core by thermal expansion of the drivelines.

\section{II.1.3 Lower Internals Structure}

The reactor core barrel assembly consists of the core barrel, core grid, and the inlet plenum structure. The core barrel and cover assembly is not leak tight, and permits a certain amount of the inlet sodium to leak into the bulk primary coolant. This feature promotes the establishment of natural circulation cooling of the core in the event of loss of the primary pumps.

The lower internals structure (LIS) supports the reactor inlet plenum, the core assemblies, the fixed radial shielding, the redan, the 4 sodium inlet pipes, and various shields, brackets and baffles as shown in Figure II.1-7. It is designed to withstand seismic events with acceptable stresses and deflections. The LIS contains the inlet coolant flow distribution system that controls the rate of flow to the core assemblies. This distribution system consists of a doughnut-shaped manifold, or torus, and associated piping, that encircles the inlet coolant plenum. The plenum or grid assembly does not have a high and low pressure plenum. The core assemblies are individually orificed to provide sufficient flow through the fuel assemblies, reflector assemblies, and shield assemblies. Four large pipes carry coolant from the primary pumps into the torus. A total of 12 short, smaller pipes distribute the coolant from the torus into the inlet plenum.

The lower internals structure consists of the core barrel, core grid, and the inlet plenum structure. The entire assembly is supported on the core support structure as shown in Figure II.17. 


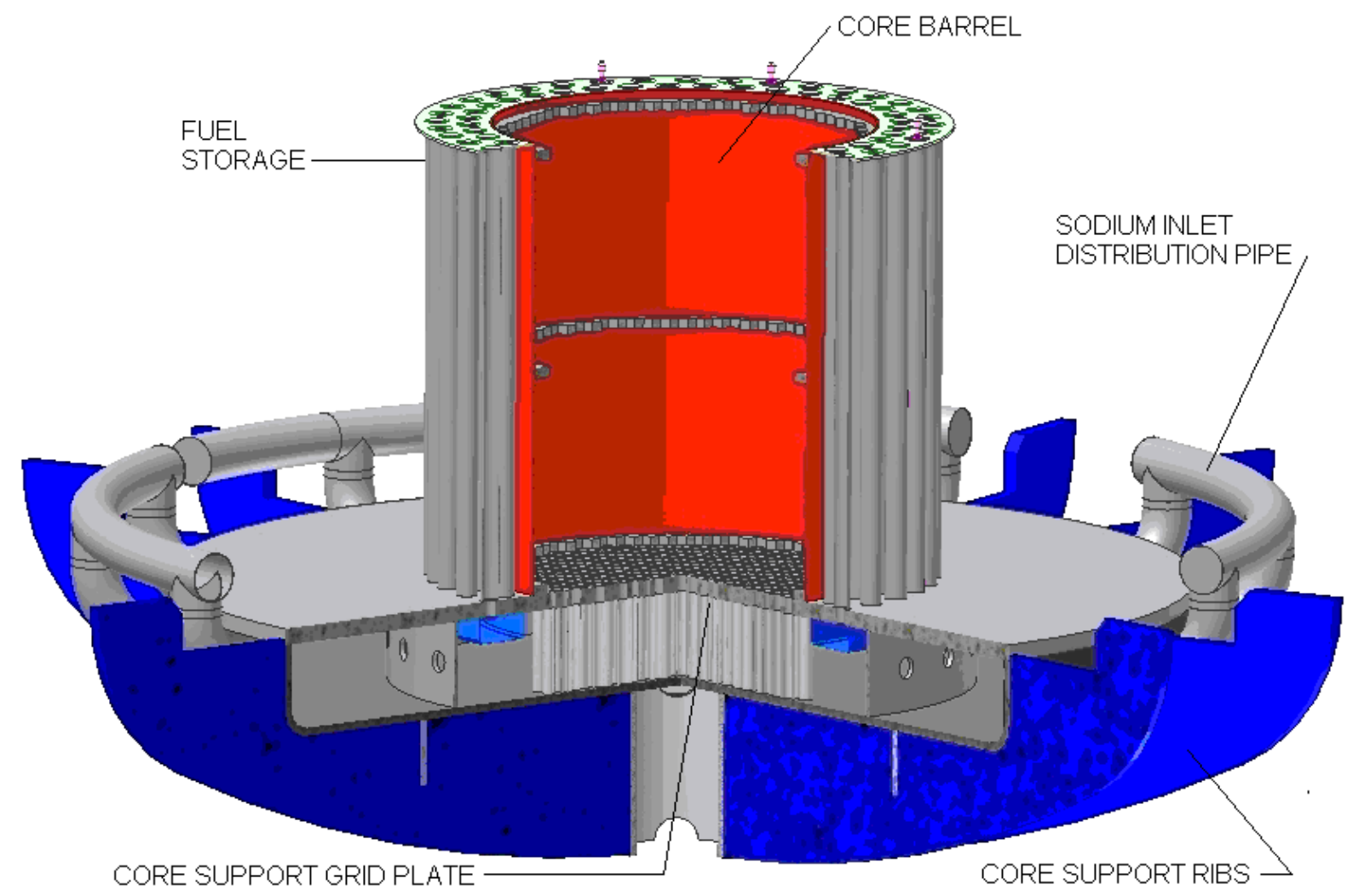

Figure II.1-7 Schematic of Lower Internal Structure

\section{II.1.4 Core Restraint System}

The core restraint system consists of distributed passive hardware features which, acting together, must meet the following functional requirements:

1. Establish the positions of the individual core assemblies in the horizontal plane;

2. Control horizontal movements of core assemblies arising from thermal expansion effects, irradiation-induced swelling, and irradiation-enhanced creep, in such a way that reactivity effects are acceptable and control rod driveline alignments are maintained within specified tolerances;

3. Accommodate horizontal seismic motions within alignment and stress specifications; and

4. Maintain sufficient clearances in the horizontal plane to allow for fuel handling within specified vertical withdrawal and insertion force limits.

The design choices representing the major decisions in the design of a passive core restraint system are:

1. Length and stiffness of the core assembly lower adapters;

2. Lower Internals Structure interface with the core assembly lower adapters clearances, seals, and number of support points (1 or 2 );

3. Number, location, configuration and height of the core assembly load pads; 
4. Rigidity of the peripheral boundary - stiff radial shield assemblies or a rigid, shaped, core former ring attached to the core barrel;

5. Allowable vertical core assembly insertion and withdrawal loads.

The major challenge in core restraint design is to find the "design window" which contains sufficient clearance for fuel handling (even when core assemblies are bowed due to swelling and creep effects) and sufficient tightness or stiffness for adequate radial-position control. The thermal contraction produced by the cool down to refueling conditions is very important in creating this window of design feasibility. 1 Under refueling conditions, the sum of these side loads, times the coefficient of friction yields the required initial withdrawal force over and above the dead weight.

The two core restraint system design strategies are referred to as the "limited free bow" approach and the "free-flowering core." The first, used in FFTF and CRBR, employs a short lower adapter horizontally restrained in the lower internals structure (LIS) at one point (the lower end), and rigid core formers at the two elevations of core assembly load pads -- one near the top end and one about 4 in. above the fueled zone. The second, used in EBR-II, Phenix and SuperPhenix, employs a long lower adapter horizontally restrained in the LIS at two points, and the "fence" of stiff and essentially isothermal radial shield assemblies constitutes the peripheral restraint.

The ABR design borrows features from both approaches. The relatively long lower adapter fits into a cylindrical perforated sleeve in the inlet plenum. A ball-and-cone seat at the transition from the cylindrical lower adapter to the hexagonal duct provides the coolant seal, load support, and a positive horizontal restraint point. Close clearance between the bottom of the lower adapter and the lower internal structure sleeve provides limited-movement horizontal restraint.

A rigid core former ring at the elevation of the top load pads provides positive peripheral restraint. The ring has enough clearance, relative to a tight array of assemblies, to permit removal and replacement of individual assemblies by the in-vessel transfer machine (IVTM), yet is tight enough so that the freedom of assemblies to move does not have adverse reactivity or alignment implications. The inner diameter of the ring is shaped so as to achieve flush contact with the outermost assemblies. The core former ring is welded to the inner diameter of the core barrel, to which the Upper Internals Structure is attached during operation with a system of retractable keys (not shown), thus assuring control and safety rod drive-train alignments. Each core assembly is equipped with a second load pad centered $4 \mathrm{in}$. above the fueled zone of the reactor, but no peripheral core former ring is present at this elevation. Normally the core assemblies contact one another only at the two load pads, which completely circumscribe the hexagonal ducts. The duct is $15.71 \mathrm{~cm}$ across flats and the lattice pitch (also the hot across-flat dimension of the load-pads) is $16.142 \mathrm{~cm}$. The $0.432 \mathrm{~cm}$ clearance between ducts is provided to prevent general contact at end of life due to swelling, creep (rounding of the flats), and differential bowing, but the adequacy of this clearance needs to be confirmed by analysis.

\footnotetext{
${ }^{1}$ A tool that can be used in core restraint design is the NUBOW-3D computer code. It calculates the elastic and inelastic effects on the shape of individual core assemblies in a 3-D representation of the whole core or typical sector thereof, the reactivity effects associated with short or long term movements from one set of equilibrium positions to another, and the side loads at all contact points. Because of time constraints, NUBOW-3D was not activated for this reference plant concept development.
} 
The IVTM, described elsewhere, has certain capabilities important to meeting the functional requirements of the core restraint system. The gripper assembly contains a "holddown and spreader" feature which facilitates fuel handling even when the core assemblies are distorted. The holddown provision prevents inadvertent lifting of any of the six assemblies surrounding the one being removed. The spreader feature, pioneered in EBR-II, has proven very useful in concentrating available clearances to the immediate vicinity of the core assembly being removed or the hexagonal hole into which a new assembly is being inserted. All components of the IVTM, and the core assemblies themselves, have ample margin beyond the planned administrative limit on insertion/withdrawal forces of greater than $1000 \mathrm{lb}$. excluding dead weight.

\section{II.1.5 Reactivity Control Requirements and Shutdown Margins}

Two independent safety-grade reactivity control systems were adopted for the reference ABR core concepts (see Figure II.1-1). The primary control system is required to have sufficient reactivity worth to bring the reactor from any operating condition to cold sub-critical at the refueling temperature with the most reactive control assembly stuck at the full power operating position. Any operating condition means an overpower condition together with a reactivity fault. The primary control system also serves to compensate for the reactivity effects of the fuel burnup and axial growth of metal fuel. The reactivity associated with uncertainties in criticality and fissile loading is accommodated by the primary control system. The secondary control system is required to shutdown the reactor from any operating condition to the cold shutdown, also with the most reactive assembly inoperative.

To estimate the control requirements and shutdown margins, the reactivity worth was calculated for various combinations of control assemblies inserted, and the stuck-assembly worth was estimated for each control assembly. The maximum single assembly reactivity faults were also estimated from the reactivity worth curves of primary system control assemblies. To account for the interaction effects of control assemblies, the maximum worth of each control assembly was determined by withdrawing it from the configuration where all the primary or secondary control assemblies are inserted. Table II.1-7 presents the calculated control assembly worth for various combinations of control assemblies.

Figures from II.1-19 to II.1-22 show the primary system reactivity worth curves. The solid curve denotes the core reactivity as a function of control rod tip position from the bottom of the active core for the case where all the primary control assemblies are moving together. At the full power operating condition, only the fuel cycle excess reactivity is held down by the primary control system. The critical primary control assembly positions are indicated by the dashed vertical lines, and the dashed curve represents the reactivity worth of the most reactive control assembly, which is one of the fourth row control assemblies. The reactivity insertion by the complete withdrawal of the most reactive control assembly from its critical position (maximum single rod fault) is similar for the metal and oxide cores as $\sim 0.70 \$$ for the startup core and $\sim 0.4 \$$ for the recycled core.

The control requirements were estimated by accounting for the axial expansion effect, temperature and power defects, overpower margin, and several uncertainties along with the burnup reactivity loss. The temperature and power defects were estimated with assumed coolant inlet temperature of $355^{\circ} \mathrm{C}$ and conservatively assumed refueling temperature of $205{ }^{\circ} \mathrm{C}$. Table II.1-8 presents the calculated temperature and power defects. 
The reactivity worth requirements and available reactivity of the primary and secondary control systems of the reference ABR core concept are provided in Tables II.1-9 and II.1-10, respectively. The axial irradiation growth of oxide fuel was ignored, while a $5 \%$ axial growth was assumed for the ternary metal fuel. The adopted overpower margin and the uncertainties were $3 \%$ overpower margin, $20 \%$ uncertainty for temperature defect, $15 \%$ uncertainty for burnup reactivity, and $20 \%$ uncertainty for fuel axial growth, and $1.0 \$$ uncertainty for each of the critical prediction, fissile loading and refueling. In addition, $1.80 \$$ and $1.30 \$$ of additional margins are included at BOEC and EOEC reactivity worth requirements.

In the metal core, the burnup reactivity loss is about $10.4 \$$ and $6.4 \$$ for the startup and recycled cores, respectively. The estimated temperature and power defects at BOEC are 1.23 and $1.05 \$$ for the startup and recycled cores, respectively. The overpower margin is allocated to permit the reactor to operate at $103 \%$ of the rated power, which is less than $0.02 \$$ for both startup and recycled cores. The axial growth reactivity is $0.45 \$$ for the startup core and $0.58 \$$ for the recycled core. The total control requirement for the primary control system is $16.9 \$$ for the startup core and $12.3 \$$ for the recycled core. The total control requirement for the secondary control systems is less than $1.6 \$$.

The oxide core has similar overpower margin, reactivity fault, and uncertainties as the metal core. On the other hand, the axial swelling effect is negligible, but the temperature and power defects are increased because of higher fuel temperature and larger Doppler feedback. The burnup reactivity loss is also slightly higher for the startup core. As a result, the reactivity control requirement for the primary control system becomes slightly larger for the startup core (17.5\$), but slightly smaller for the recycled core (11.9\$). The total reactivity control requirements for the secondary control systems are $2.4 \$$ and $1.9 \$$ for the startup and recycled cores, respectively. 


\section{Table II.1- 7 Control Assembly Worth (\$)}

\begin{tabular}{|c|c|c|c|c|c|c|c|c|}
\hline \multirow{3}{*}{$\begin{array}{c}\text { Inserted } \\
\text { assemblies }\end{array}$} & \multicolumn{4}{|c|}{ Metal Core } & \multicolumn{4}{|c|}{ Oxide Core } \\
\hline & \multicolumn{2}{|c|}{ Startup } & \multicolumn{2}{|c|}{ Recycle } & \multicolumn{2}{|c|}{ Startup } & \multicolumn{2}{|c|}{ Recycle } \\
\hline & BOEC & EOEC & BOEC & EOEC & BOEC & EOEC & BOEC & EOEC \\
\hline 15 & 39.5 & 40.2 & 37.7 & 37.8 & 38.8 & 39.3 & 36.4 & 36.2 \\
\hline 14 & 35.2 & 35.5 & 33.6 & 33.4 & 34.3 & 34.6 & 32.4 & 31.9 \\
\hline 14 & 36.1 & 37.0 & 34.5 & 34.9 & 35.2 & 35.9 & 33.1 & 33.2 \\
\hline 13 & 32.0 & 32.6 & 30.6 & 30.7 & 31.1 & 31.6 & 29.3 & 29.2 \\
\hline 4 & 9.5 & 10.4 & 9.2 & 10.1 & 8.7 & 9.4 & 8.1 & 8.9 \\
\hline 3 & 7.5 & 8.2 & 7.2 & 7.9 & 6.9 & 7.4 & 6.5 & 7.0 \\
\hline 3 & 6.9 & 7.6 & 6.8 & 7.3 & 6.2 & 6.8 & 5.8 & 6.4 \\
\hline & 4.4 & 4.7 & 4.1 & 4.4 & 4.4 & 4.7 & 4.1 & 4.3 \\
\hline & 2.6 & 2.8 & 2.5 & 2.7 & 2.4 & 2.7 & 2.3 & 2.5 \\
\hline
\end{tabular}



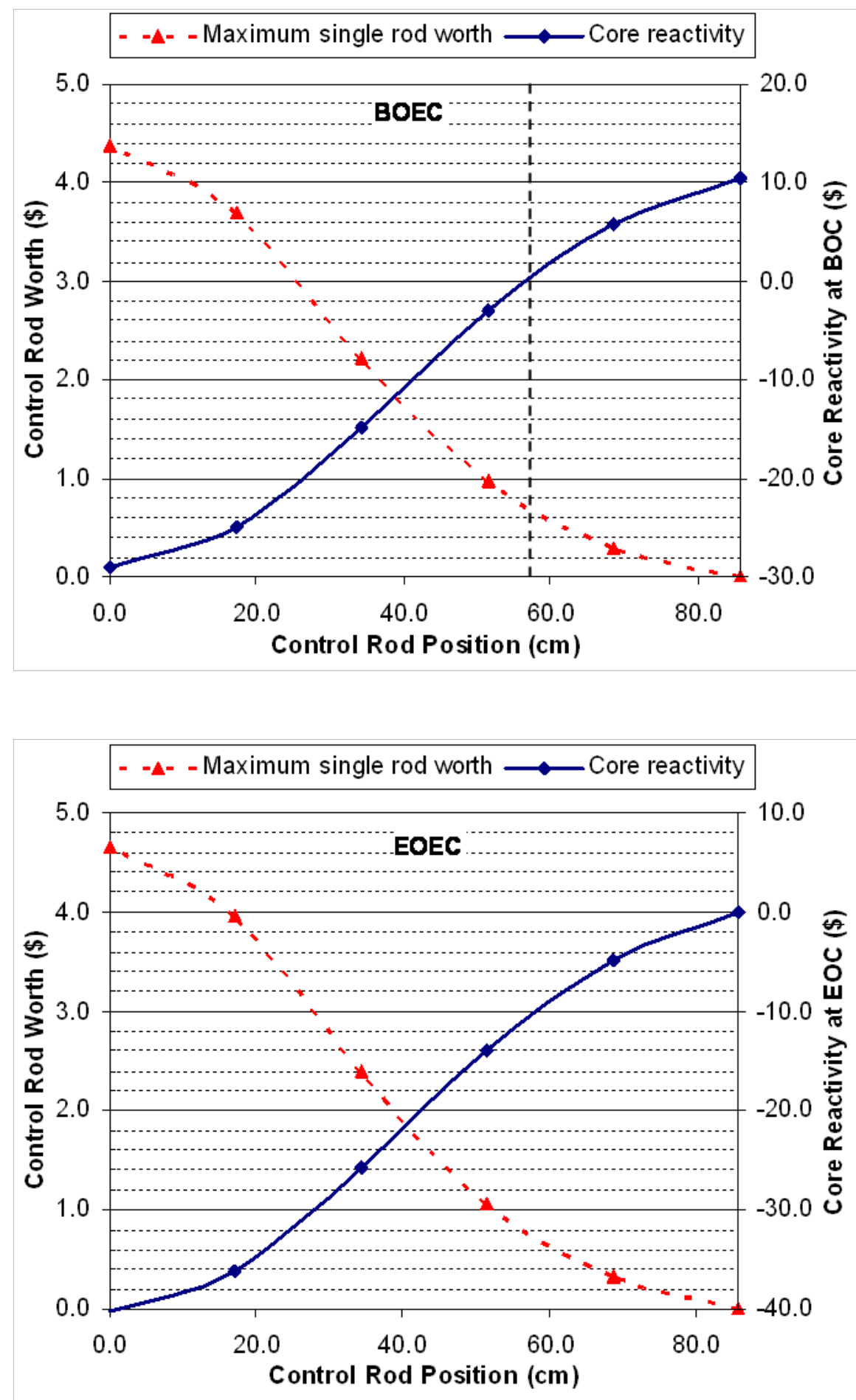

Figure II.1-19 Reactivity Worth of Primary Control System of Startup Metal Core 

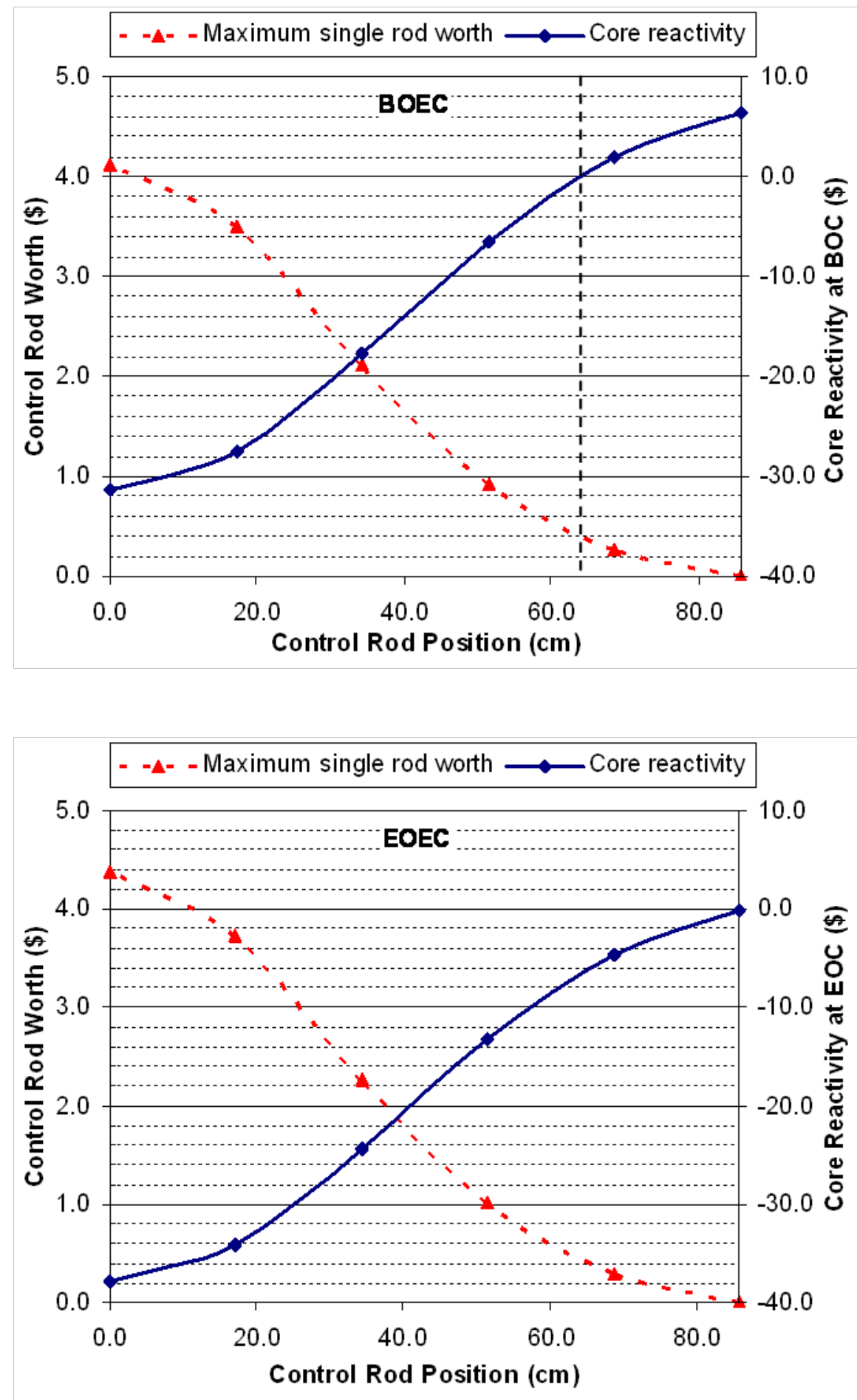

Figure II.1-20 Reactivity Worth of Primary Control System of Recycled Metal Core 

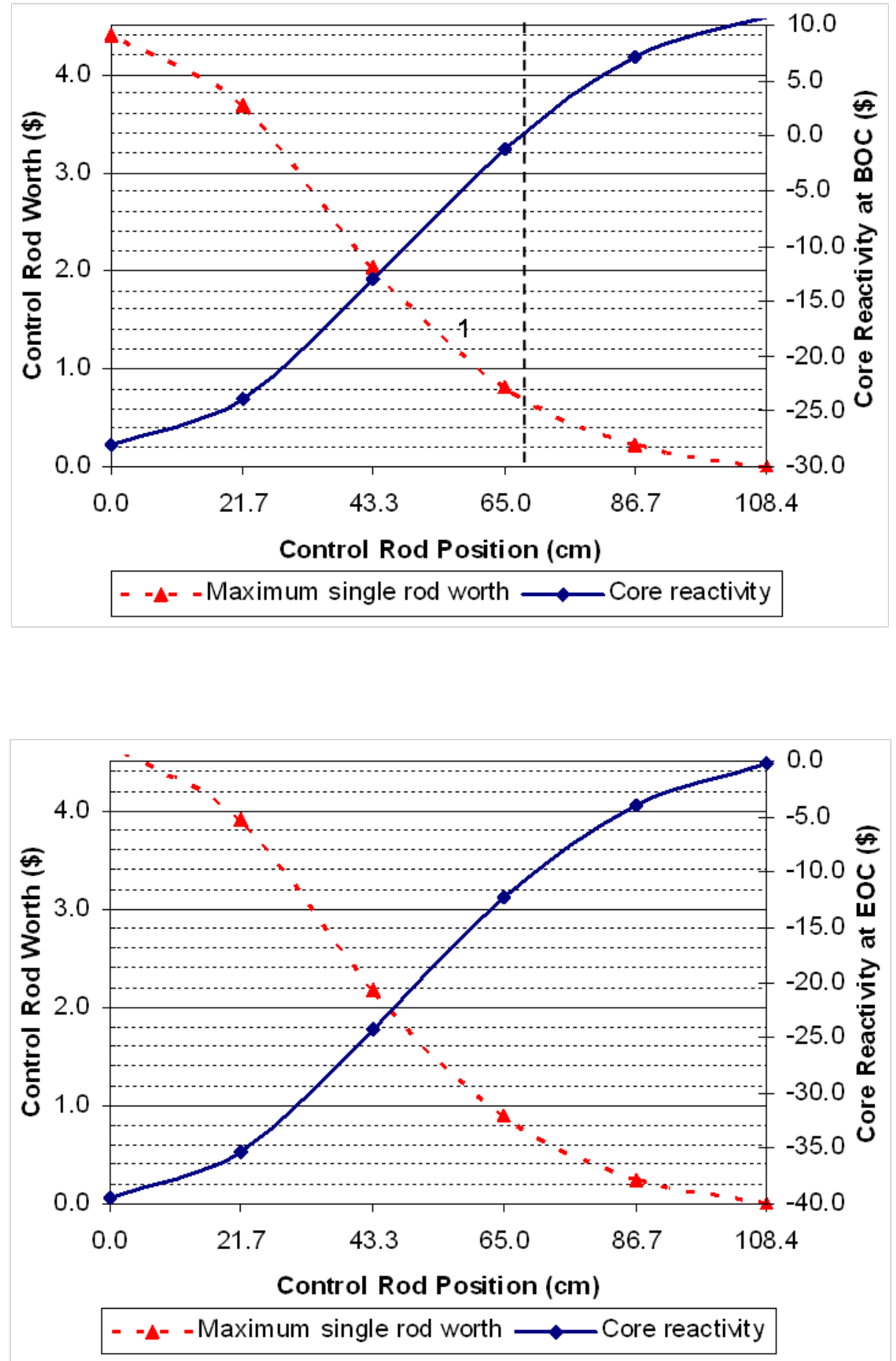

Figure II.1-21 Reactivity Worth of Primary Control System of Startup Oxide Core 

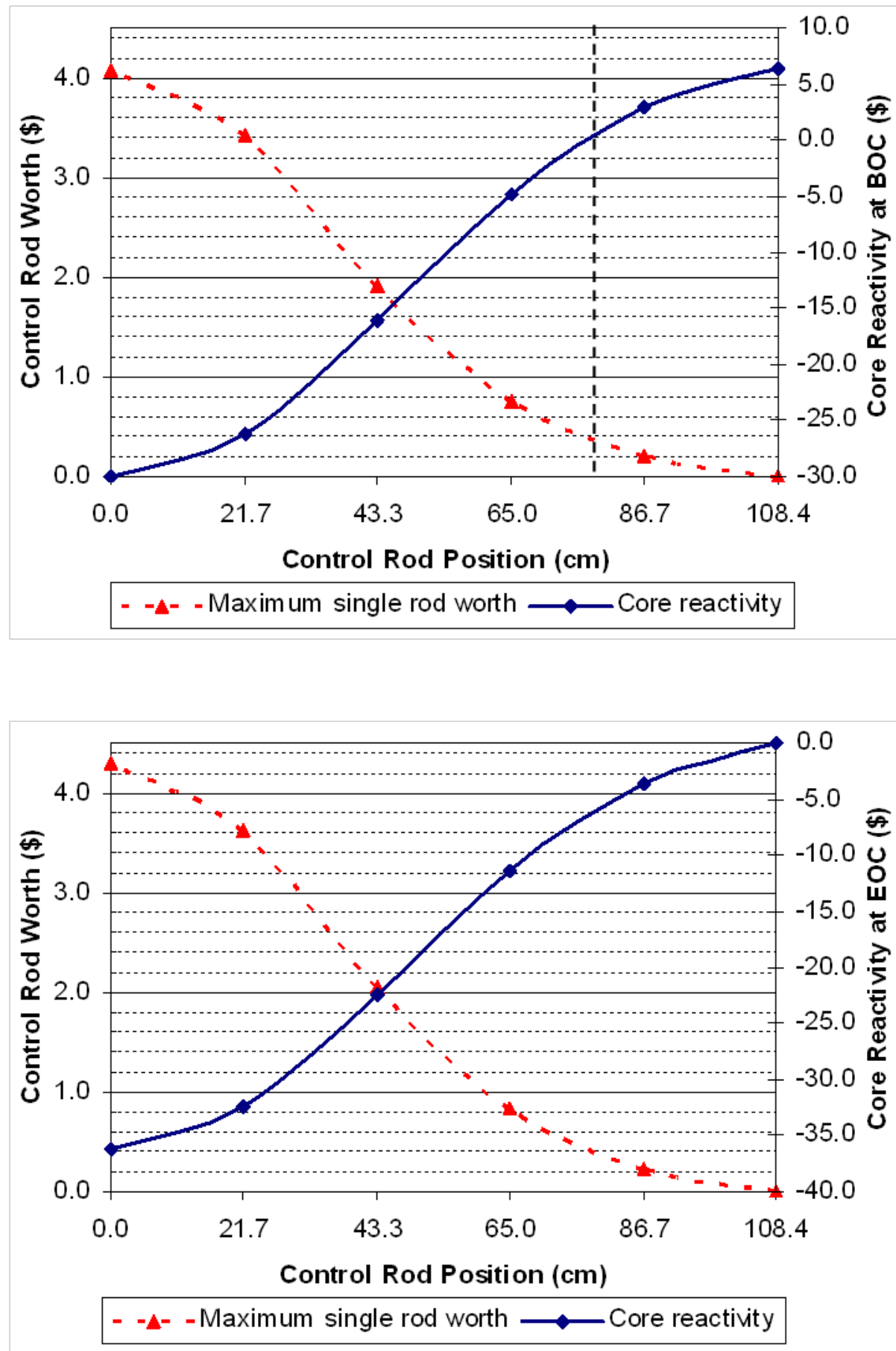

Figure II.1-22 Reactivity Worth of Primary Control System of Recycled Oxide Core 
Table II.1-8 Temperature and Power Defects (\$)

\begin{tabular}{|c|c|c|c|c|c|c|c|c|}
\hline & \multicolumn{4}{|c|}{ Metal Core } & \multicolumn{4}{|c|}{ Oxide Core } \\
\hline & \multicolumn{2}{|c|}{ Start-up } & \multicolumn{2}{|c|}{ Recycle } & \multicolumn{2}{|c|}{ Start-up } & \multicolumn{2}{|c|}{ Recycle } \\
\hline & BOEC & EOEC & BOEC & EOEC & BOEC & EOEC & BOEC & EOEC \\
\hline \multicolumn{9}{|c|}{ Hot full power to hot standby } \\
\hline Doppler & 0.30 & 0.29 & 0.23 & 0.23 & 0.92 & 0.94 & 0.69 & 0.69 \\
\hline Axial expansion & 0.04 & 0.04 & 0.05 & 0.04 & 0.03 & 0.04 & 0.04 & 0.04 \\
\hline Radial expansion & 0.30 & 0.30 & 0.30 & 0.30 & 0.24 & 0.23 & 0.24 & 0.23 \\
\hline Sodium density & -0.09 & -0.10 & -0.12 & -0.13 & -0.08 & -0.09 & -0.11 & -0.12 \\
\hline Total & 0.55 & 0.55 & 0.46 & 0.45 & 1.12 & 1.12 & 0.85 & 0.84 \\
\hline \multicolumn{9}{|c|}{ Hot standby to refueling } \\
\hline Doppler & 0.20 & 0.19 & 0.15 & 0.15 & 0.24 & 0.24 & 0.18 & 0.18 \\
\hline Axial expansion & 0.07 & 0.08 & 0.10 & 0.08 & 0.06 & 0.08 & 0.08 & 0.08 \\
\hline Radial expansion & 0.58 & 0.58 & 0.58 & 0.57 & 0.47 & 0.45 & 0.47 & 0.45 \\
\hline Sodium density & -0.17 & -0.19 & -0.23 & -0.25 & -0.16 & -0.18 & -0.22 & -0.24 \\
\hline Total & 0.68 & 0.66 & 0.59 & 0.56 & 0.61 & 0.59 & 0.49 & 0.47 \\
\hline
\end{tabular}


Table II.1-9 Shutdown Margin of Primary Control System (\$)

\begin{tabular}{|c|c|c|c|c|c|c|c|c|}
\hline & \multicolumn{4}{|c|}{ Metal Core } & \multicolumn{4}{|c|}{ Oxide Core } \\
\hline & \multicolumn{2}{|c|}{ Startup } & \multicolumn{2}{|c|}{ Recycle } & \multicolumn{2}{|c|}{ Startup } & \multicolumn{2}{|c|}{ Recycle } \\
\hline & BOEC & EOEC & BOEC & EOEC & BOEC & EOEC & BOEC & EOEC \\
\hline Number of control assemblies & 15 & 15 & 15 & 15 & 15 & 15 & 15 & 15 \\
\hline Total worth & 39.5 & 40.2 & 37.7 & 37.8 & 38.8 & 39.3 & 36.4 & 36.2 \\
\hline Worth with one stuck assembly & 35.2 & 35.5 & 33.6 & 33.4 & 34.3 & 34.6 & 32.4 & 31.9 \\
\hline Reactivity control requirement & 16.9 & 4.0 & 12.3 & 3.8 & 17.5 & 4.5 & 11.9 & 4.1 \\
\hline - Temperature defect & 1.23 & 1.20 & 1.05 & 1.01 & 1.73 & 1.71 & 1.34 & 1.30 \\
\hline - HFP to hot standby & 0.55 & 0.54 & 0.46 & 0.45 & 1.12 & 1.12 & 0.85 & 0.84 \\
\hline - Hot standby to refueling & 0.68 & 0.66 & 0.59 & 0.56 & 0.61 & 0.59 & 0.49 & 0.47 \\
\hline - Burnup reactivity loss & 10.38 & & 6.43 & & 10.83 & & 6.35 & \\
\hline - Fuel axial growth (5\%) & 0.45 & & 0.58 & & & & & \\
\hline - Overpower margin (3\%) & 0.02 & 0.02 & 0.01 & 0.01 & 0.03 & 0.03 & 0.03 & 0.03 \\
\hline - Reactivity fault & 0.70 & & 0.39 & & 0.71 & & 0.37 & \\
\hline - Uncertainties (RMS) & 2.34 & 1.43 & 1.99 & 1.43 & 2.40 & 1.45 & 1.99 & 1.44 \\
\hline - Temperature defect (20\%) & 0.25 & 0.24 & 0.21 & 0.20 & 0.35 & 0.34 & 0.27 & 0.26 \\
\hline - Burnup reactivity (15\%) & 1.56 & & 0.96 & & 1.62 & & 0.95 & \\
\hline - Fuel axial growth (20\%) & 0.01 & & 0.01 & & 0.00 & & 0.01 & \\
\hline - Criticality prediction & 1.00 & 1.00 & 1.00 & 1.00 & 1.00 & 1.00 & 1.00 & 1.00 \\
\hline - Fissile loading & 1.00 & 1.00 & 1.00 & 1.00 & 1.00 & 1.00 & 1.00 & 1.00 \\
\hline - Refueling & 1.00 & & 1.00 & & 1.00 & & 1.00 & \\
\hline - Other margin ${ }^{\text {a) }}$ & 1.80 & 1.30 & 1.80 & 1.30 & 1.80 & 1.30 & 1.80 & 1.30 \\
\hline Shutdown margin & 18.2 & 31.5 & 21.3 & 29.6 & 16.8 & 30.1 & 20.5 & 27.8 \\
\hline
\end{tabular}

a) Additional margins such as shutdown margin (1\$), ATWS reactivity (0.3\$), and fast runback margin (0.5\$). 
Table II.1-10 Shutdown Margin of Secondary Control System (\$)

\begin{tabular}{|c|c|c|c|c|c|c|c|c|}
\hline & \multicolumn{4}{|c|}{ Metal Core } & \multicolumn{4}{|c|}{ Oxide Core } \\
\hline & \multicolumn{2}{|c|}{ Startup } & \multicolumn{2}{|c|}{ Recycle } & \multicolumn{2}{|c|}{ Startup } & \multicolumn{2}{|c|}{ Recycle } \\
\hline & $\mathrm{BOE}$ & EOE & BOE & EOE & $\mathrm{BOE}$ & $\mathrm{EOE}$ & $\mathrm{BOE}$ & $\mathrm{EOE}$ \\
\hline & $\mathrm{C}$ & $\mathrm{C}$ & $\mathrm{C}$ & $\mathrm{C}$ & C & $\mathrm{C}$ & C & $\mathrm{C}$ \\
\hline $\begin{array}{l}\text { Number of control } \\
\text { assemblies }\end{array}$ & 4 & 4 & 4 & 4 & 4 & 4 & 4 & 4 \\
\hline Total worth & 9.5 & 10.4 & 9.2 & 10.1 & 8.7 & 9.4 & 8.1 & 8.9 \\
\hline $\begin{array}{l}\text { Worth with one stuck } \\
\text { assembly }\end{array}$ & 6.9 & 7.6 & 6.7 & 7.3 & 6.2 & 6.8 & 5.8 & 6.4 \\
\hline Control requirement & 1.6 & 1.6 & 1.4 & 1.3 & 2.4 & 2.4 & 1.9 & 1.8 \\
\hline - Temperature defect & 1.23 & 1.20 & 1.05 & 1.01 & 1.73 & 1.71 & 1.34 & 1.30 \\
\hline - Overpower margin (30\%) & 0.16 & 0.16 & 0.14 & 0.13 & 0.34 & 0.34 & 0.26 & 0.25 \\
\hline $\begin{array}{l}\text { - Temp defect uncertainty } \\
(20 \%)\end{array}$ & 0.25 & 0.24 & 0.21 & 0.20 & 0.35 & 0.34 & 0.27 & 0.26 \\
\hline Shutdown margin & 5.3 & 6.0 & 5.3 & 6.0 & 3.8 & 4.4 & 3.9 & 4.6 \\
\hline
\end{tabular}


The shutdown margins of primary and secondary control systems were determined with the assumption that the most reactive assembly is stuck. The minimum shutdown margin of the primary system is $\sim 18 \$$ for the metal core and $\sim 17 \$$ for the oxide core. The minimum shutdown margin of the secondary control system is $\sim 5$ for the metal core and $\sim 4$ for the oxide core. These shutdown margins are more than adequate.

The control-rod driveline expansion coefficients for reactivity feedback were estimated from Figures II.1-19 to II.1-22. Control rod expansion coefficients are governed principally by the total rod worth and the insertion depth of the assemblies. For the metal core, the calculated control-rod expansion coefficient of the startup core is $49-51 \mathrm{\$} / \mathrm{cm}$ at BOEC and $26-28 \mathrm{\$} / \mathrm{cm}$ at EOEC. For the oxide core, the coefficients are reduced because of elevated control assembly position; $35-38 థ / \mathrm{cm}$ at BOEC and $16-18 థ / \mathrm{cm}$ at EOEC.

\section{References}

1. G. L. Hofman, L. C. Walters, and T. H. Bauer, "Metallic Fast Reactor Fuels," Progress in Nuclear Energy, 31, 83 (1997).

2. U. P. Nayak, A. Boltax, R. J. Skalka and A. Biancheria, "An Analytical Comparison of the Irradiation Behavior of Fast Reactor Carbide and Oxide Fuel Pins," Proc. Topical meeting Advanced LMFBR Fuel, Tucson, AZ. October 10-13, 1977.

3. R. D. Leggett and L. C. Walters, "Status of LMR Fuel Development in the United States of America," Journal of Nuclear Materials, 204, 23 (1993).

4. L. L. Briggs, L. K. Chang, and D. J. Hill, Argonne National Laboratory, unpublished information, March 1995.

5. E. E. Feldman, "The Influence of Cyclic Fluid Temperatures on the Thermal Stresses Induced in Adjacent Structures," Intra-laboratory Memo, Argonne National Laboratory, March 10, 1975.

6. Argonne National Laboratory, unpublished information, September 1990.

7. K. L. Derstine, "DIF3D: A Code to Solve One-, Two-, and Three-Dimensional Finite Difference Diffusion Theory Problems," ANL-82-64, Argonne National Laboratory (1984).

8. B. J. Toppel, "A User's Guide to the REBUS-3 Fuel Cycle Analysis Capability," ANL-83-2, Argonne National Laboratory (1983).

9. "NSEC No. 350 ETOE-2 Documentation," National Energy Software Center Note 83-84, August 25, 1983.

10. H. Henryson II, B. J. Toppel, and C. G. Stenberg, "MC2-2: A Code to Calculate Fast Neutron Spectra and Multi-group Cross Sections," ANL-8144, Argonne National Laboratory (1976).

11. W. M. Stacey, Jr., et al, "A New Space-Dependent Fast Neutron Multigroup Cross Section Preparation Capability,” Trans. Am. Nucl. Soc., 15, 292 (1972). 
12. R. D. Lawrence, "The DIF3D Nodal Neutronics Option for Two- and ThreeDimensional Diffusion Theory Calculations in Hexagonal Geometry,” ANL-83-1, Argonne National Laboratory (1983).

13. C. H. Adams, "Specifications for VARI3D - A Multidimensional Reactor Design Sensitivity Code,” FRA-TM-74, Argonne National Laboratory (1975).

14. R. E. MacFarlane and D. W. Muir, "The NJOY Nuclear Data Processing System,” Los Alamos National Laboratory, LA-12740-M, 1994.

15. R. N. Hill, “Coupled Neutron-Gamma Heating Calculations,” Intra-Laboratory Memo, Argonne National Laboratory, February 2, 1988.

16. W. S. Yang, “Fortran 77 Version of SE2-ANL," Intra-Laboratory Memo, Argonne National Laboratory, March 3, 1993.

17. K. L. Basehore and N. E. Todreas, "SUPERENERGY-2: A Multiassembly SteadyState Computer Code for LMFBR Core Thermal-Hydraulic Analysis,” PNL-3379, August 1980.

18. R. B. Vilim, "Reactor Hot Spot Analysis,” FRA-TM-152, Argonne National Laboratory, August 1985.

19. R. B. Vilim, “A Simple Fuel Pin Model for SE2-ANL,” Intra-laboratory Memo, May 26, 1987.

20. G. Croff, "ORIGEN-2 - A Revised and Updated Version of the Oak Ridge Isotope Generation and Depletion Code,” ORNL-5621, Oak Ridge National Laboratory (1980). 


\section{II.2 Reactor Enclosure System}

\section{II.2.1 Reactor Vessel}

The reactor vessel assembly is comprised of the reactor vessel, top closure head (or deck), the rotatable plugs. the guard vessel, the vessel/deck support structure, the core support structure, core barrel, thermal barriers, shielding, the redan, and other permanent internal structures. The design is based on the experience of the reactor vessels used for the EBR-II, Phenix, PFR, and SuperPhenix LMR reactor plants. A major difference, however, is that the design concept shown here separates the reactor and guard vessels, and also employs a conical ring support structure that avoids the need for welding dissimilar metals. There are no penetrations in the reactor vessel; all equipment-IHXs, pumps, piping, instrumentation, cold traps, fuel handling equipment, and other components---penetrate the top closure head, or deck. The entire reactor vessel assembly is located in a steel-lined concrete cavity in the reactor containment building. The top of the reactor vessel deck is approximately at grade level. The reactor vessel assembly constitutes the primary sodium coolant system boundary that envelopes and supports the reactor core, reactor vessel internals, and all of the primary system components. There is an inert gas (argon) blanket between the reactor vessel deck and the bulk sodium free surface.

The design of the reactor vessel assembly incorporates appropriate measures and details that will facilitate fabrication, construction, and operational maintenance. The design incorporates recommendations made by an experienced reactor vessel fabricator on a similar concept developed at ANL. In addition, removal of any component for repair or replacement follows a common scheme, i.e., use of a heavy-shielded cask placed over the piece of equipment, sealed to the component support flange in the deck, and lifting the equipment up into the cask.

The arrangement of the reactor vessel assembly components is shown in Figures II.2-1 and II.2-2.

The reactor vessel assembly is designed to:

- Provide a very high level of reliability of primary sodium containment.

- Provide support for the reactor core, reactor vessel internals, primary system components, and certain components of the fuel handling system

- Accommodate high static loads at design temperatures.

- Accommodate loads from design basis natural hazards.

- Accommodate loads from design basis accidents, including dynamic loads and thermal transients.

- Minimize the dead load deflections and thermal distortions (e.g., bowing of the deck structure) to assure positive equipment alignment.

- Provide a structure that can be field-erected to stringent tolerances

- Ensure symmetrical radial thermal expansion of the reactor vessel assembly about its vertical center axis.

- Provide reliable positioning of supported components (e.g., pumps, IHXs, etc.). 


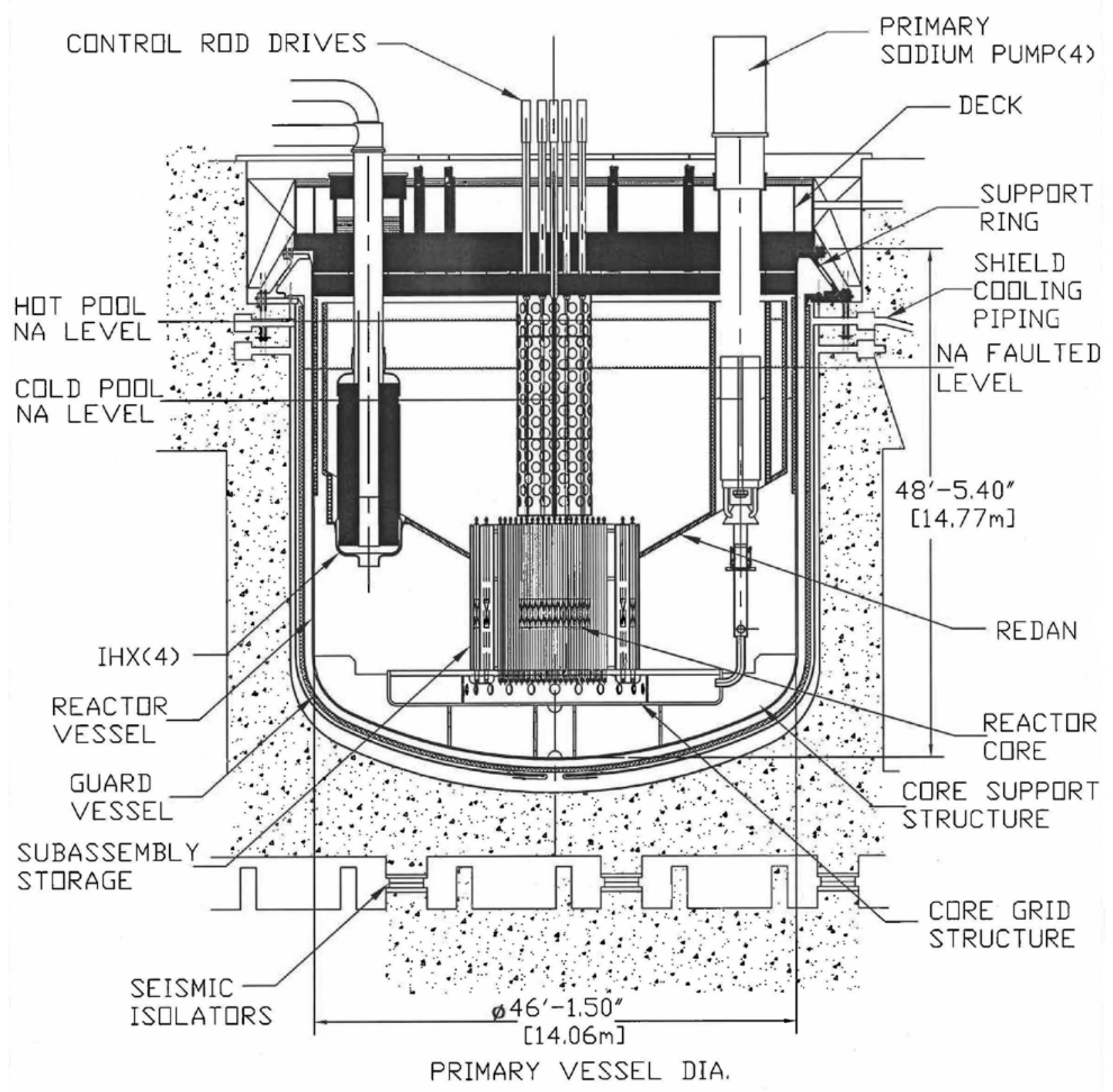

Figure II.2-1 Cross Sectional View of Primary System 


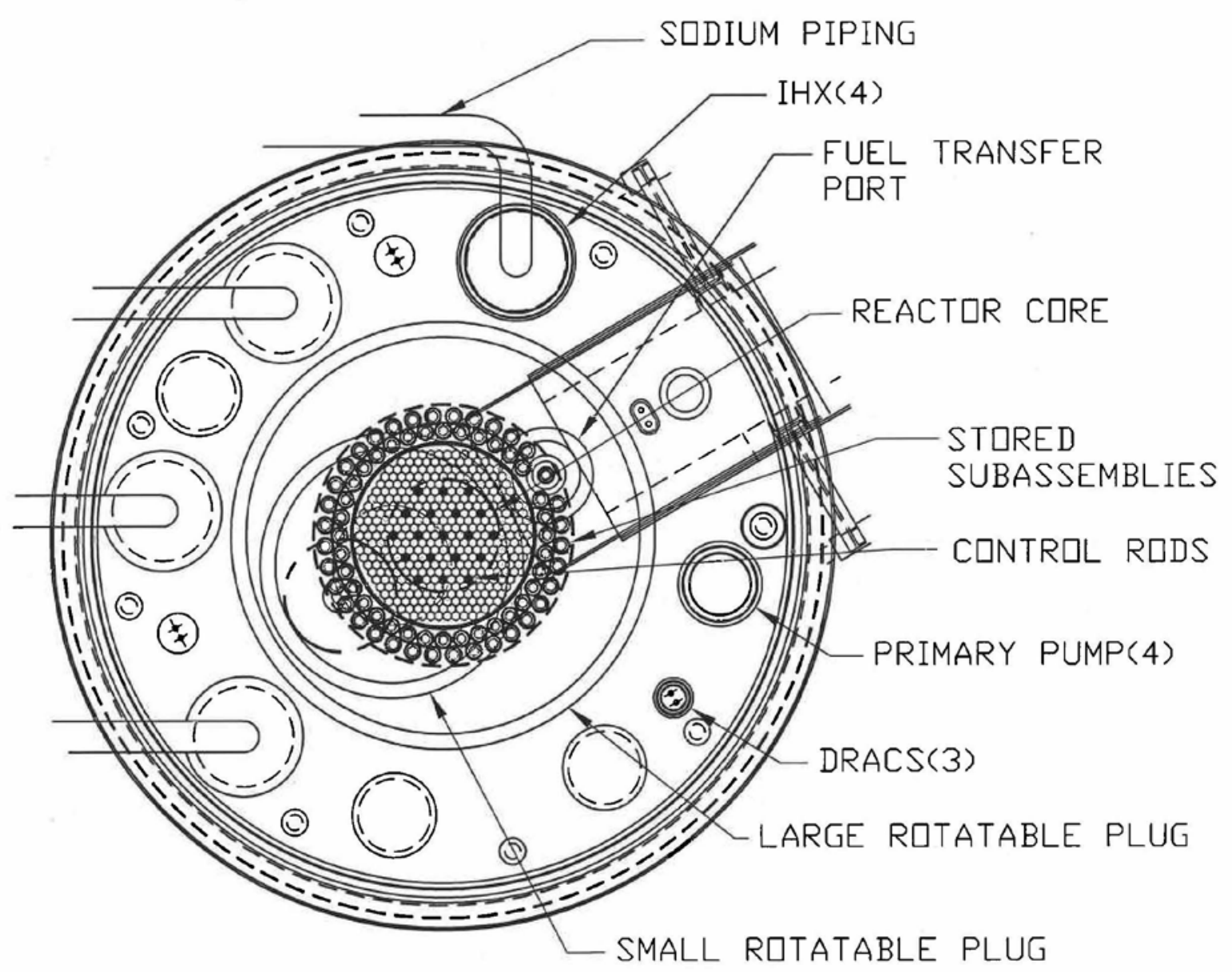

Figure II.2-2 Plan view of Primary System

\section{II.2.1 Reactor Vessel}

The reactor vessel is constructed of austenitic stainless steel, Type 304. Low carbon stainless steel will be specified for the formed bottom head of the reactor vessel. The cylindrical shell and bottom head have an inside diameter of $46 \mathrm{ft}-1 \frac{1}{2}$ in $(14.06 \mathrm{~m})$ and a thickness of 2 in $(5.08 \mathrm{~cm})$ for both the cylindrical shell and the torospherical bottom head ${ }^{2}$. The overall height of the reactor vessel is $48 \mathrm{ft}-5 \mathrm{1} / 2 \mathrm{in}(14.77 \mathrm{~m})$. The reactor vessel contains essentially all of the radioactive primary sodium coolant, except for that sodium contained in the sodium purification system. The reactor vessel is suspended at its top flange on the conical support ring (frustum) that also provides support for the RV deck. The use of bolting to attach the RV and the deck to the support frustum avoids the necessity of using bi-metallic welds to join the vessel and support.

\footnotetext{
${ }^{2}$ A torospherical head consists of a torodial transition piece connected to a spherical bottom shell. This unit is then attached to the reactor vessel vertical walls. A torospherical head is shallower than a spherical bottom head and thus will reduce the overall height depth of the reactor vessel.
} 
The height of the reactor vessel is established from the fuel assembly length, the intermediate heat exchanger (IHX) length, and the need to keep the IHX inlet covered by primary sodium in the event of a leak in the reactor vessel that would drain the primary sodium into the guard vessel, resulting in a drop in the sodium level. This "faulted" primary sodium level is indicated in Figure II.2-1. The diameter of the reactor vessel is established from the core diameter and IHX characteristics with respect to sodium hydraulic and heat transfer requirements and the requirement to have the capability to remove primary system components, such as the IHX, primary sodium pumps, and the direct reactor auxiliary cooling system (DRACS) heat exchangers.

The reactor vessel will be designed in accordance with the requirements of the ASME B\&PV Code, Section III, Division 1, Subsection NB-Class 1, Seismic Category I. ${ }^{3}$ In addition to the dead loads of the weight of the primary sodium, primary system equipment, and all loads from the core support structure, the design will meet the ASME Code requirements for normal and transient thermal loads, and from loads and displacements resulting from design basis earthquakes.

\section{II.2.2 Reactor Vessel Top Enclosure (Deck)}

The reactor vessel top enclosure (referred to as the deck), forms the top head of the reactor vessel. The major functions of the deck are: (1) to provide the upper boundary for the primary sodium coolant and cover gas; and, (2) to provide structural support for all of the equipment penetrating the top of the reactor vessel, including the rotatable plugs, IHXs, pumps, and the Direct Reactor Auxiliary Cooling System (DRACS). In turn, the deck transfers the weight of the reactor vessel and deck and all of their contents to the vessel/deck support, and then to the reactor cavity concrete structure. It also provides thermal and radiation shielding. Thus, the structural integrity of the deck is an important element of the overall safety of the reactor design.

The design goals and functions for the deck are:

- Accommodate all dead loads, including the weight of the deck and all supported equipment.

- To provide a high degree of structural redundancy.

- To provide a high level of structural stiffness, such that vertical deflections of equipment are acceptably low.

- To provide a leak-tight barrier between the primary sodium cover gas and the operating floor of the Reactor Containment Building.

- To provide adequate biological shielding to allow personnel access to the area above the reactor vessel deck structure.

- To provide for the effects of the Design Basis Earthquakes, for both horizontally and vertically applied loads.

${ }^{3}$ If it should happen that, under certain accident or upset conditions, the temperature of some regions of the reactor vessel exceeds $800 \mathrm{~F}$, the vessel will need to be designed to meet the requirements of Subsection NH of the ASME B\&PV Code, Section III. This may also lead to a decision to change the vessel material to Type 316 SST. 
- To accommodate thermal stresses and displacements resulting from the very large temperature drop across the deck.

- To provide thermal shielding between the top of the sodium cover gas and the deck structure.

- To provide a structure that is feasible to fabricate onsite, and a design that allows reasonable access for Inservice Inspection (ISI) during the life of the plant.

The design concept selected for this reference plant design is a welded stressed-skin, box type structure. It is a concept used on SuperPhenix and for some U.S. plant studies performed several years ago. The box-beam concept selected for this reference plant has several very desirable attributes:

- The box beam structure is highly redundant, i.e., failure of a local structural element does not lead to failure or collapse of the deck. In one study done by the General Electric Company (GE)4, they used this concept and found that analysis showed that complete failure of the bottom plate (or "skin") would not lead to collapse of the deck.

- Adequate stiffness is achieved with modest stress levels without resorting to massive structures that would be difficult to fabricate and erect.

- The design has considerable margin to accommodate accident conditions, such as abnormal cover gas pressures and loss of deck cooling.

- The concept is compatible with a simple conical support skirt which can accommodate both applied seismic loads and differences in temperature between the deck and the reactor building steel-lined walls.

- The design can accommodate the use of gas cooling in the interior of the deck structure.

- The design can accommodate access to important structural elements for ISI and maintenance.

- The design concept provides considerable flexibility in the arrangement of reactor components located on the deck.

- It appears that the concept has excellent potential for extrapolation to larger sizes required for large LMR plants. (Indeed, this concept was used on the French SuperPhenix reactor plant which was a 1200 MWe LMR plant. In addition, in the GE study cited above they concluded that the concept would be appropriate for LMR plants of at least 1500 MWe capacity.)

The current design of the deck is shown in Figure II.2-3. It has an outer diameter of $47 \mathrm{ft}-7 \mathrm{in}$ $(14.5 \mathrm{~m})$ and a depth of $8 \mathrm{ft}-2 \mathrm{in} .(2.49 \mathrm{~m})$. The material for the deck is SA-516 Grade 70 carbon steel. It is constructed of steel plates, rings, and penetration cylinders, all welded together to form a conservatively designed, highly redundant structure, that supports all applied loads (including seismic loads) with very small deflections. With the exception of the central cylindrical ring, all of the steel plates and rings are $1.5 \mathrm{in}(3.81 \mathrm{~cm})$ thick to avoid the need for onsite stress relieving.

\footnotetext{
4 “Pool-Type LMFBR Plant 1000 MWe, Phase A Design', by General Electric Company (GE), EPRI NP-646, April, 1978.
} 
The central ring is 6 in $(15.2 \mathrm{~cm})$ thick. The space inside the box structure is divided into many chambers by the ribs (vertical plates) that connect the top and bottom plates and the inner, outer rings (see Figure II.2-4). Under the imposed loadings, the ribs will take mainly the shear forces; whereas, the top and bottom plates are designed to take the bending moments. Allowable stress levels will be those from the ASME B\&PV Code, Section III, Division 1, Subsection NB (as applicable).

There are eleven major penetrations between the inner and outer rings. Also, there are several other small penetrations (not shown) for instrumentation, sodium purification piping, and other uses. The eleven major penetrations provide support for the IHXs, pumps, and DRACS, and have three different size diameters---76, 45, and 18 inches, $(193,114$, and $45.7 \mathrm{~cm})$ respectively. The inner ring of the deck is used to support the rotatable plugs. The ribs are discontinued when they intersect with the penetrations. The chambers will be filled with 3/8-inch $(0.95 \mathrm{~cm})$ steel balls for radiation shielding. To provide thermal shielding, a stack consisting of twelve horizontal $1 / 4$ inch $(0.64 \mathrm{~cm})$ steel plates, with a gap of 0.87 inch $(2.21 \mathrm{~cm})$ between adjacent plates, is hung from the deck bottom plate. In addition, on the top plate, there are several layers of thermal insulation to lower the temperature of the top area to allow for personnel access for maintenance and operations. This insulation also prevents the floor slab from touching the top plate directly.

A preliminary scoping stress analysis of the deck structure, given in the appendices to this report, shows that the design concept shown here is feasible and is expected to meet allowable stresses and deflections. 


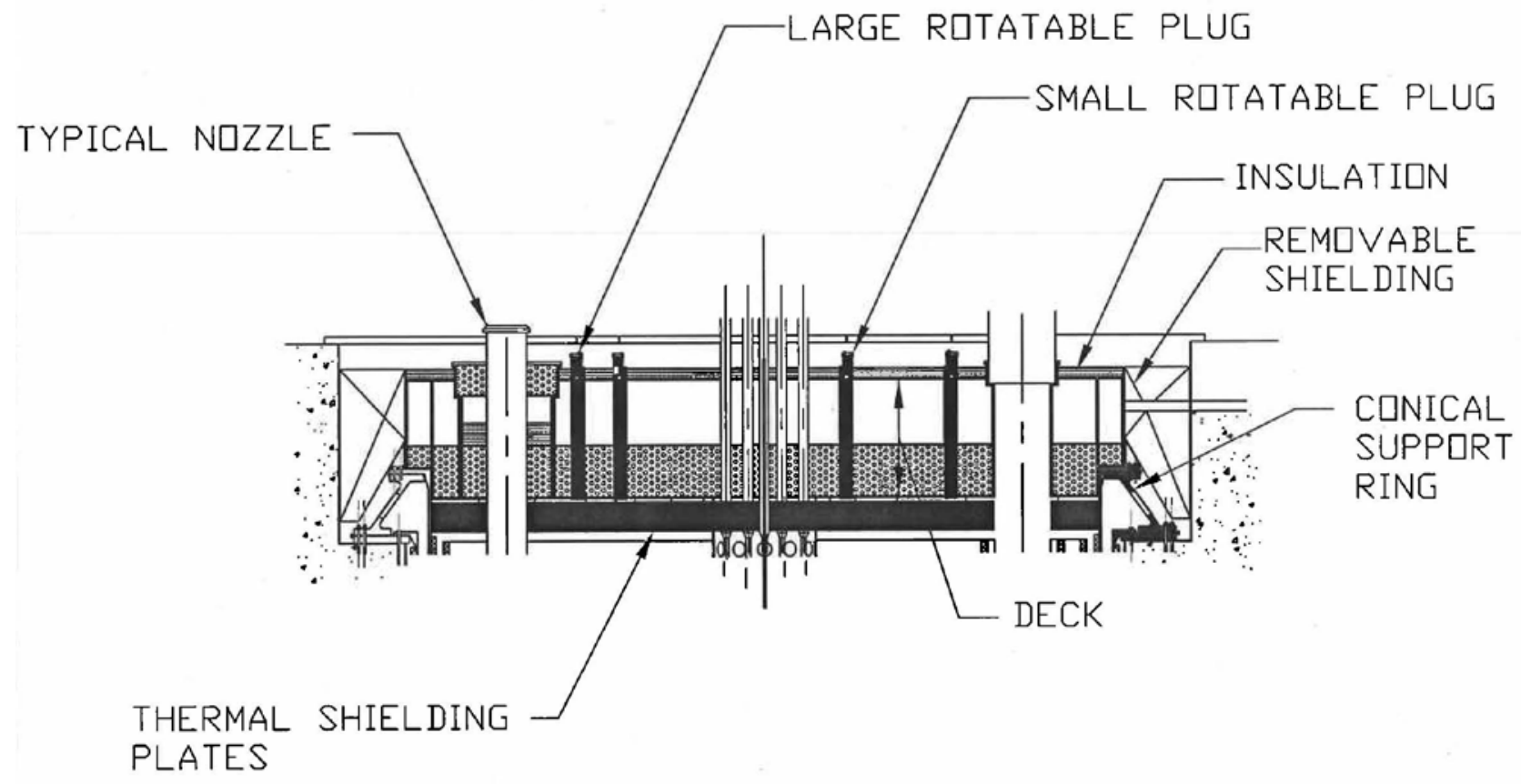

Figure II.2-3 Reactor Vessel Deck Cross Section

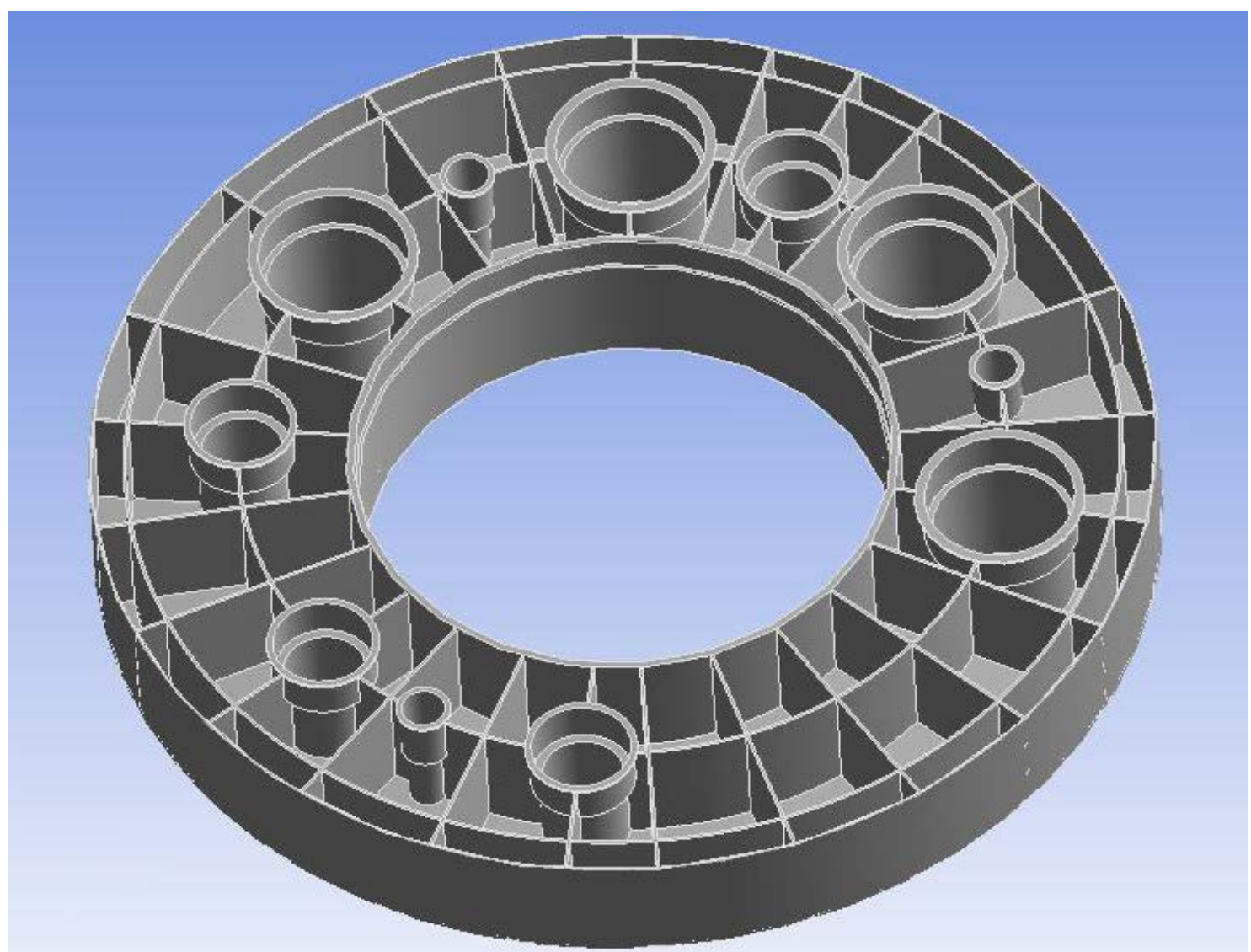

Figure II.2-4 Reactor Vessel Deck with top plate removed 


\section{II.2.3 Deck Cooling and Insulation}

The deck will be cooled to: (1) prevent the deck from having unacceptable thermal distortions and stresses caused by temperature gradients; (2) to maintain the bottom plate (skin) at temperatures low enough to allow personnel access for ISI and maintenance during plant shutdowns: and (3) to minimize radial thermal growth of the deck at the joint with the conical support ring. In conjunction with insulation on the top of the deck this cooling will function to reduce the temperature above the deck to allow personnel access at all times to the top area above the deck.

Air will be circulated within the deck structure to cool the interior of the deck. To allow the cooling gas to reach the bottom plate, a series of flat ducts separating the bottom plate and the steel shielding balls in the deck will be incorporated in the design. The design must provide this cooling while also allowing reasonable access for inspection of welds in the bottom plate. Future work is planned that will study the effects of loss of this deck cooling on temperatures of the bottom and top plates of the deck, in order to assure adequate heat removal from the primary sodium coolant.

\section{II.2.4 Deck Shielding}

Since the deck is designed to permit personnel access for ISI activities during plant shutdown conditions, it is necessary to provide adequate shielding within the pool of sodium to prevent neutron activation of the pump and IHX steel, and the intermediate sodium system. Steel shielding is placed around the core barrel and above the core, along with boron carbide around the core barrel. Local steel shielding will be placed around the pumps and IHXs, if necessary. Some shielding is provided by the laminated steel plate thermal shielding underneath the deck bottom plate.

Steel balls of 3/8 inch $(0.95 \mathrm{~cm})$ diameter are placed into each of the many chambers within the deck structure, to a depth of 42 inches $(106.7 \mathrm{~cm})$ to allow operations personnel access around the top of the deck at all times. These steel balls are removed from the chambers to allow personnel access for ISI of the interior of the deck during reactor shutdown.

\section{II.2.5 Rotatable Plugs}

The principal function of the rotatable plug is to serve as part of the fuel handling system. Their number and their size depend largely on the type of other components in the fuel handling chain. Specifically, the method used for in-vessel transfer of subassemblies and the location of the fuel transfer point determine the distance of lateral movement that the rotatable plugs have to provide. After consideration of various options, the fuel handling system described in Section II.7 was chosen as the reference technology. This system uses two rotatable plugs - a large rotatable plug and a small rotatable plug - and a straight-pull gripper mechanism with a transfer point to the ex-vessel vertical fuel unloading machine that is located on the deck structure, just radially outside the large rotatable plug. The two rotatable plugs, in concert with the gripper mechanism, have to provide a lateral movement that covers a circle that encompasses all subassemblies whether in the reactor or in the storage area around the core barrel. Further requirements affecting the rotatable plug size are derived from the reactor control drive pattern and the upper internals structure (UIS) design. This technology was successfully demonstrated in EBR-II. 
Design features regarding shielding arrangements and the interfaces among the rotatable plugs are essentially the same as described for the reactor vessel enclosure. The two plugs have a stressed-skin box-beam structure and contain 42 in. $(106.7 \mathrm{~cm})$ of 3/8 in diameter steel balls for biological shielding, which design is virtually identical to the deck structure. The underside of each plug has laminated steel sheet reflective sandwich insulation which also is similar to that used on the underside of the deck structure.. Plug interfaces are provided with elastomer seals. The UIS is attached to the bottom of the rotatable plug. The small rotatable plug supports the gripper mechanism. The rotatable plugs incorporate devices for locking their positions during reactor operation, and devices against lift-off due to excessive reactor vessel pressures. Equipment associated with, or part of the rotatable plugs require a variety of services-connections for electricity, control, instrumentation, inert gas etc., that are provided via the festoon cable system (not shown).

The large rotatable plug supports part of the festoon cable system and the eccentrically located small rotatable plug. The small rotatable plug supports the UIS. The UIS assembly penetrates the small rotatable plug through a large nozzle, forming a shielded plug that is supported from a flange on the nozzle. The UIS plug in turn is penetrated by the control drive shafts, the instrument conduits and the seismic key retracting mechanism. The large rotatable plug supports the vertical push-pull IVTM.

\section{II.2.6 Reactor Vessel and Deck Support Structure}

The current design of the reactor vessel and deck support structure is a conical frustum made of carbon steel, SA-516 Grade 70. (See Figure II.2.5). This structure provides support for the reactor vessel, its internals, and the reactor deck. The conical shell (frustum) has top and bottom flanges of 21 inches $(533.4 \mathrm{~mm})$ in width and 6 inches $(152.4 \mathrm{~mm})$ in thickness. The thickness of the conical shell is 4 inches $(101.6 \mathrm{~mm})$. The top flange provides the bearing surface for the reactor vessel and deck, and the bottom flange provides the bearing surface for transferring the loads to the reactor cavity concrete structure. If necessary, "Lubrite" sliding plates will be inserted between the RV flange and deck support flange.

The use of a bolted joint and common conical support skirt for the reactor vessel and deck at the deck-vessel interface avoids the need for a bi-metallic structural weld between these components. It is recognized that this bolted joint and conical ring presents special alignment and erection problems. An almost identical design was employed in an ANL study for a 1000 MWe LMR plant conducted in $1982^{5}$. In a detailed review of that design concept, the Chicago Bridge and Iron Company (CB\&I), a highly qualified fabricator of nuclear vessels, concluded that not only was the design feasible, but that is represented an improvement over many of the alternative approaches for the vessel/deck support. CB\&I stated that the fully machined support ring could be set into place with a single lift, aligned, leveled, and completed without the problems associated with in-containment welding, scaffolding, and temporary supports.

This conical ring concept has also been proposed in several LMR plant studies performed by GE and others (see Ref. 1). The present concept for the ABR1000 goes one step further. Rather than placing the bolting inside of the deck structure as used in previous designs, the concept presented here incorporates reinforcing gussets assemblies welded to the outer ring surface of the

5 “Conceptual Design Report-1000 MWe Pool-Type LMFBR Plant”, Argonne National Laboratory, July, 1982. 
deck and bolted to the top flange of the conical ring, thus allowing greatly improved access to all of the bolting. This not only facilitates construction but also benefits ISI and maintenance activities over the life of the plant. The gussets also provide positive structural resistance for seismic loads imposed on the support ring. An additional benefit obtains from the fact that by extending the outer diameter of the conical support ring it is possible to support the ring, and all of its loads, directly on the reinforced concrete cavity wall without the need for special heavy steel inserts used on some of the previous LMR design studies. There is an inert gas (argon) blanket between the reactor vessel cover and the bulk sodium free surface.

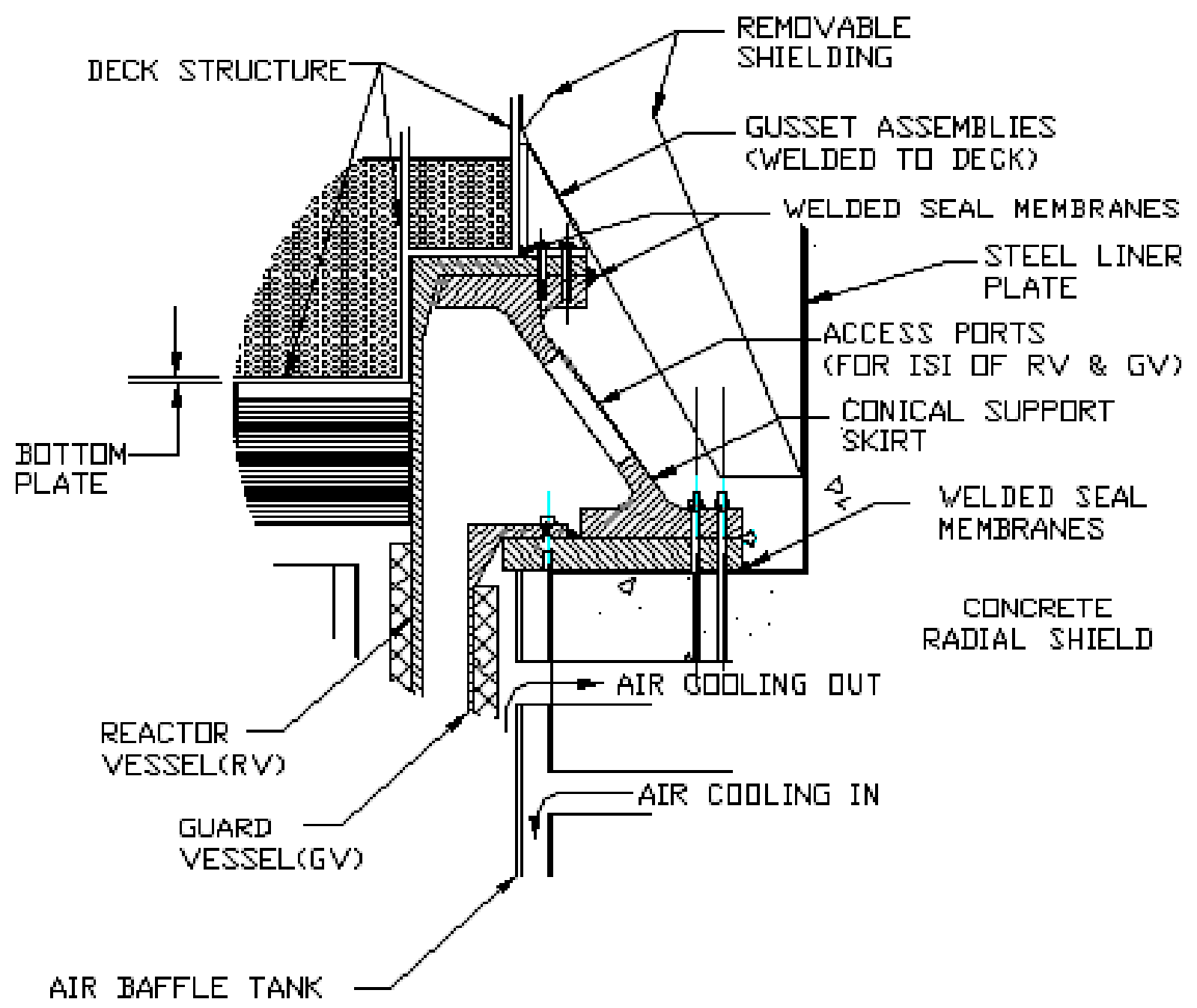

Figure II.2-5 Reactor Vessel/Deck Support Structure 


\section{II.2.7 Guard Vessel}

The guard vessel has one major function: to contain the primary sodium coolant in the very unlikely event that it should leak from the reactor vessel. No ties, attachments, penetrations, or equipment are permitted on the guard vessel wall; except perhaps for tracks used for remote ISI of the reactor vessel. The diameter of the guard vessel is $47 \mathrm{ft}-11.5$ in $(14.62 \mathrm{~m})$, and it has a height of $45 \mathrm{ft}-0.5$ in $(13.73 \mathrm{~m})$. The entire guard vessel is 1 in $(2.54 \mathrm{~cm})$ thick. The guard vessel is supported at its top by a flange bearing on a ledge in the reactor cavity wall. Suitable welded membranes will be used to seal the gas space between the guard and reactor vessels and to seal the joints between the reactor vessels and deck and conical support skirt. The guard vessel is completely independent of the reactor vessel and the deck. The annular gap between the guard vessel and the reactor vessel is 18 inches $(45.7 \mathrm{~cm})$ to allow for ISI of both vessels. This gap size results in a feasible size for access of remote viewing equipment, taking into account the need to assure that the faulted sodium level remains above the top of the IHXs, and to allow for fabrication and erection tolerances needed in construction. Carbon steel will be used for the guard vessel to permit the use of a magnetic attachment of a remote device used during the ISI of the reactor and guard vessels, and to reduce plant capital cost.

The guard vessel provides the secondary containment for the primary sodium in the very unlikely event that the reactor vessel develops a leak. The guard vessel is sized such that the gap (8.5 inches) between it and the reactor vessel is:

1. Wide enough to accommodate in-service inspection devices

2. Narrow enough to prevent the primary sodium from dropping to an unacceptable level within the reactor vessel if the reactor vessel should develop a leak. The sodium level must remain high enough to keep the IHX and DRACS heat exchanger inlets covered to provide a path for natural convection cooling of the core during a leak in the reactor vessel.

Insulation is provided on the exterior of the guard vessel to reduce the heat lost to the guard vessel cooling system. A guard cooling shroud is provided on the outside of the insulation that forces air to flow around the supporting concrete structure and up over the guard vessel insulation to prevent overheating of the concrete support structure during normal and off-normal conditions.

\section{II.2.8 Redan ${ }^{6}$}

The core barrel and redan assembly, illustrated in Figure II.2-6 is a single integrated unit that provides the internal structure for the reactor core assemblies and provides a barrier between the hot and cold sodium pools. The core barrel is a right circular cylinder fabricated from stainless steel. It is attached to the inlet plenum and lower support structure. It also provides support for the core restraint system. The upper portion of the redan consists of a conical shell and

\footnotetext{
6 The term "Redan” is a French word for "projection” or "salient” in military terms, and is a term related to fortifications. It is a structure in a V-shaped salient angle toward an expected attack. A number of French fast reactor designs use redans as the barrier between the hot and cold sodium within the reactor vessel.
} 
cylindrical shell. The primary sodium pumps and the IHXs penetrate the redan, but are sealed to avoid hot and cold sodium from mixing.

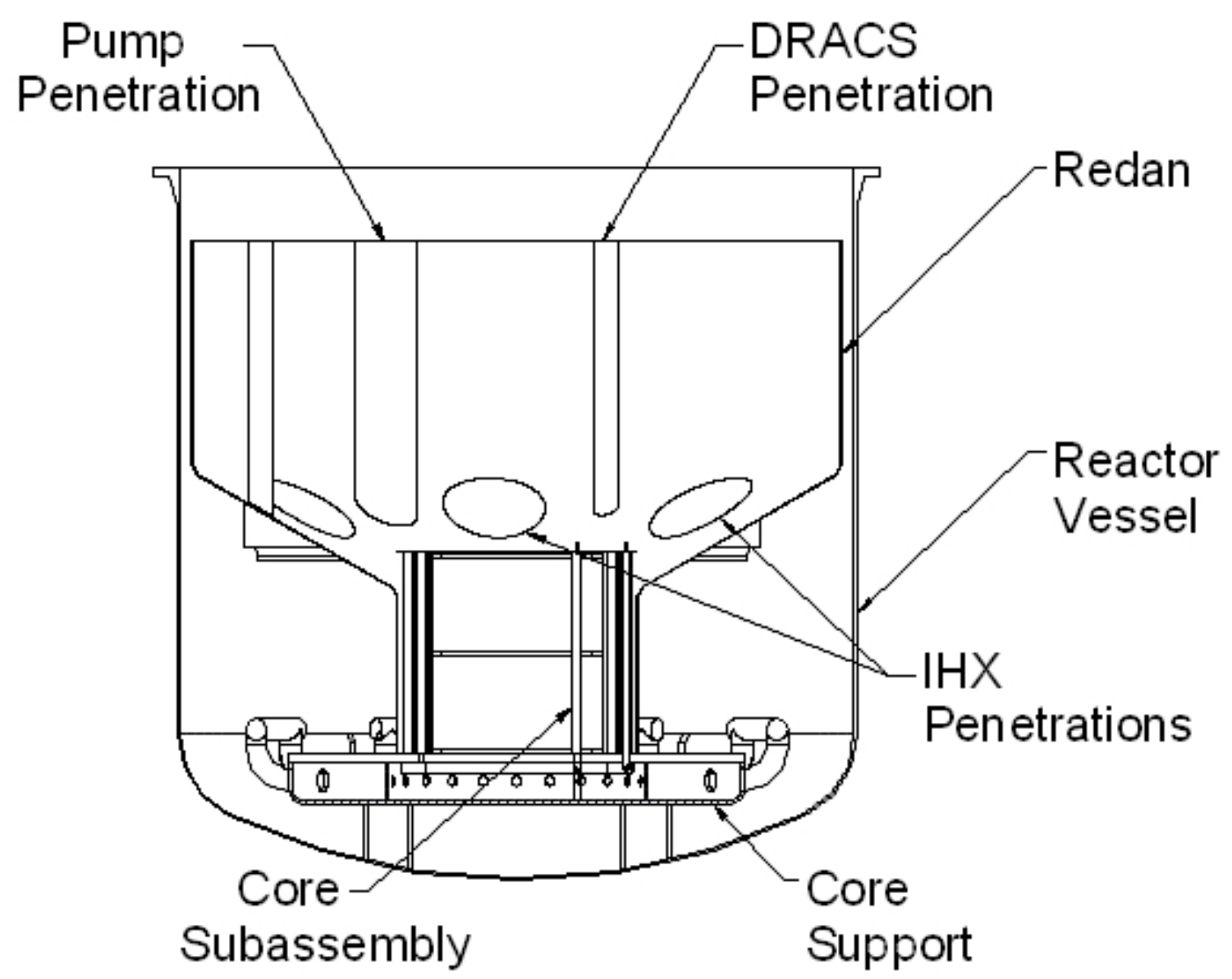

Figure II.2-6 Schematic of Redan Structure

The redan is a single integrated unit that separates the hot sodium pool from the cold sodium pool, and provides the flow path of the hot sodium from the discharge of the reactor core to the inlet of the intermediate heat exchanger. It consists of multiple formed plates welded together to form a shell that surrounds the intermediate heat exchangers and the upper internal structure. The redan is supported vertically and seal welded to the core barrel. It is essentially a cylindrical/conical vessel, but without either a top head or bottom head. The intermediate heat exchangers and upper internal structure are located within the redan. The primary pumps and DRACS heat exchangers are located in cylindrical shells (that separate the hot sodium from the cold sodium) in the conical portion of the redan. The redan is one of the permanent structures within the primary reactor vessel. A mechanical labyrinth seal between the IHX and redan reduces the leakage of hot primary sodium from the hot pool into the cold pool. Special details at the top of the redan will be incorporated in the design to accommodate the effects of sodium sloshing that may occur during seismic events. It is a design requirement for the redan that no hot 
sodium coolant spill over the top of the redan into the cold sodium pool in the event of design basis earthquakes.

The use of an open-sodium outlet-plenum concept, such as use of the redan to direct the hot outlet coolant to the IHXs results in several key design features:

- An instrument tree, or Upper Internal Structure (UIS) is required for flow, temperature, and monitoring of the core outlet coolant.

- Space used for closed outlet sodium piping (such as that used on EBR-II and Phenix) is not required, thus leading to a smaller, more economical reactor vessel.

- The vertical lift distance required for handling fuel subassemblies is reduced when using the open-outlet-plenum

- The reactor vessel top closure (deck) is exposed to hot primary sodium temperature, 950F (510C), which presents certain major structural design challenges for the reactor vessel and deck.

- Insulation and cooling of the deck is required.

- Insulation and cooling of the reactor vessel wall is a special concern.

- Flow baffles are required above the core outlet sodium in order to obtain a quiescent hot sodium pool free surface.

- Low differential liquid levels between the hot and cold sodium requires a low pressure drop through the IHX.

\section{II.2.9 Core Support Structure}

The core support structure, shown in Figure II.2-7, provides support for the lower internals structure, the core assemblies, the core barrel assembly, the primary sodium inlet pipes, brackets, and baffles. It is designed to the requirements of the ASME B\&PV Code, Section III, Division 1, Subsection NG, Core Support Structures. The core support structure consists of a steel web structure supported on ribs formed to the contours of the reactor vessel bottom head, welded to the bottom head, and, thus, becomes an integral part of the reactor vessel. Except for the bottom head of the reactor vessel, all core support structure components are under compression. Therefore there is no need for performing ISI on these components. The part of the core support structure that is in tension is that portion of the reactor vessel bottom head which is integral with the support structure. Welds in the reactor vessel bottom head in the vicinity of the core structure are subject to ISI during the life of the plant. 


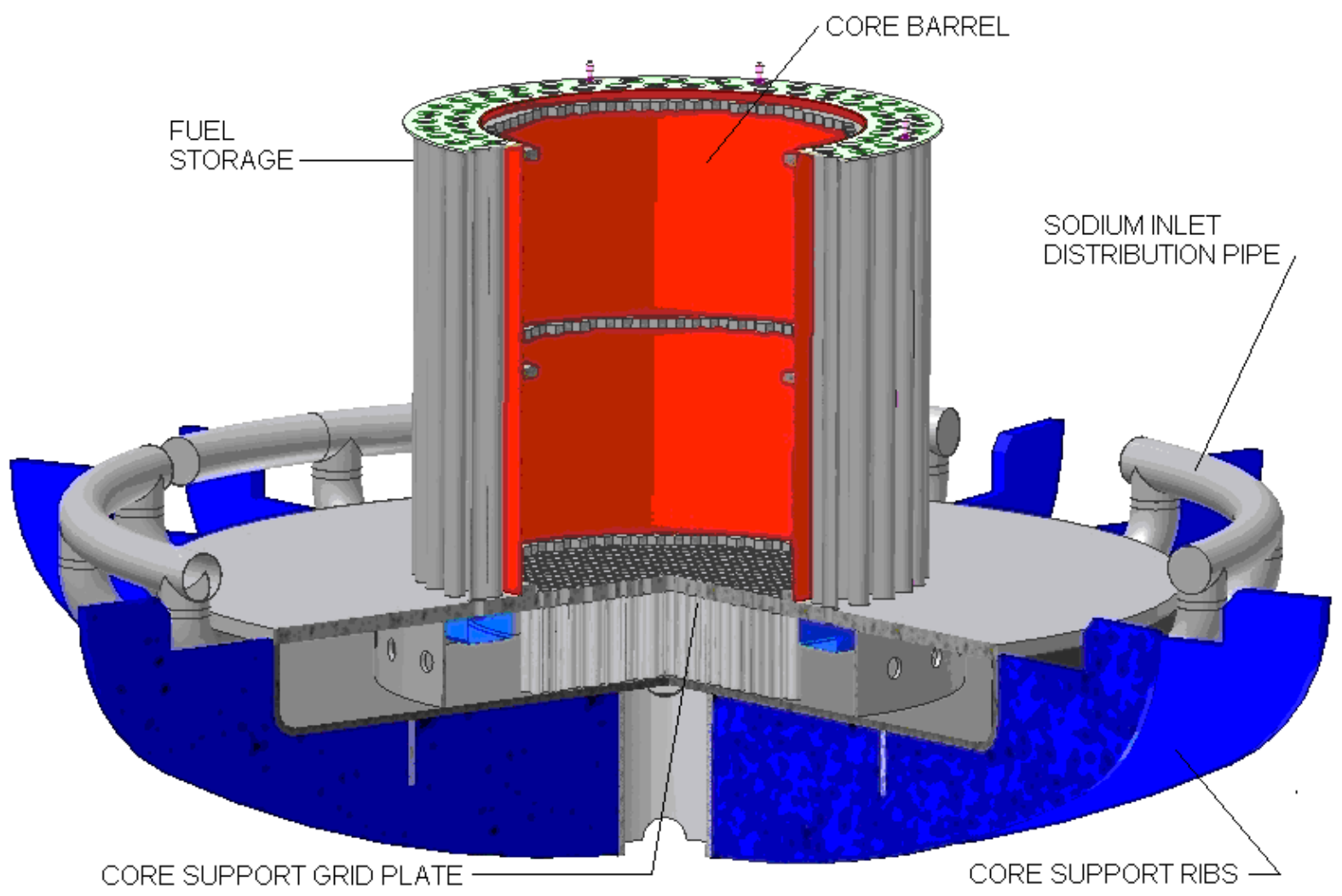

Figure II.2-7 Core Support Structure

\section{II.2.10 Reactor Containment Boundary}

The reference ABR primary containment boundary is comprised of the reactor vessel, reactor vessel enclosure, the tubes in the intermediate heat exchanger and in the direct reactor auxiliary cooling system heat exchanger, and the sodium purification piping and components. These components maintain the containment for the primary radioactive sodium and form the first containment boundary. This initial boundary also includes the instrument thimbles and the cover gas piping system. If this first boundary is breached, then the next secondary confinement is composed of the reactor guard vessel, the reactor containment, the intermediate sodium piping and steam generators, the direct reactor auxiliary cooling system intermediate piping and systems, the stainless steel-lined compartments around the reactor vessel support, the purification system cell confinement, and the reactor building (which is maintained at a negative pressure with HEPA-filtered ventilation). If there is a breach in a thimble or cover gas system, the gas operates at a slightly higher pressure than the sodium and therefore, there will be no release of sodium to the environment. The reactor building always operates at a negative pressure compared to the outside environment. All effluents are filtered via high-efficiency particulate air filters before they are released into the environment. 


\section{II.2.11 Integrity of Primary Coolant Boundary}

It has been postulated that a structural failure or leak which would develop in the reactor vessel could prove to be a major economic setback. The purpose of this discussion is to show that current, proven technology can be applied to ensure a leak-tight primary coolant boundary over the plant lifetime, reducing the probability of such a leak occurring to a very low level. In the pool-type design, the reactor and the entire radioactive primary coolant are contained in a single reactor vessel. This arrangement results in a direct and simple containment for the primary sodium, with no penetrations in the reactor vessel wall below the primary sodium surface. The ABR design avoids, wherever possible, the use of dissimilar materials and the oftentimes difficult welding techniques associated with these dissimilar materials. The surrounding environment of sodium is common to all of the primary system components, which mitigates pipe (duct) expansion stresses and minimizes effects of pipe leaks. A very high level of structural and leak-tight integrity of the reactor vessel and its surrounding guard vessel is achieved with the use of both proven design features and intrinsic characteristics of the sodium system. This basic foundation is supported by established fabrication, field construction, and quality control procedures, comprehensive testing plus inspections and finally, regular in-service monitoring and surveillance.

Inherent characteristics of the sodium system that mitigate potential challenges to the reactor vessel include non-corrosiveness of sodium to structural materials. The operating pressure of the bulk sodium is very low, generally limited to the hydrostatic head of sodium. This leads to low stresses in the reactor vessel structure and permits large design margins and the use of thinwalled plates for fabrication which, in turn, enhances the reliability of nondestructive examination methods which may be used for in-service inspection.

To ensure system safety, design features are included, such as the guard vessel, that give unimpaired capability for removal of reactor decay heat in the event of reactor vessel leakage. Design features that promote the integrity of both the reactor vessel and the guard vessel are viable due to the following basic characteristics of the pool-type arrangement:

- There are no attachments or penetrations in the shells and bottom head of either the reactor vessel or the guard vessel, except for the core support structure.

- The vessels have simple geometries, e.g., right circular cylinders and smooth, curved bottom heads.

- The core support structure is attached to the bottom head of the reactor vessel with a set of formed steel webs contoured to the shape of the reactor vessel bottom head. This eliminates welds at points of high stress.

- In addition, the austenitic stainless steel material, used throughout the reactor vessel assembly (with the possible exception of the guard vessel), has several favorable characteristics:

o Excellent compatibility with the sodium coolant.

o Excellent ductility/toughness properties, even at total neutron fluences higher than expected at the reactor vessel boundaries.

o No appreciable high temperature aging degradation with a primary sodium coolant temperature of about $510^{\circ} \mathrm{C}$.

o Excellent weldability. 
o High fracture toughness prevents rapid crack propagation and makes a leakbefore-break strategy feasible.

Another unique feature is that the reactor vessel is interfaced with the cold pool which results in a low and approximately constant temperature for the reactor vessel wall and for the guard vessel as well. This approach has two very important benefits:

- The reactor vessel temperature is almost always at the low sodium-inlet temperature (about 355 to $410^{\circ} \mathrm{C}$ ) except for the upper sections of the reactor vessel which sees the increased temperature of the hot sodium pool.

- Deleterious effects of large and frequent temperature transients are avoided by maintaining this temperature relatively constant.

With regard to radiation damage, adequate shielding in the core barrel as well as the distance from the core barrel to the reactor vessel wall results in total neutron fluences well below any level which would be of concern during the 60 year design life for the plant. 


\section{II.3 Primary Heat Transport System}

\section{II.3.1 System Requirements and Description}

The basic function of the primary heat transport system (PHTS) is to transport heat from the reactor to the intermediate heat transport system (IHTS) under normal and off-normal operating conditions. The arrangement of the PHTS is shown in Figures II.2-1 and II.2-2.

The PHTS consists of four primary pumps, four intermediate heat exchangers (IHXs) and expansion joints between the pumps and the reactor inlet plenum header. The four pumps and four IHXs are located around the reactor vessel in such a manner as to reduce the length of intermediate sodium piping in the reactor containment. The four pumps are located in the cold pool between the redan and the reactor vessel. Each of the four IHXs is located within the redan and penetrates through the redan from the hot pool to the cold pool. Each of the IHX plena is open to the reactor vessel cover gas at the top and each is closed at the bottom by a seal. The sodium level in the plena around each IHX is essentially the same as in the main redan. Thus, the IHXs have no primary piping associated with them; the hot primary sodium enters the IHX inlet through openings in the IHX shell and is discharged to the cold region at the bottom of the IHX. The sodium enters the IHX approximately 12 inches below the upper tubesheet to reduce the amount of thermal stress on the tubesheet. A baffle plate is located just above the inlet of the IHX to maintain this static layer of sodium. The tubesheet is the thickest part of the IHX and thus minimizing the temperature transients on this part increases the longevity of the IHX.

In this pool configuration the pumps as well as the IHXs are supported from the reactor vessel deck which is maintained near ambient temperature. The flexible seal between each IHX and the redan accommodates thermal and seismic motions. The flexible coupling between the discharge of each pump and the reactor inlet plenum which is fixed to the lower core support structure accommodates thermal and seismic motions. The flexible coupling is designed to accommodate the small amount of differential thermal expansion between the pump and the lower support structure, including the differential thermal expansion of these items from shutdown to full power conditions.

A total sodium flow of $5,220 \mathrm{~kg} / \mathrm{sec}$ removes the heat from the core and passes into the upper plenum at an average temperature of $510^{\circ} \mathrm{C}$. The core effluent mixes with the hot sodium in the plenum and flows up into the redan to four IHXs. Approximately $250 \mathrm{MWt}$ of heat is transferred from the primary to the secondary coolant in each IHX. The primary sodium leaves the IHX at $355^{\circ} \mathrm{C}$ and enters the cold pool region of the reactor vessel. Four pumps take the sodium from the cold pool and discharge it through inlet piping to the inlet plenum below the reactor core.

The PHTS must satisfy all normal and off-normal conditions specified in the duty cycle for the plant. In addition to full power 4-loop operation, the PHTS must provide heat removal for $50 \%$ power operation when only one IHTS loop is available or $75 \%$ when one primary pump is out-of-service. The system, along with the core and the IHX, is arranged to remove decay heat

under natural circulation conditions. Maintainability and inspectability are important considerations in the design of the PHTS. 


\section{II.3.2 Primary Sodium Mechanical Pump}

Both mechanical and electromagnetic (EM) pumps can be adopted for the ABR. In the ABR design, the location of the suction of the primary pump is critical to ensure sodium circulation under a variety of operating conditions. In addition, the diameter of the pump is an important parameter, since it affects the diameter of the reactor vessel.

Although there are many different types of mechanical pumps, only centrifugal pumps have been used in the sodium cooled fast reactors and therefore are considered as the primary candidate. Similarly, annular linear induction pumps (ALIPs) are considered as the primary candidate for the EM pump option.

\section{II.3.2.1 Mechanical pump}

In general, mechanical pumps have a longer history than that of EM pumps. Development of mechanical pumps specifically for the sodium cooled reactor application started with EBR-I, in which a 500 gpm mechanical pump was used to pump NaK. Since then, essentially all sodium cooled fast reactors have utilized mechanical pumps for the primary system, although some EM pumps were used in the secondary systems.

Mechanical pumps have the following advantages over EM pumps:

1. Mature technology,

2. High efficiency (70-85 \% for those used in nuclear reactors).

However, mechanical pumps have the following inherent disadvantages when compared to EM pumps:

1. Moving parts are involved,

2. Historically, prone to thermal distortion damage,

3. Expected to be more prone to cavitation,

4. Need of bearings,

5. Need of seals for rotating shaft,

6. Need of penetration through the reactor vessel for rotating shaft,

7. The existence of a $\mathrm{Na} /$ cover gas interface somewhere along the length of the rotating shaft,

8. Only vertical installation is practical.

The operating experience indicates that hydrodynamic and hydrostatic bearings in liquid sodium have become less of a problem [1]. Operations of BOR-60, BN-350, and BN-600 showed that wear in the bearing was virtually absent and replacements were not needed within several tens of thousands of hours of operation. The single factor requiring periodic maintenance that necessitates removal of the pump internals is erosion of the impeller due to cavitation for BN-350 and BN-600 [2].

The EBR-II mechanical pumps experienced shaft binding after less than 200 hrs of operation. Rubbing had occurred between the shaft and lower labyrinth seal. Although the initial cause was not definitely established, possible causes include insufficient initial clearance and warping of the shaft prior to operation [3-4]. In FFTF, thermal shock deformed the sodium bearing housing, 
causing a rotation problem. Other rotation problems were caused by condensation of sodium between the shaft and the thermal shields and deformation of the shaft due to a non-uniform circumferential temperature distribution in the shaft.

In Rapsodie, reduction of the gaps in the sodium bearing due to thermal shock produced a rotation problem. In Phenix, two causes of rotation problems were a failed shaft sleeve due to thermal transients and a shifting of the bearing parts. In PFR, the separation of the surface of the sodium bearing caused a rotation problem.

In BN-350, condensation of sodium vapor in the narrow gaps hindered start-up of the primary pump after long downtime due to incorrect estimate of component temperatures. Also, cavitation damage of impellers was reported. At the end of the reactor lifetime, the pump repair interval was extended, due to maturity of sodium technology, to about 10 years. In BN-600, during the initial period of operation, several problems relating to failures of the primary and secondary pumps due to their increased vibration, shaft fissuring, coupler damage, and nonreliable drive performance were experienced. Although all problems were later successfully resolved, replacement of the pump shafts was required. In BOR-60, although one primary mechanical pump was replaced due to high vibration caused by inappropriate thermal treatment of the shaft components during fabrication, maximum operation time for primary and secondary mechanical pumps was impressive: 220,000 and 180,000 hrs, respectively [5].

The long history of the mechanical pumps in sodium cooled fast reactors indicates that although some minor maintenance would be required on a periodic basis, the overall reliability has been demonstrated and the mechanical pump would be a logical choice for the ABR as well.

However, one potential issue unique for the $A B R$ is that the $A B R$ reactor vessel is much longer than vessels of other previously developed pool type reactors. This is schematically illustrated in Figure II.3-1, and the actual design data are summarized in Table II.3-1.

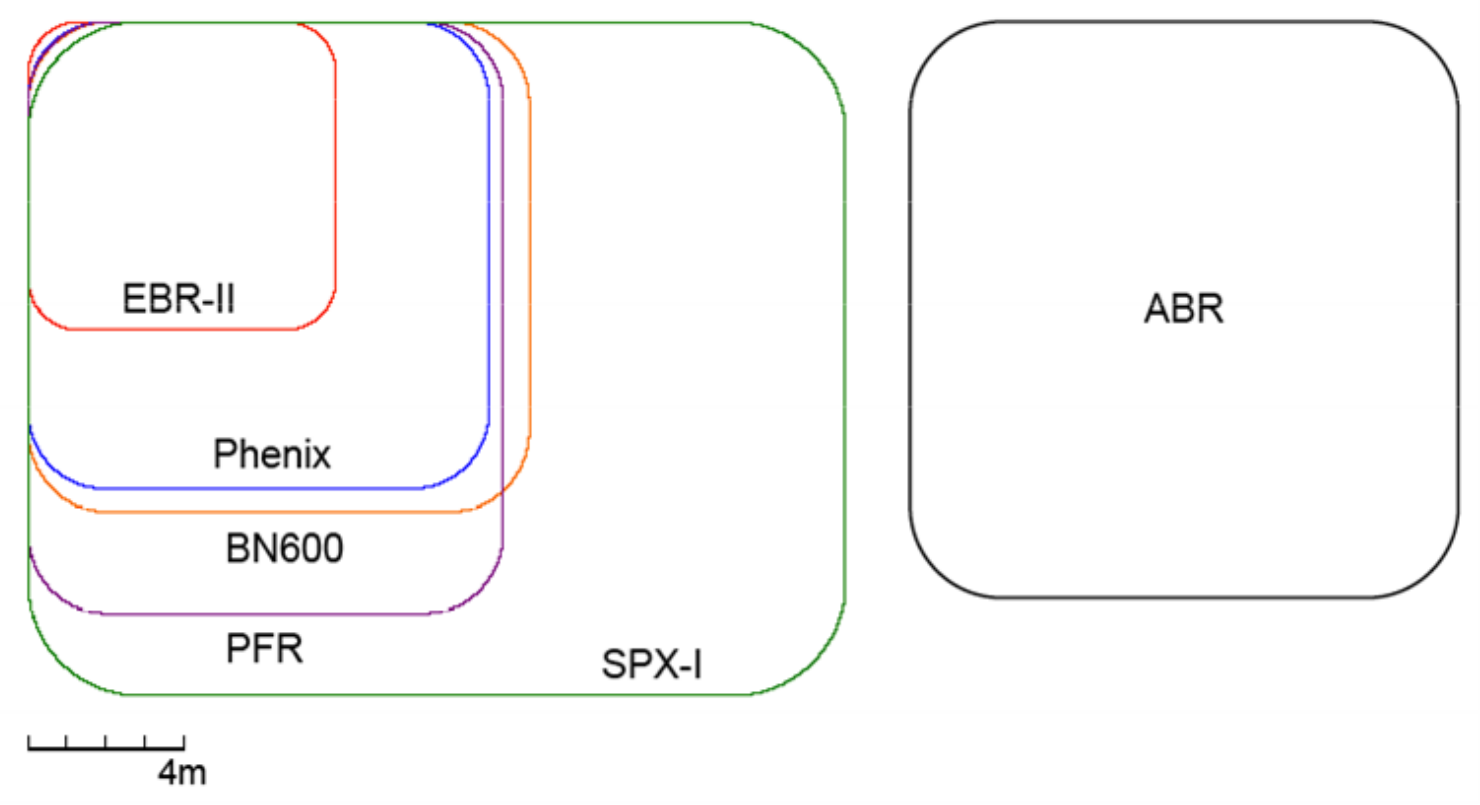

Figure II.3-1 Schematic comparison of pool type reactor vessels 
Table II.3-1 Dimensions for various pool type reactor vessels

\begin{tabular}{|l|c|c|c|}
\hline \multicolumn{1}{|c|}{ Reactor } & Diameter, $\mathrm{m}$ & Height, $\mathrm{m}$ & $\mathrm{H} / \mathrm{D}$ ratio \\
\hline EBR-II & 7.92 & 7.92 & 1.00 \\
\hline Phenix & 11.82 & 12.00 & 1.02 \\
\hline SuperPhenix & 21.00 & 17.30 & 0.82 \\
\hline PFR & 12.20 & 15.20 & 1.25 \\
\hline BN-600 & 12.86 & 12.60 & 0.98 \\
\hline PRISM & 9.07 & 19.35 & 2.13 \\
\hline ABR 1000 MWt & 14.1 & 14.8 & 1.05 \\
\hline
\end{tabular}

This fact drives the mechanical pumps for the ABR to be longer than the other pumps. In fact, values for the ratio of the pump length to the pump diameter for several mechanical pumps used in the above pool type reactors are between approximately 3 and 5, however, the L/D ratio for the mechanical pump preliminarily designed for the ABR is approximately 6 , as compared in Table III.3-2, possibly making satisfactory performance of the pump more difficult to achieve. The calculational procedure to obtain these pump dimensions for an ABR pump is presented in Section III.1.1.

Table II.3-2 Dimensions of mechanical pump for various pool type reactors

\begin{tabular}{|l|c|c|c|c|c|}
\hline \multicolumn{1}{|c|}{ Reactor } & $\begin{array}{c}\text { Flowrate, } \\
\mathrm{m}^{3} / \mathrm{min}\end{array}$ & $\begin{array}{c}\text { Pump } \\
\text { head, MPa }\end{array}$ & $\begin{array}{c}\text { Pump } \\
\text { diameter, } \mathrm{m}\end{array}$ & $\begin{array}{c}\text { Pump shaft } \\
\text { length, } \mathrm{m}\end{array}$ & $\begin{array}{c}\mathrm{L} / \mathrm{D} \\
\text { ratio }\end{array}$ \\
\hline EBR-II & 19.0 & 0.54 & 1.28 & 3.4 & 2.66 \\
\hline PFR & 70.7 & 0.73 & 1.91 & 5.5 & 2.88 \\
\hline Phenix & 67.2 & 0.70 & 1.32 & 5.0 & 3.79 \\
\hline SuperPhenix & 300.0 & 0.55 & 2.50 & 12.0 & 4.8 \\
\hline ABR 1000 MWt & 90.9 & 0.76 & 1.19 & 7.0 & 5.88 \\
\hline
\end{tabular}

As described in Section III.10, the mechanical pump was designed using a series of nomographs developed by Byron Jackson [6]. In the first nomograph, the specific speed and rotation speed are determined. In the second nomograph, the required power is determined. Then, in the third nomograph, the shaft diameter is determined. In the fourth nomograph, the pump case diameter, which is related to the impeller diameter, is determined. In the fifth nomograph the size of the pump outer barrel is determined, however, for the pool type configuration, the outer barrel is not used and the pump casing is suspended directly in the pool of Na. Finally in the sixth nomograph, the suction and discharge nozzle diameters are determined. This procedure estimates only the diameter of the pump. The length of the pump can be estimated from other considerations, namely the submergence depth should be greater than 0.9-1.2 m to prevent vortexing [7], see Figure II.3-3. A summary of input data is presented in Figure II.3-2. 


Gravity, g $\left(\mathrm{m} / \mathrm{s}^{2}\right)$
$\mu_{0}:$ Magnetic permeability $(\mathrm{Wb} / \mathrm{A}-\mathrm{m})$
Gas Constant, R (J/mol-K)
Reference
Molecular weight $(\mathrm{g} / / \mathrm{mol})$
MP $\left({ }^{\circ} \mathrm{C}\right)$
MP $(\mathrm{K})$
operating temperature, T $(\mathrm{K})$
Density, $\rho\left(\mathrm{kg} / \mathrm{m}^{3}\right)$
Dynamic Viscosity, $\eta(\mathrm{Pa}-\mathrm{s})$
Surface Tension, $\sigma_{\mathrm{s}}(\mathrm{N} / \mathrm{m})$
Thermal Conductivity, $\mathrm{k}(\mathrm{W} / \mathrm{m}-\mathrm{K})$
Specific Heat, $\mathrm{C}_{\mathrm{p}}(\mathrm{J} / \mathrm{K}-\mathrm{kg})$
Electrical Conductivity, $\sigma_{\mathrm{e}}(1-\Omega-\mathrm{m})$
Vapor Pressure, $\mathrm{P}(\mathrm{Pa})$
Vapor Pressure, $\mathrm{P}(\mathrm{Torr})$
Est. Vapor Density, $\rho_{\mathrm{a}}\left(\mathrm{kg} / \mathrm{m}^{3}\right)$
Latent Heat of Evaporation $(\mathrm{J} / \mathrm{kg})$
Dynamic Viscosity, $\eta_{\partial}(\mathrm{Pa}-\mathrm{s})$

$\begin{array}{lll}\text { Flow rate }(\mathrm{kg} / \mathrm{s}) & =1280= & \underline{23391.62} \mathrm{GPM} \\ \text { Pressure }(\mathrm{Pa}) & =756250= & \underline{291.68 \mathrm{feet}} \\ \text { Temperature }(\mathrm{K}) & =628= & \underline{670.73 \mathrm{~F}}\end{array}$

*Flow rate $\left(\mathrm{m}^{3} / \mathrm{s}\right) \quad 1.4761602$

*Pressure (psi) 109.68479

\section{Figure II.3-2 Input data for mechanical pump design nomograph}

The resulting design parameters are summarized in Table II.3-3, and the ABR mechanical pump is schematically compared with that of EBR-II in Figure II.3-3.

\section{Table II.3-3 Mechanical pump design parameters}

\begin{tabular}{|l|c|}
\hline Flow rate, $\mathrm{m}^{3} / \mathrm{s}$ & 1.51 \\
\hline Pump head, psig & 110 \\
\hline Power, $\mathrm{kW}$ & 1417 \\
\hline Efficiency, \% & 81 \\
\hline Pump shaft length, $\mathrm{m}$ & 7.0 \\
\hline Pump diameter, $\mathrm{m}$ & 1.19 \\
\hline Suction nozzle diameter, $\mathrm{m}$ & 0.81 \\
\hline Discharge nozzle diameter, $\mathrm{m}$ & 0.64 \\
\hline
\end{tabular}




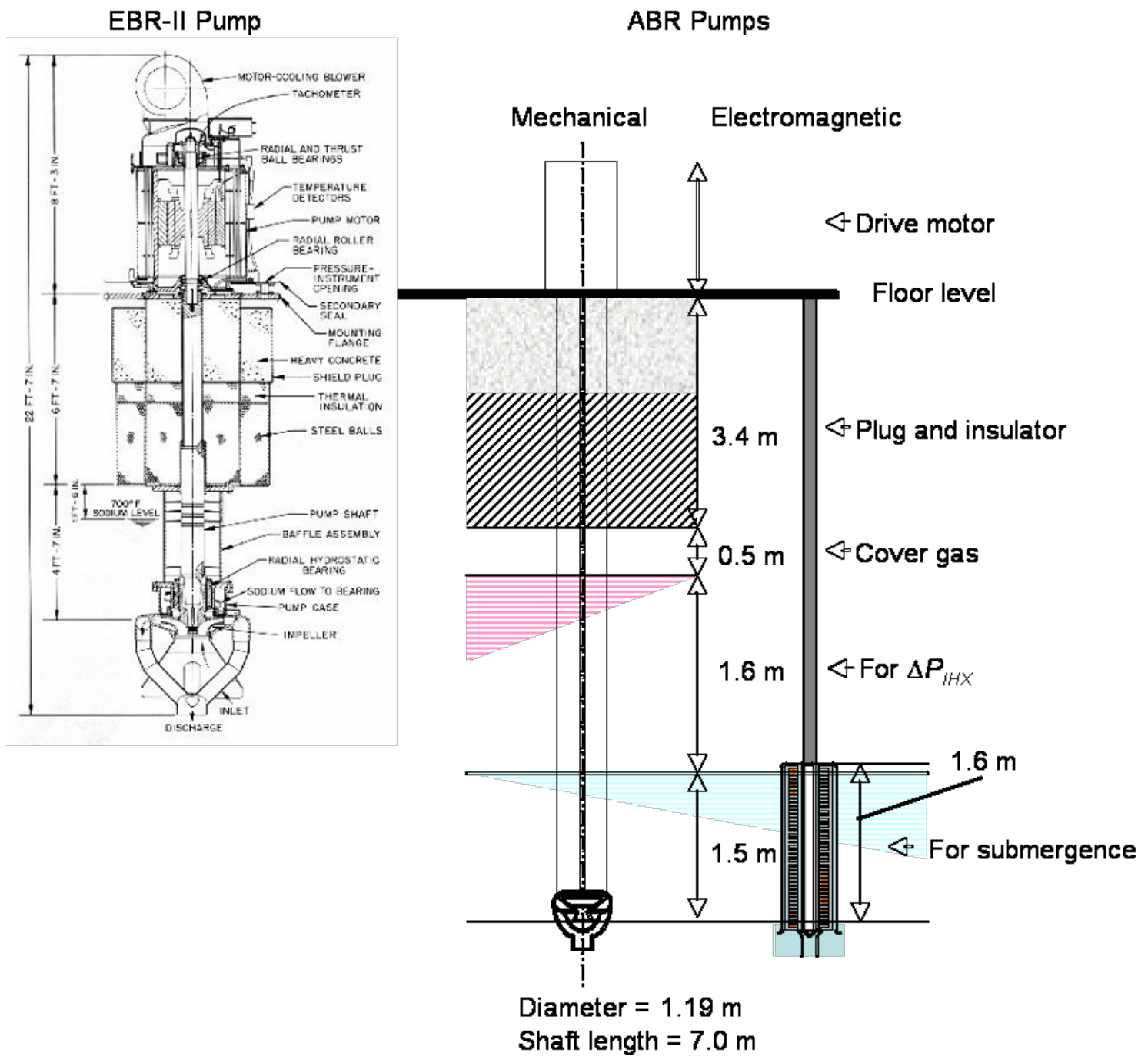

Figure II.3-3 Schematic comparison of ABR and EBR-II pumps

\section{II.3.2.2 Electromagnetic Pump}

The first application of EM pump in sodium cooled fast reactor goes back to the nuclear powered submarine Seawolf, which used four flat linear induction pumps (FLIPs) rated at 3,300 gpm at 85 psi. These 4 units were operated at $43 \%$ efficiency for 10,000 hours of maintenance free operation. The EBR-II used 5000 gpm and 6500 gpm FLIP units in the intermediate heat transport system. Although the operating history was satisfactory, the pump duct developed fatigue cracks after 1,385 hours of operation. They were caused by low inlet pressure and electrically induced pressure pulsation [3]. In DFR, 24 linear induction pumps were used as primary pumps. In BR-10, mechanical pumps were replaced with EM pumps, whose reported lifetime was 170,000 hours without any major repairs [7]. Among the many different types of EM pump designs, the EM pump type most favored for use in reactor systems is the annular linear induction pump (ALIP) type. Electromagnetic pump's advantages over mechanical pump are as follows: 
1. Design using no penetration through the boundary between working fluid and ambient, thus a design without seals is possible,

2. No moving parts are involved,

3. Installation in any orientation is possible,

4. Thermal distortion tolerant.

On the other hand, EM pumps have some disadvantages over mechanical pumps.

1. Lower pumping efficiency ( $40 \%)$,

2. Lesser reactor plant experience, especially for large EM pumps.

A known issue is that large scale EM pumps may exhibit flow instability if the wrong operating conditions are chosen. This topic is still being studied; however, the instability can be avoided by selecting the right operating parameters [8]. In addition, although no experience of using a large EM pump in the nuclear reactor exists, recently an EM pump with a capacity of 160 $\mathrm{m} 3 / \mathrm{min}(42,267 \mathrm{gpm})$ at $280 \mathrm{kPa}(40.6 \mathrm{psi})$ was tested for 2,550 hrs and confirmed applicability of the EM pump in the nuclear plants [9].

Traditionally, electrical insulators and magnetic materials in ALIPs limited the operating temperature of the pump. Until recently, ALIPs for liquid sodium service usually required external cooling to maintain acceptable temperatures in electrical insulators and magnetic materials; and removed thermal energy was wasted. However, recent development of high temperature insulators and magnetic materials that can operate at as high as 700 oC allows operation of EM pumps using those materials at temperatures as high as $350 \mathrm{oC}$ without any cooling [9-11]. This means that ALIPs immersed in a pool type reactor can utilize almost 100\% of the input power provided to the pumps, making low pumping efficiency of the ALIPs a nonissue. The impact on the overall plant efficiency due to the differences in pumping efficiency between mechanical and EM pumps is estimated to be only about $0.4 \%$ [12]. However, as shown by the history of EM pump development depicted in Figure II.3-4, the important developments occurred only recently and there exists no experience with ALIPs in sodium primary systems. 


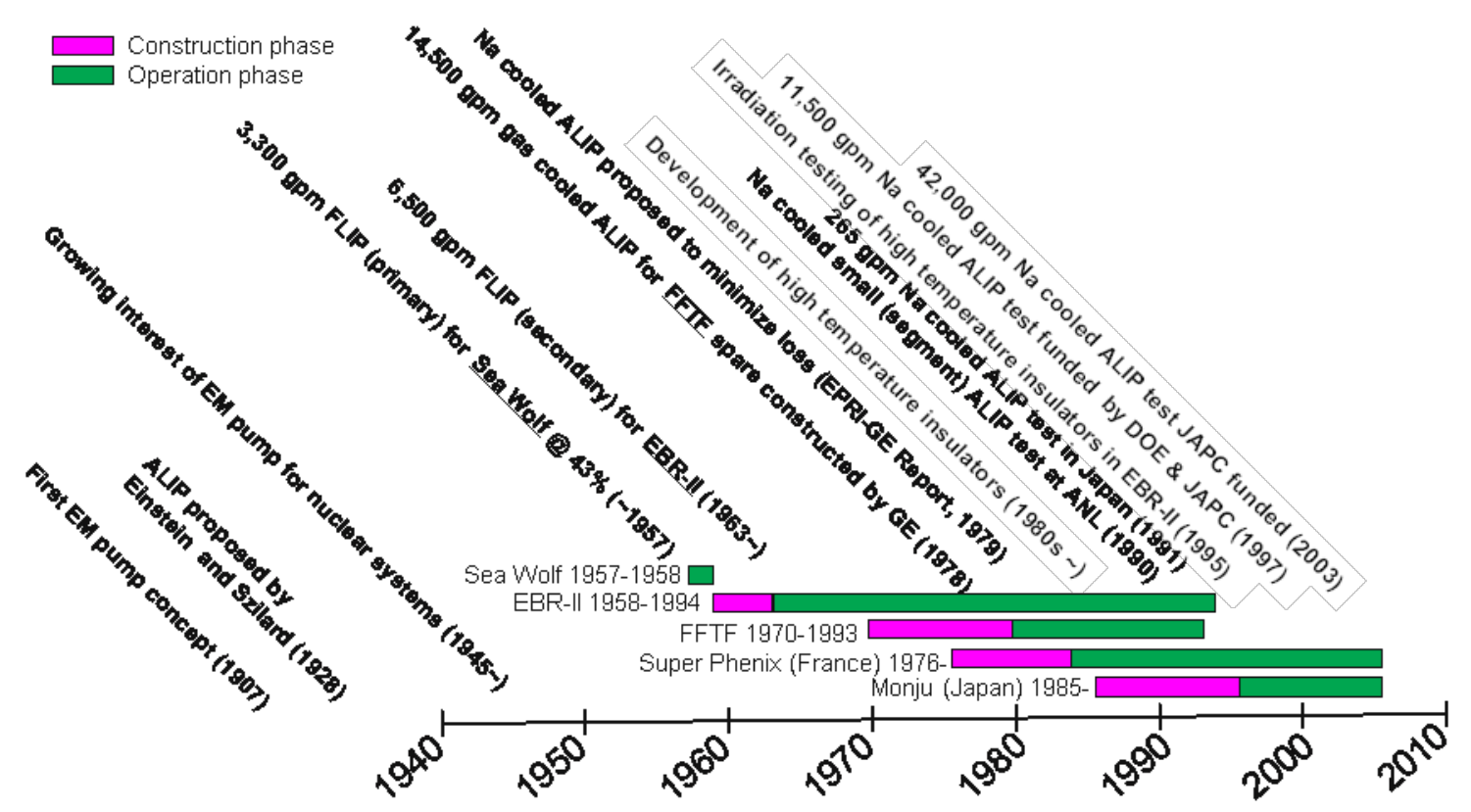

Figure II.3-4 History of EM pump development

The ALIP shown in Figure II.3-5 is a standard design obtained using a code developed at ANL. The code is based on the calculational scheme originally developed to estimate the performance of various ALIPs, for example, for the EBR-II and TREAT. An annular linear induction pump that can be calculated using the current code is a single stator design. A single stator ALIP comprises a core, coolant passage (or duct), coolant duct wall, stator core, and coils (see Figure II.3-5). For the ABR, however, the pump of choice is a return type and includes the return duct that is placed outside of the stator. All magnetic core pieces are assumed to be made of a magnetic material with high saturation value, such as Permendur 2V or other cobalt alloy. Since the coolant bulk outlet temperature is $510 \mathrm{o}$ C, typical stainless steel can be used for the coolant ducts. The conductor part of the coil is made of copper. The electrical insulation around the conductor is made of mica tape backed with alumina cloth and inorganic silicone-base binder, the state of art electrical insulation for high temperature applications. Integrity of a similar coil was experimentally demonstrated at elevated temperatures up to 800o C. Similar coils were also used in a large sodium-immersed self-cooled ALIP [9].

In this design, the magnetic structures do not need to be thermally insulated from the coolant duct, due to the low coolant temperature that is well below the curie point of Permendur 2V (940o C) [11]. In fact, the coolant duct serves as the heat sink, since some of input energy is thermally dissipated as losses within the ALIP during operation and needs to be removed to avoid overheating of various components. Since this thermal energy is transferred to the coolant, these thermal losses in the ALIP are completely recovered in the system. This is the key reason that submerged, self-cooled, ALIPs reduce the overall plant efficiency by only about $0.4 \%$ in comparison to the overall plant efficiency obtained with mechanical pumps. 


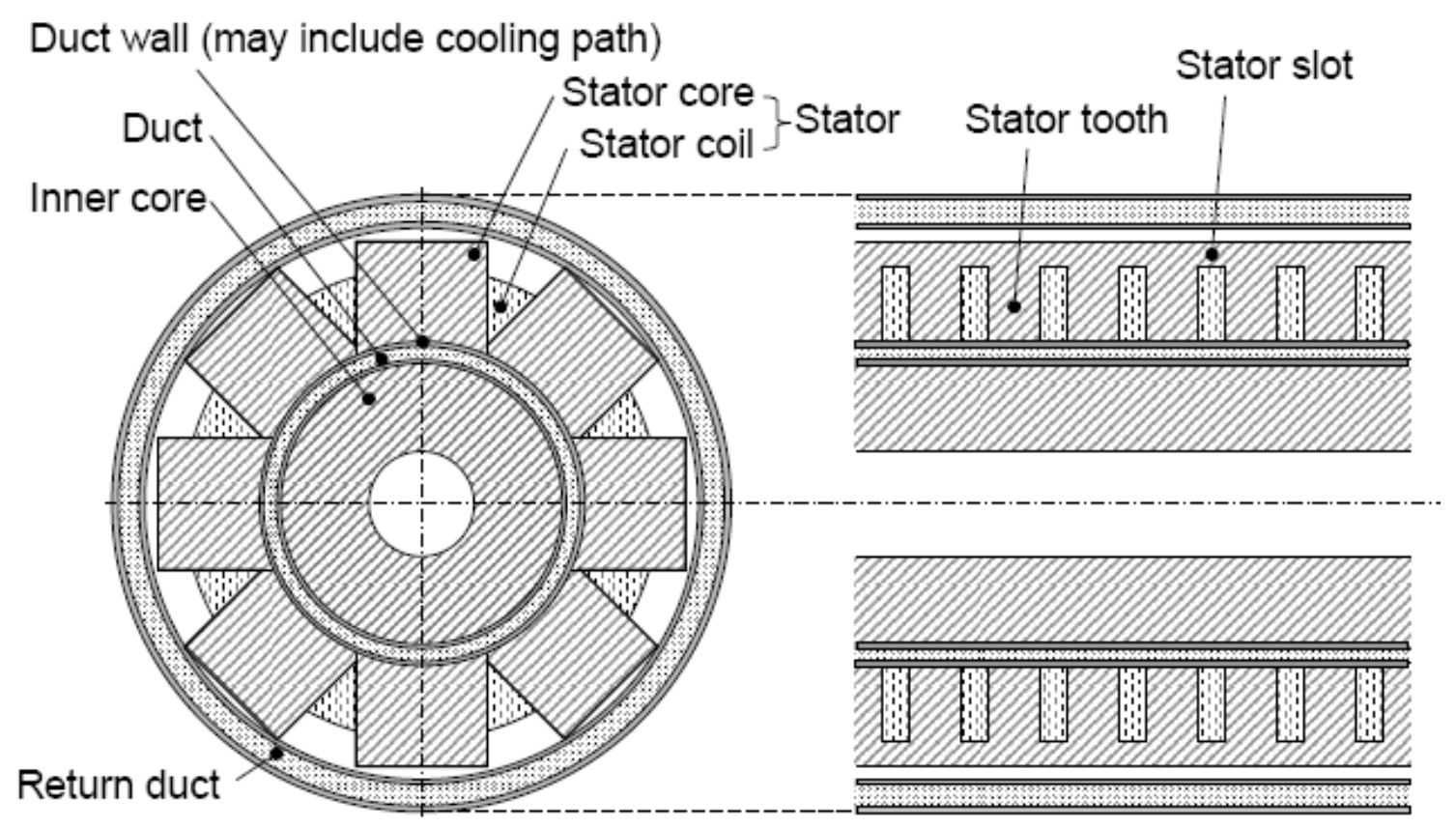

Figure II.3-5 Schematic of a single stator ALIP

The EM code described in Section III.1.2 does not include end effects, which degrade the efficiency of ALIPs [13]. However, for large ALIPs with a large number of poles, thus a large number of coils, sufficient tailoring of magnetic field at the ends of the pump can be easily achieved without altering the performance of a majority of the coils, yielding insignificant pump performance degradation [13]. In addition to the end effect, the skin effect will attenuate the electromagnetic field in the coolant, thus reducing the performance of the large scale ALIPs, unless a double stator design is employed [12], however, the current design code does not include the details of the skin effect. A schematic of a typical double stator ALIP is shown in Figure II.3-6. It should be noted that the combination of the attenuation of the electromagnetic field due to the skin effect and increase of the electromagnetic field due to the cylindrical configuration is somewhat balanced, which means that the net effect depends on the size of the pump and could be very small [14]. These effects will be investigated further in future work. Also noted is that since the ALIP for the ABR must have both inlet and outlet at the bottom of the pump (return configuration), the internal hydrodynamic pressure drop of the pump is assumed twice that for a straight design ALIP in the code.

Important inputs for the code are the flow rate, pressure head, the length of the pump, various duct dimensions (wall thicknesses), and input voltage. For the flow rate and pressure head inputs, the nominal values for the ABR design are used. Based on these inputs, the code calculates all other dimensions and parameters for the best efficiency. The calculated parameters include dimensions of the inner core, the duct gap size, and the stator assembly including number of coils and poles, mass of each component, and performance parameters including input power, dissipated power in components, and efficiency. Also obtained is the optimum drive frequency. 


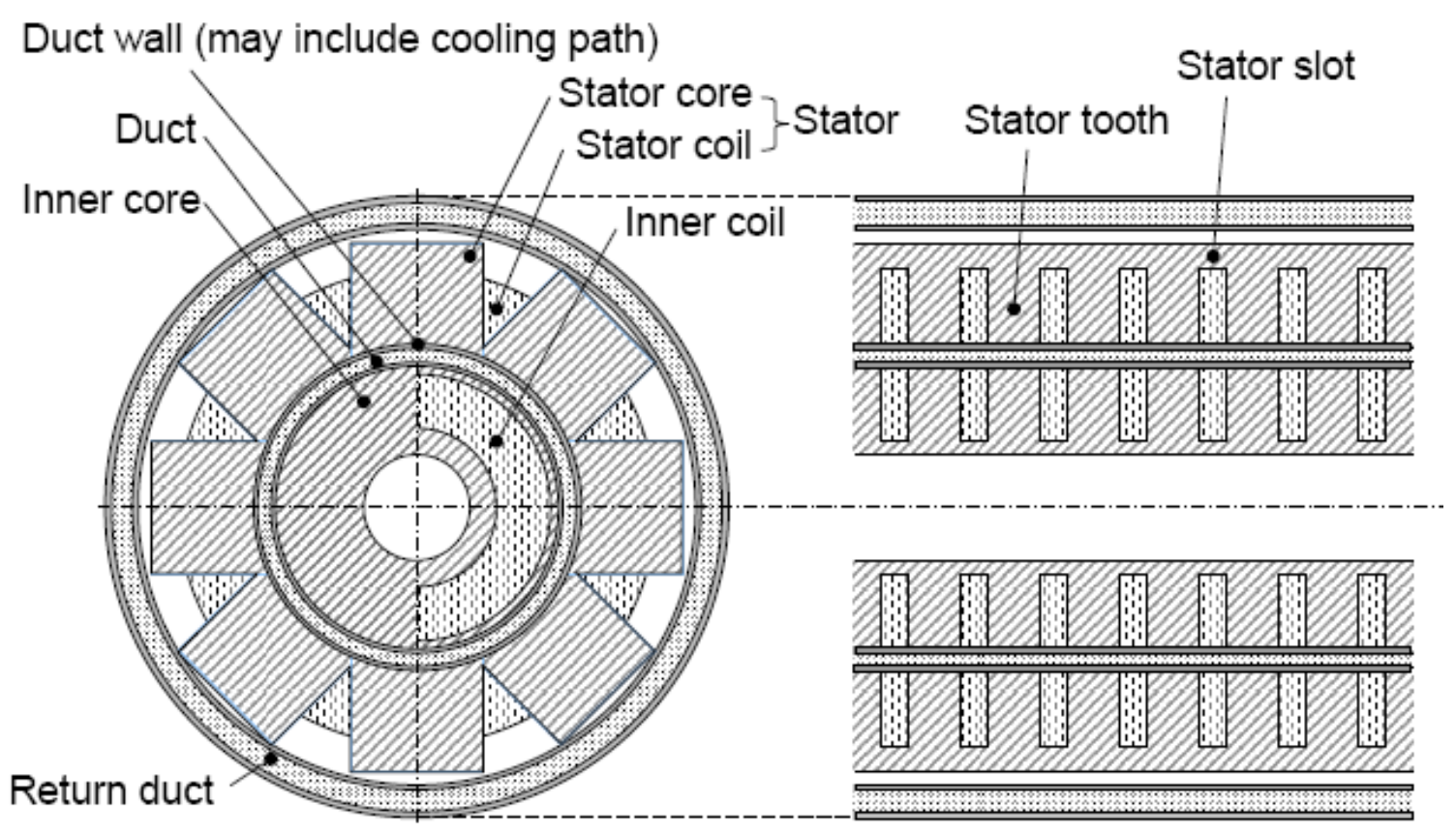

Figure II.3-6 Schematic of a double stator ALIP

Several calculations showed that most heat dissipation occurs in the coolant, duct wall, and coils. This fact allows us to construct a simple 1-D thermal model to conservatively estimate the critical temperatures, such as maximum wall and coil temperatures to ensure safe operation of the ALIP. This thermal model is integrated with the code for calculating the pump performance. The geometry for the thermal model is illustrated in Figure II.3-7.

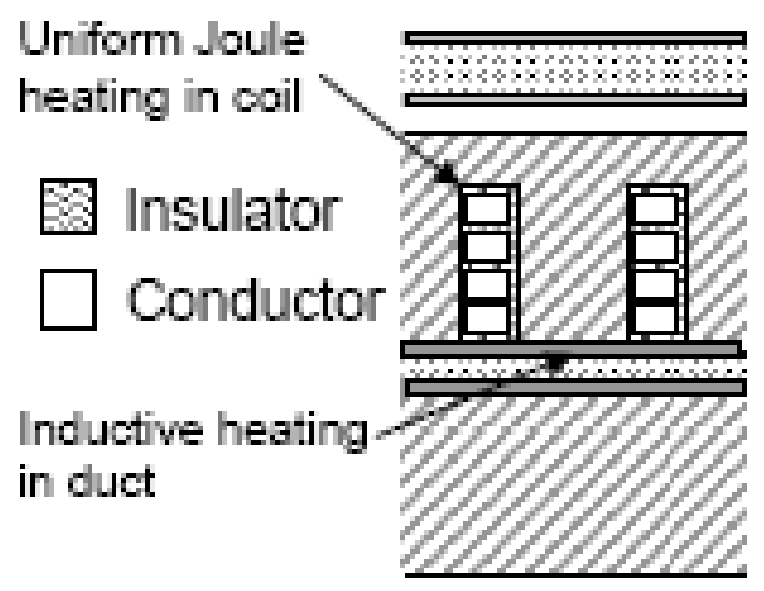

Figure II.3-7 Heat generations in the ALIP 
In this thermal model, only radial heat flow is assumed and the outside surface of the ALIP is assumed to be thermally insulated for simplicity and assuring conservative estimation. Bulk thermal conductivity of the coil is estimated from the dimensions of the coil, thermal conductivity of the coil (copper), and that of insulator (mica).

For the ABR, the diameter of the pump is limited to $1.2 \mathrm{~m}$, so that the ALIP can be installed in the opening for the mechanical pump designed in the previous section. The key design parameters for the EM pump are summarized in Table II.3-4. (Additional detailed data are presented in Section III.1.2.)

Table II.3-4 EM pump design parameters

\begin{tabular}{|l|c|}
\hline Power, kW & 2315 \\
\hline Efficiency, \% & 48.6 \\
\hline Mass, kg & 6176 \\
\hline Number of Poles & 8 \\
\hline Number of Coils & 24 \\
\hline Temperature, ${ }^{\circ} \mathrm{C}$ & 355 \\
\hline Flow Rate, $\mathrm{m}^{3} / \mathrm{s}$ & 1.51 \\
\hline Discharge Pressure, psig & 110 \\
\hline Length, m & 1.6 \\
\hline Pump Diameter, m & 1.18 \\
\hline
\end{tabular}

\section{Reference}

1. R. W. Atz, and R. S. Baker, Sodium-NaK Engineering Handbook, Vol. IV, Sodium Pumps, Valves, Piping, and Auxiliary Equipment, Chap. 1, Gordon and Breach, Science Publishers, Inc., New York, NY, 1978.

2. Status of Liquid Metal Cooled Fast Reactor Technology, Chap. 8, IAEA, Vienna, Austria, 1999.

3. Davis, J. R., et al., Operating Experience with Sodium Pumps at EBR-II, ANL/EBR027, 1970.

4. Monson, H. O., et al., “Components for sodium reactors,” Proceedings of the Third International Conference on the Peaceful Uses of Atomic Energy, Geneva, v 8, pp588-599, 1964.

5. Korolkov, A. S., "Operating experience with BOR-60: basic results and prospects,” TM-27172 and TM-26984, TWG-FR/123, Working Material, IAEA, pp218-225, 2005.

6. Charnock, H. O. and Holz, H. B., "Sodium Systems Components,” Sodium Technology, Session IV, Atomic International, North American Rockwell, 1970. 
7. Nikulin, M. P., et al., "Experience Gained during BR-10 Reactor Operation,” TM27172 and TM-26984, TWG-FR/123, Working Material, IAEA, pp211-217, 2005.

8. R. Stieglitz, "MHD-features of the Main Service and Bypass Pump in the MEGAPIE Design,” ISSN 0947-8620, Forschungszentrum Karlsruhe GmbH, Karlsruhe, 2003.

9. H. Ota et al., "Development of $160 \mathrm{~m} 3 / \mathrm{min}$ Large Capacity Sodium-Immersed SelfCooled Electromagnetic Pump,” J. of Nucl. Sci. and Tech. v41, No4, pp511-523, 2004.

10. M. Ishida, Y. Ikeda, and N. Naohara, "Development of Insulating System for FBR Electro-Magnetic Pump," Proc. of the 4th Int'l Conf. on Properties and Applications of Dielectric Materials, Brisbane Australia, pp471-474, July 3-8, 1994.

11. Abraham Weitzberg et al., Liquid Metal Cooled Reactor Concept for Nuclear Space Initiative Electric Propulsion Missions, Draft Input for DOE Report, October, 2002

12. Electromagnetic Pumps for Large Pool-Concept LMFBR, General Electric Company, NP-1265, Research Project 620-28, December 1979.

13. L. R. Blake, “Conduction and Induction Pumps for Liquid Metals,” Proc. I.E.E., Paper No. 2111U, v104A, pp49-67, July 1956.

14. Kliman, G. B., "Large Electromagnetic Pumps,” Electric Machines and Electromechanics: An International Quarterly, v.3 pp129-142, Hemisphere Publishing Corp., 1979.

\section{II.3.3 Intermediate Heat Exchanger}

The Intermediate Heat Exchangers (IHXs) transfer heat from the radioactive sodium coolant in the primary heat transport system to the nonradioactive sodium coolant in the intermediate heat transport system. Four sodium-to-sodium heat exchangers rated at $250 \mathrm{MWt}$ each are used to transfer the $1000 \mathrm{MWt}$ core power at full-power conditions corresponding to core inlet and outlet temperatures of 355 and $510^{\circ} \mathrm{C}$, respectively.

There are several factors that are important in the evaluation of the overall IHX design. These factors include material of construction, tube configuration (straight vs. bent), shell vs. tube-side primary flow, elevation of the IHX within the primary system, shape of the IHX (in plan view), primary flow-side pressure drop (i.e., low pressure loss is needed to ensure adequate natural convection primary sodium flow during loss-of-flow events), and the possible inclusion of a second internal coil near the upper region of the IHX to serve as a shutdown heat removal system. The various design choices that have been made, along with the underlying rationale for these choices, are described below.

The heat exchanger arrangement selected is a shell-and-tube counter-current flow arrangement with the primary flow on the shell-side, and secondary sodium flow on the tube side. Major features of these heat exchangers are graphically depicted in Figure II.3-8, while key design information is provided in Table II.3-5. The tube-side secondary flow was selected to simplify cleaning of the heat exchanger tubes in the event of a tube leak in a steam generator. Moreover, this configuration maximizes the ability of the IHX to accommodate any pressure transients that may arise if a steam generator tube rupture event were to occur, since for a given tube wall thickness the tubing is much stronger in tension (internal pressure source) versus compression (external pressure source). 
The installation of the IHXs inside the reactor vessel is illustrated in Figure II.3-9. Each unit is vertically suspended from two rigid pipes that are welded to the IHX shroud. These pipes extend upward from the hot pool and are welded to the underside of a removable integral plug in the reactor vessel head. The IHXs pass through horizontal sections of the redan.

Primary sodium enters the shell side of each IHX through a series of $20 \mathrm{~cm}$ diameter circular openings in the shell approximately $20 \mathrm{~cm}$ below the upper tube sheet. These openings allow sodium from the hot plenum to enter the IHX with minimal head loss. Also, introduction of the sodium below the upper tube sheet minimizes the thermal shock to this relatively thick component during transients. The sodium then flows downward through the shell and vents through a $10 \mathrm{~cm}$ wide gap around the lower tube sheet that leads to the single $61 \mathrm{~cm}$ diameter exit nozzle that discharges directly into the cold pool.

The IHX shell contains a seal where it seats on the horizontal deck of the redan to prevent sodium bypass from the hot to cold pools. Minor leakage of these seals is permissible. 
Table II.3-5 Intermediate Heat Exchanger Design Parameters

\begin{tabular}{|c|c|}
\hline Parameter & Value \\
\hline Heat transfer capacity & $250 \mathrm{MWt}$ \\
\hline Design & $\begin{array}{l}\text { Straight tube, counter- } \\
\text { flow }\end{array}$ \\
\hline Heat transfer area & $1074 \mathrm{~m}^{2}$ \\
\hline Primary sodium temperature inlet & $510^{\circ} \mathrm{C}$ \\
\hline Primary sodium temperature outlet & $355^{\circ} \mathrm{C}$ \\
\hline Primary sodium mass flowrate & $1256 \mathrm{~kg} / \mathrm{s}$ \\
\hline Secondary sodium temperature outlet & $333^{\circ} \mathrm{C}$ \\
\hline Secondary sodium temperature inlet & $488^{\circ} \mathrm{C}$ \\
\hline Secondary side sodium mass flowrate & $1256 \mathrm{~kg} / \mathrm{s}$ \\
\hline Tube outer diameter & $1.59 \mathrm{~cm}$ \\
\hline Tube wall thickness & $0.889 \mathrm{~mm}$ \\
\hline Tube pitch & $2.23 \mathrm{~cm}$ \\
\hline Active Tube Length & $4.78 \mathrm{~m}$ \\
\hline Number of tubes & 4500 \\
\hline Upper tube sheet - area & $2.25 \mathrm{~m}^{2}$ \\
\hline Upper tube sheet - thickness & $10.0 \mathrm{~cm}$ \\
\hline Lower tube sheet - area & $2.25 \mathrm{~m}^{2}$ \\
\hline Lower tube sheet - thickness & $10.0 \mathrm{~cm}$ \\
\hline Downcomer piping - OD & $61.0 \mathrm{~cm}$ \\
\hline Downcomer piping - thickness & $12.7 \mathrm{~mm}$ \\
\hline Downcomer piping - length & $10.8 \mathrm{~m}$ (est.) \\
\hline Outlet piping - OD & $86.4 \mathrm{~cm}$ \\
\hline Outlet piping - thickness & $12.7 \mathrm{~mm}$ \\
\hline Outlet piping - length & $6.6 \mathrm{~m}$ (est.) \\
\hline Shell baffle plates - thickness & $6.4 \mathrm{~mm}$ \\
\hline Shell baffle plates - number (spacing) & $5(91 \mathrm{~cm})$ \\
\hline Baffle plate cut (transverse dist. across IHX not occluded by plates) & $50 \%$ \\
\hline Baffle plate perforation (open flow area/ total axial flow area) & $45 \%$ \\
\hline Perforation hole diameter & $8.12 \mathrm{~mm}$ \\
\hline Number of perforation holes per IHX tube & 2 \\
\hline Shell (primary) side pressure drop & $18.2 \mathrm{kPa}$ \\
\hline Tube (secondary) side pressure drop & $14.8 \mathrm{kPa}$ \\
\hline Shell height & $5.88 \mathrm{~m}$ \\
\hline Shell outside circumference & $5.40 \mathrm{~m}$ \\
\hline Shell thickness & $1.3 \mathrm{~cm}$ \\
\hline Shell cross-sectional area & $2.32 \mathrm{~m}^{2}$ \\
\hline Tube material & 9Cr-1Mo \\
\hline
\end{tabular}



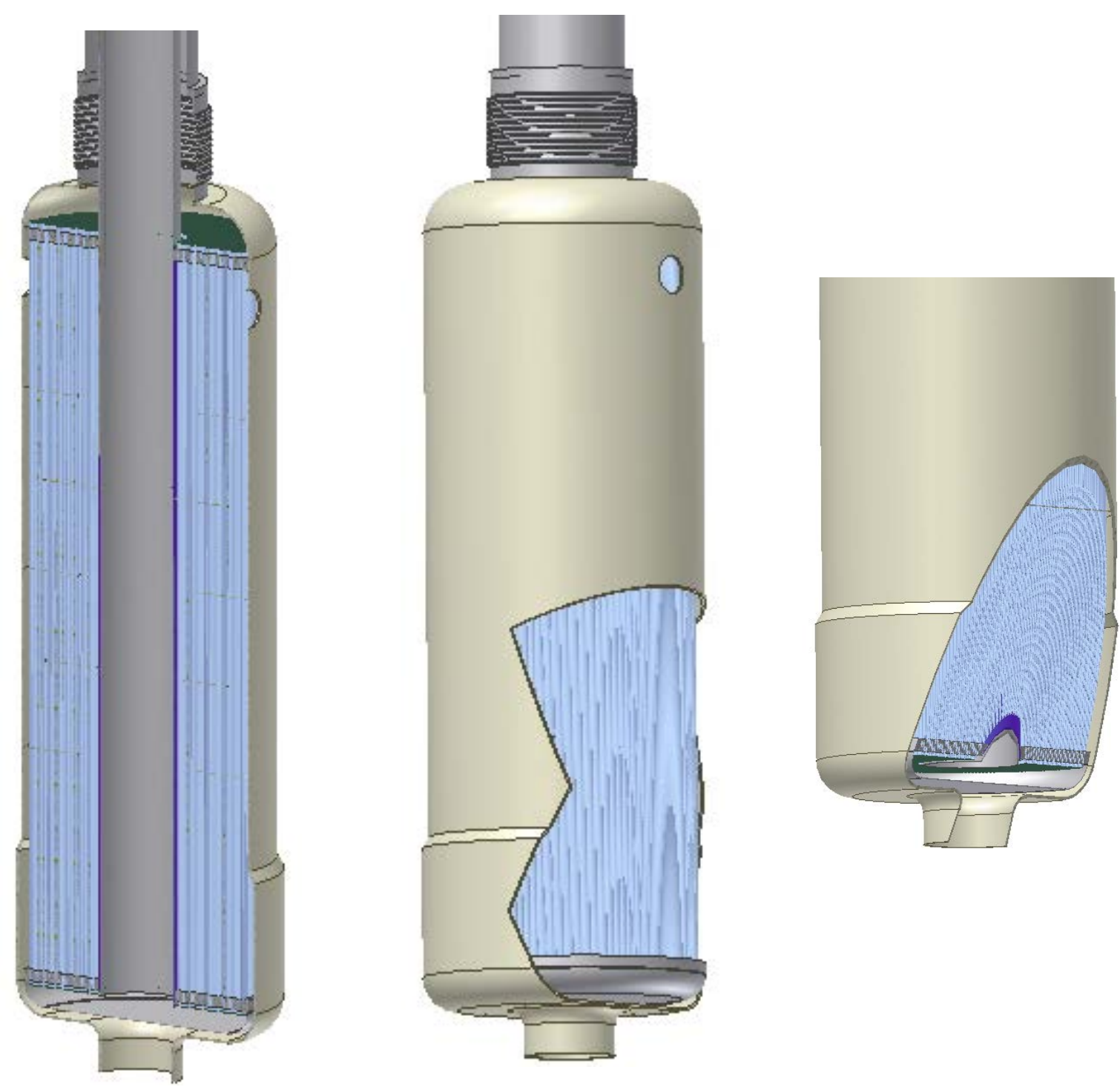

Figure II.3-8 Intermediate Heat Exchanger Design Details

The shell side of the IHX includes a series of horizontally mounted, disk and donut-type baffle plates uniformly spaced at $91 \mathrm{~cm}$ intervals along the length of the tube bank. Aside from providing lateral support for the tubes, these plates promote cross-flow and mixing that enhances thermal performance on the shell (primary) side. The plates are made from $6.4 \mathrm{~mm}$ steel plate and occlude $50 \%$ of the vertical flow path at each plate location. As shown Table II.3-5, the plates are orificed (45 \% equivalent porosity) to reduce flow pressure drop; a schematic showing the orifice design is provided in Figure II.3-10. This approach is similar to that used in the GE Prism Mod B IHX design.

As shown in Figure II.3-9, cold intermediate sodium enters the IHX through a central $61 \mathrm{~cm}$ diameter downcomer. The downcomer delivers the cold sodium through the lower tube sheet into a header manifold, where it then turns $180^{\circ}$ and rises through the tube bank in counter current flow to the shell side primary sodium. The hot intermediate sodium exits the tubes into 


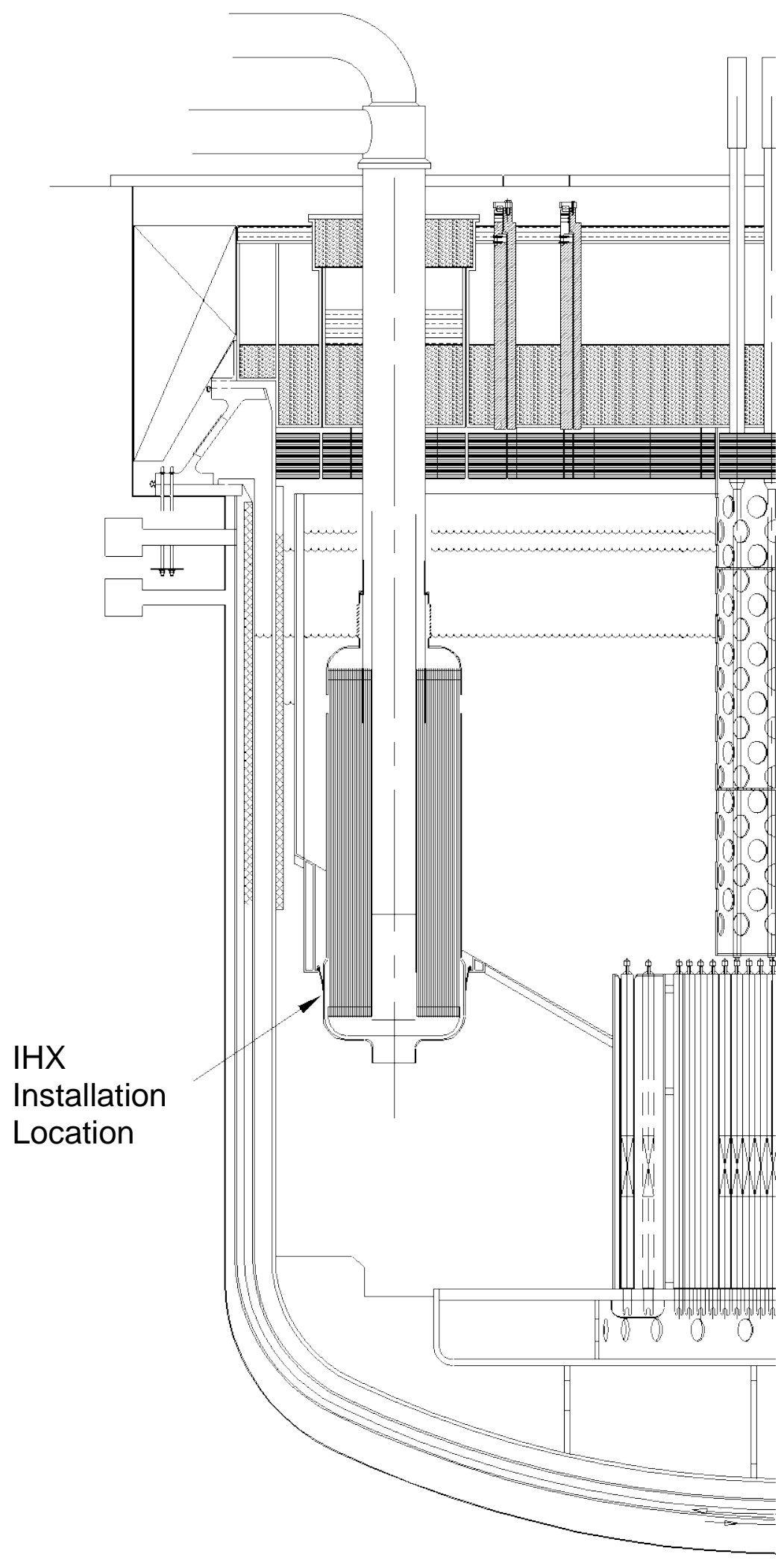

Figure II.3-9 Illustration of IHX Installation Inside the Reactor Vessel 


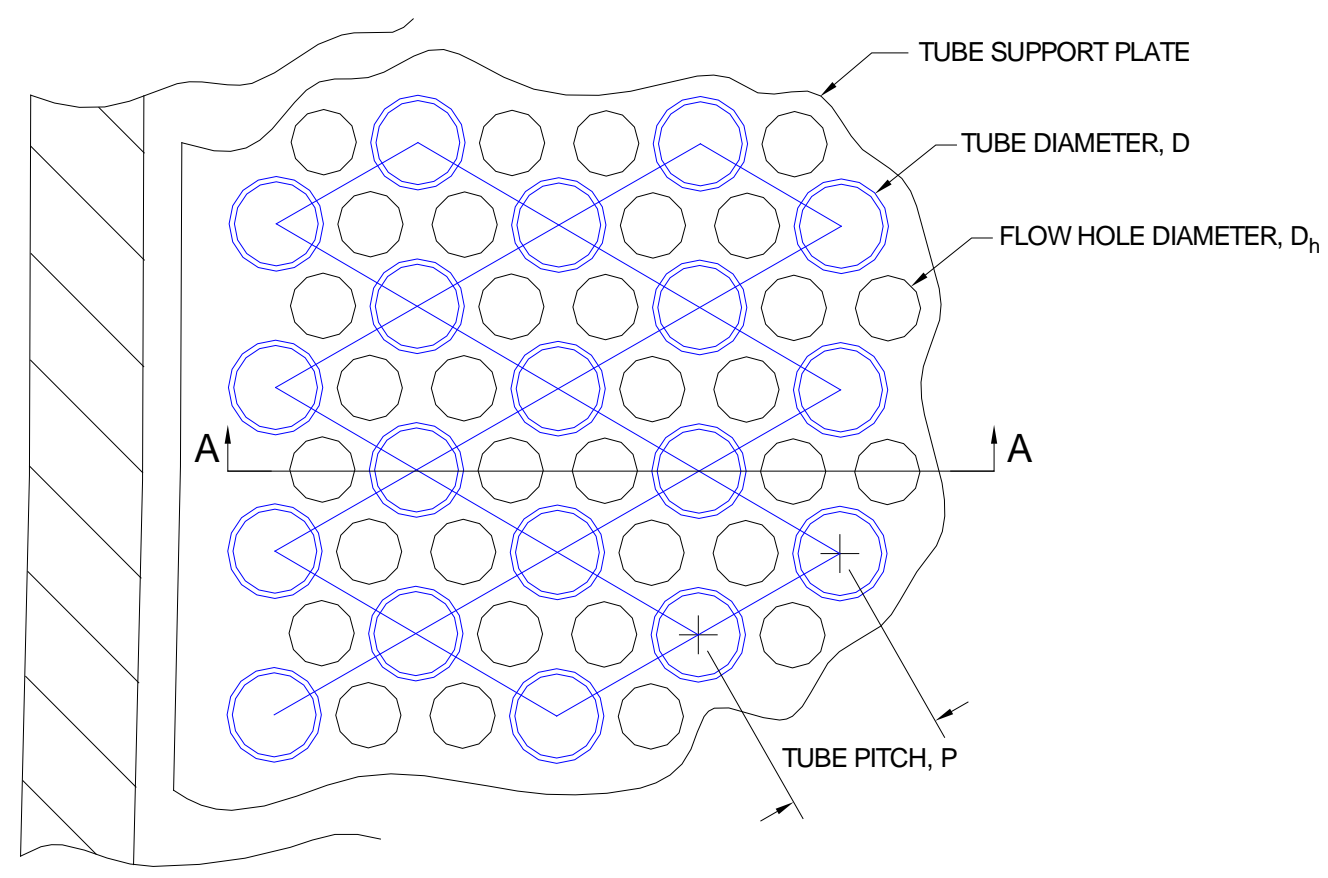

Figure II.3-10 Plan View of Orifice Baffle Plate Design

an upper header manifold, and then flows through an annular riser which is concentric to the downcomer. The downcomer is double walled with an annular gap for thermal insulation between the hot and cold streams. As shown in Figure II.3-9, both the downcomer and the 87 cm OD annular riser pipes are equipped with bellows just above the shroud to accommodate any differences in thermal expansion between the piping and the body of the IHX itself (each unit is rigidly attached to the removable plug in the reactor vessel head). The upper tube sheet is welded to the shroud, while the lower tube sheet floats. Thus, the design accommodates differential thermal expansion within the tube bank also.

The inner wall of the shell operates near the bulk temperature of the sodium in the hot plenum. Since primary sodium flow is on the shell side of the IHX, there is no need for an insulating annulus to eliminate heat losses to the bulk sodium or to alleviate high thermal stresses in the shell.

Modified 9Cr-1Mo steel was chosen as the material of construction primarily because the thermal conductivity is higher than that of the austenitic steels such as Type 304 stainless steel. Since the heat transfer in sodium-to-sodium heat exchangers can be dominated by the tube wall thermal resistance, using modified 9Cr-1Mo steel results in considerable reduction in the required heat transfer area. The use of Type 304 stainless steel tubes would result in the need for as much as $20 \%$ more heat transfer area as compared to modified 9Cr-1Mo tubes with the same design characteristics. In addition, modified 9Cr-1Mo has a lower thermal expansion coefficient compared to Type 304 stainless steel. The higher thermal conductivity material results in lower temperature differences in component sections and, coupled with the reduced thermal expansion, results in lower thermal stresses in structural members. This is advantageous during thermal transients. Straight tubes are selected to simplify fabrication and reduce flow induced vibration problems. 
The design of the IHX has been selected such that the primary flow of sodium on the shell side provides a low pressure drop. Low pressure drop on the primary side is important from two viewpoints: 1) minimizing the pressure-related structural requirements for the IHX shell, and 2) promoting the ability to establish natural circulation of the primary sodium in the case of a lossof-flow event. Adequate natural convection flow for shutdown heat removal is essential. One key element of the DRACS shutdown heat removal system relies on natural circulation of the primary sodium through the core and IHX to the sodium pool surrounding the core barrel. Thus, the IHX has been sized and positioned to locate the primary sodium inlet below the faulted sodium level (primary sodium leak from the primary vessel to the annulus between it and the guard vessel).

Aside from the overall thermal/hydraulic design of the IHXs, additional analysis is needed to verify that the thermal stresses in various regions of these units will remain below acceptable levels to ensure that the plant design lifetime requirement can be met without undue risk of stress-related failure(s). Furthermore, shielding may need to be incorporated into the design if calculations indicate excessive activation of the secondary sodium by virtue of the close proximity of the IHXs to the core if the reactor core does not already provide sufficient shielding. These additional analyses would be part of the conceptual (CD-1) design phase.

Note that as part of the design process, a set of parametric thermal/hydraulic calculations were performed that formed the technical basis for sizing of these units so that they would fit inside the available space between the redan and reactor vessel inner surface, while achieving the desired $250 \mathrm{MWt}$ rating per IHX. In addition, a specialized model was developed in order to accurately evaluate flow pressure drop on the shell side of the IHX, including the effect of the orificed baffle plates. These various design calculations are documented in Section III.8.

\section{II.3.4 Internal Piping}

There is very little primary piping within the reactor vessel assembly. The primary piping exists between the mechanical pump and the inlet plenum. The coolant that flows through the pump will flow into a multi-pipe header that connects the articulated coupling to the inlet plenum structure. This primary piping would consist of a main header for each pump with multiple pipes leading from each pump header into the inlet plenum structure. The main header has a spherical seat that is connected to a flexible coupling. The other end of the internal piping is welded to the core inlet plenum.

Each primary piping assembly is supported to take the appropriate mechanical, thermal and hydraulic loads. The pump hangs from the reactor vessel enclosure and is connected to the inlet plenum or inlet pipe header (depending upon pump application) by a special, nonrigid, easily disconnected, low leakage articulated coupling. This articulated core inlet pipe coupling is shown in Figure II.3-11. 


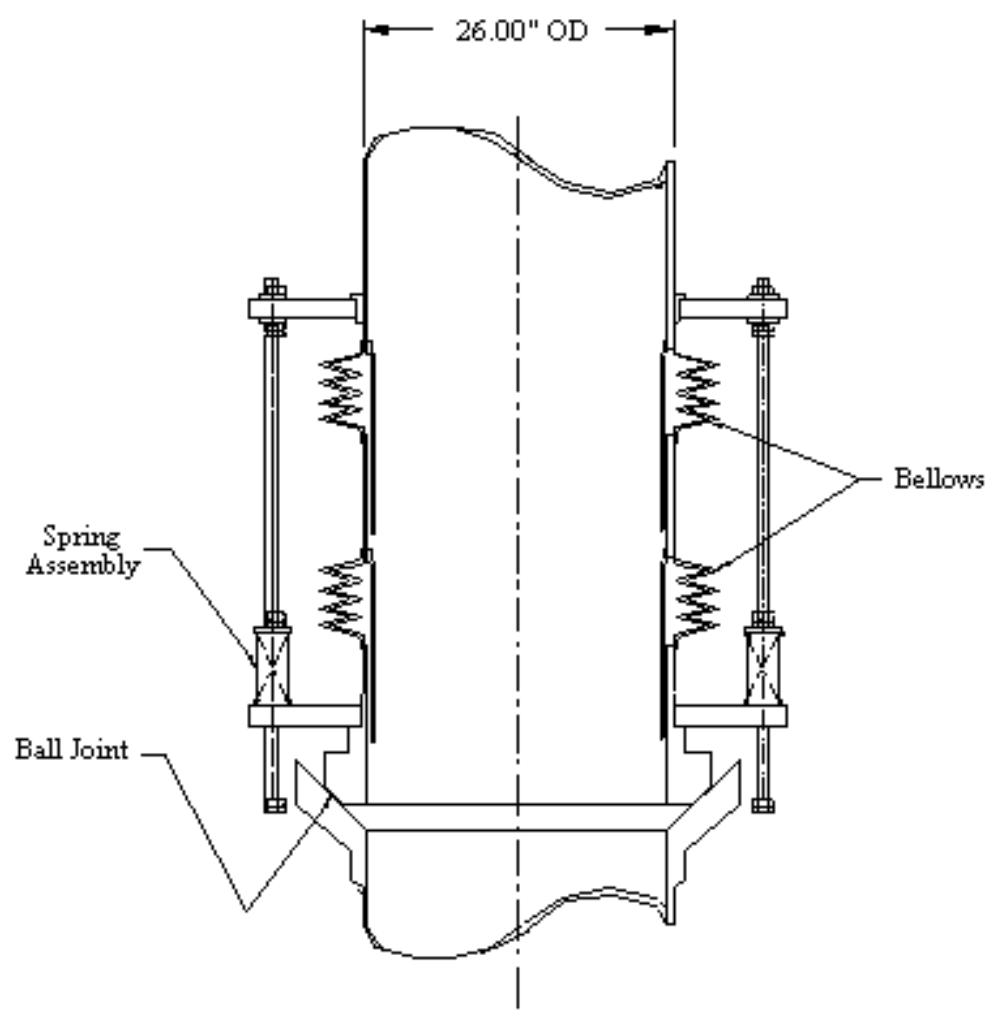

Figure II.3-11 Articulated Core Inlet Pipe Coupling (Concept)

The flexible coupling allows lateral and vertical movement without losing metal-to-metal contact and accommodates relative displacements between the pump and the piping header. The coupling has a slip joint to extend or retract the coupling length with a ball joint at the bottom end. The ball joint at the bottom end is unattached and connects with the mating seat on the main piping header. During installation, the pump is lowered vertically into the reactor vessel. When the bottom ball joint contacts the mating spherical seat on the main piping header, the coupling is compressed, creating spring pressure in the coupling. The spring pressure maintains the coupling in position and seals the ball joint against the mating joint of the main piping header.

The coupling allows the pump to be removed or installed without cutting the inlet interface. The coupling can permit some leakage but must provide flexibility to accommodate thermal movements, be sufficiently stable to avoid flow induced vibrations, and accommodate seismic loads. No valves or movable flow control devices are part of the inlet piping or flexible coupling. Pipe insulation is not required. 


\section{II.4 Intermediate Heat Transport System}

The intermediate heat transport system provides an isolation coolant circuit between the primary reactor coolant and the steam generator system. This intermediate circuit prevents leaks in the steam generator from directly impacting the reactor. The intermediate heat transport system consists of the intermediate sodium pump, the intermediate piping, the steam generator, and the auxiliary sodium systems.

\section{II.4.1 System Requirements and Description}

The Intermediate Heat Transport System (IHTS) circulates secondary sodium coolant, transporting heat from the radioactive sodium in the Primary Heat Transport System (PHTS) to the power generation system. Currently, the Rankine steam cycle is the reference power conversion system for the ABR. The IHTS is composed of four completely independent loops, as shown in Figure II.4-1. This figure provides an isometric view of the piping runs to the steam generators. The flow rate through all four loops is controlled to maintain the primary sodium coolant temperature at design operation conditions. With the exception of the short pipe runs of the hot and cold sodium piping connected to the IHX and running from the IHX to the steam generators, the entire IHTS is contained within the steam generator facility that is part of the reactor building.

The secondary sodium exits the upper portion of the IHX after being heated to $488{ }^{\circ} \mathrm{C}$. The sodium then exits the reactor containment and traverses the short distance to the adjacent steam generator compartment that is on the nuclear island. Here, the hot sodium enters the top of the steam generator and transfers heat to the steam before exiting at the bottom of these units. After exiting, the secondary sodium circulates to the intermediate pump where it is pumped back to the reactor containment and tube side of the IHX.

The tube walls of the IHX constitute the principal barrier for isolation of the activated primary sodium from the sodium in the secondary system. In the unlikely event of an IHX tube leak, sodium leakage will occur from the IHTS to the PHTS due to the fact that the secondary system is maintained at a pressure of at least 0.6 bar ( 8.5 psig) in excess of the primary system. The excess pressure is due to sodium hydrostatic head that is provided by the higher elevation of the secondary loop relative to the primary loop (see Figure II.4-1). Radiation detectors on the outlet (hot leg) piping from the IHX monitor for indications of contamination of the secondary loop sodium.

Major components in each of the four loops include the mechanical pump, steam generator, sodium storage tank, and the piping connecting these components to each as well as the IHX and steam generator. Design details of the IHX and steam generator systems are provided in Sections II.3.3 and II.5.3, respectively. Details regarding the balance of key system components are provided below. 


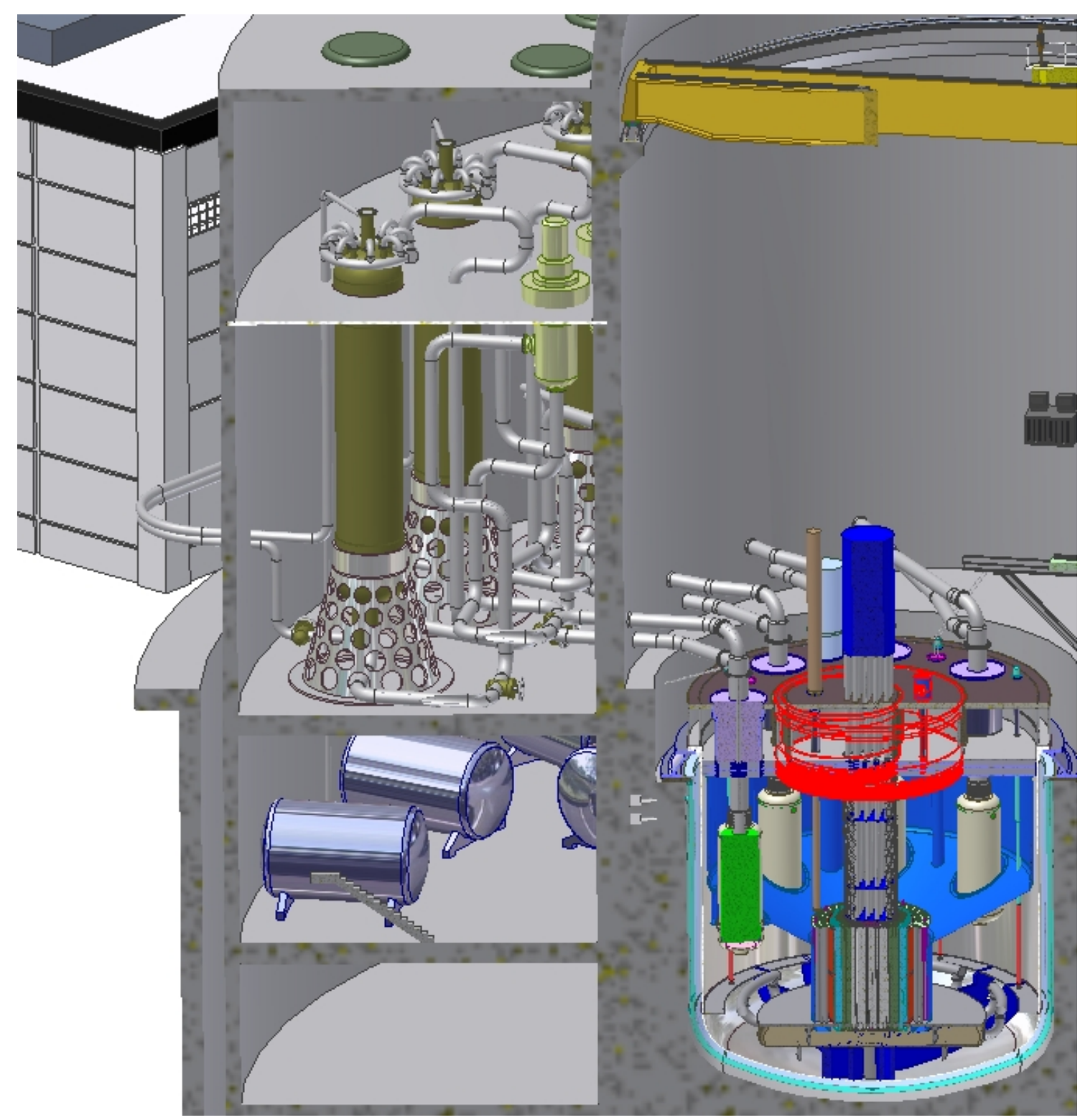

Figure II.4-1 Illustration of IHTS Layout

Auxiliary systems that connect to the IHTS main loop that are necessary to achieve operational requirements include a circulating sodium purification system, trace heating, thermal insulation, and instrumentation that monitors key system parameters including flow rate (with permanently installed magnetic flowmeter in each cold leg), and temperature differential across the IHTS (with thermocouples installed at the steam generator inlet and outlet legs).

\section{II.4.2 Intermediate Sodium Pump}

One sodium pump is used in each IHTS loop. Each unit is a vertically mounted, single stage, single suction, free surface, centrifugal mechanical pump with a lower radial hydrostatic bearing operating in sodium that is fed from the delivery side of the impeller. The pump shaft rotates in two bearings, the lower being a radial hydrostatic bearing and the upper usually a radial-axial 
roller bearing or a liquid or grease lubricated sliding bearing. The impeller is usually positioned below the hydrostatic bearing. There is an axial or radial guiding duct. A gas shaft seal is placed below the upper bearing, and a flexible coupling connects the shaft of the pump and its variable speed electric drive. The stationary part of the intermediate sodium pump, consists mainly of a stationary tank, tank cover, internal structural assembly, and casing. The tank cover and all internals are removable. A thermal shield is located just below the tank cover. A pressurized argon cover gas is used between the sodium level in the pump tank and the thermal insulation. The cover gas is controlled at a reasonable pressure to maintain the pump net positive suction head (NPSH) available and to prevent the air in-leakage into the system.

As in previous designs, each pump is installed in a tank with the piping inlet and exits at the top and bottom of the tank, respectively. The pumps are completely separate so that the IHTS loops can be operated independently to produce the desired operational characteristics.

As noted earlier, the overall pump design is quite similar to the primary mechanical pump.

\section{II.4.3 IHTS Piping}

The IHTS piping consists primarily of the main system hot and cold legs which make the necessary connections between the IHX and the steam generator. The hot leg piping connects to the secondary sodium outlet of the IHX directly to the steam generator sodium inlet. The IHTS cold leg piping connects the sodium outlet from the steam generator to the intermediate pump tank inlet and then from the pump tank discharge to the secondary cold sodium inlet to the IHX.

The IHTS piping is constructed from $61 \mathrm{~cm}$ OD, $1.74 \mathrm{~cm}$ thick-walled (24 inch Schedule 40) 316 stainless steel piping, primarily because of the lack of corrosion issues for sodium and the ease of fabrication with this material. The use of this piping diameter and schedule maintains the sodium flow velocity through the secondary piping system below $\sim 7 \mathrm{~m} / \mathrm{sec}$, which is a rule-ofthumb design criterion for these types of systems. The use of 316 stainless steel requires dissimilar metal welds connecting the austenitic Type 316 stainless steel to the ferritic steels used in the IHX. The welding technology for joining these dissimilar metals is well established.

Inside the reactor building, the IHTS piping is enclosed in secondary piping so that in the unlikely event of failure of the main system piping, the sodium is contained which greatly reduces the possibility of a major sodium leak. In addition, the steam generator building, containing the IHTS, is seismically isolated on the same platform as the reactor itself, which eliminates differential motions between these two structures during seismic events. This simplifies the design for piping hangers and supports that would otherwise need to be equipped with snubbers or other motion-dampening devices if the reactor building were not integral to the nuclear island. 


\section{Table II.4- 1 Intermediate Sodium Pump Characteristics}

$\begin{array}{lc}\text { Parameter } & \text { Value } \\ \text { Type } & \text { Mechanical } \\ \text { Quantity } & 4 \\ \text { Mass flow rate } & 1290 \mathrm{~kg} / \mathrm{s} \\ \text { Volumetric Flow rate } & 1.476 \mathrm{~m}^{3} / \mathrm{s} \\ \text { Pump head (psig) } & 0.23 \mathrm{MPa} \\ \text { Temperature } & 326^{\circ} \mathrm{C} \\ \text { Power } & 404 \mathrm{~kW} \\ \text { Efficiency } & 84.09 \%^{\mathrm{a}} \\ \text { Drive voltage } & 4160 \mathrm{~V} \\ \text { Drive current } & 100 \mathrm{~A} \\ \text { Drive frequency } & 60 \mathrm{~Hz} \\ \text { Pole count } & 4 \\ \text { Net positive suction head }- & 10.36 \mathrm{~m} \mathrm{of} \mathrm{sodium} @ \\ \text { required } & 326^{\circ} \mathrm{C} \\ \text { Net positive suction head }- & 14.2 \mathrm{~m} \mathrm{total} \\ \text { available } & 1.6 \mathrm{~m} \text { of sodium } \\ & 12.6 \mathrm{~m} \text { of cover } \\ \text { Mass } & \text { pressure at } 326^{\circ} \mathrm{C} \\ \text { Pump height (length) } & 7647 \mathrm{~kg} \\ \text { Pump outer barrel diameter } & 7.65 \mathrm{~m} \mathrm{without} \mathrm{motor} \\ \text { Pump case diameter } & 1.27 \mathrm{~m} \\ \text { shaft diameter } & 0.97 \mathrm{~m} \\ \text { Suction nozzle diameter } & 0.09 \mathrm{~m} \\ \text { Discharge nozzle diameter } & 0.86 \mathrm{~m} \\ \text { Drive motor mass } & 0.86 \mathrm{~m} \\ \text { Drive motor length } & 3700 \mathrm{~kg} \\ \text { Drive motor diameter } & 2.3 \mathrm{~m} \\ \text { Drive motor rotational speed } & 1.1 \mathrm{~m} \\ \text { Material } & 1000 \mathrm{rpm} \\ & 304 \mathrm{SS} \\ & \end{array}$




\section{II.4.4 IHTS Sodium Storage Tank, Expansion Tank, and Cleanup System}

A small secondary loop is included in the IHTS to maintain sodium volume and to provide a purification pathway for the secondary sodium. The loop supplies sodium from a cold trap to the mechanical pump tank to maintain a constant level of sodium in the IHTS loop. The recirculation system uses a fill and overflow approach to maintain the sodium level constant. Spillover into the mechanical pump tank overflow line flows through the secondary piping to the storage tank. Sodium is then pumped from the storage tank by a small recirculation pump through interconnecting piping to the cold trap. After circulating through the trap, the sodium circulates back to the pump tank.

The circulating sodium to the storage tank keeps the tank at system temperature during normal operations. When flow is not available, trace heating is used to keep system components above the sodium freezing point.

Pressurized argon cover gas is maintained in the pump tank upper plenum. Subsystems must also be provided to control the argon cover gas pressure and supply. 


\section{II.5 Power Generating System}

\section{II.5.1 System Requirements and Description}

A Rankine steam cycle is the reference power conversion system for ABR. This system utilizes heat from the Intermediate Heat Transport System (IHTS) to produce steam. The steam is delivered to the turbine, which drives the electrical generator. This is the same power conversion technology that has been used for all sodium-cooled fast reactors built to date. This technology is well developed and, with the exception of the steam generators and the Sodium Water Reaction Pressure Relief System (SWRPRS), the system components are largely off-theshelf items available from commercial vendors. At this point in the preconceptual design process, attention has been focused on the power production side of the system. However, a preliminary design for the SWRPRS has also been developed as part of this work, and this is described later in this section.

Steam generator concepts that have been developed over the years include the double-walled straight tube design that operated successfully for over 30 years for EBR-II, the hockey-stick design that was developed by Rockwell for the SAFR concept, and the Helical Coil Steam Generator (HCSG) that was developed by GE for the Prism Mod-B Concept. For the purposes of this pre-conceptual design, a modified version of the HCSG design is adopted, as it is readily scaled to different reactor power levels.

The main components of the steam generation system include four sodium heated steam generators (one for each IHTS loop), a feedwater system, and a steam distribution system. The sodium side of each steam generator is connected to the hot and cold legs of the main sodium piping on the secondary (tube) side of each IHTS loop.

The steam cycle selected for application to the ABR is a superheated cycle with dual reheats. Five feedwater heaters are used to increase cycle efficiency. The steam generator is a oncethrough design with feedwater entering the bottom of the steam generator at a temperature of 216 ${ }^{\circ} \mathrm{C}$, and superheated steam exiting from the top at a temperature of $454{ }^{\circ} \mathrm{C}$. The high pressure turbine receives steam at 2250 psia, and the low pressure turbine exhausts to a condenser at a pressure of $5 \mathrm{~cm}$ (2 inches) of mercury. The power conversion system produces approximately $380 \mathrm{MW}$ of electric power. The steam cycle system design parameters are summarized in Table II.5-1. 


\section{Table II.5-1 System Cycle System Design Parameters for 1000 MWt Design.}

$\begin{array}{ll}\begin{array}{l}\text { Parameter } \\ \text { Primary (Core) Thermal/hydraulic Data }\end{array} & \text { Value } \\ \text { Core Power } & 1000 \mathrm{MWt} \\ \text { Core Inlet Temperature } & 355^{\circ} \mathrm{C} \\ \text { Core Exit Temperature } & 510^{\circ} \mathrm{C} \\ \text { Core Temperature Rise } & 155^{\circ} \mathrm{C} \\ \text { Core Sodium Mass Flowrate } & 5028 \mathrm{~kg} / \mathrm{sec} \\ \text { IHTS Loop Operational Data } & \\ \text { Number of Intermediate Loops } & 4 \\ \text { Secondary Loop Heat Capacity } & 250 \mathrm{MWt} \\ \text { Loop Hot Leg Temperature } & 477^{\circ} \mathrm{C} \\ \text { Loop Cold Leg Temperature } & 326^{\circ} \mathrm{C} \\ \text { Loop Temperature Drop } & 151^{\circ} \mathrm{C} \\ \text { Loop Sodium Mass Flowrate } & 1290 \mathrm{~kg} / \mathrm{sec} \\ \text { Helical Coil Steam Generator (HCSG) } & \mathbf{O p e r a t i o n a l ~ D a t a} \\ \text { SG Inlet Pressure } & 167 \mathrm{bar} \\ \text { SG Feedwater Inlet Temperature } & 216^{\circ} \mathrm{C} \\ \text { SG Feedwater Mass Flowrate } & 111.8 \mathrm{~kg} / \mathrm{sec} \\ \text { Steam Exit Temperature } & 454^{\circ} \mathrm{C} \\ \text { Steam Exit Pressure } & 155 \mathrm{Bar} \\ \text { Saturation Temperature } \quad \text { at } & 345^{\circ} \mathrm{C} \\ \text { Pressure } & \\ \text { Steam Exit Superheat } & 109^{\circ} \mathrm{C}\end{array}$

\section{II.5.2 Steam Generator System}

\section{II.5.2.1 Steam Generator}

The function of the steam generator is to produce steam for the turbine-generator set from the heat transported to it via the primary heat transport system and the intermediate heat transport system. Steam is generated under normal power conditions (40-100\% power), and under decay heat conditions, using the Benson cycle. That is water is preheated, evaporated, and superheated in the single pass through the steam generator with no recirculation. Each intermediate heat transport system feeds high temperature sodium to one steam generator. The steam generator building is located adjacent to the reactor containment building and on the base isolation system. Each steam generator unit operating at full power provides the turbine with steam at $~ 450 \mathrm{C}$ and 2250 psig from approximately 250MWth transferred from the IHTS. The steam generator produces superheated steam between 40 and $100 \%$ of the power. 
As noted earlier, a steam generator design similar to that utilized in the GE Prism Mod B plant concept is proposed for the ABR. The steam generator is a helical coil, vertically oriented, sodium-to-water, countercurrent flow, shell-and-tube type unit featuring once-through operation. Four 250 MWt steam generator units are utilized for ABR; one for each IHTS loop.

The steam generator design utilizes the identical tube diameter, thickness, and pitch-todiameter ratio as for the PRISM Mod B helical-coil design. Moreover, the tube length and helical pitch are the same. Thus, if the water and sodium mass flowrates per tube are conserved, then 184 tubes per unit are selected to achieve the required $250 \mathrm{MWt}$ heat exchange rate based on a linear scaling of the Prism design (viz., 630 tubes for $845 \mathrm{MWt}$ rating). A diagram that illustrates key elements of the scaled steam generator design is provided in Figure II.5-1, while key design data are summarized in Table II.5-2. Aside from the thermal power rating, the thickness of structural elements (i.e., upper and lower tube sheets, as well as shell and elliptical head thicknesses) have been scaled to approximately preserve peak mechanical stresses for a given pressure loading. Moreover, the diameters of inlet and exit piping on both the sodium and steam sides of the HCSG have been scaled to approximately preserve flow velocities, which then approximately preserves pressure drops at the various points within the steam generator.

During full power operation, feedwater flow to the steam generator is regulated by a $20 \mathrm{~cm}$ (8 inch) main control valve. The main feedwater line breaks into four $15 \mathrm{~cm}$ ( 6 inch) feedwater nozzles located on the bottom head of each unit. These nozzles supply water to the inlet of the steam generator at $216^{\circ} \mathrm{C}$ temperature and 167 bar pressure. The GE design incorporates flow restrictors with a pressure drop of 6.5 bars at the tube inlets to increase static and dynamic flow stability over a wide load range. After entering the steam generator, the water flows up through the tube side of the $3.18 \mathrm{~cm}$ OD, $98.5 \mathrm{~m}$ long tube bank while absorbing energy from the sodium on the shell side of the unit in a counter-current flow configuration. The water then exits the steam generator as superheated steam at a temperature of $454^{\circ} \mathrm{C}$ and pressure of 155 bars through four $20 \mathrm{~cm}$ diameter (8 inch) steam nozzles located in the top head of the unit. These four nozzles are then headered into the $30 \mathrm{~cm}$ diameter (12 inch) main steam line leading to the turbine generator building. To aid in plant control and monitoring, flowrate and temperature measurements are made both at the feedwater inlet and steam exits to the steam generator. 


\section{Table II.5- 2 Scaled Once-Through HCSG Design Data for 1000 MWt Plant Design}

\author{
Parameter \\ Capacity \\ Operational Mode \\ Configuration
}

Number of Tubes

Tube OD

Tube ID

Overall Tube Length

Tube Material of Construction

Overall Tube Heat Transfer Surface Area

Heat Transfer Surface Area Margin

Tube Bundle Transverse Pitch

Tube Bundle Longitudinal Pitch

Tube Pitch Angle

Number of Tube Coil Rows

Helical Coil Bundle Height

Vessel Outside Diameter

Inner Shroud Outside Diameter

Steam Generator Height

Water Side Tube Pressure Drop

Inlet Restrictor Pressure Drop

Sodium Side Pressure Drop

Shell Thickness

Elliptical Head Thickness

Tube Sheet Thickness
Value

$250 \mathrm{MWt}$

Single Pass

Cross-Flow; Shell and Helical

Coil; Tube Side Water/Steam Flow

184

$3.18 \mathrm{~cm}$

$2.00 \mathrm{~cm}$

$98.5 \mathrm{~m}$

2-1/4 Cr-1 Mo

$1806 \mathrm{~m}^{2}$

$20 \%$

$5.72 \mathrm{~cm}$

$4.76 \mathrm{~cm}$

$7.55^{\circ}$

6

$11.6 \mathrm{~m}$

$281 \mathrm{~cm}$

$137 \mathrm{~cm}$

$20.72 \mathrm{~m}$

11.72 bar

6.55 bar

0.19 bar

$3.81 \mathrm{~cm}$

$4.45 \mathrm{~cm}$

$8.89 \mathrm{~cm}$

On the shell side of the unit, sodium is headered from the $61 \mathrm{~cm}$ diameter (24 inch) IHTS hot leg supply line from the IHX into four $30 \mathrm{~cm}$ diameter (12 inch) nozzles that provide the pathway for the sodium to enter through the upper elliptical head of the unit. The sodium then flows in a counter-current flow configuration on the shell side of the heat exchanger while delivering heat to the steam/water mixture on the interior of the tubes. The sodium then exits the steam generator through a single $61 \mathrm{~cm}$ diameter (24 inch) pipe that constitutes the cold leg of the IHTS.

The total system can be operated on either 3 or 4 loops, so steam generator maintenance can be conducted during operation at $75 \%$ power. There are sodium isolation valves that allow for isolating the steam generators for maintenance and during steam generator casualties, if needed. The units are designed to accommodate the transients specified in the duty cycle.

Particular attention is devoted to the prevention and quick detection of leakages between water and sodium in the steam generator. The overall configuration and the design details are both evaluated in terms of prevention of leaks. Very high quality is demanded of the tube-to-tube 
and tube-to-tube sheet welds. A fast response leakage detection system is included in the IHTS and the steam generator systems. In case of a large sodium-water interaction, the steam generator includes a means of isolating and dumping the water side of the steam generator and discharging the reaction products into a separate vessel. Maintainability and repairability have a high importance in the design of the steam generator. In case of a leak, the location must be readily identified and methods of plugging the leaking tube easily implemented.

\section{II.5.2.2 Steam Cycle}

A Rankine superheated steam cycle power conversion system concept has been developed for the reference ABR. Layouts of key system components, piping, and instrumentation have been roughed out for the purposes of the ABR pre-conceptual design phase. The overall system layout is shown in Figure II.5-2, while system piping and instrumentation are shown in Figure II.5-3. The power conversion system, which is based on standard technology currently employed in fossil-fueled power plants, includes both high pressure, intermediate pressure, and low pressure turbines. Other key system components include a moisture separator and two steam reheaters, as well as three low-pressure and two high-pressure feedwater heaters. The preconceptual design and analysis activities have included a calculation of the overall system electrical production cycle efficiency using the plant performance software GateCycle (GE Energy). For a given system configuration, this tool calculates design and off-design performance of power plants incorporating Rankine or gas turbine cycles. Based on the system design requirements provided in Table II.5-2, the calculated overall cycle efficiency for the system shown in Figure II.5-3 is 38 \%. The system heat balance is shown in Figure II.5-4.

The main steam system transports superheated steam from the two steam generators through a common steam header pipe to the high-pressure turbine inlet valves. Steam is extracted from the high- and intermediate- pressure turbines and is piped to the steam reheaters which condition the steam transported from the intermediate-pressure turbine exhaust to the low pressure turbine inlet. The main steam system includes a turbine bypass to the condenser to prevent a reactor trip in the event of a turbine trip. 


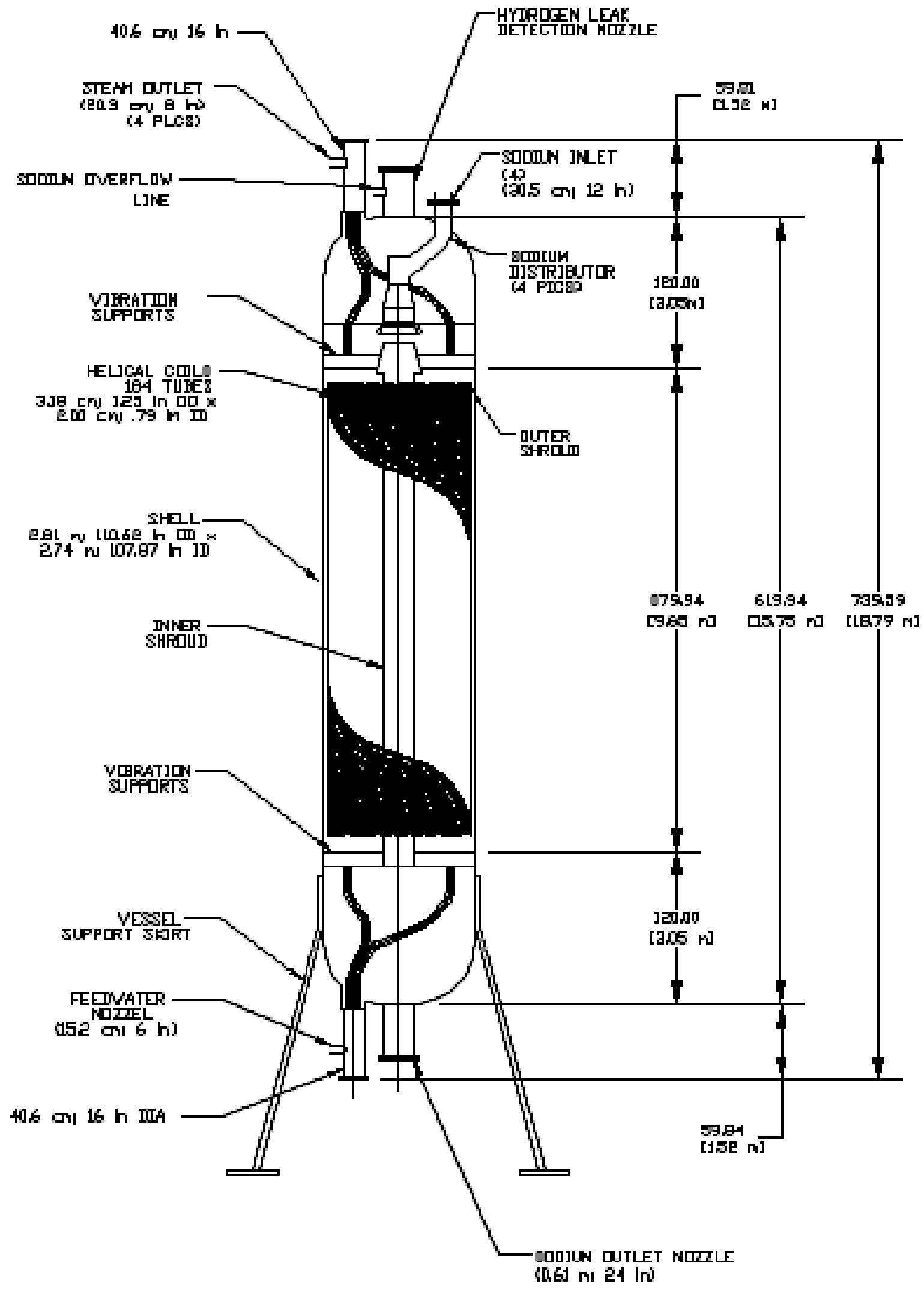

Figure II.5-1 HCSG Design Characteristics (GE Prism Mod B basis) 


\section{II.5.2.3 Auxiliary Systems}

Main auxiliary system components include the feedwater and condensate systems, and the circulating water system. The water quality and chemistry of the plant must be carefully controlled. High purity feedwater is required to prevent deposition of impurities on heat transfer surfaces and to preclude intergranular stress-corrosion cracking of the heat transfer surfaces. This requires careful attention to the design and material selection for each component of the system, i.e., on-line water monitoring, full-flow filters, full-flow demineralizers, and $\mathrm{pH}$ control.

The main function of the condensate system is to condense and collect turbine exhaust steam and water, to reject excess heat, to transport the condensate collected at the condenser hotwells to the deaerator, and to provide the initial condensate/feedwater heating. The main function of the feedwater system is to transport the feedwater collected at the deaerator storage tank to the steam generators at the required flow rate, temperature, and pressure for all operating conditions. This system not only includes the feedwater and condensate systems, but also the condensate demineralizer system. The function of the demineralizer system is to improve the chemistry of the condensate stream in order to make it acceptable for a once-through steam generation system. The feedwater and condensate systems are shown in Figure II.5-5. This design is based on standard components commonly used in the power generation industry. The design and maintainability of the feedwater system is as important as the design of the steam generator system.

A makeup water system is also provided. This system supplies makeup water that meets the feedwater chemistry requirements. The makeup water system utilizes standard components commonly used in power plants.

The circulating water system rejects waste heat from the main condenser shells to the atmosphere through a cooling tower. This system is shown in Figure II.5-6. 


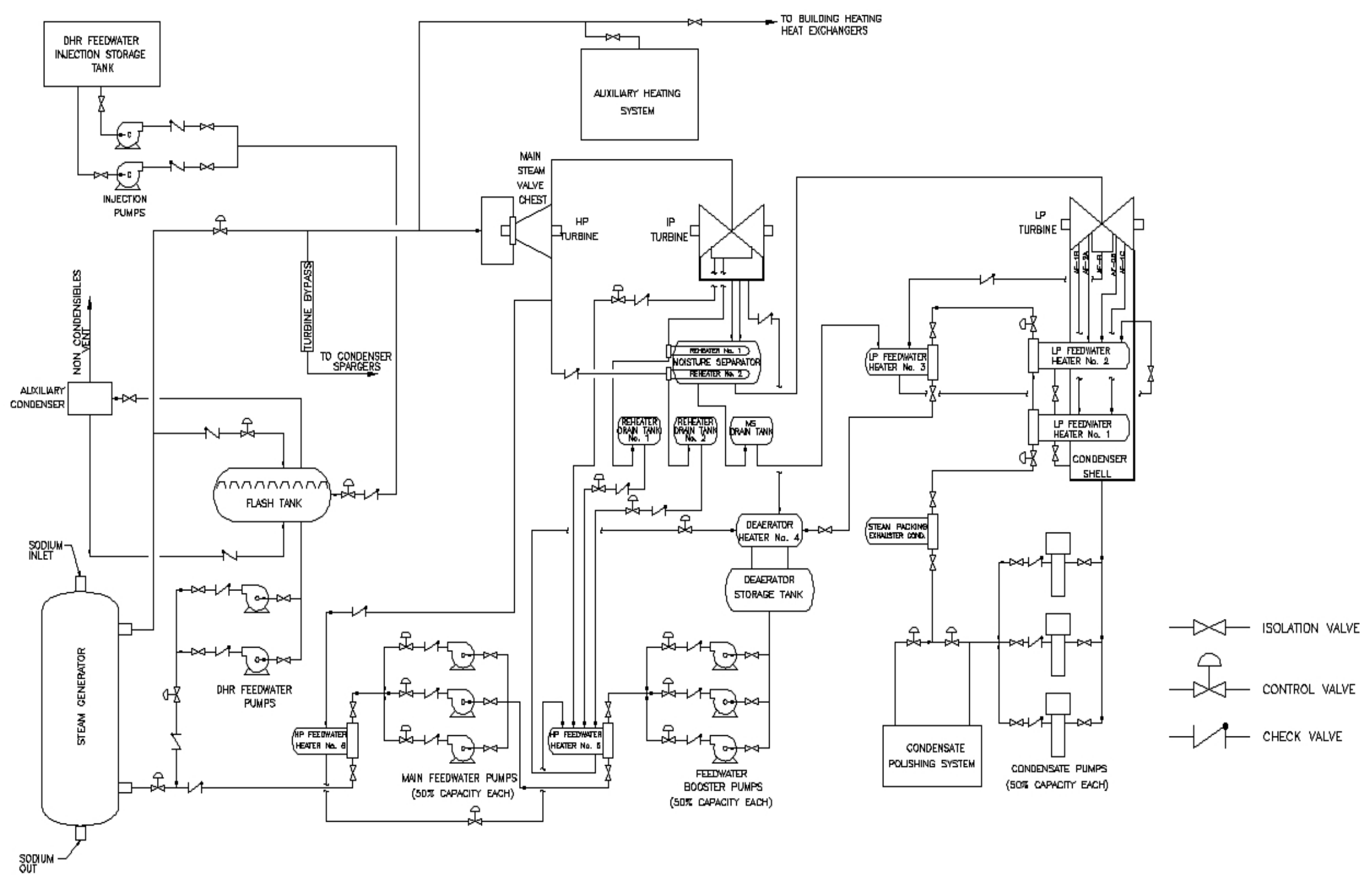

Figure II.5-2 ABR Steam Cycle Power Conversion System 


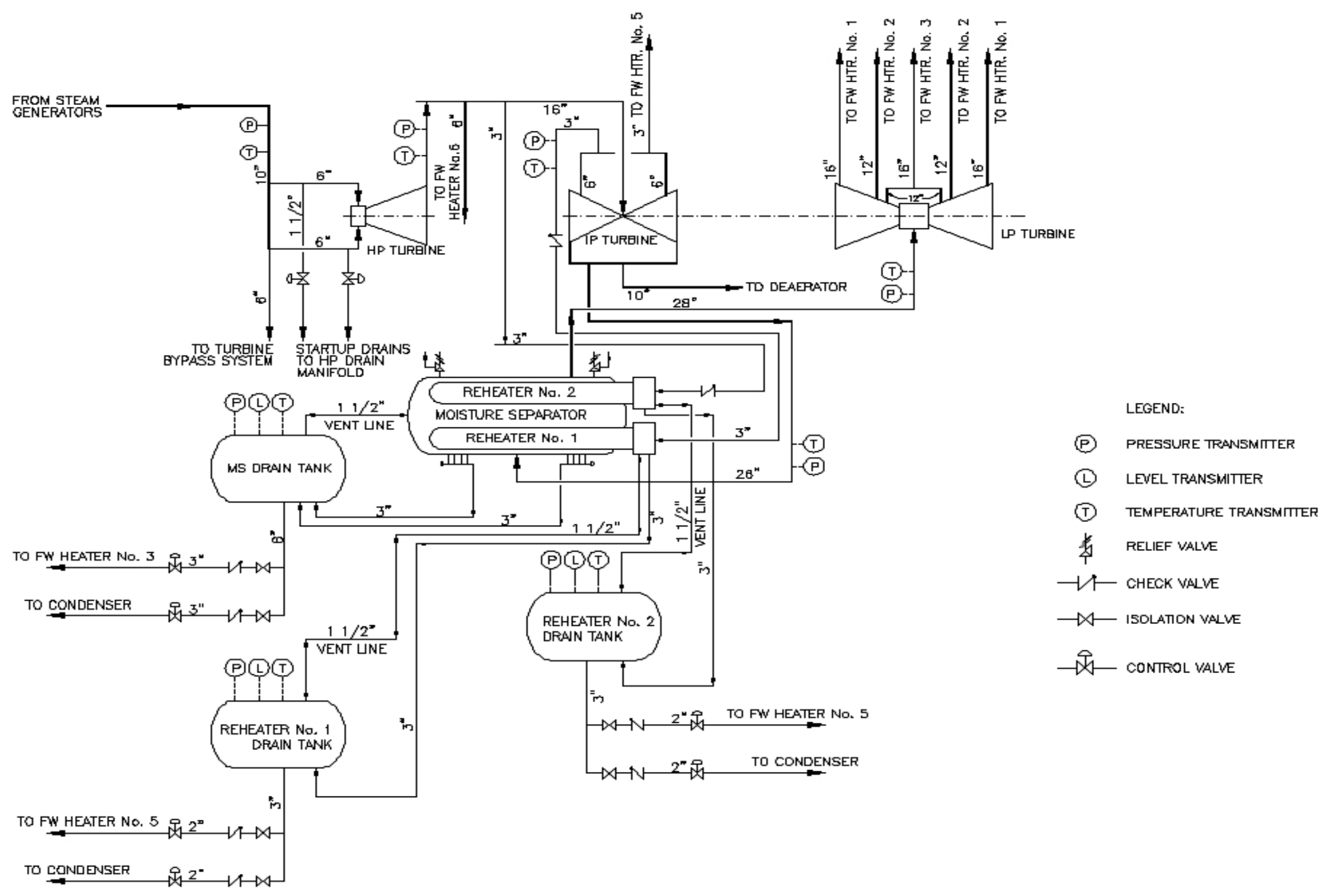

Figure II.5-3 Steam Cycle Piping and Instrumentation Drawing 


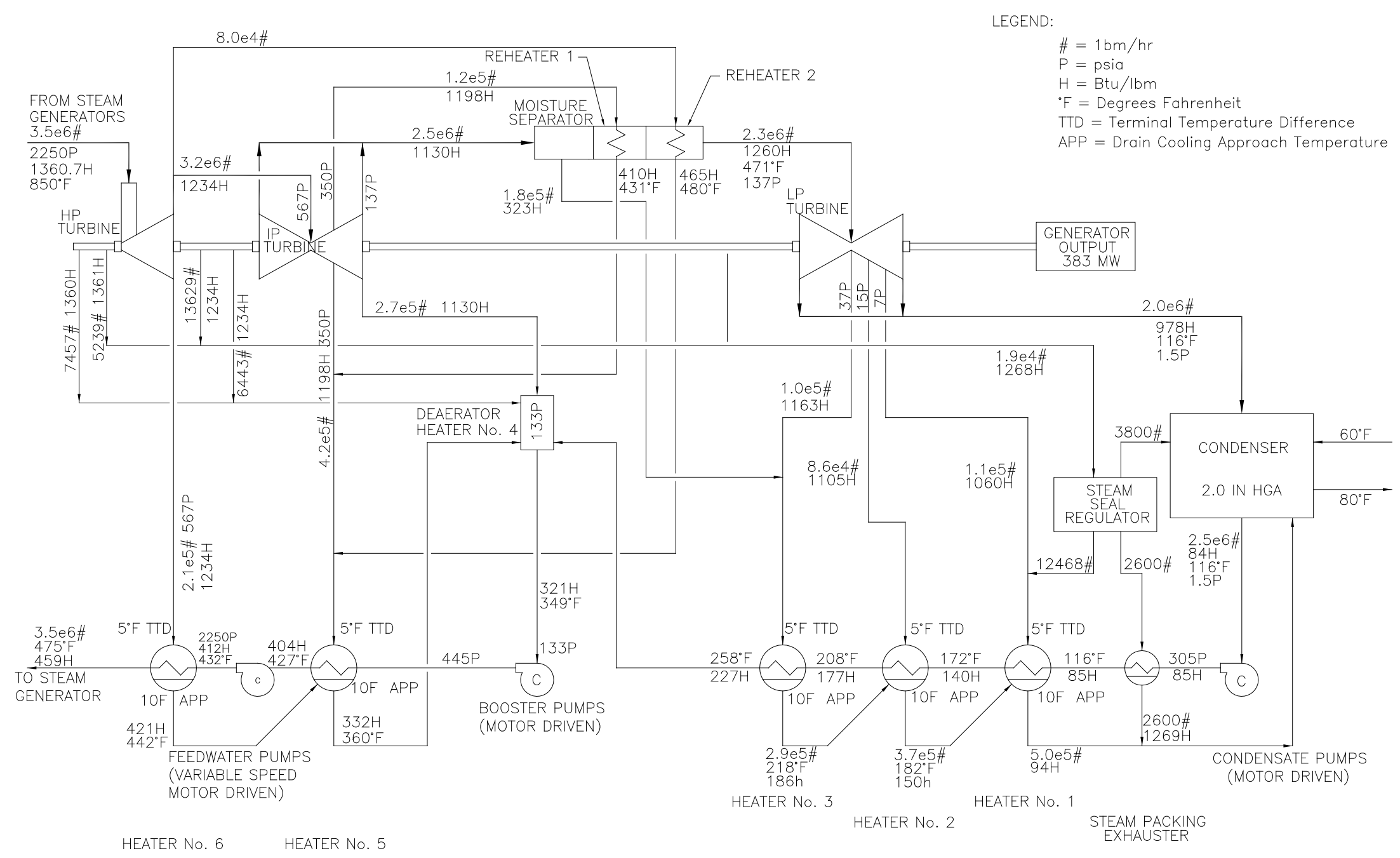

Figure II.5-4 Steam Cycle Heat Balance 


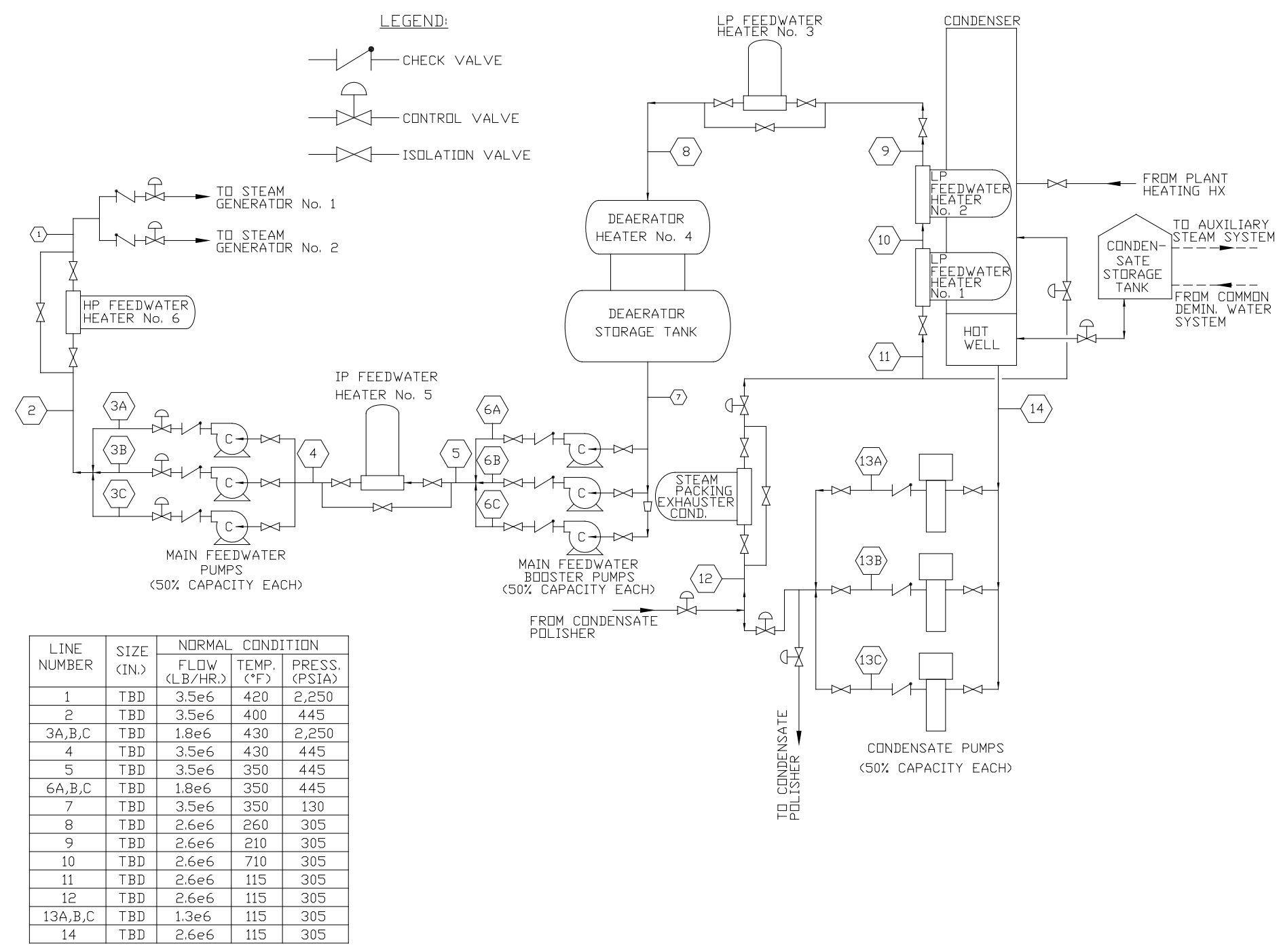

Figure II.5-5 Flow Diagram for the Condensate and Feedwater Systems 


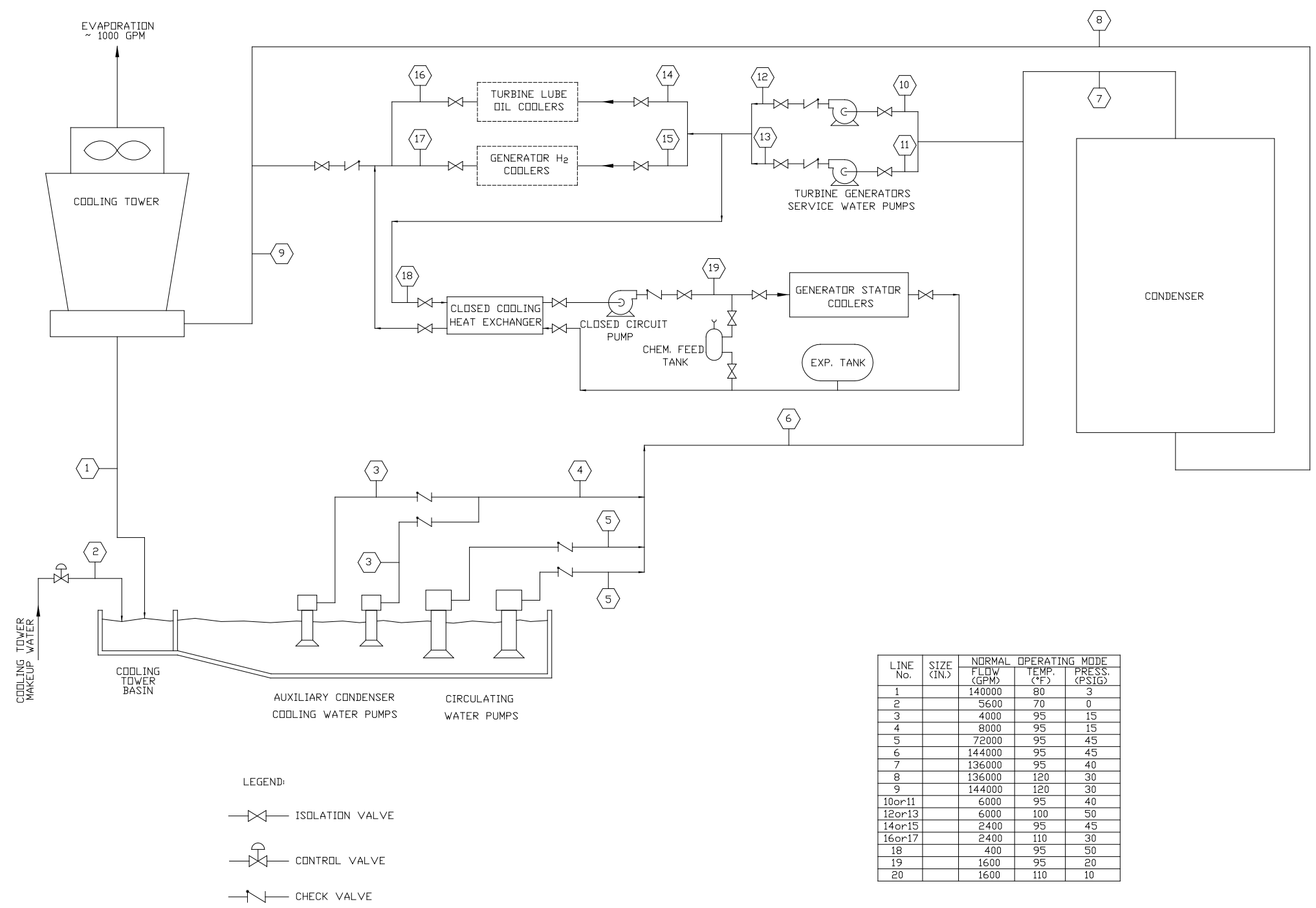

Figure II.5-6 Flow Diagram for the Circulating Water Systems 


\section{II.5.3 Sodium-Water Reaction Protection System}

The sodium-water reaction protection system provides separate relief paths for small (or intermediate size) leaks and for large leaks depending upon the quality and stability of the steam generator design. The smaller leaks are regarded as all those leaks which permit timely detection and response by the operator in systematically shutting down and draining the affected unit to terminate the reaction before leakage exceeds about $0.45 \mathrm{~kg} / \mathrm{s}$ or $1 \mathrm{lb} / \mathrm{s}$. The reaction products from these leaks can be efficiently handled by an oil sparger system of the type that has proven effective in sodium-leak testing. Such systems are inexpensive and passive in their operation, and they are relatively easily returned to readiness after a leak. Location of this system is not shown in the schematics.

A large leak cannot be handled by manual response and by such as simple system, and requires automatic sequences or rapid shutdown, blowdown, dumping of liquids, and isolation to terminate the reaction and preserve the integrity of the plant equipment. Relief and accommodation of the greater volume of reaction products requires a reaction products tank and will probably also require use of a cyclone separator to reduce the particulate emissions to an environmentally satisfactory level. The sodium-water reaction protection system will be design to handle a design-basis leak. The design-basis leak is characterized by the ID of a steam generator tube and resultant choke flow of water that can be postulated to enter sodium in the event of a sudden double-ended break, by the number of surrounding tubes that are postulated to fail similarly through wastage caused by the initial leak, and by the additional reaction products associated with the secondary tube failures.

The SWRPRS acts automatically to isolate and dump the defective steam generator when the occurrence of a large leak $(>0.45 \mathrm{~kg} / \mathrm{s}$ or $>1 \mathrm{lb} / \mathrm{s})$ causes the failure of the main rupture disc to occur within milliseconds. This is followed by reactor scram, secondary system pump and primary pump coastdown in the affected system, and pressurized inert gas blanketing of the S/G water side to prevent back-leakage of sodium into the water system.

Small leaks $\left(<4.5 \mathrm{~g} / \mathrm{s}\right.$ or $\left.10^{\wedge}-2 \mathrm{lb} / \mathrm{s}\right)$ can be handled by isolating and dumping the steam generator manually, followed by inert gas blanketing. It is anticipated that the majority of leaks in a plant fabricated with adequate quality assurance, particularly for welds, will be handled in the latter manner. Figure xxx shows a schematic of the SWRPRS.

The major components of the SWRPRS are rupture discs, reaction product tanks, centrifugal separators, interconnecting piping, igniter, check valve and stack. The components are included within the envelope defined by the rupture disc attachment flange and the sodium water reaction product discharge piping outlet to the atmosphere. A typical design approach is given in Figure II.5-7. This design includes double rupture disc assemblies on the sodium pipes just downstream of the steam generator outlet to provide overpressure protection. Pressure relief piping on each IHTS loop leads to one vertically oriented reaction product separator tanks within the steam generator building where gross separation of liquid, solid, and gaseous material is obtained. This tank is located on the main floor below the steam generators. Gaseous reaction products with entrained particles pass from the reaction products separator tank to a centrifugal separator where additional separation occurs before the hydrogen is vented and burned in the atmosphere. An igniter is located on the vent stack downstream of the atmosphere seal provides for controlled burning of the hydrogen. Provisions are made to flood the entire SWRPRS, IHTS, and failed steam generator module with nitrogen following pressure relief. The solid and liquid reaction 
products (and some sodium) which is separated from the hydrogen is contained in a drain tank below the centrifugal separation tank where it is always under an inert gas.

\section{Rupture Discs}

Rupture discs are generally employed at three different locations in a SWRPRS. They are used to protect the IHTS and SG shell from the potentially catastrophic effects of the pressure pulses associated with large sodium-water reactions that might occur within the steam generators. They are also used to protect the IHTS against over-pressure from an intermediate sodium-water reaction and other causes of over-pressure that increase the pressure of the IHTS in a gradual manner. Finally, they are used at the top of the discharge stack to provide a seal between the inert gas atmosphere within the SWRPRS and the atmosphere under normal plant operation conditions.

\section{Reaction Products Separator Tank}

The Reaction Products Separator Tank is the first in-line component in a relief system (downstream of the rupture discs) which is required to collect the bulk of the solid and liquid reaction products. These tanks should have a tangential inlet nozzle on the top. The tangential inlet nozzle causes the SWR products which enter the tank from the steam generator ${ }^{7}$ to experience centrifugal forces that separator most of the liquid and solid reaction products from the gaseous reaction products.

This tank is located as close as possible to and immediately below the steam generator. The required tank volume is dependent on the hardware configuration, but is currently sized to accommodate a full drain of one intermediate sodium loop with reserve capacity. In the case of a tube rupture, a relief nozzle at the top of a steam generator vessel opens (tube break in gas space), only part of the inventory must be accommodated, whereas, a vent in the bottom requires a larger volume. In either case, all or part of the IHTS loop sodium volume would have to be accommodated.

\section{Cyclone Separator}

The cyclone separator consists of a cylindrical shell fitted with a tangential inlet through which the dusty gas enters, an axial pipe for discharging the cleaned gas, and a conical base with dust discharge. The main objective in a centrifugal separator is to create a vortex which will centrifuge the dust particles to the walls, hence they can be transported into the dust collecting hopper out of the influence of the spinning gases.

\section{Hydrogen Burner}

The hydrogen burner is located in the exhaust stack duct and provide ignition of any hydrogen generated as a result of sodium-water reaction.

\section{Oil Sparger}

An oil sparger (not shown) is part of the sodium/water reaction protection system and can be brought on line for small steam generator leaks. If there is a small leak than the sodium water reaction products can be diverted to the oil sparger. The reaction products are bubbled through the oil sparger where water and sodium reaction products can be separated and allow hydrogen to be off-gassed to the atmosphere.

\footnotetext{
${ }^{7}$ In this case, the ABR steam generator produces superheated steam. If the proposed ABR concept has separate evaporators and superheaters, then there would be piping from each component to the SWRPRS.
} 


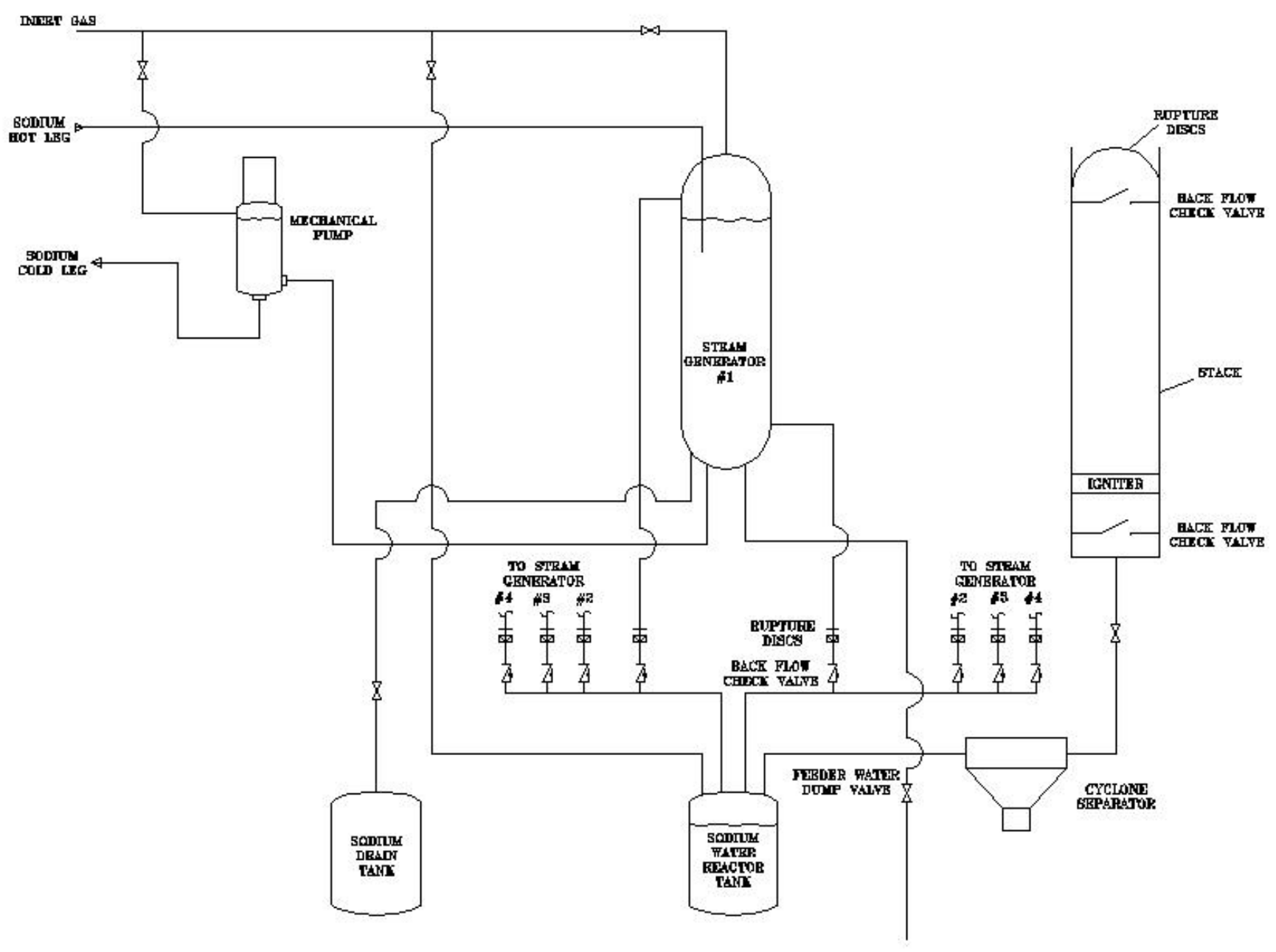

Figure II.5-7 Sodium Water Reaction Pressure Relief System 


\section{II.5.4 Generator System}

The turbine generator for the reference ABR plant was not physically sized. See steam section for thermodynamic data.

\section{II.5.5 Auxiliary Systems}

A discussion of the Balance of Plant auxiliary systems can be found in section II.12. Balance of Plant Systems. 


\section{II.6 Shutdown Heat Removal System}

\section{II.6.1 System Requirements and Description}

The shutdown heat removal system is completely independent from the normal decay heat removal through the intermediate heat transport system, and is activated only when the normal heat removal system is disabled. The system consists of three independent, redundant, and diverse heat removal loops. Each loop consists of a small in-vessel Direct Reactor Auxiliary Cooling System (DRACS), a secondary natural draft heat exchanger (NDHX), an expansion tank, and an exterior stack that forms the natural draft pathway for dissipating the decay heat to the atmosphere. A schematic diagram showing key elements of the system is shown in Figure II.6-1

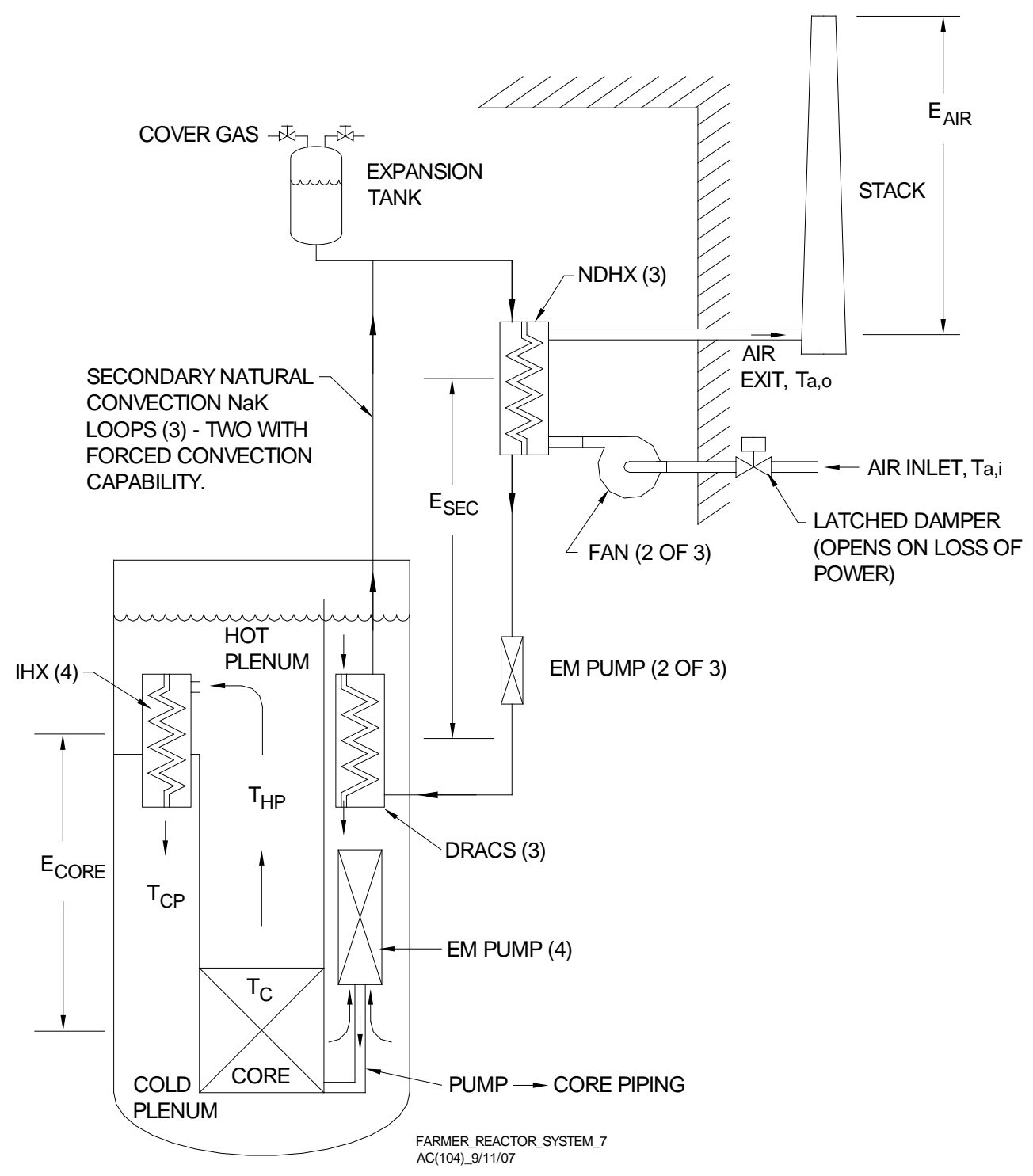

Figure II.6-1 Schematic Diagram of a Shutdown Heat Removal System 
The DRACS heat exchangers are positioned directly in the sodium cold pool. Moreover, there are no valves or other mechanical devices that isolate the primary sodium from the DRACS. Thus, during full power operation, primary cold pool sodium circulates at a modest flow rate through the shell side of the DRACS. However, when activated, buoyancy-driven natural convection flow of the primary sodium through the DRACS is initiated.

The core decay heat is transferred by natural convection flow of primary sodium from the reactor hot pool through the IHX flow path to the cold pool. Heat from the primary sodium cold pool is then transferred through the DRACS to the secondary sodium-potassium (NaK) eutectic passing through the heat exchanger tubes. All three loops are capable of operating in a natural convection mode, in which buoyancy driven convection causes the secondary NaK to circulate through the natural draft heat exchangers where the airflow transfers the heat from the NaK to the atmosphere. The secondary NaK flow circuits (one for each of the three independent systems) are completely passive without any valves or constrictions to limit the flow during normal operation or shutdown conditions.

On the tertiary (air) side of the systems, the natural convection circuits are passive except for magnetically latched dampers that prevent air flow on the air inlet side of the three NDHXs. Upon loss of electrical power to the electro-magnetic latch, the dampers fail by gravity in the open position. The DRACS are brought into full operation by opening of these dampers. The dampers are designed to provide an air leak rate that corresponds to nominally $1 \%$ of the full design flow rate in the closed position, which results in a parasitic heat loss of $\sim 18 \mathrm{~kW}$ during full power operation. This minor heat loss is included in the design to maintain the correct natural convection flow patterns in the primary, secondary, and tertiary sides of the system so that proper natural convection flow patterns are established immediately upon system activation. Moreover, continuous heat addition to the system is desirable in regions where the ambient temperature can fall below the $\mathrm{NaK}$ freezing temperature of nominally $-13^{\circ} \mathrm{C}$.

Alternatively, two of the three DRACS loops are capable of operating in a forced convection mode to control (i.e., limit) the heat removal rate from the reactor vessel during planned shutdowns (e.g., fuel shuffling operations). As shown in Figure II.6.1.1, the secondary NaK sides of these two loops are equipped with EM pumps, so that the loop flowrates can be modulated by adjusting the applied voltage to the pumps. The tertiary air loops on these two units are also equipped with fans so that the heat removal rate through the NDHXs can be adjusted as needed. The EM pumps and fans are selected since these devices have large open flow areas that do not significantly impede natural convection when the loops are operated in that manner.

The three DRACS are designed to remove 2.5 MW each at normal operating conditions. The thermal rating for each DRACS thus corresponds to $0.25 \%$ of the core full power rating of 1000 MWt. The three DRACS are thus capable of removing $7.5 \mathrm{MWt}$ of decay heat at design conditions. Since the DRACS are located inside the reactor vessel, any leakage from the DRACS or ancillary piping will not lead to coolant drain.

Key design parameters for the DRACS are summarized in Table II.6-1, while a drawing that illustrates key features is provided in Figure II.6-2. The DRACS is a shell-and-tube, countercurrent flow-type heat exchanger with primary flow on the shell-side, and NaK flow on the tube side. The DRACS HXs are mounted in vertical pipes that extended upwards through the horizontal portion of the redan into the sodium hot pool. This installation approach maintains the DRACS HXs at a high elevation in the cold pool to promote natural circulation upon activation 
of the shutdown heat removal system. Primary sodium enters the annular opening between the outer shell of the DRACS HXs and the riser pipe in the redan and flow upwards. The flow then turns $90^{\circ}$ and enters the shell side of the DRACS HXs through an annular ring opening in the shroud located just below the upper tube sheet. The sodium flows by natural convection down through the tube bundle while dissipating heat. The sodium then returns to the cold pool through a second annular ring opening located just above the lower tube sheet, and just below the redan lower surface. Cold secondary NaK enters the DRACS through a $15 \mathrm{~cm}$ diameter downcomer. The downcomer delivers the cold NaK through the lower tube sheet into a header manifold, where it then turns $180^{\circ}$ and rises through the tube bank in counter current flow to the shell side primary sodium. The hot secondary NaK exits the tubes into an upper header manifold, and then flows through an annular riser which is concentric to the downcomer. The downcomer is double walled with an annular gap for thermal insulation between the hot and cold streams.

Table II.6-1 DRACS Design Parameters

\begin{tabular}{|l|c|}
\hline Heat transfer capacity & $2.5 \mathrm{MWt}$ \\
\hline Heat transfer area & $17.4 \mathrm{~m}^{2}$ \\
\hline Primary sodium temperature inlet & $510{ }^{\circ} \mathrm{C}$ \\
\hline Primary sodium temperature outlet & $355^{\circ} \mathrm{C}$ \\
\hline Primary sodium mass flowrate & $12.6 \mathrm{~kg} / \mathrm{s}$ \\
\hline Secondary NaK temperature outlet & $484{ }^{\circ} \mathrm{C}$ \\
\hline Secondary NaK temperature inlet & $328^{\circ} \mathrm{C}$ \\
\hline Secondary NaK mass flowrate & $17.5 \mathrm{~kg} / \mathrm{s}$ \\
\hline Tube outer diameter & $2.22 \mathrm{~cm}$ \\
\hline Tube wall thickness & $0.9 \mathrm{~mm}$ \\
\hline Tube Pitch & $3.79 \mathrm{~cm}$ \\
\hline Active Tube length & $2.50 \mathrm{~m}$ \\
\hline Number of tubes & 100 \\
\hline Upper Tube Sheet - Area & $0.125 \mathrm{~m}^{2}$ \\
\hline Upper Tube Sheet - Thickness & $\mathrm{TBD}$ \\
\hline Lower Tube Sheet - Area & $0.125 \mathrm{~m}^{2}$ \\
\hline Lower Tube Sheet - Thickness & $\mathrm{TBD}$ \\
\hline Annular width of riser region to DRACS window inlets & $6.9 \mathrm{~cm}$ \\
\hline OD of unit, including riser & $46.9 \mathrm{~cm}$ \\
\hline Shell thickness & $6.4 \mathrm{~mm}$ \\
\hline Material & $9 \mathrm{Cr}-1 \mathrm{Mo}$ \\
\hline
\end{tabular}

Both the downcomer and the annular riser pipes are equipped with bellows just above the shroud to accommodate any differences in thermal expansion between the piping and the body of the DRACS itself (which is rigidly attached to the vertical piping that supports the EM pumps). The upper tube sheet is welded to the shroud, while the lower tube sheet floats. Thus, the design accommodates differential thermal expansion within the tube bank also. 


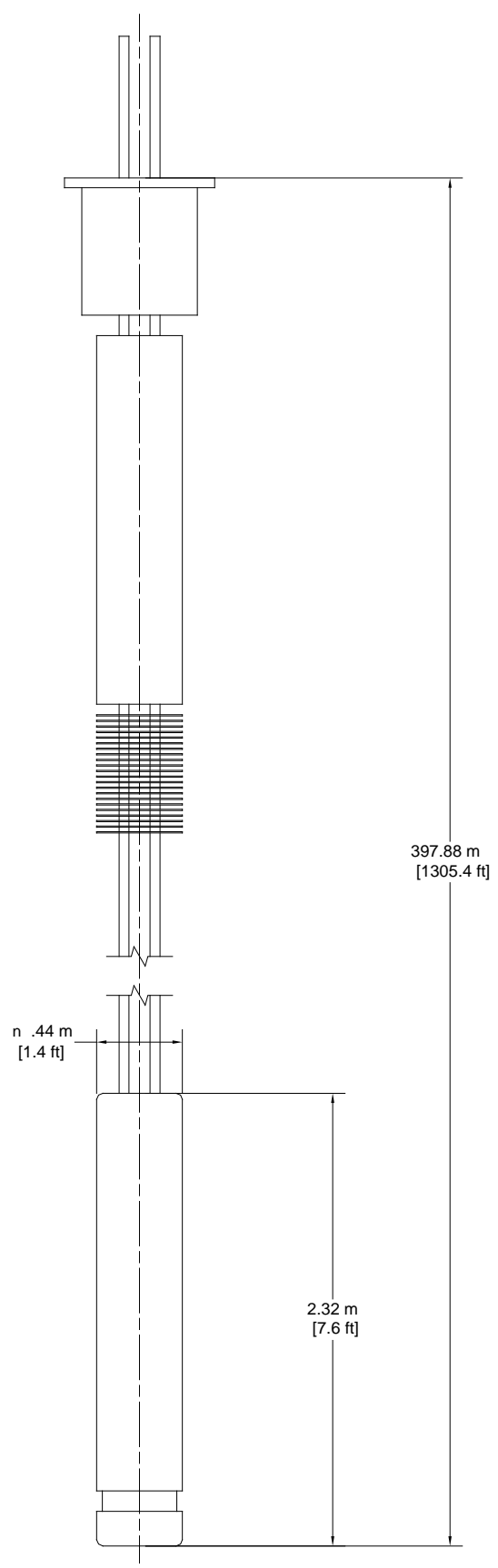

\section{Figure II.6-2 Details of DRACS Design}

The DRACS were sized for the target heat removal rate of $2.5 \mathrm{MWt}$ through a thermal/hydraulic analysis that includes models for heat transfer and pressure drop on both the primary (shell) and secondary (tube) sides. The methodology and parametric analysis results are described in Section III.8. 
Consistent with the thermal rating of the DRACS, each NaK-to-air NDHX is designed to remove 2.5 MW decay heat. The unit is a horizontal tube, cross-flow design. Key design parameters are summarized in Table II.6-2. The unit is equipped with fire suppression plate and catch basin to mitigate the effects of a $\mathrm{NaK}$ tube bundle leak. The damper to the unit is magnetically latched to fail open under gravity upon loss of electric power. The unit will also have a manual hand-wheel operation capability.

Air flow through each NDHX is induced through a dedicated exhaust stack, one for each unit. Each stack is $5 \mathrm{~m}$ high and $8.25 \mathrm{~m}^{2}$ in cross section. The stacks are of lightweight steel construction and are insulated. Each secondary NaK loop contains an expansion tank to accommodate changes in system volume due to variations in temperature. The tank has one NaK nozzle on the bottom and one gas nozzle on the top, which supplies argon cover gas to the tank and permits pressure control. The tank is located at the high point in the loop. The resultant $\mathrm{NaK}$ static head is sufficient to operate the loop with expansion tank cover gas pressures at, or slightly below, atmospheric pressure. In the event of a leak in the DRACS, loss of radioactive primary sodium into the secondary NaK loop will not occur. In the event of a leak in the NDHX, the resultant spill is minimized because of the low expansion tank operating pressure.

Table II.6-2 NDHX Design Parameters

\begin{tabular}{|l|c|}
\hline Heat transfer capacity & $2.5 \mathrm{MW}$ \\
\hline Design & Finned tube cross-flow, four pass \\
\hline Active tube length & $9.55 \mathrm{~m}$ \\
\hline Material & Type 304 stainless steel \\
\hline HX tube OD (without fins) & $3.22 \mathrm{~cm}$ \\
\hline Tube wall thickness & $3.2 \mathrm{~mm}$ \\
\hline Fin height & $3.2 \mathrm{~mm}$ \\
\hline Fin spacing & $1.0 \mathrm{~mm}$ \\
\hline Fin thickness & 72 \\
\hline Total number of tubes & $7.62 \mathrm{~cm}$ \\
\hline Tube horizontal center-to-center spacing & $10.2 \mathrm{~cm}$ \\
\hline $\begin{array}{l}\text { Tube vertical center-to-center spacing } \\
\text { (between passes) }\end{array}$ & $8.25 \mathrm{~m}^{2}$ \\
\hline Stack riser cross-sectional area & $5.0 \mathrm{~m}$ \\
\hline Stack height & \\
\hline
\end{tabular}




\section{II.7 Fuel Handling System}

\section{II.7.1 System Functions and Requirements}

The fuel handling system is a key element of any fast reactor design. The major functions of this system are to: i) receive, test, store, and then load fresh fuel into the core, and ii) unload spent fuel from the core, then clean, test, store this fuel until decay power is sufficiently low, and then ship the spent fuel. Major requirements are that the system must be reliable, replaceable components, and relatively easy to maintain. In addition, the system should be designed so that it does not adversely impact plant economics from the viewpoint of capital investment, plant operations, and overall life cycle costs.

In-vessel fuel handling includes loading and unloading of subassemblies in and from the core, as well as the transfer of fuel into temporary fuel storage locations. These operations require handling of subassemblies under sodium while maintaining the reactor vessel in a sealed state to maintain the inert atmosphere and to preclude air ingress. Ex-vessel fuel handling covers the movement of fuel assemblies from outside the reactor vessel, through air locks in the reactor building, and into other facilities on site for processing. For irradiated assemblies, as well as new assemblies containing recycled fuel with high actinide content, additional requirements are that the transfer devices (casks) used to move assemblies between the reactor building and fuel storage facilities be designed to provide adequate shielding and cooling for decay heat removal. The reduction in refueling operation time is important from the viewpoint of plant availability, since long refueling intervals increase the plant outage duration. Overall plant reliability is also important, since problems during refueling operations can also result in plant outages.

\section{II.7.2 Reference Fuel Handling System Design}

\section{II.7.2.1 Overall System Design}

The ABR fuel handling system discussed here is based upon known technology. The major components of the ABR fuel handling system are a dual rotatable plug (small rotatable plug is off-center with the large rotatable plug), a straight pull fuel handling machine with a gripper, a fuel unloading machine, in-vessel storage (located around the core barrel) area and an interbuilding cask used for transferring fuel to and from the reactor building. In-vessel transportation from the core to the in-vessel storage, and from the in-vessel storage to the transfer position is accomplished by a combination of movement of the dual rotatable plug and the fuel handling machine. There are two in-vessel storage rings located around the reactor core barrel. The invessel storage has 70 storage positions. The metal and oxide ABR cores generates 45 and 36 spent fuel subassemblies, respectively, per every refueling cycle (The both metal and oxide fuel have 180 driver fuel subassemblies and batch numbers of the both designs are four and five, respectively). The in-vessel storage accommodates spent fuel during one operation cycle (one year). After the spent fuel decays in storage for a sufficient time, it is discharged from the reactor vessel by means of the fuel unloading machine to the inter-building cask. The interbuilding cask is then moved out of the reactor building through the inter-building transfer tunnel into the fuel handling facility. 


\section{II.7.2.2 Main Components}

\section{II.7.2.2.1 Dual Rotatable Plug}

The in-vessel handling of core components are carried out under the reactor vessel plug. The ABR fuel handling system adopted a combination of a dual rotatable plug and a straight-pull fuel handling machine. Rotatable plugs make internal fuel transfers possible while maintaining the reactor vessel in a sealed and inerted condition. The fuel handling machine installed on the plug can access every subassembly position, in-vessel storage position and inter-building cask access position by means of combined motions of small and large rotatable plugs.

Located on the reactor cover is the dual rotatable plug system (Figure II.7-3). The small plug is $4.8 \mathrm{~m}(15.7 \mathrm{ft})$ in diameter and $2.5 \mathrm{~m}(8.3 \mathrm{ft})$ in height. The large rotatable plug is $7.9 \mathrm{~m}(25.9 \mathrm{ft})$ in diameter with the same height as the small plug. Along with the fuel handling capability, the rotatable plugs provide the necessary support for the control rod drive system and the fuel handling machine, and provide access for the fuel unloading machine gripper to insert and remove core assemblies.

Also supported from the small rotatable plug is the Upper Internal Structure (UIS) (Figure II.7-4). The UIS gives the control rod guide tubes their necessary support. This consists of a 52" diameter, heavy wall perforated tube with baffle plates. The control rod guide tubes penetrate these baffles and extend to just above the reactor core.

The rotatable plugs are mounted on a motor driven bearing and employ an elastomer seal around its perimeter to provide a gas tight seal with the cover. This seal maintains the integrity of the primary vessel atmosphere while the plugs are operated. It is only rotated during fuel handling operations (reactor shutdown). Once the reactor is safely shutdown, the control rod drive lines are disconnected from the control rods and raised slightly into their respective guide tubes in the UIS. Only then can the plugs be rotated and the FHM operated. 


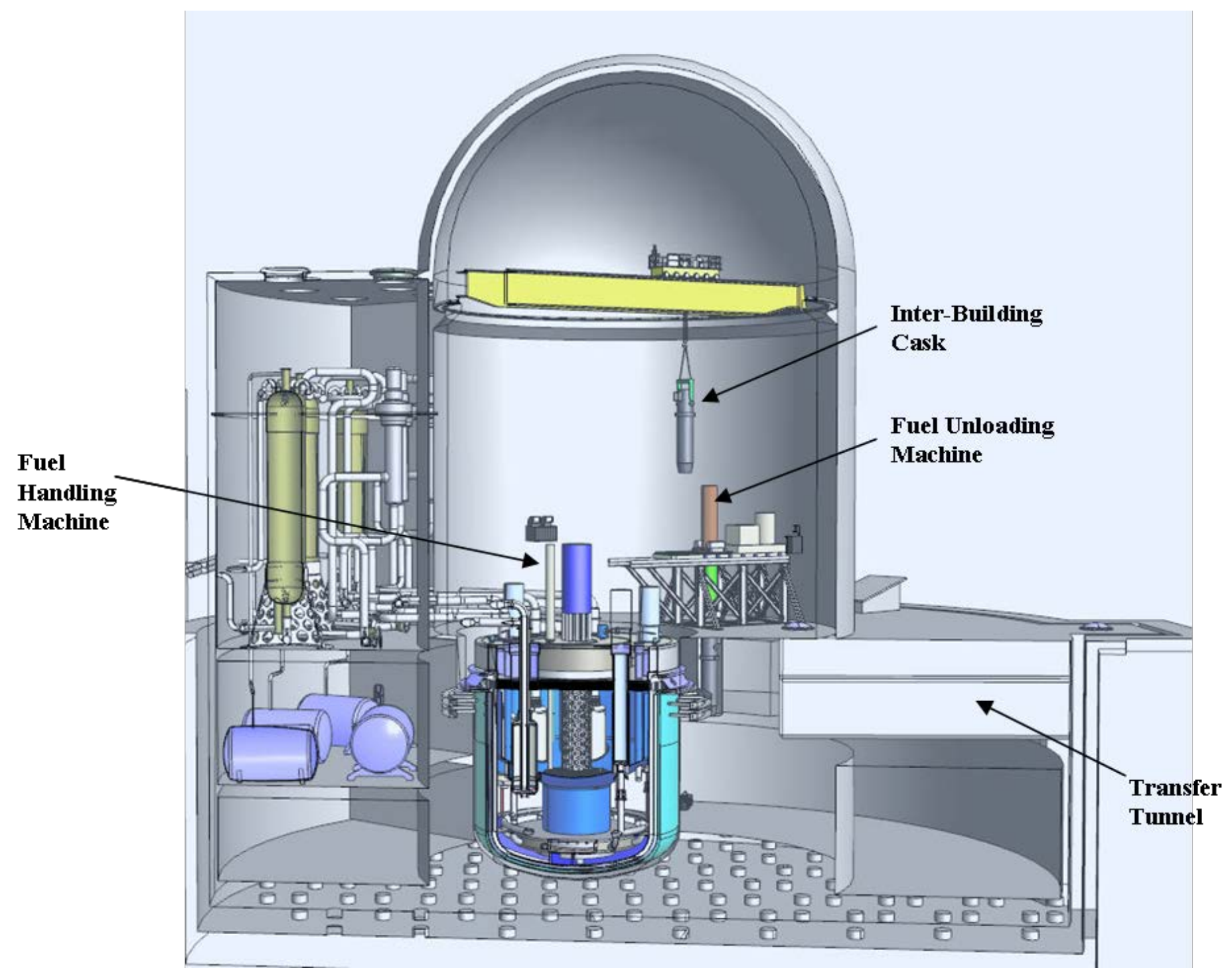

Figure II.7-1 ABR Fuel Handling 


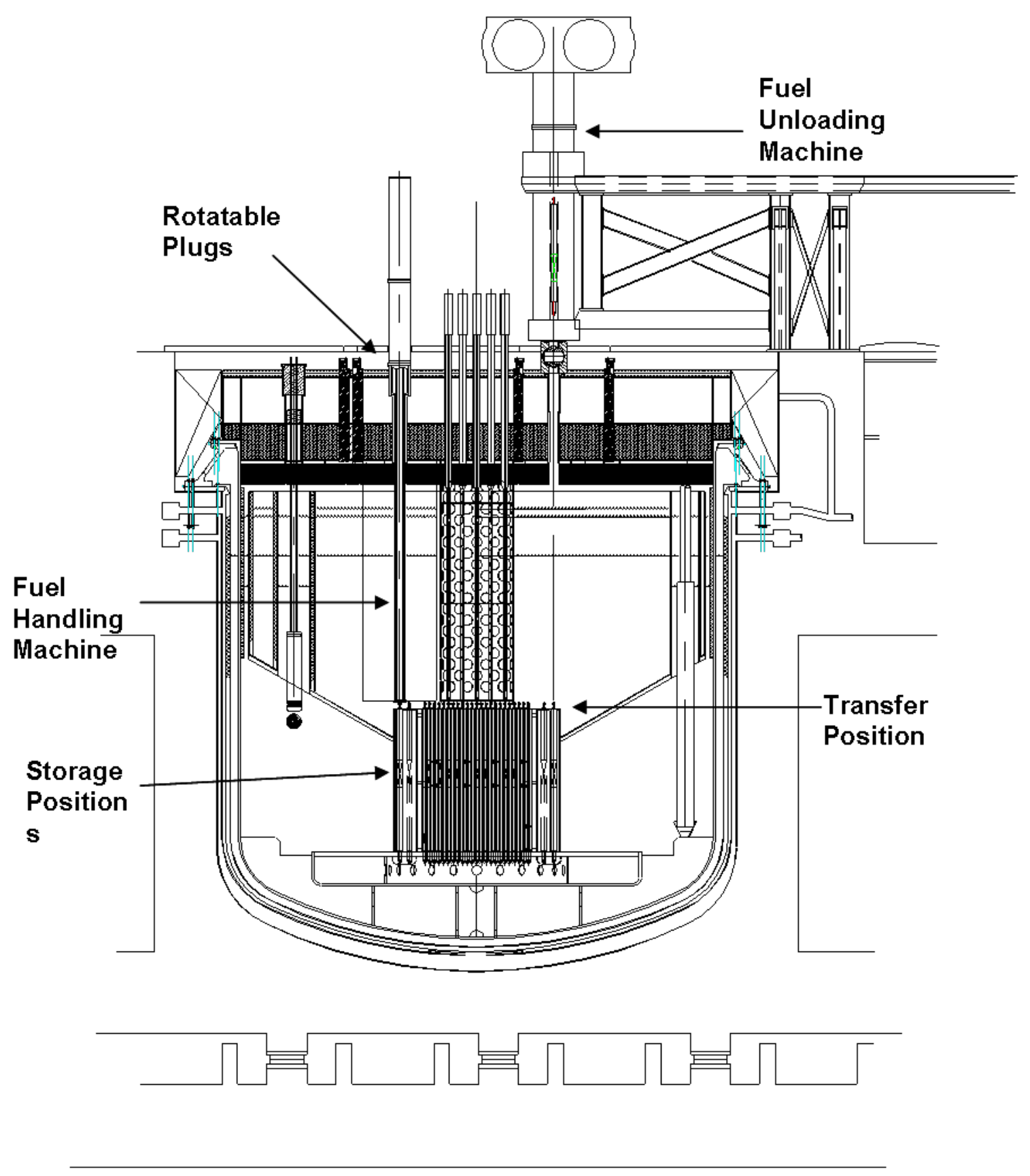

Figure II.7-2 ABR Fuel Handling 


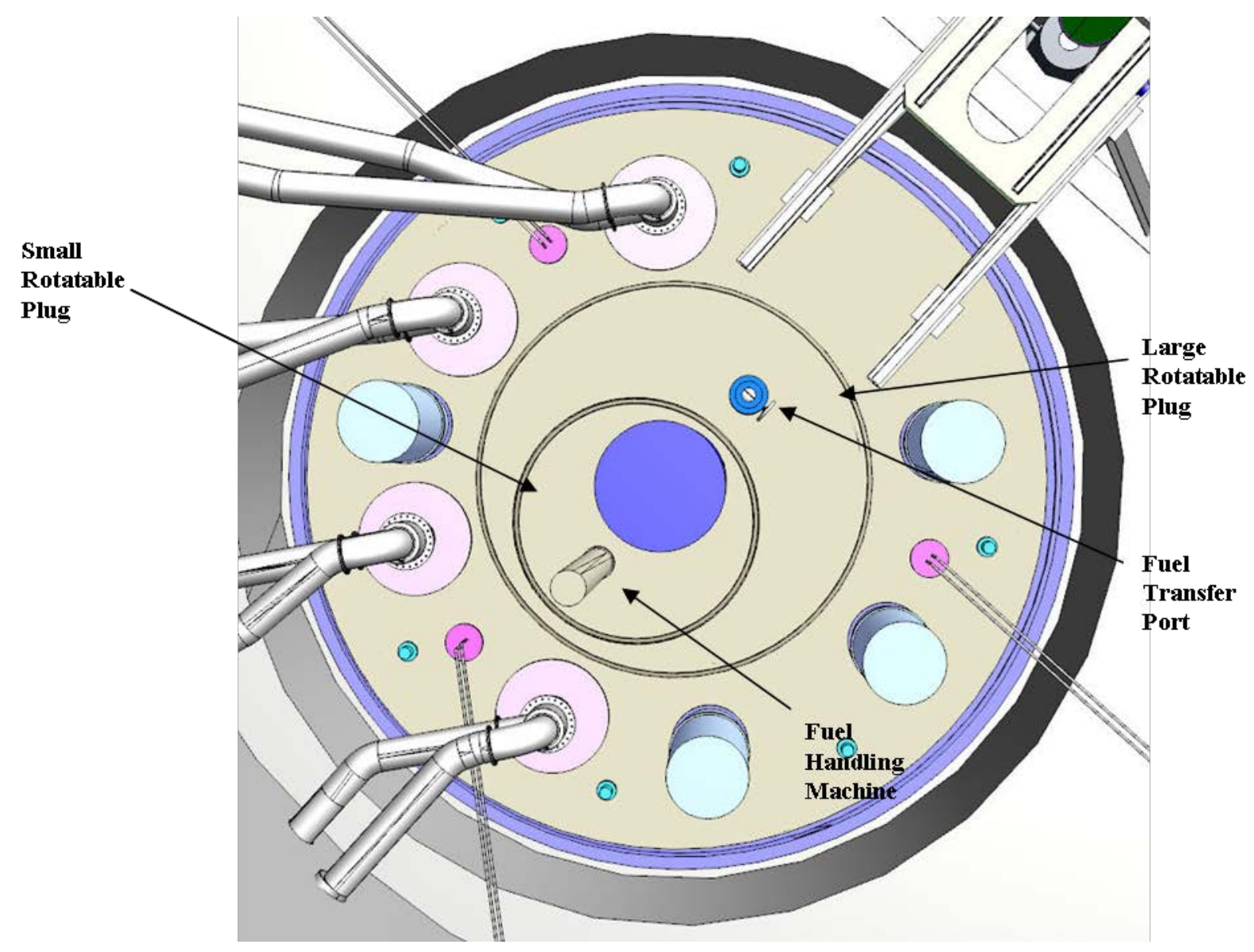

Figure II.7-3 ABR Dual Rotatable Plug Concept

The basic technology of using dual plug systems with a fuel handling mechanism has been developed through domestic and international sodium cooled reactor experience. Except for some early and/or small size reactor designs, such as EBR-I, SRE, HNPF, and SEFOR, generally sodium fast reactors utilized rotatable plugs for in-vessel fuel transfers. Early rotatable plug designs (EBR-II, Fermi, DFR, Rapsodie, KNK and Joyo) experienced buildup of sodium products in the annulus between the rotatable and stationary plugs, or oxidation of the seal alloy. These occurrences often resulted in significant reactor outages since the annuli were difficult to access and clean.

Fermi experienced sticking of the rotatable plug due to the reaction of sodium with carbon dioxide from borated graphite shielding. However, once this problem was solved, the silicon elastomer seal performed very well and required no further maintenance until the reactor was decommissioned [1]. The FFTF rotatable plug incorporated design improvements based on the Fermi experience; i.e., an inflatable seal with argon gas purge was adopted. [2,3]. The FFTF rotatable plug performed superbly with no maintenance required from 1979 to 1988 . The CRBR and SNR-300 plug designs also adopted inflatable seals. Under sodium tests with the SNR-300 triple rotatable plug design showed very good performance characteristics. 
PFR, Phenix, Superphenix and Monju all had rotatable plugs with liquid metal dip seals, and all four of these operating plants experienced no significant difficulties during fuel handling operations. These reactors all incorporated design improvements based on test reactor experiences (i.e., DFR, Rapsodie, and Joyo).

\section{II.7.2.2.2 Fuel Handling Machine}

The primary function of the fuel handling machine (FHM), also called the In-Vessel Transfer Machine (IVTM), is to transfer fresh core (fuel, shields, and reflectors) assemblies into and remove spent core assemblies from the core barrel. It is also used to place them into the storage rack and place or retrieve core assemblies from the transfer position inside the redan. (Figure II.7-2). Historically there are three types of in-vessel under-the-plug fuel handling machines: i) straight pull (EBR-II, CRBR, Superphenix, SNR-300 and Joyo), ii) offset arm (Fermi, FFTF, Phenix and Moju), and iii) pantograph (PFR). These three designs had been demonstrated in the past reactors and accumulated successful operation experiences. The straight-pull fuel handling machine was adopted for this reference concept.

The FHM can raise or lower a core assembly, it can rotate a core assembly, and it can hold down a core assembly. It can be positioned over any assembly within the core by a combination of movement of the dual rotatable plugs (Figure II.7-7).

The FHM is located and supported on the small rotatable plug. It is secured to the plug with a bolted flange to resist any loads associated with the fuel handling operation. Using its gripper mechanism, the FHM is capable of exerting up to $6000 \mathrm{lbs}$ of force to remove or insert a subassembly in the core barrel. The FHM operates using three major components, the upper section, support tube, and the gripper device.

The upper section is 36" diameter and located above the reactor cover inside a cylindrical cover. It contains all the necessary drives and controls to operate the machine. This is the only area expected to require routine maintenance. The controls used are commercially available, motion controllers. These include servo motors and resolvers for position feedback, linked to computer software with graphical depiction of position and administrative controls for every operation. Load sensing and object interference is accomplished through a system of voltage monitors on the drives. Each drive has manual override capability. 


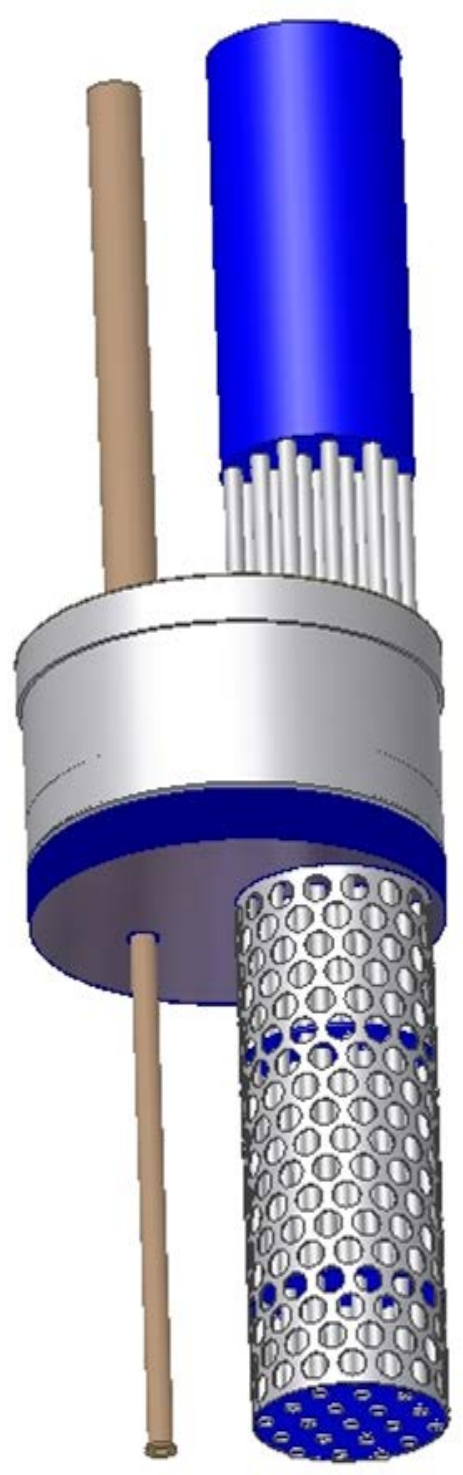

\section{Figure II.7-4 ABR FHM and UIS}

As discussed above, the straight pull type was selected as the reference baseline technology for the ABR fuel handling machine. Though the straight pull fuel handling machine was already demonstrated in past reactors. The experiences then showed that minor design changes could affect basic fuel handling functions, sodium buildup problems and tolerances. And detail design adjustment is required for newly designed subassembly handling. Therefore the ABR fuel handling machine will require careful tests with a full scale mockup in air and under sodium. 


\section{II.7.2.2.3 In-vessel Storage}

There are two in-vessel storage (IVS) rings surround the reactor core barrel. The purpose of the storage rack is to provide a location for fresh fuel assemblies to be preloaded into the reactor vessel and to store spent core assemblies removed from the core and allowing them to decay before removal from the reactor. The open construction of the rack and its proximity to the redan and cold sodium pool induce a natural convection of sodium through the assembly to facilitate adequate cooling of spent fuel assemblies. The in-vessel storage has 70 storage positions with one position in the rack located directly below the fuel unloading machine (FUM) which is located above the transfer port position on the large rotatable plug. The ABR core generates 45 spent fuel subassemblies during every refueling for metal fuel. The in-vessel storage accommodates spent fuel during one operation cycle (one year). At this conceptual design stage, the ABR does not have any plan for ex-vessel sodium cooled storage. Spent fuel assemblies are moved from the reactor building to the fuel handling facility. (Figure II.7-13).

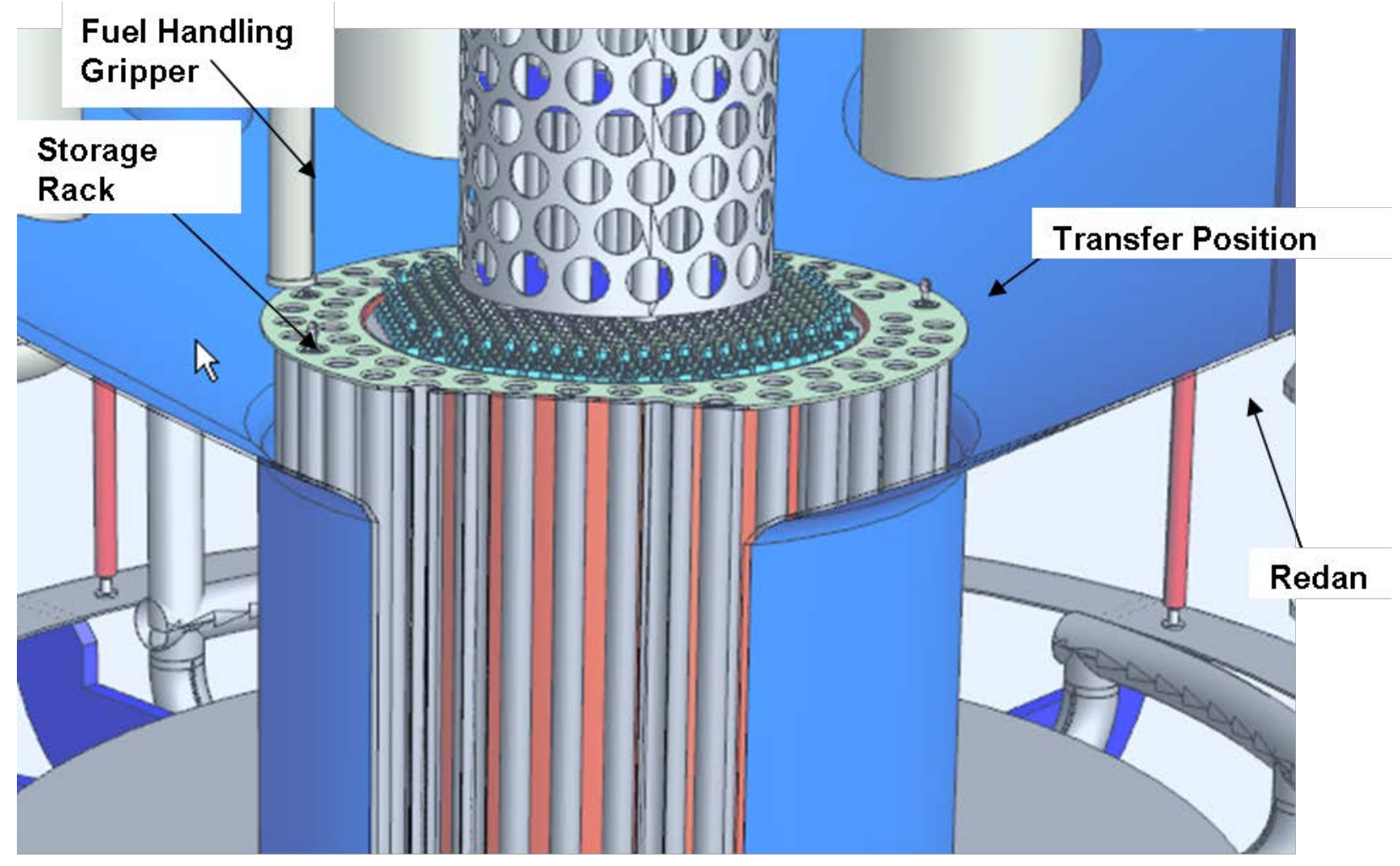

Figure II.7-5 ABR Storage Rack

In-vessel spent fuel storage systems were widely used in past reactors, like EBR-II, Joyo, Fermi, FFTF, PFR and Phenix. EBR-II and Joyo both adopted IVS without ex-vessel storage (EVS) like ABR. The EBR-II IVS could receive or send fuel subassemblies during reactor operation, because the storage rack was placed outside the core barrel, the reactor core barrel had a separate core cover, and the main primary coolant circuit was operable during refueling operations. This system significantly reduced the duration of refueling outages, since only in- 
vessel fuel transfers between the core and IVS had to be conducted when the reactor was shutdown. The Joyo IVS is placed around the core barrel and can not be accessed by the transfer machine (fuel unloading machine) during reactor operations, because access to a target subassembly requires motion of the rotatable plug. However, the design of the Joyo IVS has less impact on the reactor vessel structure.

The ABR IVS is similar to the Joyo IVS. It makes less impact to the reactor vessel structure but requires longer refueling reactor outage. The estimated refueling time is discussed in another section.

The cooling requirement for the ABR IVS depends on when the refueling starts after reactor shutdown. Figures II.7-6 and II.7-7 show that decay heat levels of metal and oxide fuels after a reactor shutdown. The decay heat from the subassemblies decreases from 350 to $20 \mathrm{~kW}$ per subassembly in ten days. It means that the cooling requirement could be approximately $16 \mathrm{MW}$ if the refueling starts immediately after a reactor shutdown. The recommended start time for refueling operations is at least ten days after reactor shutdown. In that case, the cooling requirement of the ABR IVS is approximately $1 \mathrm{MW}$ assuming all of the fuel subassemblies are discharged from the reactor vessel after one year storage.

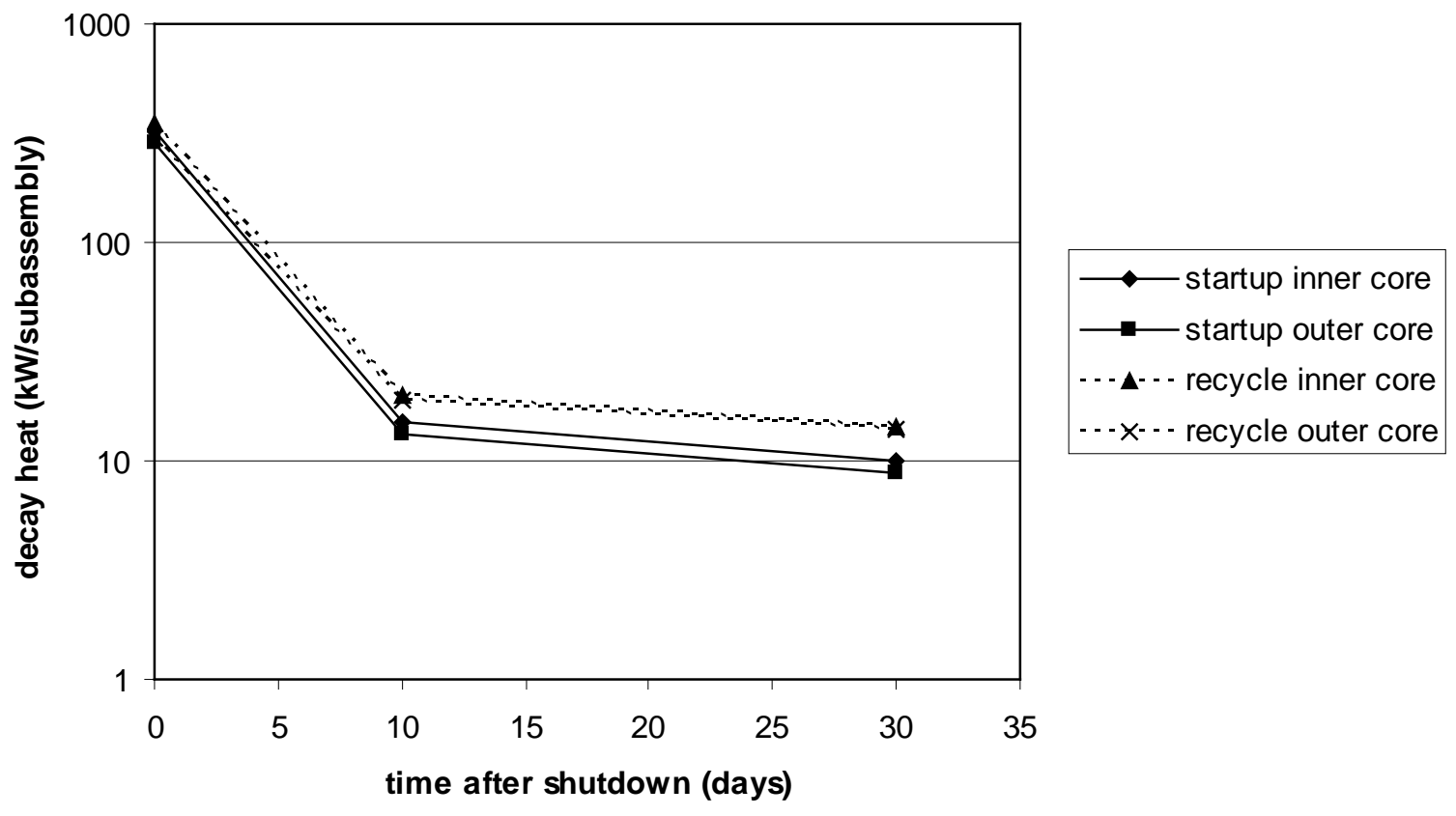

Figure II.7-6 Decay heat against time after shutdown (metal fuel case) 


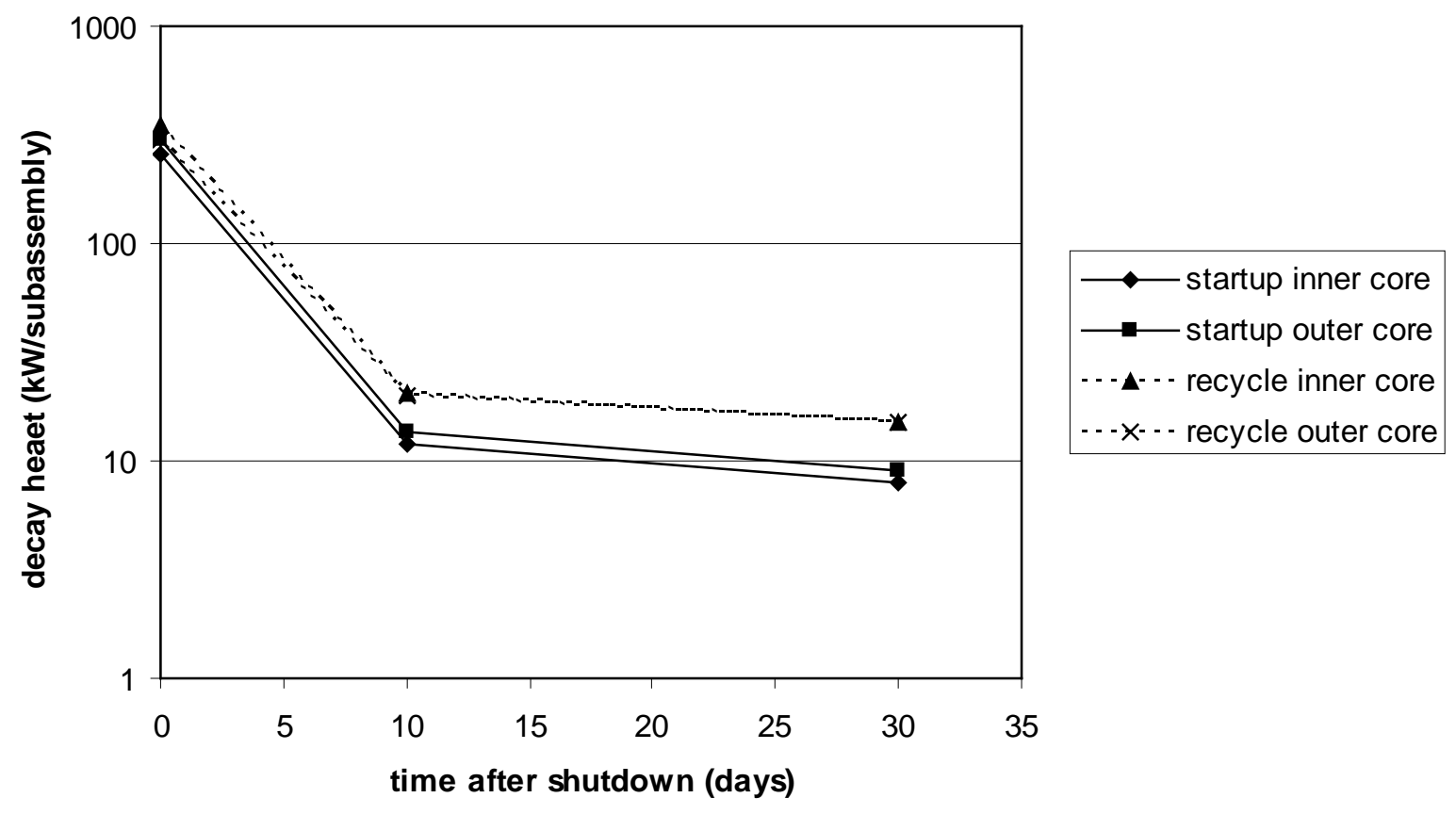

Figure II.7-7 Decay heat against time after shutdown (oxide fuel case)

Another issue of IVS is the handling of failed fuel assemblies. In the ABR IVS system, faulty fuels might stay in the IVS to reduce decay heat during certain duration including reactor operation, since the ABR does not have an EVS system and a $10 \mathrm{~kW}$ decay heat subassembly requires sodium cooling. Gas cooling of such a high decay heat fuel is still technically possible, however it involves safety and economical issue (loss of cooling could cause further fuel failure in a few minutes and cooling system requires expensive high-speed high-pressure compressors). Failed fuel assemblies stored in the primary cooling system could affect sensitivity of further failed fuel detection. Faulty fuel handling policy should be discussed in a future study.

\section{II.7.2.2.4 Inter-building Cask}

The FUM uses a shielded cask mounted on a self-propelled frame (Figure II.7-14). The frame traverses on rails between the Transfer Port and the Inter-Building Cask (IBC) pit area. The FUM is equipped with a gripper for inserting and retrieving core assemblies from the transfer position of the Storage Rack. The bottom of the machine has a movable seal to engage with the Transfer Port or an IBC. Also located in this area is a shielded door to maintain an inert atmosphere inside the FUM and to protect or shield the surrounding area from leakage and residual radioactive contamination while being transported. The FUM also provides for proper heating, cooling and inert gas atmosphere for transferring fuel assemblies between the core and an IBC. The heated inert gas is also used to blow off residual sodium during spent fuel assembly removal operations and return it to the primary vessel. 
The Transfer Port is located on the large rotatable plug. It provides an interface between the FUM and the primary vessel. The Transfer Port provides the needed radiation shielding and inert atmosphere seal to prevent oxidation of residual sodium during transfer. This seal also assists in maintaining a pure argon atmosphere inside the reactor vessel.

A potential alternate design to the FUM and the Storage Rack is a design concept similar to Phenix (CEA) (Figure II.7-15). In this arrangement a rotating arm removes a sub-assembly from the core barrel and places it in an "internal storage area". The sub-assembly is allowed to release decay heat in this area for a predetermined amount of time. The sub-assembly is removed from the reactor using an immersed handling bucket. This sodium filled bucket holds a sub-assembly which is maneuvered onto an A-framed transfer system. The bucket is raised on the A-frame, rotated over a separate sodium pool outside the reactor, and lowered into the cooling pool.

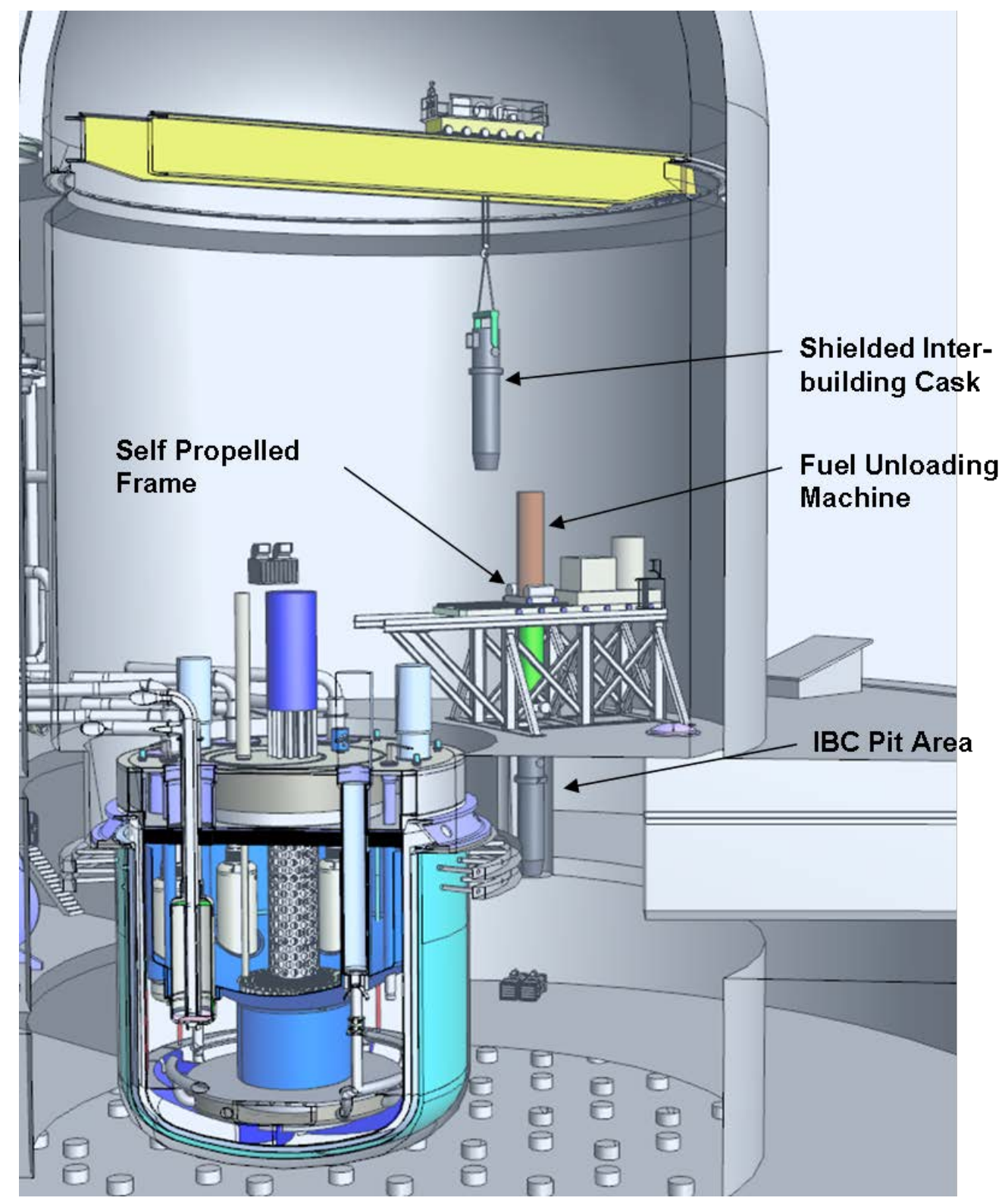

Figure II.7-8 ABR Fuel Unloading Machine 


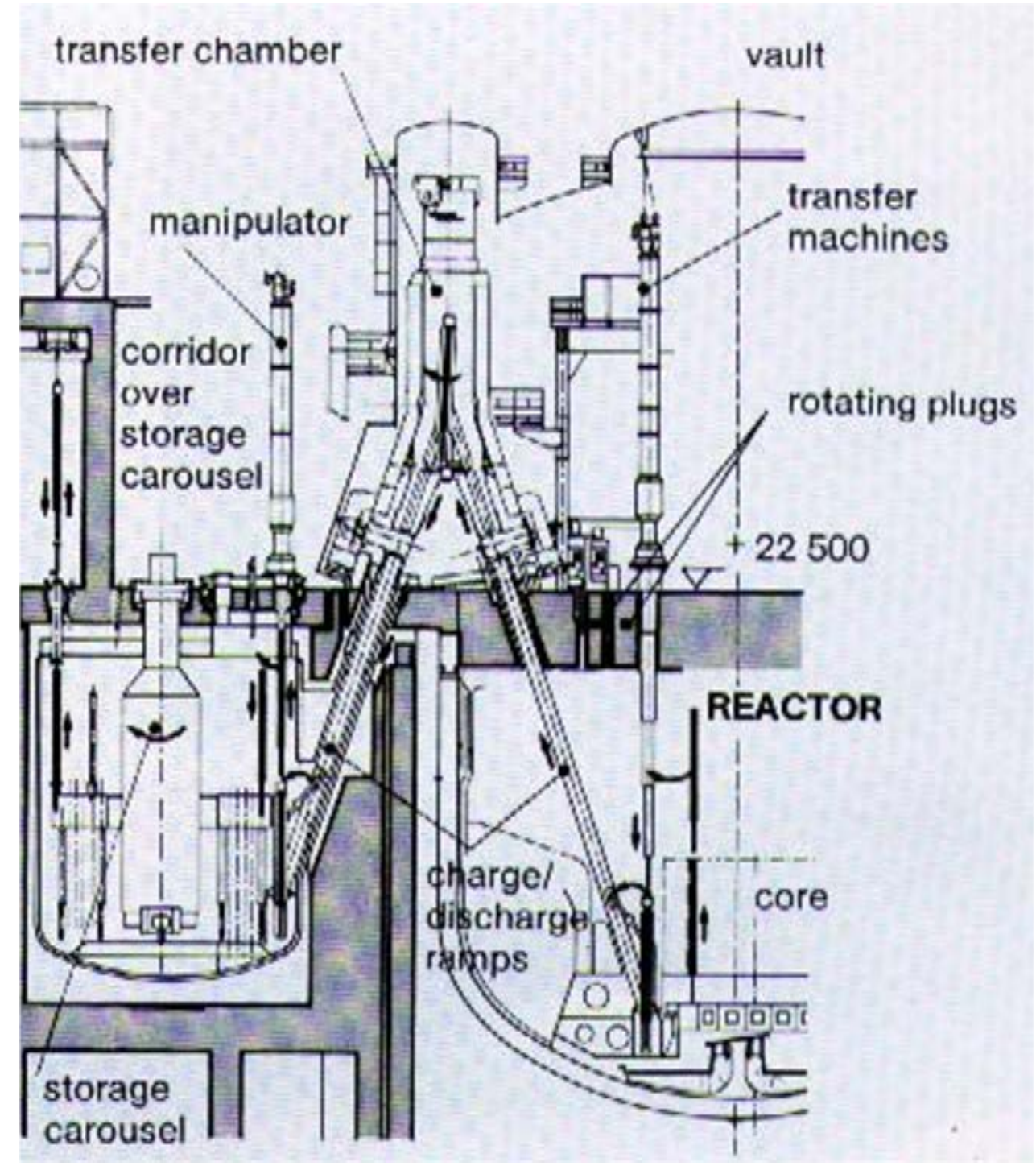

Figure II.7-9 Phenix (CEA) A-frame transfer concept

\section{II.7.2.2.5 Inter-building Cask}

An empty IBC is located in the IBC pit area under the travel of the FUM (Figure II.7-12). The core sub-assemblies that have been irradiated in the reactor are transferred by the FUM to the IBC located in the pit. Once filled, the IBC can then be moved by the reactor building overhead crane to the Intra Building Transfer Tunnel (IBTT) where it is transported from the reactor building.

There are two types of lead shielded Inter-Building Casks (Figure II.7-16). One type of IBC is used for fuel subassembly transfers that have had sufficient time to decay in their storage rack inside the reactor, such that no active cooling is required to remove decay heat. This type of IBC has the necessary shielding and inert gas atmosphere but without any active cooling for the subassembly. Another type has all the same features but with active cooling, to remove subassemblies that have not decayed adequately in the storage rack. It is expected that the actively cooled IBC will see the most use. 


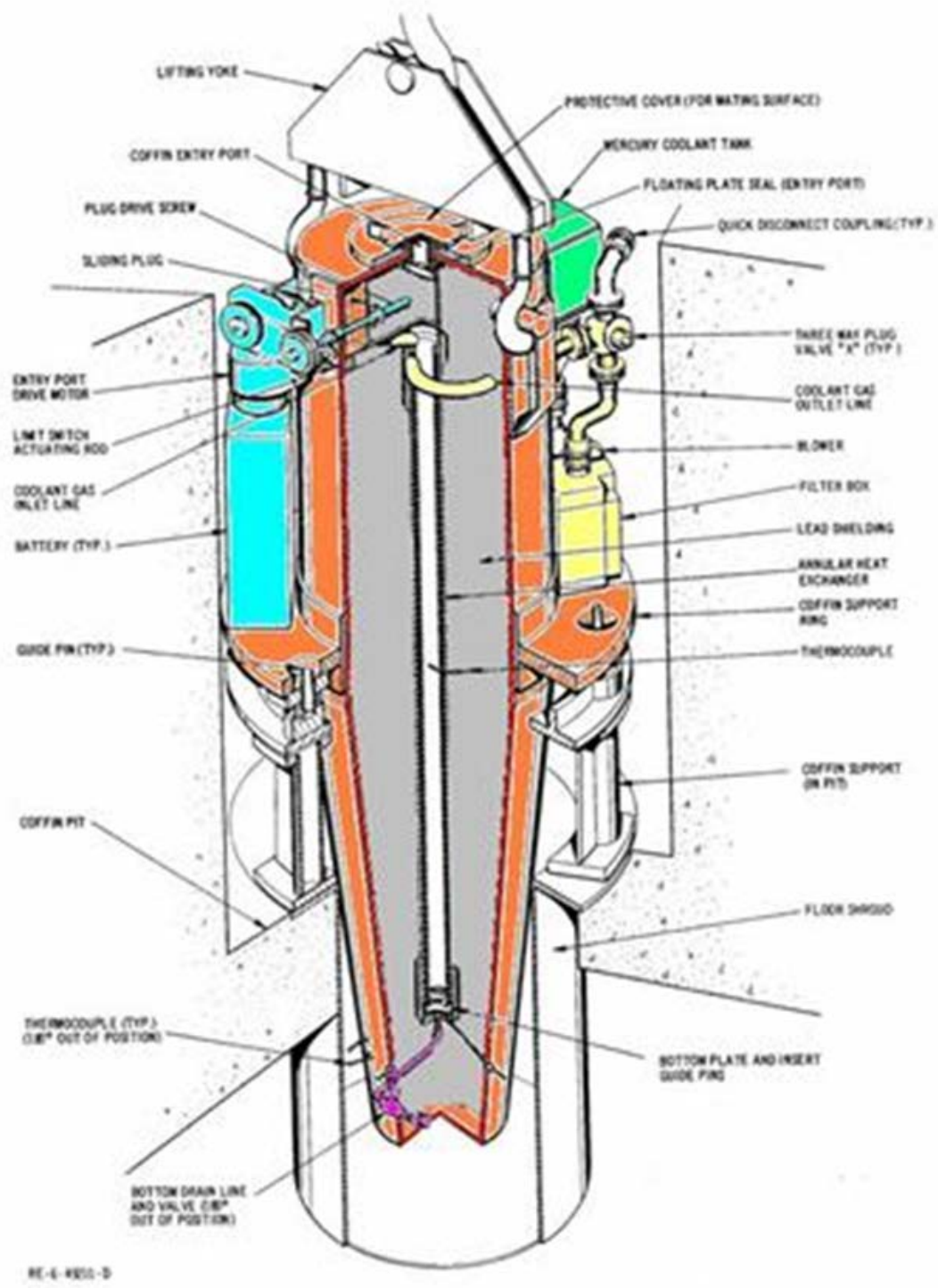

Figure II.7-10 Actively Cooled Inter-Building Cask

\section{Intra Building Transfer Tunnel (IBTT)}

The IBTT is a sealed transfer tunnel used to maintain containment of the reactor facility while transporting IBC's to and from the fuel handling facility (Figure 1). The tunnel atmospheric pressure is kept slightly below the reactor building. Each end of the tunnel has hermetically sealed and interlocked doors to maintain containment. The tunnel door in the reactor facility is opened and an IBC is brought from the IBC pit area and placed on the tunnel carriage system. The tunnel door is then closed and sealed. This carriage provides a type of conveyor system to move the IBC between facilities. Once the carriage carrying the IBC reaches the other end the door is opened and the IBC is removed. At this time another empty IBC can be transferred back to the reactor for reloading or the door can simply be closed and sealed while the empty carriage returns. The IBTT is located on the nuclear island and therefore utilizes the base isolation system. 
Long term decay heat changes of spent fuel subassemblies after a reactor shutdown are shown in Figures II.7-11 and II.7-12. After one year in-vessel storage, the spent fuels are still at levels of 2.8 and $4.3 \mathrm{~kW}$ for the startup and recycle metal fuel designs (2.6 and $5.0 \mathrm{~kW}$ for the startup and recycle oxide fuel designs). Since the ABR fuel handling system was equipped no EVS nor a sodium pot handling facility, spent fuel transportation with a sodium pot is not available. Therefore, the inter-building cask needs to be equipped with a forced gas cooling system with a cooling capacity up to $5 \mathrm{~kW}$.

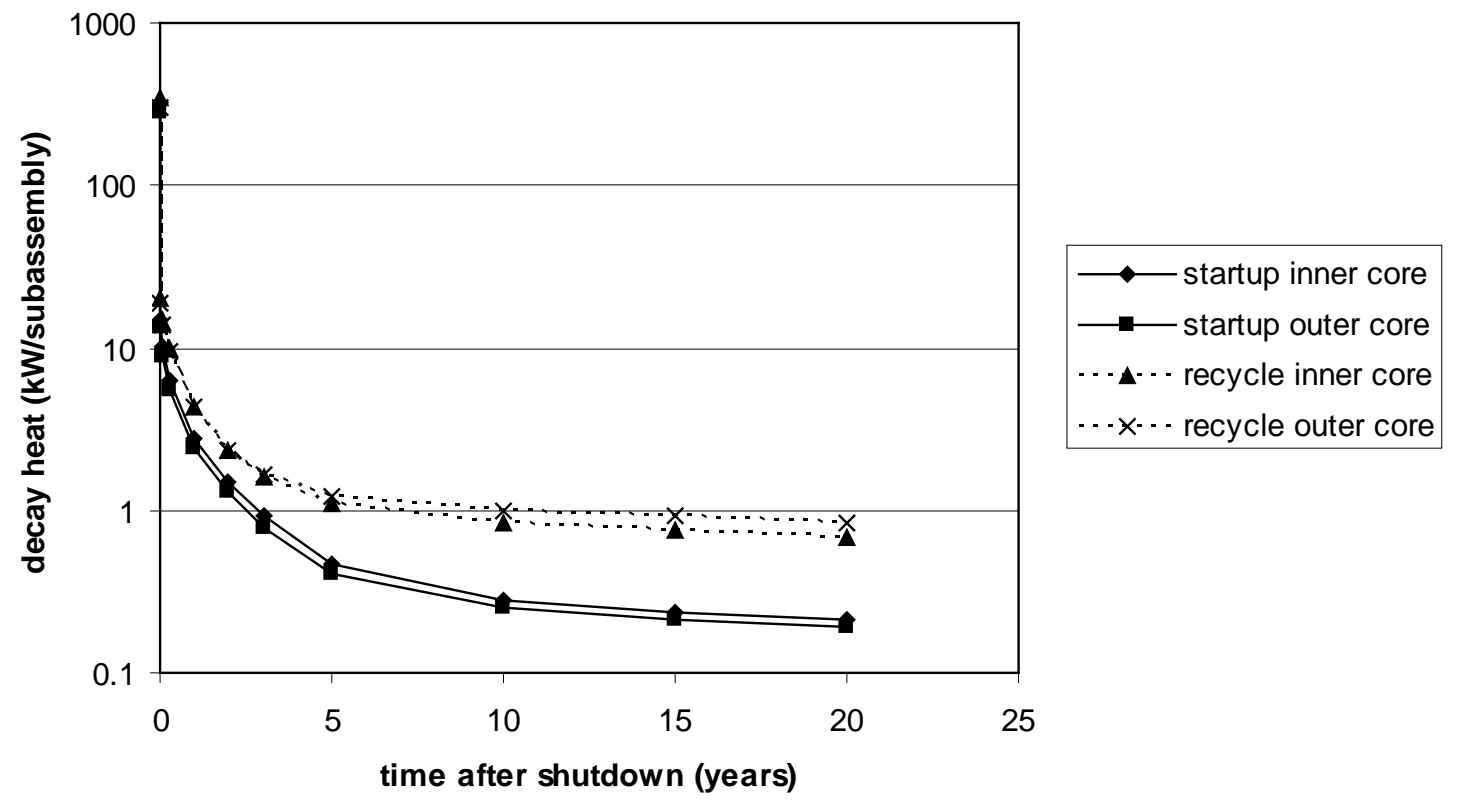

Figure II.7-11 Decay heat against time after shutdown (metal fuel case) 


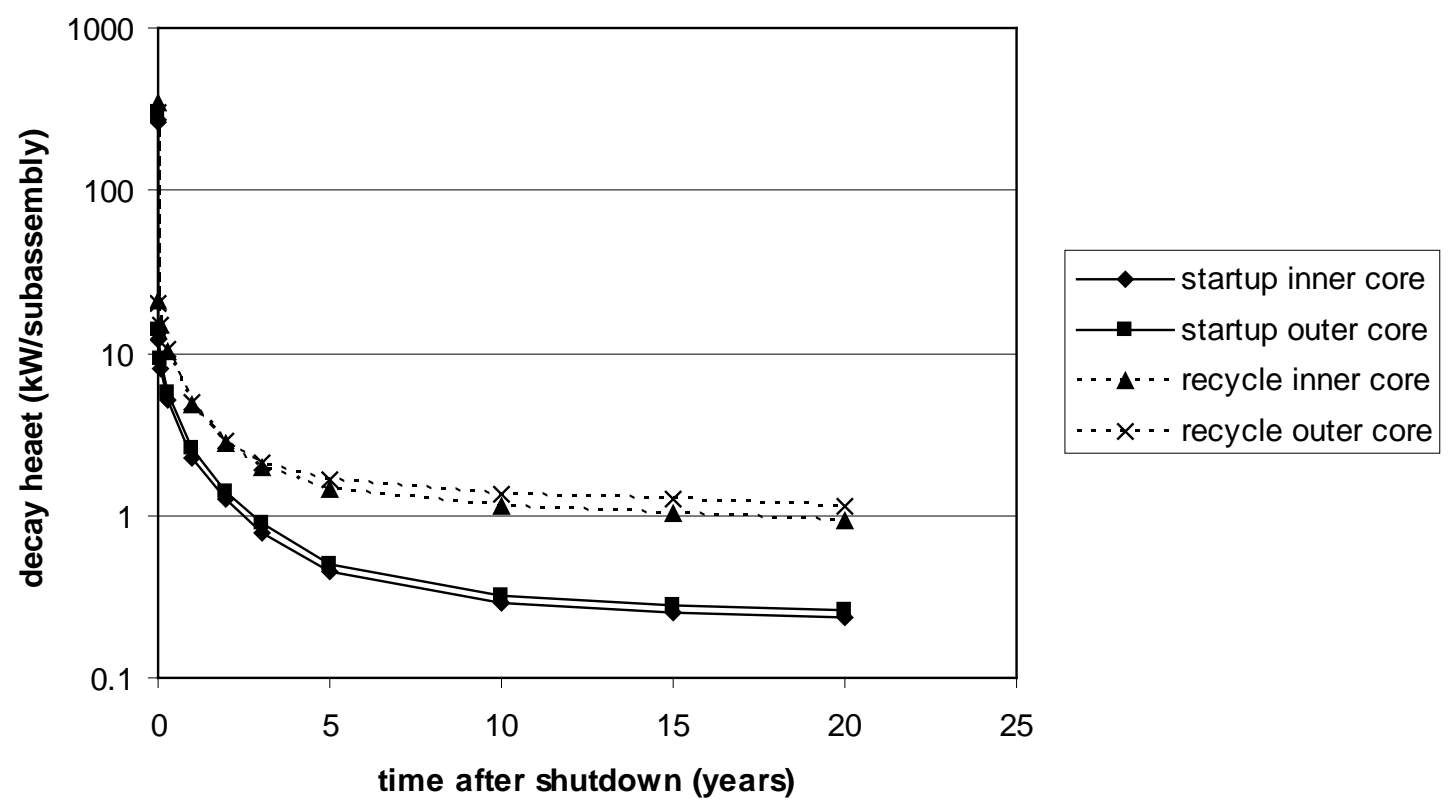

Figure II.7-12 Decay heat against time after shutdown (oxide fuel case)

Table II.7-1 shows ex-vessel transportation in existing or past reactors. The cooling capacity of forced gas cooling transportations is in the range about $2 \mathrm{~kW}$ except for the HNPF and CRBR cases. The cooling capacity of the HNPF ex-vessel fuel handling was $21 \mathrm{~kW}$. The value is much higher than the others. The major difference of the HNPF ex-vessel fuel handling was helium gas cooling while the others adopted argon and nitrogen gas. Since the HNPF was only operated from 1962 to 1964, it did not provide enough experience on ex-vessel fuel handling with helium gas cooling. In the CRBR case, the cooling capacity of the ex-vessel fuel handling with argon gas cooling was $6 \mathrm{~kW}$. The value is still higher than those of the others with argon or nitrogen gas cooling. Since the CRBR project was terminated before construction, the ex-vessel handling was not demonstrated and did not accumulate any experience.

Choosing helium as ex-vessel handling coolant means that the reactor vessel cover gas will also be helium gas. Since helium is more expensive than argon and sealing of helium is more difficult than that of argon gas, argon gas is adopted as cover gas in recent sodium cooled reactors. To adopt helium gas as ex-vessel handling coolant, a careful feasibility study is recommended before a detailed design study.

Argon gas cooling for a $6 \mathrm{~kW}$ decay heat subassembly is also a challenge. The forced convection cooling of subassemblies during transfers has experienced maintenance problems, since the forced gas convection carries sodium vapor throughout the cooling system and the vapor deposits on cold surfaces. A strong gas velocity can cause dripping and splashing problems to occur. For example, at the Rapsodie reactor, forced circulation cooling within the cask car was abandoned due to these problems. Fermi and SRE first adopted forced gas cooling but did not adopt forced gas cooling in their Mark-II casks according to their experiences. The EBR-II FUM replaced the argon gas blower with a new blower with sealed bearings to prevent sodium buildup problems. In the case of the ABR inter-building coffin, since decay heat from the spent fuel $(6 \mathrm{~kW})$ is relatively higher than the past experiences $(2 \mathrm{~kW})$, it is supposed that sodium vapor and sodium splashing problems encountered previously may be worse. 
There are also known sodium dripping and buildup at the bottom of transfer casks that resulted in malfunctions of the bottom valves in the Fermi, EBR-II, FFTF, KNK and Monju reactors. Some of the casks were modified to include larger drip pans or new valves. These actions generally reduced the problems, but continuous maintenance was still required. The EBR-II FUM and Fermi FTF grippers that traveled through both sodium and inert gas experienced malfunctions caused by the buildup of sodium oxides. The gripper designs were modified to include larger clearances that reduced the maintenance frequency.

According to past experience and the relatively higher decay heat, the inter-building cask will require a careful design study and detailed design adjustments with mockup tests under sodium.

Table II.7-1 Ex-vessel Fuel Handling in Past and Existing Sodium Cooled Reactor

\begin{tabular}{|l|l|r|l|r|}
\hline & & max decay & & \multicolumn{2}{l|}{ max decay } \\
plant & heat $(\mathrm{kW})$ & gas cooling & heat $(\mathrm{kW})$ \\
\hline EBR-I & - & - & $?$ & $?$ \\
\hline SRE & - & - & helium & $?$ \\
\hline HNPF & - & - & helium & 21 \\
\hline EBR-II & - & - & argon & 1.2 \\
\hline Fermi & sodium pot with forced gas cooling & $?$ & argon & $?$ \\
\hline SEFOR & - & - & argon & $?$ \\
\hline FFTF & sodium pot with indirect cooling & 10 & argon & 1.4 \\
\hline CRBR & $?$ & 20 & argon & 6 \\
\hline DFR & - & - & argon & 2.5 \\
\hline & & & nitrogen \\
PFR & sodium port with forced gas & - & argon & 2 \\
\hline Rapsodie & - & 5 & nitrogen & 2 \\
\hline Phenix & sodium pot (shoot) & 35 & nitrogen & 2.5 \\
\hline Superphenix & sodium pot (shoot) & - & argon & $?$ \\
\hline KNK & - & 12 & argon & $?$ \\
\hline SNR-300 & sodium pot & $?$ & argon & 2 \\
\hline Joyo & sodium pot & 17 & argon & $?$ \\
\hline Monju & sodium pot & & 2.2 \\
\hline
\end{tabular}




\section{II.8 Instrumentation and Control Systems}

\section{II.8.1 Flux Monitoring System}

The neutron flux monitoring system has several functions: 1) to provide measurements that aid in reactor startup and enable efficient plant control, 2), to monitor reactivity changes during core loading or servicing and 3 ) to detect abnormal conditions that could threaten the integrity of the fuel. As part of a test reactor, this system also provides neutron flux measurements to characterize irradiation conditions for the test fuel. Thus the flux monitoring system provides control signals for normal plant operation, signals to the plant protection system, and measurements for the fuel testing program. The system is capable of measuring the neutron flux level at all times and power levels while the core is loaded with fuel.

The main monitoring system functions over a reactor power range of $\sim 1 \mathrm{~W}$ to over $1200 \mathrm{MWt}$ and it consists of three subsystems, each having three redundant neutron detectors designed to measure flux over a specific range. The source range (SR) subsystem employs detectors with the lowest flux range and is used when the core power is less than a few kilowatts. The detectors are high sensitivity, $\mathrm{BF}_{3}$-filled counters that generate a signal proportional to the thermal flux level. The SR subsystem is used during low flux operations such as initial core loading, startup, shutdown, and refueling. The system issues a warning if there is an unplanned approach to criticality such as might occur with a refueling error.

The power range (PR) subsystem is active when the reactor is near its nominal full power rating. The detectors are fission chambers optimized for high flux levels and a reactor power in the range of $\sim 100 \mathrm{~kW}$ to $1200 \mathrm{MW}$. An intermediate range (IR) subsystem with the same type of detectors bridges the other two and is used at power levels between about $100 \mathrm{~W}$ and $1 \mathrm{MW}$. The range overlap between subsystems allows the operator to verify detector function before switching to a new subsystem.

The detectors of the main monitoring system are located below the reactor vessel at the end of guide tubes that enhance neutron flow to the sensors. Lead shielding is used to block gamma rays from ${ }^{24} \mathrm{Na}$ and a layer of borated graphite shields the detectors from $(\gamma, \mathrm{n})$ reactions. The precise size and orientation of the guide tubes will be selected to optimize the sensor signal/noise ratio. Previous experience with Superphénix has shown that such an ex-vessel detector configuration can be designed to have very little sensitivity to coolant temperature.

A system of in-vessel flux monitors supplements the main system. These detectors are held within steel thimbles mounted on the rotating plug. The system includes $\mathrm{BF}_{3}$ detectors for low flux levels to be used during the initial fueling and startup when the reactor shutdown power, along with the neutron flux to the ex-vessel detectors, is at a minimum. These sensors may be retracted from the thimbles for high power operation to prevent a loss of sensitivity due to activation. Additional thimbles can be reserved to test detector designs or record flux levels at a particular location as an element of a fuels test. 


\section{II.8.2 Heat Transport Instrumentation System}

The instrumentation system provides information on the state of the heat transport system so that the operator can control the reactor in a safe, economic, and efficient manner. A subset of the instruments is dedicated to the plant protection system so that the reactor can be shut down automatically following excursions beyond accepted operating set points.

\section{Temperature}

The majority of the sodium coolant temperature measurements are made with chromelalumel, insulated junction type thermocouples. The sensors are mounted within stainless steel thermowells for protection and to provide a robust boundary for the sodium. Both the thermocouples and thermowells are swaged at the tip to create the desired time response for each measuring station. In addition, the sensors are spring loaded against the bottom of the thermowell to improve time response.

Individual thermocouples will be used to measure the outlet temperature of each core subassembly. A total of 180 chromel-alumel type thermocouples are used for the 180 fuel subassemblies. These sensors provide protection against local cooling deficiencies caused by flow blockages or equipment malfunctions. Abnormal conditions within a subchannel can be indicated by either excessively high temperatures or the presence of increased signal noise caused by subchannel boiling. In addition to the safety function, the thermocouples on each assembly permit determination of the core power distribution through the use of an energy balance.

Certain system calibration operations benefit from higher sensor accuracy than that obtainable with thermocouples. These include in-situ flow meter calibration and measurements to establish an accurate relationship between neutron flux readings and core power output. Higher accuracy is obtained by using resistance temperature detectors (RTDs). Like the thermocouples, the RTDs are mounted within stainless steel thermowells for protection against the sodium.

\section{Level}

Level is measured in the hot and cold pools, the intermediate loop expansion tanks, and the two main system dump tanks. A subset of the pool sensors is made up of short range units to measure level under normal operating conditions when the reactor vessel sodium inventory is at its nominal level. Some of these sensors are reserved for the plant protection system to measure hot-to-cold pool level difference. This is one of the methods used to confirm proper operation of the primary pumps. Other sensors are long range, extending down near the bottom of the reactor vessel, and are used for filling and draining operations. Level meters are also used in the intermediate loop to monitor sodium inventory and provide an early alert to large-scale leaks. Each level meter uses an inductive sensor mounted within a steel well for protection.

Flow rate

At this conceptual design stage, both mechanical and electromagnetic (EM) type pumps are being considered for the main coolant pumps (although the reference is mechanical pumps). If mechanical pumps are chosen, the flow rate will be inferred from measurements of motor speed and the pump performance curve. For EM pumps, the flow rate is a function of the supplied current, which can be accurately measured with electrical equipment outside the vessel. Level meters in the hot and cold pools are used as backup flow indicators. For full flow operating conditions, the level difference between the hot and cold pools is about $2 \mathrm{~m}$. 
Flow rates through the intermediate heat transport loops are measured with permanent magnet flow meters located on the cold legs. A U-shaped magnet assembly provides the magnetic field and electrodes attached directly to the pipe wall carry the flow rate signal. The electrodes are made of the same material as the pipe to avoid thermally-induced potentials.

\section{Core thermal power}

Thermal power of the entire core is found using flow rate and the average temperature rise of the coolant:

$$
P_{\text {core }}=\dot{m} c_{p} \Delta T_{a v g}
$$

where $\dot{m}$ is the core mass flow rate and $\mathrm{c}_{\mathrm{p}}$ is the specific heat of the sodium. The power outputs of individual subassemblies can be calculated using coolant temperature measurements at the subassembly outlets:

$$
P_{i}=\alpha_{i} \dot{m} c_{p} \Delta T_{i}
$$

where $\Delta \mathrm{T}_{\mathrm{i}}$ is the difference between the measured outlet temperature for the $\mathrm{i}^{\text {th }}$ subassembly and the average inlet temperature. The parameter $\alpha_{i}$ accounts for the flow distribution through the various subassemblies and it is determined by a core design code.

\section{Pressure}

The intermediate loop will be operated at a pressure slightly higher than that of the primary system so that tube leaks in the IHX will not result in a transfer of radioactive sodium from the primary to secondary side. The operating pressure of the intermediate loop will be measured with pressure transducers connected to the main piping via NaK-filled capillaries. The pressure of the cover gas within the reactor vessel is measured with conventional pressure transmitters.

\section{II.8.3 Radiation Monitoring System}

The radiation monitoring system measures and records radiation levels in the plant and surroundings to ensure the safety of plant personnel and the general public during normal operating conditions and in the event of a plant malfunction. The system detects and measures the concentrations of airborne radioactivity and monitors activity levels in liquid and gaseous effluents. The system also provides alarms when radiation levels exceed acceptable limits.

A portion of the radiation monitoring system serves the plant safety system by providing sensor signals that can be used as trips in the event of accidental radiation release. Radiation monitors trigger containment isolation in the event of a sizable radiation release within the containment. The radiation monitoring system is also used to survey the reactor cover gas to check for elevated fission gas levels that could indicate failed fuel, and to detect fuel handling and criticality accidents.

\section{II.8.4 Impurity Monitoring and Analysis System}

The overall performance and reliability of the plant can be affected by the level of impurities within the sodium coolant. This occurs through its influence on the properties of structural materials and cladding, component performance, the malfunction of mechanisms, and other factors. The role of the impurity monitoring system is to continuously measure the sodium impurity level within the pool and intermediate heat transport loop. The impurity monitoring 
system is able to enhance plant availability and economic performance by predicting or preventing problems associated with chemical impurities.

The principal measurement system for impurity monitoring is the well-known plugging meter. With this system, a small amount of sodium is shunted to a bypass loop where it passes through a temperature-regulated section containing an orifice. Under a constant driving pressure supplied by an electromagnetic pump, the flow rate through the bypass loop is determined by the size of the orifice. Cooling the sodium within the temperature-regulated section causes impurities to precipitate and collect within the orifice, which reduces the flow rate. The saturation temperature of the impurities is close to the temperature at which the initial decline in flow rate is observed and this temperature is an indication of the sodium impurity level. Once the flow decline is detected, the cooling system is deactivated to allow an increase in sodium temperature, dissolution of the impurities, and reestablishment of the nominal flow rate in preparation of another measurement. The system automatically cycles between flow rate set points and can repeat the measurements indefinitely. The plugging meter can also be run in a continuous, rather than cyclic mode in which the temperature is regulated to maintain partial plugging.

Figure II.8-2 shows the basic layout of the impurity monitoring and analysis system. Plugging temperature indicators (PTI) are used for both the pool and intermediate loop. To monitor sodium from the pool, fluid is pumped continuously from the reactor vessel cold pool. A fraction of the flow is shunted to the PTI and returned to the sampling line. A similar system is operated on the intermediate heat transport loop cold leg. PTIs are placed both before and after the cold trap to measure the general level of system impurities and to monitor cold trap performance.

The PTI can generally provide only the saturation temperature of the precipitating impurities, but not their chemical composition. For a more detailed analysis of the coolant, a sodium sampling system (SSS) is used. The system diverts a portion of the sample stream to a system of valves that permit the collection of the sample in a receptacle that is then taken to the laboratory for detailed laboratory analysis. The analysis provides the concentrations of tritium, corrosion products, particulates, and fission products. Because the hot pool sodium is radioactive, the corresponding SSS must be operated remotely and shielded sample receptacles must be used to protect personnel.

Figure II.8-2 also shows a cover gas sampling system (CSS) that is used to monitor the impurity level in the argon cover gas within the reactor vessel. Cover gas is pumped continuously in a circuit with a filtering stage to eliminate sodium vapor and aerosols. A portion of the clean gas is shunted to the CSS where sensors such as oxygen and humidity sensors measure the air and water content of the gas. These sensors are able to provide a prompt indication of air leakage into the vessel. The system is also equipped to extract samples for laboratory analysis by filling evacuated bottles. The bottles are shielded to protect personnel since fission gases may be present in the cover gas.

\section{II.8.5 Leak Detection System}

The leak detection system is required to assure safe reactor operation, protect equipment, and enhance plant availability through early fault detection. This system is not required to shutdown the plant, remove decay heat, or prevent off site radiation exposure and so it is not classified as a safety system. 


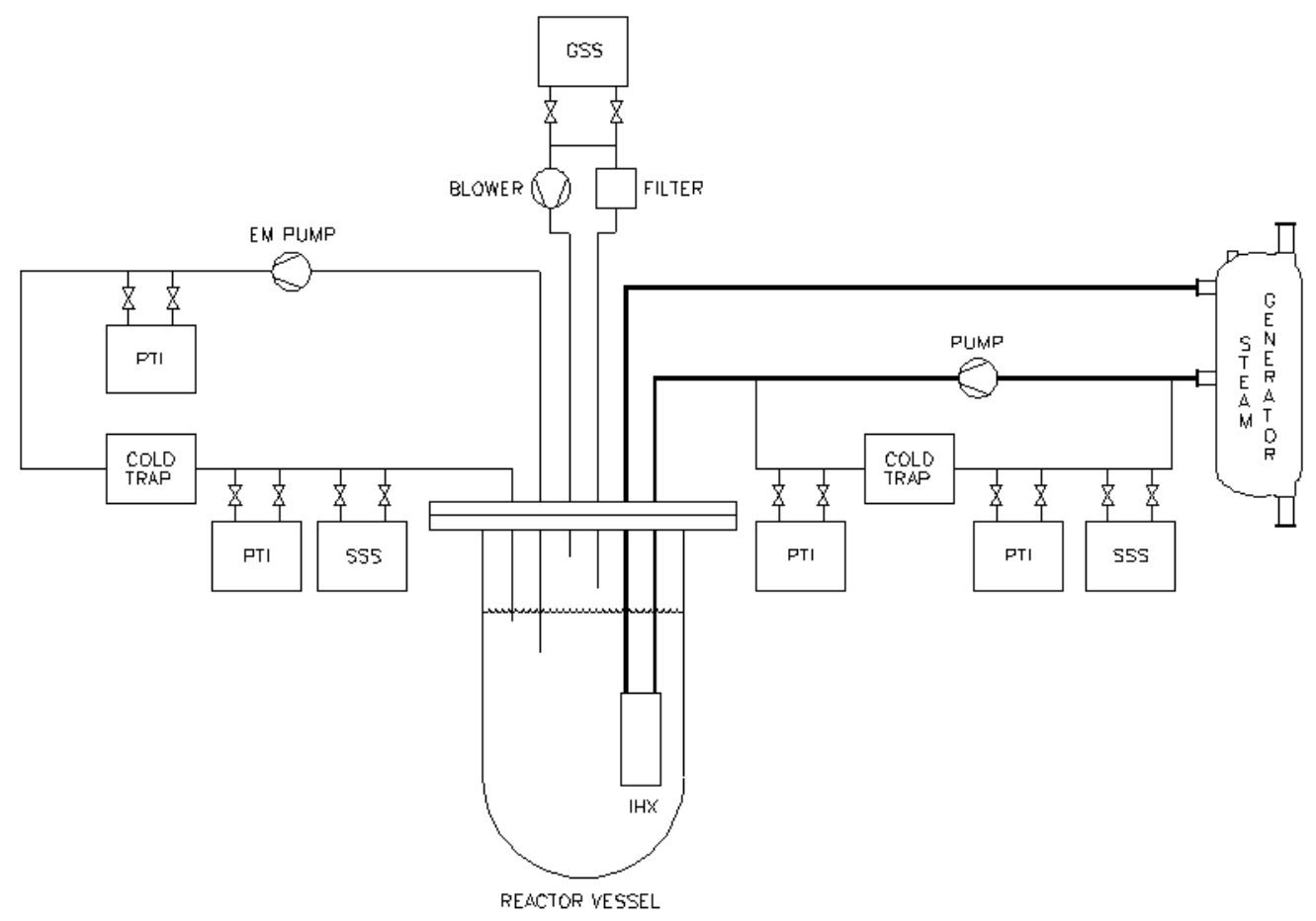

Figure II.8-1 Schematic of impurity monitoring system

The leak detection system is required to detect small leaks (10-100 g/hr) within a time frame of tens of hours, and large leaks (> $1 \mathrm{~kg} / \mathrm{min})$ within minutes. The system is also designed to provide the general location of the leak. A wide assortment of sensors is necessary to monitor the diverse elements of the plant and to provide prompt detection.

Leakage faults can be divided into three general categories, each requiring a particular set of instruments for fault detection:

- Loss of sodium from the primary system or intermediate heat transport loop into the reactor enclosure

- Water leakage from the tube side of the steam generator into sodium

- Sodium leakage between the intermediate heat transport loop and the reactor pool

The first class of faults is dealt with using a combination of level sensors, cable detectors, and an assortment of atmospheric monitors distributed throughout the reactor enclosure. Level sensors in the pool and intermediate loops register large-scale losses of sodium. Cable detectors, which are placed in the guard vessel and below tanks and intermediate loop piping, can discern more moderate leaks. These detectors are stainless steel sheathed cables with a central conductor and mineral oxide insulator. Holes in the sheath allow leaked sodium to come in contact with the conductor, causing an electrical short between the electrode and sheath which alters the signal from the no fault condition. Aerosol detectors are used to identify both small leaks and moderate-sized ones that are missed by cable detectors. Two types of aerosol detectors are employed. One is a sodium ionization detector that uses a hot filament to ionize liquid metal aerosols and vapor. An increase in aerosol concentration produces a corresponding rise in ion current, indicating the presence of a leak. The second type of detector operates as an aerosol collection system in which sample gas is passed through a membrane filter at a constant flow 
rate. Any aerosols present in the sample gas are filtered, which raises the pressure drop across the filter and provides an indication of a leak.

Water leakage from the high pressure secondary side into the sodium of the intermediate heat transport loop is detected by measuring the level of hydrogen within the sodium. Hydrogen detection is based upon the diffusion of hydrogen through a thin-walled nickel membrane. A carrier gas flows by the membrane at a low rate so that an equilibrium hydrogen concentration is established within the gas. The carrier then flows to a hydrogen measurement system. Hydrogen concentration within the sodium is proportional to the square root of the measured hydrogen pressure. The system measures hydrogen concentrations in the range of $\sim 0.1-10 \mathrm{ppm}$. Oxygen is measured with ceramic electrolytes that act as electrochemical cells to provide a low level voltage signal that can be related to oxygen concentration. These sensors operate on the same principle as conventional electrochemical oxygen sensors with one electrode immersed in the sodium and the other a reference gas (or oxide).

Leakage between the intermediate heat transport loop and the reactor pool can, in principle, occur in either direction through a breach in the IHX. However, the operating pressure of the intermediate loop is, under normal conditions, higher than the pool pressure to prevent leakage of radioactive sodium into the intermediate loop. Though such leakage is unlikely, radiation monitors on the cold legs between the steam generator and IHXs are able detect this type of leak. A significant increase in measured activity would provide an indication of probable leaks in the IHX. For the more likely case of leakage from the intermediate loop into the pool, level meters are relied upon to show the accompanying changes in coolant inventory.

\section{II.8.6 Data Handling and Signal Transmission System}

The data handling and transmission system (DHTS) is a distributed computing system linking the plant control system with all remaining aspects of plant monitoring, trending, and data analysis. It also provides remote access to plant data and operational status.

Figure II.8-2 shows the basic architecture of the DHTS. The system uses an Ethernet-based network to link PCs to plant databases containing both current and historical plant data. The databases are updated continually by the plant control system (PCS) and are for the use of supervisors, maintenance personnel, regulatory agencies, etc. Though the PCS computers have access to the databases via the Ethernet, other computers will not, in general, be able to access the PCS via the network. In general, access to plant data by anyone other than the operators within the control room is obtained through the data bases on the LAN and not directly through the main control room computers. Remote access privileges to the PCS computers are available to a very limited number of authorized personnel. In all cases, the type of plant data available for access depends upon user privileges.

\section{II.8.7 Plant Control System}

The purpose of the PCS is to maintain the neutron flux, coolant flow rates, and other plant processes at levels that meet requirements imposed to ensure safety, efficiency, and protection of plant equipment. The PCS is responsible for plant control during start-up, power generation, shutdown, and standby. It monitors and regulates the neutron flux and compensates reactivity changes resulting from fuel burnup, temperature effects, and other factors. The PCS is responsible for coordinating the operation of nuclear island systems with those of the power conversion system and the auxiliary systems. 
The lowest level of the PCS hierarchy is made up of sensors, actuators, and programmable logic controllers (PLCs) linked through a high-speed data bus. Low level control of the actuators is handled by the PLCs while programming of the PLCs and higher level computing functions are managed by computers within the main control room. A main console within the control room provides operator access to the PCS. Redundant systems are used to ensure that operators are able to monitor and direct the PCS under both normal operating and accident conditions.

\section{II.8.8 Plant Protection System}

The plant protection system is designed to shut down the reactor when one or more process variables move beyond predetermined thresholds. The system therefore limits damage to the reactor, including the fuel, and prevents the release of radioactive materials to the environment by retaining them within either the reactor vessel or primary containment. The plant protection system operates independently of the plant control system.

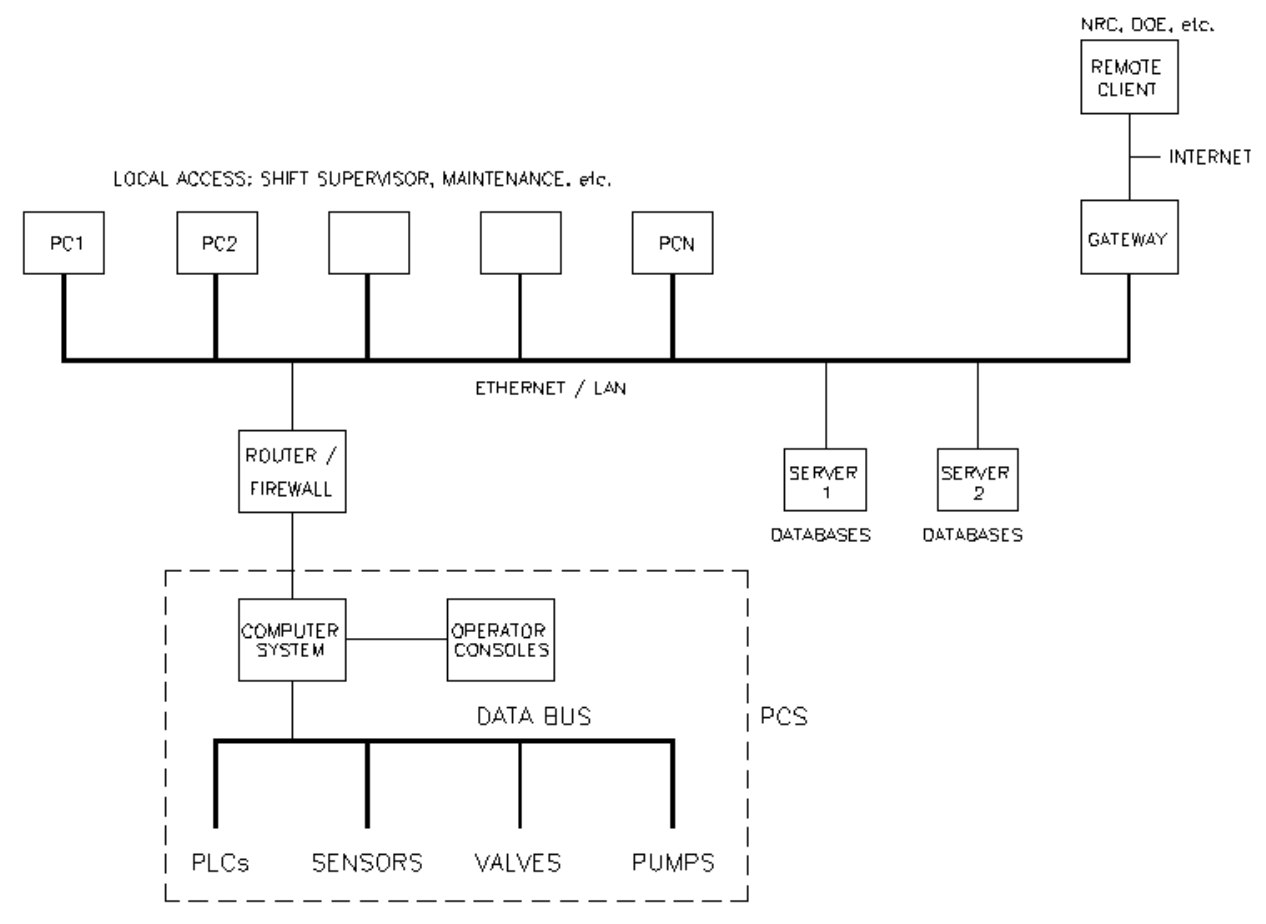

Figure II.8-2 Schematic of data handling and signal transmission system

The sensors used for the plant protection system are separate from those used for plant control. The following parameters are monitored by the plant protection system and used to trip the reactor when an abnormal condition is detected:

- Neutron flux and coolant temperature at core outlet. Protect against overpower transients and prevent high fuel temperatures.

- Sodium level. Trip reactor upon low pool level indicating coolant leakage from reactor vessel into guard vessel. Trip on low level in the intermediate heat transport loop expansion tank indicating loss of coolant from the loop.

- Pump status and rate of change of flow rate. Protect against loss of flow and 
uncontrolled flow changes.

- Intermediate loop pressure and SG hydrogen levels. Loss of SG integrity would be indicated by an abnormal pressure increase on the shell side of the SG and/or detection of hydrogen produced by sodium/water reactions.

- Delayed neutron emission levels in the primary coolant and fission gas concentration in the cover gas. These parameters are indicators of fuel integrity.

- Acoustic signals. Ultrasonic sensors detect the presence of subcooled boiling, which can indicate flow blockages within subassemblies.

The plant protection system also allows the reactor to be scrammed manually. Switches in the control room can be used by the operator to shutdown the reactor at any time.

\section{II.8.9 Communications}

A diverse set of equipment will be available for communication among plant personnel and between the plant and outside agencies. Diversification ensures the ability to communicate during both normal plant operations and in the event of severe plant disruption. The communications system consists of:

- Public address system for intra-plant communications. The system delivers site-wide or localized messages along with alarms such as fire, high radiation, and evacuation alarms.

- Microwave communications system to provide a wireless link between the plant and neighboring DOE facilities.

- Portable radio systems for two-way communications at any plant location. These systems are used for maintenance operations, traffic control, and fire protection. A separate system is used by plant security personnel.

- Security intercom system for closed communications between security personnel at the main security station and remote security stations.

- Offsite law enforcement radio system for communication with outside law enforcement agencies.

- Conventional telephone network for routine intra-plant and inter-office communications.

\section{II.8.10 Industrial Security and Safeguards System}

The industrial security and safeguards system is designed to protect plant equipment and personnel, and to prevent the theft of special nuclear materials. The system is designed to defend against the design basis threats specified in regulations. The key requirements for the security and safeguards systems are:

- Allow plant access only to authorized personnel and material

- Prevent the theft of special nuclear materials

- Prevent the sabotage of critical plant equipment

- Deter, detect, and delay unauthorized activities and assaults on the plant

To minimize the required size of the security force, the plant is divided into nuclear and non nuclear areas, each operated with appropriate levels of security. All safety grade components 
and systems are located within the nuclear island to minimize the area requiring the highest level of protection. 


\section{II.9 Primary Plant Auxiliary Systems}

In order to ensure long term, safe operations of the sodium systems, the chemistry of the system must be controlled and monitored by a set of auxiliary systems that are common to all liquid metal reactors. Although the chemistry does not affect the nuclear operation directly, it is important for the hydrodynamics of the system as well as for corrosion and contamination control. One goal is to ensure stable hydrodynamics on the long term to promote an efficient and constant heat transfer, which can be affected by oxide formation or mass transfer within the nonisothermal circuit. Another goal is to ensure the maintenance and component handling easiness by reducing the activated corrosion products.

The following auxiliary sodium systems are included:

- Sodium purification system for purification and monitoring of sodium circuits, such as crystallization and plugging indicators

- Cover gas purification system for purification and monitoring of the argon cover gas

- Sodium sampling and analysis system for contamination monitoring

- Primary and secondary sodium storage system

- Sodium reaction system for component handling, cleaning, and decontamination (located in the Maintenance Building)

The sodium purification deals not only with the hydrogen and oxygen impurities introduced during the initial startup or maintenance operations, but also with other potential source of impurities such as the sodium/water interaction products.

\section{II.9.1 Reactor Vessel Heating System}

Four electrical reactor vessel immersion heaters provide supplementary heating of the bulk primary sodium to maintain a minimum sodium temperature of 120EC whenever the reactor is not operating at sufficient power to make up for the total heat losses from the primary system. Heaters are also required during the initial sodium fill and startup.

Each heater is installed through a heater nozzle in the reactor vessel cover. Power to the heaters is supplied from 480V 3-phase AC power or, if necessary, by an emergency diesel generator.

\section{II.9.2 Guard and Thimble Cooling System}

Normally, the heat induced in the biological shield, air baffle tank, and primary support structure, by losses from the reactor vessel bulk sodium, is dissipated by the shield cooling system. The shield cooling system consists of two basic systems operating in parallel: an air recirculating and cooling system and an exhaust air system. Power is normally supplied by the $480 \mathrm{~V}$ distribution system. If this were to fail, power to the exhaust air system would be supplied by an emergency generator, and to the air recirculating and cooling system by another diesel generator. This shield cooling system also has alternate blowers and air conditioning equipment which is automatically switched on if a failure of the primary units were to occur.

A total heat load generated for the most part by heat losses from the reactor vessel sodium to the surrounding biological shields, is dissipated by the forced circulation of cooling air provided by the shield cooling system. The reactor vessel top support structure, the insulated top surface 
of the reactor vessel cover, and component nozzles are cooled primarily by the reactor building air which is drawn into the system and flows through ducts to cool these areas.

Recirculated air is made available from the cooling coils and blower and provides cooling for the radial and lower biological shields and the reactor vessel. Since the shield cooling system operates at a slightly lower pressure than the building atmosphere, a certain amount of air inleakage occurs which simplifies the cooling of certain areas which cannot be connected to a closed system.

A thimble cooling system is provided to maintain the neutron detection instruments, which are positioned in thimbles (not currently shown) next to the core barrel, at a temperature of less than 65EC. Backup cooling includes a standby turbo compressor. If this also fails, thimble cooling can be manually transferred to the shield cooling exhaust system. Operating power for the thimble cooling system is normally supplied from the $480 \mathrm{~V}$ power distribution system. The thimble cooling loads are automatically transferred to the emergency diesel generator during a loss of electrical power. If neutron detection instruments can be found that withstand ambient core conditions, then the thimble cooling system can be eliminated.

\section{II.9.3 Primary Sodium Purification System}

The primary sodium purification system provides continuous purification of the primary sodium and consists of the following loops: 1) primary sodium recirculating loop - cold trapping loop, 2) sodium-potassium alloy (NaK) loop, and 3) silicone loop.

The purification system provides control of impurity concentrations of sodium hydride, sodium monoxide, cesium, and possible other fission products at temperatures just above the melting point of sodium. The control of impurities in sodium is required to lesson the corrosion rate of reactor vessel, piping, and materials since sodium impurities could cause metal surface removal (which could affect the load-carrying capability of reactor materials), fouling of heattransfer surfaces through surface deposits, and reduced mechanical properties of materials caused by changes in base alloy composition.

Major components of the sodium purification system are the primary sodium purification plug containing the sodium suction and return lines, sodium surge tank, electromagnetic pump, nuclide trap, cold trap (an economizer and crystallizer tank), a NaK loop, and a silicone loop.

The nuclide trap is designed to trap Cs-137 and possibly other fission products from the incoming sodium, upstream of the cold trap. The nuclide trap consists of a type 304 stainless steel cylinder that contains reticulated vitreous carbon, a very porous, low-density carbon filter material, to adsorb fission products. Depending upon shielding design, the trap can contain a significant curie count of Cs-137. A second filter mounted inside the cylinder contains a sintered stainless steel filter to prevent any of the carbon material from being carried out of the nuclide trap and into the main sodium coolant.

The cold trap consists of a regenerative heat exchanger to minimize overall heat losses and a crystallizer tank to cold trap the sodium impurities. The heat exchanger is on the wall near the crystallizer within the shielded cell. The regenerative heat exchanger is intended to reduce the heat losses resulting from cooling the primary sodium from $510^{\circ} \mathrm{C}$ down to cold-trapping temperatures. It is also intended to heat up the purified sodium from the cold trap to approximately bulk sodium temperature for return to the primary tank. The sodium crystallizer 
tank removes the sodium oxide and other impurities from the primary sodium through precipitation on wire-mesh cylinders and in stilling baffles. It is cooled by the NaK loop. 


\section{II.10 In-Service Inspection}

In-service inspection (ISI) is the examination required during the plant operation lifetime for early detection of potential failures. Its objectives are to 1) confirm the integrity of the function of systems, structures and components necessary to safety, and 2) satisfy the need to protect plant investment and to assure high plant availability. In this regard, the in-service inspection approach for $\mathrm{ABR}$ is established in such a way that inspectability of all components of the reactor system is ensured.

\section{II.10.1 In-Service Inspection Requirements}

The in-service inspection approach used for $A B R$ is based on the rules and requirements delineated in Division 3, Section XI of the ASME Boiler and Pressure Vessel Code, "Rules for Inspection and Testing of Components of Liquid-Metal Cooled Plants". The Code sections specify the identification and classification of components subject to inspection, provisions for accessibility, examination methods and procedures, inspection plans and schedules, and repair requirements.

\section{II.10.1.1 Components Subject to Inspection}

The ASME Code specifies inspection requirements for nuclear safety grade components containing liquid-metal and cover-gas in liquid-metal cooled nuclear plants. The rules are established based upon anticipated service environments of the systems which contain sodium coolant and upon protection against failure of the liquid-metal coolant boundaries. For example, it is anticipated that, due to the low vapor pressure of sodium and the inherent ductility and toughness of the coolant boundary material, the reactor vessel and reactor internal structures will not fail with sudden release of energy. Also, double walled pipes and vessels are used for protection against loss of core cooling, and inert gas is introduced within all the plant components containing free liquid metal surfaces.

In-service inspection of safety grade components of a liquid-metal reactor that contain other fluids, such as water, may be provided by references to articles in Section II, Division 1 of the Code.

\section{II.10.1.2 Accessibility}

Providing adequate access for inspection and maintenance is an important factor in the reactor design. Article IMA-1500 of the Code specifies that accessibility provisions shall be made with the following considerations:

1. Access for the inspector, examination personnel, and equipment necessary to conduct the examination.

2. Capability for removal and storage of structural members, shielding components, insulating materials, and other equipment and components required to perform the visual observations, examinations, and tests.

3. Installation and support of handling machinery (e.g. hoists or other handling equipment) where required to facilitate removal, disassembly, and storage of equipment, components, and other examinations. 
4. Performance of alternative or additional examinations to those specified herein in the event structural defects or indications are revealed that may require such alternative examinations.

5. Performance of necessary operations associated with repair or replacements of system components in the event structural defects or indications are revealed that may require such repair or replacements.

\section{II.10.1.3 Examination Methods}

The ASME Code specifies four types of examination methods used during in-service inspection, namely visual, surface, continuous monitoring, and volumetric examinations.

Visual Examination is the primary method of inspecting for liquid-metal retaining components. The ASME Code defines the following categories of visual examination:

1. VTM1: close range examination to detect discontinuities and imperfections on the surface of components, including such conditions as cracks, wear, corrosion, or erosion.

2. VTM2: examination of exterior surfaces in such a way that accumulation of liquids, liquid drops and smoke are discernable. The removal of external covering, such as insulation, is not required for VTM-2 visual examination.

3. VTM3: examination to determine the general mechanical and structural conditions of components and their supports by verifying parameters such as clearance, settings, and physical displacements; and to detect discontinuities and imperfections, such as loss of integrity of bolted or welded connections, loose or missing parts, debris, corrosion, wear, or erosion.

Continuous monitoring (CM) of the region outside the liquid-metal and cover-gas containment boundary can provide detection of leakage. Other operation parameters, such as pressure, coolant flow, temperature, flux, vibration can be monitored to supplement other examination methods.

Surface examination is used to detect the presence of surface or near-surface cracks. It may be conducted by either a magnetic particle or a liquid penetrant method.

Volumetric examination is used to detect cracks and discontinuities throughout the volume of the material. The recognized techniques are radiographic examination, ultrasonic examination, and eddy-current examination.

These examination methods may be substituted by alternative examination methods, a combination of methods, or newly developed techniques, if they are demonstrated to be equivalent or superior, and accepted by the inspector.

\section{II.10.1.4 Inspection Plans and Schedules}

The Code requires that inspection plans shall be developed, which delineate the following:

1. Classification of the components and the boundaries of the system.

2. Identification of components subject to examination and testing.

3. Code requirements by category, item number for each component and the examination or test to be performed. 
4. The Code Cases proposed for use and the extent of their application.

5. Schedule for performance of examination and tests.

It is also required that all the liquid metal retaining components shall be continuously monitored for leakage. These requirements are delineated in this section for reactor internals and vessel, and summarized in Table II.10-1.

Reactor internals are required to be inspected by VTM-3 visual examination methods. VTM3 inspections may be conducted by direct or remote visual examination, dimensional gauging, and ultrasonic scanning. The key guidelines and principles for the inspection requirements are summarized as following:

1. For integrally welded components, observe dimensional or positional relationships that are required to ensure a coolable and controllable core geometry and adequate coolant circulation.

2. Observe the location of each removable or non-welded component and verify the asinstalled location of visible fasteners.

3. Components for which the applicable requirements above cannot be met shall be examined by some other methods capable of determining the general condition and functionality of the component.

The reactor vessel is required to be continuously monitored for liquid-metal leakage and periodically inspected by VTM-2 visual inspection. This visual inspection is required for all weld joints in the reactor vessel---longitudinal and circumferential shell welds, meridional, and circumferential head welds, shell-to-flange welds, and shell-to-head welds.

The Code specifies inspection time intervals during which the inspection should be performed. Accordingly, the inspection schedules for ASME Class 1 liquid metal or cover gas retaining components may follow the programs given in Table II.10-2 or II.10-3. The in-service inspection of the subject components shall be completed during each of the inspection intervals for the service lifetime of the plant. Each inspection interval may be decreased or extended by as much as 1 year. Deferral of inspections to the end of the interval is permitted. During each interval, $100 \%$ of the reactor internal structures must be examined. For the reactor vessel, 33\% of the vessel welds must be examined, so that essentially $100 \%$ of the vessel welds are inspected by the end of the third interval. 
Table II.10- 1 In-Service Inspection plan Requirements for Liquid-metal Cooled reactor Components

\begin{tabular}{|c|c|c|c|c|}
\hline $\begin{array}{l}\text { Code } \\
\text { Item }\end{array}$ & Components Examined & ISI Method & \multicolumn{2}{|c|}{ Schedule \& Extent } \\
\hline B1.10 & Reactor Vessel (RV) & VIM-2 & $33 \%$ & $33 \%$ \\
\hline \multirow[t]{3}{*}{ B5.10 } & Reactor Vessel: Circumferential shell welds joining dissimilar metals & *Volumetric & $100 \%$ & $100 \%$ \\
\hline & \multirow[t]{2}{*}{ Guard Vessel } & VTM-3 & $100 \%$ & $100 \%$ \\
\hline & & Cont. monitoring & $100 \%$ & $100 \%$ \\
\hline B12.10 & $\begin{array}{l}\text { Integrally welded Reactor Internals: } \\
\text { Core support/Retainer/Inlet Manifold and plenum/Thermal barriers }\end{array}$ & VTM-3 & $100 \%$ & $100 \%$ \\
\hline \multirow[t]{2}{*}{ B12.20 } & Removable reactor internal components & VTM-3 & $100 \%$ & $100 \%$ \\
\hline & EM Pumps, IHX & Cont. monitoring & $100 \%$ & $100 \%$ \\
\hline $\begin{array}{l}\text { B3.10 } \\
\text { B3.20 } \\
\text { B3.30 } \\
\text { B3.40 } \\
\text { B6.10 }\end{array}$ & $\begin{array}{l}\text { Reactor Cover (rotating plugs and deck) } \\
\text { Control rod drive and other above cover mechanisms containing cover gas } \\
\text { Primary cover gas systems - vessel welds, piping welds, valves and stem } \\
\text { seals, other gas tight components) } \\
\text { Reactor Closure Bolting }\end{array}$ & Cont. monitoring & $\begin{array}{l}\text { External or } \\
\text { adjacent to } \\
\text { surface } \\
\text { monitored }\end{array}$ & $\begin{array}{l}\text { External } \\
\text { or adjacent } \\
\text { to surface } \\
\text { monitored }\end{array}$ \\
\hline \multirow{2}{*}{$\begin{array}{l}\text { B8.10 } \\
\text { B8.14 } \\
\text { B9.10 }\end{array}$} & \multirow{2}{*}{$\begin{array}{l}\text { Liquid metal retaining heat transport loop piping protected by guard pipe } \\
\text { - circumferential/longitudinal welds } \\
\text { - branch pipe connection welds** } \\
\text { - piping boundary }\end{array}$} & VTM-2 & $\begin{array}{l}33 \% \\
(100 \% \text { for } * *)\end{array}$ & $\begin{array}{l}33 \% \\
(100 \% \text { for } \\
* *)\end{array}$ \\
\hline & & Cont. monitoring & $100 \%$ & $100 \%$ \\
\hline C2.30 & $\begin{array}{l}\text { Guard pipes on intermediate liquid metal boundary } \\
\text { - all guard pipe welds }\end{array}$ & VTM-3 & $100 \%$ & $100 \%$ \\
\hline
\end{tabular}


Table II.10- 2 Inspection Program A (Table IMB-2411-1)

\begin{tabular}{|c|c|c|c|}
\hline $\begin{array}{c}\text { Inspection } \\
\text { Interval }\end{array}$ & $\begin{array}{c}\text { Inspection } \\
\text { Period, Calendar } \\
\text { Years of Plant } \\
\text { Service }\end{array}$ & $\begin{array}{c}\text { Minimum } \\
\text { Examinations } \\
\text { Completed, \% }\end{array}$ & $\begin{array}{c}\text { Maximum } \\
\text { Examinations } \\
\text { Credited, \% }\end{array}$ \\
\hline $1^{\text {st }}$ & 3 & 100 & 100 \\
$2^{\text {nd }}$ & 7 & 33 & 67 \\
$3^{\text {rd }}$ & 10 & 100 & 100 \\
& 13 & 16 & 34 \\
& 17 & 40 & 50 \\
$4^{\text {th }}$ & 20 & 100 & 75 \\
& 23 & 8 & 100 \\
& 30 & 25 & 16 \\
& 33 & 50 & 67 \\
& 37 & 75 & 100 \\
& 40 & 100 & $\ldots$ \\
\hline
\end{tabular}

Table II.10- 3 Inspection Program B (Table IMB-2411-1)

\begin{tabular}{|c|c|c|c|}
\hline $\begin{array}{c}\text { Inspection } \\
\text { Interval }\end{array}$ & $\begin{array}{c}\text { Inspection } \\
\text { Period, Calendar } \\
\text { Years of Plant } \\
\text { Service }\end{array}$ & $\begin{array}{c}\text { Minimum } \\
\text { Examinations } \\
\text { Completed, \% }\end{array}$ & $\begin{array}{c}\text { Maximum } \\
\text { Examinations } \\
\text { Credited, \% }\end{array}$ \\
\hline $1^{\text {st }}$ & 3 & 16 & 34 \\
& 7 & 50 & 67 \\
$2^{\text {nd }}$ & 10 & 100 & 100 \\
& 13 & 16 & 34 \\
& 17 & 50 & 67 \\
$3^{\text {rd }}$ & 20 & 100 & 100 \\
& 23 & 50 & 34 \\
& 27 & 100 & 67 \\
$4^{\text {th }}$ & 30 & 16 & 34 \\
& 33 & 50 & 100 \\
& 37 & 100 & $\ldots$ \\
\hline
\end{tabular}

\section{II.10.2 Examination Techniques}

\section{II.10.2.1 Description of Examination Techniques}

The following examination techniques are adopted for the conduct of in-service inspection of ABR:

Continuous Monitoring: The in-service inspection approach heavily utilizes continuous monitoring of various operating conditions. Sodium vapor sensors can monitor the presence of sodium vapor in the atmosphere, and in the annular space between the reactor vessel and the guard vessel. Sodium liquid detectors can monitor the presence of liquid sodium in bottom of a vessel. Gas pressure gauges are used for leakage testing of a vessel by direct pressurization. Radiation sensors monitor an area for cover-gas leaks. In addition, various sensors are available for monitoring the conditions of reactor internals-flow, pressure, vibration, temperature, coolant level, and pump power. These technologies are readily available. 
Remote Operated Robotic Vehicle (ROV): These vehicles can be equipped with cameras and light sources to inspect parts of the components inaccessible by human, such as the reactor/guard vessel surfaces and the reactor support skirt. This robotic device should be able to navigate in small areas of vertical vessel walls, to precisely position the camera, and to endure high temperature and radiation. Currently, this technology is not available on a commercial basis.

Electromagnetic Acoustic Transducer (EMAT): These transducers can be used for volumetric inspection of structural elements, such as the reactor vessel. Compared to ultrasonic transducers, this device is particularly suitable for remote inspection because it does not require corpulent fluid and is adequate for high temperature use.

Eddy Current Examination: This method can be used for detection of structural failures in pipes and tubes of NSSS systems.

Periscope/Camera: Periscope or camera systems, inserted through the access port inthe reactor head closure, can be used to visually observe the cover gas area within the containment boundary. Special provision is needed to keep the camera lens clear from sodium vapor build-up.

Dimensional Gauging Probe: In areas where dimensional gauging is not possible with direct visual observation, positioning mechanisms can be used to indirectly verify structural integrity of structural components. In-vessel fuel handling gripper mechanism, or an equivalent indexing mechanism can be used for this purpose.

Under-Sodium Viewer (USV): Due to the opacity of sodium, the reactor internals below sodium level cannot be observed with optical means. To this end, conceptually, under-sodium viewing devices may be effectively utilized to carry out visual inspection of the components submerged under sodium. Typically, these are ultrasonic metrology devices that emit high frequency sound waves and receive the reflecting waves to perceive environmental objects. Special design provisions are made to match the acoustic impedance with sodium, and to withstand high temperature use. This device can be used for mapping the core, horizontal baffle and vessel liner during and following fuel handling, and also be used as a core sweep at the start of refueling. Currently, the benefits of using this device are uncertain because of the immature state-of-the art of the technology.

\section{II.10.2.2 Evaluation of Examination Techniques}

To ensure inspectability of the reactor system, it is important to adopt proven and readily available inspection techniques. While most of the above techniques are well developed, some of the techniques are based on a relatively immature technology basis, and require improvement. In particular, the following techniques need special and increased attention:

\section{Under-sodium Viewing}

Due to the opacity of the sodium coolant, the under-sodium viewing technique is important for ensuring full inspectability of reactor internals and internal components. Visual inspection is needed not only for the normal VTM-3 inspection, but also for supplemental close-up viewing. With available USV technology, many difficult inspection processes will be made possible or simplified. Otherwise, inspections may require complex design provisions and procedures, which may involve draining of sodium to expose the parts. In spite of the apparent benefits, however, the current state-of-the-art of the technology exhibits some significant limitations against its deployment. 
The first limitation attributes from unavailability of high temperature ultrasonic transducer. The typical transducer materials have relatively low Curie temperature, and thus they will lose the piezoelectric characteristics in the high temperature environment inside the reactor. Some piezoelectric material, such as LiNbO3 (Lithium Niobate), can withstand high temperature, but their sensitivity is known to be impractically low. In addition, the bonding between the piezoelectric element and the face plate tends to fail easily at high temperature.

Another limitation is the result of the inaccuracy of measurements taken. To make accurate ultrasonic measurement, the object's surface has to be flat and in the transducer should be faced normal to the object surface. It is difficult to measure the pose of the parts with curved or oblique surfaces, especially when the surface is specular. Since most parts are of such nature, it is not possible to use current ultrasonic techniques for general imaging. To circumvent such technical difficulty, flat plates may be provided at predefined locations in order to facilitate accurate positional measurement at these locations. However, such design provisions require substantial installations and may be impractical for general purpose inspection. Due to such difficulties, the current ultrasonic viewing techniques have limited success only in ranging mode operation, which intends to identify the presence of an object, such as for path clearance during fuel handling.

To this end, technology development is needed to develop sensor configuration for high temperature use, and to improve measurement accuracy. Such improvements may benefit from recent advances in sensor and signal processing technologies.

\section{Remote Operated Robotic Vehicle for Reactor Vessel Inspection}

The ASME code requires visual inspection of the reactor vessel (RV) welds. The annular gap between the reactor vessel and the guard vessel (GV) is made large enough to allow access for the remote operated robotic vehicle to perform remote visual inspection. Since this ISI gap contains sodium in case the reactor vessel leaks, it determines the faulted sodium level in the reactor. Therefore, minimizing the ISI gap will bring about significant benefit of reducing the sodium level variations in faulted conditions. This is a significant benefit in terms of reactor design, because it allows for reducing the height of the reactor, resulting in a more compact reactor, and, thus, alleviating design requirements for many reactor internal components. In this regard, the successful development of a small size robotic vehicle will be a significant benefit to economical plant designs.

Presently, no commercially available robotic system is able to meet the unique requirements of reactor vessel inspection, such as having small size, and being tolerant to high temperature and radiation. Recently, reactor vessel inspection robots have been developed for a few sodium cooled reactors, such as Monju in Japan and Super Phoenix in France, but the developed systems are much larger in size. Therefore, development of a new robotic system will be necessary, with emphasis on size-reduction, adaptation to high temperature use, and design of the locomotion mechanism and the camera system. Recent advances in robotics and inspection technologies should be useful for the needed development.

\section{II.10.3 In-service Inspection Approach for ABR}

In-service inspection approaches for ABR are established based on the following general guidelines, which are consistent with the ASME Code, Section XI: 
1. In-service inspections will be conducted during refueling or service outage.

2. Available examination technologies are adapted to the unique needs of the ABR.

3. Access provisions are provided for all components of the reactor system.

4. Design provisions are made to reduce possible areas and modes of failures.

5. All liquid metal and cover gas containment boundaries will be continuously monitored for leakage.

6. Continuous monitoring of reactor operating variables will be utilized to supplement or replace other inspection methods, whenever possible.

7. Problem areas observed in the normal in-service inspection will be further investigated.

The in-service inspection approaches provide for both normal and supplemental inspections. The supplemental inspections may be required to follow-up on these examinations in the event the normal inspections indicate deviations from normal operating or structural conditions.

\section{II.10.3.1 Approaches for Accessibility}

The ABR design has incorporated access provisions for in-service inspection according to the code requirements. These design provisions include:

1. Annular space (ISI gap) between reactor vessel and guard vessel for inspection.

2. Entry ports in the reactor support skirt for access to the ISI gap.

3. Access provisions to allow inspection of the reactor deck structure.

4. Space for installing work platforms and lighting and shielding and insulation during inspection and maintenance.

5. Verification that welds requiring inspection are not in inaccessible locations.

6. Access provisions for inspection within the reactor vessel. These provisions include access ports in the reactor deck and the rotating plug, passage holes in the horizontal baffle plates, and passage to the bottom of the core support structure.

In addition to accessibility, a major design focus was directed to reducing the possible areas and modes of failure. By reducing the possible failure modes, it is possible to reduce inspection requirements. For example, the core support load path is arranged in such a way that critical invessel structures are normally in compression, while the principal tensile load path is only in the reactor vessel wall or bottom head, which will be continuously monitored for leaks that would indicate a crack formation and propagation.

\section{II.10.3.2 Proposed Inspection Methods and Procedures}

This section specifies the in-service inspection approaches for the reactor system components, with emphasis given to nuclear safety grade components of the reactor internals, the reactor vessel and the reactor closure, which are shown in Figure II.10-1. The inspection of the non-nuclear safety grade components of the remaining nuclear steam generation system, such as intermediate heat transport system and steam generation system, is considered far less difficult, and is not detailed in this report. 
The inspection methods and procedures are established in accordance with possible failure modes of associated components. While the ASME Code provides general guidelines, each individual examination that makes up the inspection has to be established in consideration of its own unique limitations on access and geometry. Even a single component can fail in various ways, and each case may require different inspection techniques. These aspects cannot be generalized, and inspection methods must be considered individually associated with possible failure modes. Therefore, the failure modes of the reactor components are first postulated, and subsequently the inspection techniques are established that are capable of detecting the onset of these failures. Table II.10-4 presents a list of failure modes and the suggested in-service inspection approaches for ABR. Both normal examination methods and follow-up examination methods are presented for each failure mode.

\section{Inspection of Reactor Internals}

Normal in-service inspection of reactor internals and internal components require VTM-3 visual inspection. The sodium surface and the in-reactor vessel components above the sodium level will be examined using a periscope or camera, inserted through the multiple access ports in the closure head deck. For components below the sodium level, the normal in-service inspection approach is based on continuous monitoring, dimensional gauging and under-sodium viewing. Continuous monitoring of sodium levels, outlet temperature of a limited number of core assemblies, vibration and the pressure in the pump discharge manifold will provide indication of the integrity of the pressurized components (core barrel, inlet plenum, and primary piping discharge manifolds) in hard-to-access areas of the reactor pool as will statistical analysis of primary pump power.

The structural components of the core support and restraints will be inspected with dimensional gauging. Dimensional gauging will be performed by indexing with the fuel handling gripper, or other dedicated indexing mechanism, on several specified locations on the top of the former ring and other structural components in the hot pool. This technique can determine possible shifts in elevation of components. The verification of the proper location of a component, such as the top of the core barrel, can also imply a proper location for the core support structure. 


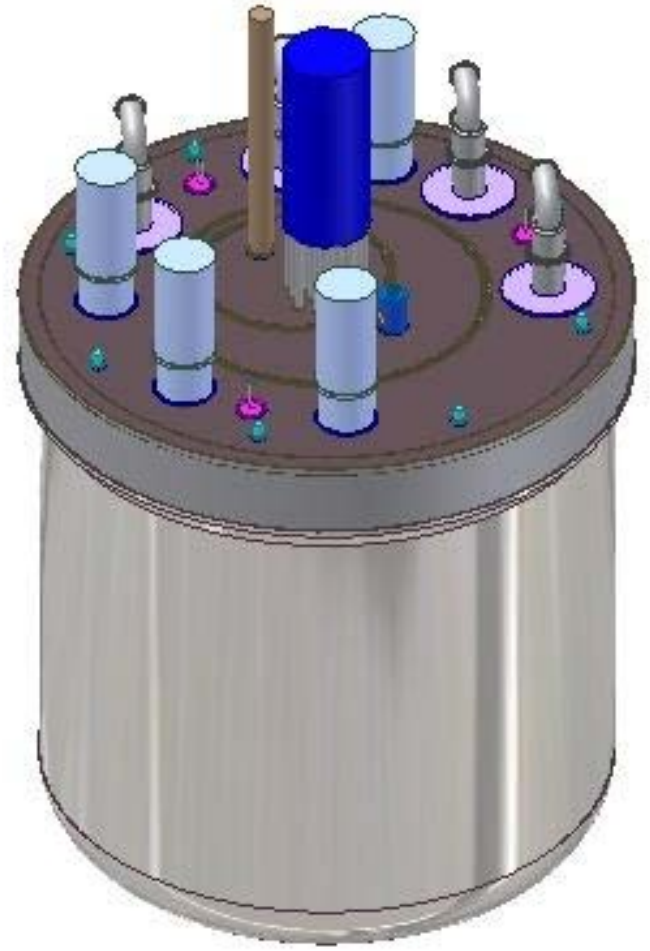

(a) Reactor Vessel and Deck

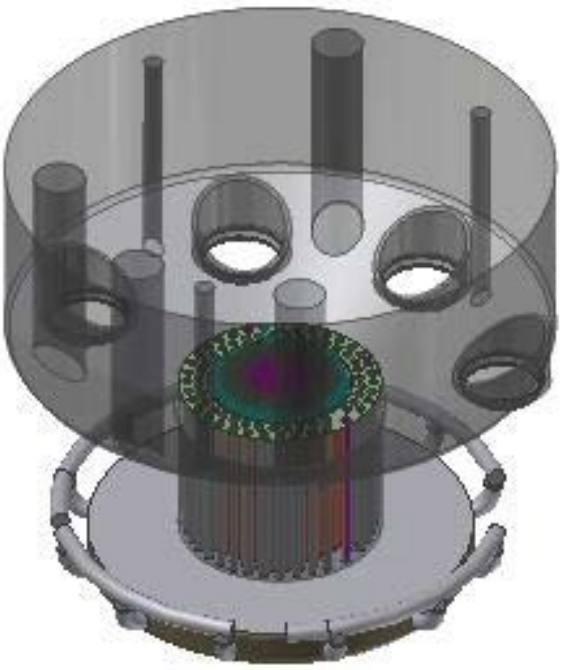

(b) Reactor Internal Structures

Figure II.10-1 Areas Subject to Inspection

An under-sodium viewer can also be used to map the core top, and be used to investigate the condition of many difficult to reach areas. The under-sodium viewer is inserted through ports in the rotating plug, and additional access to vessel internals components are provided through ports in the outer fixed part of the closure head deck. For accurate measurement, gauge blocks and gauge marks are set at key locations to serve as reference marks for the under-sodium viewer. The rotating plug can move the fuel-handling gripper and the under-sodium viewer to various locations inside the reactor vessel.

Large cracks or separation of the reactor vessel liner will be detected by continuous monitoring of vessel temperatures and pump well level. The inspection will be supplemented with visual inspections, for which a camera/periscope will be used for above sodium-level portion, while an under-sodium viewer will be used for below sodium-level portion.

Failures in the redan, such as at various connections, at the intermediate heat exchangers, and at pump wells, are checked by dimensional gauging. This can be performed with the fuel handling gripper mechanism or under-sodium viewer, and indexing various wells and penetrations.

The Upper Internals Structure (UIS) is associated with various failure modes as delineated in Table II.10-4. Its structural integrity is checked primarily by continuous monitoring with acoustic sensors, and use of an under-sodium viewer. Anomalies in the control rod drives can be tested through periodic test operations and by monitoring the drive motor current. The integrity of the 
instrument drywells will be verified by continuous monitoring for leakage with reactor cover radiation monitors, and visually inspecting for distortion using periscope/camera. Continuous monitoring will be the primary source of information on the operation of the primary pumps and intermediate heat exchanger. For this purpose, cover gas leaks will be monitored with radiation monitors, and pump oil leakage will also be monitored.

DRACS units may fail by sodium leakage, sodium blockage, and air-side blockage. Currently, the requirements for its inspection are not explicitly specified in the ASME Code. To ensure safe and reliable operation, continuous monitoring of the relevant operation parameters is included in the ABR design. Detailed scope and procedures shall be developed. Problems observed in the normal in-service inspections will be further investigated by follow-up examinations. These follow-up examinations may include close-up viewing with optical instruments and under-sodium viewing of difficult-to-access areas. Such procedures require more extensive access and may involve removal of components and lowering of sodium levels, as delineated in Table II.10-4, supplemental inspection column.

Such extensive procedures may be alleviated if accurate under-sodium imaging technology is available. However, the current state-of-the-art of the technology does not meet such expectations. The results of the follow-up examination will be used to verify structural failure and plan detailed maintenance procedures. Maintenance approaches are identified for most of the components in the reactor vessel.

\section{Inspection of Reactor Vessel, Guard Vessel, and Deck Structure}

Inspection of the reactor vessel will be conducted by visual examination (VTM-2) of all welds and continuous monitoring of the inert gas in the annular gap between the reactor vessel and the guard vessel. The reactor vessel will be continuously monitored for sodium and gas leakage. Sensors and measurement techniques are readily available for this purpose. The visual inspection is performed using a camera system mounted on a remotely operated robotic vehicle. This robotic device will be installed in the annulus between the reactor vessel and the guard vessel, as shown in Figure II.10-2. Currently, there is no commercially available system for this purpose.

VTM-3 visual inspection is required for the guard vessel. This is conducted using a TV camera mounted on the remote operated robotic vehicle. Continuous monitoring of pressure in the reactor vessel/guard vessel annulus will provide initial detection of leakage from the reactor vessel. Follow-up examinations will consist of close-up visual examination of the annulus, and analyzing a gas sample from the annulus. Sodium vapor sensors are adapted to monitor the annulus region between the reactor vessel and the guard vessel for sodium vapor. Sodium liquid detectors monitor the presence of liquid sodium in the bottom head of the guard vessel. Gas pressure gauges are used for leakage tests, during which time the reactor vessel/guard vessel annulus is pressurized. Radiation sensors monitor the reactor closure for cover-gas leaks. In addition, various sensors are utilized to monitor the conditions of reactor internals - flow, pressure, vibration, temperature, coolant level, and pump power.

VTM-3 visual inspection is required for normal inspection of the conical reactor vessel/deck support skirt in the head access area. For this purpose, the reactor support skirt welds and bolts will be inspected with remote visual examination using a camera on the remote operated robotic vehicle. In addition, the design of the conical support skirt provides improved direct access to the bolting for personal close-up inspections and for any repair or replacement of the bolting. (Removable shielding blocks are located in this area to allow such close-up inspections.) 
The reactor closure, including the deck and rotatable plug, will be continuously monitored for leaks, using radiation detectors in the head access area. Analysis of reactor cover gas and the primary sodium purity will provide follow-up indications. Further inspection will be performed if abnormal conditions are detected during normal inspection. Such supplemental inspection will involve broader TV viewing to pinpoint the problem area, and subsequent close-up visual inspection (VTM-1) and volumetric inspection with electromagnetic acoustic transducer to characterize the condition of the defects. The shielding in the deck consists of loose steel balls placed in each of the compartments in the deck. These balls can be removed to allow close-up inspection of the structural welds.

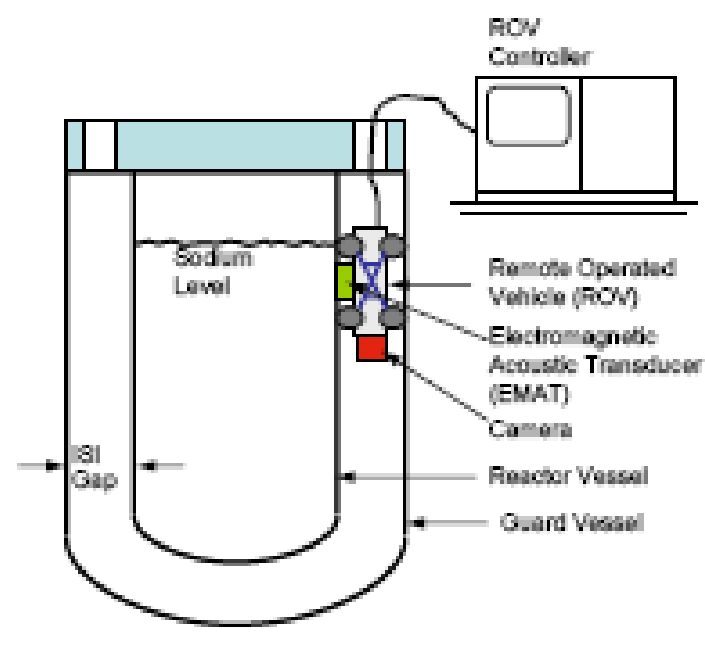

(a) Wheel type ROV

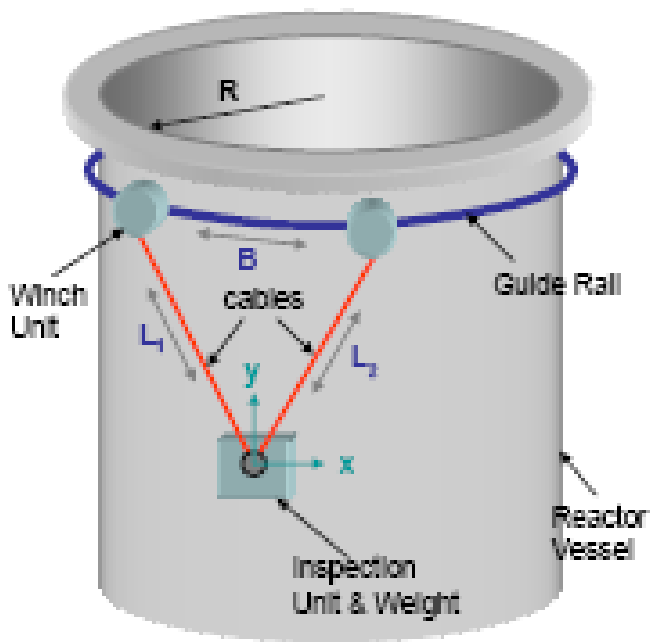

(b) Cable and Winch Type ROV

Figure II.10- 2 Reactor Vessel Inspection with ROV 
Table II.10-4 Postulated Failure Modes and In-Service Inspection Considerations (1/6)

\begin{tabular}{|c|c|c|c|c|}
\hline Component/System & Postulated Failure & $\begin{array}{l}\text { Normal Inspection (NI) } \\
\text { Method, to detect problem }\end{array}$ & $\begin{array}{l}\text { Code Requirements } \\
\text { (Div.3, Section XI, ASME } \\
\text { B\&PV) }\end{array}$ & $\begin{array}{l}\text { Supplemental Inspection } \\
\text { (SI), if a problem is } \\
\text { indicated }\end{array}$ \\
\hline 1. Reactor Vessel & $\begin{array}{l}\text { Weld crack } \\
\text { (below Na level) } \\
\text { Weld crack } \\
\text { (cover-gas region) } \\
\text { Exterior surface deterioration }\end{array}$ & $\begin{array}{l}\text { 1.1 CM: Sodium leak, } \\
\text { Radiation, RV/GV annulus } \\
\text { pressure } \\
\text { 1.2 Periodic visual inspection } \\
\text { with ROV and camera }\end{array}$ & $\begin{array}{l}\text { VTM-2 inspection on all } \\
\text { welds plus Continuous } \\
\text { monitoring }\end{array}$ & $\begin{array}{l}\text { 1.1 Broader TV viewing to } \\
\text { pinpoint the area } \\
\text { 1.2 VTM-1 inspection with } \\
\text { zoom lens or fiberscope } \\
\text { 1.3 Localized inspection } \\
\text { w/ ultrasonic transducer }\end{array}$ \\
\hline 2. Guard Vessel & $\begin{array}{l}\text { Weld crack } \\
\text { Interior surface deterioration }\end{array}$ & $\begin{array}{l}\text { 2.1 CM: Cover gas leak, } \\
\text { Radiation, Pressure } \\
2.2 \text { same as } 1.2\end{array}$ & $\begin{array}{l}\text { VTM-3 inspection for low } \\
\text { alloy steel }\end{array}$ & $\begin{array}{l}2.1 \text { Same as } 1.1-1.3 \\
2.2 \text { additional inspection } \\
\text { from the outside }\end{array}$ \\
\hline 3. RV/GV Support & Crack or distortion & $\begin{array}{l}\text { 2.1 Cm: Cover gas leak, } \\
\text { Radiation, Pressure } \\
\text { 2.2. same as } 1.2 \\
\end{array}$ & $\begin{array}{l}\text { VTM-3 inspection for low } \\
\text { alloy steel }\end{array}$ & $\begin{array}{l}\text { 2.1 Same as } 1.1-1.3 . \\
2.2 \text { additional inspection } \\
\text { from the outside }\end{array}$ \\
\hline $\begin{array}{l}\text { 4. Reactor Internals } \\
\text {-Core support }\end{array}$ & Massive weld failure & $\begin{array}{l}\text { 4.1 Check height of core } \\
\text { barrel with IVHM or USV } \\
4.2 \text { Measure loads to remove } \\
\text { fuel assy. } \\
4 / 3 \text { Test operation of control } \\
\text { rods }\end{array}$ & VTM-3 inspection & $\begin{array}{l}4.1 \text { Access to the bottom } \\
\text { of the reactor vessel, and } \\
\text { use USV in the core } \\
\text { support region if there has } \\
\text { been structural movement. }\end{array}$ \\
\hline & $\begin{array}{l}\text { Support to reactor vessel weld } \\
\text { failure }\end{array}$ & $\begin{array}{l}4.4 \text { same as } 1.24 .5 \text { same as } \\
4.1 \text { thru } 4.3\end{array}$ & & 4.2 Same as 4.1 \\
\hline
\end{tabular}


Table II.10-5 Postulated Failure Modes and in-Service Inspection Consideration (2/6)

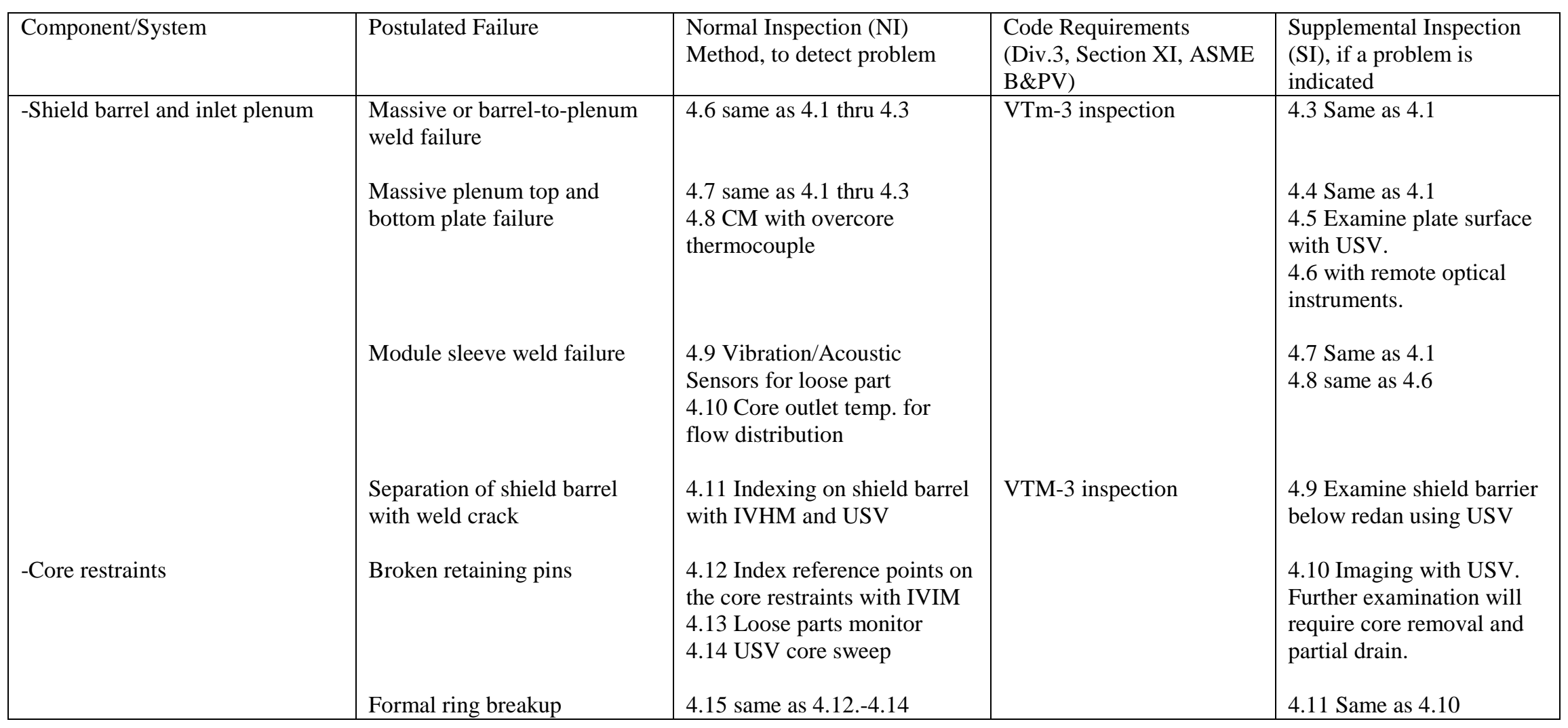


Table II.10-6 Postulated Failure Modes and In-Service Inspection Considerations (3/6)

\begin{tabular}{|c|c|c|c|c|}
\hline Component/System & Postulated Failure & $\begin{array}{l}\text { Normal Inspection (NI) } \\
\text { Method, to detect problem }\end{array}$ & $\begin{array}{l}\text { Code Requirements } \\
\text { (Div.3, Section XI, ASME } \\
\text { B\&PV) }\end{array}$ & $\begin{array}{l}\text { Supplemental Inspection } \\
\text { (SI), if a problem is } \\
\text { indicated }\end{array}$ \\
\hline -Pump well & Well weld failure & $\begin{array}{l}\text { 4.16 examine well position } \\
\text { with Periscope/camera above } \\
\text { the Na level. Use USV to heck } \\
\text { position below Na level. }\end{array}$ & VTM-3 inspection & $\begin{array}{l}4.12 \text { Remove pump } \\
\text { examine interior of well } \\
\text { with camera/periscope } \\
\text { above Na level, and USV } \\
\text { below Na surface }\end{array}$ \\
\hline -Pump discharge pipes & $\begin{array}{l}\text { Large Na leak (weld fail at } \\
\text { pump or plenum) }\end{array}$ & $\begin{array}{l}\text { 4.17 Monitor pump well level, } \\
\text { pump speed, pump discharge } \\
\text { flow, core outlet temperature }\end{array}$ & & $\begin{array}{l}\text { 4.13 Remove pump, } \\
\text { examine pipes from within } \\
\text { using USV. } \\
4.14 \text { If needed, remove } \\
\text { fuel, drain } \mathrm{Na} \text {, and } \\
\text { examine pipe optically }\end{array}$ \\
\hline \multirow[t]{2}{*}{-Vessel thermal liner } & Large liner crack & $\begin{array}{l}4.18 \text { Monitor pump well level } \\
\text { and vessel temp. } \\
4 ; 19 \text { Examine above Na level } \\
\text { region with camera/periscope }\end{array}$ & & $\begin{array}{l}4.16 \text { Remove core, drain } \\
\mathrm{Na} \text {, and examine areas } \\
\text { with periscope }\end{array}$ \\
\hline & Spearation of liner from vessel & $\begin{array}{l}4.20 \text { same as } 4.18 \\
4.21 \text { USV of selected points } \\
\text { on the liner and on top of } \\
\text { horizontal baffle assembly }\end{array}$ & & 4.16 Same as 4.15 \\
\hline
\end{tabular}


Table II.10-7 Postulated Failure Modes and In-Service Inspection Consideration (4/6)

\begin{tabular}{|c|c|c|c|c|}
\hline Component/System & Postulated Failure & $\begin{array}{l}\text { Normal Inspection (NI) } \\
\text { Method, to detect problem }\end{array}$ & $\begin{array}{l}\text { Code Requirements } \\
\text { (Div.3, Section XI, ASME } \\
\text { B\&PV) }\end{array}$ & $\begin{array}{l}\text { Supplemental Inspection } \\
\text { (SI), if a problem is } \\
\text { indicated }\end{array}$ \\
\hline \multirow[t]{2}{*}{-Redan } & $\begin{array}{l}\text { Fail at rdan connections to } \\
\text { fuel storage basket, IHX, and } \\
\text { pump well. }\end{array}$ & $\begin{array}{l}\text { 4.21 Dimensiosn gauging with } \\
\text { IVHM and USV on the redan } \\
\text { and various wells and } \\
\text { penerations } \\
4.22 \text { Monitor level in pump } \\
\text { well }\end{array}$ & VTM-3 inspections & $\begin{array}{l}\text { 4.17 Remove high burnup } \\
\text { assemblies, drain Na to } \\
\text { partially expose the redan } \\
\text { and examine with optical } \\
\text { devices. }\end{array}$ \\
\hline & $\begin{array}{l}\text { Lower baffle support skirt } \\
\text { structural failure }\end{array}$ & 4.23 same as 4.21 & & 4.18 Same as 4.17 \\
\hline \multirow[t]{2}{*}{-Instrumental drywells } & Drywells leackage & $\begin{array}{l}4.24 \mathrm{CM} \text { wish reactor cover } \\
\text { radiation monitors }\end{array}$ & & $\begin{array}{l}\text { 4.19 Pressurize drywell } \\
\text { and check for pressure } \\
\text { decary } \\
4.20 \text { remove probe and } \\
\text { examine for Na leakage }\end{array}$ \\
\hline & Drywell distortion & 4.25 Camera/periscope, USV & & -- \\
\hline \multirow[t]{2}{*}{-Inlet modules } & Module weld failure & $\begin{array}{l}4.26 \text { same as } 4.1-4.3 \\
4.27 \mathrm{CM} \text { of core outlet } \\
\text { temperature }\end{array}$ & & $\begin{array}{l}4.21 \text { Remove any hot or } \\
\text { binding fuel assay, and } \\
\text { inspect with USV }\end{array}$ \\
\hline & Binding on fuel assay & 4.28 same as 4.2 & & 4.22 Same as 4.21 \\
\hline
\end{tabular}


Table II.10-8 Postulated Failure Modes and In-Service Inspection Considerations (5/6)

\begin{tabular}{|c|c|c|c|c|}
\hline Component/System & Postulated Failure & $\begin{array}{l}\text { Normal Inspection (NI) } \\
\text { Method, to detect problem }\end{array}$ & $\begin{array}{l}\text { Code Requirements } \\
\text { (Div.3, Section XI, ASME } \\
\text { B\&PV) }\end{array}$ & $\begin{array}{l}\text { Supplemental Inspection } \\
\text { (SI), if a problem is } \\
\text { indicated }\end{array}$ \\
\hline \multirow[t]{5}{*}{-Upper internals Structure (UIS) } & $\begin{array}{l}\text { UIS support cylinder weld fail } \\
\text { or distortion }\end{array}$ & $\begin{array}{l}\text { 4.29 Examine exposed surface } \\
\text { above Na level with } \\
\text { camera/periscope. Check } \\
\text { location and general shape } \\
\text { with USV below Na level }\end{array}$ & & $\begin{array}{l}4.23 \text { Remove core, lower } \\
\text { Na level, and inspect } \\
\text { cylinder with remote optics } \\
\text { and periscope. }\end{array}$ \\
\hline & $\begin{array}{l}\text { Distorted control rod drive } \\
\text { (CRD), shroud tubes, weld \& } \\
\text { bearing failures }\end{array}$ & $\begin{array}{l}\text { 4.30 Periodic testing of CRDs } \\
\text { 4.31 Monitor drive motor } \\
\text { current } \\
\text { 4.32 Loose part monitor } \\
\text { 4.33 USV core sweep }\end{array}$ & & $\begin{array}{l}4.24 \text { Remove driveline and } \\
\text { insert fiber-optic device in } \\
\text { opening to examine shroud } \\
\text { tubes. }\end{array}$ \\
\hline & $\begin{array}{l}\text { Failure of spokes or } \\
\text { connection to drywells at } \\
\text { support plates }\end{array}$ & 4.34 same as $4.30-4.33$ & & \\
\hline & Erosion of UIS surface & $\begin{array}{l}4.35 \text { Loose parts monitor } \\
4.367 \text { analyze particulate from } \\
\text { Na purification sys. for metal } \\
\text { particle }\end{array}$ & & \\
\hline & $\begin{array}{l}\text { Failure of connection pins, } \\
\text { loose sleeves on instrument } \\
\text { hold-down, loose shock liner } \\
\text { plates }\end{array}$ & $\begin{array}{l}\text { 4.37 Loose part monitor } \\
\text { 4.38 USV core sweep }\end{array}$ & & $\begin{array}{l}\text { 4.25 Scan top of core for } \\
\text { loose parts with USV. } \\
4.26 \text { Lower Na level, and } \\
\text { examine underside of } \\
\text { redan with optics }\end{array}$ \\
\hline
\end{tabular}


Table II.10-9 Postulated Failure Modes and In-Service Inspection Considerations (6/6)

\begin{tabular}{|c|c|c|c|c|}
\hline Component/System & Postulated Failure & $\begin{array}{l}\text { Normal Inspection (NI) } \\
\text { Method, to detect problem }\end{array}$ & $\begin{array}{l}\text { Code Requirements } \\
\text { (Div.3, Section XI, ASME } \\
\text { B\&PV) }\end{array}$ & $\begin{array}{l}\text { Supplemental Inspection } \\
\text { (SI), if a problem is } \\
\text { indicated }\end{array}$ \\
\hline $\begin{array}{l}5 . \text { Reactor Cover (deck and } \\
\text { rotatable plug) }\end{array}$ & $\begin{array}{l}\text { Cover gas seal leakage } \\
\text { Dip seal loss } \\
\text { Inflatable seal failure }\end{array}$ & $\begin{array}{l}\text { 4.39 Reactor cover radiation } \\
\text { monitor }\end{array}$ & Continuous monitoring & $\begin{array}{l}\text { 4.27 Liquid penetrant } \\
\text { examination of seal and } \\
\text { support flange. } \\
4.28 \text { Periodically examine } \\
\text { and clean seal }\end{array}$ \\
\hline 6. Primary Pump & $\begin{array}{l}\text { Deck seal leak } \\
\text { Pump shaft seal leak } \\
\text { Support flange failure }\end{array}$ & $\begin{array}{l}\text { 4.40 Reactor cover radiation } \\
\text { monitor } \\
4.41 \text { Pump oil leakage monitor }\end{array}$ & Continuous monitoring & $\begin{array}{l}\text { 4.29 Liquid penetrant } \\
\text { examination of seal and } \\
\text { support flange }\end{array}$ \\
\hline 7. IHX & $\begin{array}{l}\text { Deck seal leak } \\
\text { IHX inlet/outlet leak } \\
\text { IHX to primary Na leak }\end{array}$ & $\begin{array}{l}4.42 \text { Reactor cover radiation } \\
\text { monitor }\end{array}$ & Continuous monitoring & 4.30 Same as 4.28 \\
\hline 8. Primary cover gas & $\begin{array}{l}\text { Cover gas leak } \\
\text { Gas valve failure }\end{array}$ & $\begin{array}{l}4.43 \text { Reactor cover radiation } \\
\text { monitor }\end{array}$ & Continuous monitoring & $\begin{array}{l}\text { 4.31 Pressure test and } \\
\text { visual }\end{array}$ \\
\hline 9. DRACS & $\begin{array}{l}\text { Na leakage Sodium blockage } \\
\text { Air-side blockage }\end{array}$ & $\begin{array}{l}\text { 4.44 CM (details to be } \\
\text { developed) }\end{array}$ & $\begin{array}{l}\text { None } \\
\text { (NI exceeds Code } \\
\text { requirements) }\end{array}$ & \\
\hline
\end{tabular}




\section{II.11 Buildings and Structures}

Figure II.11-1 is a pictorial view of the overall site for the ABR1000. The sodium generator building, shown to the left of the reactor containment building, is located on a common concrete base mat with the reactor containment building. Natural draft cooling towers are shown as well as the turbine-generator building, control room building and office and support buildings. Table II.11-1 lists all the site buildings and provides their overall dimensions and footprint.

Table II.11-1 Site Buildings with Dimensions

\begin{tabular}{|l|c|c|c|c|}
\hline Building Name & Footprint $\left(\mathrm{ft}^{2}\right)$ & Length $(\mathrm{ft})$ & Width $(\mathrm{ft})$ & Height $(\mathrm{ft})$ \\
\hline Security Gate House & 900 & 30 & 30 & 16 \\
\hline Control/Personnel Building & 12,576 & 131 & 96 & 30 \\
\hline Reactor Building & 7,775 & 100 & 100 & 166 \\
\hline Nuclear Island & 34,361 & 209 & 209 & \\
\hline BOP Building & 41,860 & 260 & 161 & 49 \\
\hline Emergency Generator Building & 3000 & 100 & 30 & 12 \\
\hline Balance of Plant Service Building & 9,000 & 100 & 90 & 20 \\
\hline Cooling Towers (each) & 19,113 & 156 & & 60 \\
\hline Radwaste/ Maintenance Facility & 24,000 & 120 & 200 & $40 / 80$ \\
\hline Lift Station & 1,200 & 40 & 30 & 16 \\
\hline Wastewater Treatment Plant & 4,800 & 80 & 60 & 16 \\
\hline Interior Security Perimeter Fence & 736,308 & 1,086 & 678 & - \\
\hline Exterior Security Perimeter Fence & $1,181,700$ & 1,313 & 900 & - \\
\hline
\end{tabular}

\section{II.11.1 Reactor Building}

The reactor building, as schematically indicated in Figures II.11-2(a) and II.11-2(b), encloses the entire primary reactor system. The entire reactor building is seismically-isolated from earthquake events using seismic isolators located between the base slab of the building and a separate, independent reinforced concrete slab structure. The reactor building is a conventional, steel-lined, reinforced concrete containment structure. It is designed to accommodate the pressures and temperatures due to a large sodium fire caused by the unlikely event of a significant sodium leak within the containment. The containment is designed to have a maximum leak rate of $0.1 \%$ day of building volume at an internal pressure of 10 psig (0.069 $\mathrm{MPa}$ ). The top of the reactor vessel assembly is located at approximately grade level. All of the primary radioactive systems are located at or below grade within the reactor building.

The major functions of the reactor building are as follows:

- Contain radioactive material following the unlikely event of an accidental radioactivity release from the primary reactor system.

- House and structurally support the reactor vessel, guard vessel, the shield/air baffle cooling system, support structure of the primary system and temporary fuel handling equipment, biological shielding, and associated equipment and structures.

- Provide adequate space for the operation, maintenance, and removal of equipment housed within the containment structure during periodic maintenance. 
- Facilitate sodium and non-sodium fire protection for all safety equipment; this includes separation of redundant systems required for safe shutdown and for maintaining the reactor in safe shutdown condition.

- Provide protection for all safety equipment from the environment and natural phenomena such as floods, winds, tornadoes, and earthquakes, as well as loads from man-made hazards, including an aircraft crash.

- Maintain pressure within the containment boundary at less than 0.5 psig $(0.0034$ $\mathrm{MPa}$ ) negative with respect to the exterior, except during pressurization accidents.

- Limit leakage from the containment boundary to no more than $0.1 \%$ of its contained volume per day at an internal pressure of $10 \mathrm{psig}(0.069 \mathrm{MPa})$.

- Maintain the integrity of the containment boundary during all design loadings, including a maximum long term containment atmosphere temperature of $122^{\circ} \mathrm{F}\left(50^{\circ}\right.$ C) under normal operating and design basis accident containment atmosphere conditions.

\section{II.11.1.1 Containment Design Requirements}

The reactor building will be designed to the rules of the current ASME Boiler and Pressure Vessel Code, Section III, Division 2, "Code for Concrete Reactor Vessels and Containments," Subsection CC for concrete containment. These rules provide for: material, design, fabrication, construction, examination, testing, marking, stamping, and preparation of reports for prestressed and reinforced concrete containment. The containment components covered by the ASME B\&PV Code include: (1) structural concrete pressure resisting shells and shell components; (2) shell metallic liners; (3) and penetration liners extending the containment liner through the surrounding shell concrete.

Additionally, the reactor building must be designed for natural phenomena hazards, such as an earthquake, wind, snow loading, and flood, etc. The building is also designed for the loads and fires from an aircraft crash. The design will conform to NRC regulations (Federal Regulations 10 CFR 50 and 10 CFR 100) and applicable NRC Regulatory Guides, for seismic and other natural phenomena and man-made hazards.

\section{II.11.1.2 Penetrations and Transfer Openings}

A large number of penetrations through the reactor building shell are required for access of personnel, equipment, freight, electrical conductors, and service fluids. These penetrations are grouped into three broad classifications: large mechanical penetrations, small mechanical penetrations, and electrical penetrations. These penetrations use pressure-tight seals consisting of appropriate materials. These seals are protected from the building atmosphere since this atmosphere could become hot enough to destroy the seals should a major sodium/air reaction occur. All seals are designed to withstand the same maximum pressure of $10 \mathrm{psig}$ as for the building. To provide adequate assurance that the total leak rate of the reactor containment boundary remains less than the design value of $0.1 \%$ of the free volume per day, selected penetrations are leak tested annually. 


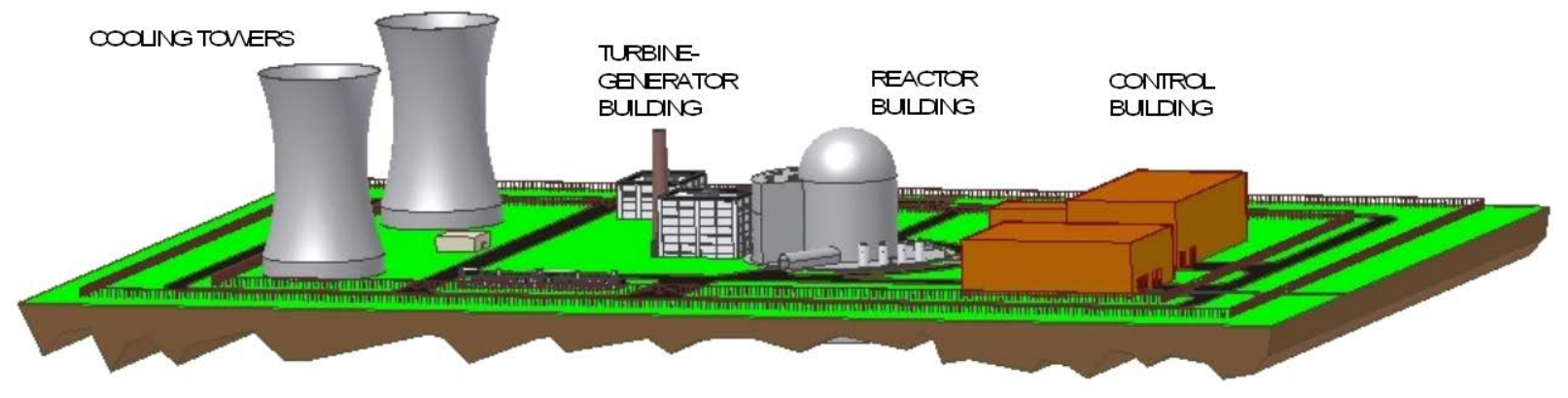

Figure II.11-1 Site Pictorial

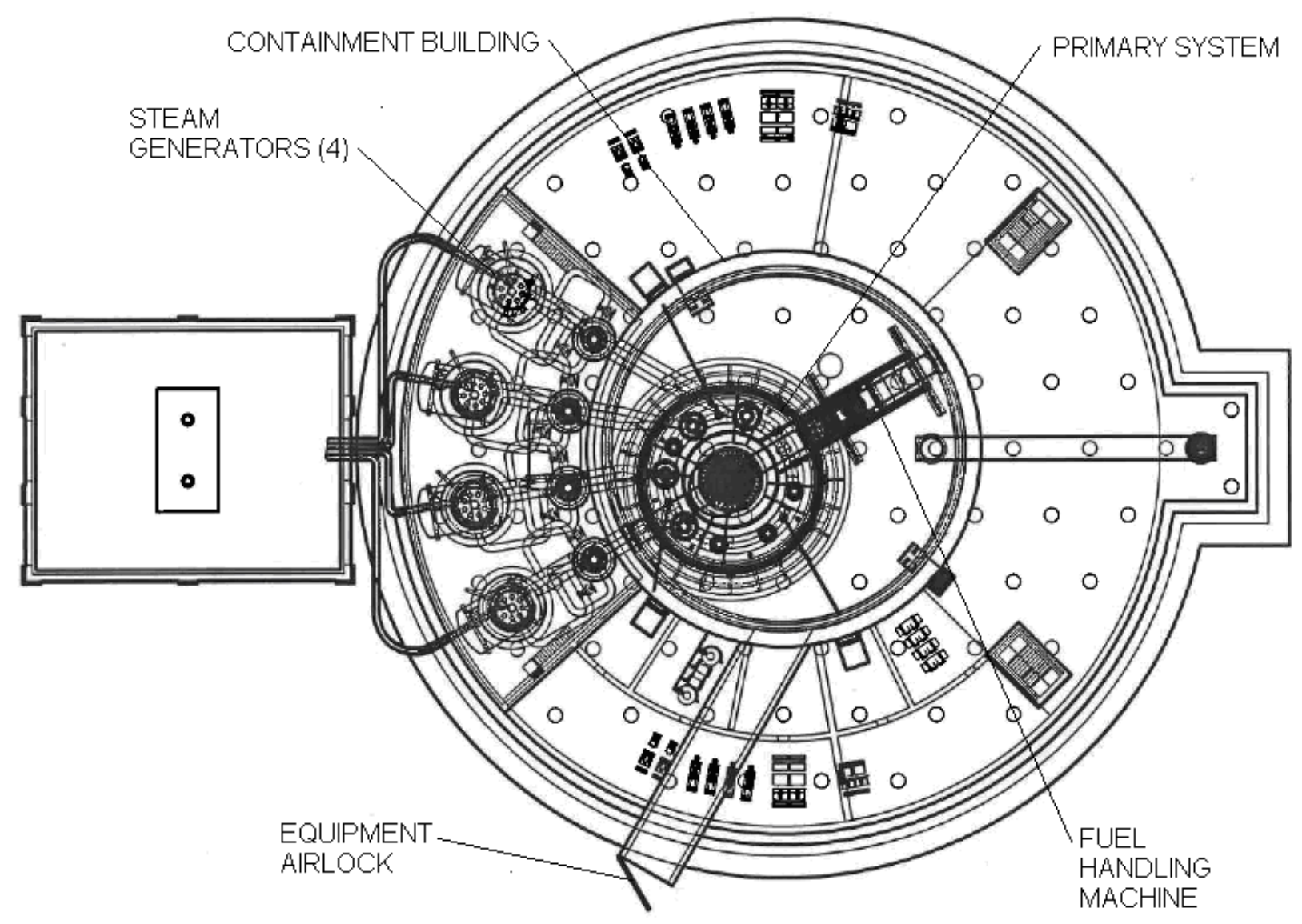

Figure II.11-2(a) Plan View of the Reactor Building 


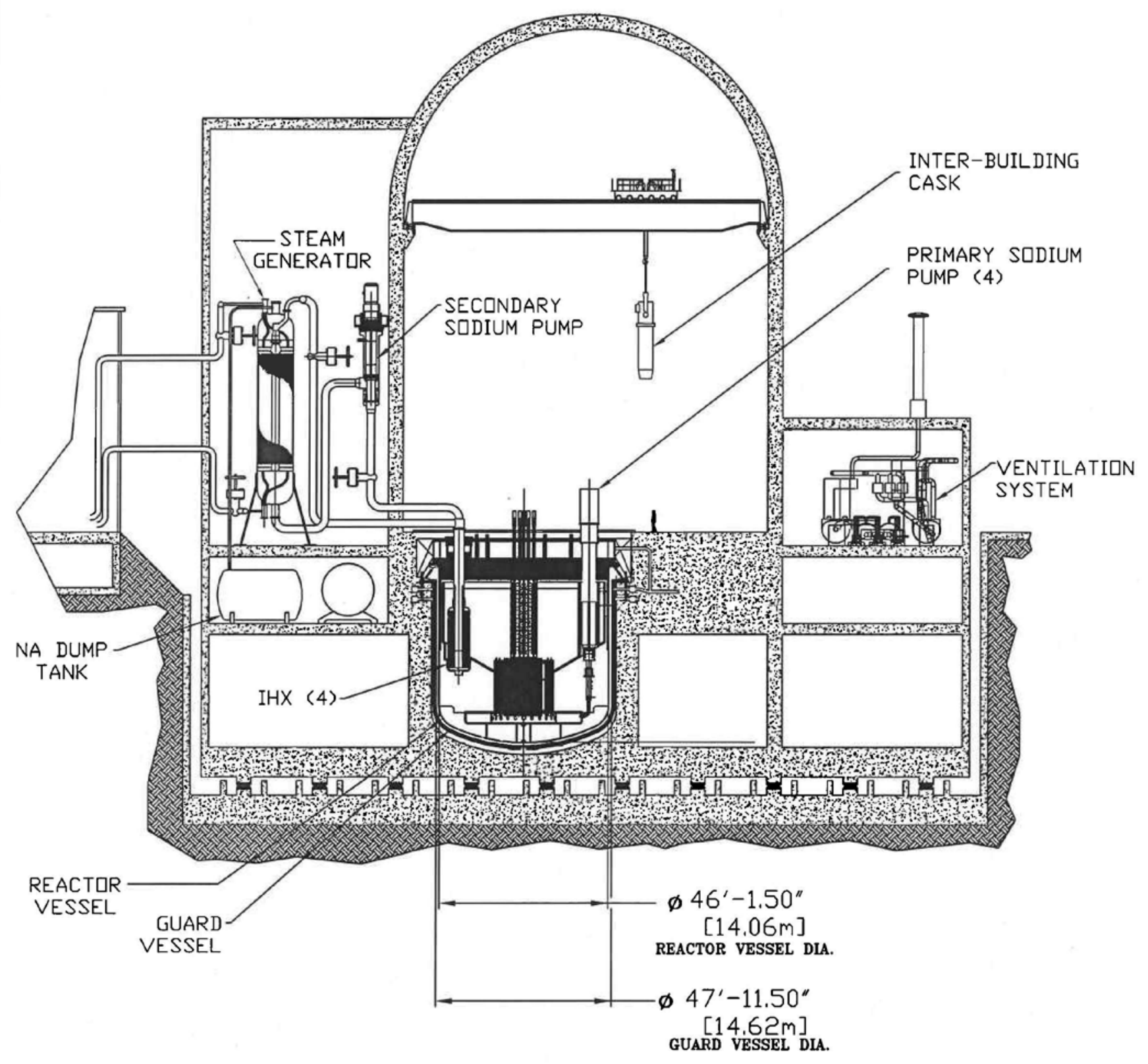

Figure II.11-2(b) Cross Section of the Reactor Building

The large penetrations are comprised of three airlocks (personnel, emergency personnel, and equipment airlocks). The airlocks allow equipment and personnel access to the reactor plant while maintaining building containment integrity at all times. All airlocks are cylindrical steelwelded shells that have a sealed door at each end. The doors are electrically or mechanically interlocked to allow only one door at a time to be opened.

The equipment airlock is the largest of the three; it connects the reactor building to an equipment transfer location outside the facility. The personnel airlock is smaller than the 
equipment airlock; it connects the operating floor area of the reactor plant to the control/personnel service building and serves as the normal personnel entrance and exit.

The emergency airlock is the smallest of the three. It provides an emergency exit from the reactor building should the personnel airlock become blocked. All airlock doors are periodically pressure tested.

\section{II.11.1.3 Cooling Requirements}

The shutdown heat removal system transfers the decay heat from the bulk primary sodium in the reactor vessel directly to the atmosphere through heat exchangers located on the outside of the reactor building. Therefore, there are no unusual cooling requirements for the reactor building internal atmosphere. A standard heating, ventilation and air conditioning (HVAC) system maintains the internal atmosphere around $72^{\circ} \mathrm{F}\left(22^{\circ} \mathrm{C}\right)$ at all times.

\section{II.11.2 Balance of Plant Building System}

The Balance of Plant building is multi-story building that consists of an upper and lower level. The upper level is fabricated on a slab or grate with sheet metal over frame construction and no windows. The building's heating and air conditioning system maintains an ambient temperature for the enclosed equipment and maintains a slightly negative atmosphere pressure relative to outside. This negative pressure ensures that minor steam leaks are contained within the structure. The building also includes an overhead bridge crane with a capacity sufficient to provide maintenance or removal of equipment. The BOP building contains all the plant steam cycle equipment except for the steam generator and the cooling tower equipment.

All ventilation equipment is located adjacent to the building to deliver air for cooling of the generator and ambient temperature control for the building.

The lower level is located below grade. It is sized to contain the main condenser and other auxiliary equipment.

\section{II.11.3 Control Room and Personnel Building}

The reactor control building is a multi-story building adjoining the reactor building. This concrete and steel tornado-hardened, Seismic Category 1 structure, houses the control room, technical support center, and the central computer for the overall plant. It also includes space for switchgears, cable routing rooms, motor-generator sets, heating, ventilating and air conditioning equipment, compressed air and other auxiliary systems. Fire protection/suppression systems are also provided.

\section{II.11.4 Radwaste/Maintenance Building}

The radwaste/maintenance building is a slab-on-grade sheet metal high bay structure that provides two areas, a waste management area and a maintenance area. The waste management area is sized and designed to handle the collection, treatment, staging and shipment for disposal of all regulated wastes generated at the site. Waste will be generated from on-going and periodic maintenance work during the life of the plant. Equipment will exist in this building to condition the waste streams that are expected to be generated from this plant.

The maintenance side of the building provides space and equipment for the routine and planned maintenance of the facility and equipment. The maintenance building also has a location in the structure where large components will be assembled prior to installation in the reactor 
building. A rail spur provides easy access and delivery of components such as the reactor vessel module, primary pumps, intermediate heat exchangers, and steam cycle equipment to the maintenance area during installation and checkout of the primary and secondary systems. After the facility has been constructed, then the maintenance building has space and equipment for performing routine and non-routine maintenance of the reactor primary and secondary systems.

\section{II.11.5 Security Building}

The security building is a single- story reinforced concrete non-seismic category structure located outside of the administration and service building with a reinforced concrete slab located at grade. The windows are made from bullet-proof glass.

The security building provides a controlled means of access to the plant site to prevent inadvertent access, industrial sabotage or the theft of nuclear materials. This facility will be in a remote area with minimal staff so it is essential that there be adequate site security and only one normal site entrance. All personnel must pass through this building and be checked by the associated security systems for ingress and egress to sensitive plant structures/areas or areas where radioactive materials are stored. The plant security system is monitored and operated from this building. A truck trap is located adjacent to this building that allows for security force control and containment of trucks requiring access to the site for deliveries or pickups.

\section{II.11.6 Emergency Generator Building}

A gas or diesel generator building is located adjacent to the reactor control building. It houses a modular 1MWe generator that provides emergency power to the primary and secondary systems upon demand. The emergency generator building is shipped as a single integrated unit that can be quickly installed at that site and made operable to support the construction activities during the facility construction, emergency power during reactor operations, and as an alternative source of power during reactor decommissioning.

\section{II.11.7 Balance of Plant Services Building System}

The balance of plant service building provides space for equipment that supports the Brayton cycle building, cooling towers, and other services. This includes recirculation pumps, water conditioning equipment, air compressors, electrical switchgears, motor control centers, plant heating systems, and other support equipment.

\section{II.11.8 Lift Station and Waste Treatment Plant Building}

The lift station building provides pumps and filtration system to pump water from the river to the plant for use in cooling and domestic water services. All wastewaters go through the wastewater treatment plant where the water is conditioned prior to being discharged into the river.

\section{II.11.9 Seismic Isolation System}

The seismic isolation system is discussed in Section III.1. 


\section{II.12 Balance of Plant Systems}

In general, most of the effort for this report was focused on sizing the nuclear steam supply system components from the reactor core to the steam generator. The balance of plant systems were not size for this reference ABR plant concept. A general discussion follows of the various balance of plant systems that should be located on the reference ABR site. ${ }^{8}$

\section{II.12.1 Compressed Gas System}

The compressed gas system distributes compressed gases throughout the reactor plant from the point of storage or compression to the point of use. Four subsystems comprise this compress gas system. They are 1) Service Air System, 2) Instrument Air System, 3) Hydrogen System, and 4) Carbon Dioxide System.

\section{System Requirements}

The service air system shall provide the following:

- Air for various plant fuel handling systems.

- Air for maintenance systems, unloading devices, tools, miscellaneous cleaning, and inspection services.

- Breathable air for required stations.

The instrument air system shall supply the following:

- Clean dry air to instrumentation, controls, pneumatic piston and diaphragm valve operators, and air locks located in all areas of the plant.

- The service air system.

The hydrogen system shall provide hydrogen gas to the main generator for cooling the stator core and rotating field. The carbon dioxide system shall provide carbon dioxide gas for generator purging.

\section{System Description}

The service air system provides compressed air for use by various plant systems such as the fuel handling system, maintenance systems, unloading devices, tools, miscellaneous cleaning, and inspection services. The system also delivers breathable air to required stations. The service air system receives compressed air from the instrument air system. The service air system is a distribution system of piping and valves that takes compressed air from the instrument air header and distributes it to the users.

The service air system operates at all times providing compressed air during normal plant operation or when the plant is shut down. The service air system is not safety related.

The instrument air system provides clean, oil-free dry air for operation of instruments and controls, pneumatic piston and diaphragm valve operators and air locks in all areas of the plant. The instrument air system operates at all times to provide compressed air during normal plant operation or when the plant is shutdown, since compressed air is required during plant shutdown as well as during normal plant operation.

${ }^{8}$ A major portion of the description of the balance of plant systems were taken from a previous 1000MWe design study prepared at Argonne National Laboratory. 
The instrument air system also supplies the service air system. The instrument air system is not safety related. Safety-related items requiring instrument air are supplied from local safety-class accumulators. The hydrogen system stores and supplies hydrogen gas to the main generator for cooling of the generator stator core and rotor during operation. The hydrogen system is not safety related.

The carbon dioxide system stores carbon dioxide in liquid form, processes the carbon dioxide liquid to carbon dioxide gas, and supplies the gas to the main generator for purging.

The carbon dioxide system is not safety related.

\section{II.12.2 Chilled Water System}

The chilled water system provides chilled water during normal plant operation for air conditioning and removal of heat from the following areas or systems:

- Nuclear island (NI) HVAC and balance of plant (BOP) HVAC system.

- Process cooling in the NI maintenance building.

- Recirculating gas system.

The system includes chillers, pumps, expansion tanks, piping, valves, and related components. A refrigerant pumpout and storage system is also provided for maintenance. The system does not include air cooling coils in air conditioning units or heat transfer equipment in process cooling systems. Cooling water for the chiller condensers is provided by the plant service water system.

\section{System Requirements}

The chilled water system is not safety grade and is only needed for routine plant operation. The total loads have not been evaluated in detail, but based on previous LMFBR designs it is estimated that the total cooling load will be $\sim 4000$ tons of refrigeration. The system will consist of three 2000 ton cross-connected chiller systems. Two of the systems will normally be in operation with one system on standby.

\section{System Description}

The chilled water system consists of a single large central system to serve buildings with both constant and seasonal cooling loads. The system has three centrifugal chillers each of approximately 50 percent total system capacity and connected in parallel so that one chiller is available as a standby. Each chiller has a circulating pump, piping, valves and controls. The normal chilled water system has a single loop with branches to several buildings. The chillers and their accessory equipment are located in the turbine building auxiliary bay. The piping supply loop runs from the turbine building through the control building around the reactor containment building with branches to the buildings served by the system. Branch piping within the buildings is direct return except where long runs would make balancing difficult.

The type of system selected is consistent with large industrial type systems that are commercially available today. Because the reference ABR concept here is a pool type design, the chilled water system size has been greatly reduced compared with a loop plant. The reduced system size results from elimination of the primary heat transport system cell cooling load. 


\section{II.12.3 Essential Chilled Water System}

The essential chilled water system provides chilled water for removal of heat during plant normal and off-normal operation from the following systems:

- Portions of the nuclear island (NI) HVAC system.

- Portions of the reactor service building heat removal system.

\section{System Requirements}

The essential chilled water system shall be designed as two independent closed chilled water loops each with a 100 percent capacity water chiller, heat exchangers, recirculating pump, expansion tank, piping, valves, instrumentation, and controls. The components of one loop shall be separated from the components of the other loop, in accordance with single failure criteria. The essential chilled water chillers shall be designed with a minimum of 25 percent refrigeration margin. The essential chilled water system shall be available during or following all normal, upset, emergency, and faulted events of plant operation.

\section{System Description}

The essential chilled water system supplies and distributes chilled water $\left(-42^{\circ} \mathrm{F}\right)$ to the various components that are required for maintenance of the safe shutdown condition. This system operates during normal and emergency plant operation.

The essential chilled water system has two 100 percent capacity redundant closed loops, each with one mechanical refrigeration centrifugal water chiller, one circulating pump, one expansion tank, and one air separator, complete with interconnecting piping, valves, instrumentation, and controls. Chillers and pumps are located in the auxiliary building east for one system and the auxiliary building west for the redundant system. Piping loops with separation as required are run with branches to the various buildings served. All returns are direct. Temperature control valves are located near each piece of equipment served.

\section{II.12.4 Radioactive Waste System}

The radioactive waste system (RWS) provides the equipment and facilities for collecting, processing, monitoring, storing, and disposing of liquid and solid radioactive wastes. The RWS processes both liquid radioactive wastes and solid radioactive wastes.

\section{System Requirements}

The following functions shall be provided by the RWS.

1. Liquid Radioactive Waste Subsystem (LRWS)

a. Collect radioactive liquid waste for processing, recycle or disposal.

b. Provide sample points and equipment to monitor the chemical and radioactive content of liquid wastes.

c. Process and concentrate radioactive liquid waste.

d. Store processed or reclaimed liquid for reuse or disposal.

e. Control discharges to assure compliance with applicable regulatory standards. 


\section{Solid Radioactive Waste Subsystem (SRWS)}

Radwaste Solidification System - 1) Receive and measure the radiation levels of small low activity level components from other systems and determine temporary storage, processing or disposal requirements. 2) Compact, package, and prepare compactible radioactive solid wastes for disposal.

Radwaste Disposal Management System - 1) Establish the necessary interfaces with other systems for packaging and disposal of large non-compactible radioactive solids. 2) Develop an inventory control system that provides for accountability of radioactive materials.

The following general design requirements shall apply:

- The general system design shall provide as-low-as-reasonably achievable (ALARA) radiation protection for maintenance and plant operating personnel.

- The system shall have the capability to monitor and record discharges to the environment.

- The equipment and its associated supports are to be classified as nonseismic.

- The quality of the discharged water shall comply with the governing water quality standards.

Prior to discharge to the environment, a laboratory analysis of all liquids to be discharged to the environs shall be performed to assure that the activity requirements for such discharges are met. An acid and caustic solution supply, neutralization and $\mathrm{pH}$ control system shall be provided, with the capability to adjust the $\mathrm{pH}$ of all liquid wastes which are collected, prior to further processing.

Receive radioactive sodium generated by the fuel handling and fuel receiving, storage and shipping systems.

Provide control functions for the LRWS and SRWS to maintain the design, operating and performance parameters during all operating modes.

\section{System Description}

The RWS provides the equipment and facilities for collecting, processing, monitoring, storing and disposing of liquid and solid radioactive waste. The RWS consists of two subsystems: (1) the LRWS and (2) the SRWS. The LRWS collects and processes radioactive liquid wastes in order to reduce the volume of radioactive wastes which must ultimately be disposed of. This volume reduction is performed by extracting the solid contaminants from the collected liquids and then concentrating the extracted contaminants. The liquids, less solid content, are processed to produce a purified, decontaminated effluent in accordance with the regulatory requirements for discharge off-site. The SRWS collects and processes both concentrated liquid and solid wastes which are radioactive and prepares these waste substances for storage and disposal.

The RWS facilities are located adjacent within the radwaste/maintenance building. Equipment within the buildings has been located and arranged in a manner which isolates the operating and maintenance galleries from the equipment to achieve as low a level of personnel radiation exposure as is reasonably achievable. 


\section{II.12.5 Nuclear Island Heating, Ventilating, and Air Conditioning System}

The nuclear island (NI) heating, ventilating, and air conditioning (HVAC) system maintains environmental conditions within design limits throughout the noninert areas of the NI buildings, and in conjunction with the radiation monitoring system and plant protection system (PPS) limits the release of radioactive materials from the NI buildings.

System Requirements

The NI HVAC system shall serve the following buildings:

- Reactor Building

- Control Building (CB)

- Steam Generator Building

and shall perform the following functions:

- Maintain the temperature, humidity, pressure, and cleanliness of the air atmospheres in the various building spaces during normal plant operation.

- Maintain, as necessary, the temperature, humidity, pressure, and cleanliness of the air atmospheres in the areas containing safety-related equipment during normal plant operation and during off-normal conditions.

- In conjunction with the radiation monitoring system and PPS, maintain the release of airborne radioactive materials to the outside environment below the acceptable limits during normal operation and off-normal conditions.

- Limit the intake of hazardous airborne materials into certain safety-related and occupied areas, such as the control room.

All safety-related ventilation trains shall consist of two redundant $100 \%$ capacity equipment trains, and designed to Seismic Category I requirements. No single failure of any active component of the safety-related subsystems will result in loss of that subsystem's safety-related functions.

\section{System Description}

The nuclear island HVAC system serves the Reactor Building, Control Building, and Steam Generator Building. The NI HVAC system provides heating, ventilating, and air conditioning for the various non-inerted atmospheric areas of the Nuclear Island buildings during normal and abnormal conditions. The system maintains the temperature, humidity, pressure, and air cleanliness as required. The NI HVAC system, in conjunction with the radiation monitoring system and PPS, limits the release of radioactive materials from the buildings by filtration of exhausted air and/or by isolation of the buildings. 


\section{II.12.6 Balance of Plant (BOP) Heating, Ventilating, and Air Conditioning System}

The BOP HVAC systems provide heat, ventilation, cooling, humidity control, and control of airborne contamination as required for the equipment and activities in the BOP buildings.

\section{System Requirements}

The BOP HVAC systems shall provide heat, ventilation, cooling, humidity control, pressure, and control of airborne contamination or any combination of these functions to the BOP buildings or portions of buildings as required for proper operation of equipment and for personnel comfort.

\section{System Description}

The BOP HVAC system consists of several subsystems serving various areas of the BOP buildings, each performing specialized functions as required for the area served. Each subsystem consists of combinations of various basic components as required to satisfy the performance requirements for that sub-system. These basic components include air handling units, fans or blowers, cooling and heating coils, filters of various efficiencies, duct work, dampers, isolation valves, carbon absorbers, instrumentation and controls, and other accessories as required.

Facilities served by the BOP HVAC System include:

- Turbine Generator Building

- Balance of Plant Services Building

- Radwaste/Maintenance Building

- Cooling Tower Pumphouse

- Fire Protection Pumphouse

- Switchyard Relay House

- Circulating Water Pumphouse

- Gate House

- Emergency Generator Buildings

- Auxiliary Boiler House

- Water Treatment Plant

- Sewage Treatment Plant

- Well Water Pumphouse

\section{II.12.7 Fire Protection System}

The FPS provides the means to detect, and in some cases, anticipate, fires throughout the reactor plant, and to minimize the fire hazard to personnel and equipment.

\section{System Requirements}

The FPS shall provide the means to prevent, control, and mitigate the consequences of plant fires through the following general functions: 
- Detect fires, or incipient fires, activate alarms, suppress fires, extinguish fires, isolate and confine fires, protect personnel from smoke and heat and protect safety-related systems and components to assure continued readiness and operation.

The FPS shall be comprised of two subsystems: the sodium fire protection system (SFPS), which addresses sodium or sodium-potassium fires; and the non-sodium fire protection system (NSFPS), which addresses fires of non-alkali metal origin or involvement.

\section{System Description}

The SFPS provides the means for detecting, locating, confining, suppressing, and extinguishing sodium and $\mathrm{NaK}$ fires. Special requirements imposed by the presence of sodium and NaK in the plant result in employing special fire protection methods in the SFPS. These methods include passive catch pans and fire-suppression decks, and manually operated, portable fire extinguishers.

Fire detectors and associated instrumentation are used to inform the plant operator of the existence and location of a fire. The effectiveness of these fire protection methods for limiting fire losses and the spread of airborne contaminants is augmented by fire barriers, fire doors, fire dampers, low-leakage penetrations, and similar isolation devices provided by the building design, and by the heating and ventilation system.

The fire protection water supply system provides water at the design pressure and quantity to the sprinkler, deluge, and water spray systems, and the yard hydrants. The water storage tanks, fire pumps, and hydrants are all nonseismic Category I components. One (1) seismically qualified (Seismic Category I) pump, taking its suction from two (2) seismically qualified (Seismic Category I) storage tanks, provides water at the design pressure and quantity to special service wet standpipes located in buildings and areas of the plant containing systems, equipment and components essential for reactor safe shutdown.

\section{II.12.8 Recirculating Gas Cooling System}

The recirculating gas cooling system (RGCS) provides gas cooling capability to the inerted fuel handling machine - fuel transfer tubes system in the reactor building and to the fuel handling cell in the fuel handling facility. The RGCS provides heat removal from cell and equipment by forced circulation in closed loops.

System Requirements

The RGCS shall provide the following function and requirements:

- Recirculate inert atmospheres through cooling units to remove heat and maintain the required atmospheric temperature.

- Limit potential release of radioactive materials to the environment.

- Provide a means to inert and deinert.

- Condition recirculated air to maintain the required atmospheric air temperature during inspection and maintenance operations.

- Monitor the performance of each subsystem in maintaining the design operating conditions.

- In the event of a sodium or NaK spill or leak, means shall be provided to isolate the affected portion of the system. 
- Those safety-related cooling circuits shall consist of two-100 percent capacity equipment trains that are redundant, separated from each other, and are designed to Seismic Category I requirements. No single failure of any active component of a safety-related cooling circuit shall result in loss of the cooling.

\section{System Description}

The RGCS provides heat removal from the inert gas atmospheres of the following cells and equipment.

- Fuel handling and storage cells in the Fuel Handling Facility.

- Fuel handling machine in the Reactor Building and fuel handling tubes in the Reactor Building and Fuel Handling Facility.

Each RGCS subsystem acts as an extension of the cell pressure and leakage boundary and thereby limits potential release of radioactive materials to the environment. Connections are provided to the Inert Gas System for pressure control and gas sampling and to the nuclear island heating, ventilating and air conditioning system for de-inerting air supply and exhaust. The RGCS is capable of recirculating and cooling cell atmosphere air during periods when the cells are de-inerted and occupied.

The fuel handling and storage cells subsystem operates to maintain temperatures within the cell. Heat loads include decay heat from fuel assemblies and lighting loads. Cell temperatures are also maintained within design limits in the event of a design basis fuel handling accident. This subsystem together with the cell liner and penetrations seals, maintains a very low leakage barrier to contain the products of a design basis accidental release of radioactive materials.

The RGCS System for this pool plant will be smaller in size than for a loop plant. The principal reason for this reduction in size is the elimination of the primary heat transport system cells and the use of an air atmosphere in the reactor vessel cavities and in some other cells.

\section{II.12.9 Fuel Receiving, Storage, and Shipping System}

The fuel receiving, storage, and shipping system (FRSSS) provides for the receiving and unloading of new core assemblies, inspection, temporary storage, provision for inventory control of all core assemblies, examination of irradiated core assemblies, and preparation and loading of irradiated core assemblies for shipment off-site. Also provided is the instrumentation and control system that is used to operate the FRSSS and the fuel handling system. This system interfaces closely with the fuel handling system, the fuel handling facility, and the liquid metal auxiliary system.

\section{System Requirements}

The primary requirements of the fuel receiving, storage, and shipping system (FRSSS) are as follows:

- Receive, inspect, store, and prepare new core assemblies for insertion into the reactor. Core assemblies are defined as fuel blanket, control, and radial shield assemblies.

- Make selected measurements on irradiated core assemblies and prepare irradiated core assemblies for shipment to an off-site location.

- Provide inventory control for all core assemblies at all times within the plant.

- During transfer operations, the FRSSS shall be capable of cooling fuel assemblies for an indefinite period. 
- Total storage capacity in air cell shall be about 1200 positions.

- A fuel handling cell (FHC) shall provide for preparation of irradiated core assemblies for shipment.

\section{System Description}

The fuel receiving, storage, and shipping system (FRSSS) provides the means of receiving, storing, inspecting, and shipping reactor core assemblies, including fuel, blanket, control, and radial shield assemblies, within the reactor facility. The FRSSS also assists in the refueling of the reactor. The FRSSS is located in the Fuel Handling Facility. New core assemblies enter the fuel handling facility, are unloaded from their shipping containers and inspected, and are temporarily stored in the FHC. See fuel handing section for more details on refueling operations.

\section{II.12.10 Feedwater and Condensate System}

The feedwater and condensate system collects water from the main turbine and auxiliaries, where its available thermal energy has been extracted, conditions it, and returns. it to the steam generator at design temperature and pressure. The feedwater and condensate system is not safety grade.

\section{System Requirements}

- The feedwater and condensate system shall perform the following major functions:

- Provide conditioned feedwater at design temperature, pressure, and flow rate to steam generators for startup.

- Provide preheated, deaerated, demineralized, and pressurized feedwater at the required flow rate for partial- and full-power operation.

- Store varying quantities of condensate to provide operating flexibility.

- Provide redundancy as required to achieve an acceptable level of plant reliability.

\section{Description}

The feedwater and condensate system provides high purity feedwater to the steam generators. Condensate from the hotwell is delivered via the condensate pumps to the deaerating heater after being processed by the condensate polishing subsystem and the low-pressure heaters. The deaerating heater storage tanks provide the source of feedwater for the main feedwater pumps. The main feed pumps take the fluid, increase the pressure, pump it through the high-pressure heater shells to increase the temperature to the specified level, and deliver it to the steam generator system where it is converted to superheated steam. Some steam is returned to the feedwater heaters via the extraction steam subsystem, for heating condensate and feedwater, and to the main feedwater pump turbine drives. The exhaust from these turbines, along with the cascaded drains from the feedwater low- and high-pressure heaters, is returned to the main condenser. All heater drains are cascaded to the condenser to ensure that feedwater chemistry specifications are met.

Because the steam generators are not required for shutdown heat removal, emergency feedwater pumps, protected condensate storage, and attendant safety-grade components, structures, and buildings are not required. 


\section{II.12.11 Main and Auxiliary Steam System}

The main and auxiliary steam system transports the steam produced by the steam generators to its point of use and extracts its available thermal energy, primarily in the main turbine, but also in auxiliary equipment. Included within this system are those systems that are needed to support the operation of the main turbine generator.

\section{System Requirements}

The main and auxiliary steam system, which is not safety grade, shall perform the following functions:

- Transport the steam from the steam generators to its point of use.

- Convert thermal energy to electrical energy in a turbine generator.

- Provide turbine generator auxiliary systems including:

o lube oil system

o hydrogen seal oil system

o gland steam system

o stator winding cooling water system

o electrohydraulic control system

o high pressure hydraulic system

- Provide steam to steam-operated auxiliaries such as pumps, feedwater heaters, air ejectors, etc.

- Provide steam for plant requirements such as space and process heating.

- Provide capability to dump steam directly to the main condenser.

\section{System Description}

The main steam system takes live steam from the steam generator system and transports it to the high pressure turbine of the turbine generator. The system includes provisions for steam dump to the condenser following a turbine generator load rejection or turbine trip to prevent a reactor trip. The steam dump also provides a flow path for main steam during plant startup until the steam reaches conditions or quality required for other uses. The steam dump portion of this system takes steam from the manifold, bypasses the turbine, and dumps the steam into the condenser. The steam dump arrangement is a part of the turbine bypass system. The steam dump system includes desuperheaters and steam dump control valves to desuperheat the steam and control the flow of steam into the condenser in accordance with the control of the reactor.

The extraction steam system conducts steam from the high pressure, the intermediate pressure, and the low pressure turbines to the reheat tube bundles in the moisture separator/reheaters and the feedwater heaters. The extraction steam system also supplies steam to the auxiliary steam system. The extraction steam is required for feedwater heating to increase cycle efficiency.

The system also removes moisture from the turbine to provide water erosion protection for the low pressure turbine blades.

The turbine-generator converts the thermal energy generated in the reactor to electrical energy. The turbine is provided with two moisture separator/reheaters with two stages of reheat in each moisture separator/ reheater unit. The generator is a liquid-cooled stator type with a capability of 
400 MWe gross at rated turbine conditions of inlet steam at $\sim 2,200$ psig and $850^{\circ} \mathrm{F}$ exhausting at 1.6 in. of mercury absolute mean effective exhaust pressure with zero makeup and five stages of extraction.

The turbine generator auxiliary systems provide supportive services to the turbine-generator to provide needed cooling, sealing, lubricating, and control functions to sustain the operation and assure the maximum efficiency of the turbine generator.

The auxiliary steam system provides steam for various process uses and heating throughout the plant during plant startup, normal plant operation, and plant shutdown. The auxiliary steam system receives its steam from one of three sources. During normal operation, the steam is supplied from the extraction steam system through a pressure reducing valve. When the extraction steam pressure is too low, auxiliary steam is supplied from the main steam system through a pressure reducing valve. If neither extraction steam nor main steam is available, auxiliary steam is supplied by the auxiliary boiler.

\section{II.12.12 Circulating Water System}

The circulating water system provides cooling water flow to the main condenser and the turbine plant component cooling water heat exchangers to remove waste heat rejected by the turbine-generator cycle. The circulating water rejects this waste heat to the atmosphere by means of mechanical draft cooling towers.

\section{System Requirements}

- The circulating water system shall be capable of removing the design heat load placed on the turbine condenser. The system shall also remove the heat load rejected through the turbine plant component cooling water heat exchangers.

- The circulating water system shall be capable of removing the heat load from a 50 percent steam dump occurring at summer design condition.

The safety classification of the circulating water system piping, equipment, and components shall be nonsafety. Provisions shall be made to isolate portions of the system for major maintenance without removing the generating unit from operation.

\section{System Design}

The circulating water system provides cooling water for the main con-denser and the turbine plant component cooling water heat exchangers. Circulating water pumps in separate bays in a pump house take suction from a flume connecting the cold water basins of the mechanical draft cooling towers. Cooled water is pumped through a pipeline to the main condenser and back to the cooling towers. Turbine plant component cooling water pumps, in a separate bay, pump water through a pipeline to the turbine plant heat exchangers and discharge into the circulating water pipe downstream of the main condenser.

\section{II.12.13 Service Water System}

The service water system consists of three subsystems, namely: the turbine plant water, the nuclear island service water, and the turbine plant component cooling water. All three of these subsystems are closed loop cooling systems and in total their function is to provide cooling for all plant systems and components except the main turbine condenser. With the exception of the nuclear island service water subsystem, the heat load is rejected to the recirculating water system. The nuclear island service water subsystem rejects heat to two separate cooling towers. 


\section{$\underline{\text { System Requirements }}$}

- The turbine plant water and turbine plant component cooling water subsystems are nonsafety grade.

- The NI service water subsystem is safety grade.

- It shall be possible to isolate portions of the system for major maintenance without removing the generating unit from operation.

- The NI service water subsystem shall be designed to meet the single failure criteria.

\section{System Design}

It is expected, based upon prior concepts, that the service water flowrate of approximately 5,000 gpm will suffice for a 1000MWth pool-concept plant. A loop concept would require a high flowrate to accommodate primary heat transport system cell cooling.

\section{II.12.14 Treated Water System}

The treated water system receives raw water from the well water system and supplies various levels of treated water for the entire plant needs. The principle plant water needs are: (1) makeup water for the main cooling towers, (2) demineralized water, and (3) potable water.

\section{System Requirements}

To provide water at the appropriate specifications to the following systems and components:

1. Potable water

2. Fire Protection Water Demineralized Water Condensate

3. Makeup

4. Close Cooling Systems Makeup

5. Auxiliary Boiler Makeup

6. Turbine Component Cooling Makeup

7. Sodium Cleanup System Decontamination Facility

The treated water system is not safety grade.

\section{System Design}

This system consists of piping, tanks, valves, instrumentation, and controls necessary to supply the quantity and quality of water to operate, maintain, and protect the plant.

\section{II.12.15 Industrial Waste Water Treatment System}

The waste water treatment system collects, treats, and disposes of all nonradioactive liquid plant wastes originating from the plant floor drain system, lube oil storage area drains, auxiliary boiler, chemical storage area drains, the makeup water treatment system, the condensate polishing system, and sanitary waste system.

\section{System Requirements}

- This system is not safety grade.

- The system shall receive, handle, and treat the following liquid wastes:

o All demineralizer regeneration waste water 
o M/U gravity mixed media filter backwash

o $\quad \mathrm{M} / \mathrm{U}$ activated carbon filter backwash

o All plant floor drains

o Cooling coil drainage

o $\mathrm{M} / \mathrm{U}$ clarifier blowdown

o Auxiliary boiler blowdown

o All plant sewage during plant construction and operating lifetime.

\section{System Design}

The system consists of a system of piping, valves, storage tanks, polishers, and component for treating the industrial wastewater generated on the ABR site

\section{II.12.16 Liquid Metal Auxiliaries System}

The liquid metal auxiliaries system (LMAS) consists of the following:

1. Sodium and NaK Receiving System - Provides for receipt, transfer to storage, and unloading of plant liquid metals.

2. Primary Auxiliary Liquid Metal System - Provides reactor sodium purification and facilities for EVST sodium storage and draining.

3. EVST Auxiliary Liquid Metal System - Provides EVST sodium purification and cooling for fuel stored in the EVST.

4. Intermediate Auxiliary Liquid Metal System - Provides purification of intermediate sodium.

\section{System Requirements}

The liquid metal auxiliaries system shall be designed to meet the following general requirements:

- Receives, melts, and transfers to storage all sodium delivered to the site.

- Receives, stores, purifies, and distributes all NaK.

- Provides the pumps and piping to transfer sodium and NaK.

- Provides the pumps and piping to fill and drain all sodium and NaK systems.

- Separate, independent, purification capability shall be provided, as necessary, for the primary sodium and for the sodium in each of the four intermediate heat transport system loops. There shall be no permanent connection between primary sodium components and intermediate sodium components.

\section{System Description}

The various subsystems of the liquid metal auxiliaries system are described in the following sections:

- Sodium and NaK Receiving System - The sodium and NaK receiving system receives all sodium and $\mathrm{NaK}$ needed by the plant. The sodium receiving system provides the capability to melt the contents of a sodium tank car, or sodium drum, and transfer it to the system to be filled. The capability to transfer $\mathrm{NaK}$ from drums to storage vessels 
and to circulate and purify NaK during system loading and cleanup is also provided by the system.

- $\quad$ Primary Auxiliary Liquid Metal System - The primary auxiliary liquid metal system provides purification (cold trapping) for sodium used in the reactor vessel and the primary sodium plugging temperature indicator (PTI) system and primary sodium sampling system. Two NaK-cooled, primary sodium cold traps are located in the reactor building below grade hot cell. An EM pump is located between the shield plug and the cold trap in each unit. During normal plant operation, one trap is in use and the second is on standby.

- Intermediate Auxiliary Liquid Metal System - The intermediate auxiliary liquid metal system provides for purification of the intermediate sodium in each of the intermediate loops. Capabilities exist to check for impurities in the intermediate sodium. Two NaK-cooled, sodium cold traps are located in the steam generator building below grade. An EM pump is used for circulating sodium in the sodium purification system. During normal plant operation, one trap is in use and the second is on standby.

\section{II.12.17 Inert Gas Receiving and Processing System}

The inert gas receiving and processing system (IGRPS) provides inert gases and vacuum, as required by other systems of the Reference ABR, including: (1) cover gas, (2) cell-inerting atmospheres, (3) valve actuation gas in inerted cells, (4) cooling gas, (5) gas for certain seals, for component cleaning, and other services, and (6) vacuum for liquid metal transfer and gas analysis purposes.

The system consists of five subsystems, as follows:

1. Helium Gas Distribution Subsystem

2. Argon Gas Distribution Subsystem

3. Nitrogen Gas Distribution Subsystem

4. Radioactive Helium Processing Subsystem (RHPS)

\section{System Requirements}

The IGRPS shall meet the following general requirements:

- $\quad$ Receive, store, transfer, and distribute inert gas used on the site.

- Provide cover gas for the intermediate heat transport system (IHTS).

- Provide inert gas to the fuel transfer machine (FTM) and fuel transfer tubes

- Provide cover gas for the reactor shutdown heat removal systems.

- Provide a nitrogen atmosphere for the space between the reactor vessel its guard vessel.

- Process all vented gas prior to reuse in the plant or release to the environment.

Those portions of the IGRPS classified safety grade are the piping, vapor traps, and the first two valves which form a part of the reactor vessel boundary or the fuel storage tank boundary. 


\section{System Description}

The IGRPS is composed of the following subsystems:

- The radioactive argon gas distribution subsystem, which distributes fresh and recycled argon principally to the reactor vessel cover gas space and portions of the fuel handling system.

- The intermediate argon gas distribution subsystem, which distributes fresh argon mainly to the cover gas spaces of the IHTS.

- The nitrogen gas distribution subsystem, which primarily distributes nitrogen to the inerted spaces between the reactor vessel and its guard vessel.

Radioactive Argon Gas Distribution Subsystem - The radioactive argon gas distribution subsystem is composed of high pressure storage vessels (tube banks), pressure regulating valves, stop valves, piping, emergency gas bottles, vapor traps, filters, and relief systems. All the cover gas cell and equipment spaces that require argon use recycled argon to minimize consumption. The level of radioactivity in the recycled argon gas is reduced by the RHPS to a level such that it will not cause a radiation hazard.

Intermediate Argon Gas Distribution Subsystem - The intermediate argon gas distribution subsystem is composed of liquid argon storage tanks, pressure control valves, stop valves, piping, vapor traps, filters, and relief systems. Argon is distributed to the Steam Generator building for use as a cover gas in the intermediate heat transport system (IHTS), IHTS cold traps, and SWRPS rupture disc areas. Argon is distributed to the auxiliary buildings for use as the cover gas for DRACS NaK expansion and drain vessels. The fuel handling facility is supplied with argon gas for various fuel handling system uses.

Nitrogen Gas Distribution Subsystem - The nitrogen gas distribution subsystem is composed of liquid nitrogen storage tanks, vaporizers, pressure control valves, stop valves, piping, filters, and relief systems. Nitrogen gas is used for inerting the annulus between the Reactor Vessel and its guard vessel. It is also used for steam generator water-side purging.

Radioactive Argon Processing Subsystem - The radioactive argon processing subsystem is composed of a vacuum vessel, vacuum compressors, a surge vessel, charcoal absorber vessels, piping, pressure control valves, stop valves and particulate filters. It is located in shielded cells in the lower level of the reactor building. The RHPS cleans up the argon gas that is normally vented from the reactor cover gas space and the FTM and FHC such that the gas can be safely recycled with an acceptable radiation hazard to personnel external to the recycled helium piping. 


\section{II.13 Electrical Power Systems}

\section{II.13.1 Power Transmission System}

The power transmission system provides the electrical interties for the control and distribution of power between the off-site power system and the station service AC power systems. The generating station can receive AC power from the main generator; from off-site backfeed through the main generator step-up transformers; or from off-site ties through reserve station service transformers.

The power transmission system provides the means for the control and transmission of generator output power to the utility grid through the main generating step-up transformers and the station switchyards.

\section{System Requirements}

The system shall be designed so that power can be supplied from the generator output during normal operation, or from the off-site power sources during startup and shutdown, or during maintenance or repair of parts of the normal system. An automatic transfer scheme shall be used to transfer from the unit station service transformer to the off-site source for certain loss of power events. The preferred transfer will be to the off-site supply feed through the generator step-up transformers. If this latter supply is lost or unavailable, station service system will automatically transfer to the reserve power supply. The generator load break switch shall be capable of full load current circuit interruption and fault current circuit interruption. The power transmission system is a nonsafety-grade system and is classified as Quality Assurance Group D in accordance with Regulatory Guide 1.26.

\section{System Description}

The power transmission system supplies three alternate power connections for the plant station service load. A reserve supply is provided from the off-site system, via two three-winding reserve station service transformers, each $161 \mathrm{kV}$ to $13.8 \mathrm{kV}-4.16 \mathrm{kV}$. The two reserve sources are developed in a $161 \mathrm{kV}$ switchyard. Each reserve source connection from the switchyard is installed in a duct bank or in a concrete lined trench, set in a sand filled earthen furrow, and bridged with precast concrete covers designed with drainage provisions, and protected along roadways, access ways, and removed from major building foundations. Either of these installation methods offers accessibility and protection and is able to accommodate future circuits. Separation of duct banks (or trenches) is required for each reserve source connection (i.e., each has a dedicated installation). Within a duct bank (or trench), control circuits are specified as separate from power circuits. The electrical power interface of the Class IE AC power systems and the power transmission system is located at the Class IE $4 \mathrm{kV}$ switchgear breaker terminals.

\section{II.13.2 Station Power System}

The station power system receives power from the power transmission system, provides standby AC and DC power supplies, and distributes power to all building and equipment loads of the power station. It also provides the plant grounding grids for safety grounding of electrical circuits and building structures. The electrical load of this system is commonly called "the hotel load." 


\section{$\underline{\text { System Requirements }}$}

- The station power system shall receive power from the power transmission system and provide the transformers and associated switching and control equipment necessary to supply the maximum design station electrical power load.

- The station power system shall provide standby AC and DC electrical power capacity sufficient to supply essential loads upon complete loss of power at the interfaces between the power transmission system and the station power system.

- Those portions of the Station Power System that provide power to Class IE power systems as defined in IEEE Standard 308 shall meet the requirements of this Standard.

- The interfaces between the Station Power System and the systems that receive power from the Station Power System shall be established by interface agreements between the responsible design organizations. These agreements shall be prepared with due consideration for minimizing fragmentation of design and construction responsibility. Similar interface agreements shall be prepared for connection of structures and equipment to the plant grounding grids.

\section{System Description}

The station power system provides electrical power to all station loads, grounding for electrically energized equipment and plant structures, and lightning protection for the plant. This system consists of standard industrial power plant type equipment necessary to distribute power to the loads. This equipment includes medium voltage and low voltage switchgear buses, medium voltage to low voltage step-down unit substations, distribution transformers and panels, station batteries, and standby turbine-generator units. The station power system is designed to supply the plant loads at the appropriate utilization voltages. The nominal bus voltages selected are:

1. $13.8 \mathrm{kVAC}$, nominal, 3-phase, 3-wire, $60 \mathrm{~Hz}$ - Power supply for very large motordriven equipment rated $13.2 \mathrm{kVAC}$, resistive loads, and unit substation transformers with transformer primary rated $13.8 \mathrm{kVAC}$ nominal.

2. $4.16 \mathrm{kVAC}$, nominal, 3-phase, 3-wire, $60 \mathrm{~Hz}$ - power supply for large motor-driven equipment rated $4.0 \mathrm{kVAC}$ and unit substation trans-formers with transformer primary rated $4.16 \mathrm{kVAC}$ nominal.

3. $480 \mathrm{Y} / 277 \mathrm{VAC}$, nominal, 3-phase, 4-wire, $60 \mathrm{~Hz}$ - power supply for heat tracing control panels and plant lighting.

4. $480 \mathrm{VAC}$, nominal, 3-phase, 3-wire, $60 \mathrm{~Hz}$ - power supply for small motor-driven equipment, lighting, heating loads, and welders.

5. 120/208 VAC, nominal, 3-phase, 4-wire, $60 \mathrm{~Hz}$ - power supply for control systems, instrumentation, lighting, communications, and small (generally fractional horsepower) motor-driven equipment.

6. 250 VDC, nominal 2-wire - power supply for DC motor-driven equipment.

7. 125 VDC, nominal 2-wire - power supply for control systems, instrumentation and annunciation. 
8. 120/208 VAC nominal 3-phase, 4-wire, $60 \mathrm{~Hz}$, uninterruptibel power supply for control and instrumentation which must be continuously energized under all plant operating modes.

The station power system is radial in configuration, that is, there are no bus ties, double-ended substations, or primary selector switches. Each medium voltage bus $(13.8 \mathrm{kV}$ and $4.16 \mathrm{kV}$ nominal) has at least two transferable incoming supplies. One supply is from one of the two Unit station service transformers (USST); the second supply is from one of the two reserve transformers. Power can be supplied to the $13.8 \mathrm{kV}$ and $4.16 \mathrm{kV}$ buses via the USSTs from the main turbine-generator (Plant Power Supply) with the generator circuit breaker closed, or from an off-site power source via the generating switchyard and main transformer with the generator circuit breaker open or from the reserve transformer. The off-site power via the generating switchyard is considered the preferred power supply, because it serves as the first access to the utility power transmission grid upon plant trip and is consistent with utility terminology.

The station power system delivers the maximum total continuous plant power requirements from the plant, preferred or reserve power supplies. All 480 VAC nominal unit substations (USS) are fed from medium voltage buses through unit substation transformers; and 480 VAC nominal motor control centers (MCC) are fed from 480 VAC USS. Voltage at USS and MCC buses is adjusted by no-load tap changers at the USS transformers to ensure acceptable voltage regulation.

The 120/208 VAC and 277/480 VAC nominal power and lighting panels are fed from the 480 VAC nominal motor control centers through dry type distribution transformers. Voltage at the power and lighting panels is adjusted by no-load tap changers at the distribution transformers to ensure acceptable voltage regulation.

The standby AC power system is divided into two load groups known as Division 1 and 2 that are powered from two gas turbine-generator units. One generator unit feeds Division 1 and one generator unit feeds Division 2. Division 1 and 2 are physically and electrically independent power systems. The gas turbine-generator units are housed in separate buildings as shown on Fig. II.9-1, and the buildings and equipment are Seismic Category I and Safety Grade.

Standard LWR practice is to use diesel-generator units for on-site standby power. Gas turbinedriven generators are an acceptable alternative for a pool-type LMFBR because the large thermal capacity in the sodium pool eliminates the fast starting requirements of the LWR plants. Tests conducted on sodium-cooled fast-reactor plants in the United States and in Europe have demonstrated that properly designed systems can safely withstand loss of normal power without operation of standby generators for extended periods of time. One standby generator has the capacity to supply the Class IE and other essential loads if one generator is inoperative during the loss of off-site power. The gas turbine-generator units and buildings shown on Fig. II.9-1 are the same as a unit recently installed at the Fast Flux Test Facility.

A past loop designs for a 1,000 MWe plant requires about 80 MWe of hotel power. Although a detailed system load analysis has not yet been made, reductions in primary system pumping power, elimination of the recirculating gas system for the primary heat transport system, reductions in HVAC system size and other attendant reductions suggest that the potential exists for reducing the hotel load for the ABR pool-type plant significantly. For purposes of comparison, Phenix a 254 MWe plant has a hotel load of 16 MWe. 


\section{II.13.3 Sodium Piping and Equipment Heating and Insulation System}

The sodium piping and equipment heating and insulation system provides the piping and equipment insulation and insulation hardware, electrical heaters, the heater mounting devices, the electrical power controllers and the temperature sensors, required to insulate and heat the sodium or the NaK containing components of the following process systems:

1. Reactor Enclosure System

2. Fuel Receiving, Storage, and Shipping System

3. Intermediate Heat Transport System

4. Direct Reactor Auxiliary Cooling Systems

5. Steam Generator System

6. Liquid Metals Auxiliaries System

7. Inert Gas System

8. Impurity Monitoring and Analysis System

This system also provides a heating system for preheating the reactor vessel prior to sodium loading.

\section{System Requirements}

The sodium piping and equipment heating and insulation system provides the following heating and insulation functions for those process systems that contain sodium or NaK:

- Preheat the sodium process systems solid metal parts, before the systems are filled with liquid metal sodium, from ambient conditions up to $450^{\circ} \mathrm{F}$.

- Heat the sodium process systems from plant extended shutdown conditions to reactor startup conditions, with the process systems filled with sodium.

- The piping and equipment electrical heating system shall provide controlled heat to melt sequentially the frozen sodium.

- Insulate to limit piping and equipment heat losses to the building and maintain insulation surface temperatures at or below $140^{\circ} \mathrm{F}$.

The sodium piping and equipment heating and insulation system is nonsafety grade.

\section{System Description}

Electrical power is used for the piping and equipment heaters. The heating cable is either wrapped around the component or piping or placed in zig-zag pattern on the surface of the component. The heat rate required by different components is controlled by thermocouples which monitor piping and component temperatures and adjust the power supplied to the heaters.

The insulation system consists of an inner jacket of stainless steel, insulation layer of either mineral or reflective insulation depending on the operating temperature, and an outer protective jacket of stainless steel. For smaller piping or components, the heaters are wrapped around the component. For tanks and large components, the heaters are arranged in banks.

Because the primary pump guard vessels, IHX guard vessels, primary piping check valves, overflow vessel and primary piping are eliminated, the system size will be approximately $60 \%$ of a comparable loop plant design. 


\section{Part III \\ Detailed Design and Engineering Analyses}

\section{III.1 Seismic Isolation}

When an earthquake occurs, the seismic waves travel through the ground where the reactor is supported causing the reactor structures to shake back and forth along with the ground. For this reason, reactor structures must be designed to sustain the inertia force generated by the vibration. The earthquake motion obviously depends on the characteristics of the site where the reactor is located. This makes the seismic design of the reactor site-dependent. One of ABR design requirements is to modularize the reactor construction to reduce capital costs. In other words, the reactor structure design has to be standardized regardless of seismic conditions. To make this standardization feasible, the site specific seismic design has to be decoupled from the structural design. This can be achieved by incorporating the base isolation into the reactor structure. These base isolators can be tuned (adjusting its period of vibration) so that the reactor structure will experience about the same magnitude of the seismic loading irrespective the site.

The fundamental principle of base isolation is to provide a layer with low horizontal stiffness between the structure and the foundation so that the structure is decoupled from the horizontal components of the earthquake motion. This layer gives the reactor structure a fundamental frequency that is much lower than that of the same reactor structure without this soft layer. Also, this fundamental frequency is much lower than the predominate frequencies of the ground motion. As a result, the ground motion transmitted into the structure is modified (the isolator functions as a low pass filter). The high frequency contents in the ground motion will be filtered out along with the high energy associated with these frequencies. Since the base isolator has low horizontal stiffness, a large horizontal displacement will take place during the earthquake. This large displacement needs to be accommodated in the structural design; therefore, in ABR a trench with a seismic gap of $90 \mathrm{~cm}$ is built around the reactor structure.

There are three technical challenges for designing a base isolation system for ABR. These challenges are listed below.

1. The footprint of ABR reactor structure is small. Therefore, the number of base isolators that can be installed is limited, and the size of the base isolator is also limited by the footprint. As a result, the load carried by each base isolator is high. The preliminary design of ABR indicates that this load is about 600 metric tons. If a safety factor of 1.5 is considered, 900 metric tons would be the design load for each isolator.

2. The ABR site will be used in a remote rural area where the weather temperature may be below freezing during winter. The mechanical properties of the isolator must be stable during the temperature change.

3. The weight of the ABR reactor structure is not a constant during the whole life of the ABR. The fundamental natural frequency of the base isolator system must be insensitive to this weight change. 
Seismic isolators are classified as either elastomeric or sliding. Elastomeric isolators include high-damping rubber bearings (HDR), low-damping rubber bearings (RB) or low-damping rubber bearings with a lead core (LRB). Sliding isolators include flat assemblies, the frictionpendulum system (FPS) or the multiple friction-pendulum system (MFPS). The difference between the FPS and MFPS is that the FPS has a single concave surface whereas the MFPS consists of two facing concave surfaces. As a result, the MFPS can accommodate a larger relative displacement at the isolator level.

In ABR the sliding isolators are chosen in favor of the elastomeric isolators for the following reasons.

1. With the design load of 900 metric tons, the size of the elastomeric isolator would be about two meters in diameter. This may cause difficulties for the manufacturer of the rubber bearing because the damping of the rubber bearing comes from a chemical compound added into the natural rubber. The bigger the rubber bearing, the longer the curing time. This makes quality control of the bearing difficult.

2. The mechanical properties of the rubber bearing will be degraded in a cold temperature environment. This is based on the actual performance of rubber bearings in real earthquakes. During the 1995 Kobe earthquake in Japan, the rubber bearings installed in the Matsumura Gumi Laboratory building did not attenuate the ground motion because the ambient temperature was $0{ }^{\circ} \mathrm{C}$ and the isolator area was not heated (Ref. 1).

3. The fundamental natural frequency of the elastomeric isolator is a function of the weight of the structure. As a result, the performance of the base isolator system depends on the weight of the structure.

Among the sliding isolators, the MFPS is chosen to be used in the ABR. The MFPS consists of doubled concave sliding surfaces and an articulated slider with a hinge mechanism. The hinge mechanism is to guarantee that the super structure always remains vertical during an earthquake. The system mimics the kinematics of a pendulum, forcing the structure to rise slightly as it moves horizontally. This movement generates a restoring force to return the super structure back to its original position. The energy is dissipated by friction. The isolator is made of stainless steel and is capable of carrying a large load. The fundamental frequency of the isolator is independent of the weight of the structure (it depends on the radius of curvature of the concave surface), and the operating temperature of the isolator is from $-125^{\circ} \mathrm{C}$ to $250^{\circ} \mathrm{C}$. Therefore, the MFPS meets all the three challenges mentioned above. In addition, since the movement of the structure always starts from the mass center of the structure, the torsional response is minimized (Ref. 2). Note that the MFPS possesses characteristics of both elastomeric and sliding type base isolators in reducing the seismic response. The advantage of MFPS over HDRB is summarized below.

1. Period is independent of the structural weight

2. Design is simpler

3. Torsional effect is minimum

4. No buckling concerns

5. Durable (made of stainless steel)

6. Wider working temperature range 


\section{Quality control is easier}

The reactor design can be standardized by incorporating the MFPS in the reactor structure. The period of the MFPS may be adjusted for the same reactor design to be used at a different site.

The weight of the ABR reactor structure is estimated to be 11000 metric tons. If each isolator carries 600 metric tons, a total of twenty (20) bearings are to be used to support the reactor structure. A preliminary design shows that for a load of 900 metric tons (600 metric tons with a safety factor of 1.5), the size of the isolator is 1 meter in width by 1 meter in length (square shape) with the height of $35 \mathrm{~cm}$. The radius of curvature is designed such that the period of the isolator is 3 second, and the maximum allowable seismic displacement is $30 \mathrm{~cm}$. These isolators are housed in between the basement concrete slab and the foundation concrete slab shown in Figure III.1-1. The arrangement of these isolators is shown in Figure Y, and the cross section of a MFPS isolator is shown in Figure III.1-3. Each seismic isolator is inserted at the mid-height of a $120 \mathrm{~cm}$ by $120 \mathrm{~cm}$ reinforced concrete column that transfers the weight of the super-structure through the isolator to the foundation. These columns provide a $152 \mathrm{~cm}$ high space for inspection and maintenance. Each isolator unit is surrounded by four $50 \mathrm{~cm}$ by $50 \mathrm{~cm}$ concrete pedestals. The clear distance from the isolator to the pedestal is $85 \mathrm{~cm}$. These pedestals function as a physical barrier, in case of a higher than expected structural response. This is to avoid collision of the reactor structure and the moat wall. These pedestals also serve as temporary supports for the reactor structure when an isolator unit needs to be replaced.

Note that the MFPS is a 2D isolator. It means that it provides reduction for the horizontal seismic loading only. It does not reduce the vertical seismic loading. A three-dimensional seismic isolation is not proposed herein for the following two reasons.

1. The reason for inserting the steel shim plates into the laminated rubber bearing is to increase the vertical stiffness of the isolator. However, isolation in vertical direction is achieved by reducing the vertical stiffness. These two ideas contradict each other.

2. The vertical isolators will introduce additional rocking vibration to the structure. In 1969, the Swiss Full Base Isolation-3D system was installed for an elementary school in Skopje, Yugoslavia. The building bounced and rocked backward and forward in an earthquake (Ref. 3). Therefore, the vertical isolator is not recommended in any building codes.

However, several 3D isolators were developed in Japan recently (Ref. 4). These 3D isolators are under reviewing now. 3D isolators may be proposed in the future if they are proved to be effective in reducing the seismic loadings with no adverse side effects.

\section{Reference}

1. Trevor E Kelly, "Base Isolation Structures - Design Guidelines", Holmes Consulting Group Ltd., July 2001.

2. C. S. Tsai, T. C. Chiang and B. J. Chen, "Seismic Behavior of MFPS Isolated Structure under Near-Fault Sources and Strong Ground Motions with Long Predominant Periods”, Seismic Engineering-2003, PVP Vol. 466, ASME, Cleveland, Ohio, 2003.

3. Naeim, F. and Kelly, J. M., Design of Seismic Isolated Structures, John Wiley, New York, 1999. 
4. Takahashi, Kenji, et, al, “A Development of 3D Seismic Isolation for Advanced Reactor System in Japan - Part 2”, SMiRT 18-K10-2, Beijing, China, 2005

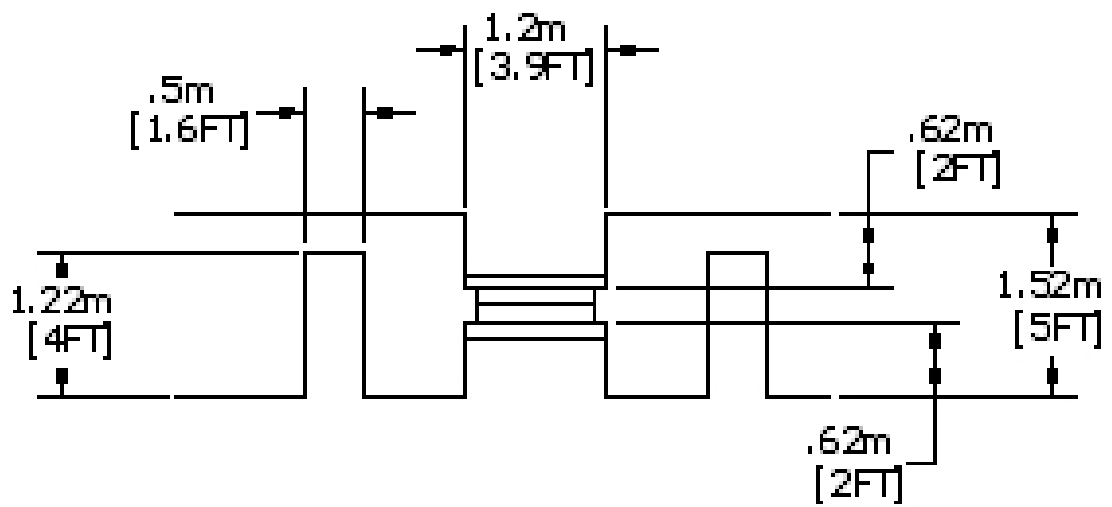

Figure III.1-1 A Typical MFPS Installation - One MFPS isolator with Four Concrete Pedestal Stopper

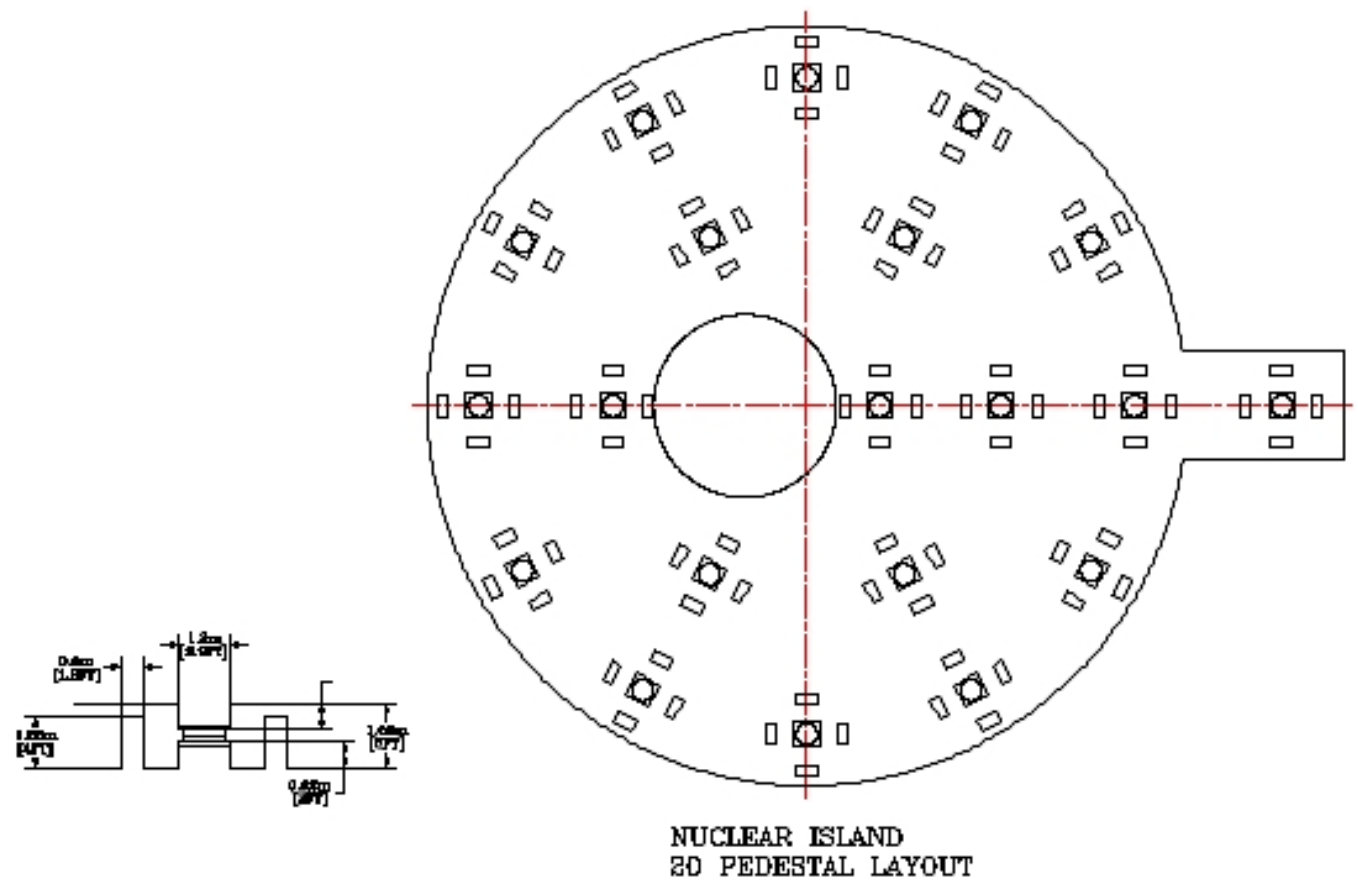

Figure III.1-2 Seismic Bearing Layout 


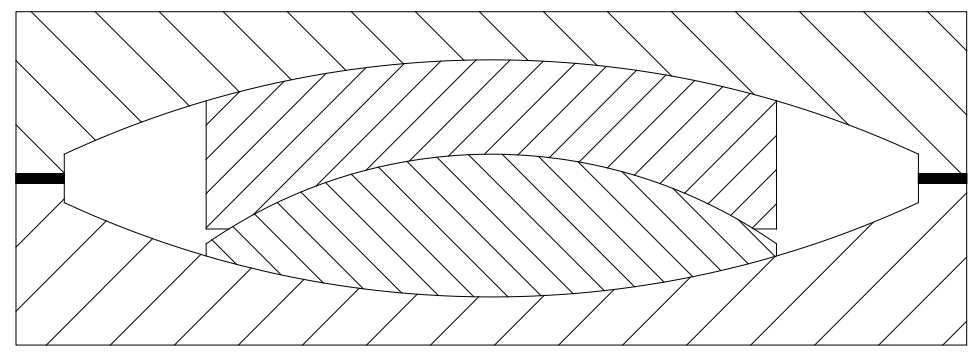

Figure III.1-3 Multiple Friction Pendulum System - Cross Section 


\section{III.2 Structural Analyses}

\section{III.2.1 Structural Analysis of Reactor Vessel Deck}

The annular deck forms the top closure head (or cover) of the reactor vessel. Functionally, it provides support for the rotatable plugs, IHXs, pumps, and the Direct Reactor Auxiliary Cooling System (DRACS). In turn, it transfers the weight to the vessel support and then to the reactor cavity concrete structure. It also provides thermal and radiation shielding. Thus, the structural integrity of the deck is an important element of the overall safety of the reactor design.

The current design of the deck is shown in Figure III.2-1. It is a stressed-skin, box-type structure construction that consists of top and bottom plates, and inner and outer rings. The space inside the box structure is divided into many chambers by the ribs (vertical plates) that connect the top, bottom plates and the inner, outer rings (see Figure III.2-2). Under the imposed loadings, the ribs will take mainly the shear forces; whereas, the top and bottom plates are designed to take the bending moments. There are eleven major penetrations between the inner and outer rings. Also, there are several other small penetrations (not shown) for instrumentation, sodium purification piping, and other uses. The eleven major penetrations provide support for the IHXs, pumps, and DRACS, and have three different size diameters---76 (1.93), 45 (1.14), and 18 (0.46) inches (meters), respectively. The inner ring of the deck is used to support the single rotatable plug. The ribs are discontinued when they intersect with the penetrations. The chambers will be filled with 3/8-inch $(0.95 \mathrm{~cm})$ steel balls for radiation shielding. To provide thermal shielding, a stack consisting of twelve horizontal $1 / 4$-inch $(0.635 \mathrm{~cm})$ steel plates with a gap of 0.87 inch $(2.21$ $\mathrm{cm}$ ) between each plate is hung from the deck bottom plate. In addition, on the top plate, there are several layers of thermal insulation that prevent the floor slab from touching the top plate directly, and lower the temperature of the top area to allow for personnel access for maintenance and operations.

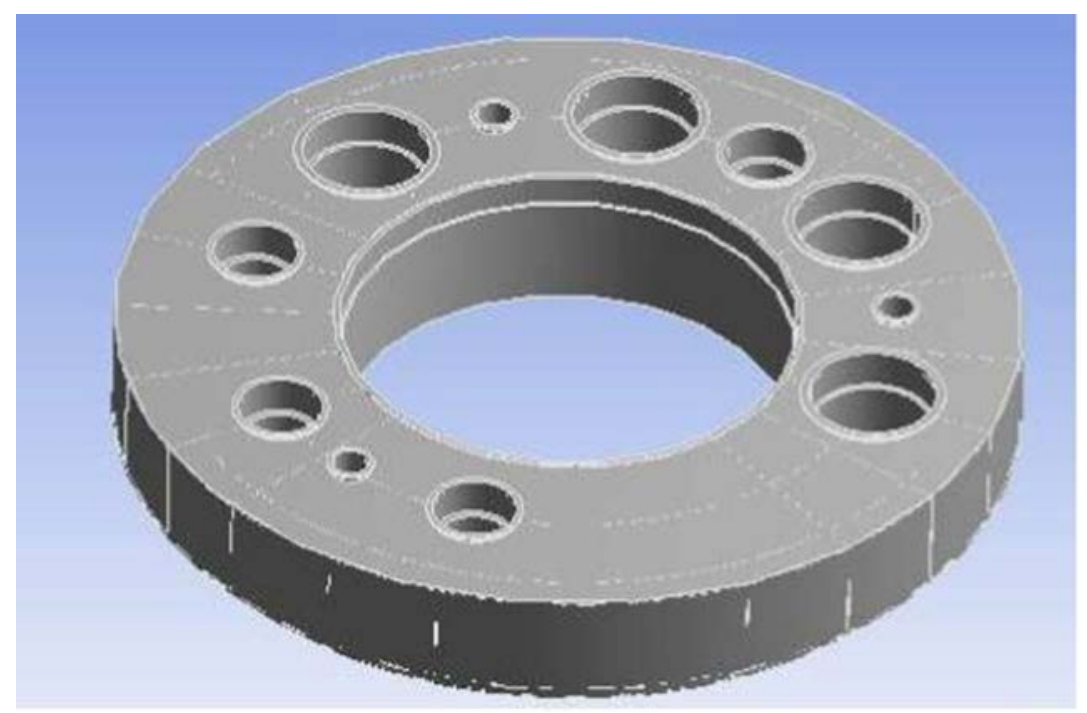

Figure III.2-1 Reactor Vessel Deck (Top View) 


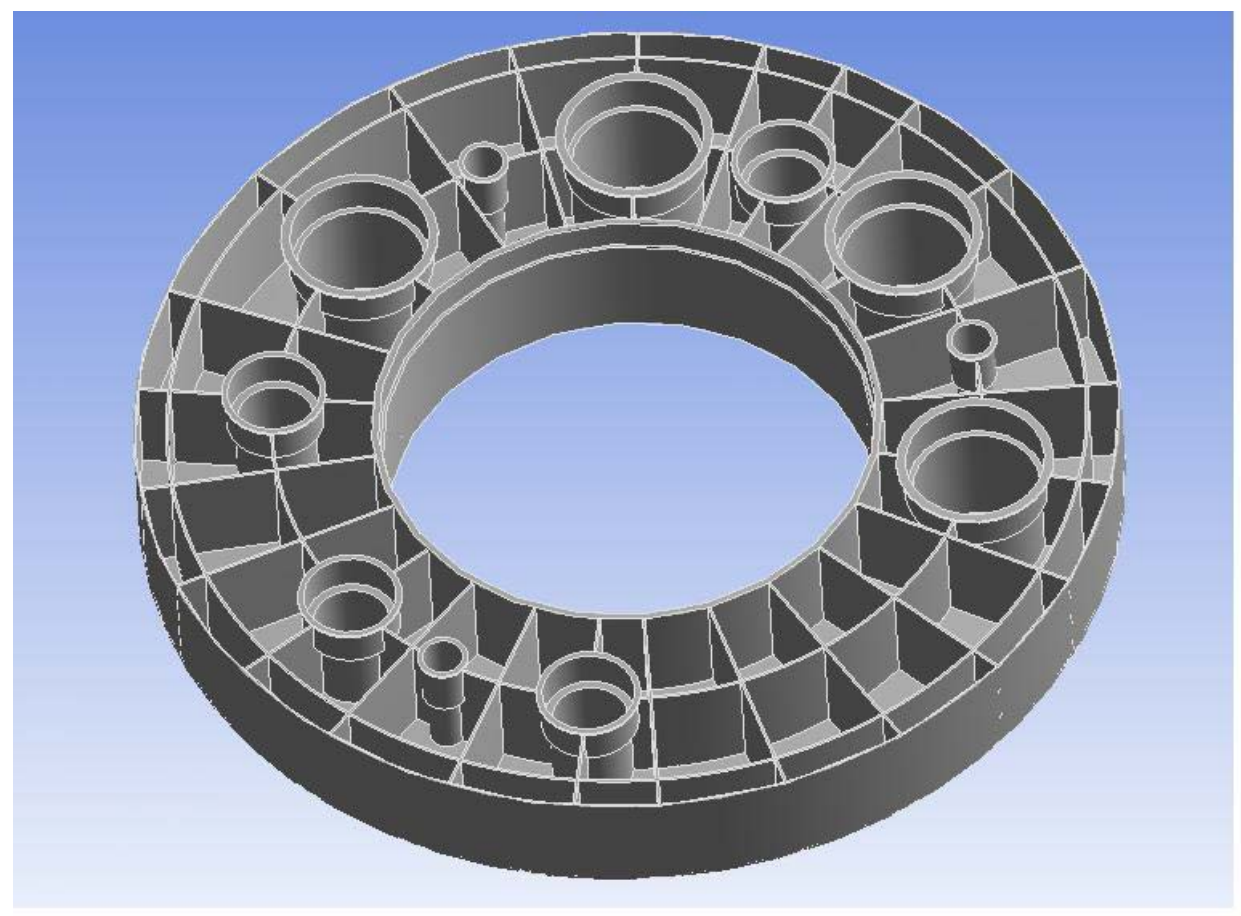

Figure III.2-2 Reactor Vessel Deck with Top Plate removed

The deck has a height of ninety-eight $(98)$ inches $(2.49 \mathrm{~m})$. The outer diameter of the deck is a step function. The outer diameter is 571 inches $(14.5 \mathrm{~m})$ for the upper 73 inches $(1.85 \mathrm{~m})$ and narrows to 528 inches $(13.41 \mathrm{~m})$ for the lower 25 inches $(63.5 \mathrm{~cm})$. This recess of the deck provides the bearing surface for support of the deck on the top flange of the reactor vessel conical support structure. All structural plate elements, with the exception of the center cylinder, are 1.5 inches $(3.81 \mathrm{~cm}$ ) thick to avoid the post-weld heat treatment required by the ASME code for carbon steel plate thicknesses greater than 1.5 inches $(3.81 \mathrm{~cm})$. The center cylinder has a thickness of 6 inches $(15.24 \mathrm{~cm})$. The material for the deck is SA-516 Grade 70 carbon steel. The current deck design is chosen from four different designs based on the results of the stress analyses performed. The stress analyses will be briefly described hereinafter. The four designs are referred as Model 1, 2, 3, and 4 in the following description.

Model 1: This design is the current design without the top plate. An additional 1.5-inch (3.81 $\mathrm{cm})$ plate is located at 25 -inch $(63.5 \mathrm{~cm})$ above the bottom plate. The idea of this design is to move the layers of thermal insulation on the top plate inside the deck to reduce the overall height of the deck.

Model 2: This design is to add 12-inch $(30.48 \mathrm{~cm})$ wide flanges to all the ribs of the Model 1 design. The idea of this design is to increase the bending resistance of the vertical ribs.

Model 3: This design is the Model 1 design with the top plate added. The idea of this design is to further increase the bending rigidity of the deck.

Model 4: This design is the Model 3 design with the mid-plane plate removed. The idea of this design is to assess the effectiveness of the mid-plane plate. 
The ANSYS computer code was used to perform the stress analyses of these four models. The loadings considered in the analyses are:

- Weight of the structure

- Four IHXs, each weight of 102,000 lbs (46,266 kg)

- Four pumps, each weight of 92,600 lbs (42,003 kg)

- Three DRACS, each weight of 5,300 lbs (2,404 kg)

- Weight of the thermal and radiation shielding is 8.05 psi (55.5 kPa) (applied to the base plate)

- Weight of the dual Rotatable Plug system of 1,045,000 lbs (474,004 kg)

Note that the twelve $1 / 4$-inch $(0.635 \mathrm{~cm})$ plates hung at the deck bottom plate are not modeled in the finite element analysis. However, the weight of these plates is included in the loading calculation. The total weight of the deck structure, including all supported equipment, is about 4,000 kips (1,818,182 kg). Two computer output quantities are examined and used as the index for the assessment. They are the maximum deflection and the maximum Von Mises stress (the so-called equivalent stress). The results of the stress analyses are tabulated in the following table.

Table III.2-1

\begin{tabular}{|l|l|l|}
\hline Model & Deflection (in)/mm & $\begin{array}{l}\text { Von Mises Stress } \\
(\mathrm{ksi}) / \mathrm{MPa}\end{array}$ \\
\hline 1 & $0.0625 / 1.59$ & $8.47 / 58.4$ \\
\hline 2 & $0.0624 / 1.58$ & $9.17 / 63.2$ \\
\hline 3 & $0.041 / 1.04$ & $3.53 / 24.3$ \\
\hline 4 & $0.037 / 0.94$ & $3.07 / 21.2$ \\
\hline
\end{tabular}

The data presented in the table above clearly indicates that Model 4 is the most effective design. Therefore, Model 4 is chosen to be the current design. The vertical deflection and Von Mises stress plots for Model 4 are presented in Figures III.2-3 and III.2-4 respectively. 


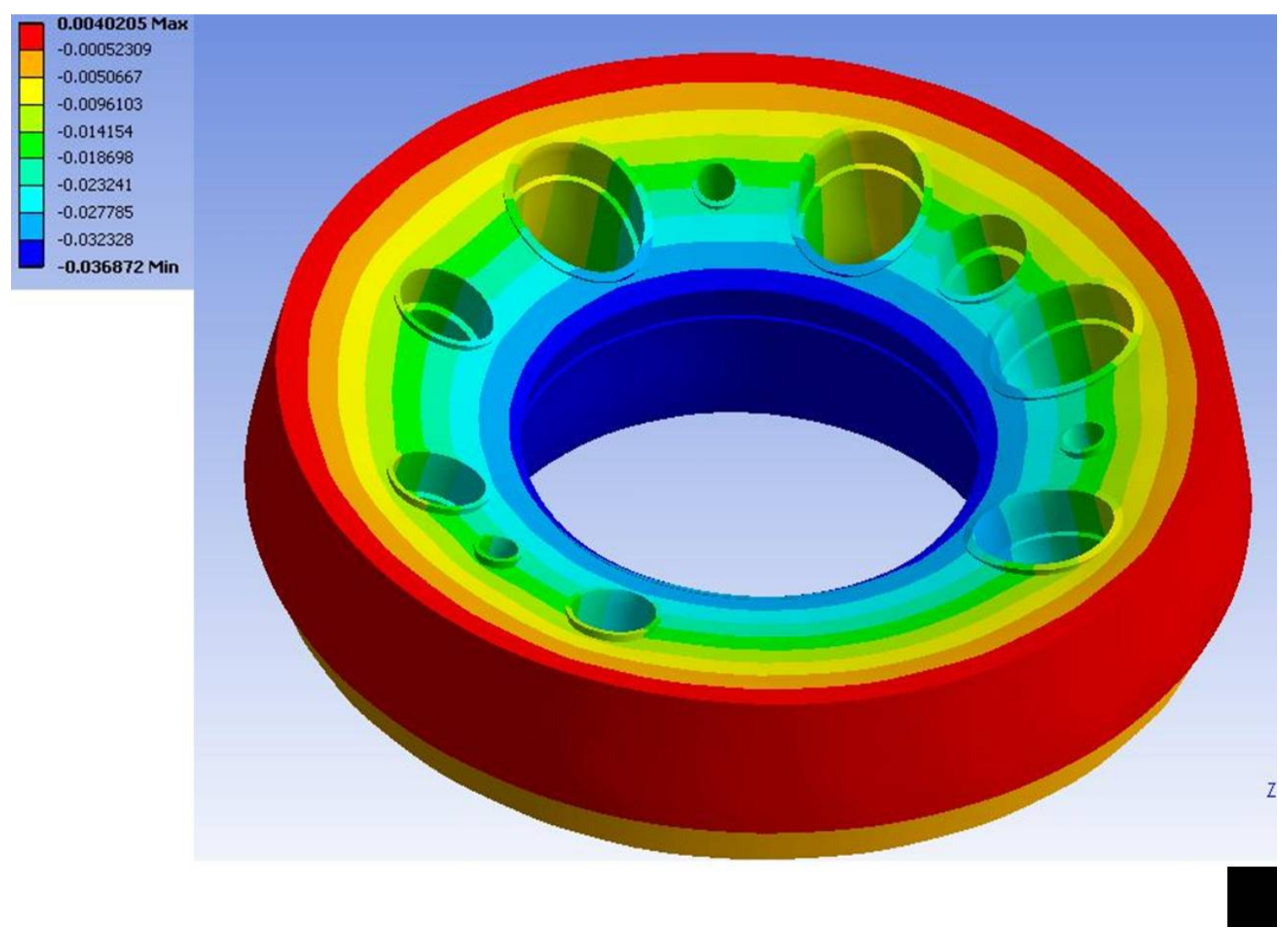

Figure III.2-3 Vertical Deflection of Model 4

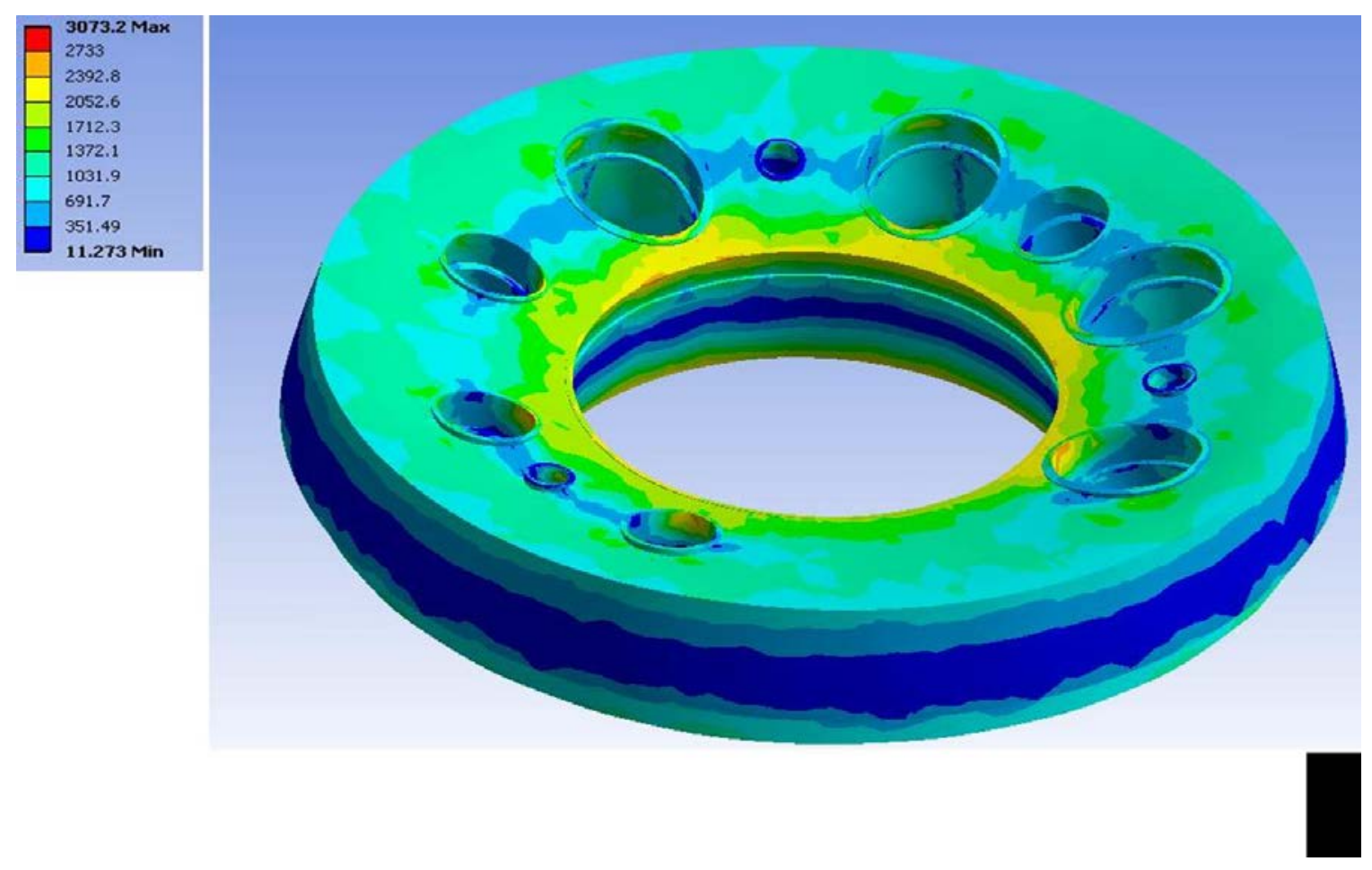

Figure III.2-4 Von Mises Stress of Model 4 


\section{III.2.2 Structural Analysis of Reactor Vessel}

This section describes the preliminary structural/stress analyses of the reactor vessel. The purpose of this analysis is to determine the thickness of the vessel and the knuckle radius for the torispherical vessel head. The design presented is not the optimal solution. Additional studies are being carried out. The results will be reported in future reports. In the current design, the reactor vessel is a cylindrical shell with a torispherical bottom. A torispherical bottom is a spherical cap with a toroidal segment that connects to the cylindrical shell. The inside radius of the cylindrical shell is 276.75 inches $(7,029.45 \mathrm{~mm})$. The radius for the spherical shell portion of the bottom head is 549.5 inches $(13,957.3 \mathrm{~mm})$. The radius of the toroidal segment of the bottom head is referred to as the knuckle radius. Two knuckle radii are assumed in the study presented herein. They are 33 inches $(838.2 \mathrm{~mm})$ and 72 inches $(1828.8 \mathrm{~mm})$ which are about $6 \%$ and $13 \%$ of the spherical radius, respectively. The stress analysis presented here is for only the static loads imposed on the vessel which include:

1. The vessel weight

2. The weight of the sodium (hydrostatic pressure)

3. The weight of the internal structures including the redan, reactor core, and the core support structure

The material for the entire vessel is Type 304 stainless steel. The stress-related quantities examined include the stress intensity, the equivalent stress, the shear stress, and the vertical deflection of the bottom head. The stress intensity and equivalent stress are the indicators for material yielding. They are used in the Tresca and Von Mises yield conditions, respectively. The shear stress is the indicator for the degree of discontinuity at the boundary of the cylindrical and toroidal shells and the boundary of the toroidal and spherical shells. The vertical deflection (at the center of the torispherical shell) is the indicator for the overall structural rigidity. In this study, the entire vessel is assumed to have the same thickness throughout. The thicknesses considered are 1.375, 1.5, 1.625, 1.75, and 2 inches (34.92, 38.1, 41.28, 44.45, $50.8 \mathrm{~mm}$ ). The ANSYS finite element code is used to perform the stress analyses. The results are tabulated in the table below. 
Table III.2-2

Knuckle radius $=72$ in $(1828.8 \mathrm{~mm})(13 \%$ of spherical radius $)$

$\begin{array}{lllll}\begin{array}{l}\text { Thickness } \\ \text { (in)/(mm) }\end{array} & \begin{array}{l}\text { Von Mises } \\ (\mathrm{ksi}) /(\mathrm{MPa})\end{array} & \begin{array}{l}\text { Stress Intensity } \\ (\mathrm{ksi}) /(\mathrm{MPa})\end{array} & \begin{array}{l}\text { Shear Stress } \\ (\mathrm{ksi}) /(\mathrm{MPa})\end{array} & \begin{array}{l}\text { Vertical } \\ \text { Deflection } \\ \text { (in)/(mm) }\end{array} \\ 1.375 / 34.92 & 16 / 110.3 & 17.5 / 120.7 & 6.5 / 44.8 & 0.26 / 6.60 \\ 1.5 / 38.1 & 13.6 / 93.8 & 15.6 / 107.6 & 4.7 / 32.4 & 0.23 / 5.84 \\ 1.625 / 41.28 & 12.4 / 85.5 & 14.1 / 97.2 & 4.2 / 28.9 & 0.21 / 5.33 \\ 1.75 / 44.45 & 11.5 / 79.3 & 13.1 / 90.3 & 3.9 / 26.9 & 0.2 / 5.08 \\ 2 / 50.8 & 9.4 / 64.8 & 10.6 / 73.1 & 3.1 / 21.4 & 0.18 / 4.57\end{array}$

Knuckle radius $=33$ in ( $6 \%$ of spherical radius)

$\begin{array}{lllll}\begin{array}{l}\text { Thickness } \\ \text { (in)/(mm) }\end{array} & \begin{array}{l}\text { Von Mises } \\ (\mathrm{ksi}) /(\mathrm{MPa})\end{array} & \begin{array}{l}\text { Stress Intensity } \\ (\mathrm{ksi}) /(\mathrm{MPa})\end{array} & \begin{array}{l}\text { Shear Stress } \\ (\mathrm{ksi}) /(\mathrm{MPa})\end{array} & \begin{array}{l}\text { Vertical } \\ \text { Deflection } \\ (\mathrm{in}) /(\mathrm{mm})\end{array} \\ 1.375 / 34.92 & 29.8 / 205.5 & 34 / 234.4 & 11.5 / 79.3 & 0.39 / 9.91 \\ 1.5 / 38.1 & 25.5 / 175.8 & 29 / 199.9 & 9 / 62.1 & 0.36 / 9.14 \\ 1.625 / 41.28 & 23.7 / 163.4 & 26.9 / 185.5 & 8.4 / 57.9 & 0.32 / 8.13 \\ 1.75 / 44.45 & 20.7 / 142.7 & 23.6 / 162.7 & 7.3 / 50.3 & 0.3 / 7.62 \\ 2 / 50.8 & 17 / 117.2 & 19.3 / 133.1 & 6.3 / 43.4 & 0.25 / 6.35\end{array}$

The data in the table clearly indicates that the knuckle radius of 72 inches $(1828.8 \mathrm{~mm})$ is better than that of 33 inches $(838.2 \mathrm{~mm})$, and that the thicker the shell, the lower the stresses. Therefore, in the preliminary study, a vessel with the torispherical knuckle radius of 72 inches and the shell thickness of 2 inches is recommended. Notice that the Von Mises stress for this case is $9.4 \mathrm{ksi}(64.8 \mathrm{MPa})$ which is less than the allowable stress, $16.6 \mathrm{ksi}$ (114.4 MPa) given in ASME code for $304 \mathrm{SS}$ at the temperature of $600{ }^{\circ} \mathrm{F}\left(316^{\circ} \mathrm{C}\right)$. The stress intensity for the vessel with the knuckle radius of 72-in (1828.8-mm) and thickness of 2-in (50.8-mm) thickness is shown in Figure III.2-5. One can see clearly that the high stress occurs at the containing the toroidal segment. It is also seen from Figure III.2-5 that the cylindrical shell has low stress intensity at the top and middle sections. The thickness of shell at these regions may be reduced to save the material and fabrication cost. This will be evaluated in future work. 


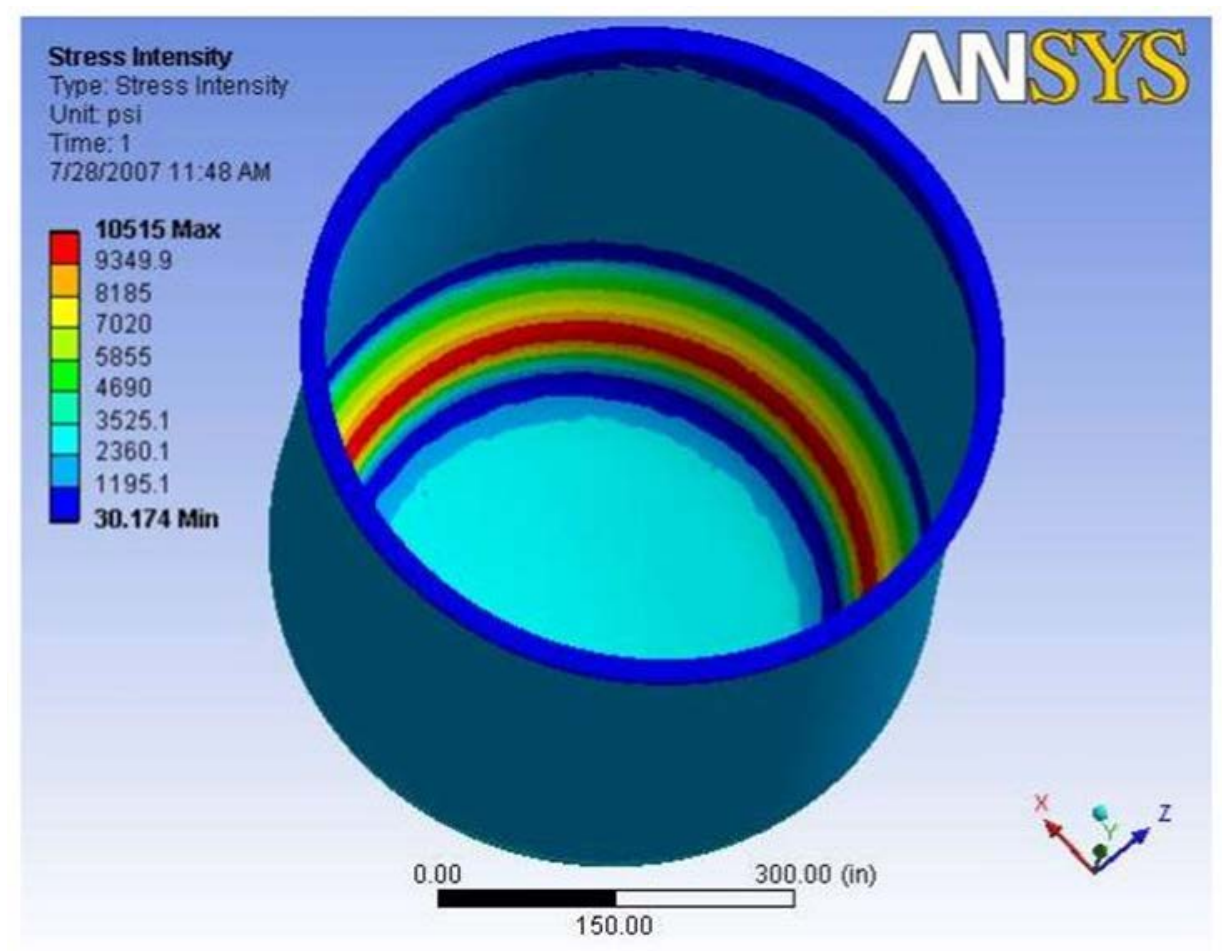

Figure III.2-5 Stress Intensity for Reactor Vessel with Knuckle radius of 72-in (1828.8-mm) and thickness of 2 -in $(50.8-\mathrm{mm})$

The shear stress plot for the same vessel is presented in Figure III.2-6. Again, the maximum shear stress is at the transition region containing the toroidal segment. This result parallels the same information as that of the stress intensity---namely, the high stress is caused by the curvature discontinuity. Examination of the data for the shear stress listed in the table above clearly shows that the shear stress monotonically decreases with the increase of the thickness. This is because the thicker the shell, the less deformation of the shell and, hence, the less shear stress developed to eliminate the discontinuity of the displacement. 


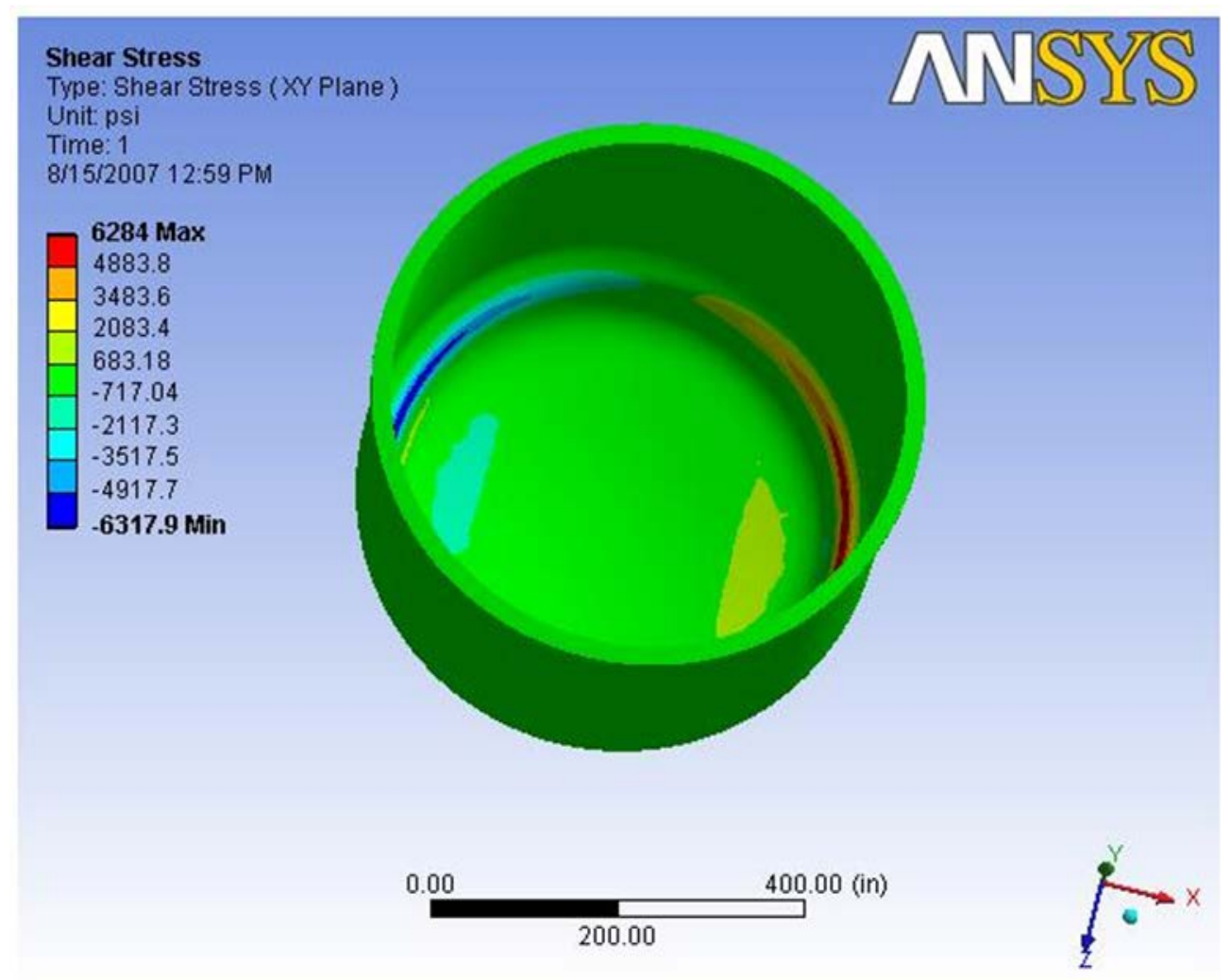

Figure III.2-6 Shear Stress for Reactor Vessel with Knuckle radius of 72-in (1828.8-mm) and thickness of 2-in (50.8-mm)

\section{III.2.3 Stress Analysis of the Sodium Piping in the Intermediate Heat Transfer System (IHTS)}

The layout of the IHTS sodium piping needs to be designed so that it is sufficiently flexible to accommodate the thermal expansion due to the sodium high temperature, and that the piping layout is practical and appears feasible from a construction and maintainability viewpoint. The preliminary stress analysis performed and presented herein was used to arrive at a preliminary sodium piping layout. The purpose of this analysis is not to qualify the piping system according to the ASME code, but to design a piping layout that can be reasonably expected to meet all functional and code requirements. Subsequent structural analysis in the next phase of work will develop these piping systems further to meet all requirements of applicable codes, and to provide cost effective piping layouts. These more detailed analyses will consider loads not included in this preliminary analysis, such loadings from design earthquakes and possible sodium-water accidents.

There are four (4) piping loops used in the secondary sodium system. Each piping loop consists of a "Hot Leg" that transports the hot sodium $\left(900{ }^{\circ} \mathrm{F} / 482{ }^{\circ} \mathrm{C}\right)$ from the IHX in the reactor vessel to the steam generator and a "Cold Leg" that returns the cold sodium $\left(620^{\circ} \mathrm{F} / 327\right.$ ${ }^{\circ} \mathrm{C}$ ) back to the IHX. The Cold Leg of the piping system can be further divided into two segments. The first is the piping segment which runs from the steam generator to the secondary 
sodium mechanical pump, and the other segment which runs from the mechanical pump to the IHX. These two segments are referred to as "Cold Leg 1" and "Cold Leg 2", respectively. The mathematical models for the piping systems are generated from the piping module of the ANSYS computer code. All piping is 24" schedule 40, and the material is Type 316 stainless steel. The following loadings are considered in the analyses:

- The piping material weight

- The sodium weight

- The insulation and jacket weight assuming 4 inches $(102 \mathrm{~mm})$ of insulation with a density of $4.34 \mathrm{e}-5 \mathrm{lb}-\mathrm{s}^{2} / \mathrm{in}\left(7.74 \mathrm{e}-6 \mathrm{~kg}-\mathrm{s}^{2} / \mathrm{cm}\right)$

- Internal pressure of 325 psi (2.24 MPa)

- The valve weight of $500 \mathrm{lbs}$ (227 kg)(assumed)

Based on the ANSYS stress analyses, the following quantities are calculated.

- $\quad$ Sustained stress, $\mathrm{S}_{\mathrm{L}}$, calculated by

$$
\begin{aligned}
& \mathrm{S}_{\mathrm{L}}=\mathrm{PD}_{\mathrm{o}} / 4 \mathrm{t}+\mathrm{iM}_{\mathrm{A}} / \mathrm{Z} \\
& \text { where } \mathrm{P}=\text { internal pressure }=325 \mathrm{psi}(2.24 \mathrm{MPa}) \\
& \qquad \begin{array}{l}
\mathrm{D}_{\mathrm{o}}=\text { outside diameter of pipe } \\
\mathrm{t}=\text { wall thickness of pipe }=0.687 \text { inch }(17.4 \mathrm{~mm}) \\
\mathrm{i}=0.75 \text { of the stress intensification factor (output from ANSYS) } \\
\mathrm{M}_{\mathrm{A}}=\text { resultant moment on cross section (output from ANSYS) } \\
\mathrm{Z}=\text { section modulus of pipe }=285.1 \mathrm{in}^{3}\left(4792 \mathrm{~cm}^{3}\right)
\end{array}
\end{aligned}
$$

- $\quad$ Thermal stress, $S_{t}$, given by

$$
\mathrm{S}_{\mathrm{t}}=\mathrm{i} \mathrm{M}_{\mathrm{C}} / \mathrm{Z}
$$

$$
\text { where } \mathrm{M}_{\mathrm{C}}=\text { resultant moment due to thermal expansion (output from ANSYS) }
$$

The sustained stress, $S_{L}$, will be compared with the material allowable stress at the sodium temperature, $S_{h}$, and the thermal stress will be compared with the stress, $S_{\mathrm{a}}$, calculated by

$$
\mathrm{S}_{\mathrm{a}}=1.25\left(\mathrm{~S}_{\mathrm{c}}+\mathrm{S}_{\mathrm{h}}\right)-\mathrm{S}_{\mathrm{L}}
$$

were $S_{c}$ is the material allowable stress at room temperature.

A pipe layout is admissible if $\mathrm{S}_{\mathrm{L}} \leq \mathrm{S}_{\mathrm{h}}$ and $\mathrm{S}_{\mathrm{t}} \leq \mathrm{S}_{\mathrm{a}}$. Note that these two criteria are derived from the ANSI/ASME B31.1 power piping code. By satisfying these two criteria, it is very likely that the piping will meet the Section III of the ASME Boiler and Pressure Vessel code as well. Trial-and-error was used to determine the layouts. The final layouts and the associated stresses for three pipe segments are presented below.

Hot Leg: from IHX to Steam Generator (length is about 173.5 feet $(53 \mathrm{~m})$ )

The layout is shown in Figure III.2-7, and the stresses are tabulated in the following table. 
Table III.2-3

\begin{tabular}{|c|c|c|c|c|c|c|c|}
\hline $\begin{array}{c}\text { Cross } \\
\text { Section }\end{array}$ & $\begin{array}{c}\text { 0.75 Stress } \\
\text { Intensificatio } \\
\text { n Factor, i }\end{array}$ & $\begin{array}{c}\mathrm{M}_{\mathrm{A}} \text { (in- } \\
\mathrm{kip} /(\mathrm{m}-\mathrm{kg})\end{array}$ & $\begin{array}{c}\mathrm{M}_{\mathrm{C}}(\mathrm{in}- \\
\mathrm{kip}) /(\mathrm{m}-\mathrm{kg})\end{array}$ & $\begin{array}{c}\mathrm{S}_{\mathrm{L}}, \\
\mathrm{ksi} / \mathrm{Mpa}\end{array}$ & $\begin{array}{c}\mathrm{S}_{\mathrm{h}}, \\
\mathrm{ksi} / \mathrm{MPa}\end{array}$ & $\begin{array}{c}\mathrm{S}_{\mathrm{t}}, \\
\mathrm{ksi} / \mathrm{MPa}\end{array}$ & $\begin{array}{c}\mathrm{S}_{\mathrm{a}}, \\
\mathrm{ksi} / \mathrm{MPa}\end{array}$ \\
\hline Straight & 1 & $\begin{array}{c}1462.5 / 1685 \\
0\end{array}$ & $\begin{array}{c}3446.5 / 3970 \\
9\end{array}$ & $7.97 / 55$ & $\begin{array}{c}14.6 / 100 . \\
7\end{array}$ & $\begin{array}{c}32.09 / 83.4 \\
3\end{array}$ \\
\hline Elbow & 2.1 & $898.1 / 10347$ & $\begin{array}{c}3204.8 / 3692 \\
4\end{array}$ & $\begin{array}{c}9.45 / 65 . \\
2\end{array}$ & $\begin{array}{c}14.6 / 100 . \\
7\end{array}$ & $\begin{array}{c}23.61 / 162 . \\
8\end{array}$ & $33.8 / 233$ \\
\hline
\end{tabular}

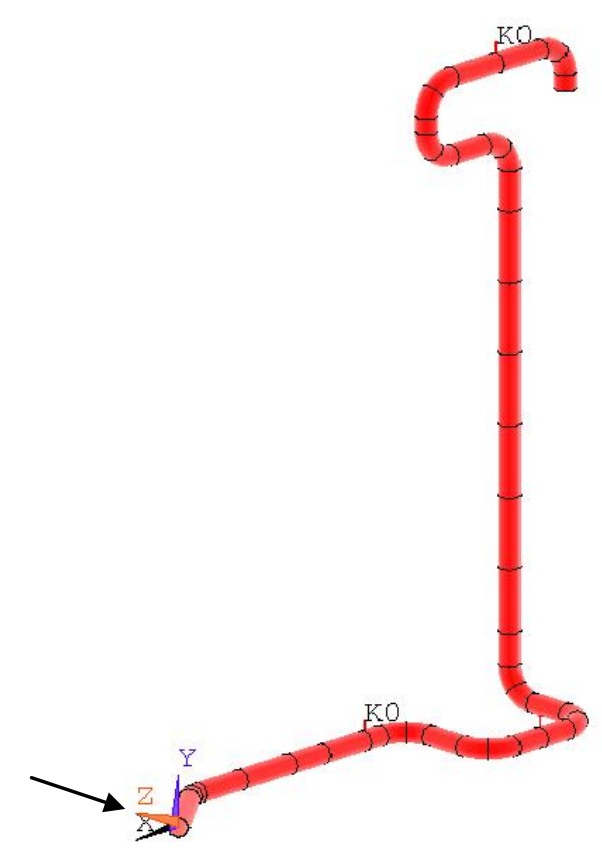

Figure III.2-7 Piping from IHX to Steam Generator Layout

Cold Leg 1: from Steam Generator to Mechanical Pump (length is about 129 feet (39 m)) The layout for this pipe line is shown in Figure III.2-8. The stresses are tabulated in the following table.

Table III.2-4

\begin{tabular}{|c|c|c|c|c|c|c|c|}
\hline $\begin{array}{c}\text { Cross } \\
\text { Section }\end{array}$ & $\begin{array}{c}0.75 \text { Stress } \\
\text { Intensification } \\
\text { Factor, i }\end{array}$ & $\begin{array}{c}\mathrm{M}_{\mathrm{A}} \text { (in- } \\
\text { kip)/(m-kg) }\end{array}$ & $\begin{array}{c}\mathrm{M}_{\mathrm{C}} \text { (in- } \\
\text { kip)/(m-kg) }\end{array}$ & $\begin{array}{c}\mathrm{S}_{\mathrm{L}}, \\
\text { ksi/MPa }\end{array}$ & $\begin{array}{c}\mathrm{S}_{\mathrm{h}}, \\
\text { ksi/MPa }\end{array}$ & $\begin{array}{c}\mathrm{S}_{\mathrm{t}}, \\
\text { ksi/MPa }\end{array}$ & $\begin{array}{c}\mathrm{S}_{\mathrm{a}}, \\
\text { ksi/MPa }\end{array}$ \\
\hline Straight & 1 & $1570.9 / 18099$ & $1271.2 / 14646$ & $8.35 / 57.6$ & $17 / 117$ & $4.46 / 30.8$ & $37.9 / 261.3$ \\
\hline Elbow & 2.1 & $844.57 / 9731$ & $1294.6 / 14916$ & $9.06 / 62.5$ & $17 / 117$ & $9.54 / 65.8$ & $37.2 / 256.5$ \\
\hline
\end{tabular}




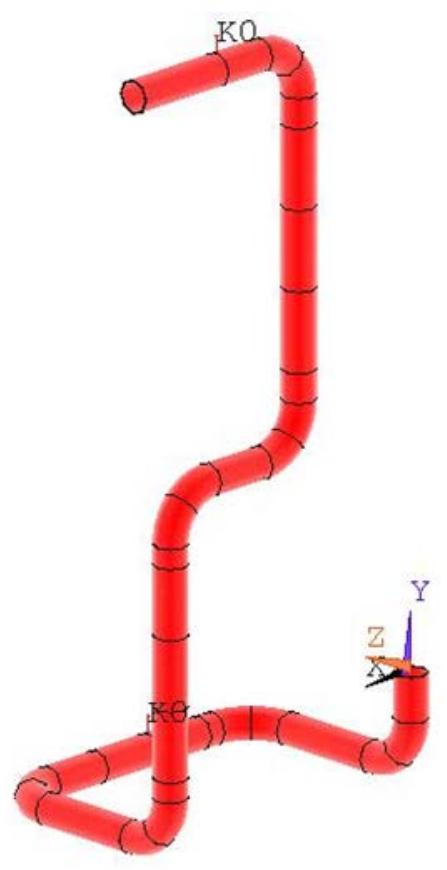

Figure III.2-8 Piping from Pump to IHX Layout 


\section{III.3 Thermal-Hydraulic Analysis}

A computational fluid dynamics (CFD) analysis was performed for a reference ABR primary system to assess at steady-state flow patterns in the primary reactor system, sodium pool temperature distributions, and temperature distributions in the reactor vessel, guard vessel and the reactor vessel closure structures. For these analyses the CFD code STAR-CD was used.

The CFD model has about 630,000 computational cells, and includes the reactor pumps, the reactor plenum, the reactor core, lower reflector, upper shield, radial reflector, and radial shielding, the redan, the intermediate heat exchangers (IHXs), the reactor vessel, the guard vessel, the structures that form the closure of the reactor vessel, the argon cover gas, the argon gas space between the reactor and guard vessel and between the reactor vessel closure and the concrete support structures. The reactor and the IHXs were modeled as porous media. The heat generation in the core was modeled as a volumetric heat source, and the IHXs were modeled as volumetric heat sinks. Because at this time the STAR-CD code does not allow radiation from a liquid surface, a solid of zero thickness and zero thermal resistance was placed on the top of the sodium pool (separating the sodium from the argon cover gas). The emissivity of this solid was set equal to that of the sodium pool surface (Ref.1).

Because the auxiliary cooling systems have not been sized yet, the following boundary conditions were used on the outer boundary of the model: a temperature of $90^{\circ} \mathrm{C}$ on the outer surface of the guard vessel insulation, on the top of the structure supporting the reactor vessel skirt, and on the outer surface in the radial direction of the concrete blocks surrounding the upper structures of the reactor vessel; and a temperature of $32^{\circ} \mathrm{C}$ on the top of the insulation above the reactor vessel cover, and on the top surface of the concrete blocks surrounding the upper structures of the reactor vessel. For the reference case a very effective insulation (foam glass ) has been used in the redan $(10 \mathrm{~cm}$ thick), the upper section of the reactor vessel ( $10 \mathrm{~cm}$ thick), the guard vessel (12 cm thick), and the top of the reactor cover (42 cm thick).

Figure III.3-1 shows the computational grid in the fluid domain for one half of the model. Figure III.3-2 shows the pump shells, the pipes from the pumps to the inlet plenum, and the outer IHX shells. Figures III.3-3 and III.3-4 show the reactor vessel, and the guard vessel, respectively.

Figure III.3-5 shows the sodium flow in the hot pool on a vertical plane passing through one of the IHXs. The figure also shows part of the top of the hot pool. The main flow pattern is a strong upward flow towards the inlet of the IHXs. There is a weak recirculation zone on the side of the pumps. Figure III.3-6 shows the velocity field on a horizontal plane passing through the inlet of the IHXs.

Figure III.3-7 shows the temperature distribution in the cold pool and the reactor. As expected, there is a thermal stratification in the cold pool, with a decreasing sodium temperature as we move from the top to the bottom of the pool. Figure III.3-8 shows the temperature distribution in the hot pool on a vertical plane passing through the center of one of the IHXs. There is a hot plume at the center of the pool, and a small temperature variation between the side of the IHXs and the side of the pumps.

Figure III.3-9 shows the temperature distribution in the reactor vessel and Figure III.3-10 shows the temperature distribution in the reactor vessel and the reactor cover structures above the reactor cover gas and below the reactor cover insulation on a vertical reactor vessel symmetry plane. Because a very effective insulation has been used, most of the heat loss is through the 
reactor vessel skirt and the large temperature gradients are in the radial direction. Figure III.3-11 shows the temperature distribution in the guard vessel.

To assess the impact of the insulation placed all around the redan and on the upper section of the reactor vessel (starting $1.7 \mathrm{~m}$ below the top of the cold pool), simulations were performed with and without the insulation. With an insulated redan, about $13 \mathrm{Kw}$ of heat are transferred from the hot pool to the cold pool. Without insulation, the heat transferred from the hot pool to the cold pool is about $5 \mathrm{Mw}$. This underscores the significance of the redan insulation.

Figure III.3-12 shows the temperature distribution in the reactor vessel when its insulation is removed. As expected, with insulation (Figure III.3-9) the upper section of the reactor vessel is colder, and. above the cold pool its temperature is reduced by about 35oC. Because the maximum temperature is the same (with and without insulation), the impact of the insulation should be assessed in terms of the related thermal stresses.

To provide an estimate of the heat load to be removed by the reactor cover cooling system, and the guard vessel cooling system, the heat removed out of the boundaries cooled by these systems was computed for the boundary conditions used in this analysis. A load of $155 \mathrm{kw}$ was computed for the guard vessel cooling system, and a load of $40 \mathrm{kw}$ for the reactor cover cooling system.

To provide an estimate for the guard vessel cooling system requirements, a CFD model of this system was generated. This system provides forced air flow that removes heat from the guard vessel insulation and the steel-liner of the concrete structure that surrounds the guard vessel. The air flows down in the gap $(12.7 \mathrm{~cm})$ between the concrete liner and a $2.54 \mathrm{~cm}$ thick baffle surrounding the guard vessel, and upwards in the gap $(9.84 \mathrm{~cm})$ between this baffle and the guard vessel insulation. In this preliminary analysis, an air flow rate of $7.11 \mathrm{~kg} / \mathrm{s}$ and an inlet temperature of $30 \mathrm{oC}$ were used. An adiabatic boundary condition was used at the outer surface of the concrete liner. This is a conservative assumption. The air heat transfer coefficient was computed directly by the turbulence model (standard high-Reynolds number k- $\varepsilon$ model). In this analysis, the grid in the fluid (air) is very coarse, and further analysis is needed to compare the heat transfer coefficient computed by the model with that computed from correlations.

Figure III.3-13 shows the temperature distribution predicted in the concrete liner. This distribution is quite uniform with a maximum temperature of $460 \mathrm{C}$. It should be pointed out that

this initial simulation is based on a coarse grid and the impact of the grid structure on predicted temperatures needs further investigation.

\section{References}

1. A. Yamaguchi and Y. Tajima, "Numerical Investigation of Mass and Heat Transfer in Sodium Pool Combustion,” Numerical Heat Transfer, Part A, 41:697-709, 2002. 

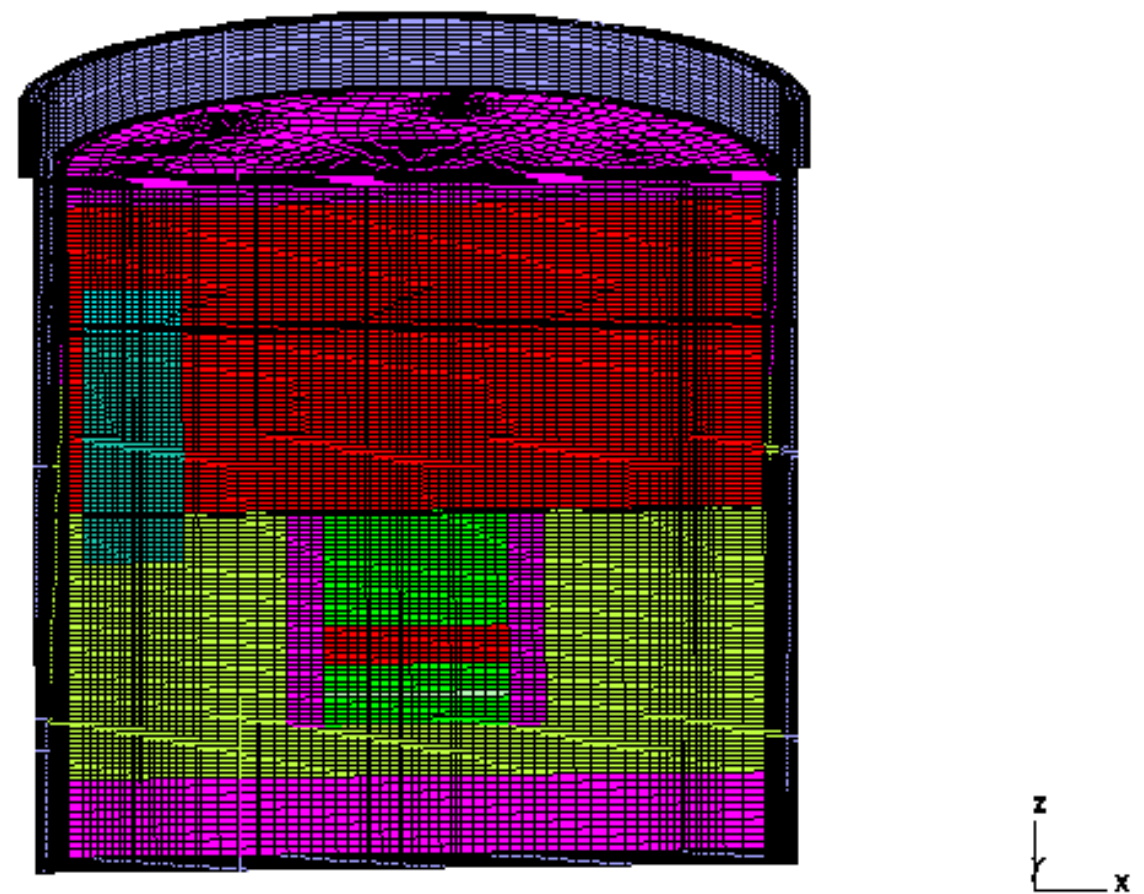

Figure III.3-1 Computational grid in fluid.
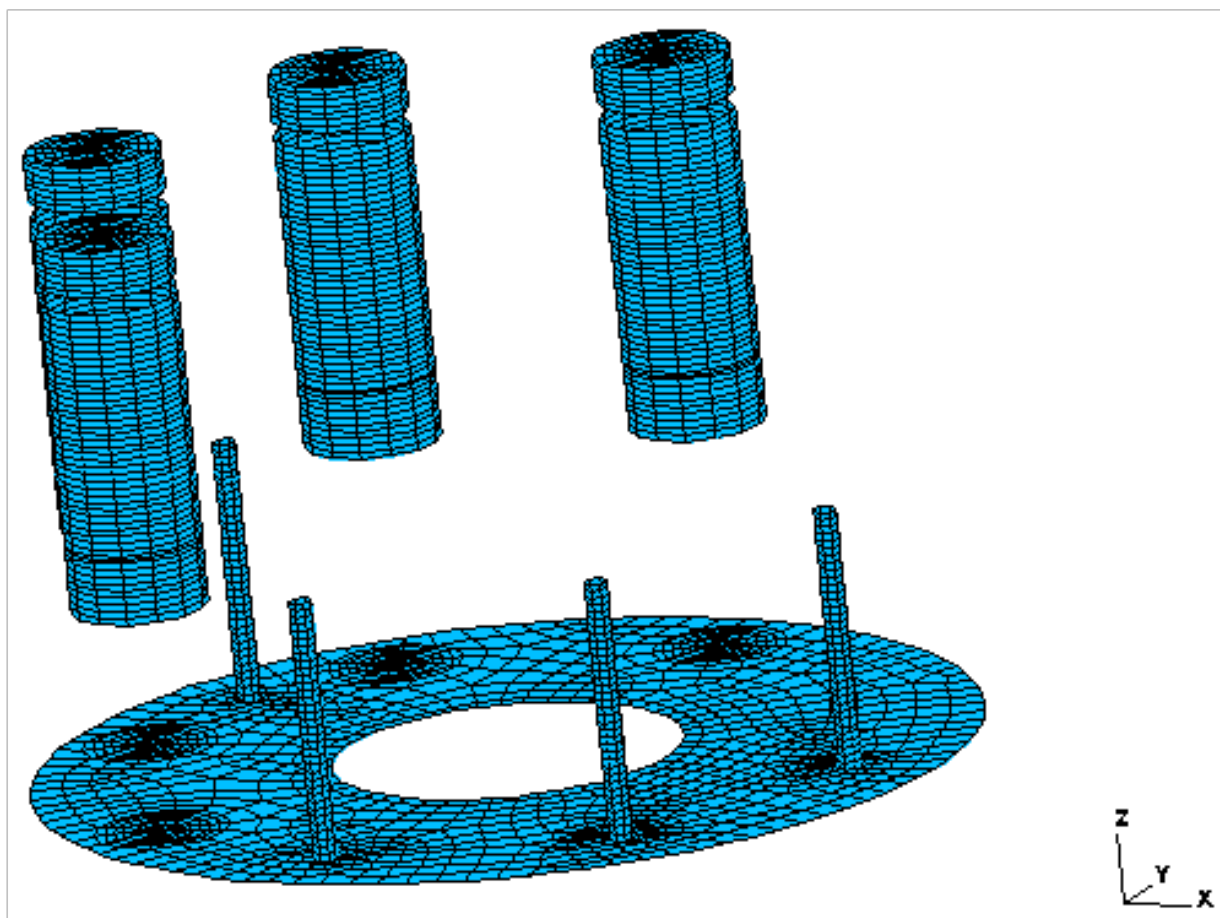

Figure III.3-2 Pump pipes and IHX shells 

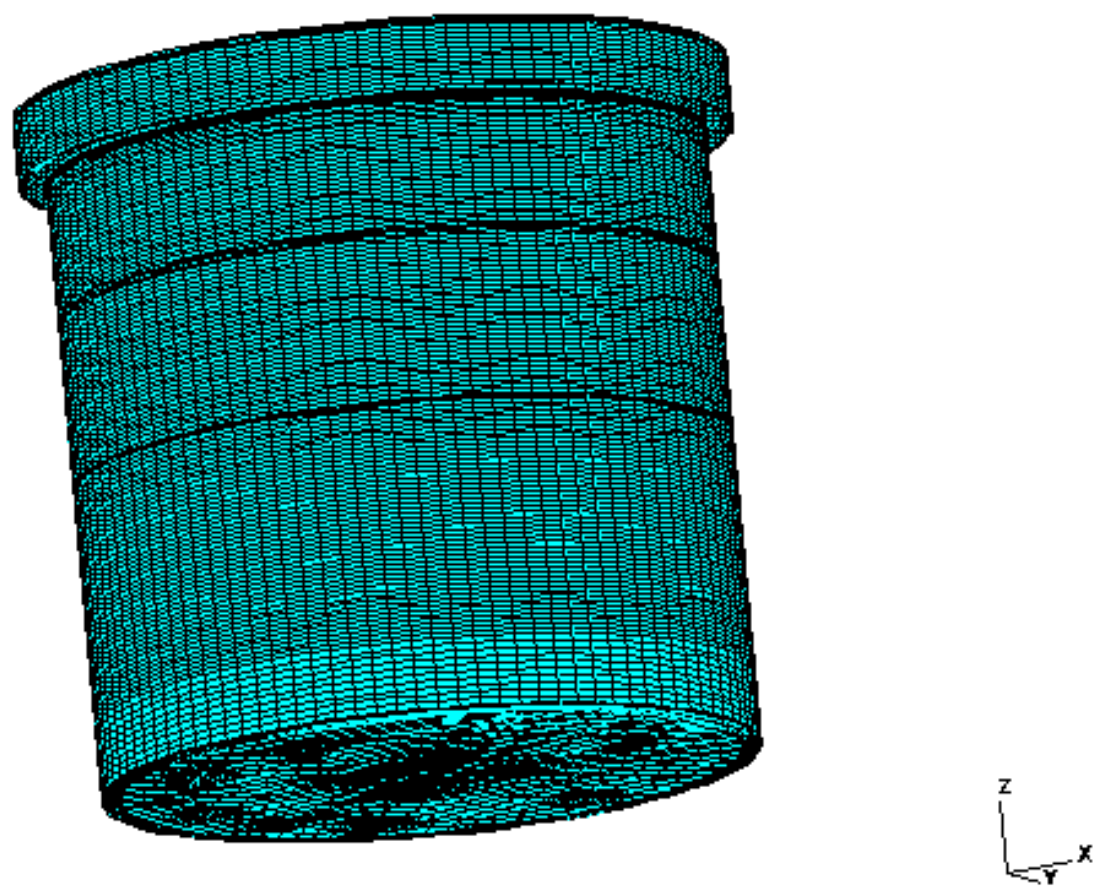

Figure III.3-3 Reactor vessel 

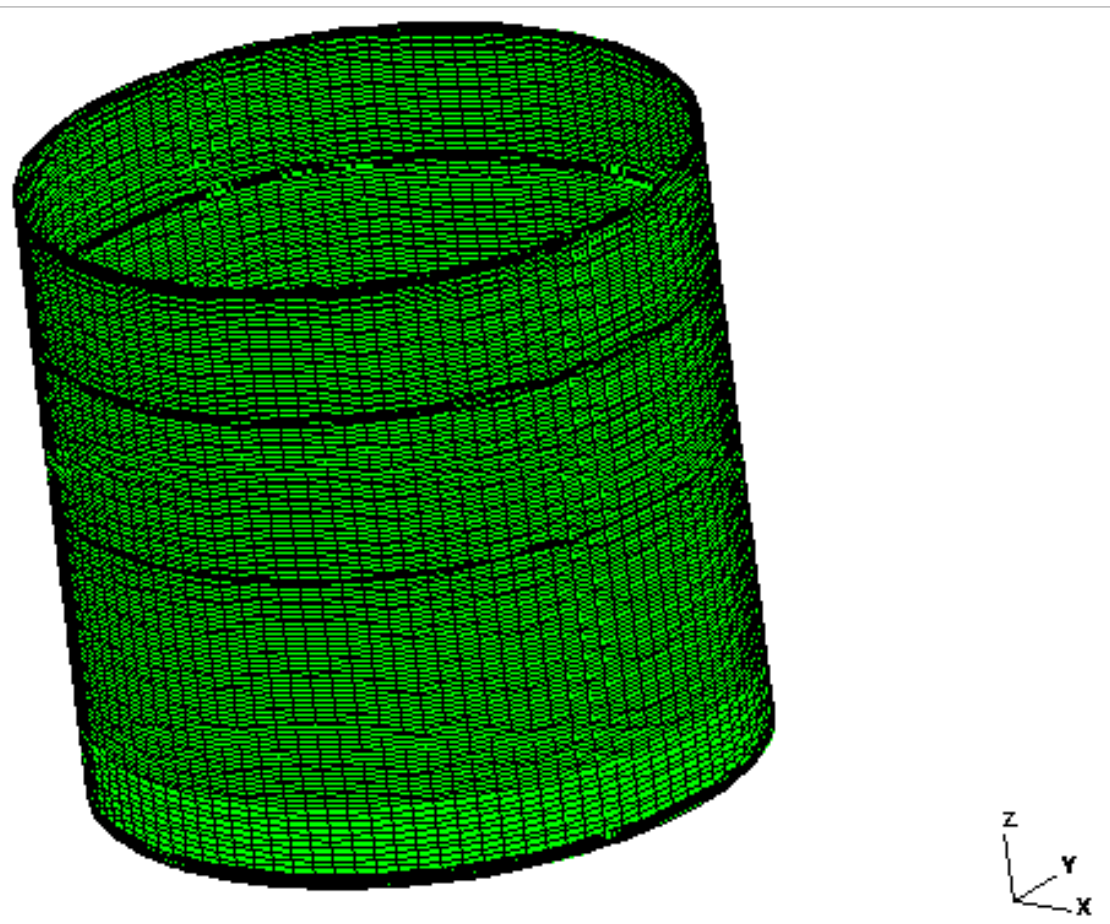

Figure III.3-4 Guard vessel

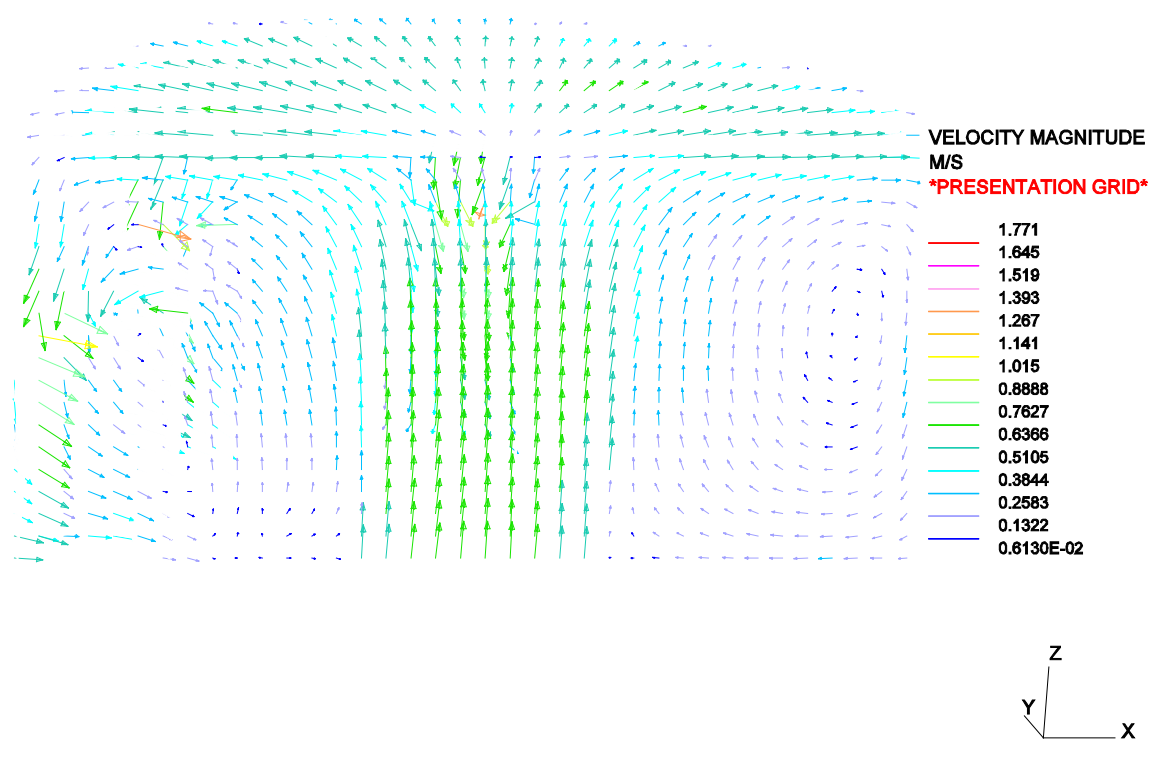

Figure III.3-5 Sodium velocity in the hot pool. 


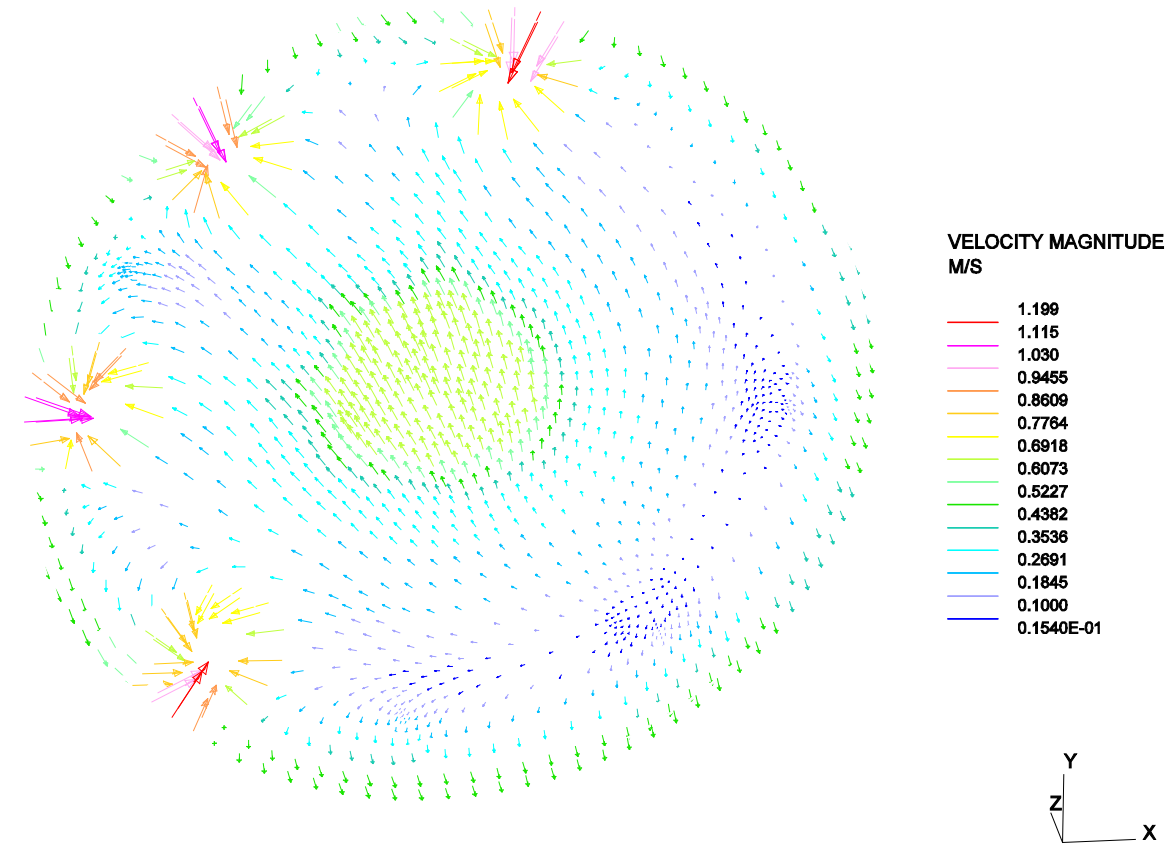

Figure III.3-6 Sodium velocity on a horizontal plane passing through the inlet of the IHXs

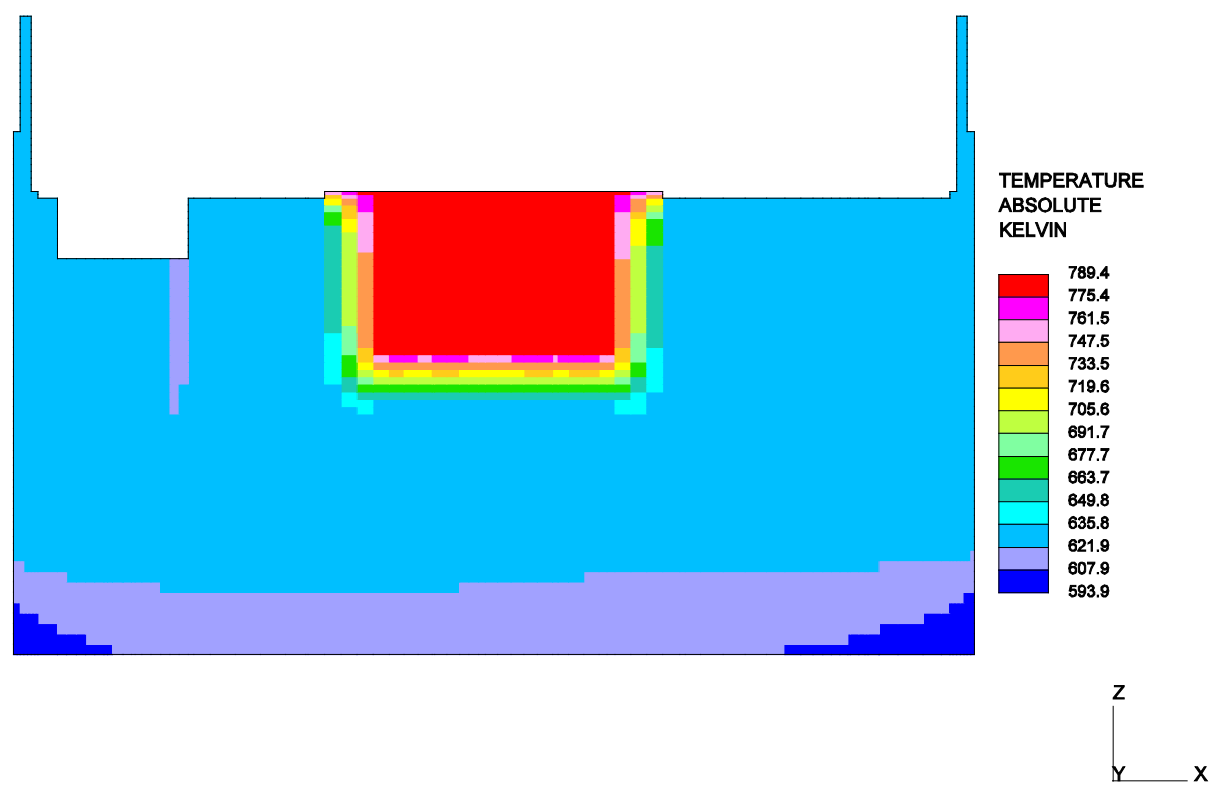

Figure III.3-7 Temperature distribution in cold pool and reactor 


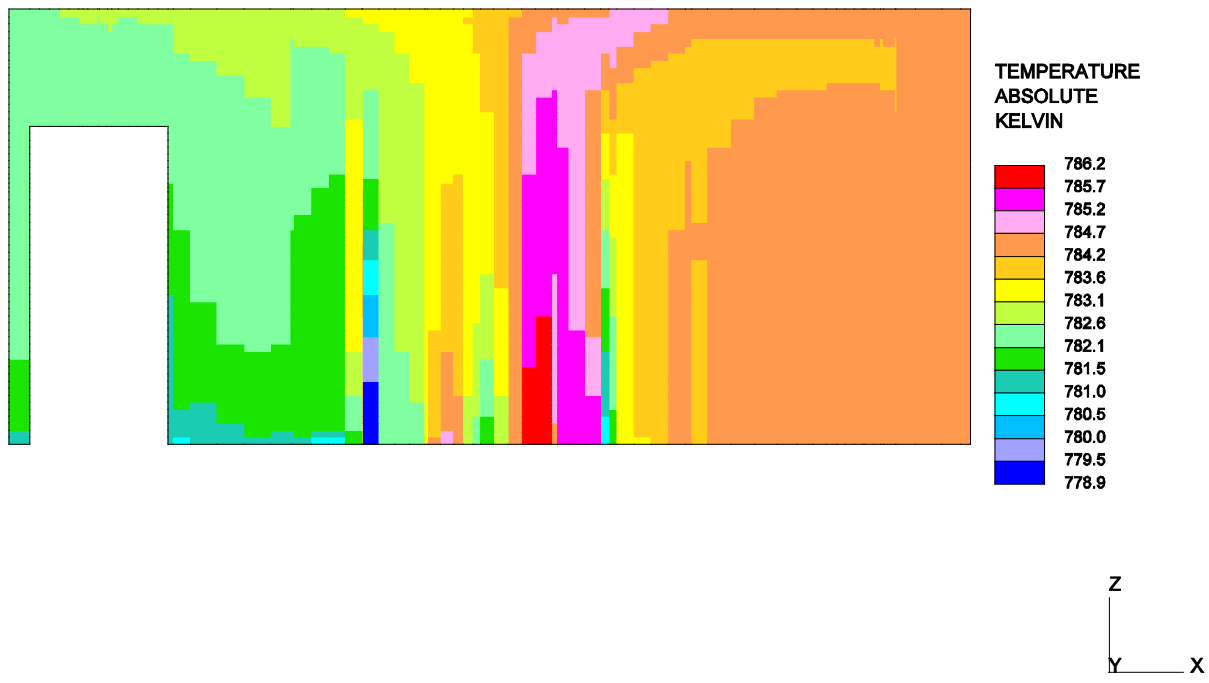

Figure III.3-8 Hot pool temperature distribution
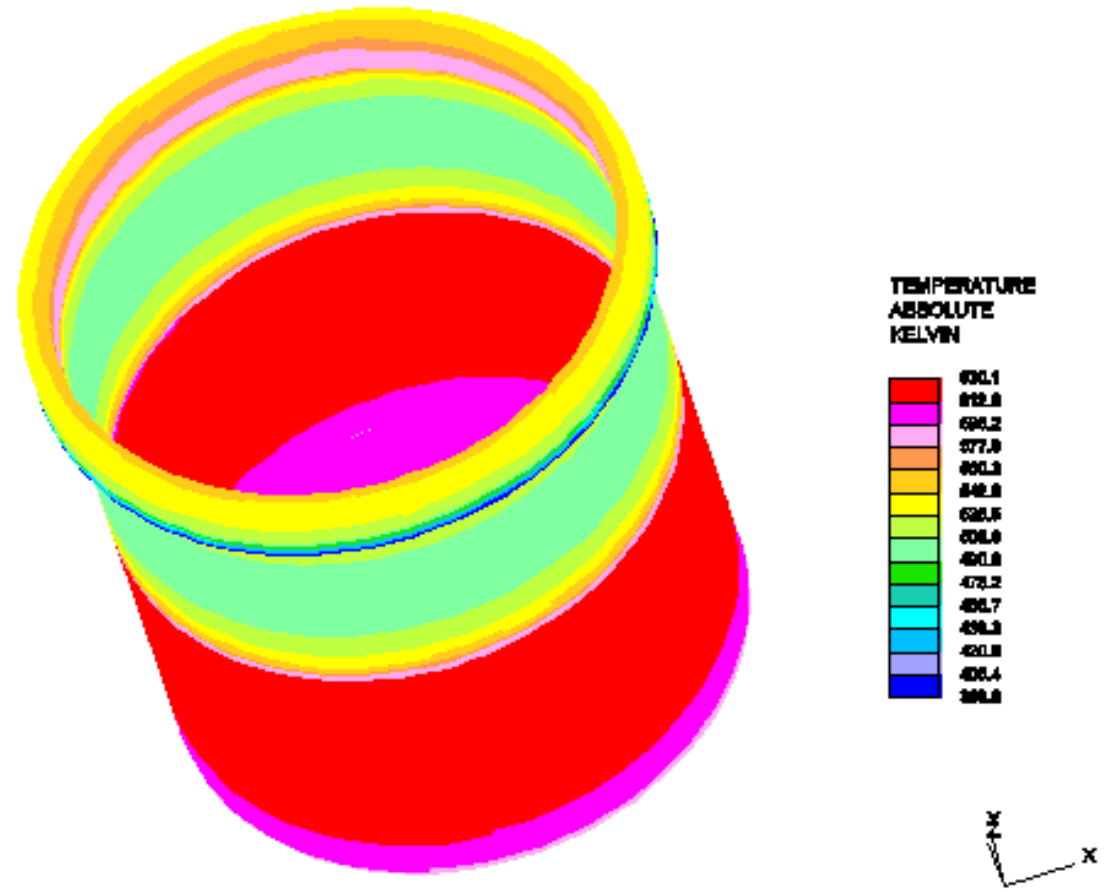

Figure III.3-9 Reactor vessel temperature 

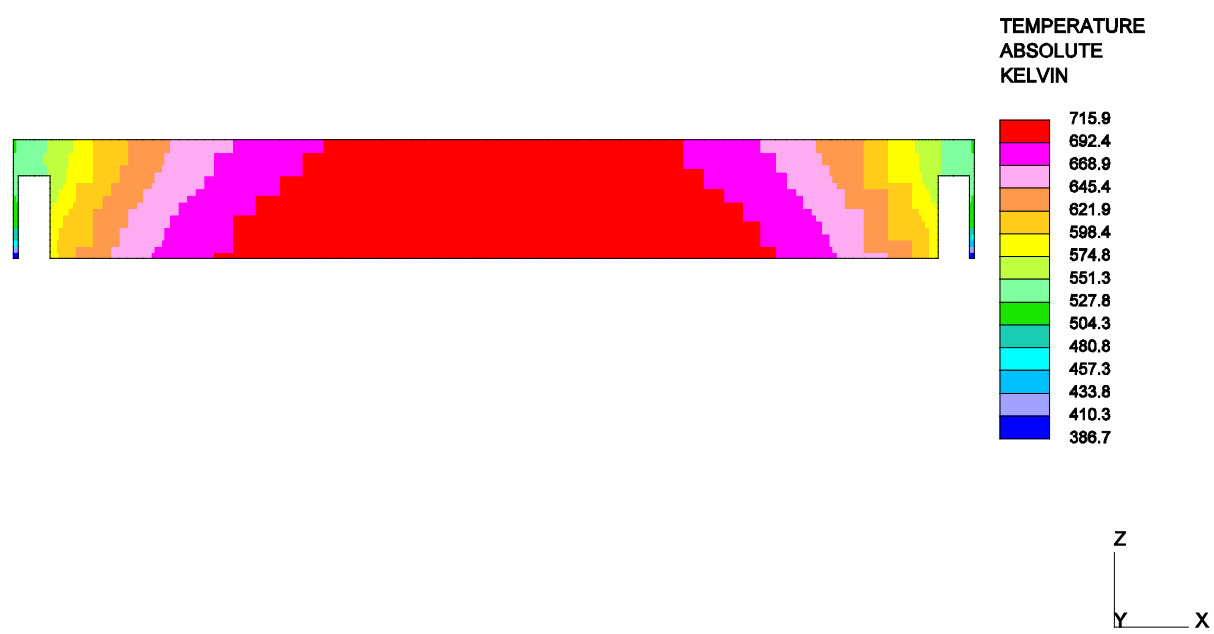

Figure III.3-10 Temperature distribution in reactor cover
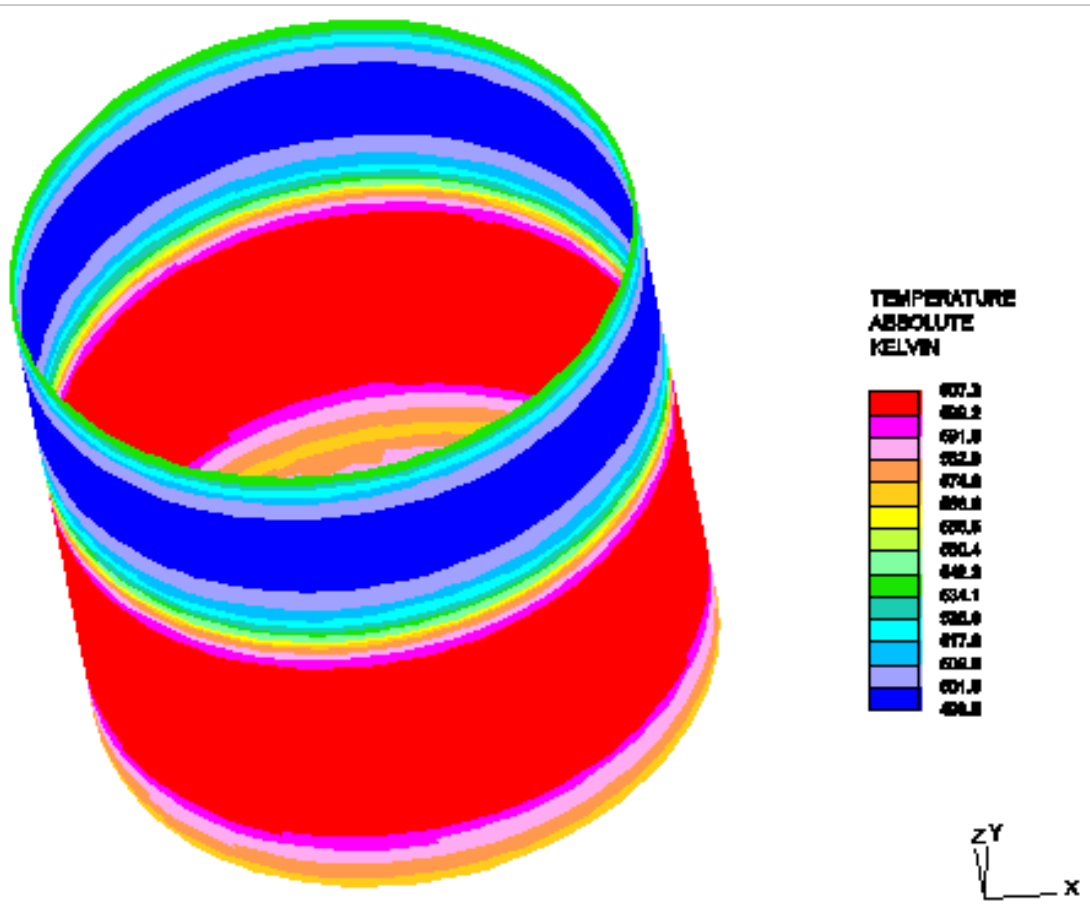

Figure III.3-11 Guard vessel temperature 

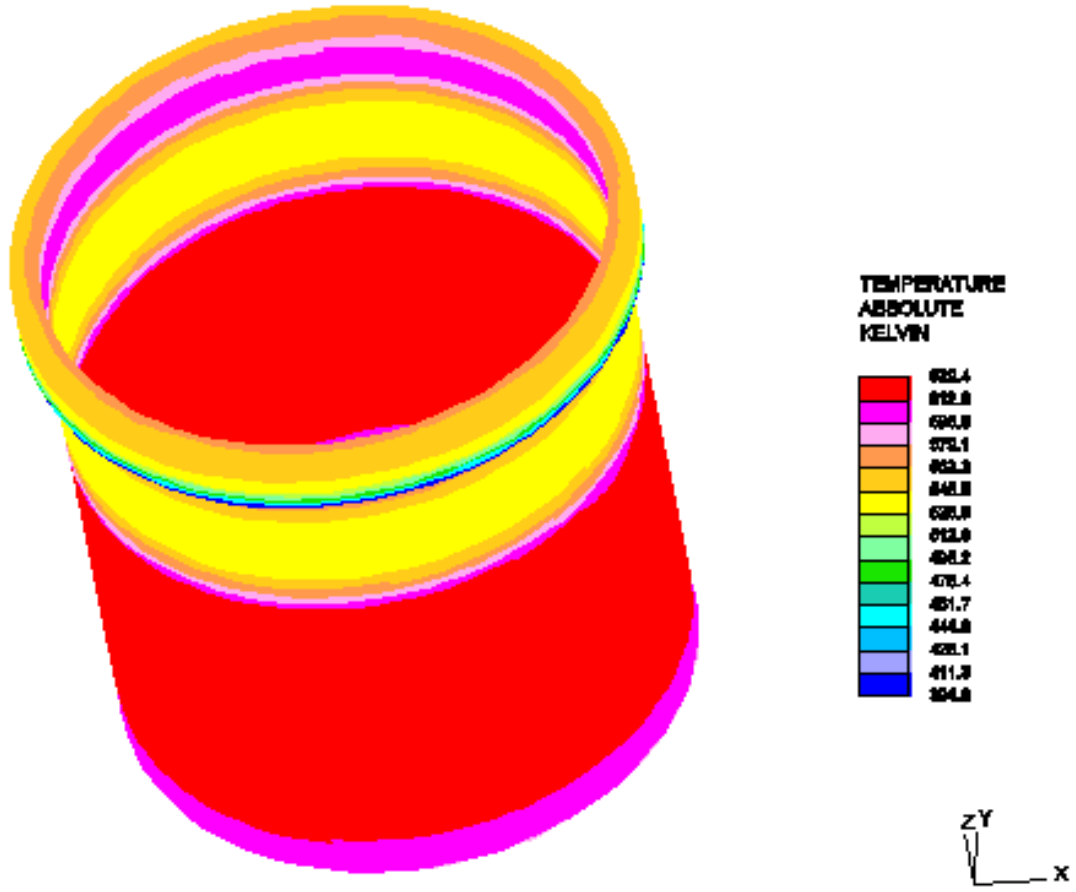

Figure III.3-12 Reactor vessel temperature - no insulation

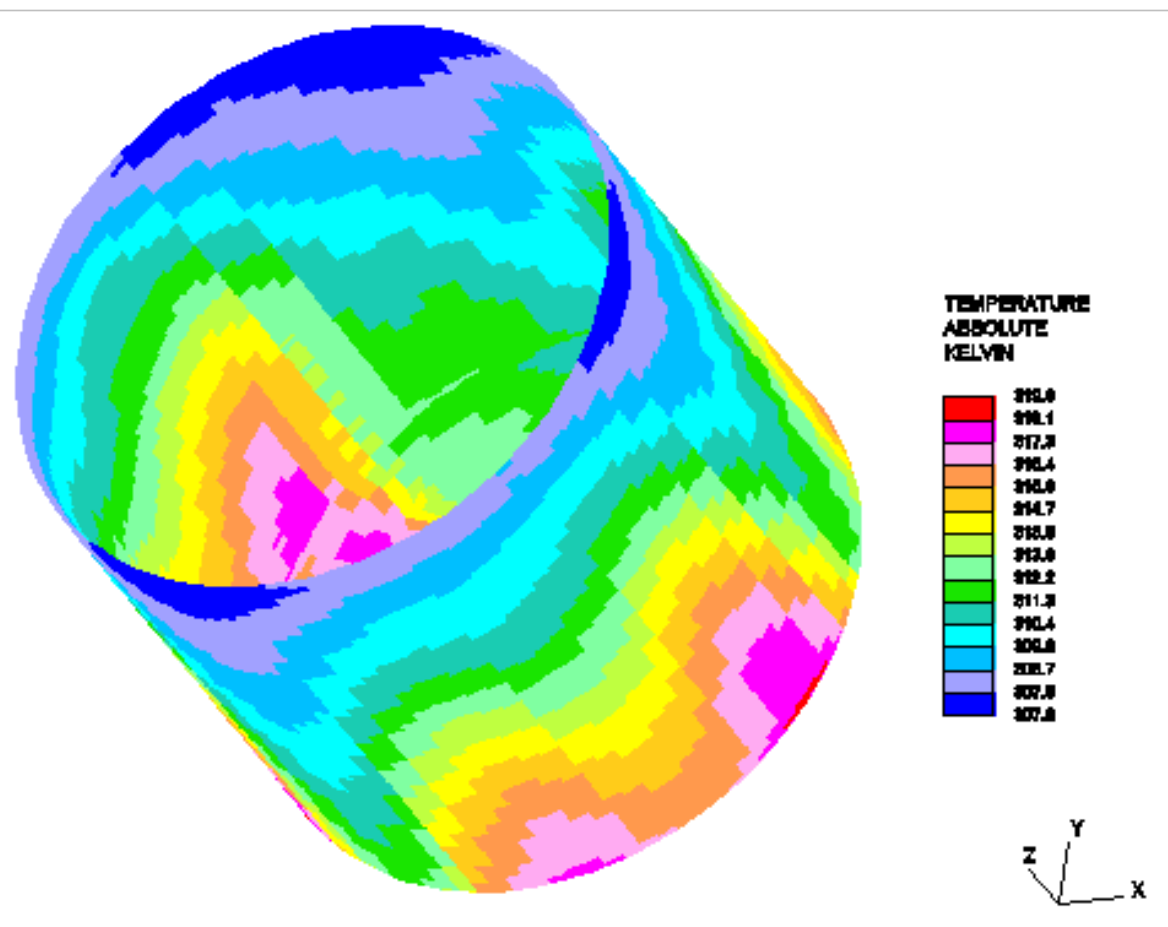

Figure III.3-13 Concrete liner temperature 


\section{III.4 Shielding Analysis}

\section{III.4.1 Evaluation of Dose Rate in Reactor Building}

To determine the biological shield thickness of the reactor closure, the radiation dose rate in the reactor building of the ABR plant was evaluated. The reference ABR core concepts of 1000 MWth power rating have been developed using ternary metal (U-TRU-10Zr) and mixed oxide $\left(\mathrm{UO}_{2}+\mathrm{TRUO}_{2}\right)$ fuels and the detailed design description and core performances parameters are provided in Section II.1. Two core concepts were developed for both metal and oxide cores: startup and TRU recycled equilibrium cores. The weapons-grade plutonium was used as the TRU feed of the startup core without recycling the spent fuel, while TRUs recovered from the ABR and LWR spent fuels were used as the primary and makeup TRU feeds for the recycled core, respectively. Among these core concepts, the recycled metal core was used for the dose rate calculations expecting a conservative result from high flux level and TRU enrichment. It is noted that the average core flux of the reference ABR metal core is $~ 17 \%$ higher than that of the oxide core and the TRU enrichment is about $22 \%$.

The typical maximum permissible radiation dose rates are provided in Table III.4-1, which were determined using the constraints for the biological shield of reactor personnel stated in the Code of Federal Regulations (10CFR20 and 10CFR50). For example, the maximum permissible radiation dose rate of $2.5 \mathrm{mrem} / \mathrm{hr}$ in the reactor building, which is classified as Zone-II, was determined from the maximum radiation dose rate of 100 mrem for a 40-hour work per week. It is noted that the measured radiation dose rate in the EBR-II reactor building above the reactor closure with the reactor operation at $72 \%$ full power rating (45 MW) is $0.4 \mathrm{mrem} / \mathrm{hr}$ [1]. For the $\mathrm{ABR}$ plant, the target radiation dose rate in the reactor building above the reactor closure was decided to be less than $0.1 \mathrm{mrem} / \mathrm{hr}$ during a full power operation.

Table III.4-1 Maximum permissible Radiation Dose Rates

\begin{tabular}{|l|l|l|l|}
\hline $\begin{array}{l}\text { Zone } \\
\text { Class }\end{array}$ & Zone description & $\begin{array}{l}\text { Weekly } \\
\text { Access time } \\
\text { (hr) }\end{array}$ & $\begin{array}{l}\text { Criteria } \\
\text { (mrem/hr) }\end{array}$ \\
\hline I & Unrestricted area, routinely occupied & Unlimited & $<0.25$ \\
\hline II & Restricted area, non-routinely occupied & 40 & $<2.5$ \\
\hline III & Radiation area, limited access for routine task & 6 & $<15$ \\
\hline IV & Radiation area, access for specific non-routine task & 1 & $<100$ \\
\hline
\end{tabular}

Figure III.4-1 shows the primary system of the reference $1000 \mathrm{MWt}$ ABR plant. The reference ABR core consists of 180 driver fuel assemblies, 114 reflectors, 66 shields, 15 primary control assemblies and 4 secondary control assemblies. The equivalent diameters of the active core and core barrel are $2.39 \mathrm{~m}, 3.47 \mathrm{~m}$, respectively. The in-vessel storage is located between the core barrel and redan: there are 66 positions for the interim storage of the fresh and spent fuels. By assuming structural occupation of $20 \%$ in the primary vessel, the primary vessel contains coolant sodium of 1309 MT with the radius of $7.03 \mathrm{~m}$ and height of about $13.25 \mathrm{~m}$. The average primary coolant flow rate is $\sim 5080 \mathrm{~kg} / \mathrm{sec}$, and the primary coolant circulation time is less than few minutes [2]. 


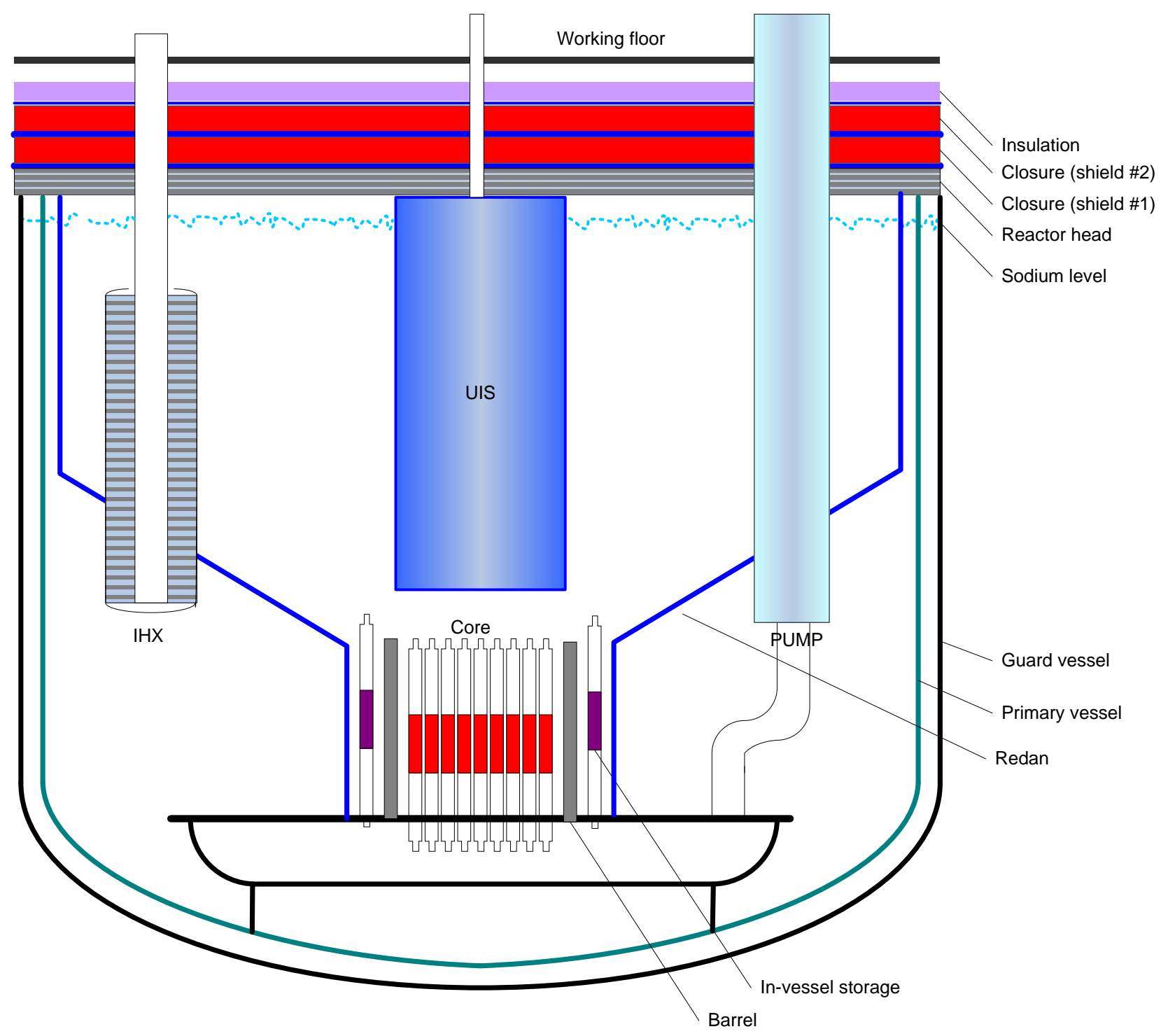

Figure III.4-1 Schematics of 1000 MWth ABR Reactor

The primary vessel is covered by a reactor head which is composed by multiple steel plates filling insulating material between the plates. The reactor head thickness was designed to be 61 $\mathrm{cm}$ with 22 steel plates. However, the reactor head was ignored in the dose rate calculation because the detailed design parameters of the reactor head have not been fixed [3]. There are two closures are designed for the biological shield of the reactor building. The reactor closure is made with steel balls. The volume fraction of steel balls in the shield was determined to be $60 \%$, based on the theoretical maximum volume fraction of $\sim 68 \%$ for a body centered cubic (BCC) distribution. The total thickness of the lower and upper closures was determined to be $\sim 107 \mathrm{~cm}$ in the recent ABR plant design. A thermal insulation of $\sim 50 \mathrm{~cm}$ thickness is covered the reactor closure. Three steel plates are located between the reactor head, the lower closure, the upper closure, and the insulation. The total thickness of these steel plates is $\sim 7.0 \mathrm{~cm}$. 
Sodium in the primary system is activated during a reactor operation and the activation products are the primary radiation sources to be shielded by a biological shield. Natural sodium is composed entirely by Na-23 and reactions of $(n, \gamma)$ and $(n, 2 n)$ produce radioactive Na-24 and Na-22 isotopes, respectively. Na-24 decays with 14.95 hour half-life and emits both $1.37 \mathrm{MeV}$ and $2.75 \mathrm{MeV}$ photons. Na-22 has a relatively longer half-life of 2.60 year and emits $1.27 \mathrm{MeV}$ photon. Thus, Na-24 is the dominant activation product during a reactor operation, while Na-22 becomes dominant after 10 days following a reactor shutdown. By assuming that the primary coolant is activated in the activating zone (i.e., core and vicinity) and mixed up in the primary vessel, the equilibrium activity per unit volume of the primary coolant is determined as

$$
S_{x}=R_{x} \frac{\left(1-e^{-\lambda_{x} t_{1}}\right)}{\left(1-e^{-\lambda_{x} t_{2}}\right)} \cong R_{x} \frac{m_{\text {activation }}}{m_{\text {total }}}
$$

where

$$
\begin{aligned}
& x=\text { reaction type, } \\
& R_{x}=\text { reaction rates }\left({ }^{\Sigma_{x} \phi}\right), / \mathrm{cm}^{3} \text {-sec, } \\
& \lambda_{x}=\text { decay constant of activated sodium, /sec, } \\
& t_{1}=\text { transit time in activating zone, sec, } \\
& t_{2}=\text { circulation time of primary coolant, sec, } \\
& m_{\text {activation }}=\text { flowing sodium mass in activating zone, } \mathrm{kg}, \\
& m_{\text {total }}=\text { circulating sodium inventory of primary system, } \mathrm{kg} .
\end{aligned}
$$

It is noted that the equilibrium sodium activity of Eq (1) is simplified by the reaction rate multiplied by the sodium mass ratio between flowing sodium in the activating zone and sodium inventory of the primary system because the primary coolant circulation time is much shorter than the half-life of the activated sodium isotopes. Eq (1) indicates that the sodium activity is dependent on the flux level (i.e., reaction rate) and the primary system sodium inventory. Reference 4 indicates that the calculated specific activity of the CRBRP primary coolant is about $\sim 30 \mathrm{Ci} / \mathrm{kg}$ based on the primary system sodium inventory of $\sim 640 \mathrm{MT}$ and the core average flux level of $\sim 3.6 \times 10^{15} / \mathrm{cm}^{2}$-sec.

The activating zone in the ABR plant was determined from the radial and axial flux distributions at the equilibrium cycle shown in Figures III.4-2 and III.4-3, respectively. The fluxes were obtained from the TWODANT calculations using a RZ geometry model: the inside of the core barrel and in-vessel storage were modeled in detail, but major components outside core were simplified. It was assumed conservatively that 60 locations of the in-vessel storages were occupied by the spent fuels. The flux levels of the reflectors and gas plenum are much smaller than that of the active core, but sufficiently high to activate the sodium. Thus, the reflector and gas plenum were included into the activating zone. For the conservative estimation of the dose rate in the reactor building, however, the average core flux of beginning (BOEC) and end of equilibrium cycle (EOEC) was used as the flux level of the activating zone.

The calculated flowing sodium mass in the activating zone is $\sim 740 \mathrm{~kg}$ with the coolant volume fraction of $36 \%$. The stagnation sodium fraction in the primary system was assumed to be about $5 \%$, which results in the circulating primary sodium inventory of 1243 MT. The core 
average fluxes and neutron-sodium reaction rates in the activating zone were also obtained from the TWODANT calculations. Table III.4-2 provides the estimated sodium activities in the

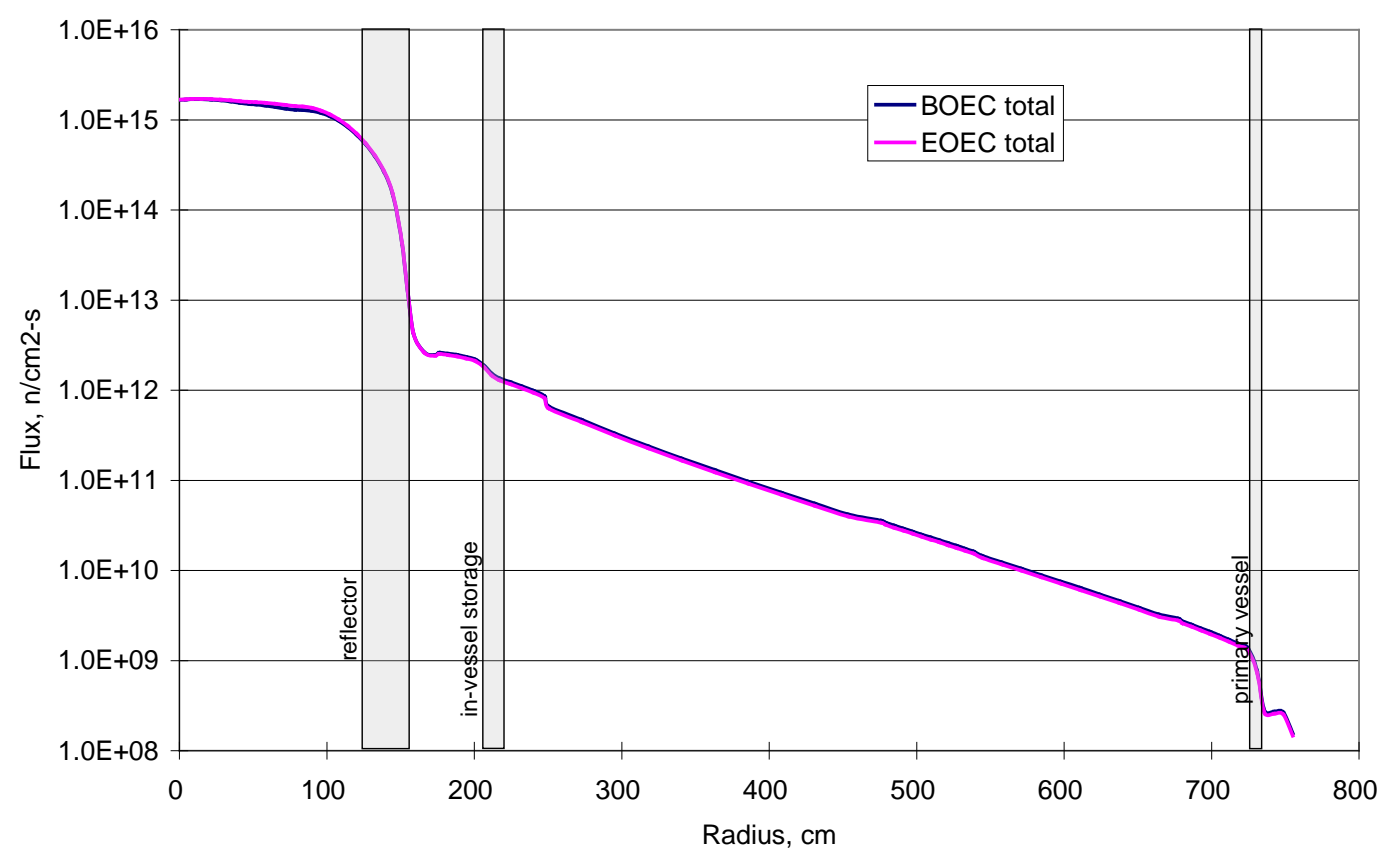

Figure III.4-2 Radial Flux Distributions at Mid-plane of Core

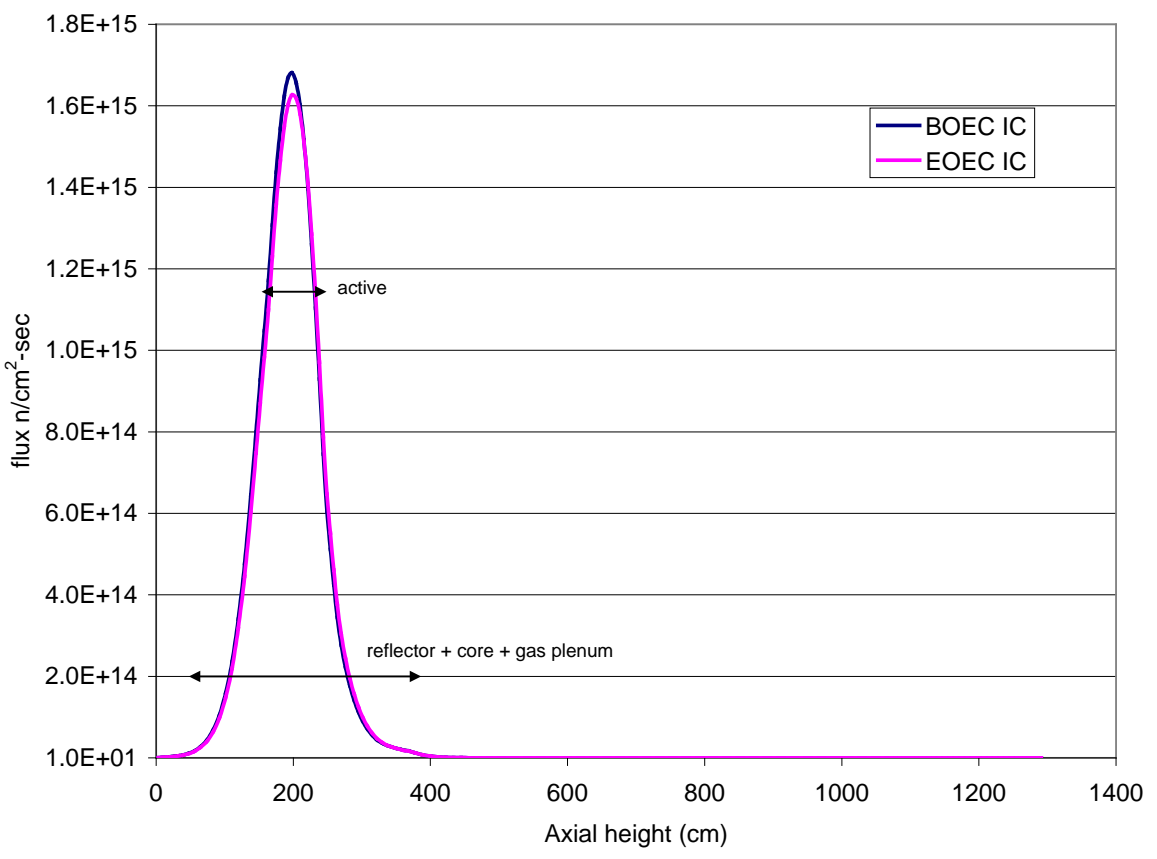

Figure III.4-3 Axial Flux Distributions at Inner Core 
Table III.4-2 Equilibrium Sodium Activities of Primary Sodium

\begin{tabular}{|c|c|c|c|c|}
\hline & \multicolumn{2}{|l|}{ ABR } & \multicolumn{2}{|c|}{ CRBRP [4] } \\
\hline Power rating, MWt & \multicolumn{2}{|l|}{1000} & \multicolumn{2}{|l|}{975} \\
\hline Primary sodium inventory, MT & \multicolumn{2}{|l|}{1309} & \multicolumn{2}{|l|}{640} \\
\hline \multirow[t]{2}{*}{ Core average flux, $10^{15} / \mathrm{cm}^{2}$-sec } & \multicolumn{2}{|l|}{3.3} & \multicolumn{2}{|l|}{3.6} \\
\hline & $\mathrm{Na}-24$ & Na-22 & $\mathrm{Na}-24$ & Na-22 \\
\hline Volumetric activity, $\mathrm{mCi} / \mathrm{cm}^{3}$ & 9.5 & $9.3 \mathrm{E}-4$ & 25.1 & $1.9 \mathrm{E}-3$ \\
\hline Specific activity, Ci $/ \mathrm{kg}$ & 11.2 & $1.1 \mathrm{E}-3$ & 29.6 & $2.3 \mathrm{E}-3$ \\
\hline
\end{tabular}

primary system of the ABR plant. For comparison, the corresponding values of CRBRP plant are also provided in Table III.4-2. Compared to the CRBRP, the flux level of the ABR core is smaller by $9 \%$ and the primary sodium inventory is doubled. As a results, the specific activity of primary sodium of the ABR core is smaller than that of the CRBRP $(11.3 \mathrm{Ci} / \mathrm{kg}$ versus 29.6 $\mathrm{Ci} / \mathrm{kg}$ ). The photon dose rate in the reactor building was estimated using a so-called "disc slab geometry with constant volume source" [5] model shown in Figure III.4-4.

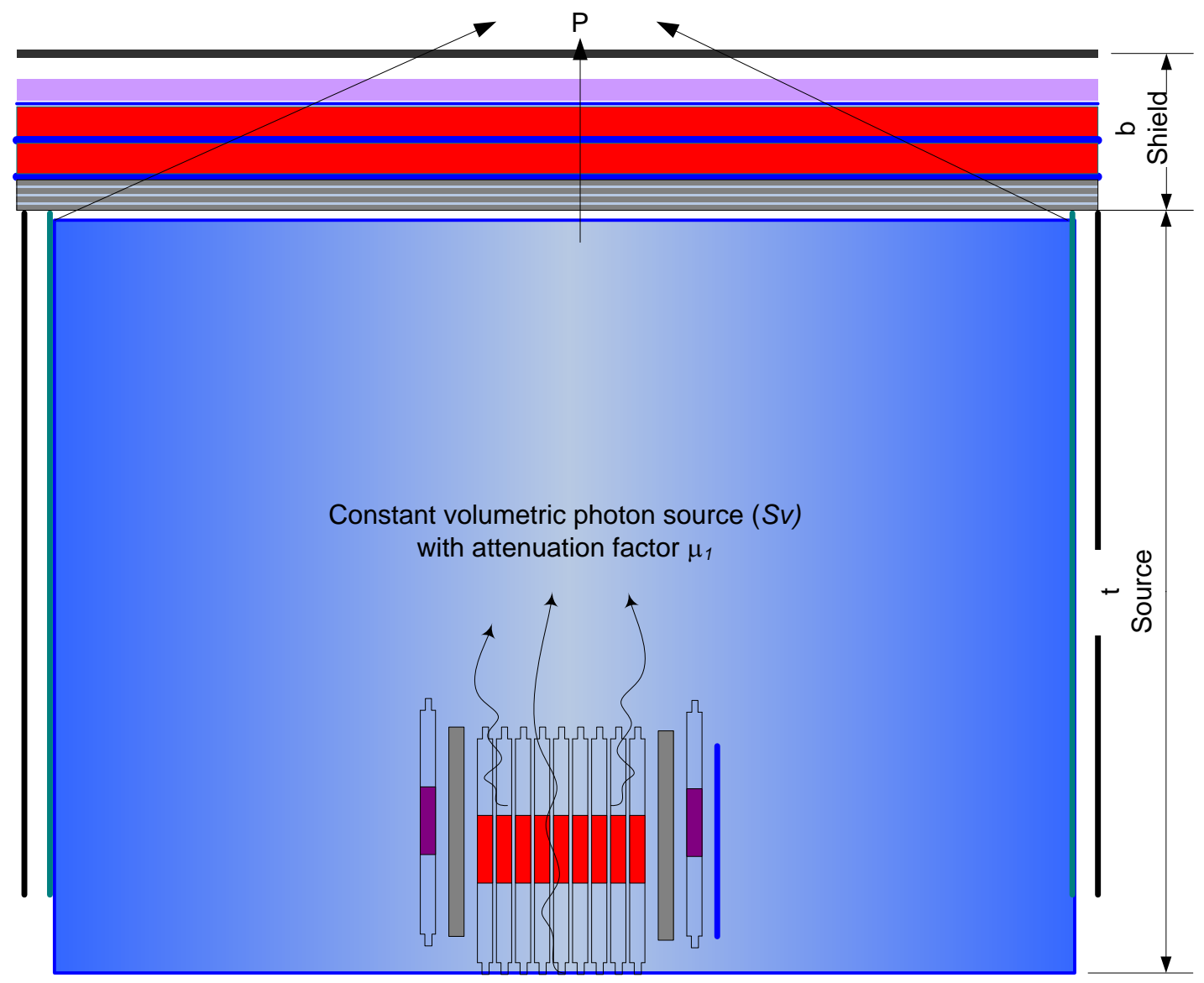

Figure III.4-4 Disc Slab Model for Dose Calculation in Reactor Building 
A disc slab of thickness $t$ contains a uniform volumetric photon source, and it is shielded by a disc slab of thickness $b$. The volumetric photon source was obtained form the equilibrium primary sodium activity of Eq (1). Since the radius of the primary vessel is much larger than the thickness of biological shield, the problem can be simplified to be infinite slab geometry with a constant volume source. Then, the photon flux at the center point on the floor of the reactor building is determined as:

$$
\phi \cong \frac{S_{x}}{2 \mu_{1}} B \cdot E_{2}\left(\mu_{2} b\right) \text {, }
$$

where

$$
\begin{aligned}
& S_{x}=\text { volumetric photon source, } / \mathrm{cm}^{3} \text {-sec, } \\
& B=\text { overall build-up factor in shield, } \\
& E_{2}=\text { second order exponential integral function, } \\
& \mu_{1}=\text { photon attenuation factor of sodium, /cm, } \\
& \mu_{2}=\text { effective photon attenuation factor of shield, } / \mathrm{cm} .
\end{aligned}
$$

The attenuation factors of the sodium and shielding materials are provided in Table III.4-3, and the overall build-up factor was assumed conservatively to be 20 based on the build-up factors provided in Ref. 6. The calculated radiation dose rates are provided in Table III.4-4, including the contribution of the neutron dose rates. It is noted that the neutron dose rate in the reactor building was estimated using the neutron flux obtained from the TWODANT calculations. The flux-to-dose conversion factors of the ANSI/ANS-6.1.1-1997 [6] were used to obtain the dose rate from the calculated photon and neutron fluxes.

Table III.4- 3 Material Data for Shielding Calculations

\begin{tabular}{|l|l|l|l|l|}
\hline \multirow{2}{*}{ Region } & \multirow{2}{*}{$\begin{array}{l}\text { Material } \\
\text { (volume fraction) }\end{array}$} & Density, g/cm & Attenuation factor, /cm \\
\cline { 4 - 5 } & & $\begin{array}{l}1.27 \text { or } 1.37 \\
\mathrm{MeV}\end{array}$ & $2.75 \mathrm{MeV}$ \\
\hline Primary coolant & Sodium & 0.85 & 0.043 & 0.031 \\
\hline Steel plates & Iron & 7.86 & 0.384 & 0.294 \\
\hline Reactor closure & Steel balls (60\%) & 4.72 & 0.230 & 0.176 \\
\hline
\end{tabular}

Table III.4- 4 Radiation Dose Rate on Floor of Reactor Building

\begin{tabular}{|l|l|l|l|l|l|l|}
\hline \multicolumn{2}{|l|}{ Thickness, cm } & \multicolumn{5}{l|}{ Dose rate (mrem/hr) } \\
\hline $\begin{array}{l}\text { Steel } \\
\text { plates }\end{array}$ & $\begin{array}{l}\text { Reactor } \\
\text { closure }\end{array}$ & $\begin{array}{l}1.27 \mathrm{MeV} \\
\text { Photon }\end{array}$ & $\begin{array}{l}1.37 \mathrm{MeV} \\
\text { Photon }\end{array}$ & $\begin{array}{l}2.75 \mathrm{MeV} \\
\text { Photon }\end{array}$ & Neutron & Total \\
\hline 0.0 & 0.0 & $2.23 \mathrm{E}+03$ & $1.04 \mathrm{E}+07$ & $1.43 \mathrm{E}+07$ & $2.80 \mathrm{E}-01$ & $2.47 \mathrm{E}+07$ \\
\hline 0.0 & 107 & $2.20 \mathrm{E}-08$ & $1.64 \mathrm{E}-04$ & 0.1 & N/A & 0.1 \\
\hline 7.0 & 107 & $1.37 \mathrm{E}-09$ & $1.02 \mathrm{E}-05$ & $1.28 \mathrm{E}-02$ & N/A & 0.01 \\
\hline
\end{tabular}

a) Not available. 
The dose rate on the surface of the primary coolant is significantly high $(\sim 2.5 \mathrm{E}+07 \mathrm{mrem} / \mathrm{hr})$, but it decreases below the target value of $0.1 \mathrm{mrem} / \mathrm{hr}$ by introducing the $107 \mathrm{~cm}$ thick biological shield. The steel plate of $7.0 \mathrm{~cm}$ thickness reduces radiation dose rate by $89 \%$, additionally. It is noted that $2.75 \mathrm{MeV}$ photon emitted from Na-24 is the dominant contributor of the radiation dose in the reactor building during the reactor operation

\section{III.4.2 In-Vessel Shielding Analysis}

Preliminary in-vessel shielding calculation for the reference 1000 MWt ABR core concept was performed to estimate the fission power of spent ABR fuels in the in-vessel spent fuel storage and the neutron irradiation damage of the in-vessel structures. For a conservative estimation, the recycled equilibrium metal core was used in the in-vessel shielding analysis. Using the TWODANT transport code, the neutron flux distributions were calculated for the BOEC and EOEC configurations. A cylindrical- $Z$ geometry model and $\mathrm{S}_{16}$ angular discretization were used. For neutron damage calculations, 28-group dpa (displacement per atom) cross sections were generated using the NJOY code based on the ENDF/B-VI data. The time integrations were evaluated using the average values of BOEC and EOEC fluxes.

In this preliminary analysis, the inside of the core barrel was modeled in details, but only major components were modeled outside the core barrel. The core barrel is made of SS-316, and its radius and thickness are $174 \mathrm{~cm}$ and $2.54 \mathrm{~cm}$, respectively. The in-vessel spent fuel storage is located between core barrel and redan for 69 spent fuel assemblies. It was assumed that 60 storage positions were occupied by the ABR spent fuel assemblies. Primary pumps and intermediate heat exchangers (IHX) are radially located at $490 \mathrm{~cm}$ from the core center and elevated to $484 \mathrm{~cm}$ from the bottom of reactor. They are modeled by homogeneous mixtures of SS-316 steel and sodium. The steel volume fractions of the pump and IHX are assumed to be $37.2 \%$ and $10.7 \%$, respectively. Direct reactor auxiliary cooling system (DRACS) and other internal structures were not modeled. The primary vessel is made of SS-316, and its radius and thickness are about $703 \mathrm{~cm}$ and $5.1 \mathrm{~cm}$, respectively. The radius and thickness of the guard vessel are about $731 \mathrm{~cm}$ and $2.5 \mathrm{~cm}$, respectively, and it is made of SS-304.

Table III.4-5 provides the region-wise power distribution determined from the neutronics calculations only, in which the gamma energy is locally deposited at the position of its creation. Most of the power is generated inside the core, but non-negligible amount of power is generated outside the core. The spent fuel assemblies located in the in-vessel storage generates $\sim 0.1 \%$ of the rated power. The fission power generated by each spent fuel assembly is $\sim 14 \mathrm{~kW}$.

The neutron irradiation damage of the in-vessel structures is the loss of ductility and fracture resistance due to the atom displacement within the material. The dpa is used to measure the neutron irradiation damage instead of the traditional fluence calculations, since it provides a simpler correlation with ductility that is generally only material dependent. It is recommended to ensure that the neutron fluence on the reactor vessel and core support structure during its service life does not reduce the residual total elongation of the material below 10\%. For SS-316, based on the presently available data, this condition corresponds to $\sim 5 \mathrm{dpa}$. 
Table III.4- 5 Region-wise Power Distribution of Reference ABR Design

\begin{tabular}{|l|l|l|l|l|l|}
\hline & & Inner core & Outer core & Spent fuel & Other \\
\hline \multirow{2}{*}{ Power (MW) } & BOEC & 439.8 & 538.7 & 0.9 & 20.5 \\
\cline { 2 - 6 } & EOEC & 446.4 & 533.4 & 0.8 & 19.4 \\
\hline \multirow{2}{*}{$\begin{array}{l}\text { Power fraction } \\
(\%)\end{array}$} & BOEC & 44.0 & 53.9 & 0.1 & 2.1 \\
\cline { 2 - 6 } & EOEC & 44.6 & 53.3 & 0.1 & 2.0 \\
\hline $\begin{array}{l}\text { Power per } \\
\text { assembly(MW) }\end{array}$ & BOEC & 5.64 & 5.28 & 0.01 & \\
\cline { 2 - 6 } $\begin{array}{l}\text { Peak power } \\
\text { density }\left(\mathrm{W} / \mathrm{cm}^{3}\right)\end{array}$ & EOEC & 5.72 & 5.23 & 0.01 & \\
\cline { 2 - 6 } & EOEC & 394.5 & 401.8 & 2.1 & \\
\hline
\end{tabular}

The neutron irradiation damages of the in-vessel structures are summarized in Table III.4-6, which were calculated for an assumed life time of 30 years with $90 \%$ capacity factor. As can be seen, the peak dpa occurs at core barrel and the dpa of the grid plate is lower that that of the core barrel. This peak value is $2.44 \mathrm{dpa}$. The peak fast fluence of the core barrel $\left(6.41 \times 10^{21} \mathrm{n} / \mathrm{cm}^{2}\right)$ is about $58 \%$ of the peak fast fluence of the grid plate of the ABTR design $\left(\sim 1.1 \times 10^{22} \mathrm{n} / \mathrm{cm}^{2}\right)$ [8]. These results suggest that the irradiation damages of grid plate and core barrel are well within the limits. The irradiation damages of primary vessel and guard vessel appear to be negligible.

Table III.4- 6 In-Vessel Structure Irradiation Damage

\begin{tabular}{|l|l|l|l|l|l|}
\hline & & Grid plate & Core barrel & $\begin{array}{l}\text { Primary } \\
\text { vessel }\end{array}$ & $\begin{array}{l}\text { Guard } \\
\text { vessel }\end{array}$ \\
\hline \multirow{2}{*}{$\begin{array}{l}\text { Fast fluence } \\
\left(\mathrm{n} / \mathrm{cm}^{2}\right)\end{array}$} & Average & $1.88 \mathrm{E}+20$ & $7.75 \mathrm{E}+20$ & $2.95 \mathrm{E}+12$ & $1.41 \mathrm{E}+12$ \\
\cline { 2 - 6 } & Peak & $1.44 \mathrm{E}+21$ & $6.41 \mathrm{E}+21$ & $1.55 \mathrm{E}+13$ & $7.31 \mathrm{E}+12$ \\
\hline \multirow{2}{*}{$\mathrm{dpa}$} & Average & 0.13 & 0.30 & $5.51 \mathrm{E}-07$ & $1.18 \mathrm{E}-07$ \\
\cline { 2 - 6 } & Peak & 0.99 & 2.44 & $2.89 \mathrm{E}-06$ & $6.14 \mathrm{E}-07$ \\
\hline
\end{tabular}

\section{References}

1. Argonne National Laboratory, unpublished information, (1991).

2. Private communication with Mitchell Farmer

3. Private communication with Chris Grandy

4. A. E. Walter, A. B. Reynolds, “Fast Breeder Reactors,” Pergamon Press (1981).

5. J. Wood, “Computational Methods in Reactor Shielding,” Pergamon Press (1982).

6. “Reactor Physics Constant,” ANL-5800, second edition, Argonne National Laboratory (1963).

7. “MCNP - A General Monte Carlo N-Particle Transport Code, Version 5,” LA-CP03-0245, Los Alamos National Laboratory (2003). 
8. Y. I. Chang, P. J. Finck, and C. Grandy, “Advanced Burner Test Reactor Preconceptual Design Report,” ANL-ABR-1, Argonne National Laboratory (Sept. 2006). 


\section{III.5 Response during Natural Circulation Transients}

The ABR primary system design is configured to provide natural circulation shutdown heat removal. The capability to remove shutdown decay heat with natural circulation provides a means to maintain reactor component temperatures at acceptable levels even in the event of loss of all off-site and emergency on-site power supplies. This section presents a discussion of the design features that contribute to this capability. A quantitative assessment of ABR emergency decay heat removal by natural circulation in a protected (i.e., with scram) loss of flow accident sequence is provided in Section III.7.

Liquid sodium and its alloys are excellent fluids for natural circulation heat removal because of their thermo-physical properties. Due primarily to its high thermal conductivity, liquid sodium is capable of very high convective heat transfer rates, even at the modest fluid velocities characteristic of natural circulation. This tends to minimize the temperature differences between the heat source and the fluid, and between the fluid and the heat sink, and to reduce the overall source-to-sink temperature difference required for natural circulation cooling.

Natural circulation flow arises due to the effect of gravity on a continuous fluid with a density difference along the elevation. Heavy fluid sinks to displace lighter fluid. Buoyancyinduced flow can be established when a fluid is heated, decreasing its density, at an axial position below the elevation at which the fluid is cooled, increasing its density. In a one-dimensional model, flow occurs when the buoyancy force is great enough to overcome form, friction, and shear losses. The natural circulation flow rate is regulated by the balance between the buoyancy force and the flow-related pressure losses. When the buoyancy force is provided by a thermallydriven density difference, the fluid flow rate will be determined by the fluid properties, the elevation difference between the heat sink and the heat source, and the temperature difference in the fluid between the heat source and the heat sink.

In a pool plant, the hot sodium pool is a large configuration. Sodium enters the hot pool at the radial center and at a vertical location which depends on the upper internals structure (UIS) design. Sodium leaves the hot pool by gravity flow into the IHX entrances. The locations of the IHXs determine what volume of the hot-pool sodium is contained between their radial locations and the center of the hot pool, the remainder being contained between the IHXs and the vertical thermal barriers. Past calculations indicate that the main part of the steady state flow pattern is complex in the region between the UIS and the ring of IHXs, especially after a reactor scram when the temperature of the sodium entering the hot pool can drop by approximately $250^{\circ} \mathrm{F}$ in a few seconds. The large diameter of the hot pool produces low velocities as the sodium flows outward, and the cooler, heavier post-scram sodium drops to the hot pool bottom before reaching the IHX. Thus only part of the hot pool sodium may be involved in the main flow pattern and there may be other flow cells in a higher temperature volume. There can also be recirculation flow patterns between the core and the plena. This, therefore involves the mixing of cold sodium, leaving the core after a scram, with hot-pool sodium above the core. But the pool plant has the large, hot-pool sodium volume which can mitigate thermal transients following reactor scram.

However, the temperature distributions in the large plenums of the reactor tank can be highly stratified. This means that the sodium temperature in the upper parts of the plenum will not be lower than the temperature below it. Where sodium flows into a plenum at a temperature higher 
than the local temperature of the plenum, it will rise in the plenum to a higher level where it is above all sodium having a lower temperature. A similar but opposite effect will occur when the sodium flows into a plenum at a lower temperature than the local plenum temperature. Even at full pump flow, the flow velocities in the plenums will be low, and stratification may be an important phenomenon.

During normal steady state reactor operation, the major plenums will remain essentially isothermal, with the lower plenum at the reactor inlet temperature and the upper plenum at reactor outlet temperature. After reactor shutdown, the core outlet temperature may be higher or lower than normal depending upon whether the flow decay is greater or less than the reactor power response. A higher outlet temperature due to undercooling of the core will tend to form a high temperature stratified layer at the upper free surface of the sodium in the top outlet plenum. A lower reactor outlet temperature due to overcooling of the core will result in cool sodium flowing into the bottom of the outlet plenum. This lower temperature sodium will then pass into the IHX inlets after the volume of the lower part of the plenum has been displaced to that exit level. In this case, the remainder of the upper plenum will remain at its steady state temperature until its heat is slowly removed by downward conduction and radial and upward heat transfer. Stratification in the lower plenum of the vessel will not be important when heat is removed by the normal heat removal path through the intermediate sodium loops and the steam generators. On the other hand, loss of the intermediate sodium system, or the closing of isolation valves in that system will cause a rise in the sodium temperature entering the lower plenum. This higher temperature sodium will stratify at the upper part of this plenum and eventually reach the pump inlets and the reactor core inlet. The sodium in the lower plenum below the level of the pump inlets will not be heated (except very slowly by sodium conduction), and will therefore contribute little to the energy storage ability of the system.

The temperature distribution during reactor shutdown transients will be significantly affected by the elevations of the entrances and exits to the large plenums. The entrance elevations to the plenums will have an effect on the convective heads developed by the components feeding the plenum, but will otherwise have little effect upon the temperature distribution in the plenums. A low exit from the intermediate heat exchangers to the lower plenum will tend to decrease the natural convection flow through the core when temperature leaving the heat exchangers is higher than that in the surrounding space in the plenum. Another similar but positive effect occurs if the reactor flow passes through "chimneys" between the core and the outlet plenum. The elevation of a plenum exit will determine the temperature passing into the next component. A low entrance to the intermediate heat exchangers will receive sodium from that elevation above the core outlet into the heat exchanger. A higher heat exchanger entrance will considerably affect the system temperature and flow transient after shutdown, since it will decrease the volume of hot sodium that can remain stratified at the upper part of the vessel. However, this may not be practical because of the necessity of keeping this heat exchanger inlet supply below the free sodium surface. A very low entrance to the primary sodium pumps from near to the bottom of the primary vessel will delay the entrance of hotter sodium into the reactor core inlet after a shutdown transient where heat removal by the intermediate heat exchangers is lost. On the other hand, a low pump inlet may permit the reactor support structure to overheat more in such a transient, since hot sodium will stratify on its lower surface.

The ABR design is configured to promote natural circulation shutdown heat removal. The key design parameters are 1) provision for a relatively free-flowing fluid natural circulation path, and 2) provision for sufficient elevation difference between the heat source and the heat sink. In 
the ABR emergency shutdown heat removal design, there are two natural circulation loops joined by a heat exchanger. Acting together, these loops remove heat from the reactor to the ultimate heat sink, atmospheric air.

In the primary coolant circuit, natural circulation flow for shutdown heat removal is established along the same flow path used for normal operation. Coolant is heated in the reactor, rises to the hot pool, and flows through the intermediate heat exchangers (IHX) to the cold pool. In accidents or emergency shutdown conditions in which no heat is removed from the coolant in the IHXs, heat is removed by multiple, independent heat exchangers in the direct reactor auxiliary cooling system (DRACS). The DRACS heat exchangers are mounted high in the cold pool.

Primary coolant chilled in the DRACS heat exchangers falls to near the bottom of the cold pool, where it travels into the primary coolant pump inlet and back to the reactor. This completes the primary coolant natural circulation circuit with heat added in the reactor and removed at the DRACS heat exchangers.

Heat removed from the cold pool at a DRACS heat exchanger is transferred to a second natural circulation loop. In ABR, the working fluid in this second loop is sodium-potassium alloy $(\mathrm{NaK})$. The NaK loop carries the heat through piping to a second heat exchanger located at a high elevation outside the containment building, where the heat is rejected to environmental air. The relatively low melting point of NaK minimizes the potential for freezing in the secondary loop.

The ABR DRACS circuits are designed to operate at reduced flow during power operation, to minimize parasitic heat losses. However, maintaining some flow at all times avoids the high transient DRACS start-up temperatures that would occur upon natural circulation initiation from stagnant conditions. 


\section{III.6 Core Performance Analysis}

Using ternary metal and mixed oxide fuels, the reference ABR core concepts of $1000 \mathrm{MWt}$ power rating were developed for the study on future fast rector design options under GNEP. The design description and core performance characteristics of the reference ABR core were discussed in Section II.1.1. Compact core concepts of medium TRU conversion ratio were developed by trade-off between the burnup reactivity loss and TRU conversion ratio. The burnup reactivity loss over a one-year cycle length was constrained to $\sim 3.5 \% \Delta \mathrm{k}$ in order to achieve a sufficient shutdown margin with a reasonable number of primary control assemblies that can be accommodated in a compact core and to limit a single assembly reactivity fault less than $\sim 0.7 \$$. The resulting TRU conversion ratio was $\sim 0.8$ for the startup core with weapons-grade plutonium feed and $\sim 0.7$ for the TRU recycled equilibrium core.

Using these reference ABR core concepts, sensitivity studies were performed to assess the impacts of low and high TRU conversion ratios on core design and performance parameters. Within the reference core configuration and assembly size, the intra-assembly design parameters and active core layout were varied to achieve a low or high TRU conversion ratio. As in the reference core concepts, the same assembly size was used for all core concepts in order to allow the interchange between metal and oxide fuel assemblies.

The TRU conversion ratio is inversely proportional to the TRU enrichment. To achieve a low conversion ratio, therefore, the TRU enrichment should be increased significantly from those of the reference medium conversion ratio designs, which in turn requires a significant decrease in fuel volume fraction. On the other hand, to meet the constraints on the burnup reactivity swing and the single assembly reactivity fault, the cycle length needs to be reduced. Based on the results of a parametric study performed with varying fuel volume fraction, TRU enrichment, and number of fuel batches, low conversion ratio metal and oxide cores were developed with a reduced cycle length. As an alternative design approach to achieve a low TRU conversion ratio without sacrificing the reference cycle length of one year, the use of fixed absorber was also investigated.

For the high conversion ratio core, the active core arrangement was modified while retaining the overall core configuration. Three design options were investigated: homogeneous core without blanket, homogeneous core with radial blankets, and heterogeneous core with internal and radial blankets. In the first case, the reference core layout was retained, and only the intraassembly design parameters and fuel management scheme were varied to increase the conversion ratios. For the second and third cases, a fraction of driver assemblies were replaced with blanket assemblies in addition to the changes in the driver assembly design and fuel management scheme.

Reactor performances were evaluated for the recycled equilibrium cores using the TRU recovered from the ABR spent fuel as the primary TRU feed and the TRU recovered from the LWR spent fuel as the makeup TRU feed. Fuel cycle performance parameters and reactivity feedback coefficients were calculated in detail. The maximum single assembly reactivity fault, reactivity control requirements and shutdown margins were also evaluated. The quasi-state reactivity balance analysis was also performed to estimate the passive safety features.

The thermal margins of the low and high TRU conversion ratio cores were evaluated by comparing the peak liner power to the linear power limit. The peak liner power was determined 
from simple thermal-hydraulic calculations based on a single channel model. The coolant inlet and bulk outlet temperatures were $355{ }^{\circ} \mathrm{C}$ and $510{ }^{\circ} \mathrm{C}$, respectively. The average flow rate was determined such that the coolant temperature rise across the core is $155^{\circ} \mathrm{C}$. A chopped cosine shape was assumed for the axial power distribution. Hot channel factors of 2.10, 1.19, 1.48 and 1.26 [1] were used for the film, cladding, gap and coolant regions, respectively. The linear power limit was determined such that the peak fuel centerline temperature is lower than the fuel melting temperature. For metal fuel, linear power was additionally limited such that the peak fuel-clad interface temperature is lower than the fuel cladding eutectic temperature of $650^{\circ} \mathrm{C}$.

\section{III.6.1 Low Conversion Ratio Core Concepts}

\section{III.6.1.1 Feasible Design Domain of Low Conversion Ratio Cores}

Using the reference ABR core concepts, a feasible design domain was investigated to determine the appropriate design parameters for a wide range of low TRU conversion ratios. By varying the intra-assembly design parameters (i.e., volume factions), the TRU enrichment, and the number of fuel batches, a feasible domain of fuel volume fraction and cycle length was determined such that the core satisfies two design constraints on the maximum burnup reactivity loss and the peak fast fluence of HT9 cladding. The maximum burnup reactivity loss was limited to $\sim 3.5 \% \Delta \mathrm{k}$ in order to limit the maximum single rod reactivity fault to $0.7 \$$ for 15 primary assemblies. The peak fast fluence limit of HT9 cladding was assumed $\sim 4 \times 10^{23} \mathrm{n} / \mathrm{cm}^{3}$. Although additional design constraints on the thermal margin, peak discharge burnup, etc., are expected to reduce the feasible design domain, those were not employed to determine the feasible design domain. For the metal core case, the weight fraction of $\mathrm{Zr}$ in the ternary metal fuel was fixed as $10 \%$ without considering the thermal conductivity changes with increasing TRU enrichment.

For different TRU enrichments and fuel volume fractions, the cycle lengths were determined such that k-effective value at the end of equilibrium cycle is 1.0. The impacts of the fuel management scheme were also investigated by varying the number of fuel batches. Since four and five batch fuel management schemes were adopted for the reference metal and oxide cores, respectively, four and six batch schemes were considered for the metal core, and five and seven batch schemes were considered for the oxide core. Figures III.6-1 and III.6-2 provide the contour plots for three core performance parameters of the recycled equilibrium cores: TRU conversion ratio $\left(\mathrm{CR}_{\mathrm{TRU}}\right)$, k-effective at the beginning of equilibrium cycle $\left(\mathrm{k}_{\mathrm{BOEC}}\right)$, and peak fast fluence (PFF). In these figures, the solid contour lines denotes the results obtained from the lower number of batch schemes, while the dotted contour lines for the higher number of batch schemes.

For a fixed cycle length, the TRU conversion ratio decreases as the fuel volume fraction decreases, but the burnup reactivity loss (i.e., $\mathrm{k}_{\mathrm{BOEC}}$ ) increases. The peak fast fluence increases with increasing cycle length, but it is almost independent of the fuel volume fraction. For a fixed TRU conversion ratio, the cycle length is proportional to the fuel volume fraction. As can be seen, the maximum achievable cycle length is determined by the burnup reactivity loss limit for lower TRU conversion ratios (less than $\sim 0.5$ ), while it is determined by the peak fast fluence limit for higher TRU conversion ratios (greater than $\sim 0.5$ ). When the number of batches is increased, the contour lines of $\mathrm{k}_{\mathrm{BOEC}}$ and TRU conversion ratio are shifted in the direction of higher fuel volume fraction and shorter cycle length, and the peak fast fluence contour lines of are shifted in the direction of shorter cycle length. 


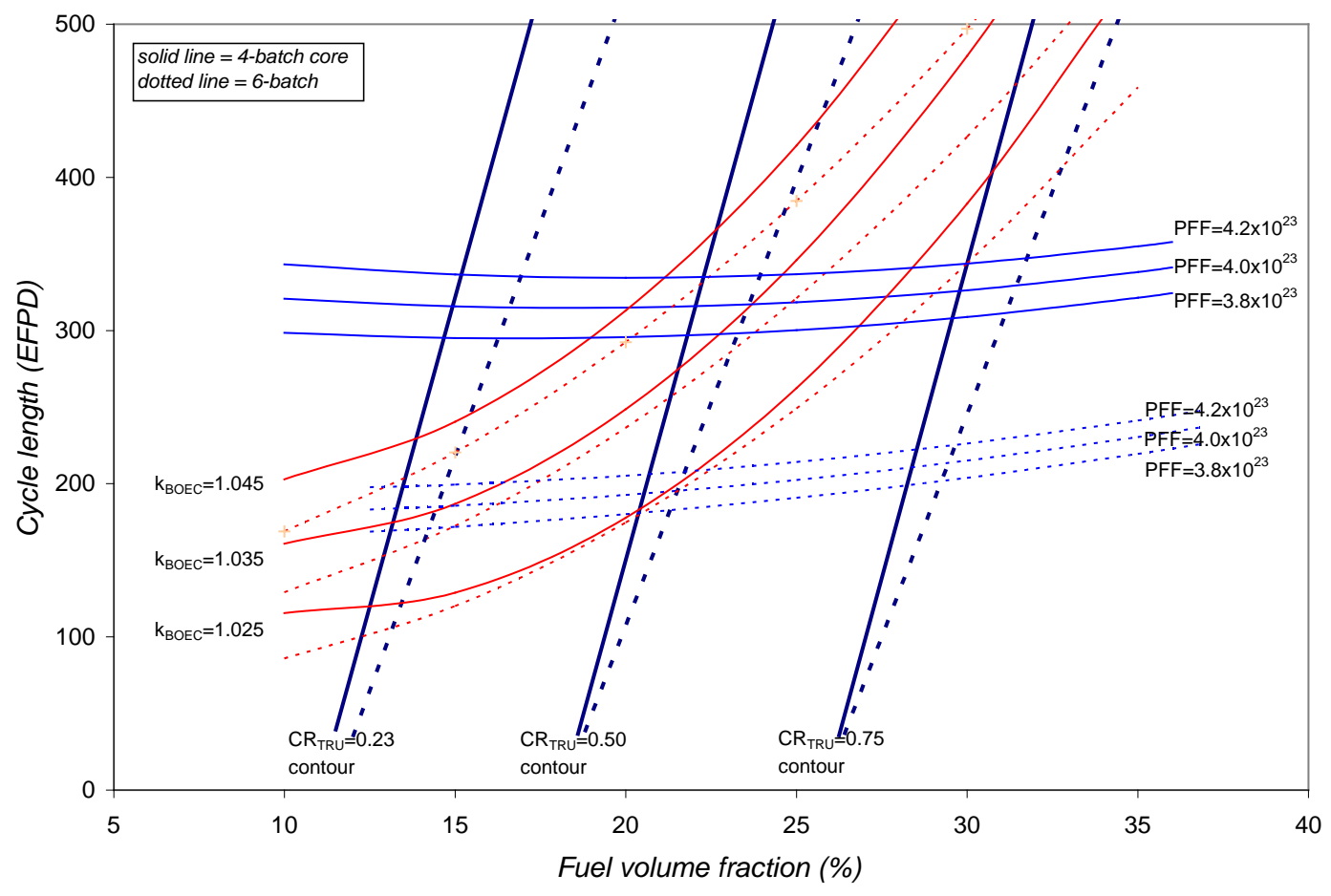

Figure III.6-1 Core Performance Parameter Contour Lines of Metal Core

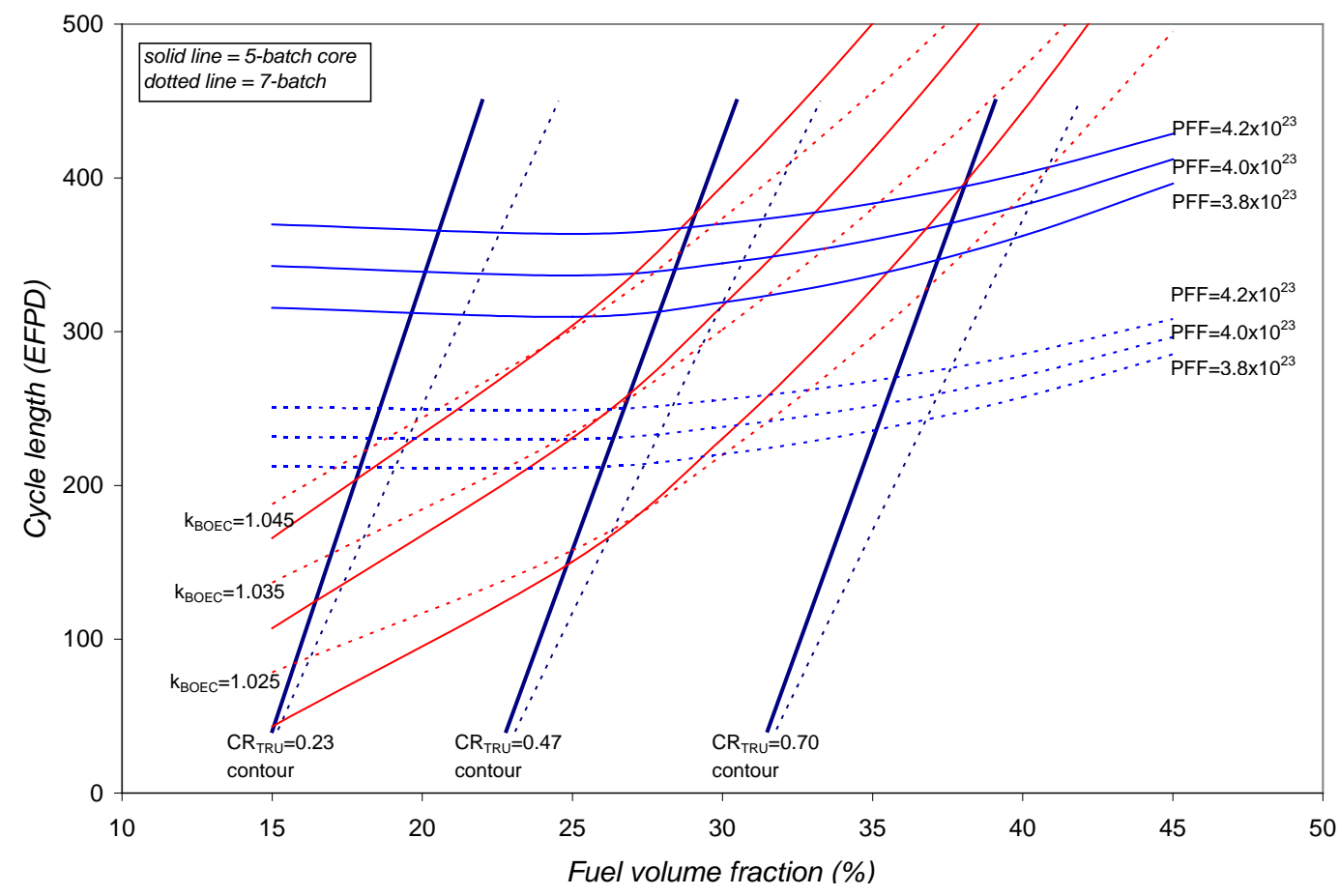

Figure III.6-2 Core Performance Parameter Contour Lines of Oxide Core 
The feasible design domains of fuel volume fraction and cycle length were determined from these contour plots. Figures III.6-3 and III.6-4 show the feasible design domains for the metal and oxide cores, respectively. The lower regions of the bold solid lines denote the feasible design domains and the reference $1000 \mathrm{MWt}$ ABR cores are also indicated in these figures for comparison. It is noted that the reference metal core design is located at the boundary of feasible domain, while the reference oxide core design is located inside the feasible domain. This indicates that the cycle length of the the reference oxide core can be increased somewhat within the peak fast fluence limit while maintaining the medium TRU conversion ratio. Figures III.6-3 and III.6-4 indicate that the TRU conversion ratio of reference metal and oxide cores could be reduced to $\sim 0.6$ by reducing the fuel volume fractions without changing the cycle length of oneyear. In order to reduce the TRU conversion ratio further, it is necessary to reduce the cycle

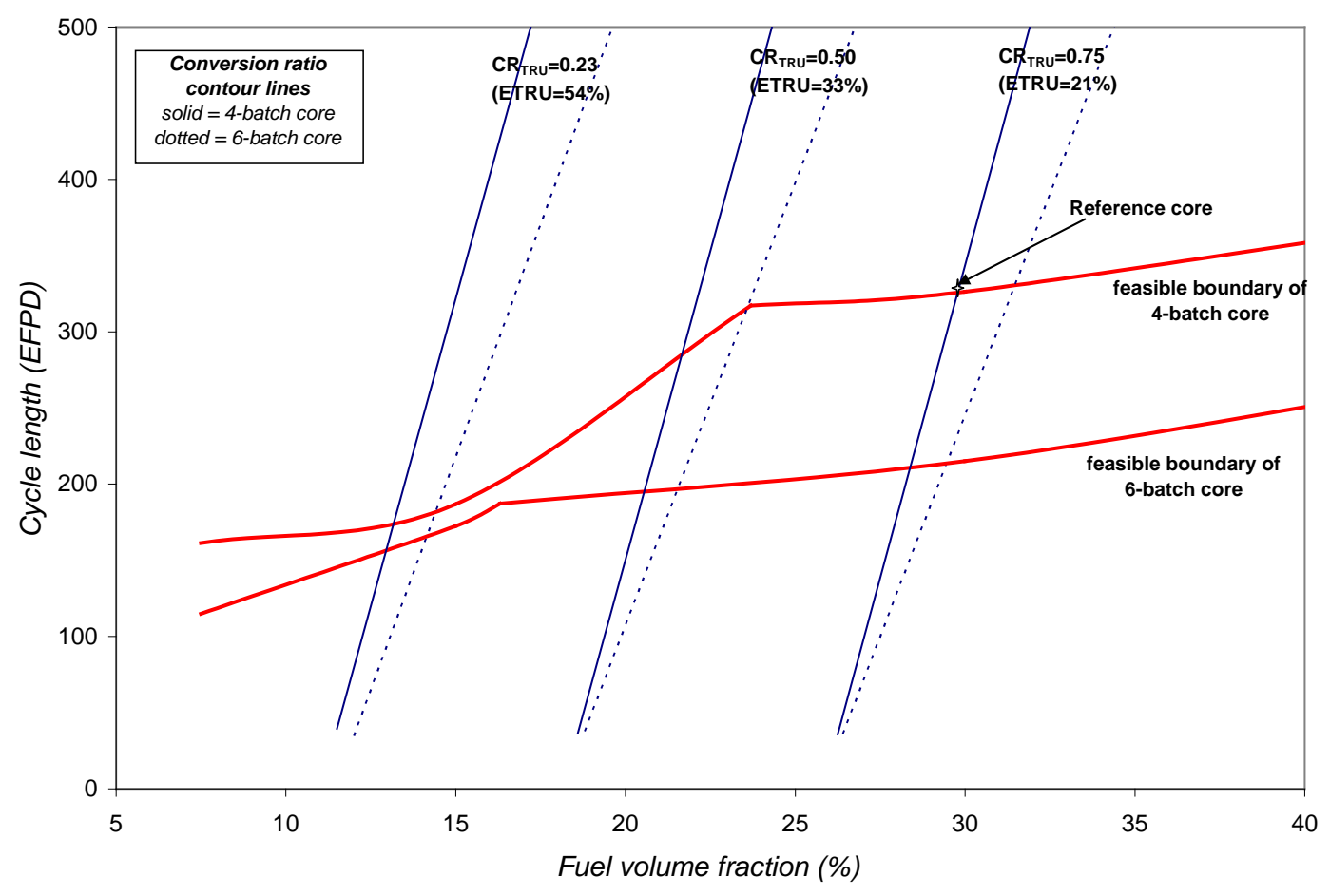

Figure III.6-3 Feasible Design Domain of Low Conversion Ratio Metal Core 


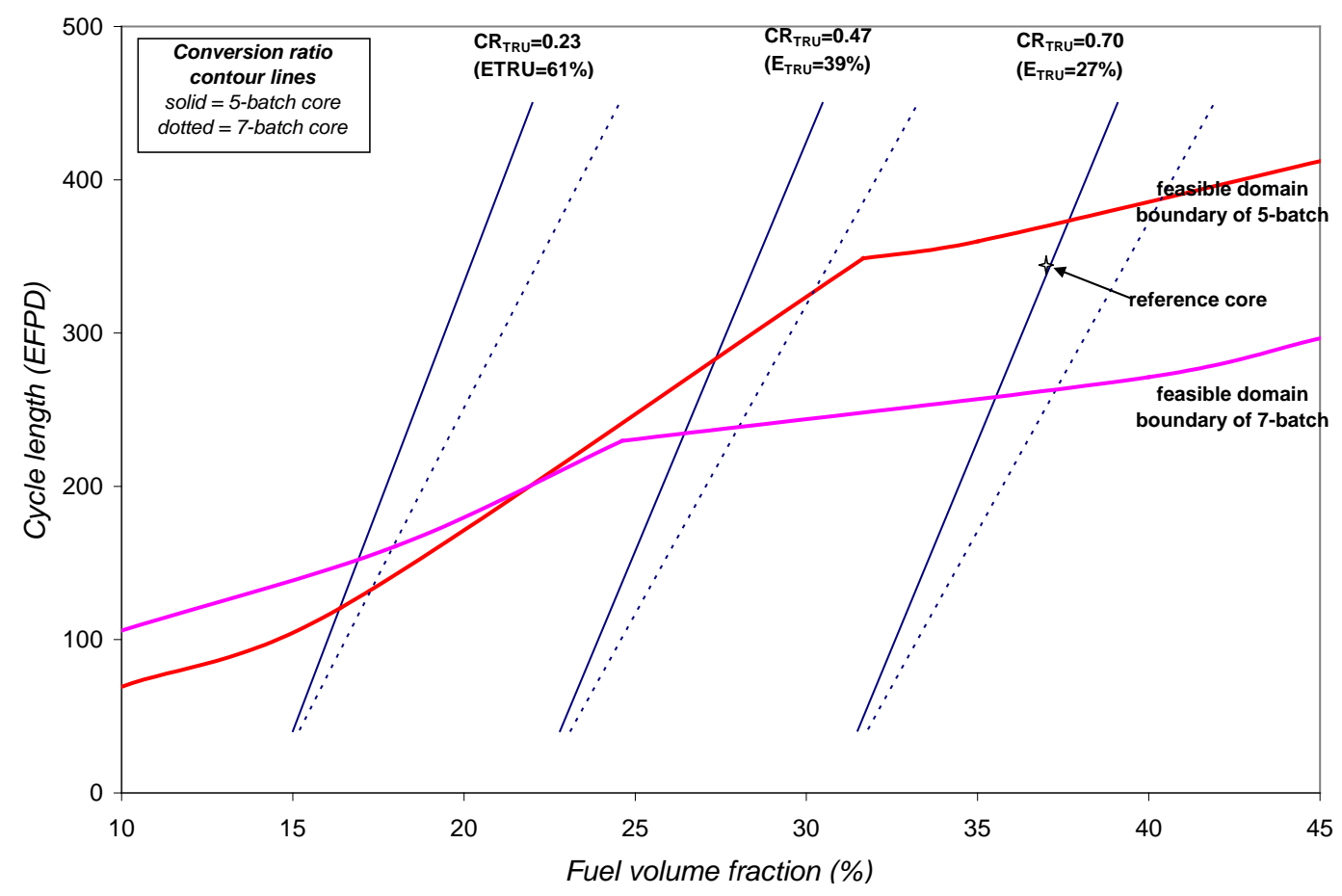

Figure III.6-4 Feasible Design Domain of Low Conversion Ratio Oxide Core

length. Otherwise, more control assemblies should be employed to increase the burnup reactivity loss limit or the power density should be reduced by increasing the core size. The feasible design domain is reduced with increasing number of batches mainly because of increased fast fluence. For low TRU conversion ratio cores, there exists a sufficient margin to the peak fast fluence limit, and hence the number of batches can be increased to increases the discharge burnup.

\section{III.6.1.2 Low Conversion Ratio Cores}

TRU conversion ratio is inversely proportional to TRU enrichment. To achieve a low TRU conversion ratio, therefore, the TRU enrichment should be increased significantly from the reference core design, which in turn requires a significant decease in fuel volume fraction. As in the reference ABR design, the maximum burnup reactivity loss was constrained to $\sim 3.5 \% \Delta \mathrm{k}$ in order to limit the maximum single rod reactivity fault to $0.7 \$$, and the peak fast fluence limit of HT9 cladding was assumed at $4 \times 10^{23} \mathrm{n} / \mathrm{cm}^{3}$. The feasible design domains determined by employing these two design constraints indicate that the TRU conversion ratio of both metal and oxide cores could be reduced to $\sim 0.6$ for a one-year cycle length by reducing the fuel volume fractions. However, to achieve further lower TRU conversion ratio, it was necessary to reduce the cycle length.

Based on these observations, metal and oxide fuel assembly designs and fuel management schemes were determined to achieve a low TRU conversion ratio. The fuel volume fraction was reduced by decreasing the fuel pin diameter and increasing the number of fuel pins per assembly. Because of the resulting large pin pitch-to-diameter ratio, grid spacers were assumed instead of wire-wrap spacers. For the U-TRU-Zr metal fuel, the Zr mass fraction was raised to $20 \%$ from $10 \%$ in order to compensate for the decreased thermal conductivity with increasing TRU enrichment. The active core height was also increased to reduce the peak linear power so that 
sufficient margins to fuel melting or fuel-cladding eutectic temperature could be achieved with increased TRU enrichment. Since there exists a sufficient margin to the peak fast fluence limit for low TRU conversion ratio cores as indicated in the feasible design domain, the number of batches was increased to eight for the metal core and nine for the oxide core. Table III.6-1 provides the driver assembly design parameters of the low TRU conversion ratio core concepts.

Table III.6-1 Assembly Design Parameters of Low Conversion Ratio Cores

\begin{tabular}{|l|r|r|}
\hline & Metal & Oxide \\
\hline Assembly data & & \\
- Number of pins & 324 & 324 \\
- Structural pins & 7 & 7 \\
- Spacer type & Grid & Grid \\
- Assembly pitch, cm & 16.142 & 16.142 \\
- Inter-assembly gap, cm & 0.432 & 0.432 \\
- Duct material & HT9 & HT9 \\
- Duct thickness, cm & 0.394 & 0.394 \\
- Overall duct length, cm & 477.5 & 477.5 \\
\hline Pin data & & \\
- Pin material and type & U-TRU-Zr & MOX \\
- Bond material & Na & He \\
- Overall pin length, cm & 387.4 & 381.00 \\
- Active core height, cm & 109.2 & 106.7 \\
- Pellet smeared density, \% TD & 75.0 & 85.0 \\
- Pellet diameter, cm & 0.372 & 0.415 \\
- Cladding material & HT9 & HT9 \\
- Clad outer diameter, cm & 0.550 & 0.540 \\
- Pin pitch-to-diameter ratio & 1.590 & 1.617 \\
- Cladding thickness, cm & 0.060 & 0.057 \\
- Wire wrap diameter, cm & - & - \\
\hline Volume fraction at cold, \% & & \\
- Fuel or Absorber & 15.6 & 19.4 \\
- Bond & 5.7 & 1.5 \\
- Structure & 22.8 & 22.0 \\
- Coolant & 55.9 & 57.1 \\
\hline
\end{tabular}

Compared to the reference core concepts, the fuel volume fraction is reduced to $15.6 \%$ for the metal core and $19.4 \%$ for the oxide core. The number of fuel pins is increased to 324 from 271 to reduce the peak linear power, and seven structural pins are introduced to support and space the grids. The fuel pin diameter is reduced to $5.5 \mathrm{~mm}$ for metal fuel and $5.4 \mathrm{~mm}$ for oxide fuel. The active core height of the metal core was increased by $34 \%$ to reduce the peak linear power, whereas the reference height is maintained for the oxide core.

Table III.6-2 summarizes the key core performance parameters of the low TRU conversion ratio cores obtained from REBUS-3 equilibrium cycle analyses. For both the metal and oxide cores, the TRU conversion ratio is decreased to $\sim 0.22$. To limit the burnup reactivity swing within $\sim 3.5 \% \Delta \mathrm{k}$, however, the cycle length is reduced to 6.6 months for the metal core and 7.1 months for the oxide core. Even with the reduced cycle length, the increased number of batches 
increases the fuel residence time from 48 to 53 months for the metal core and from 60 to 64 months. The required TRU enrichment is $58.5 \%$ for the metal core and $61.3 \%$ for the oxide core, which are beyond the current irradiation experiences (28.9\% of the metal fuel in EBR-II [2] and $33 \%$ of the oxide fuel [3]). Compared to the reference cores, the heavy metal inventory is reduced to 7.1 MT for the metal core and 6.7 MT for the oxide core. The increased active core height of the metal core reduces the power density from $303 \mathrm{~kW} / \mathrm{l}$ to $225 \mathrm{~kW} / \mathrm{l}$, while the oxide core power density is not changed. The increased residence time and the reduced heavy metal loading increase the average discharge burnup from 93 to $185 \mathrm{MWd} / \mathrm{kg}$ for the metal core and from 111 to $226 \mathrm{MWd} / \mathrm{kg}$ for the oxide core.

Table III.6-2 Key Core Performance Parameters of Low Conversion Ratio Cores

\begin{tabular}{|l|r|r|}
\hline & Metal & Oxide \\
\hline Cycle length, month & 6.6 & 7.1 \\
\hline Number of batches & 8 & 61.3 \\
\hline Average TRU enrichment, \% & $0.50 / 0.22$ & 0.52 / 0.22 \\
\hline Fissile/TRU conversion ratio & $7.1 / 3.9$ & $6.7 / 3.9$ \\
\hline HM/TRU inventory at BOEC, MT & $185 / 278$ & $226 / 339$ \\
\hline Discharge burnup (ave/peak), MWd/kg & 132.4 & 135.6 \\
\hline Specific power density $(\mathrm{kW} / \mathrm{kg})$ & 225 & 231 \\
\hline Average core power density , kW/l & 3.74 & 3.92 \\
\hline Peak discharge fast fluence, $10^{23} / \mathrm{cm}{ }^{2}$ & 3.4 & 15.7 \\
\hline Burnup reactivity loss, $\% \Delta \mathrm{k}$ & 14.5 & 28.4 \\
\hline Average linear power, $\mathrm{kW} / \mathrm{m}$ & 25.8 & 36.7 \\
\hline Peak linear power density, $\mathrm{kW} / \mathrm{m}$ & 25.9 & 3.13 \\
\hline Linear power density limit, $\mathrm{kW} / \mathrm{m}$ & 3.32 & 0.59 \\
\hline Core average flux, $10^{15} / \mathrm{cm}{ }^{2}-\mathrm{sec}$ & 0.63 & 250.3 \\
\hline Fast flux fraction, $>0.1 \mathrm{MeV}$ & 241.3 & \\
\hline TRU consumption rate, $\mathrm{kg} / \mathrm{year}$ & & \\
\hline
\end{tabular}

The peak linear power is reduced due to the increased number of fuel pins and increased active core height. Nevertheless, the margin to the linear power limit is reduced because of the reduced linear power limit. In particular, the linear power limit of the metal core is reduced significantly since both thermal conductivity and melting temperature decrease with increasing TRU enrichment.

The fast flux fraction of the metal core is reduced from 0.68 to 0.63 because of the increased coolant volume fraction. On the other hand, the neutron spectrum of the oxide core becomes 
slightly harder since the reduced slowing down by oxygen due to reduced fuel volume fraction dominates the increased slowing down by sodium due to the increased coolant volume fraction. The reduced TRU conversion ratio increases the TRU consumption rate from 81.6 to 241.3 $\mathrm{kg} /$ year for the metal core and from 86.5 to $250.3 \mathrm{~kg} /$ year for the oxide core.

Table III.6-3 presents the kinetics parameters and reactivity coefficients calculated for the low TRU conversion ratio core configurations at the beginning of equilibrium cycle (BOEC) and the end of equilibrium cycle (EOEC). In these calculations, the primary control assemblies were positioned at critical locations and the secondary control assemblies were positioned at the top of the active core. At BOEC, the critical position of the primary control assemblies is $84 \mathrm{~cm}$ from the bottom of the active core for the metal core and $75 \mathrm{~cm}$ for the oxide core.

Table III.6-3 Kinetics Parameters and Reactivity Coefficients of Low Conversion Ratio Cores

\begin{tabular}{|c|c|c|c|c|}
\hline & \multicolumn{2}{|c|}{ Metal } & \multicolumn{2}{|c|}{ Oxide } \\
\hline & BOEC & EOEC & BOEC & EOEC \\
\hline Effective delayed neutron fraction & 0.00263 & 0.00264 & 0.00262 & 0.000264 \\
\hline Prompt neutron lifetime, $\mu \mathrm{s}$ & 0.52 & 0.54 & 0.56 & 0.59 \\
\hline Radial expansion coefficient, $\mathbb{\Phi} /{ }^{\circ} \mathrm{C}$ & -0.54 & -0.53 & -0.50 & -0.48 \\
\hline Axial expansion coefficient, $\mathbb{\leftarrow} /{ }^{\circ} \mathrm{C}$ & -0.10 & -0.10 & -0.08 & -0.09 \\
\hline Fuel density coefficient, $\mathbb{\Phi} /{ }^{\circ} \mathrm{C}$ & -1.01 & -0.97 & -0.69 & -0.64 \\
\hline Structure density coefficient, $\mathbb{\mathbb { }} /{ }^{\circ} \mathrm{C}$ & 0.09 & 0.09 & 0.08 & 0.09 \\
\hline Sodium density coefficient, $\mathbb{C} /{ }^{\circ} \mathrm{C}$ & 0.30 & 0.32 & 0.24 & 0.26 \\
\hline Doppler constant, \$ & -0.45 & -0.48 & -0.58 & -0.61 \\
\hline Doppler coefficient, $\mathbb{\Phi} /{ }^{\circ} \mathrm{C}$ & -0.08 & -0.08 & -0.07 & -0.07 \\
\hline $\begin{array}{l}\text { Sodium voided Doppler coefficient } \\
, \mathbb{\Phi} /{ }^{\circ} \mathrm{C}\end{array}$ & -0.04 & -0.04 & -0.04 & -0.04 \\
\hline
\end{tabular}

The kinetic parameters and reactivity coefficients of the low conversion ratio cores are significantly different from those of the reference cores because of the increased TRU enrichment and coolant volume fraction. The increased TRU enrichment reduces the U-238 fraction proportionally, and hence the effective delayed neutron fraction decreases by $\sim 19 \%$ for the metal core and by $\sim 15 \%$ for the oxide core. The increased scattering to absorption ratio due to the reduced heavy metal loading and increased coolant volume fraction increases the prompt neutron lifetime by $\sim 60 \%$ for the metal core and by $\sim 36 \%$ for the oxide core. The uniform radial and axial expansion coefficients become more negative since the leakage increases with decreasing fuel volume fraction. The sodium void worth becomes more positive because of the increased coolant volume fraction. The Doppler constant of the oxide core becomes $\sim 48 \%$ less negative due to the significantly reduced amount of U-238 and the slightly hardened spectrum. For the metal core, the Doppler constant becomes $~ 18 \%$ less negative because of the combined effects of the reduced amount of U-238 and the softened spectrum.

The integral reactivity parameters for the quasi-static reactivity balance [4] are presented in Table III.6-4. Compared to the reference cores, the transient overpower initiator is increased for both the metal and oxide cores, as a result of increased burnup reactivity loss. As in the reference 
core, the integral reactivity parameters of the metal core satisfy all the three sufficient conditions for acceptable asymptotic core outlet temperatures for possible unprotected accident scenarios (loss of flow, loss of heat sink, transient overpower, etc). The reference oxide core does not meet the passive safety condition for the unprotected loss of flow accident $(\mathrm{A} / \mathrm{B}<1)$ because of the large power coefficient $(A)$ due to high fuel temperature and large Doppler constant. However, the less negative Doppler coefficient of the low conversion ratio design yields a less negative power coefficient (A) and a more negative power/flow coefficient (B). As a result, the passive safety condition for the unprotected loss of flow accident is satisfied. These results indicate that the low conversion ratio core concepts have favorable passive safety features, although detailed safety analyses are required to verify passive safety behavior.

Table III.6-4 Integral Reactivity Parameters of Low Conversion Ratio Cores

\begin{tabular}{|c|c|c|c|c|c|}
\hline & & \multicolumn{2}{|c|}{ Metal } & \multicolumn{2}{|c|}{ Oxide } \\
\hline & & BOEC & EOEC & BOEC & EOEC \\
\hline \multicolumn{2}{|c|}{ A, power coefficient (\$) } & -11.1 & -11.7 & -35.7 & -37.5 \\
\hline \multicolumn{2}{|c|}{ B, power/flow coefficient (\$) } & -86.9 & -65.8 & -83.7 & -62.7 \\
\hline \multicolumn{2}{|c|}{$\begin{array}{l}\mathrm{C} \text {, inlet temperature coefficient } \\
\left(\$ /{ }^{\circ} \mathrm{C}\right)\end{array}$} & -0.68 & -0.53 & -0.66 & -0.51 \\
\hline \multicolumn{2}{|c|}{$\begin{array}{l}\text { Transient overpower initiator, } \Delta \rho_{\text {TOP }} \\
(\$)\end{array}$} & 67 & 0.0 & 0.73 & 0.0 \\
\hline \multirow{3}{*}{$\begin{array}{l}\text { Sufficient } \\
\text { conditions for } \\
\text { passive safety }\end{array}$} & $\mathrm{A} / \mathrm{B}<1.0$ & 0.13 & 0.18 & 0.43 & 0.60 \\
\hline & $\begin{aligned} 1.0 & <\mathrm{C} \Delta T_{c} / \mathrm{B} \\
& <2.0\end{aligned}$ & 1.21 & 1.25 & 1.22 & 1.27 \\
\hline & $\begin{array}{c}\Delta \rho_{\mathrm{TOP}} /|\mathrm{B}|< \\
1.0\end{array}$ & 0.77 & 0.00 & 0.87 & 0.00 \\
\hline
\end{tabular}

The evaluated shutdown margin is presented in Tables III.6-5 and III.6-6 for the primary and secondary control systems, respectively. It is noted that the total control assembly worth is significantly larger in the low conversion ratio cores than in the reference cores because of the softer neutron spectrum for the metal core and the increased flux level for the oxide core. This is in part due to the reduced effective delayed neutron fraction. The minimum shutdown margin of the primary system is $\sim 48 \$$ for the metal core and $\sim 41 \$$ for the oxide cores. The minimum shutdown margin of the secondary control system is $\sim 10 \$$ for the metal core and $\sim 8$ for oxide core. These shutdown margins are more than adequate. In the low TRU conversion ratio metal core, the calculated control-rod driveline expansion coefficient is $65 \$ / \mathrm{cm}$ at BOEC and $35 \$ / \mathrm{cm}$ at EOEC. For the oxide core, the coefficient is $63 \AA / \mathrm{cm}$ at BOEC and $33 \$ / \mathrm{cm}$ at EOEC. 
Table III.6-5 Shutdown Margin of Primary Control System for Low Conversion Ratio Cores

\begin{tabular}{|l|r|r|r|r|}
\hline & \multicolumn{2}{|c|}{ Metal } & \multicolumn{2}{|c|}{ Oxide } \\
\cline { 2 - 5 } & BOEC & EOEC & BOEC & EOEC \\
\hline Number of control assemblies & 15 & 15 & 15 & 15 \\
\hline Total worth, \$ & 74.8 & 77.3 & 67.7 & 69.9 \\
\hline Worth with one stuck & 67.7 & 69.9 & 60.8 & 62.8 \\
assembly, \$ & & & & \\
\hline Reactivity control & 19.3 & 3.7 & 19.6 & 3.9 \\
requirement, \$ & & & & \\
\hline - Temperature defect & 1.04 & 0.99 & 1.22 & 1.18 \\
- Burnup reactivity loss & 12.3 & & 13.2 & \\
- Fuel axial growth (5\%) & 0.94 & & & \\
- Overpower margin (3\%) & 0.01 & 0.01 & 0.02 & 0.02 \\
- Reactivity fault & 0.67 & & 0.73 & \\
- Uncertainties (RMS) & 2.54 & 1.43 & 2.64 & 1.43 \\
- Other margin & 1.80 & 1.30 & 1.80 & 1.30 \\
\hline Shutdown margin, \$ & 48.4 & 66.2 & 41.2 & 58.9 \\
\hline
\end{tabular}

Table III.6-6 Shutdown Margin of Secondary Control System for Low Conversion Ratio Cores

\begin{tabular}{|l|r|r|r|r|}
\hline & \multicolumn{2}{|c|}{ Metal } & \multicolumn{2}{|c|}{ Oxide } \\
\cline { 2 - 5 } & BOEC & EOEC & BOEC & EOEC \\
\hline Number of control assemblies & 4 & 4 & 4 & 4 \\
\hline Total worth, \$ & 15.2 & 16.1 & 13.1 & 14.0 \\
\hline Worth with one stuck assembly, \$ & 11.0 & 11.7 & 9.4 & 10.1 \\
\hline Control requirement, \$ & 1.37 & 1.31 & 1.65 & 1.60 \\
\hline - Temperature defect & 1.04 & 0.99 & 1.22 & 1.18 \\
- Overpower margin (30\%) & 0.12 & 0.12 & 0.19 & 0.18 \\
- Temp defect uncertainty (20\%) & 0.21 & 0.20 & 0.24 & 0.24 \\
\hline Shutdown margin, \$ & 9.6 & 10.4 & 7.7 & 8.5 \\
\hline
\end{tabular}




\section{III.6.1.3 Impacts of Fixed Absorber}

As discussed in the previous section, within the reference core configuration and burnup reactivity loss limit of $\sim 3.5 \% \Delta \mathrm{k}$, a low TRU conversion ratio of $\sim 0.22$ can be achieved only with reducing the cycle length to six to seven months. As an alternative design approach to achieving a low TRU conversion ratio without sacrificing the reference cycle length of one year, the use of fixed absorber (FA) was investigated. The rationale is to decrease the effective fuel volume fraction in the core by replacing a fraction of fuel pins with absorber rods. The reduced fuel volume fraction and the residual reactivity penalty at EOEC of partially depleted absorber rods would increase the TRU enrichment to maintain the criticality for a fixed cycle length, which in turn would reduce the TRU conversion ratio.

By introducing $\mathrm{B}_{4} \mathrm{C}$ rods into every driver assembly, the impacts of a fixed absorber on the core performance and reactivity coefficients were evaluated. All the other core and assembly design parameters of the reference ABR core designs were retained, except for those described below. Based on preliminary trade-off studies between the burnup reactivity swing and conversion ratio, 13 and 7 fuel pins of each driver assembly were replaced with $90 \%$ enriched $\mathrm{B}_{4} \mathrm{C}$ rods for the metal and oxide fuel core, respectively. The TRU enrichment was redetermined to achieve the one-year cycle length of the reference designs. The $\mathrm{Zr}$ mass fraction in the ternary metal fuel was raised to $20 \%$ to compensate for the decreasing thermal conductivity of ternary metal fuel with increasing TRU fraction. To meet the imposed thermal design criteria, the active core height was increased to $96.5 \mathrm{~cm}$ for the metal core and to $111.8 \mathrm{~cm}$ for the oxide core.

The key design and performance parameters of the reference metal and oxide cores with $\mathrm{B}_{4} \mathrm{C}$ absorber are compared in Table III.6-7. In the metal core, the heavy metal inventory is reduced because the fuel rods are replaced with absorber rods and $\mathrm{Zr}$ fraction is increased. The reduced heavy metal inventory and the residual reactivity penalty at EOEC of absorber rods increase the TRU enrichment from $22 \%$ to $43 \%$ to achieve the fixed cycle length of one year. The increased TRU enrichment decreases the TRU conversion ratio to 0.30 from 0.73 , with an increased reactivity loss from $2.2 \% \Delta \mathrm{k}$ to $3.0 \% \Delta \mathrm{k}$, which is within the targeted value. The reduced heavy metal inventory also increases the specific power density to $83.5 \mathrm{~kW} / \mathrm{kg}$ and the discharge burnup to $107 \mathrm{MWd} / \mathrm{kg}$.

In the oxide core, the increased active core height increases the heavy metal inventory slightly although seven fuel rods are replaced by absorber rods in every driver assembly. As a result, the specific power density and discharge burnup are slightly reduced relative to the reference oxide core. However, the residual reactivity penalty at EOEC of absorber rods increases the TRU enrichment from $27.1 \%$ to $38.1 \%$. As a result, the TRU conversion ratio is reduced to 0.43 from 0.71 , although the burnup reactivity loss is decreased to $1.8 \% \Delta \mathrm{k}$ from 2.0 $\% \Delta \mathrm{k}$.

The fractional burnup of B-10 is $47 \%$ in the oxide core versus $37 \%$ in the metal core. The introduction of fixed absorber increases the fast flux fraction from 0.68 to 0.73 for the metal core and from 0.58 to 0.64 for the oxide core. The reduced TRU conversion ratio increases the TRU consumption rate from 81.6 to $221.1 \mathrm{~kg} /$ year for the metal core and from 86.5 to $176.8 \mathrm{~kg} /$ year for the oxide cores.

The kinetics parameters and reactivity coefficients of the fixed absorber bearing cores are provided in Table III.6-8. Compared to the reference ABR cores without absorber rods, the 
effective delayed neutron fraction is reduced by $~ 20 \%$ because of the reduced U-238 fraction. The prompt neutron lifetime is reduced by $\sim 40 \%$ due to the increased absorption by absorber rods. The thermal expansion coefficients are increased slightly because of the reduced fuel volume fraction that increases the leakage. The hardened spectrum and increased TRU enrichment result in more negative fuel density effect coefficient. The sodium void worth becomes more positive because of the hardened spectrum. Due to the hardened spectrum, the magnitude of the Doppler constant is reduced by $\sim 5$ times for the metal core and by $\sim 4$ times for the oxide core.

Table III.6-7 Core Performance Parameters of Low Conversion Ratio Cores with Fixed Absorber

\begin{tabular}{|l|r|r|}
\hline & Metal & Oxide \\
\hline Make up TRU Feed & LWR-SNF & LWR-SNF \\
\hline Cycle length, month & 12 & 12 \\
\hline Number of batches & 4 & 5 \\
\hline Active core height, cm & 96.5 & 111.8 \\
\hline Average TRU enrichment, \% & 43.1 & 38.1 \\
\hline Fissile/TRU conversion ratio & $0.53 / 0.30$ & $0.65 / 0.43$ \\
\hline HM/TRU inventory at BOEC, MT & $11.5 / 4.7$ & $13.7 / 5.1$ \\
\hline Discharge burnup (ave/peak), MWd/kg & $107 / 174$ & $110 / 175$ \\
\hline Specific power density (kW/kg) & 83.5 & 69.9 \\
\hline Average core power density at cold, $\mathrm{kW} / \mathrm{l}$ & 255 & 231 \\
\hline Peak discharge fast fluence, $10^{23} / \mathrm{cm}{ }^{2}$ & 3.35 & 3.17 \\
\hline Burnup reactivity loss, \% $\Delta \mathrm{k}$ & 3.0 & 1.8 \\
\hline Power peaking factor $(\mathrm{BOEC} / \mathrm{EOEC)}$ & $1.65 / 1.61$ & $1.60 / 1.57$ \\
\hline Average linear power, $\mathrm{kW} / \mathrm{m}$ & 19.8 & 18.0 \\
\hline Peak linear power density, kW/m & 37.0 & 32.8 \\
\hline Linear power density limit, kW/m ${ }^{\text {a) }}$ & 38.2 & 40.0 \\
\hline Core average flux, $10^{15} / \mathrm{cm}{ }^{2}$-sec & 2.57 & 2.19 \\
\hline Fast flux fraction & 0.73 & 0.64 \\
\hline B-10 depletion, \% & 37 & 47 \\
\hline TRU consumption rate, $\mathrm{kg} / \mathrm{year}$ & 221.1 & 176.8 \\
\hline
\end{tabular}

To evaluate the passive safety features, the integral reactivity parameters for the quasi-static reactivity balance analysis were estimated and presented in Table III.6-9. For both the metal and oxide cores, the passive safety condition for the unprotected transient overpower accident is not satisfied because of the increased transient overpower initiator or reduced power/flow coefficient. Further study needs to be performed to determine the minimum conversion ratio that can be achieved while satisfying the sufficient conditions for acceptable asymptotic core outlet temperatures for possible unprotected accident scenarios. 
Table III.6-8 Kinetics Parameters and Reactivity Coefficients of Low Conversion Ratio Cores with Fixed Absorber

\begin{tabular}{|c|c|c|c|c|}
\hline & \multicolumn{2}{|c|}{ Metal } & \multicolumn{2}{|c|}{ Oxide } \\
\hline & BOEC & EOEC & BOEC & EOEC \\
\hline$\beta$ eff & 0.00265 & 0.00267 & 0.00276 & 0.00277 \\
\hline Prompt neutron lifetime, $\mu \mathrm{s}$ & 0.19 & 0.21 & 0.23 & 0.25 \\
\hline $\begin{array}{l}\text { Radial expansion coefficient, } \\
\mathbb{\$} /{ }^{\circ} \mathrm{C}\end{array}$ & -0.41 & -0.40 & -0.31 & -0.31 \\
\hline $\begin{array}{l}\text { Axial expansion coefficient, } \\
\mathbb{\$} /{ }^{\circ} \mathrm{C}\end{array}$ & -0.07 & -0.08 & -0.06 & -0.06 \\
\hline 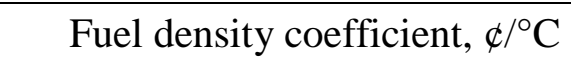 & -1.05 & -1.01 & -0.62 & -0.59 \\
\hline $\begin{array}{l}\text { Structure density coefficient, } \\
\mathbb{\$} /{ }^{\circ} \mathrm{C}\end{array}$ & 0.12 & 0.12 & 0.11 & 0.11 \\
\hline $\begin{array}{l}\text { Sodium density coefficient, } \\
\mathbb{\$} /{ }^{\circ} \mathrm{C}\end{array}$ & 0.33 & 0.34 & 0.26 & 0.27 \\
\hline Doppler constant, \$ & -0.11 & -0.13 & -0.29 & -0.34 \\
\hline Doppler coefficient, $\mathbb{C} /{ }^{\circ} \mathrm{C}$ & -0.02 & -0.02 & -0.03 & -0.04 \\
\hline
\end{tabular}

Table III.6-9 Integral Reactivity Parameters of Low Conversion Ratio Cores with Fixed Absorber

\begin{tabular}{|c|c|c|c|c|c|}
\hline & \multicolumn{2}{|c|}{ Metal } & \multicolumn{2}{|c|}{ Oxide } \\
\hline & & BOEC & EOEC & BOEC & EOEC \\
\hline \multicolumn{2}{|c|}{ A, power coefficient (\$) } & -2.96 & -3.58 & $\begin{array}{c}-14.8 \\
\end{array}$ & \begin{tabular}{c|}
-17.3 \\
\end{tabular} \\
\hline \multicolumn{2}{|c|}{ B, power/flow coefficient (\$) } & -50.2 & -36.5 & -35.0 & -28.2 \\
\hline \multicolumn{2}{|c|}{$\begin{array}{l}\text { C, inlet temperature coefficient } \\
\left(\Phi /{ }^{\circ} \mathrm{C}\right)\end{array}$} & -0.34 & -0.25 & -0.24 & -0.20 \\
\hline \multicolumn{2}{|c|}{$\begin{array}{l}\text { Transient overpower initiator, } \\
\Delta \rho_{\text {TOР }}(\$)\end{array}$} & 0.60 & 0.0 & 0.40 & 0.0 \\
\hline \multirow{3}{*}{$\begin{array}{l}\text { Sufficient } \\
\text { conditions } \\
\text { for } \\
\text { passive } \\
\text { safety }\end{array}$} & $\mathrm{A} / \mathrm{B}<1.0$ & 0.06 & 0.10 & 0.42 & 0.61 \\
\hline & $1.0<\mathrm{C} \Delta T_{c} / \mathrm{B}<2.0$ & 1.06 & 1.06 & 1.07 & 1.09 \\
\hline & $\Delta \rho_{T O P} /|\mathrm{B}|<1.0$ & 1.20 & 0.0 & 1.14 & 0.00 \\
\hline
\end{tabular}




\section{III.6.2 High Conversion Ratio Core Concepts}

Feasibility studies were also performed to convert the reference core designs into a breakeven or breeder core within the reference core configuration and assembly size. While retaining the overall core configuration, only the active core arrangement was modified. Three design options were investigated: homogeneous core with no blanket, homogeneous core with radial blankets, and heterogeneous core with internal and radial blankets. In the first case, the reference core layout was retained, and only the intra-assembly design parameters and fuel management scheme were varied to increase the TRU conversion ratio. For the second and third cases, a fraction of driver assemblies were replaced with blanket assemblies in addition to the changes in the driver assembly design and fuel management scheme.

Parametric studies were performed to determine assembly designs and core arrangements that yield the higher TRU conversion ratios within the thermal and material related design constraints. The resulting radial core layouts for the homogeneous core with radial blankets and the heterogeneous core with internal and radial blankets are shown in Figures III.6-5 and III.6-6, respectively. In both the homogeneous and heterogeneous cores, 30 driver assemblies at the core periphery are replaced with blanket assemblies. For the heterogeneous core configuration, 42 internal driver assemblies are additionally replaced with blanket assemblies. Consequently, the homogeneous core with radial blankets consists of 150 drivers and 30 radial blankets, and the heterogeneous core is composed of 108 drivers, 42 internal blankets and 30 radial blankets. In order to allow the interchange of metal and oxide fuel assemblies, the same core layout is used for both metal and oxide cores. It is noted that the metal and oxide cores would have different optimum core layouts. For example, the S-PRSIM design [3] employs different core layouts for metal and oxide cores; the metal core consists of 138 drivers, 49 internal blankets and 48 radial blankets, and the oxide core is composed of 162 drivers, 73 internal blankets and 60 radial blankets. It can be noticed that the planar area of the oxide core is $25 \%$ bigger than that of the metal core. 


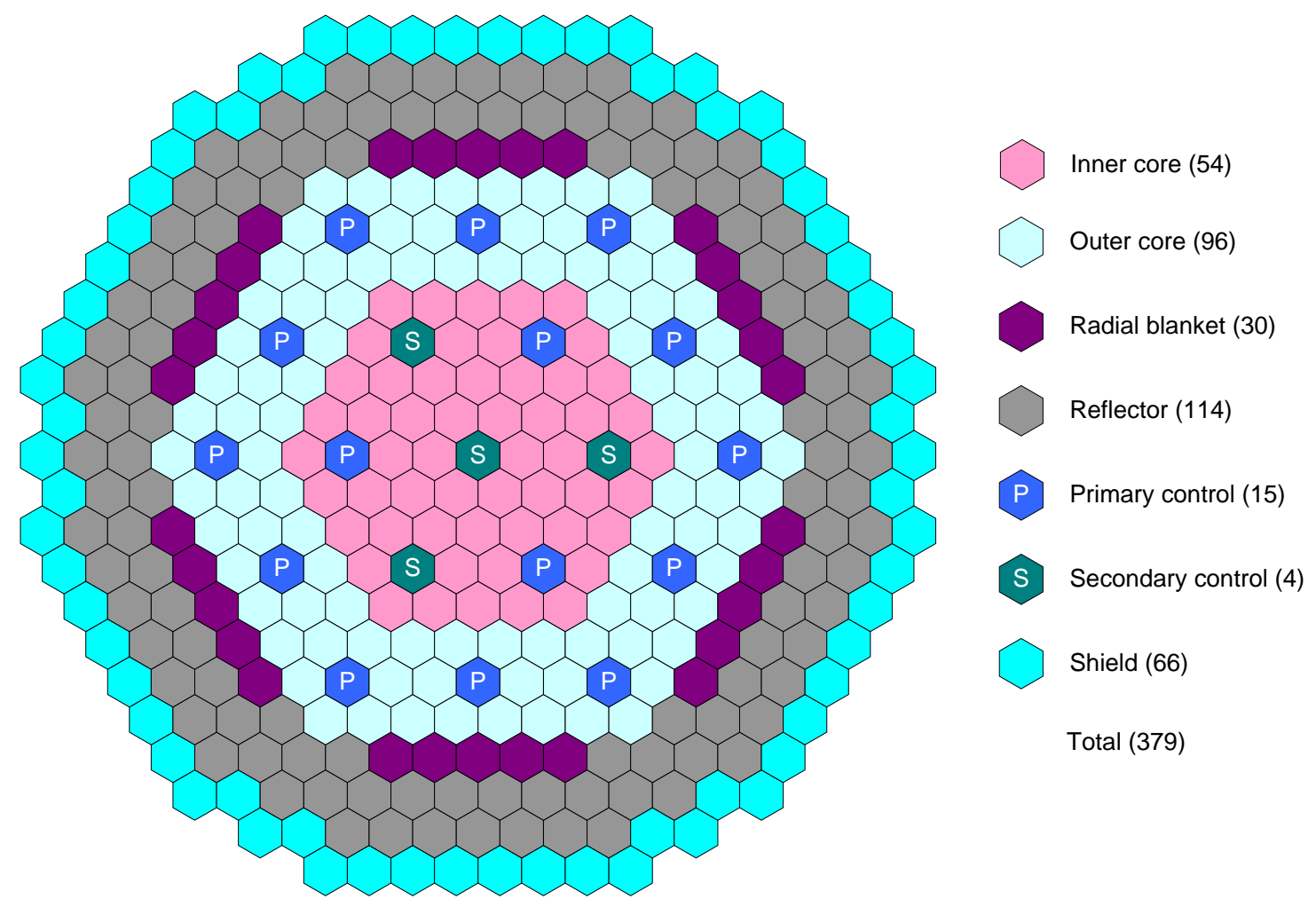

Figure III.6-5 High Conversion Ratio Core Layout with Radial Blankets
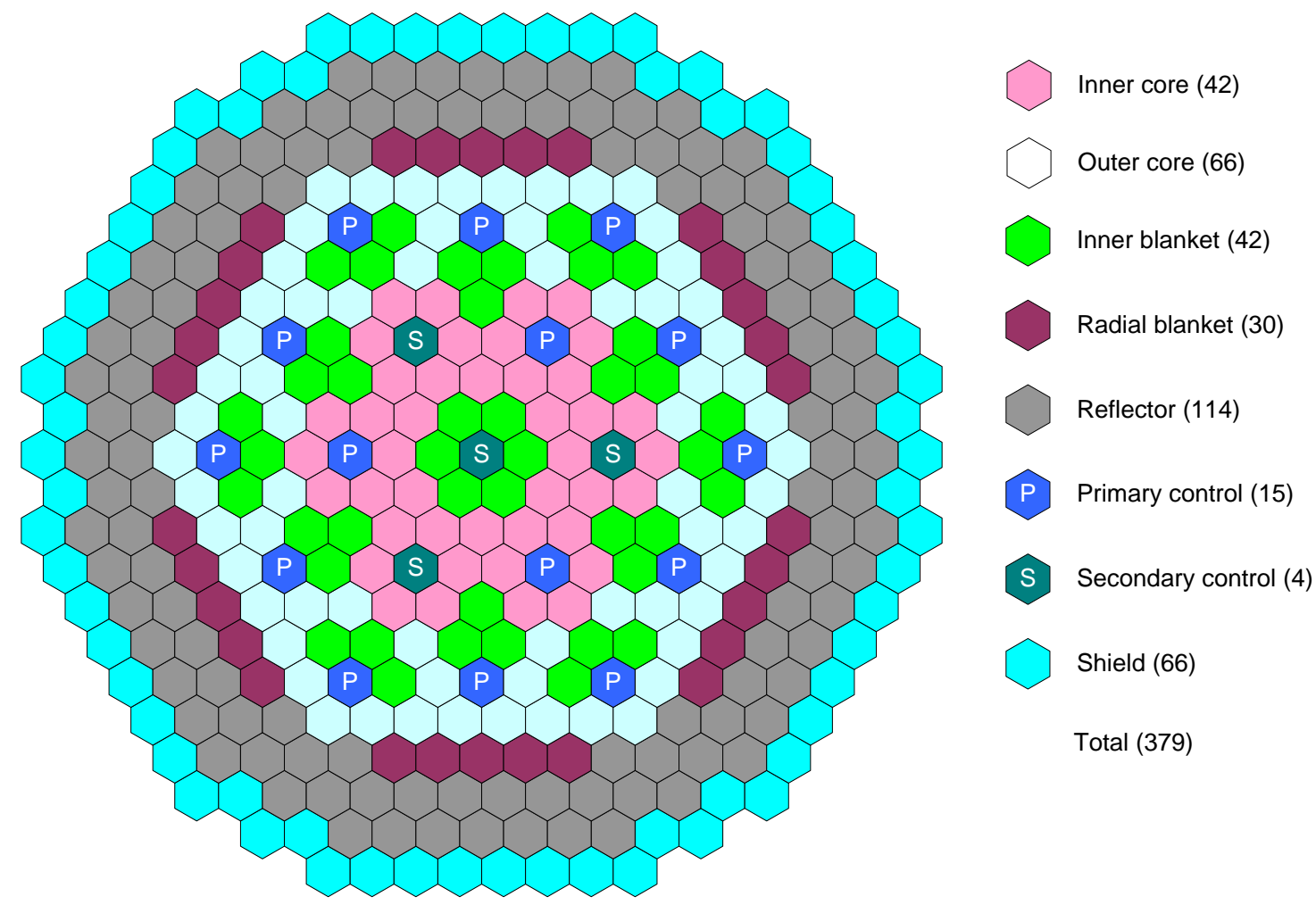

Figure III.6-6 High Conversion Ratio Core Layout with Internal and Radial Blankets 
The driver and blanket design parameters are summarized in Tables III.6-10 and III.6-11, respectively. The driver and blanket design parameters of the breakeven S-PRISM cores are also included for comparison. To reduce the required TRU enrichment, the heavy metal loading is increased by increasing the fuel pin diameter and active core height. Relative to the reference design, the fuel pin diameter is increased from $7.55 \mathrm{~mm}$ to $8.08 \mathrm{~mm}$. The thicker pin diameter increases the fuel volume is increased from $29.2 \%$ to $34.3 \%$ for the metal fuel driver and from $37 \%$ to $41.7 \%$ for the oxide fuel driver. The active core height is increased from $81.3 \mathrm{~cm}$ to 96.5 $\mathrm{cm}$ for the metal core, and from $106.7 \mathrm{~cm}$ to $132.1 \mathrm{~cm}$ for the oxide core. It is noted that the active core height is shorter than that of the S-PRISM drivers while the overall assembly length is the same. The blanket assembly designs are similar to the S-PRISM blanket assemblies. U-Zr binary metal and uranium oxide fuels with depleted uranium are assumed. The blanket assembly is composed of 127 fuel pins of $1.02 \mathrm{~cm}$ diameter. The fuel volume fraction is $44.6 \%$ for the metal core and $51.1 \%$ for oxide core.

The core performance parameters of the high TRU conversion ratio cores are summarized in Tables III.6-12 and III.6-13 for the metal and oxide cores, respectively. The one-year cycle length of the reference core designs is retained, but the number of batches is changed to maximize the discharge burnup within the peak fast fluence limit of the HT9 cladding. In the metal core, four- and six-batch fuel management schemes are used for driver and blanket assemblies, respectively. For the oxide core, six- and nine-batch schemes are used for driver and blanket assemblies, respectively. 
Table III.6-10 Driver Assembly Design Parameters of High Conversion Ratio Cores

\begin{tabular}{|l|r|r|r|r|}
\hline & \multicolumn{2}{|c|}{ Metal Core } & \multicolumn{2}{c|}{ Oxide Core } \\
\cline { 2 - 5 } & $\begin{array}{c}\text { High } \\
\text { Conversion }\end{array}$ & S-PRISM & $\begin{array}{c}\text { High } \\
\text { Conversion }\end{array}$ & S-PRISM \\
\hline Assembly data & & & & \\
- Number of pins & 271 & 271 & 271 & 217 \\
- Assembly pitch, cm & 16.142 & 16.142 & 16.142 & 16.142 \\
- Inter-assembly gap, cm & 0.432 & 0.432 & 0.432 & 0.432 \\
- Duct material & HT9 & HT9 & HT9 & HT9 \\
- Duct thickness, cm & 0.394 & 0.394 & 0.394 & 0.394 \\
- Overall duct length, cm & 477.52 & 477.52 & 477.52 & 477.52 \\
\hline Pin data & & & & \\
- Bond material & Na & Na & He & He \\
- Lower reflector, cm & 111.8 & 111.8 & 111.8 & 96.5 \\
- Active core height, cm & 96.5 & 101.6 & 132.1 & 137.2 \\
- Gas plenum, cm & 144.9 & 191.1 & 167.6 & 170.8 \\
- Pellet smeared density, \% TD & 75.0 & 75.0 & 85.0 & 85.0 \\
- Clad outer diameter, cm & 0.808 & 0.744 & 0.794 & 0.851 \\
- Pin pitch-to-diameter ratio & 1.106 & N/A & 1.120 & N/A \\
- Cladding thickness, cm & 0.056 & 0.056 & 0.056 & 0.064 \\
- Wire wrap diameter, cm & 0.081 & 0.142 & 0.094 & 0.140 \\
\hline Volume fraction at cold, \% & & & & \\
- Fuel or Absorber & 34.3 & 28.3 & 41.7 & 37.6 \\
- Bond & 11.4 & 9.4 & 2.2 & 2.0 \\
- Structure & 25.8 & 25.7 & 25.7 & 25.9 \\
- Coolant & 28.5 & 36.6 & 30.4 & 34.6 \\
\hline
\end{tabular}

As shown in Table III.6-12, breakeven fissile and TRU conversion ratios can be achieved in the metal fuel core without a blanket. Relative to the reference core design, the required TRU enrichment is reduced to $14.4 \%$ from $22.1 \%$, and the heavy metal loading is increased from 13.2 MT to $18.5 \mathrm{MT}$. The increased heavy metal loading reduces the average discharge burnup from 93 to $67 \mathrm{MWd} / \mathrm{kg}$. Since the fissile conversion ratio is almost unity, the burnup reactivity swing is significantly reduced from $2.2 \%$ to $0.6 \%$. The replacement of 30 drivers at the core periphery with blanket assemblies (as in Figure III.6-5) increases the fissile and TRU conversion ratios further without a noticeable change in TRU enrichment; the fissile and TRU conversion ratios are 1.03 and 1.07, respectively. Relative to the homogeneous core without a blanket, the average discharge burnup of driver assemblies is increased to $77 \mathrm{MWd} / \mathrm{kg}$ due to the reduced number of drivers. Additional replacement of 42 internal drivers with blankets as in Figure III.6-6 increases the fissile and TRU conversion ratios to 1.07 and 1.12, respectively. The TRU enrichment is $19.3 \%$, and the average discharge burnup of driver assemblies is $92 \mathrm{MWd} / \mathrm{kg}$. 
Table III.6-11 Blanket Assembly Design Parameters of High Conversion Ratio Cores

\begin{tabular}{|l|r|r|r|r|}
\hline & \multicolumn{2}{|c|}{ High Conversion Core } & \multicolumn{2}{|c|}{ S-PRISM } \\
\hline & \multicolumn{1}{|c|}{ Metal } & Oxide & \multicolumn{1}{|c|}{ Oetal } & \\
\hline Assembly data & & & & 127 \\
- Number of pins & 127 & 127 & 127 \\
- Assembly pitch, cm & 16.142 & 16.142 & 16.142 & 16.142 \\
- Inter-assembly gap, cm & 0.432 & 0.432 & 0.432 & 0.432 \\
- Duct material & HT9 & HT9 & HT9 & HT9 \\
- Duct thickness, cm & 0.394 & 0.394 & 0.394 & 0.394 \\
- Overall duct length, cm & 477.52 & 477.52 & 477.52 & 477.52 \\
\hline Pin data & & & & \\
- Fuel material & U-Zr metal & UO 2 & U-Zr metal & UO 2 \\
- Bond material & Na & He & Na & He \\
- Pellet smeared density, \% TD & 85.0 & 87.0 & 85.0 & 93.0 \\
- Clad outer diameter, cm & 1.201 & 1.201 & 1.201 & 1.201 \\
- Pin pitch-to-diameter ratio & 1.083 & 1.083 & N/A & N/A \\
- Cladding thickness, cm & 0.056 & 0.056 & 0.056 & 0.056 \\
- Wire wrap diameter, cm & 1.300 & 0.094 & 1.300 & 0.094 \\
\hline Volume fraction at cold, \% & & & & \\
- Fuel or Absorber & 44.6 & 51.1 & 44.6 & 51.2 \\
- Bond & 7.8 & 1.3 & 7.9 & 1.3 \\
- Structure & 21.0 & 21.0 & 21.0 & 21.0 \\
- Coolant & 26.6 & 26.6 & 26.5 & 26.5 \\
\hline
\end{tabular}

The results in Table III.6-13 for the oxide cores show similar trends for the metal cores. For a given core layout, however, the oxide core requires a higher TRU enrichment than the metal core. As a result, the fissile and TRU conversion ratios are lower than those of the metal core. On the other hand, the average discharge burnup is significantly higher since the low flux level and softer spectrum allow an increased fuel residence time.

The key performance parameters of the S-PRISM designs [3] are also included in these tables for comparison. By comparing the S-PRISM designs with the heterogeneous designs with radial and internal blankets, it can be seen that the heavy metal loading of the S-PRISM cores is 27\% larger for metal core and $\sim 70 \%$ larger for oxide core. It is noted that the S-PRISM core volume is $\sim 37 \%$ bigger for metal core and $\sim 70 \%$ bigger for oxide core. Despite the significantly higher heavy metal loading, however, the TRU enrichment is higher both for metal and oxide cores. The fissile conversion ratio of the S-PRISM designs is lower for metal core and slightly higher for oxide core. On the other hand, the cycle length is significantly longer ( $\sim 80 \%$ for metal core and $\sim 88 \%$ for oxide core). The ratio of peak fast fluence to average discharge burnup is $\sim 20 \%$ lower for both metal and oxide cores. However, the combined effects of lower power density and longer cycle length of the S-PRISM designs yield a higher discharge burnup for metal core and a lower discharge burnup for oxide core. 
Table III.6-12 Core Performance Parameters of High Conversion Ratio Metal Cores

\begin{tabular}{|c|c|c|c|c|}
\hline & \multicolumn{3}{|c|}{ High Conversion Core } & \multirow{2}{*}{$\begin{array}{c}\text { S-PRISM } \\
\text { Heterogeneous }\end{array}$} \\
\hline Core configuration & Homogeneous & Homogeneous & Heterogeneous & \\
\hline Blanket usage & No & $\begin{array}{c}\text { Radial } \\
\text { blanket only }\end{array}$ & $\begin{array}{l}\text { Internal and } \\
\text { radial blanket }\end{array}$ & $\begin{array}{c}\text { Internal and } \\
\text { radial blanket }\end{array}$ \\
\hline Cycle length, month & 12 & 12 & 12 & 21.7 \\
\hline No. assemblies (driver/blanket) & 180 & $150 / 30$ & $102 / 72$ & $138 / 97$ \\
\hline Number of batches (core/internal/radial) & $4 /-/-$ & $4 /-/ 6$ & $4 / 4 / 6$ & $3 / 4 / 4$ \\
\hline TRU enrichment of fresh fuel, \% & 14.4 & 14.8 & 19.3 & 21.4 \\
\hline Fissile/TRU conversion ratio & $0.99 / 1.00$ & $1.03 / 1.07$ & $1.07 / 1.12$ & a) 1.05 \\
\hline HM/TRU inventory at BOEC, MT & $18.5 / 2.8$ & $19.3 / 2.4$ & $20.5 / 2.5$ & $26.1 / 3.1$ \\
\hline $\begin{array}{l}\text { Driver discharge burnup (ave/peak), } \\
\mathrm{MWd} / \mathrm{kg}\end{array}$ & $67 / 103$ & $77 / 123$ & $92 / 146$ & $106 / 149$ \\
\hline Driver specific power density, W/g & 52.6 & 60.7 & 72.5 & b) 53.0 \\
\hline Driver power density, $\mathrm{kW} / \mathrm{l}$ & 234 & 270 & 321 & b) 218 \\
\hline Peak fast fluence (drive/blanket), $10^{23} / \mathrm{cm}^{2}$ & $3.49 /-$ & $3.83 / 2.98$ & $3.85 / 3.64$ & $3.71 / 3.90$ \\
\hline Burnup reactivity loss, $\% \Delta \mathrm{k}$ & 0.14 & 0.57 & 0.0 & 0.12 \\
\hline Average linear power, $\mathrm{kW} / \mathrm{m}$ & 19.8 & 22.8 & 28.2 & 18.9 \\
\hline Peak linear power density, kW/m & 32.6 & 39.4 & 52.5 & 30.4 \\
\hline Linear power density limit of fuel, $\mathrm{kW} / \mathrm{m}$ & 40.3 & 46.3 & 55.8 & N/A \\
\hline Core average flux, $10^{15} / \mathrm{cm}^{2}$-sec & 2.92 & 3.28 & 3.12 & $\mathrm{~N} / \mathrm{A}$ \\
\hline Fast flux fraction & 0.67 & 0.69 & 0.69 & $\mathrm{~N} / \mathrm{A}$ \\
\hline Fissile $\mathrm{Pu}$ gain rate, $\mathrm{kg} /$ year & 2.3 & 15.0 & 25.0 & 19.3 \\
\hline
\end{tabular}

a) Breeding ratio

b) Estimated because the data was not available 
Table III.6-13 Core Performance Parameters of High Conversion Ratio Oxide Cores

\begin{tabular}{|c|c|c|c|c|}
\hline \multirow[b]{2}{*}{ Core configuration } & \multicolumn{3}{|c|}{ High Conversion Core } & \multirow{2}{*}{$\begin{array}{c}\text { S-PRISM } \\
\text { Heterogeneous }\end{array}$} \\
\hline & Homogeneous & Homogeneous & Heterogeneous & \\
\hline Blanket usage & No & $\begin{array}{c}\text { Radial } \\
\text { blanket only }\end{array}$ & $\begin{array}{l}\text { Internal and } \\
\text { radial blanket }\end{array}$ & $\begin{array}{l}\text { Internal and } \\
\text { radial blanket }\end{array}$ \\
\hline Cycle length, month & 12 & 12 & 12 & 22.6 \\
\hline No. assemblies (driver/blanket) & 180 & $150 / 30$ & $102 / 72$ & 162/133 \\
\hline Number of batches (core/internal/radial) & $5 /-/-$ & $6 /-/ 9$ & $6 / 6 / 9$ & $3 / 4 / 5$ \\
\hline TRU enrichment of fresh fuel, \% & 20.4 & 20.5 & 28.6 & 29.8 \\
\hline Fissile/TRU conversion ratio & $0.96 / 0.92$ & $0.99 / 0.99$ & $1.02 / 1.04$ & a) 1.03 \\
\hline HM/TRU inventory at BOEC, MT & $18.7 / 3.9$ & $19.5 / 3.4$ & $20.5 / 3.5$ & $34.9 / 5.2$ \\
\hline $\begin{array}{l}\text { Driver discharge burnup (ave/peak), } \\
\mathrm{MWd} / \mathrm{kg}\end{array}$ & $96 / 156$ & $111 / 178$ & $131 / 210$ & $116 / 178$ \\
\hline Driver specific power density, W/g & 51.1 & 59.0 & 69.5 & b) 42.4 \\
\hline Driver power density, kW/l & 181 & 208 & 249 & b) 188 \\
\hline Peak fast fluence (driver/blanket), $10^{23} / \mathrm{cm}^{2}$ & $3.45 /-$ & $3.91 / 2.68$ & $4.02 / 3.73$ & $2.96 / 2.44$ \\
\hline Burnup reactivity loss, $\% \Delta \mathrm{k}$ & 0.67 & 1.01 & 0.55 & 0.98 \\
\hline Average linear power, $\mathrm{kW} / \mathrm{m}$ & 15.2 & 17.6 & 21.6 & 16.0 \\
\hline Peak linear power density, kW/m & 27.3 & 31.3 & 40.6 & 30.1 \\
\hline Linear power density limit of fuel, kW/m ${ }^{\mathrm{c})}$ & 41.8 & 42.1 & 41.8 & N/A \\
\hline Core average flux, $10^{15} / \mathrm{cm}^{2}$-sec & 2.17 & 2.39 & 2.35 & N/A \\
\hline Fast flux fraction & 0.57 & 0.58 & 0.58 & N/A \\
\hline Fissile Pu gain rate, kg/year & -8.2 (loss) & 0.7 & 8.6 & 11.2 \\
\hline
\end{tabular}

a) Breeding ratio 
Among three design options, the kinetics and reactivity coefficients of the homogeneous core concept with radial blankets were evaluated. The kinetics and reactivity coefficients are provided in Table III.6-14. It is noted that the sodium void worth and the sodium voided Doppler coefficient were calculated by voiding the flowing coolant in the active core and above, but not voiding in the radial blanket.

Table III.6-14 Kinetics and Reactivity Coefficients of High Conversion Ratio Cores

\begin{tabular}{|c|c|c|c|c|}
\hline & & & & \\
\hline & BOEC & EOEC & BOEC & EOEC \\
\hline Effective delayed neutron fraction & 0.00352 & 0.00349 & 0.00328 & 0.00326 \\
\hline Prompt neutron lifetime, $\mu \mathrm{s}$ & 0.30 & 0.30 & 0.40 & 0.40 \\
\hline Radial expansion coefficient, $\mathbb{\Phi} /{ }^{\circ} \mathrm{C}$ & -0.31 & -0.31 & -0.24 & -0.24 \\
\hline Axial expansion coefficient, $\mathbb{\$} /{ }^{\circ} \mathrm{C}$ & -0.05 & -0.05 & -0.05 & -0.05 \\
\hline Fuel density coefficient, $\mathbb{\$} /{ }^{\circ} \mathrm{C}$ & -0.59 & -0.58 & -0.38 & -0.36 \\
\hline Structure density coefficient, $\mathbb{\mathbb { C }} /{ }^{\circ} \mathrm{C}$ & 0.07 & 0.07 & 0.08 & 0.08 \\
\hline Sodium void worth, \$ & 5.28 & 5.55 & 5.38 & 5.62 \\
\hline Sodium density coefficient, $\mathbb{\$} /{ }^{\circ} \mathrm{C}$ & 0.12 & 0.13 & 0.13 & 0.13 \\
\hline Doppler constant, \$ & -0.57 & -0.56 & -1.29 & -1.26 \\
\hline Doppler coefficient, $\mathbb{\Phi} /{ }^{\circ} \mathrm{C}$ & -0.10 & -0.10 & -0.15 & -0.14 \\
\hline Sodium voided Doppler coefficient, $\mathbb{\$} /{ }^{\circ} \mathrm{C}$ & -0.07 & -0.07 & -0.12 & -0.12 \\
\hline
\end{tabular}

Compared to the reference cores, the effective delayed neutron fraction increases by $5-7 \%$ because of the increased U-238 fraction. The increased active core height decreases the radial expansion coefficient by $18 \%$ for the metal core and $23 \%$ for the oxide core. The Doppler coefficient of the metal core is comparable to the reference core, but the coefficient of the oxide core increases by $25 \%$. The sodium void coefficient decreases by $16 \%$ for the metal core and $12 \%$ for the oxide core. The calculated shutdown margins of the primary and secondary control systems are more than adequate. Due to the low burnup reactivity loss, the primary control assemblies are almost withdrawn from the active core at BOEC, and as a result, the control-rod

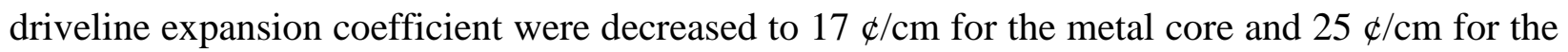
oxide core.

The integral reactivity parameters for the quasi-static reactivity balance analysis were estimated and provided in Table III.6-15. Compared to the reference cores, the integral parameter $B$ of both metal and oxide cores was decreased significantly, and the increased Doppler coefficient of the oxide core increases the integral parameter $A$. All combined effects result in that the integral reactivity parameters of the metal core concept satisfy all three sufficient conditions for passive safety. However, the oxide core concept does not meet the unprotected loss of flow accident, while better for unprotected transient overpower accident. Further studies need to determine design fixes for acceptable asymptotic core outlet temperatures for possible unprotected accident scenarios. 
Table III.6-15 Integral Reactivity Parameters of High Conversion Ratio Cores

\begin{tabular}{|c|c|c|c|c|c|}
\hline & \multicolumn{2}{|c|}{ Metal } & \multicolumn{2}{|c|}{ Oxide } \\
\hline & & BOEC & EOEC & BOEC & EOEC \\
\hline \multicolumn{2}{|c|}{ A, power coefficient (\$) } & -10.0 & -9.9 & -81.7 & -79.7 \\
\hline \multicolumn{2}{|c|}{ B, power/flow coefficient (\$) } & -45.4 & -44.1 & -45.8 & -35.4 \\
\hline \multicolumn{2}{|c|}{$\begin{array}{l}\text { C, inlet temperature coefficient } \\
\left(\Phi /{ }^{\circ} \mathrm{C}\right)\end{array}$} & -0.40 & -0.40 & -0.41 & -0.34 \\
\hline \multicolumn{2}{|c|}{$\begin{array}{l}\text { Transient overpower initiator, } \\
\Delta \rho_{\text {TOP }}(\$)\end{array}$} & 0.18 & 0.0 & 0.21 & 0.0 \\
\hline \multirow{3}{*}{$\begin{array}{l}\text { Sufficient } \\
\text { conditions } \\
\text { for } \\
\text { passive } \\
\text { safety }\end{array}$} & $\mathrm{A} / \mathrm{B}<1.0$ & 0.22 & 0.22 & 1.78 & 2.25 \\
\hline & $\begin{aligned} 1.0 & <\mathrm{C} \Delta T_{c} / \mathrm{B} \\
& <2.0\end{aligned}$ & 1.41 & 1.40 & 1.39 & 1.48 \\
\hline & $\begin{array}{c}\Delta \rho_{\text {TOP }} /|\mathrm{B}|< \\
1.0\end{array}$ & 0.40 & 0.00 & 0.46 & 0.00 \\
\hline
\end{tabular}

\section{References}

1. E. Walter, A. B. Reynolds, “Fast Breeder Reactors,” Pergamon Press (1981).

2. Y. I. Chang, “Technical Rationale for Metal Fuel in Fast Reactors," Nuclear Engineering and Technology, Vol 39, 161 (2007).

3. E. Dubberley, K. Yoshida, C. E. Bardman, T. Wu, "Super PRISM Oxide and Metal Fuel Core Designs,” Proceedings of ICONE 8, April 2-6, Baltimore, MD (2000).

4. D. C. Wade and E. K. Fujita, "Trends versus Reactor Size of Passive Reactivity Shutdown and Control Performance,” Nuclear Science and Engineering, 103, 182 (1989). 


\section{III.7 Safety Analyses}

Preliminary analyses have been completed to assess the potential safety performance characteristics of the Advanced Burner Reactor (ABR) $1000 \mathrm{MW}_{\text {th }}$ design. The scope of the analyses presented here focuses on the ability of ABR to provide inherent protection against damaging consequences in low probability accident sequences involving multiple equipment failures.

\section{III.7.1 Background and Summary}

A summary description of the accident sequences analyzed and the results obtained are first presented. Detailed descriptions of the analyses are provided in subsequent sections. In particular, Section III.7.2 contains a description of the analysis scope and initial reactor state, while Section III.7.3 describes the analysis methods modeling data and Section III.7.4 contains a more detailed account of the transient results.

\section{III.7.1.1 Analysis Background}

One of the primary goals in the ABR design is to provide not only the customary safety margins in design basis events, but also to deliver superior safety performance in beyond design basis events involving multiple equipment failures or unplanned operator actions. Consequently, the preliminary analyses presented here examine the behavior of the ABR in response to an accident initiator that is normally considered to have a low occurrence frequency but potentially severe consequences, especially when engineered safety systems are assumed to fail. The analyses consider the response of both metal-fueled and oxide-fueled cores to the transient conditions.

The accident initiator examined here is the total loss of normal power to the reactor cooling system while the plant is operating at full rated power. Within the plant, the effect of this initiator is the loss of normal operation of all reactor and feed-water coolant pumps. According to design, the plant responds with a reactor scram, with activation of emergency power supplies (diesel generators and batteries), and with activation of the normal shutdown heat removal mode. The normal shutdown heat removal path is through the reactor coolant system and steam generators, with auxiliary power supplied by the emergency power supplies. As a backup, a low-capacity emergency heat removal system is provided to remove heat directly from the reactor without the need for emergency power.

For the transient accident sequences analyzed here, the loss of power is accompanied by a complete failure of the emergency power supply system, resulting in a total loss of power to the reactor and intermediate coolant pumps. It is also assumed that the power generation plant immediately ceases operation, and provides no heat rejection capacity through the steam generators. The sole heat removal path following the loss of forced coolant flow is through the emergency heat removal system by natural circulation. This sequence was analyzed for the case with an immediate reactor scram and for the case without reactor scram. These cases are identified as the protected loss-of-flow (PLOF) and the unprotected loss-of-flow (ULOF) cases respectively. The PLOF and ULOF accident sequences both assume multiple equipment failures, failures of safety grade protection and cooling systems, and no operator actions. These sequences 
are an extreme test of the ABR to provide inherent self-protection against the consequences of the most severe accident initiators.

\section{III.7.1.2 Results Summary}

The base cases for both the metal and oxide core designs are for the beginning of equilibrium cycle conditions. The detailed analysis results for the PLOF and ULOF accident sequences are presented in Section III.7.4, below. In addition to the base cases, parametric variations have also been performed for the ULOF accident sequence for both the metallic-fueled and the oxidefueled cores. Parametric variations for the metal core involve changes to the control-rod driveline (CRDL) expansion reactivity feedback. Parametric variations for the oxide core also involve the CRDL reactivity feedback, but additional results are presented for the case where a self-actuated shutdown system (SASS) is employed. The SASS could be a secondary or a tertiary shutdown system. The specific SASS device selected for the analysis presented here is based on a safety rod passive de-latch mechanism utilizing magnetic resistance change at the Curie point temperature of the sensing alloy. Although these sequences simulate accidents that for some reactor designs may cause damage to the fuel and possibly progress into severe accident conditions, in the ABR these events cause no damage. During the accident sequences, reactor fuel, cladding, and coolant temperatures remain below safety limits.

In the PLOF sequence, the loss of forced coolant flow and normal heat removal is accompanied immediately by a reactor scram, which quickly brings the reactor power to decay heat levels. Early in the sequence, the emergency decay heat removal system does not have sufficient capacity to remove all the heat being produced, so system temperatures rise. However, due to the large heat capacity of the sodium-cooled pool-type concept, the ABR is able to absorb a significant amount of energy with only a slight temperature increase, and the natural circulation capability of the ABR promotes heat removal through the available emergency heat sink. After about six hours, the reactor decay heat falls to the capacity of the emergency heat removal system, and system temperatures begin to decrease. The analysis predicts that short coolant and cladding temperature spikes occur during the transition to natural circulation, but no fuel damage or cladding failures would occur. These conclusions hold for both the metal core and the oxide core, although some quantitative differences exist due to differences in core pressure drop (due to differences in the hydraulic resistances of the respective pin bundles) and differences in the initial stored energy (due to the higher operating temperature of oxide fuel).

In the ULOF accident, the reactor safety system fails to scram the reactor upon loss of forced coolant flow and normal heat removal, so the reactor remains at full power initially. Within the first minute, reactor temperatures increase as the coolant flow rate decreases, and inherent reactivity feedbacks reduce the reactor power. During this time, peak cladding temperatures rise to approximately $700^{\circ} \mathrm{C}$ for the metal core and $750^{\circ} \mathrm{C}$ for the oxide core. As coolant flow continues to decline, a second temperature peak occurs, and peak cladding temperatures reach approximately $550^{\circ} \mathrm{C}$ for the metal core and $800^{\circ} \mathrm{C}$ for the oxide core. This increase in temperature provides the necessary driving force to establish natural circulation flow. The development of natural circulation holds the slowly-rising peak cladding temperatures to around $800^{\circ} \mathrm{C}$ for the oxide core, but even after six hours into the transient the fission power remains a significant part of the total power and the DRACS heat removal capability is still below the total heat production rate. The oxide system continues slowly heating up. The situation is quite different for the metal core where the peak fuel and cladding temperatures are reduced to about $520^{\circ} \mathrm{C}$ after six hours and the decay heat dominates. At this point the DRACS heat removal 
capability is reaching the total heat production rate, after which temperatures can be expected to remain stable. There is also a difference in the coolant boiling margin. The ULOF parametric feedback cases show a boiling margin of around $220^{\circ} \mathrm{C}$ to $250^{\circ} \mathrm{C}$ for the metal cases, but of only $50^{\circ} \mathrm{C}$ to $150^{\circ} \mathrm{C}$ for the oxide cases without the benefit of a SASS scram. The results also show that with the use of a SASS scram, the coolant boiling margin for the most challenging oxide case can be increased from $50^{\circ} \mathrm{C}$ to $170^{\circ} \mathrm{C}$.

The primary significance of the analysis results is that both metal and oxide core designs demonstrate significant safety margins to coolant boiling and fuel damage in PLOF accident sequences. The metal core design also exhibits significant safety margins in the ULOF accident sequence. This is a direct consequence of the high thermal conductivity and low operating temperature of metal fuel, and the favorable negative feedback due to thermal expansion. However, the ULOF analyses for the oxide core design indicate that margins to coolant boiling will not be adequate without additional accident mitigation features. Inadequate margins exist for the oxide core design despite the use of a significantly longer flow halving time (20 seconds) compared to that used for the metal core (five seconds). The initially high oxide operating temperatures result in significant positive Doppler feedback when trying to reduce temperatures. Additional enhancements, such as a self-actuating shutdown (SASS) device will be required for the oxide core to increase safety margins so that no fuel damage or cladding failures would occur, even when multiple safety systems are assumed to malfunction. Nevertheless, the neutronic, thermal, and hydraulic performance characteristics of the ABR design provide a defensive barrier to reactor damage for accident initiators that otherwise progress into severe accident conditions. Such superior safety characteristics are inherent to a sodium-cooled, pooltype reactor concept.

\section{III.7.2 Analysis Scope}

The analysis results reported here were selected on the basis that they show the safety margins and the inherent ability of a sodium-cooled, pool-type reactor system to provide protection against severe, damaging consequences. The accident sequences analyzed are near the end of the spectrum of the most pessimistic, challenging, and potentially damaging events. The analysis results demonstrate the passive safety performance advantages of the ABR. This performance is possible because of the favorable natural circulation shutdown heat removal capability that is possible with low pressure sodium coolant in a pool configuration. In addition, metallic fuel provides significant inherent safety performance due to its superior heat transfer and reactivity feedback characteristics.

\section{III.7.2.1 Accident Sequences}

The basic accident sequence analyzed is the loss of normal power to the reactor and intermediate coolant pumps, with failure of the emergency power supplies. The result is a loss of forced flow in the primary and intermediate coolant circuits, although mechanical pump inertia will provide for a coast-down in the coolant flow during the very early part of the transient. In addition, it is assumed that heat removal through the steam generator ceases, so that the only heat removal path is through the emergency direct reactor auxiliary cooling system (DRACS).

The natural circulation DRACS consists of heat exchangers located in the cold pool region within the reactor vessel, air dump heat exchangers located outside containment, and the connecting piping. The working fluid in the DRACS is NaK, and fluid flow is by natural 
circulation. Multiple independent DRACS units are provided for defense in depth. The DRACS is designed to remove $0.5 \%$ of full power $(5000 \mathrm{~kW})$ at normal operating temperatures assuming failure of one DRACS unit. The DRACS system operates continuously, with heat losses limited in normal operation by dampers on the NaK-to-air heat exchangers. In all the accident sequences analyzed here, one DRACS unit is assumed to fail, leaving a nominal system heat rejection capacity of $5000 \mathrm{~kW}$.

The initial condition for the accident sequence is normal operation at full power and flow. With the loss of pumping power, flow in the primary circuit coasts down according to the spinning inertia of the pumps and motors. Following flow coast down, natural circulation flow is established.

With the loss of power, forced flow in the intermediate coolant system is also lost. Further, it is assumed that heat rejection through the steam generator ceases. The intermediate heat transport system (IHTS) is alternately a heat sink or source in the accident sequence, depending on its temperature and the primary system temperature at the intermediate heat exchanger (IHX). During the transient, natural circulation flow in the IHTS may reverse, depending on transient temperature conditions.

Two variations of the loss-of-flow accident sequence have been analyzed. In the first, it is assumed that the reactor safety system acts as designed to insert control rods and reduce reactor power immediately to decay heat. This sequence is called the protected loss-of-flow (PLOF) accident. In the second analysis, it is assumed that the reactor safety system fails to insert the scram control rods, and the loss of forced flow proceeds at full power. This sequence is called the unprotected loss-of-flow (ULOF).

In the PLOF sequence, the absence of normal shutdown heat removal through the reactor coolant system causes a slow system temperature rise following the reactor scram. This temperature increase occurs because the DRACS has insufficient heat removal capacity to overcome both the early decay heat production rate and the stored heat in the primary and intermediate systems. Eventually, the decay heat falls below the DRACS capacity, and the system temperature declines.

In the ULOF sequence, the system temperature rises significantly with the flow coast down, but the core temperature rise introduces negative reactivity that acts to reduce the reactor fission power. In general, negative reactivity feedback effects will cause the reactor to seek equilibrium with the available heat sink by reducing power, but there are important differences between the metal and oxide cores. For the metal core, the reduction in power has the effect of reducing the reactor temperature and establishing a quasi-equilibrium condition. However, until the decay heat falls below the available heat rejection capacity, the reactor system will continue to heat slowly, with the long term temperature rise buffered by the thermal heat inertia of the system. When decay heat production falls below the DRACS capacity, the system temperature declines. For the oxide core, the positive Doppler feedback tends to limit the net reduction in power, thereby inhibiting the reduction in reactor temperatures.

\section{III.7.2.2 Reactor State}

Safety analyses were performed for the beginning-of-equilibrium-cycle (BOEC) reactor conditions described in Section II.1.1. For the BOEC safety analysis of the metal core, it was assumed that sufficient irradiation had taken place to swell the fuel radially into contact with the cladding. Examination of EBR-II irradiated fuel has indicated that fuel-cladding contact will 
occur early in fuel life (1\% burnup), depending on the initial geometry and local specific power. Fuel-cladding contact has the impact of lowering thermal resistance by eliminating the sodiumfilled fuel-cladding gap. For the oxide core, calculations were carried out to determine preaccident conditions of the fuel, including radial expansion, central void formation, and fuel porosity and cracking.

In both the PLOF and ULOF analyses, the decay heat curve was taken as $100 \%$ of the ANSI 5.1 standard [1] for Pu-239. For the ULOF analysis, the decay heat curve was combined with the computed fission power calculated using the reactivity feedback parameters determined by the reactor physics analysis of Section II.1.1.

\section{III.7.3 Analysis Methods and Input Data}

The analytical methods used to evaluate the ABR performance are incorporated into the SAS4A/SASSYS-1 computer code [2] which was used to produce all the safety analysis results presented here. In the following sections, the reactor and coolant system thermal-hydraulic models are described along with key input data relevant to the determination of ABR safety performance.

\section{III.7.3.1 Reactor Thermal Hydraulics}

The thermal-hydraulic performance of the reactor core is analyzed with a geometric model consisting of a number of single-pin channels. In a multiple-channel, whole-core model, each channel represents a single fuel pin and its associated coolant and structure. The single pin is assumed to characterize the average pin in a fuel subassembly, and subassemblies with similar reactor physics and thermal-hydraulics characteristics are grouped, so a number of channels are selected to represent all the pins in the reactor core.

The geometry assumed in the channel thermal-hydraulic model is shown in Figure III.7-1. Heat generated in the fuel is assumed to travel through the cylindrically-symmetric pin to the upward-flowing coolant. The structure field is used to represent part of the hexagonal duct and the wire wrap. One-dimensional, radial heat transfer calculations are performed at many axial locations to model heat transfer from the fuel through the cladding to the coolant, and from the coolant to the structure, the gas plenum, and the reflectors. One-dimensional (axial) coolant mass flow is modeled with a momentum equation solution for the axial pressure profile, and convective heat transfer conditions are assumed at the interfaces between the coolant and the cladding, the reflectors, and the structure. Temperatures are calculated at multiple radial nodes in the fuel, the cladding, the reflectors, and the structure. A single bulk coolant temperature is calculated at each axial location. Axial heat conduction is neglected. 

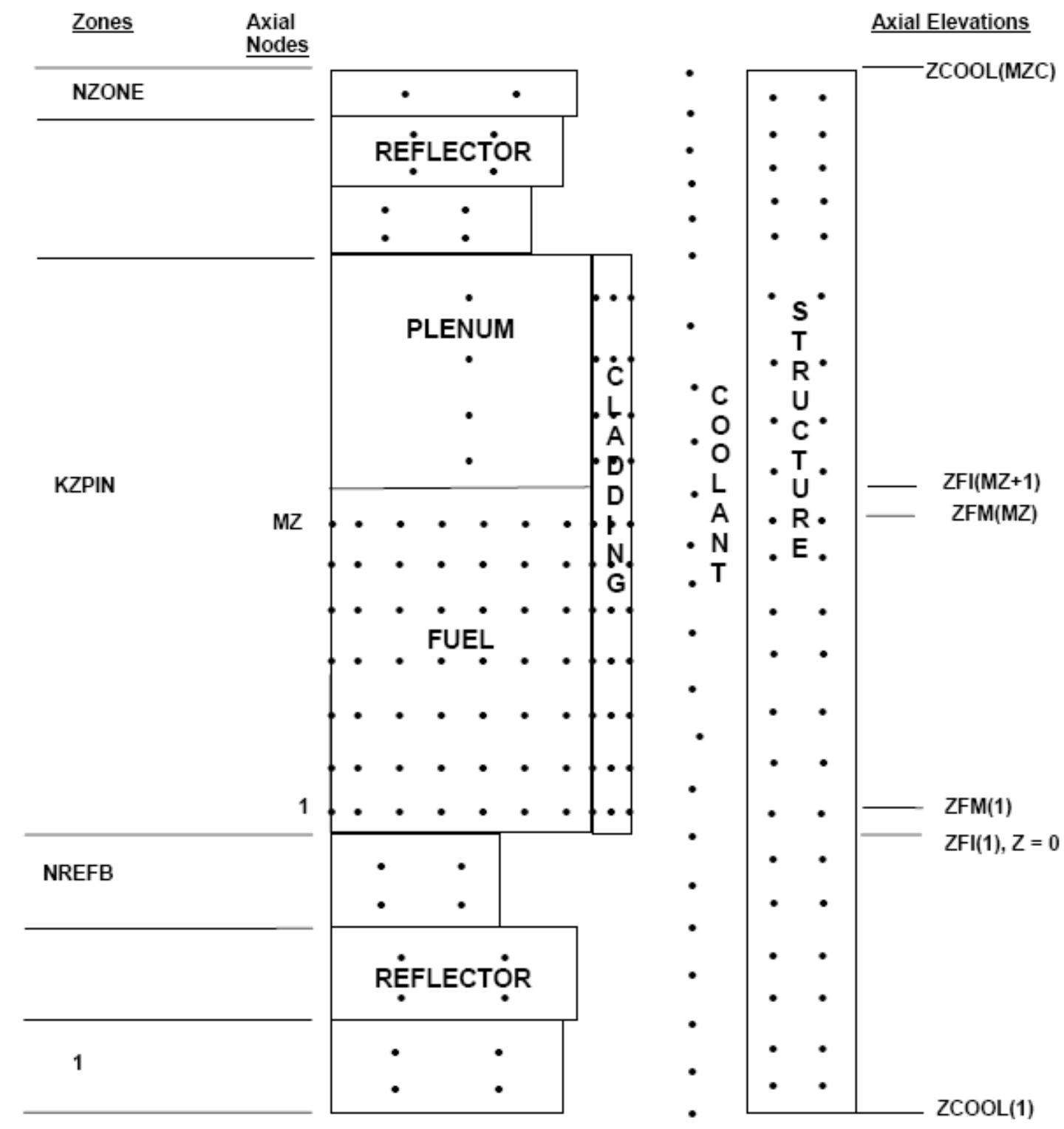

Figure III.7-1 SAS4A/SASSYS-1 Single-Pin Channel Model

Thermal, transport, and physical properties data for the coolant were taken as the temperature-dependent liquid sodium properties available in SAS4A/SASSYS-1. Cladding properties were taken as the HT9 data presented in Reference 3. Fuel properties for metal fuel were taken from the SSCOMP correlations in SAS4A/SASSYS-1 Version 3.0; these correlations are based on data generated in the Integral Fast Reactor (IFR) Program. Fuel properties for both pre-transient and transient behavior of oxide fuel are based on the DEFORM-4 module of SAS4A/SASSYS-1.

On the basis of the reactor physics calculations, the multiple-channel model depicted in Figure III.7-2 was selected for safety analysis calculations. This model approximates the full heterogeneity of the reactor physics model by assigning channels to represent each fuel 
enrichment zone. Channels 1 and 2 represent the average subassemblies in the inner and outer enrichment zones, respectively. Channel 3 represents all of the non-fuel subassemblies, including reflector and control assemblies. A fourth channel is used to represent the peak power-to-flow subassembly containing fresh fuel. The peak assembly is selected based on the highest power-tocoolant-flow ratio. For both metal and oxide cores, the peak power-to-flow assembly is one of the inner-core assemblies although the location of the peak assembly is different, as shown in Figure III.7-2.

Table III.7-1 shows the geometric input data employed in the multiple-channel whole-core model. For the metal fuel, it has been assumed that the irradiated fuel has swollen into contact with the cladding. For the oxide fuel, the DEFORM-4 model in SAS4A/SASSYS-1 is used to calculate the radial swelling of the oxide fuel under steady-state radiation. The swelling is calculated for each axial node such that in some axial location the gap between the fuel and cladding could be closed while in other locations it could still be present. The thermal conductivity of the gap, initially filled with helium, is calculated by DEFORM-4 at each axial location.

The reflector channel power and flow rate represent average power and flow for all non-fuel assemblies in the core, including primary and secondary control rods and reflectors. The reflector channel is assumed to be represented by the reflector assembly geometry.

Axial power profiles in the core region are assumed to be identical for all assemblies. The power profile for the SAS4A/SASSYS-1 axial mesh, presented in Figure III.7-1, was calculated based on the five-region core power density for core assembly (IC34). The five-region power density was fitted with a fourth-degree polynomial in a way that the region-average values are conserved. Figure III.7-3shows the five-region core average and peak power densities as well as the polynomial fit to the power profile for metal and oxide fuel cores.

Figure III.7-4 shows the initial subassembly powers for the BOEC condition. The subassembly power for the peak assembly assumes fresh fuel. All other subassembly powers represent channel averages from BOEC fuel compositions at various stages of depletion. Subassembly coolant flow rates at steady-state conditions are shown in FigureIII.7-5 Coolant flow rates are from the flow-orifice calculations described in Section II.1.1.

Based on the above input conditions, SAS4A/SASSYS-1 calculates the initial, steady-state, thermal-hydraulic conditions in the reactor core prior to the onset of a transient. Peak coolant outlet, peak cladding, and peak fuel temperatures are shown in Figures III.7-6, III.7-7, and III.7-8, respectively. Coolant, cladding, and fuel temperatures that arise during a transient are discussed in the relevant sections below. 

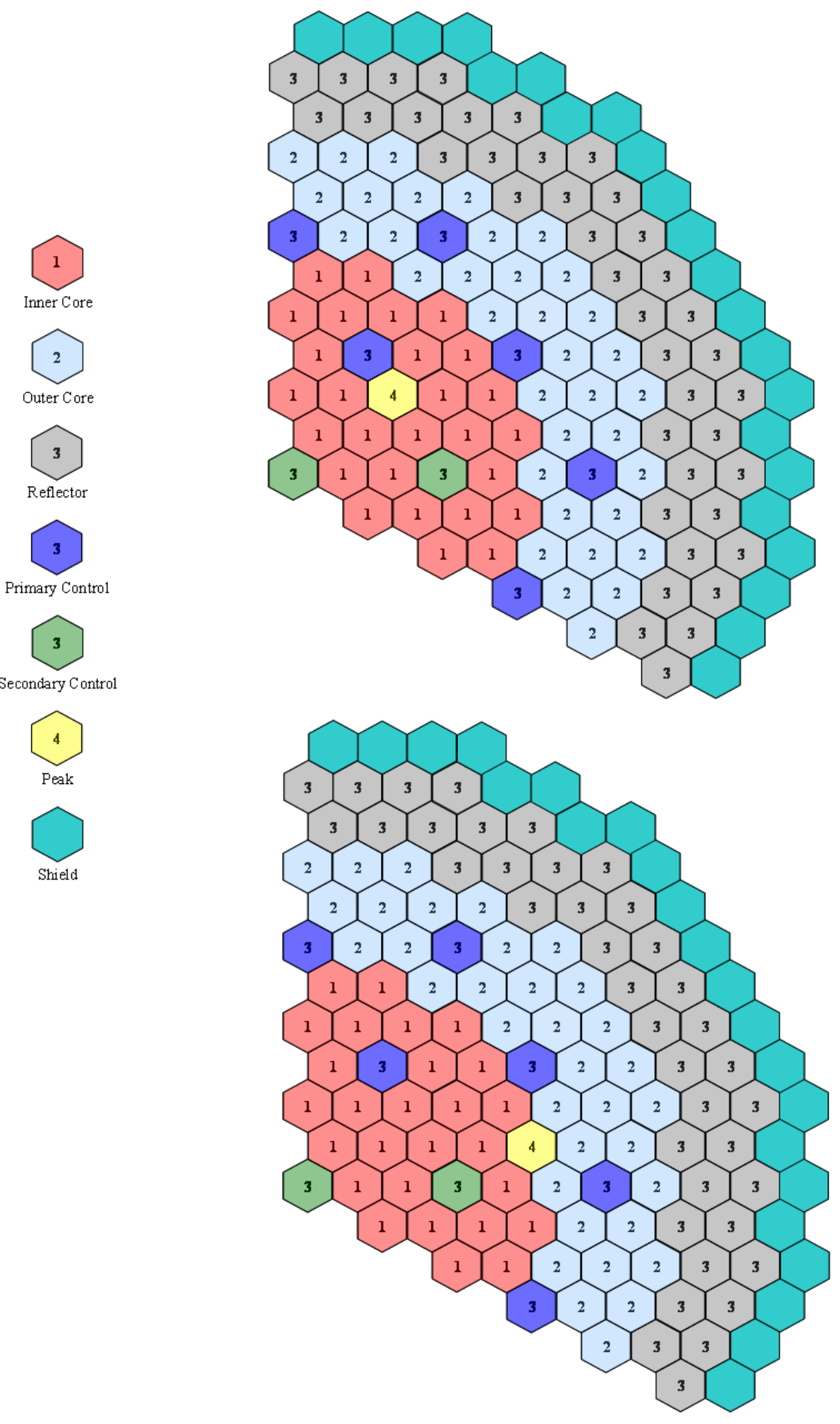

Figure III.7-2 Channel Assignment for Reactor Core Thermal-Hydraulic Model 
Table III.7-1 Fuel Assembly, Pin, and Coolant Channel Model Data

\begin{tabular}{lccc}
\hline & $\begin{array}{c}\text { Metal } \\
\text { Fuel }\end{array}$ & $\begin{array}{c}\text { Oxide } \\
\text { Fuel }\end{array}$ & Reflector \\
\hline Pins per Assembly & 271 & 271 & 91 \\
Number of Assemblies & 180 & 180 & 133 \\
$\quad$ Channel 1 (Inner Core) & 77 & 77 & - \\
$\quad$ Channel 2 (Outer Core) & 102 & 102 & - \\
$\quad$ Channel 3 (Reflector) & - & - & 133 \\
$\quad$ Channel 4 (Peak) & 1 & 1 & - \\
Fuel Height, mm & 812.8 & 1066.8 & - \\
Gas Plenum Height, mm & 1244.6 & 1600.2 & - \\
Upper Reflector Height, mm & 1117.6 & 635 & - \\
Lower Reflector Height, mm & 1244.6 & 1117.6 & - \\
Axial Node Height, mm & & & \\
$\quad$ Core & 40.6 & 53.3 & same as \\
$\quad$ Gas Plenum & 207.4 & 266.7 & fuel \\
$\quad$ Upper Reflector & 223.5 & 127.0 & assembly \\
$\quad$ Lower Reflector & 248.9 & 223.5 & \\
Hydraulic Diameter, mm & 3.361 & 3.361 & 1.878 \\
Coolant Flow Area per Pin, mm ${ }^{2}$ & 25.03 & 25.03 & 25.40 \\
Outer Fuel Radius, mm & 3.22 & 3.13 & - \\
Inner Cladding Radius, mm & 3.22 & 3.22 & - \\
Outer Cladding Radius, mm & 3.78 & 3.78 & 7.71 \\
Structure Thickness ${ }^{\mathrm{a}}$, mm & 4.50 & 4.50 & 4.50 \\
Structure Perimeter ${ }^{\mathrm{b}}$ per Pin, mm & 2.21 & 2.21 & 5.68 \\
Reflector Thickness, mm & 1.89 & 1.89 & 3.85 \\
Reflector Perimeter, mm & 23.72 & 23.72 & 48.41 \\
\hline
\end{tabular}

a. Structure thickness includes weighted contribution from the inter-assembly gap sodium.

b. Structure perimeter includes contribution from wire wrap spacers. 


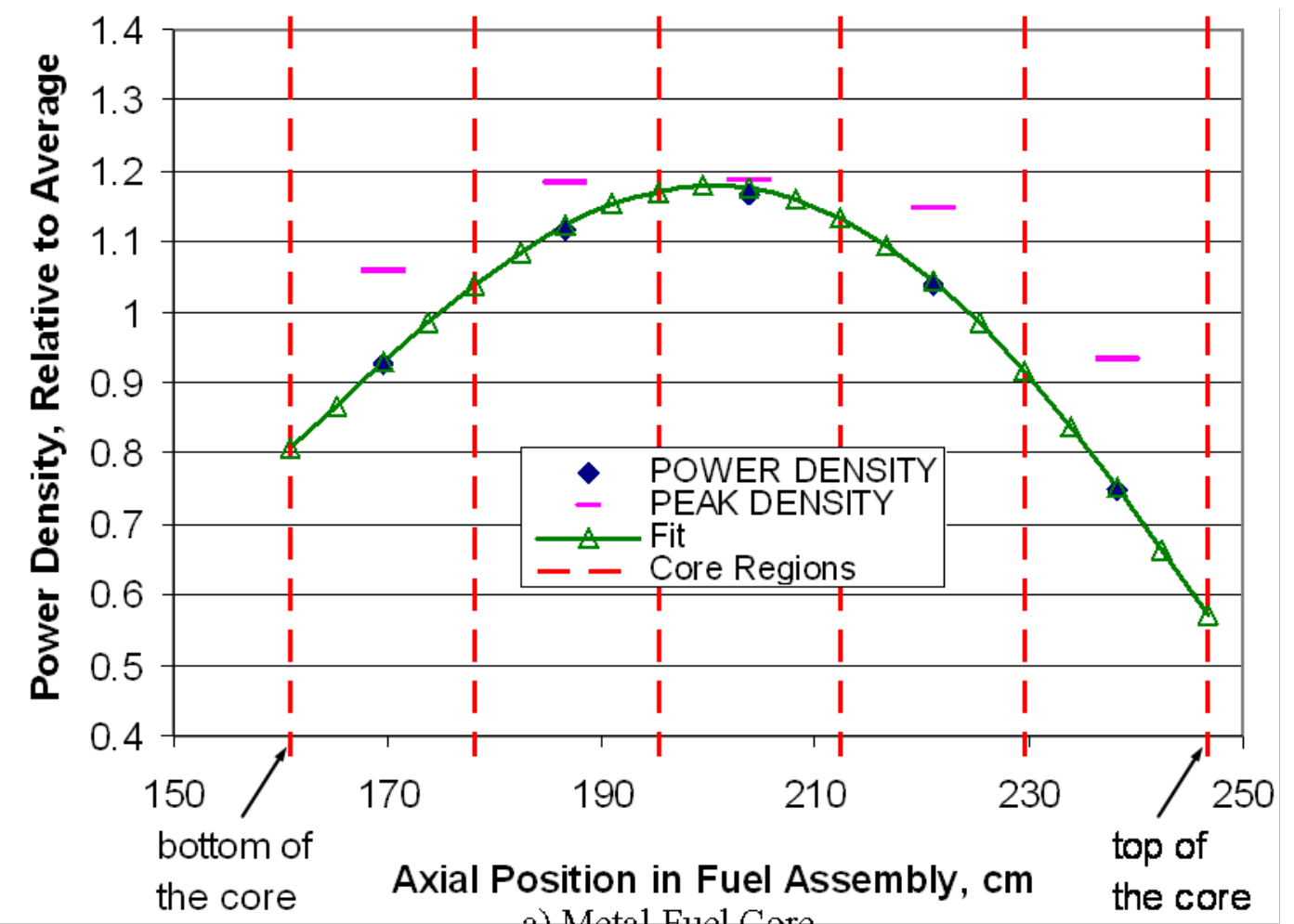

a) Metal Fuel Core

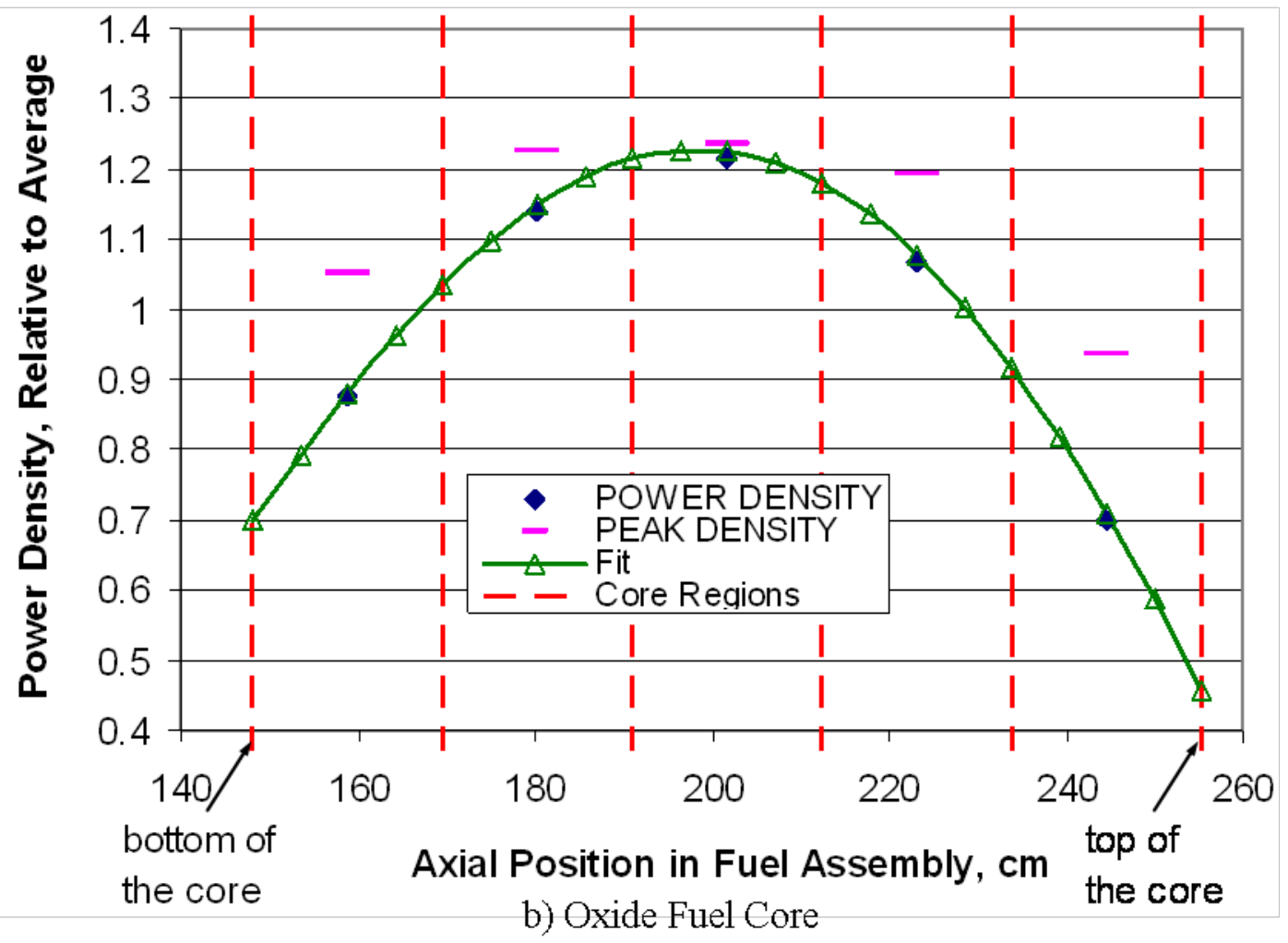

Figure III.7-3 Core Power Profiles for SAS4A/SASSYS-1 Model 


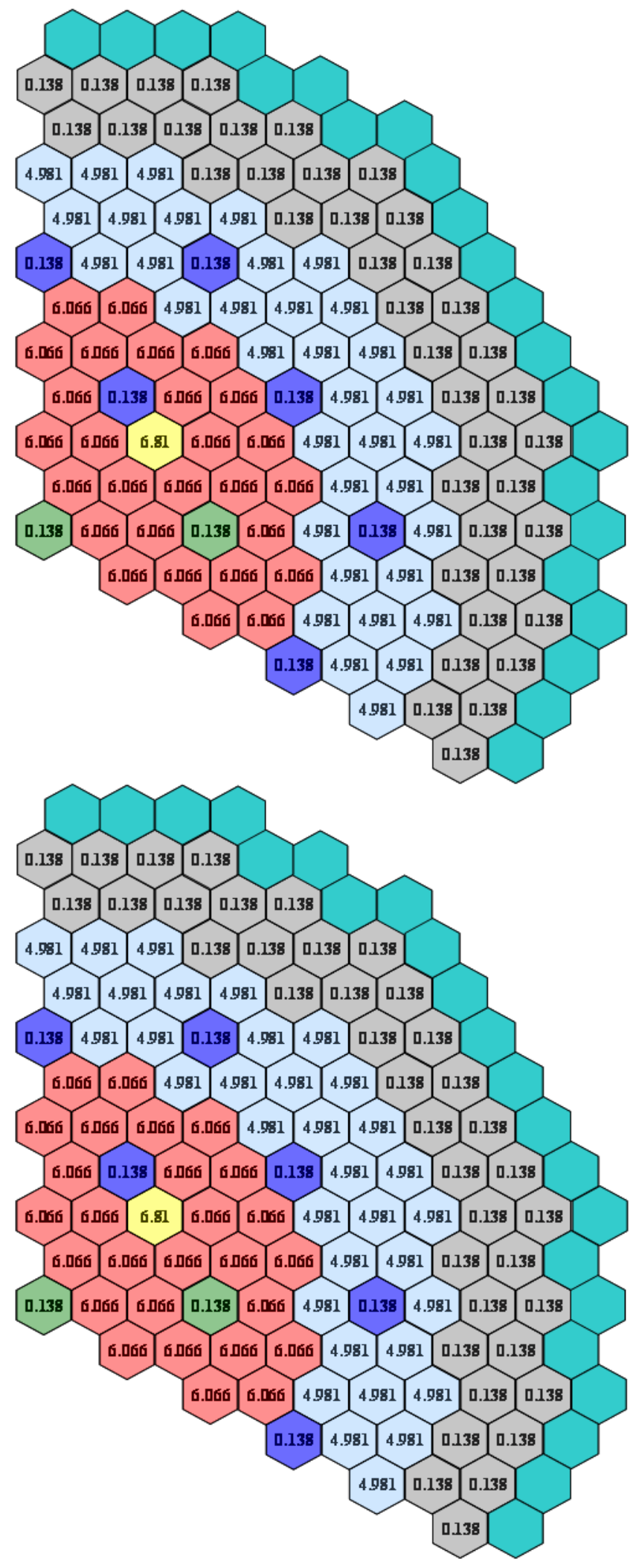

Figure III.7-4 BOEC Initial Subassembly Power (MW) 


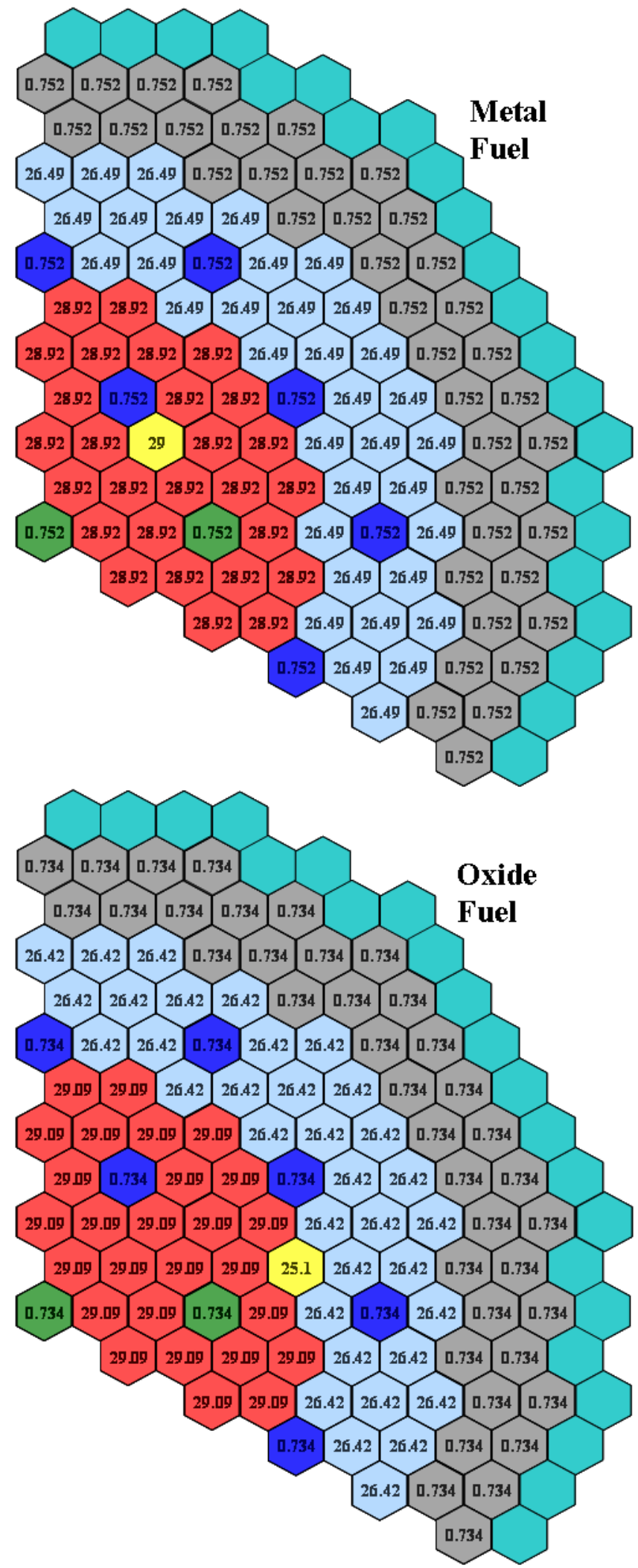

Figure III.7- 5 Initial Subassembly Coolant Flow (kg/s) 


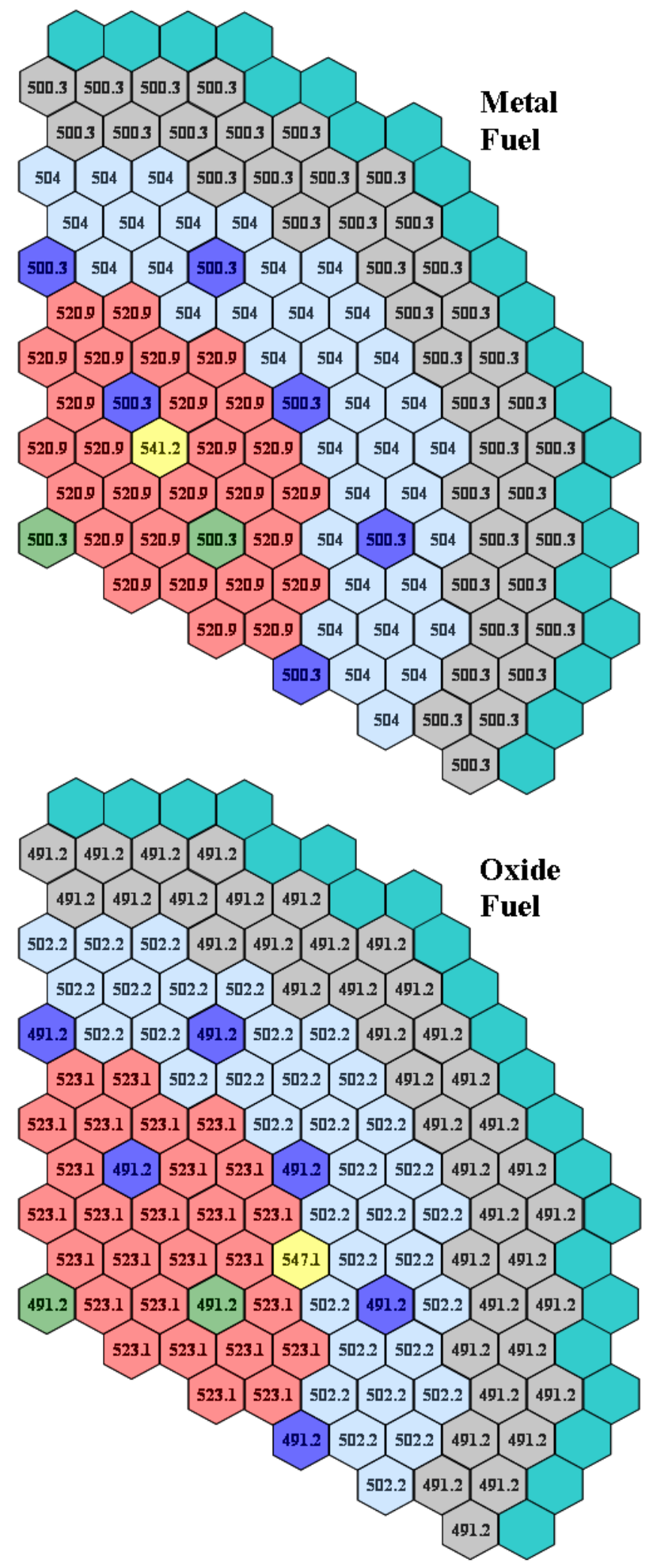

Figure III.7-6 BOEC Initial Coolant Outlet Temperature $\left({ }^{\circ} \mathrm{C}\right)$ 


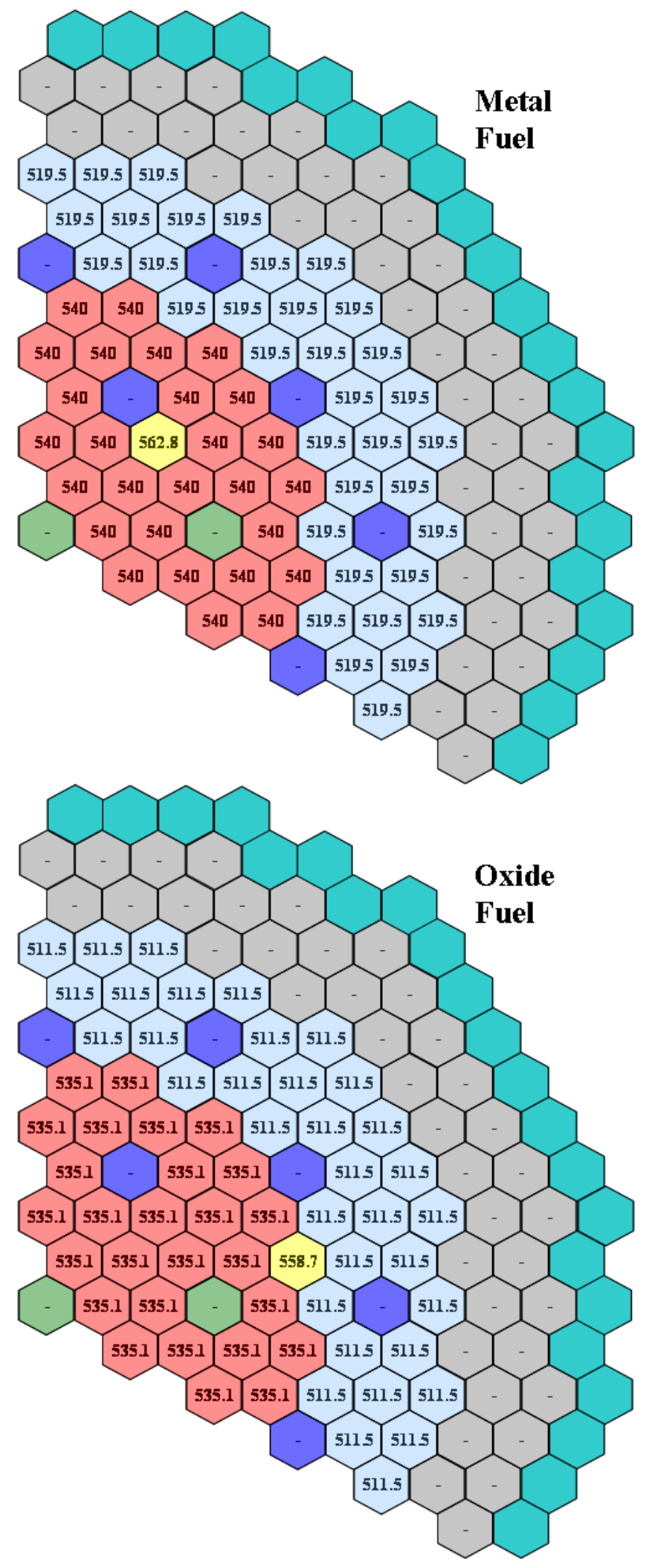

Figure III.7-7 BOEC Initial Peak Cladding Temperature $\left({ }^{\circ} \mathrm{C}\right)$ 

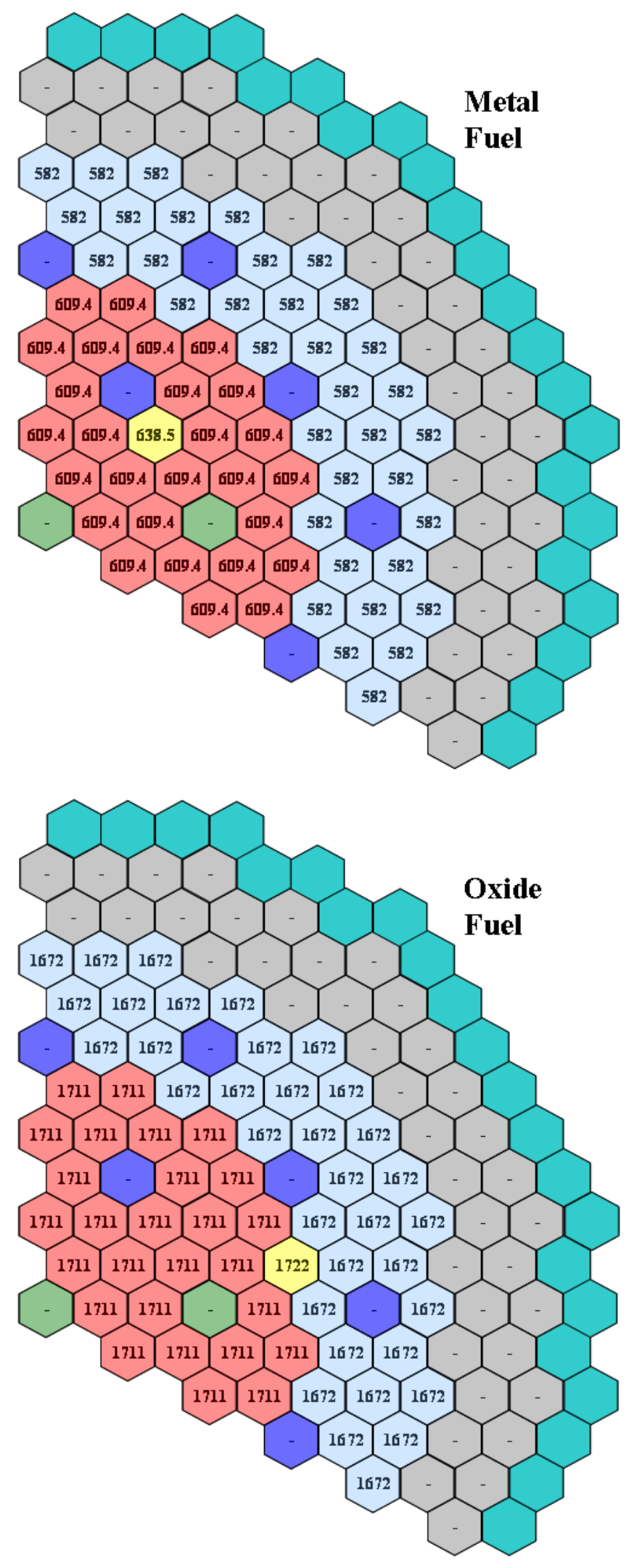

Figure III.7-8 BOEC Initial Peak Fuel Temperature $\left({ }^{\circ} \mathrm{C}\right)$ 


\section{III.7.3.2 Coolant Systems Thermal Hydraulics}

The coolant systems thermal hydraulics model represents coolant flow and heat transfer in the primary and intermediate sodium systems, and in the emergency decay heat removal system, with a network of volumes and components connected by flow paths. The coolant systems model is shown in Figure III.7-9. From the inlet plenum, cold coolant flows through the core and is heated, then exits to the outlet plenum and travels through the shell side of the intermediate heat exchangers (IHXs), where it gives up its heat. Cold primary coolant exits the IHXs and flows to the cold pool. The primary coolant pumps take suction from the cold pool and deliver the coolant back to the inlet plenum. Emergency decay heat removal is provided by the direct reactor auxiliary cooling system (DRACS), a natural circulation system that removes heat by means of a heat exchanger in the upper region of the primary circuit cold leg and rejects heat through an air dump heat exchanger outside the containment. The working fluid in the DRACS system is NaK.

In the primary coolant circuit, volumes 1 and 2 represent the inlet and outlet plenums. Volumes 3, 4, and 5 represent regions of the cold pool. Volume 3 represents the annular region between the redan and the vessel wall. Volume 4 represents the coolant below and around the inlet plenum. The coolant in volume 4 is essentially stagnant. Volume 5 represents the bulk of the cold pool. Volumes 6 and 9 simulate the gas expansion volumes in the intermediate loop and decay heat removal system, respectively. Volumes 7 and 8 represent the sodium in the active part of the steam generator. Design parameters assumed for the volumes in the model are shown in Table III.7-2. All of the volumes in the model are perfectly mixed (i.e. characterized by a single temperature) except for volumes 2 and 5 which are treated by a stratification model for low flow conditions. Most of the temperature stratification in the system is expected to occur in volumes 2 and 5.

Volumes in the model are connected by one-dimensional flow segments, which are further subdivided into temperature elements for heat transfer calculations. Table III.7-3 shows the parameters assumed for the liquid segments. Flow segment 1 stands for the core channels, and flow segment 2 represents the shell side of the IHX. The ABR has four IHXs, but only a single IHX is modeled, and it is assumed in this work that all primary circuits behave identically. Segment 3 represents the four primary coolant pumps and the discharge pipes connected to the inlet plenum. Segment 4 represents the primary coolant flow path through the decay heat removal heat exchanger. Segment 5 connects the annular part of the cold pool with the main part, and segment 6 connects the main region of the cold pool with the near stagnant lower region. Segments 7, 8 and 9 represent the intermediate heat transfer loop including the loop piping, the intermediate heat exchanger, the steam generator and the intermediate coolant pump. Segment 10 represents natural circulation flow in the DRACS loop. In normal operation, heat addition takes place in segment 1 , and heat is rejected in segments 2 and 4 . Segment 2 is thermally connected through the IHX to the intermediate loop, and segment 4 is thermally connected to the DRACS loop through the DRACS heat exchanger.

In the model, liquid flow segments are divided into a number of elements for the purpose of heat transfer and pressure drop calculations. The liquid elements in the coolant systems model are described in Table III.7-4. 


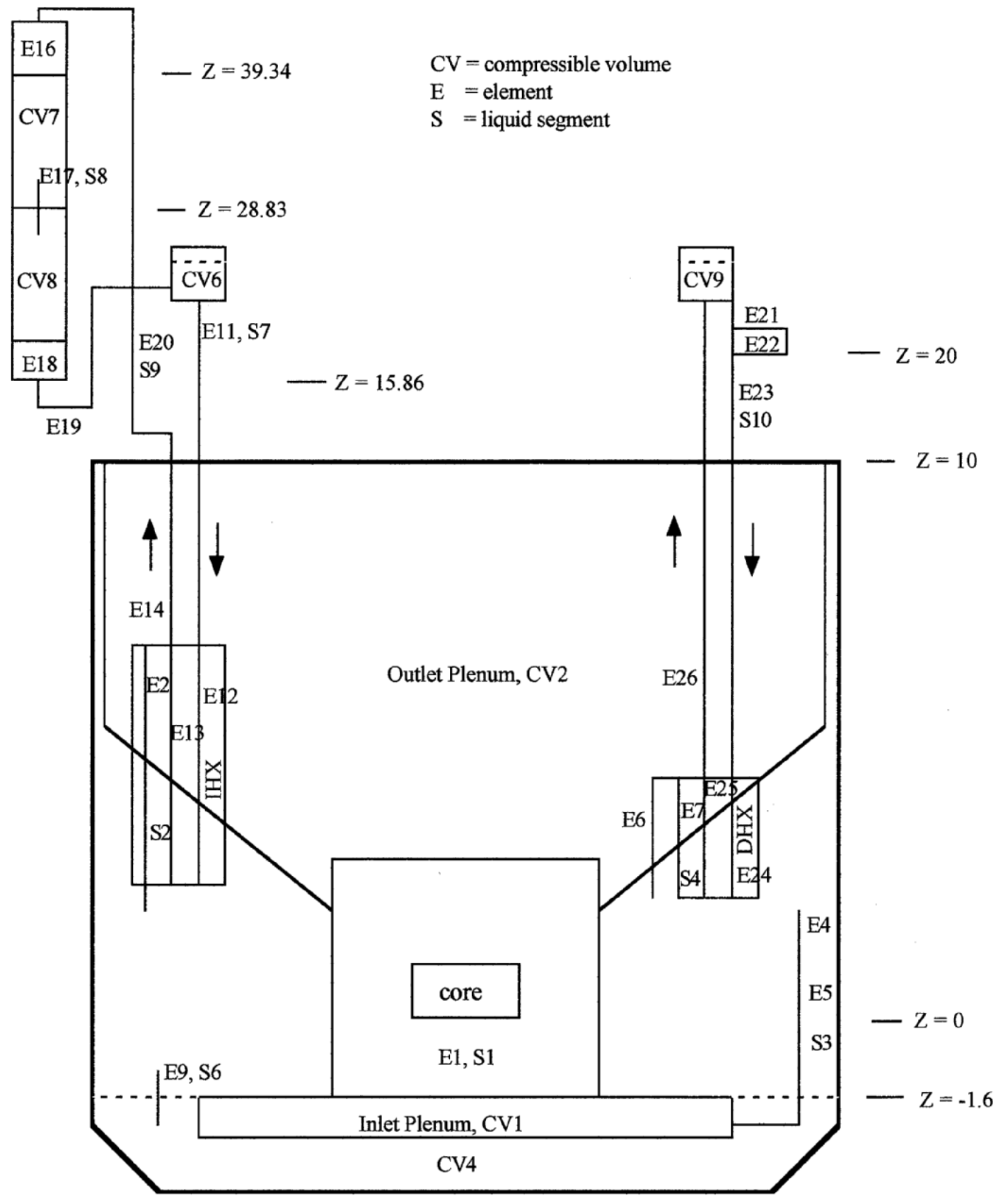

Figure III.7-9 Coolant Systems Thermal Hydraulics Model 
Primary and intermediate circuit flows are driven by both forced circulation at the pumps and by buoyancy due to temperature-induced density changes. Transient natural circulation flows adjust to changes in heat generation and heat transfer. DRACS loop natural circulation flow changes due to temperature changes in the DRACS heat exchanger and in the air dump heat exchanger. Heat transfer at the air dump heat exchanger can be enhanced by opening air flow dampers, which are closed during normal operation.

Table III.7-2 PRIMAR-4 Compressible Volumes Input Data

\begin{tabular}{clcc}
$\begin{array}{c}\text { Compressible } \\
\text { Volume }\end{array}$ & Description & $\begin{array}{c}\text { Total Volume } \\
\mathrm{m}^{3}\end{array}$ & $\begin{array}{c}\text { Gas Volume } \\
\mathrm{m}^{3}\end{array}$ \\
\hline 1 & Inlet plenum & 7.7 & 0. \\
2 & Outlet plenum, stratified & 895.9 & 140. \\
3 & Cold pool annulus & 76.4 & 26.7 \\
4 & Cold pool, stagnant region below & 1.64 & 0. \\
& the inlet plenum & 581.8 & 0. \\
5 & Bulk of cold pool, stratified & 20. & 10. \\
7 & Intermediate loop expansion volume & 0. \\
& Sodium in upper half of active & 38. & 0. \\
& region of the steam generator & & \\
& Sodium in lower half of active & 38. & 2. \\
\hline
\end{tabular}

Table III.7-3 PRIMAR-4 Liquid Segments Input Data.

\begin{tabular}{ccc}
\hline $\begin{array}{c}\text { Segment } \\
\text { Number }\end{array}$ & Elements & $\begin{array}{c}\text { Initial Coolant Flow Rate } \\
\mathrm{kg} / \mathrm{s}\end{array}$ \\
\hline 1 (core) & 1 & 5029.2 \\
2 & $2-3$ & 1257.3 \\
3 & $4-5$ & 1257.3 \\
4 & 7 & 0. \\
5 & 8 & 0. \\
6 & 9 & 0. \\
7 & $11-16$ & 1290. \\
8 & 17 & 1290. \\
9 & $18-20$ & 1290. \\
10 & $21-26$ & 0. \\
\hline
\end{tabular}


Table III.7-4 Liquid Elements Input Data

\begin{tabular}{|c|c|c|c|c|c|c|c|}
\hline Element & Usage & $\begin{array}{c}\text { Inlet } \\
\text { Elevation } \\
\text { m } \\
\end{array}$ & $\begin{array}{c}\text { Outlet } \\
\text { Elevation } \\
\mathrm{m} \\
\end{array}$ & $\begin{array}{c}\text { Length } \\
\mathrm{m}\end{array}$ & $\begin{array}{c}\text { Area } \\
\mathrm{m}^{2}\end{array}$ & $\begin{array}{r}\text { Hydraulic } \\
\text { Diameter }\end{array}$ & $\begin{array}{c}\text { Orifice } \\
\text { Coef. }\end{array}$ \\
\hline 1 & $\begin{array}{l}\text { Core } \\
\text { channels }\end{array}$ & -1.6 & 2.973 & 4.573 & \multicolumn{3}{|c|}{ (See Table III.7-1) } \\
\hline 2 & $\begin{array}{l}\text { IHX, shell } \\
\text { side }\end{array}$ & 7.31 & 2.53 & 4.780 & 1.044 & .0186 & 15.9 \\
\hline 3 & IHX outlet & 2.53 & 1.813 & .717 & .79 & 1.0 & 1.0 \\
\hline 4 & $\begin{array}{l}\text { Primary } \\
\text { pump }\end{array}$ & 2.016 & 2.016 & .10 & .255 & .57 & 0. \\
\hline 5 & Pipe & 2.016 & -1.65 & 4.0 & .255 & .57 & 1.6 \\
\hline 6 & $\begin{array}{l}\text { DRACS inlet } \\
\text { annulus }\end{array}$ & 2.6 & 4.783 & 2. & .0807 & .13 & 1.6 \\
\hline 7 & $\begin{array}{l}\text { DRACS shell } \\
\text { side }\end{array}$ & 4.783 & 2.263 & 2.5 & .0807 & .0491 & 0 . \\
\hline 8 & pipe & 2.75 & 2.650 & .1 & .2 & .2 & 0. \\
\hline 9 & pipe & -1.55 & -1.65 & .1 & .2 & .2 & 0. \\
\hline 10 & $\begin{array}{l}\text { Intermediate } \\
\text { pump }\end{array}$ & 23.58 & 23.58 & .1 & .25 & .5 & 0. \\
\hline 11 & pipe & 23.58 & 7.310 & 25. & .255 & .57 & 0. \\
\hline 12 & pipe & 7.31 & 2.53 & 4.78 & .255 & .57 & 0. \\
\hline 13 & $\begin{array}{l}\text { IHX, tube } \\
\text { side }\end{array}$ & 2.53 & 7.31 & 4.78 & .7048 & .0141 & 0. \\
\hline 14 & pipe & 7.31 & 10.0 & 6.8 & .255 & .57 & 0. \\
\hline 15 & pipe & 10.0 & 42.36 & 76. & .255 & .57 & 0. \\
\hline 16 & $\begin{array}{l}\text { pipe, SG } \\
\text { inlet section }\end{array}$ & 42.36 & 39.34 & 3.0 & 5.2 & 1. & 0. \\
\hline 17 & $\begin{array}{l}\text { steam } \\
\text { generator }\end{array}$ & 28.88 & 28.78 & .1 & .255 & 1. & 1.3 \\
\hline 18 & $\begin{array}{l}\text { pipe, SG } \\
\text { outlet section }\end{array}$ & 15.86 & 15.86 & 3.0 & 5.2 & 1.0 & 0 . \\
\hline 19 & pipe & 15.86 & 15.86 & 6.8 & .255 & .57 & 0. \\
\hline 20 & pipe & 15.86 & 24.88 & 30. & .255 & .57 & 0. \\
\hline 21 & pipe & 22. & 21. & 1.0 & .255 & .2 & 0. \\
\hline 22 & $\begin{array}{l}\text { air dump heat } \\
\text { exchanger }\end{array}$ & 21. & 20. & 11.3 & .255 & .035 & 0 . \\
\hline 23 & pipe & 20. & 4.763 & 27. & .255 & .2 & 0. \\
\hline 24 & pipe & 4.763 & 2.263 & 2.5 & .255 & .2 & 0. \\
\hline 25 & $\begin{array}{l}\text { DRACS, } \\
\text { tube side }\end{array}$ & 2.263 & 4.763 & 2.5 & .255 & .0204 & 0. \\
\hline 26 & pipe & 4.763 & 22. & 30. & .255 & .2 & 1.5 \\
\hline
\end{tabular}




\section{III.7.3.3 Reactor Kinetics and Reactivity Feedback}

A point kinetics model is employed to calculate the response of reactor fission power to transient reactivity feedbacks as well as to reactor scram conditions. At any time, the net reactivity is the sum of a number of individual reactivity feedbacks that are determined by the transient thermal, hydraulic, mechanical, and neutronic state of the reactor. The feedback reactivities considered are fuel Doppler, coolant density, fuel and cladding axial expansion, radial core expansion, and control rod driveline thermal expansion. In addition to tracking fission power, a decay heat model is integrated with the point kinetics model to track thermal power in sub-critical conditions.

Fuel Doppler feedback is calculated from the spatially-dependent fuel temperature distribution and the input spatial distribution of the fuel Doppler reactivity coefficient. In each single-pin channel, the axial distribution of the radial pin-average fuel temperature is used to calculate the reactivity feedback.

Coolant density reactivity feedback is calculated from the spatially dependent coolant density distribution and the input distribution of the coolant density reactivity coefficient calculated from perturbation theory. The reactivity feedback data is entered as a coolant void worth (the negative of the coolant mass worth).

Transient fuel and cladding temperatures are used to predict fuel and cladding axial dimension changes, and in each single-pin channel the reactivity feedbacks associated with fuel and cladding axial expansion are computed from first order perturbation theory.

A simple radial core expansion model accounts for core dilation due to thermal expansion of the above-core load pads and thermal expansion of the core support grid plate. Reactivity feedback is then calculated from the computed core dimension change and an input linear reactivity coefficient based on stand-alone reactor physics eigenvalue calculations.

For the control-rod driveline feedback model, it is assumed that the control rod drivelines are washed by the outlet coolant from the core. Thermal expansion of the drivelines due to a rise in core outlet temperature will cause the control rods to be inserted further into the core, providing a negative reactivity component. On the other hand, when the control rod drives are supported on the vessel head and the core is supported by the vessel walls, then heating the vessel walls will lower the core, leading to a positive reactivity component. In the present model, both control-rod driveline expansion and vessel wall expansion are treated.

For the parametric variation on the oxide core design where a self-actuated shutdown system (SASS) is employed, the analysis here assumes the presence of a safety rod passive de-latch mechanism utilizing magnetic resistance change at the Curie point temperature of the sensing alloy. The SASS is assumed to be coupled to the secondary control-rods with a total scram worth of \$8.7. The insertion is initiated by the coolant outlet temperature of channel 1 (inner driver subassembly) reaching $727^{\circ} \mathrm{C}(1000 \mathrm{~K})$.

\section{III.7.4 Analysis Results}

Analyses of the protected loss-of-flow (PLOF) and unprotected loss-of-flow (ULOF) accident sequences were performed with coupled heat transfer, thermal-hydraulics, and reactor kinetics models available in the SAS4A/SASSYS-1 code. These models have been validated 
through many applications to EBR-II and FFTF transient tests. Additionally, temperature criteria for assessments of cladding damage thresholds have been established by results from testing of fuel in EBR-II, TREAT, and FFTF. Consequently, there is high confidence that the detailed results from the PLOF and ULOF accident analyses presented here give a true characterization of the physical performance that could be obtained in the ABR design.

The base case results for both metal and oxide core designs are for the beginning of equilibrium cycle conditions. Detailed analysis results for the PLOF and ULOF accident sequences are presented below. In addition to the base cases, parametric variations have also been performed for the ULOF accident sequence for both the metallic-fueled and the oxidefueled cores. Parametric variations for the metal core involve changes to the control-rod driveline (CRDL) expansion reactivity feedback. Parametric variations for the oxide core also involve the CRDL reactivity feedback, but additional results are presented for the case where a self-actuated shutdown system (SASS) is employed. The SASS could be a secondary or a tertiary shutdown system. The specific SASS device selected for the analysis presented here is based on a safety rod passive de-latch mechanism utilizing magnetic resistance change at the Curie point temperature of the sensing alloy.

\section{III.7.4.1 Metal Fuel}

\section{Protected Loss-of-Flow (PLOF) Accident Sequence}

Results from analysis of the metal fuel PLOF accident sequence are shown in Figures III.7-10, III.7-11, III.7-12, and III.7-13. Figures III.7-10 and III.7-11 show the history of the reactor power, the decay heat power, the DRACS heat removal rate and the coolant flows through the highest temperature subassembly (channel 4) and an average temperature subassembly (channel 1) in the inner core. This transient is initiated by a complete loss of forced coolant flow in the primary and intermediate loops, and is driven in the early transient period largely by the pump coast-down. During the later period when a long-term quasi-equilibrium is being reached, the response is driven more by temperature distributions with-in the hot and cold pools and the thermal boundary conditions. The discussion in this section is separated into these two time periods of the transient history. Both the primary pumps and the intermediate pumps have enough inertia to give initial flow halving times of five seconds. Primary pump rotors completely stop turning at about 150 seconds after the start of the transient, leading to a transition to natural circulation. The transition to natural circulation goes as smoothly in the cooler channel 1 as in the hotter channel 4. Almost immediately at initiation, the reactor protection system scrams the reactor, beginning the power reduction to decay heat shown in Figure III.7-10, and the dampers on the DRACS air dump heat exchangers open, permitting the DRACS to operate at its full capacity of $0.5 \%$. As the cold pool temperature rises, DRACS heat removal capacity increases, eventually reaching the equivalent of $0.7 \%$ of normal reactor power. Not indicated in this figure is the loss of heat removal to the balance-of-plant, which is assumed to occur after the first 10 seconds. Figure III.7.-10 shows that the reactor decay heat power equals the DRACS heat removal capacity at about 6 hours into the transient. 


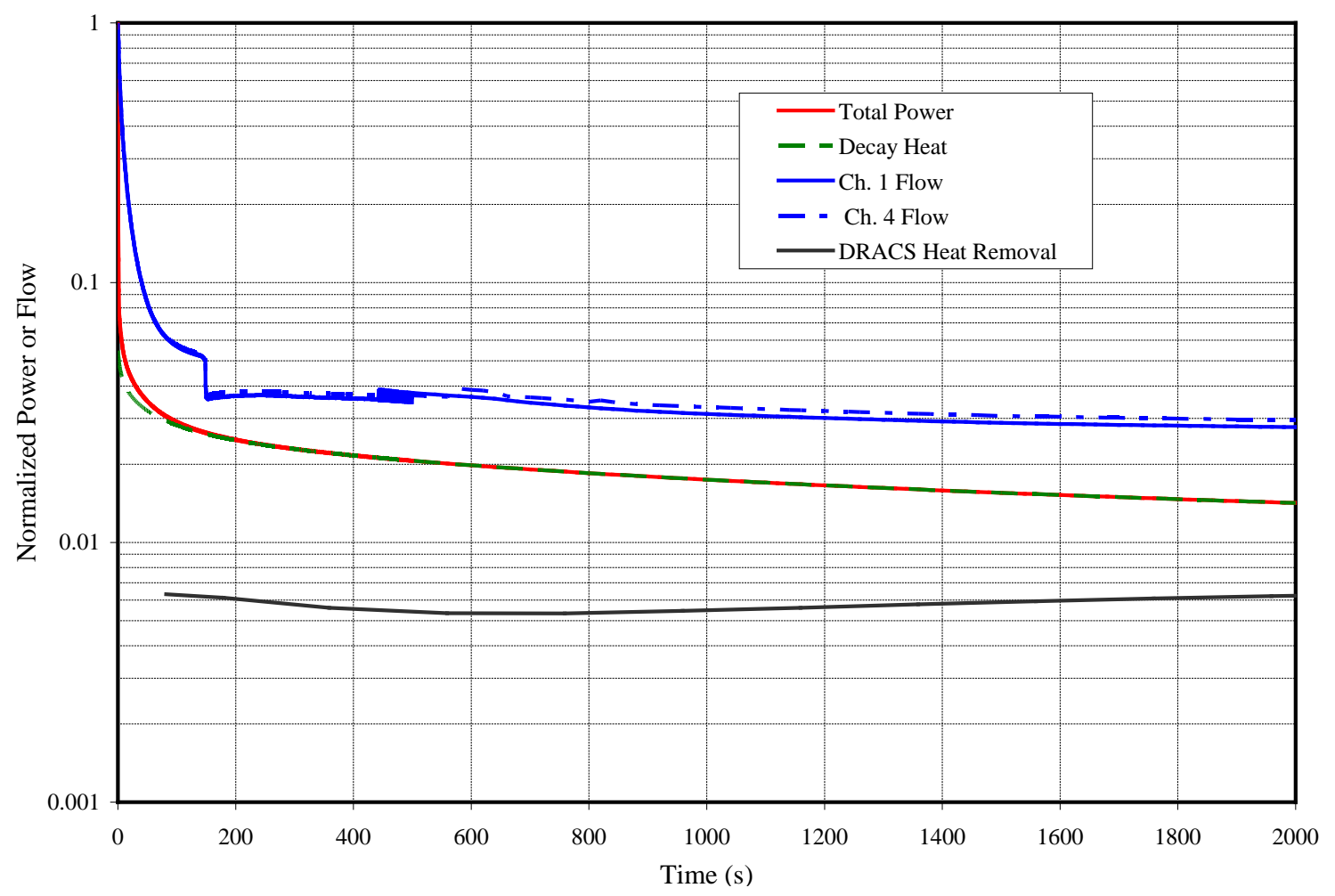

Figure III.7-10 Metal Fuel PLOF Power and Flow History, Early Times

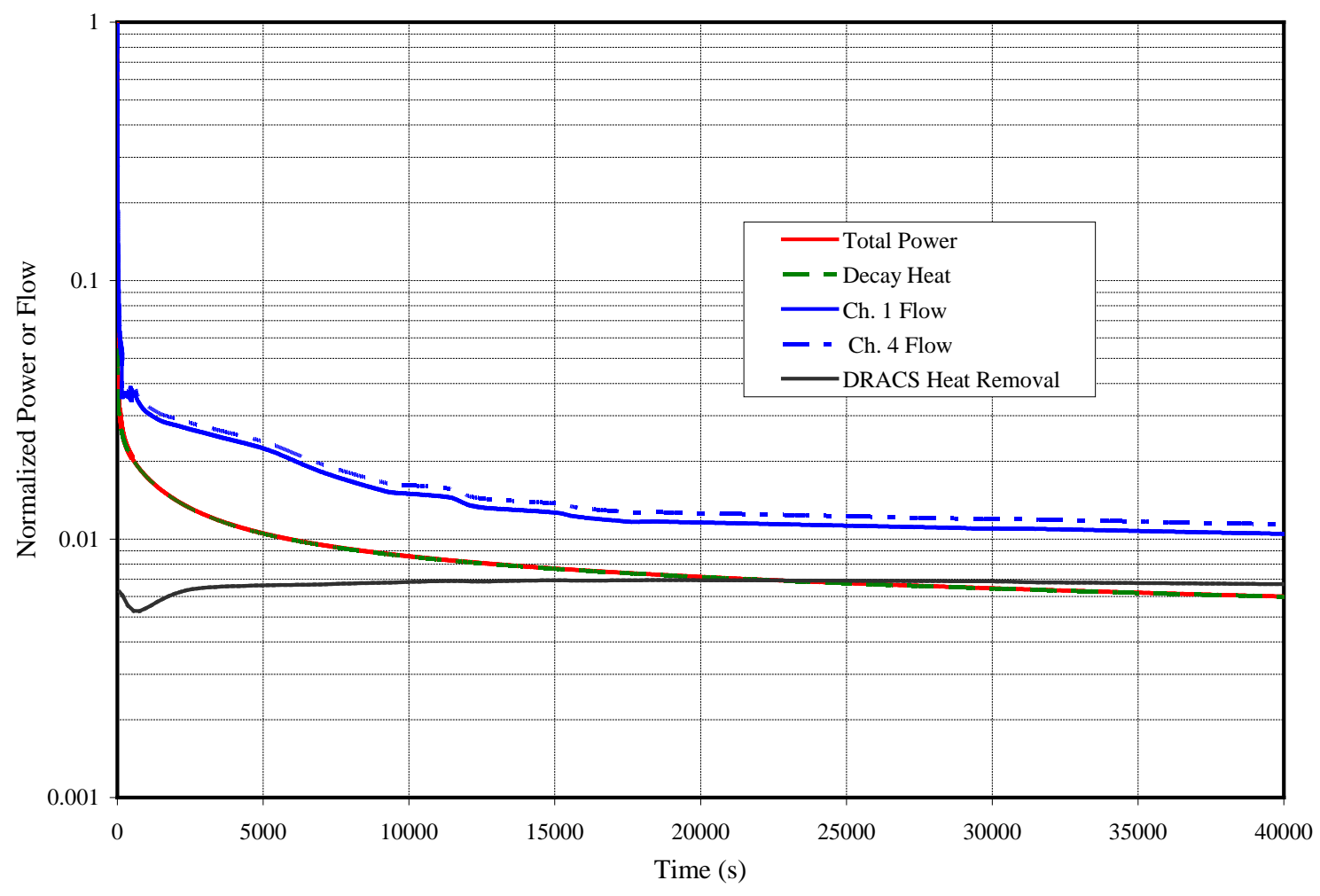

Figure III.7-11 Metal Fuel PLOF Power and Flow History, Extended Times 


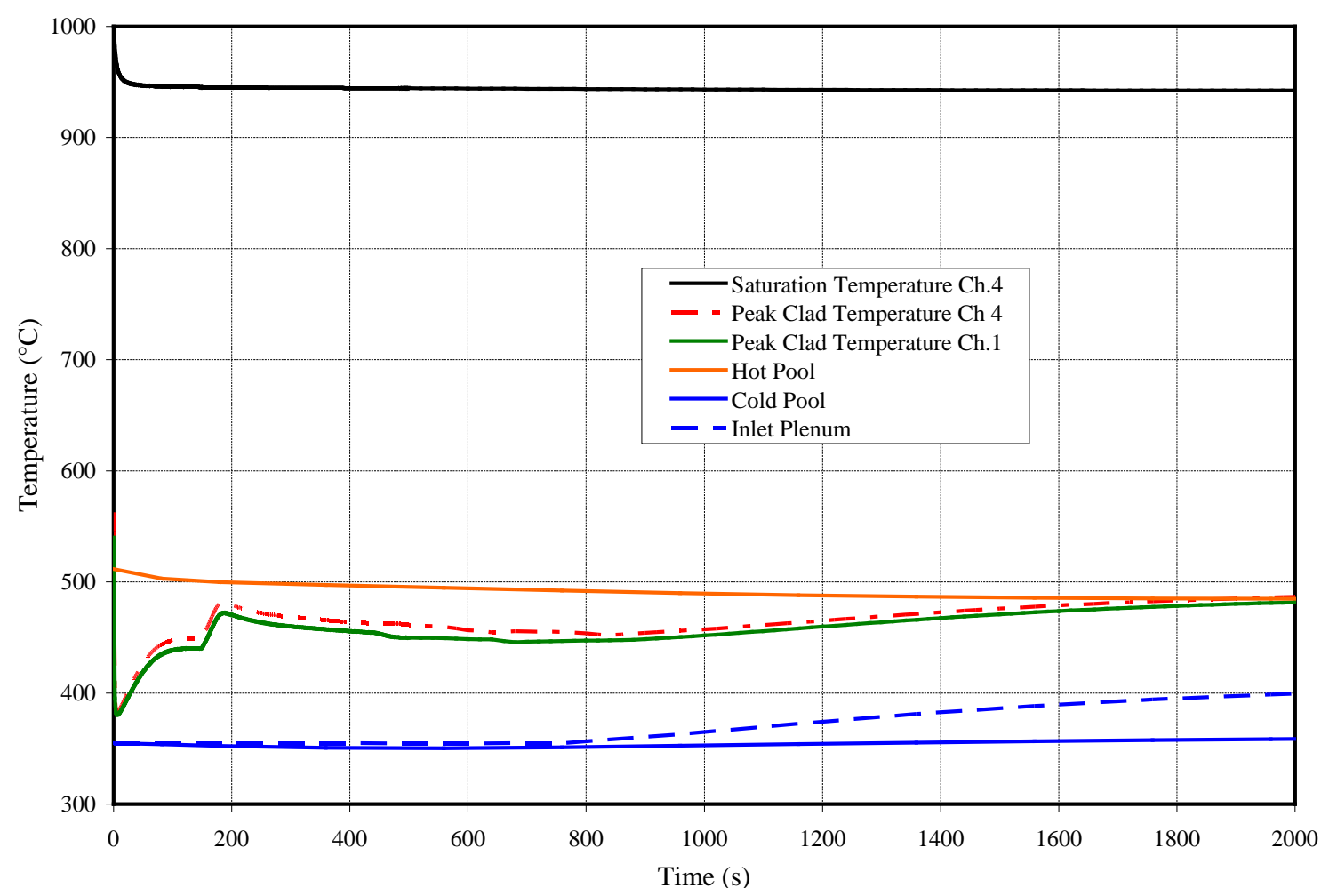

Figure III.7-12 Metal Fuel PLOF Temperature History, Early Times

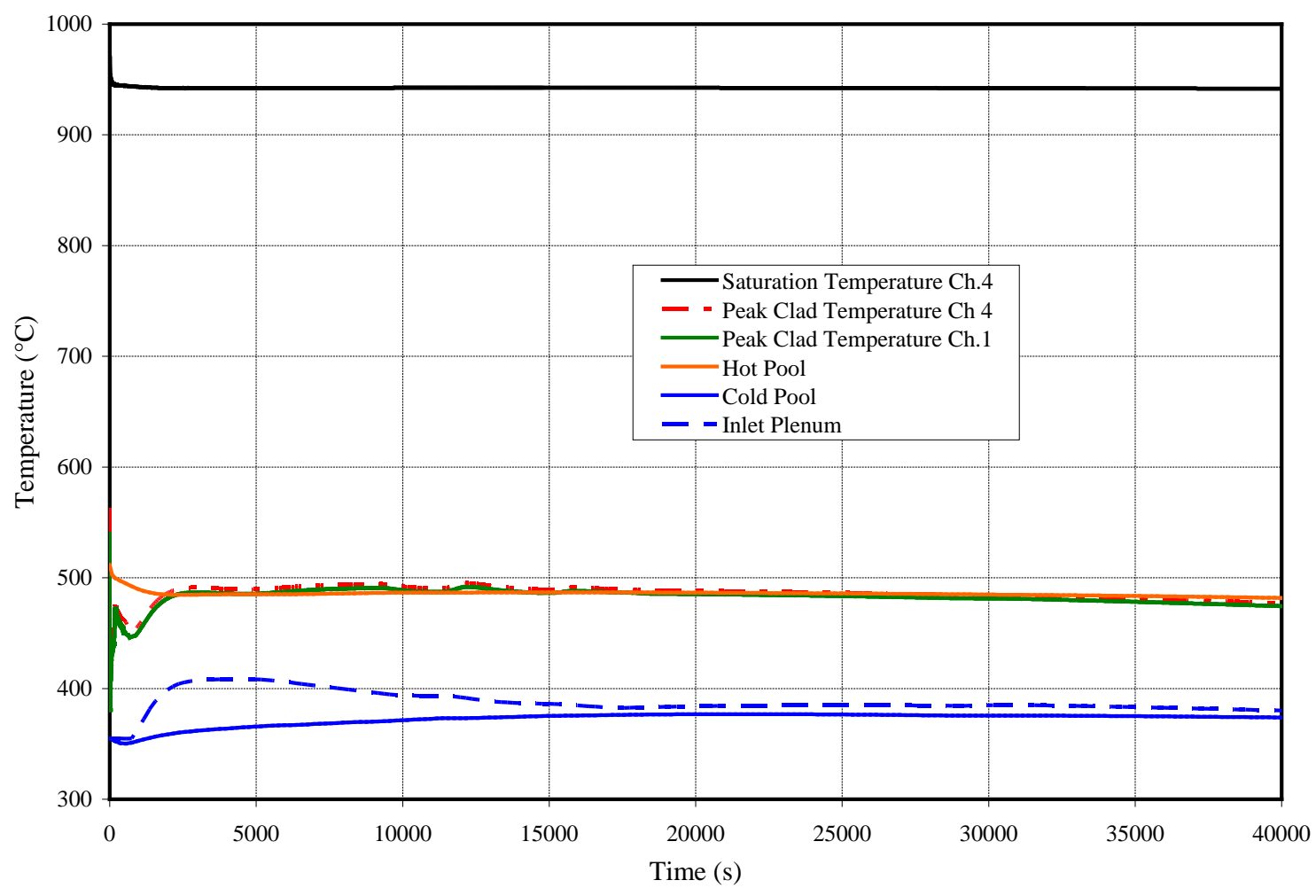

Figure III.7-13 Metal Fuel PLOF Temperature History, Extended Times 
Figure III.7-12 shows the early reactor temperature histories during the coolant flow coast down and transition to natural circulation. During this and the following time, the only heat removal is through the DRACS. The rapid reactor power decrease due to the scram initially lowers the coolant and cladding temperatures in the core. Then the drop in core flow as the pumps coast down leads to a rise in core coolant and cladding temperatures. The transition to natural circulation is about as smooth in channel 1 as in channel 4 . In both channels as the primary pumps stop turning the coolant flow rate abruptly drops to very low values, leading to higher peak coolant and cladding temperatures at around 180 seconds. This short-term cladding temperature rise however is still lower than the initial peak clad temperatures so no cladding failure would occur. There is a large margin to coolant boiling.

Note that the "cold pool” temperature shown in Figures III.7-12 and III.7-13 is the average temperature of the axial distribution computed with the volume stratification model. The pump inlet is at a certain elevation in the cold pool, and at this location the coolant temperature is different from the average for the cold pool.

In the longer term transient history when the system temperature rises, as shown in Figure III.7-13, the DRACS heat removal capability increases due to an increase in NaK flow and heat rejection at the air dump heat exchangers. Decay heat production declines throughout the transient, until it becomes equal to the DRACS heat removal. Hot pool and cold pool temperatures reach long-term peak values at about this time which is around 6 to 7 hours into the transient, and then uniformly decrease as the whole system cools. The DRACS begins to remove more heat than the decay heat rate production. Channel cladding temperatures follow the inlet plenum temperature history to a large part, as the inlet channel flows start to stabilize and a longterm natural convection quasi-equilibrium is reached. During this period the results show the effect of the stratification within the hot and cold pools on this flow and the channel temperatures in the core.

The significance of the PLOF analysis results is emphasized in Figure III.7-12 which shows the initial peak cladding temperature $\left(563^{\circ} \mathrm{C}\right)$ in channel 4 , compared to the short-term transient peak cladding temperature of $490^{\circ} \mathrm{C}$ and the long-term peak cladding temperature just above $470^{\circ} \mathrm{C}$ at around 5 hours before the cool down begins to take effect. In the PLOF transient, no cladding failures would occur, and the long-term peak temperatures in the accident are lower than the normal operating temperatures. Stated in another way, the long-term temperature safety margins in the accident are greater than the margins at the normal operating conditions. This very significant result is obtained as a result of the natural circulation capabilities of the reactor coolant system and the DRACS.

\section{Unprotected Loss-of-Flow (ULOF) Accident Sequence}

\section{a) Base Case}

The metal fuel ULOF transient is initiated by the same set of failures as for the PLOF accident (loss of forced flow and loss of normal heat rejection). However, for the ULOF case, the reactor protection system also fails to scram the reactor; so the accident proceeds from full power. All heat rejection is through the DRACS, with a design heat rejection of $0.5 \%$ of full power at nominal conditions. Results from the analysis of the ULOF accident sequence are shown in Figures III.7-14, III.7-15, and III.7-16. Similar to the PLOF results, there is an early transient period which is driven mostly by the pump coast-downs. Then, during a later period when a long-term quasi-equilibrium is being reached, the response is driven more by temperature distributions within the hot and cold pools and the thermal boundary conditions. However, unlike 
the PLOF case, in these results there is coupling between the power history and the thermal response through the passive core reactivity feedbacks since the core is not shutdown through a scram. This two-way coupling is reflected in the results presented below.

Figure III.7-14 shows the histories for the total reactor power, the decay heat production, and the coolant flow in channel 4 (the peak inner core assembly). The power-to-flow imbalance during the first 50 seconds results in significant transient reactor heating. Peak fuel, peak clad, and coolant outlet temperatures for channel 4 are shown in Figure III.7-15. Coolant and cladding temperatures increase to approximately $700^{\circ} \mathrm{C}$ within the first 30 seconds. The fuel temperature increases to about $730^{\circ} \mathrm{C}$. This heating causes the reactivity feedbacks shown in Figure III.7-16. The net reactivity reaches 80 cents sub-critical. Control-rod driveline (CRDL) and radial expansion are the main contributors to the initial negative reactivity feedback, which causes power and fuel temperatures to decline. Shortly after the onset of the transient, highertemperature coolant begins washing over and heating the control-rod drivelines. As the drivelines expand, an additional negative reactivity component is introduced, as shown in Figure III.7-16. Although reduced fuel temperatures provide a positive Doppler feedback, the magnitude is modest due to the high thermal conductivity and relatively low operating temperatures of metallic fuel. As a consequence, by 100 seconds the total power is significantly below 10\% nominal. The power-to-flow ratio initially increases, but due to the net negative reactivity, peaks and then decreases. Beyond 1000 seconds, the power-to-flow ratio remains below one for the duration of the transient.

The flow coast-down provided by the inertia of the primary pumps ends at approximately 150 seconds when the shafts stop turning. At this point, natural circulation has not yet been fully established, so with the abrupt drop in flow the cladding and local coolant temperatures begin to rise to form a second temperature peak of about $550^{\circ} \mathrm{C}$ at approximately 200 to 250 seconds. The increased temperatures provide additional driving force to aid in establishing natural circulation flow. But the second temperature peak also causes additional thermal expansion and negative reactivity feedback and this starts a period of oscillations between the fission power and the thermal hydraulic feedback effects. The oscillations are damped oscillations and the conditions eventually reach a quasi-equilibrium. By 2000 seconds, fission power has been significantly reduced, and residual heating is dominated by decay heat. Therefore, the changes in reactivity feedback (particularly CRDL expansion) have negligible impact on subsequent transient development.

As natural circulation is being established, and with changes in the channel inlet temperatures, the peak in the coolant temperature moves up through the core and to the subassembly outlet. Similarly, the delay in observing the temperature peak at the outlet is partly due to the low flow conditions, but a bigger contributor to the delay is the large thermal inertia of the structural materials above the core, which must all be heated before the temperature peak reaches the outlet. This affects the hot pool temperature distributions for establishing a quasiequilibrium natural circulation. But the development of natural circulation holds peak coolant and cladding temperatures to around $520^{\circ} \mathrm{C}$. The peak fuel temperatures follow. 


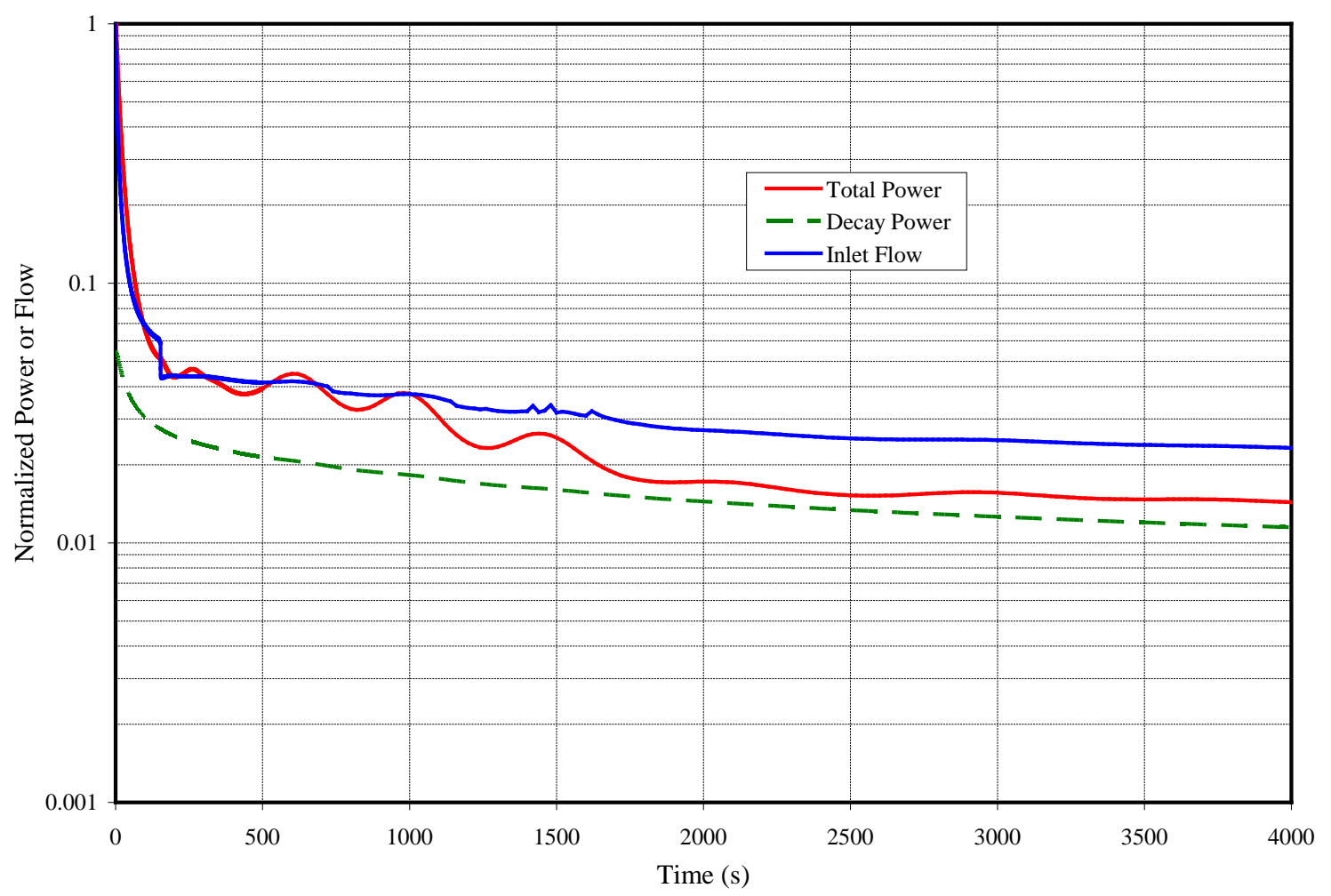

Figure III.7-14 Metal Fuel ULOF Transient Total Power and Channel 4 Flow

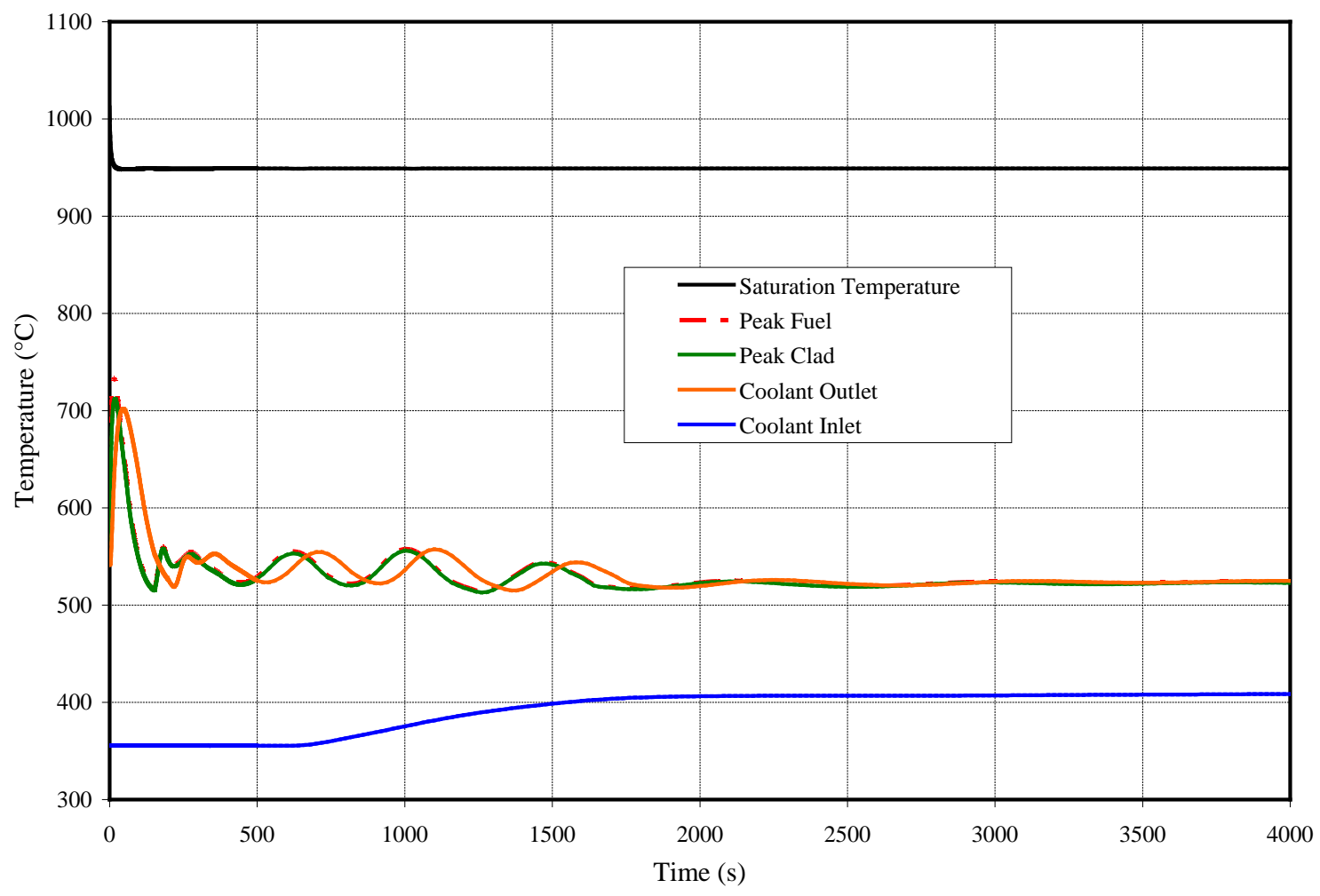

Figure III.7-15 Metal Fuel ULOF Transient Temperatures for Channel 4 


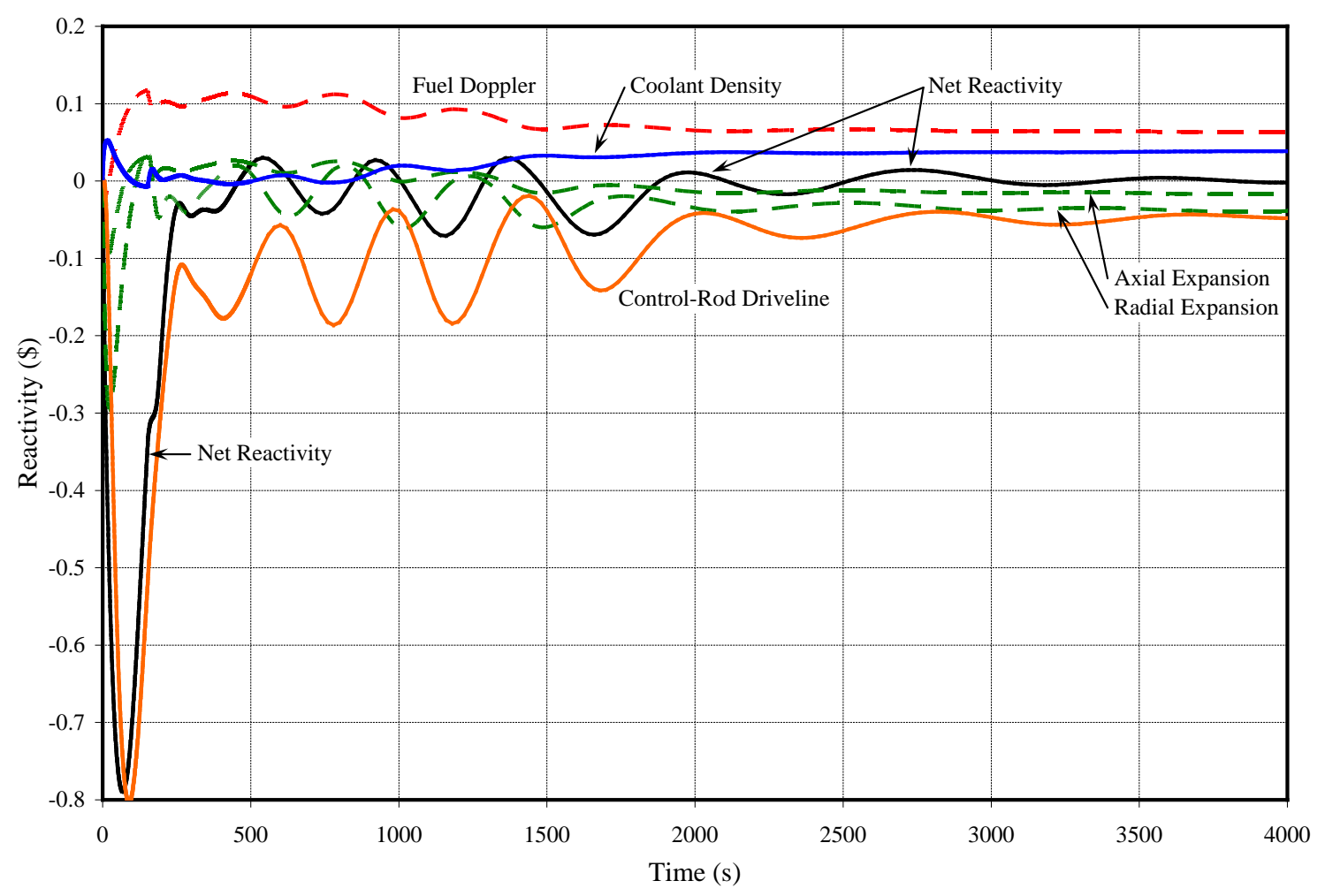

Figure III.7-16 Metal Fuel ULOF Transient Reactivity Feedback

Beyond 6000 seconds, the normalized power-to-flow remains quasi-constant, and the channel temperatures do not increase significantly but are driven by the inlet core temperature as the system slowly heats up. In the long term beyond 20,000 seconds, when the total power approaches the heat rejection capability of the DRACS, overall system temperature rise begins to level off.

The significance of the ULOF accident analysis results for the metal-fueled core is confirmed by Figure III.7-15. As shown for channel 4, the peak fuel, cladding, and coolant temperatures remain well below the coolant saturation (boiling) temperature, with a minimum margin to coolant boiling of about $250^{\circ} \mathrm{C}$. The analysis suggests that the core would survive an unprotected loss-of-flow accident without pin failures or fuel damage. This very favorable result comes about because of 1) the high thermal conductivity and relatively low operating temperature of metallic fuel, 2) the capability of a sodium-cooled reactor in a pool-type primary system to remove decay heat in natural circulation, and 3) the beneficial negative reactivity feedback coefficients and reactor physics performance of metallic fuel.

\section{b) Reduced CRDL Feedback Case}

A parametric variation has been performed for the metal-fueled core with reduced CRDL feedback. Reference should be made to the discussion above for the base case (a). In this parametric, the worth fitting coefficients for the CRDL expansion feedback model have also been reduced from the metal-fueled core base case (a) by $50 \%$. This is approximately equivalent to reducing the worth by a factor of two and could be viewed as more appropriate for an EOEC case with the rods withdrawn. The ULOF transient is initiated by the same set of failures as for 
the base case (loss of forced flow and loss of normal heat rejection) and the reactor protection system also fails to scram the reactor; so the accident proceeds from full power. All heat rejection is through the DRACS, with a design heat rejection of $0.5 \%$ of full power at nominal conditions. Results from the analysis of the ULOF accident sequence are shown in Figures III.7-17, III.7-18 and III.7-19 The same overall trends can be seen when compared with the metal-fueled core base case (a), although the core temperature margins are slightly reduced.

Figure III.7-17 shows the histories for total reactor power, decay heat production, and coolant flow in channel 4 (the peak inner core assembly). The power-to-flow imbalance during the first 50 seconds results in significant transient reactor heating. Peak fuel, peak clad, and coolant outlet temperatures for channel 4 are shown in Figure III.7-18. Coolant and cladding temperatures increase to approximately $720^{\circ} \mathrm{C}$ within the first 30 seconds. The fuel temperature reaches $740^{\circ} \mathrm{C}$. These temperatures are all slightly higher than the metal-core base case. This heating causes the reactivity feedbacks shown in Figure III.7-19. As with the base case, CRDL and radial expansion are the main contributors to the initial negative reactivity feedback, which causes power and fuel temperatures to decline. As can be anticipated, the CRDL feedback is reduced in comparison to the base case though the reduction is not proportionate to the assumed reduction in the worth fitting coefficients. Reduced fuel temperatures provide a positive Doppler feedback, although the magnitude is modest due to the high thermal conductivity and relatively low operating temperatures of metallic fuel. The magnitude of this feedback component is quite similar to that of the base case. Consistent with these feedbacks, the total power is slightly higher at this point in the transient.

The flow coast-down provided by the inertia of the primary pumps ends at approximately 150 seconds when the shafts stop turning. As in the base case, natural circulation has not yet been fully established, so with the abrupt drop in flow the fuel, cladding, and local coolant temperatures begin to rise to form a second temperature peak of about $560^{\circ} \mathrm{C}$ at approximately 180 to 200 seconds. The increased temperatures provide additional driving force to aid in establishing natural circulation flow. But as in the base case, the second temperature peak also causes additional thermal expansion and negative reactivity feedback and this starts a period of oscillations between the fission power and the thermal hydraulic feedback effects. However, the oscillations are damped significantly faster and the conditions reach a quasi-equilibrium much sooner than in the base case. By 2000 seconds, fission power has been significantly reduced, and residual heating is dominated by decay heat. Therefore changes in reactivity feedback (particularly CRDL expansion) have negligible impact on subsequent transient development.

As a consequence, during the quasi-equilibrium phase as natural circulation is being established, the same behavior as in the base case can be observed and reference should be made to that discussion. The development of natural circulation holds peak coolant and cladding temperatures to around $520^{\circ} \mathrm{C}$. The peak fuel temperatures follow. Beyond 6000 seconds, the normalized power-to-flow remains quasi-constant and the channel temperatures do not increase significantly. In the long term beyond 20000 seconds, the total power approaches the heat rejection capability of the DRACS, and the overall system temperature rise begins to level off. 


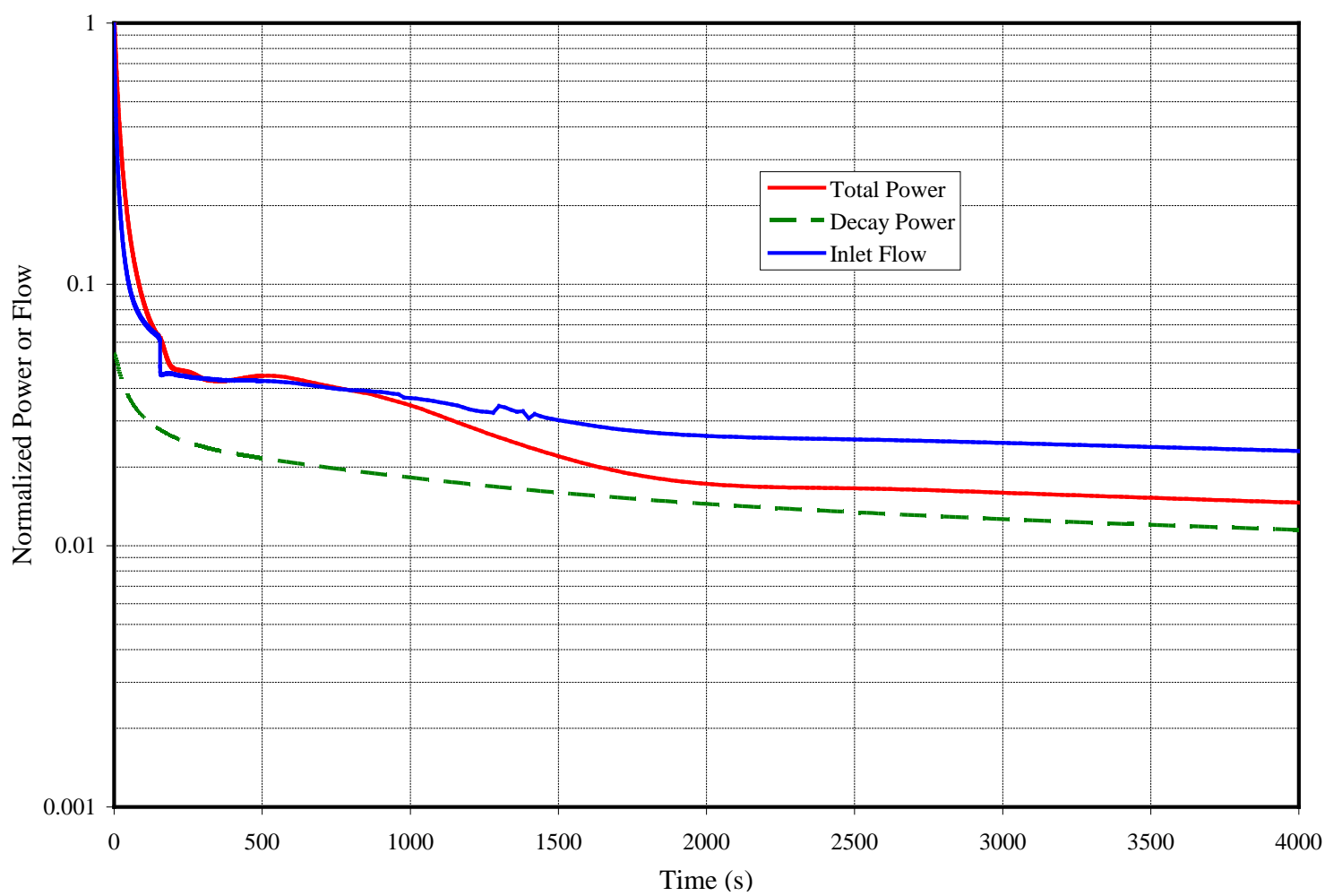

Figure III.7-17 Metal Fuel ULOF Transient Total Power and Channel 4 Flow (50\% CDRL Feedback)

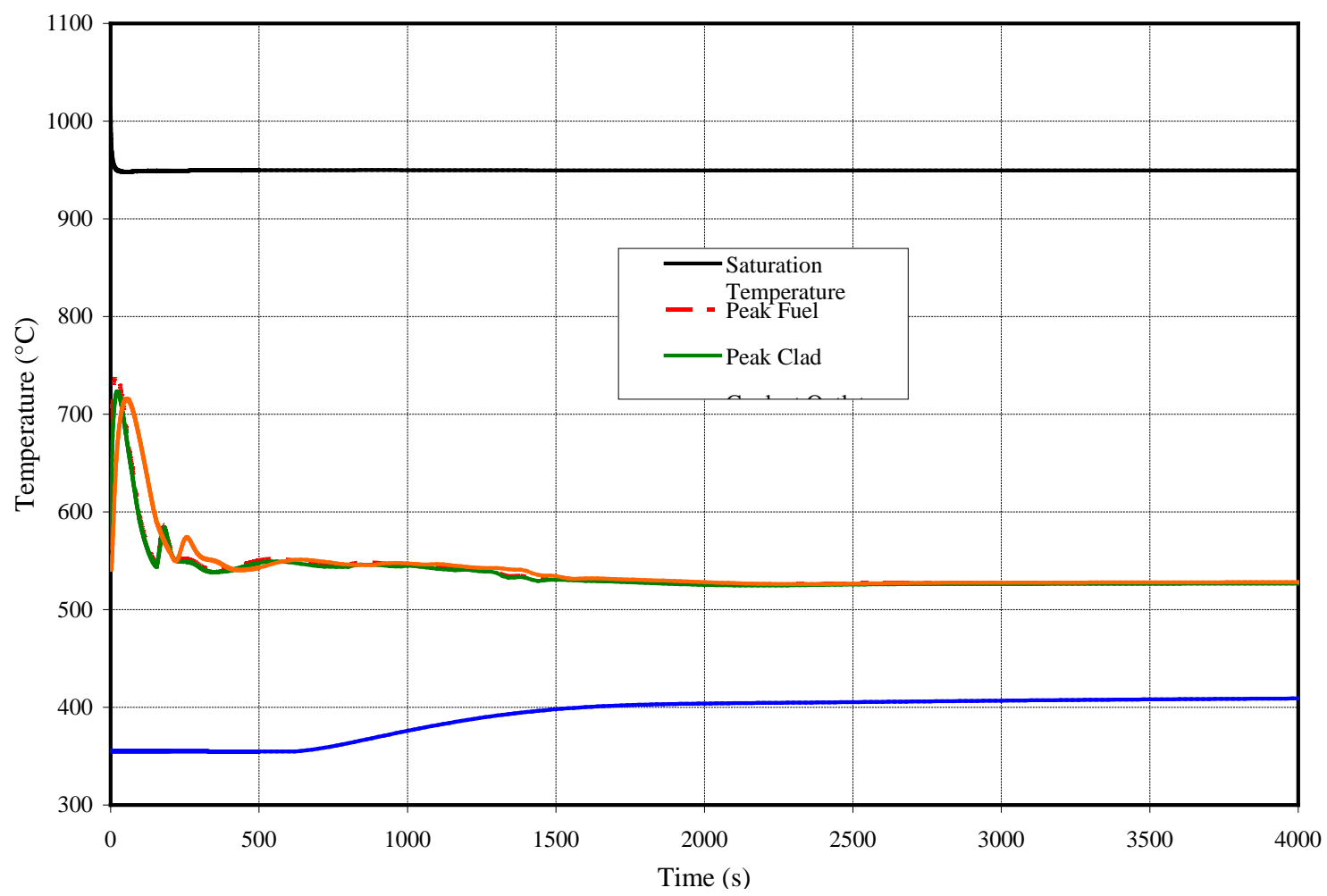

Figure III.7-18 Metal Fuel ULOF Transient Temperatures for Channel 4 (50\% CDRL Feedback) 


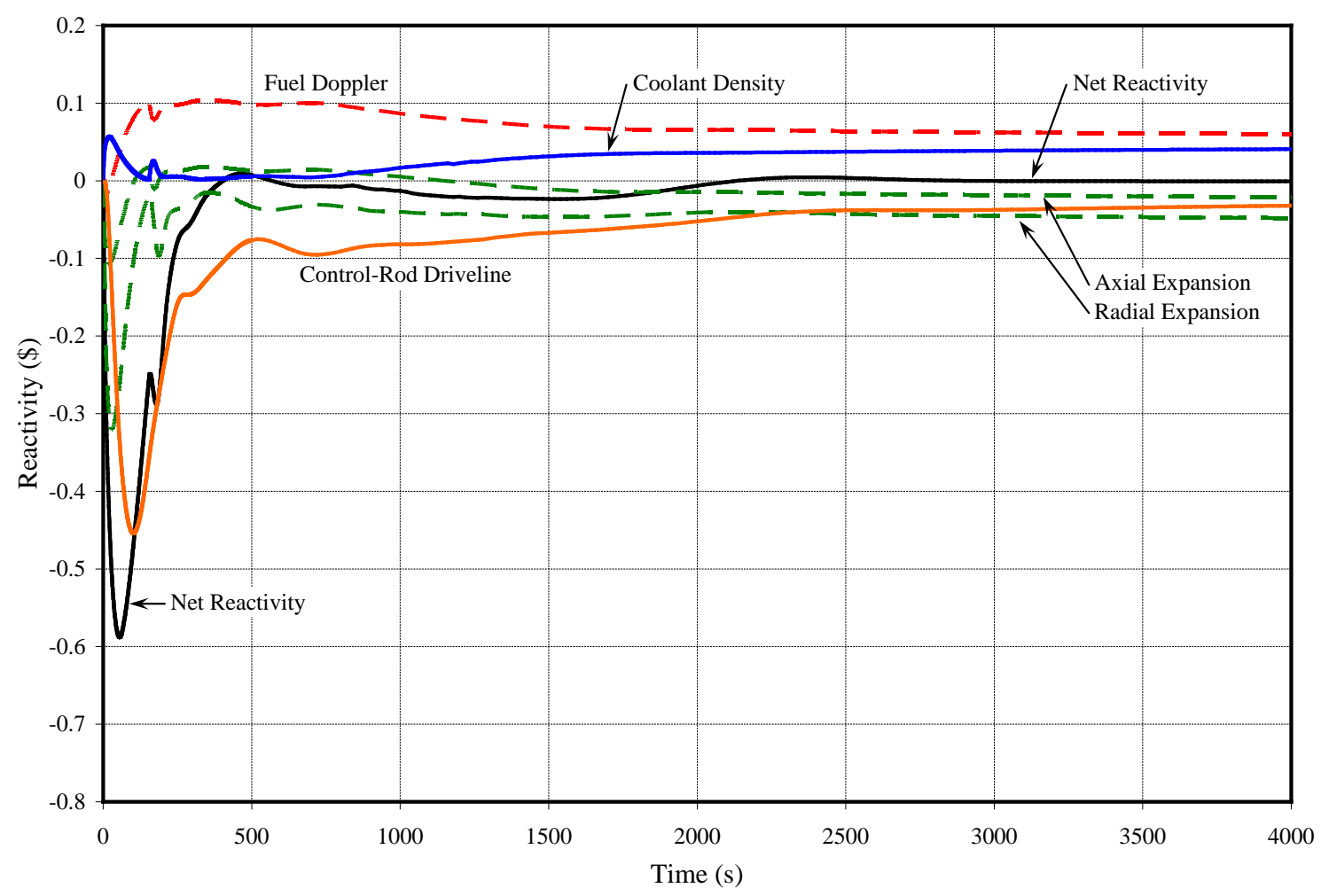

Figure III.7-19 Metal Fuel ULOF Transient Reactivity Feedback (50\% CDRL Feedback)

The significance of these ULOF accident analysis results for the reduced CRDL feedback is that the margins are only slightly different from those of the metal-fueled core base case (a). A change of $50 \%$ only affects the large margins by about $20^{\circ} \mathrm{C}$. This is confirmed in Figure III.7-18. As shown for channel 4, the peak fuel, cladding, and coolant temperatures remain well below the coolant saturation (boiling) temperature, and the minimum margin to coolant boiling is about $220^{\circ} \mathrm{C}$. The conclusions for the base case are still valid here. The metal-fueled core would survive an unprotected loss-of-flow accident without pin failures or fuel damage. As with case (a), this very favorable result comes about because of 1) the high thermal conductivity and relatively low operating temperature of metallic fuel, 2) the capability of a sodium-cooled reactor in a pool-type primary system to remove decay heat in natural circulation, and 3) the beneficial negative reactivity feedback coefficients and reactor physics performance of metallic fuel.

\section{III.7.4.2 Oxide Fuel}

\section{Protected Loss-of-Flow (PLOF) Accident Sequence}

Results from analysis of the oxide fuel PLOF accident sequence are shown in Figures III.7-20, III.7-21, III.7-22, and III.7-23. Figures III.7-20 and III.7-21 show the history of the reactor power, the decay heat power, the DRACS heat removal rate and the coolant flows through the highest temperature subassembly (channel 4) and an average temperature subassembly (channel 1) in the inner core. This transient is initiated by a complete loss of forced coolant flow in the primary and intermediate loops, and is driven in the early transient period largely by the pump coast-down. The later period when a long-term quasi-equilibrium is being reached, the response is driven more by temperature distributions within the hot and cold pools 
and the thermal boundary conditions. The discussion in this section is separated into these two time periods of the transient history. The primary pumps are assumed to have enough inertia to give initial flow halving times of 20 seconds. The intermediate pump inertia is sufficient for an initial flow halving time of five seconds. The primary pump rotors completely stop turning at about 650 seconds after the start of the transient, leading to a transition to natural circulation. The transition to natural circulation goes as smoothly in the cooler channel 1 as in the hotter channel 4. Almost immediately at initiation, the reactor protection system scrams the reactor, beginning the power reduction to decay heat shown in Figure III.7-20, and the dampers on the DRACS air dump heat exchangers open, permitting the DRACS to operate at its full capacity of $0.5 \%$. As the cold pool temperature rises, DRACS heat removal capacity increases, eventually reaching the equivalent of $0.7 \%$ of normal reactor power. Not indicated in this figure is the loss of heat removal to the balance-of-plant, which is assumed to occur after the first ten seconds. Figure III.7-21 shows that the reactor decay heat power equals the DRACS heat removal capacity at about six hours into the transient.

Figure III.7-21 shows the early reactor temperature histories during the coolant flow coast down and transition to natural circulation. During this and the following time, the only heat removal is through the DRACS. The rapid reactor power decrease due to the scram initially lowers the coolant and cladding temperatures in the core. The total power history essentially follows the decay heat curve as the fission power is negligible. Then the drop in core flow as the pumps coast down leads to a rise in core coolant and cladding temperatures. The transition to natural circulation is about as smooth in channel 1 as in channel 4, so the temperature behavior in the two channels tends to mirror each other. In both channels as the primary pumps stop turning the inlet coolant flow rate abruptly drops to very low values, leading to higher peak coolant and cladding temperatures at around 680 seconds. This short-term cladding temperature rise is however still lower than the initial peak clad temperatures so no cladding failure would occur. There is a large margin to coolant boiling.

Note that the "cold pool” temperature shown in Figures III.7-22 and III.7-23 is the average temperature of the axial distribution computed with the volume stratification model. The pump inlet is at a certain elevation in the cold pool, and at this location the coolant temperature is different from the average for the cold pool.

In the longer term transient history while the system temperature rises, as shown in Figure III.7-23, the DRACS heat removal capability increases due to an increase in NaK flow and heat rejection at the air dump heat exchangers. Decay heat production declines throughout the transient, until it becomes equal to the DRACS heat removal. Figure III.7-23 shows that this occurs around 6 to 7 hours after the initiation of the transient. The elevated pool temperatures at this time have led to both an increase in the DRACS natural convection flow and in the driving temperature difference to the air dump heat exchangers, which together contribute to the increase in the DRACS heat removal capability over the design value. Hot pool and cold pool temperatures reach long-term peak values at about this time, and then uniformly decrease as the whole system cools. The DRACS begins to remove more heat than the decay heat rate production. Channel cladding temperatures follow the inlet plenum temperature history to a large part, as the inlet channel flows start to stabilize and a long-term natural convection quasiequilibrium is reached. 


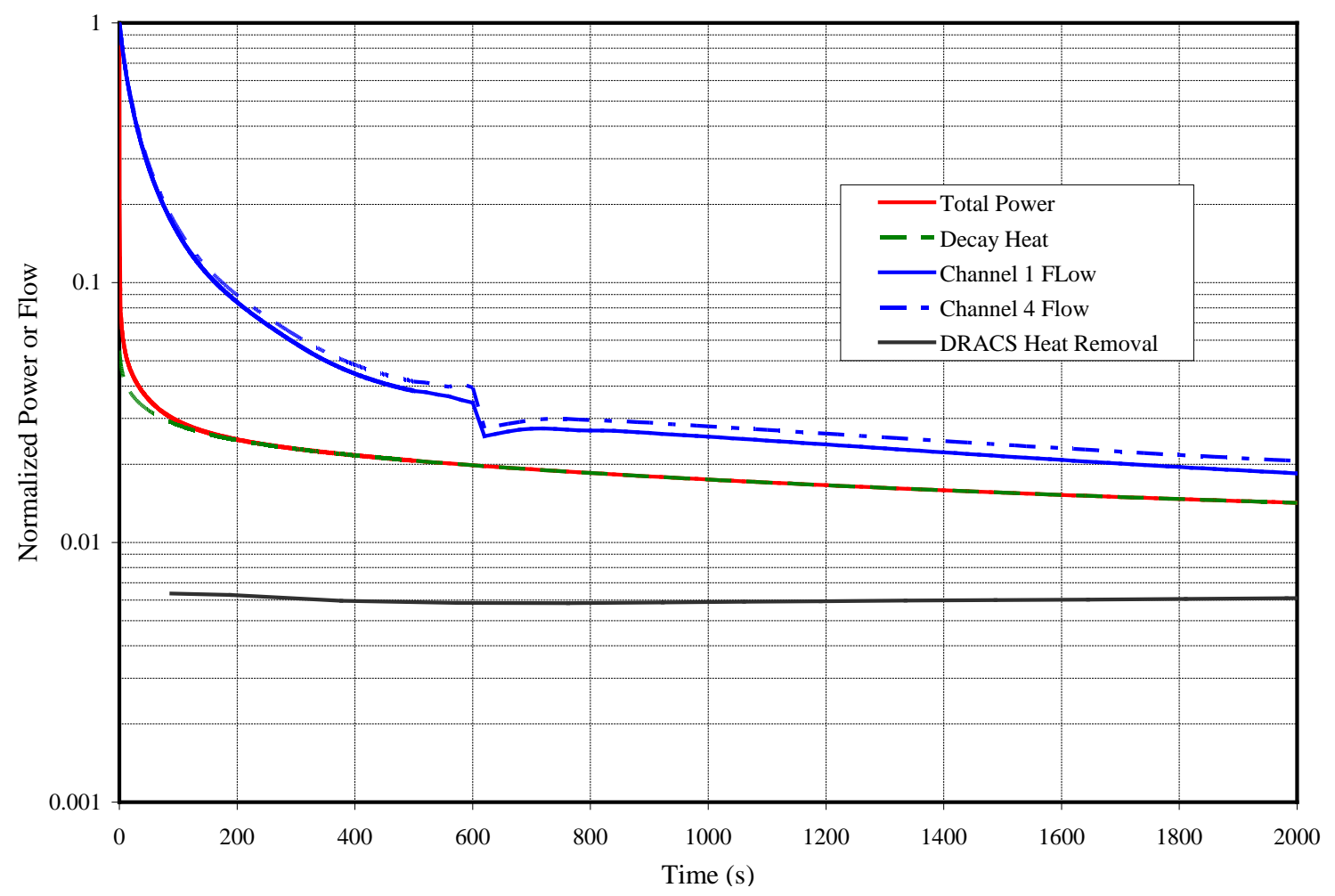

Figure III.7-20 Oxide Fuel PLOF Power and Flow History, Early Times

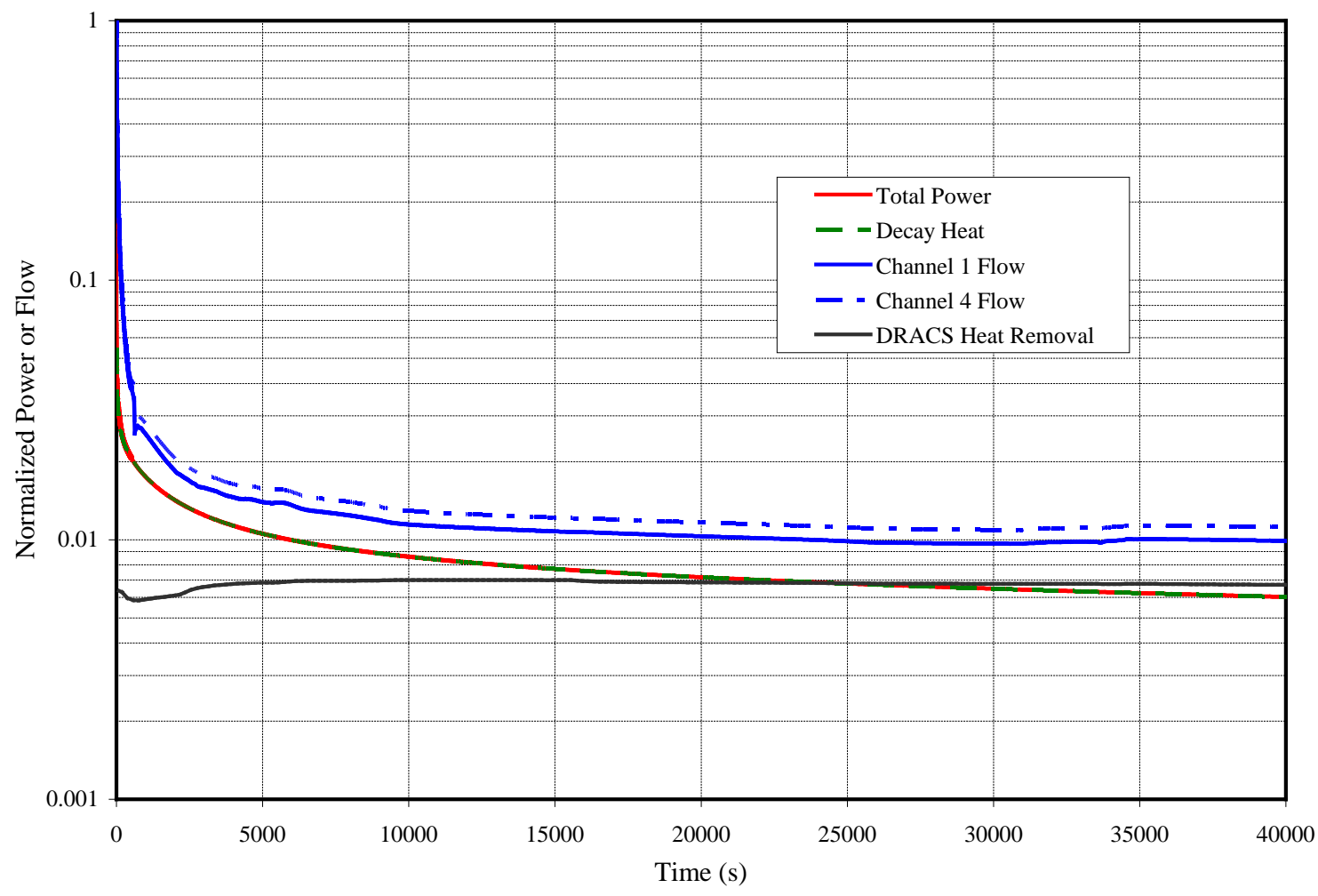

Figure III.7-21 Oxide Fuel PLOF Power and Flow History, Extended Times 


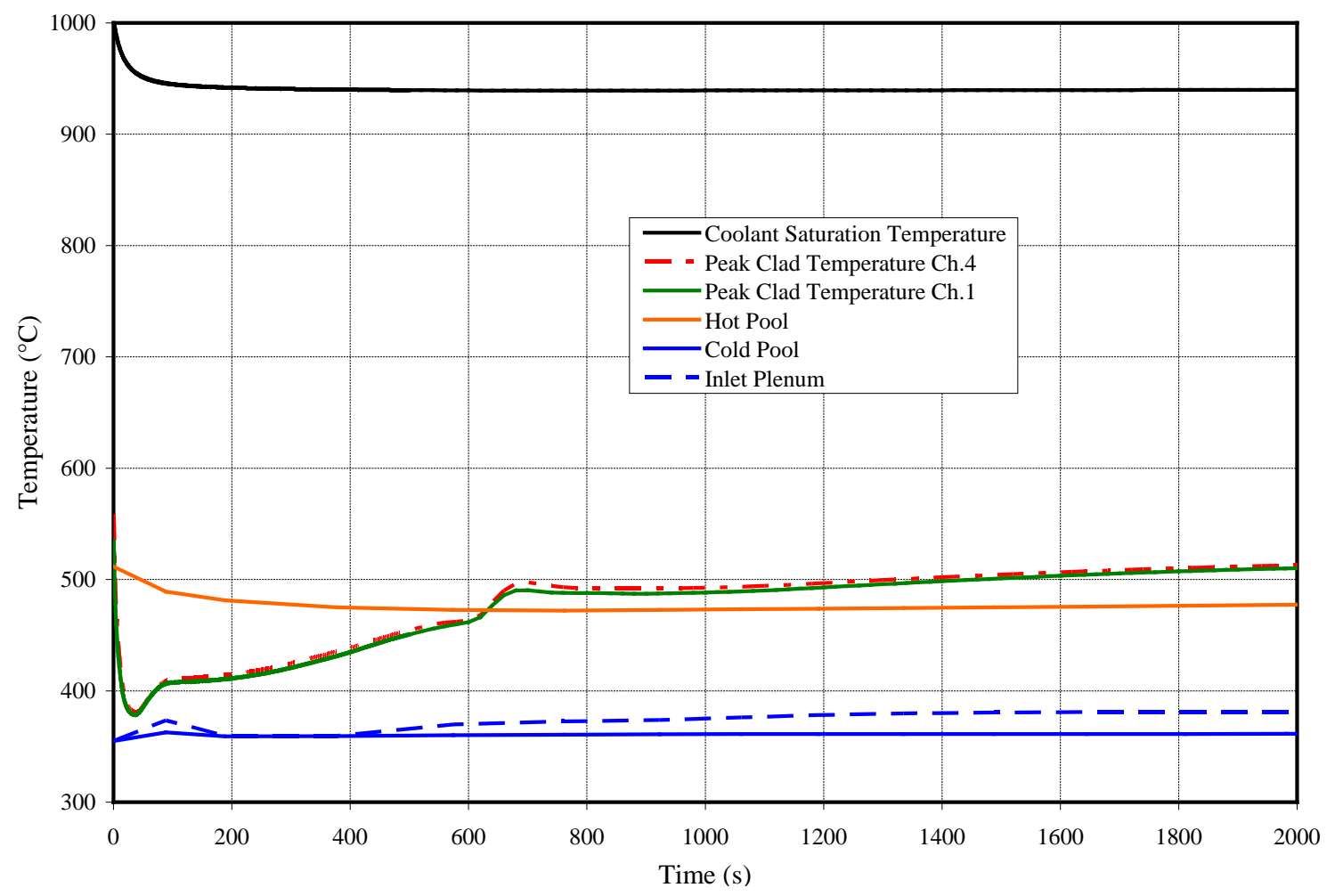

Figure III.7-22 Oxide Fuel PLOF Temperature History, Early Times

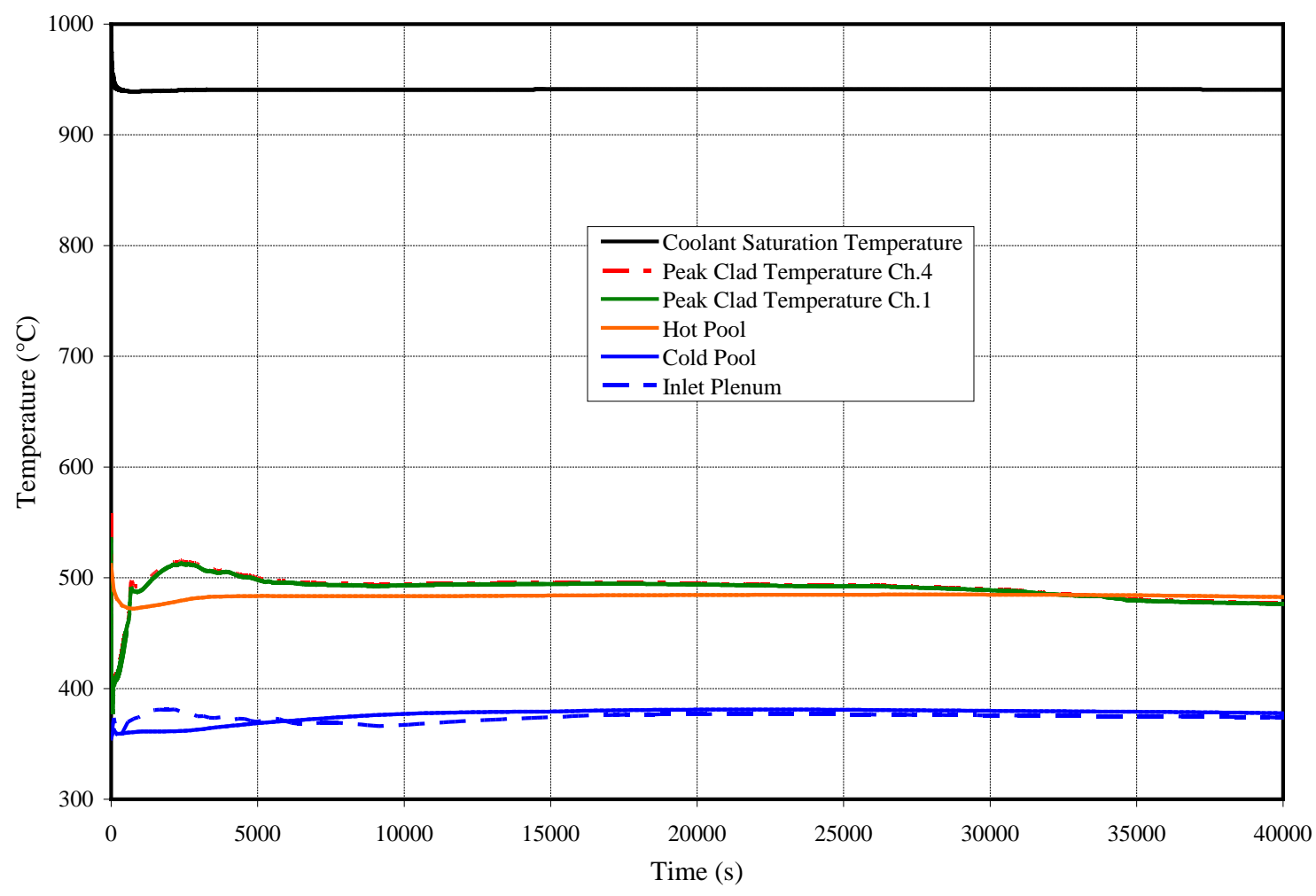

Figure III.7-23 Oxide Fuel PLOF Temperature History, Extended Times 
The significance of the PLOF analysis results is emphasized in Figure III-7-22 which shows the initial peak cladding temperature $\left(558^{\circ} \mathrm{C}\right)$ in channel 4 , compared to the short-term transient peak cladding temperature of $510^{\circ} \mathrm{C}$ and the long-term peak cladding temperature just above $470^{\circ} \mathrm{C}$ at around 5 hours before the cool-down begins to take effect. In the PLOF transient, no cladding failures would occur, and the long-term peak temperatures in the accident are lower than the normal operating temperatures. Stated in another way, the long-term temperature safety margins in the accident are greater than the margins at the normal operating conditions. This very significant result is obtained as a result of the natural circulation capabilities of the reactor coolant system and the DRACS.

\section{Unprotected Loss-of-Flow (ULOF) Accident Sequence}

\section{a) Base Case}

The oxide fuel ULOF transient is initiated by the same set of failures as for the PLOF accident (loss of forced flow and loss of normal heat rejection). However, for the ULOF case, the reactor protection system also fails to scram the reactor; so the accident proceeds from full power. All heat rejection is through the DRACS, with a design heat rejection of $0.5 \%$ of full power at nominal conditions. Results from the analysis of the ULOF accident sequence are shown in Figures III.7-24, III.7-25, and III.7-26. Similar to the PLOF accident, there is an early transient period which is driven mostly by the pump coast down. Then, there is a later period when a long-term quasi-equilibrium is being reached, the response is driven more by temperature distributions within the hot and cold pools and the thermal boundary conditions. However unlike the PLOF, in this accident there is coupling between the power history and the thermal response through the passive core reactivity feedbacks since the core is not shutdown through a scram. This two-way coupling is reflected in the results presented below.

Figure III.7-24 shows the histories for the total reactor power, the decay heat production, and the coolant flow in channel 4 (the peak inner core assembly). The power-to-flow imbalance during the first 100 seconds results in significant transient reactor heating. Core outlet fuel, peak clad, and coolant outlet temperatures for channel 4 are shown in Figure III.7-25. Coolant and cladding temperatures increase to approximately $750^{\circ} \mathrm{C}$ within the first 100 seconds. In contrast, the fuel temperature drops to $1100^{\circ} \mathrm{C}$ as the coolant heating causes the reactivity feedbacks shown in Figure III.7-26 with the net reactivity dropping to about 15 cents subcritical, which is a considerably smaller feedback than in the metal-fueled case. Control-rod driveline and radial expansion are the main contributors to the initial negative reactivity feedback, which causes power and fuel temperatures to decline. Shortly after the onset of the transient, highertemperature coolant begins washing over and heating the control-rod drivelines. As the drivelines expand, an additional negative reactivity component is introduced, as shown in Figure III.7-26. Reduced fuel temperatures provide a significant positive Doppler feedback due to the low thermal conductivity and therefore the relatively high initial operating temperatures of oxide fuel. The power-flow ratio reaches a quasi-asymptotic value higher than the initial value of one towards the end of this period, so the channel coolant and clad temperatures which are initially driven by this, reach a quasi-equilibrium higher than the initial temperatures. The net reactivity has reestablished a quasi-equilibrium around critical, and the fission power remains by far the largest component of the total power. At 400 seconds into the transient, the total power is still above $10 \%$ nominal.

The flow coast-down provided by the inertia of the primary pumps ends at approximately 650 seconds when the shafts stop turning. At this point, natural circulation has not yet been fully 
established, so with the abrupt drop in flow the cladding, and local coolant temperatures begin to rise to form a second temperature peak of about $800^{\circ} \mathrm{C}$ at approximately 700 seconds. There is less of a rise in the fuel temperature. The increased temperatures provides additional driving force to aid in establishing natural circulation flow and the coolant flow rate in channel 4 begins to increase. But the second temperature peak also causes additional thermal expansion and negative reactivity feedback and this starts a period of oscillations between the fission power and the thermal hydraulic feedback effects. The oscillations are damped oscillations and the conditions eventually reach quasi-equilibrium. At 6000 seconds the fission power is still a large part of the total power and the residual heating is still affected by the fission power. Decay heat now makes a significant contribution but changes in reactivity feedback (particularly the control rod drive line expansion) still have impact on subsequent transient development.

As natural circulation is being established, and with changes in the channel inlet temperatures, the peak in the coolant temperature moves up through the core and to the subassembly outlet. While the delay in observing the temperature peak at the outlet is partly due to the low flow conditions, a bigger contributor to the delay is the large thermal inertia of the structural materials above the core, which must all be heated before the temperature peak reaches the outlet. This affects the hot pool temperature distributions for establishing a quasi-equilibrium natural circulation. Nevertheless, the development of natural circulation holds the peak coolant and cladding temperatures to around $800^{\circ} \mathrm{C}$. The peak fuel temperatures follow. Beyond 6000 seconds, the normalized power-to-flow remains quasi-constant and the channel temperatures do not increase significantly. In the long term beyond 20000 seconds, the total power still remains above the heat rejection capability of the DRACS, and overall system temperatures is still slowly rising. The core inlet temperature continues to rise and with it, the channel temperatures. The fission power is still a significant contributor.

The significance of the ULOF accident analysis results is captured in Figure III.7-26. As shown for channel 4, fuel temperatures are well below the melting point. In contrast, the peak cladding and coolant temperatures approach but remain below the coolant saturation (boiling) temperature, with a minimum margin to coolant boiling of about $150^{\circ} \mathrm{C}$. This result comes about because of the low thermal conductivity and relatively high operating temperature of oxide fuel. The analysis suggests that parametric variations on the reactivity feedback coefficients, in particular the control rod drive line expansion worth and enhanced scram capability, would be advisable. Results from these parametric variations are presented below. 


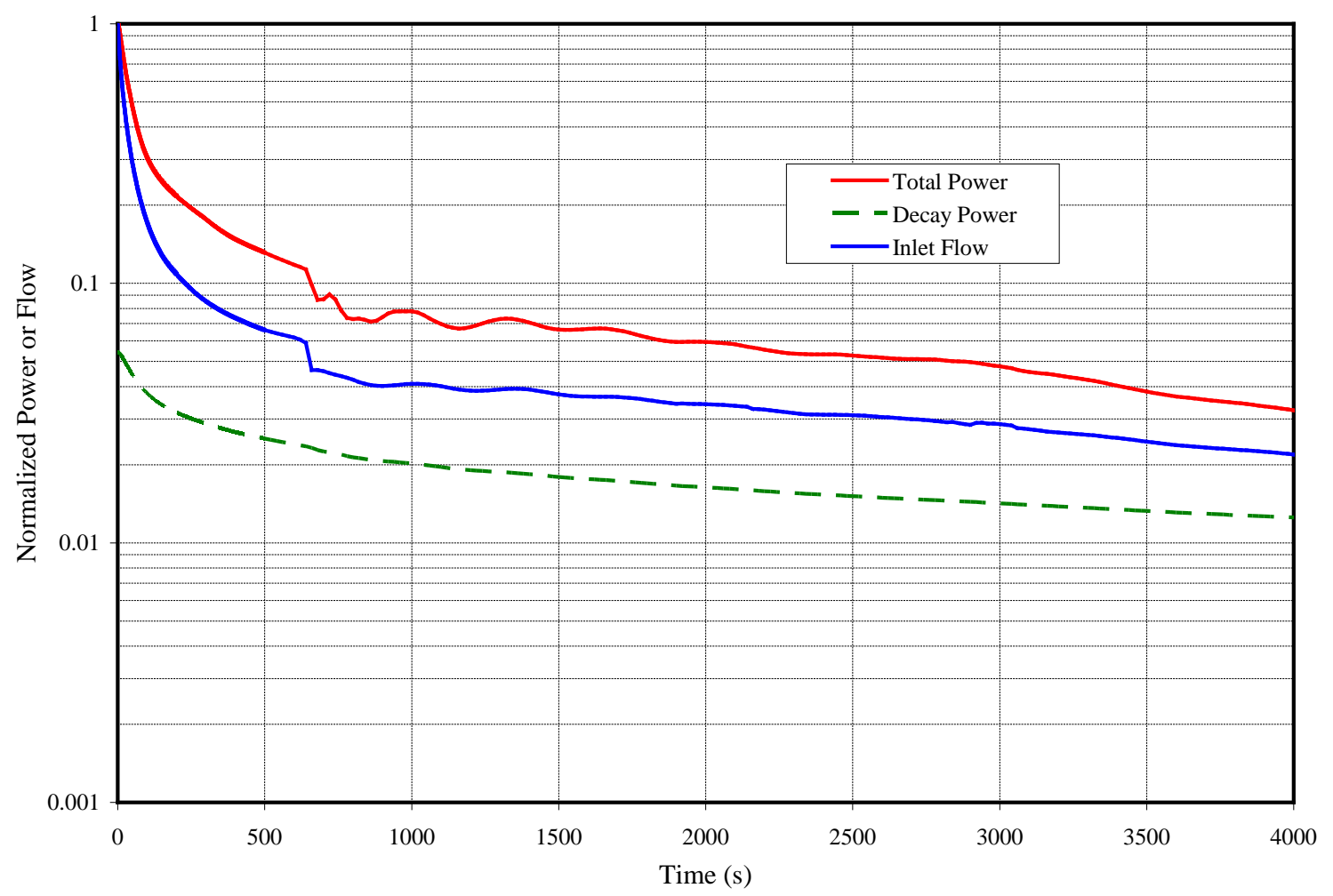

Figure III.7-24 Oxide Fuel ULOF Transient Power and Channel 4 Flow

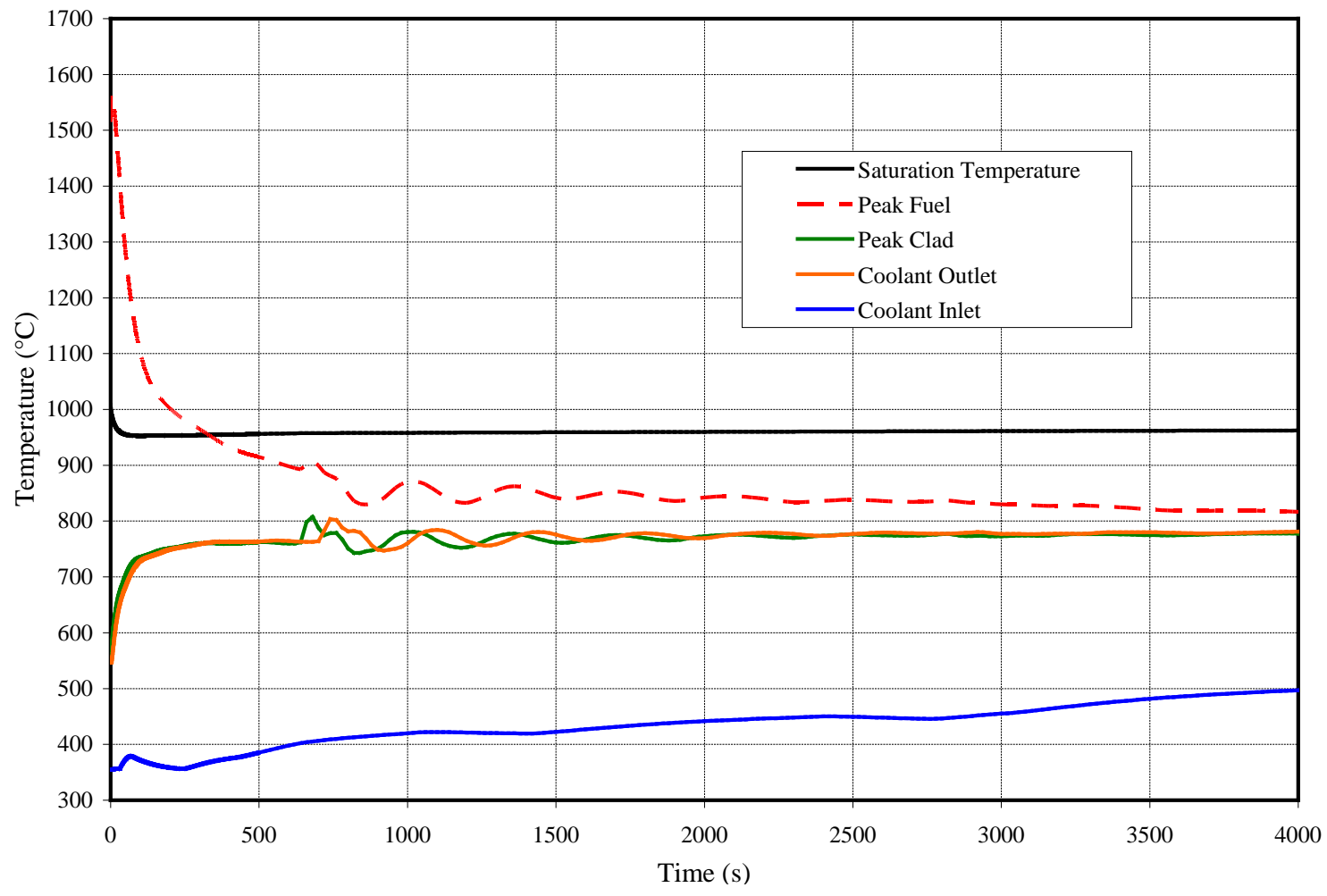

Figure III.7-25 Oxide Fuel ULOF Transient Temperatures for Channel 4 


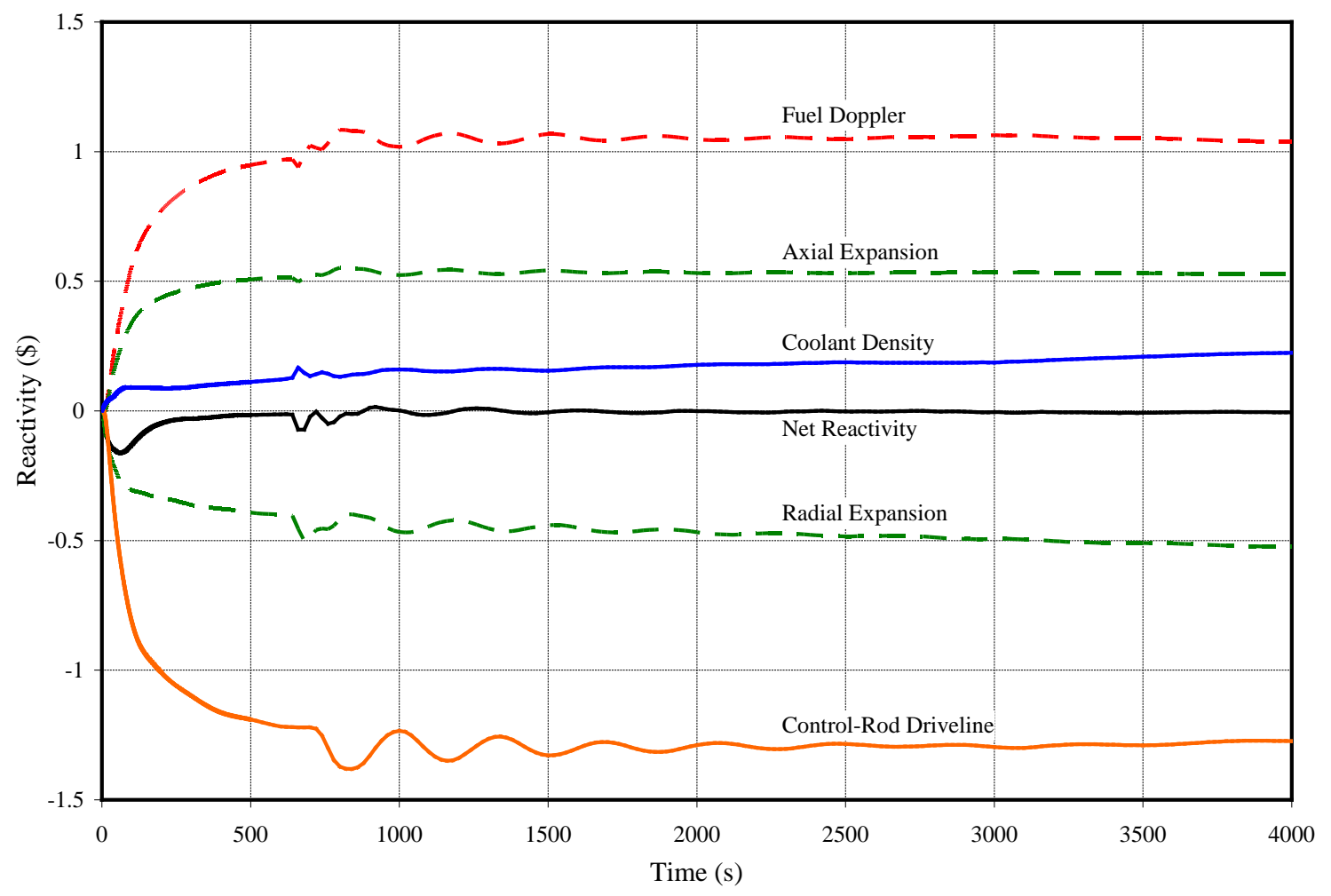

Figure III.7-26 Oxide Fuel ULOF Transient Reactivity Feedback

\section{b) Reduced CRDL Feedback Case}

A parametric variation has been performed for the oxide-fueled core with reduced CRDL feedback. Reference should be made to the discussion above for the base case (a). In this parametric case, the worth fitting coefficients for the control rod driveline expansion feedback model have been reduced from the base case by $50 \%$. This is approximately equivalent to reducing the worth by a factor of two and could be viewed as more appropriate for an EOEC case with the rods withdrawn. The ULOF transient is initiated by the same set of failures as for the base case (loss of forced flow and loss of normal heat rejection) and the reactor protection system also fails to scram the reactor; so the accident proceeds from full power. All heat rejection is through the DRACS, with a design heat rejection of $0.5 \%$ of full power at nominal conditions. Results from the analysis of this ULOF accident sequence are shown in Figures III.727, III.7-28, and III.7-29. The same overall trends can be seen when compared with the base case; however the core temperature margins are significantly reduced.

Figure III.7-27 shows the histories for the total reactor power, the decay heat production, and the coolant flow in channel 4 (the peak inner core assembly). It can be seen that the fission power decreases as the transient proceeds but is noticeably higher than in case (a). Core outlet fuel, peak clad, and coolant outlet temperatures for channel 4 are shown in Figure III.7-28. Coolant and cladding temperatures increase to approximately $850^{\circ} \mathrm{C}$ within the first 200 seconds. In contrast, the fuel temperature drops to about $1100^{\circ} \mathrm{C}$ as the heating causes the reactivity feedbacks shown in Figure III.7-29 with the net reactivity dropping to about the same as in case (a). The channel 4 temperatures are higher than in case (a). 


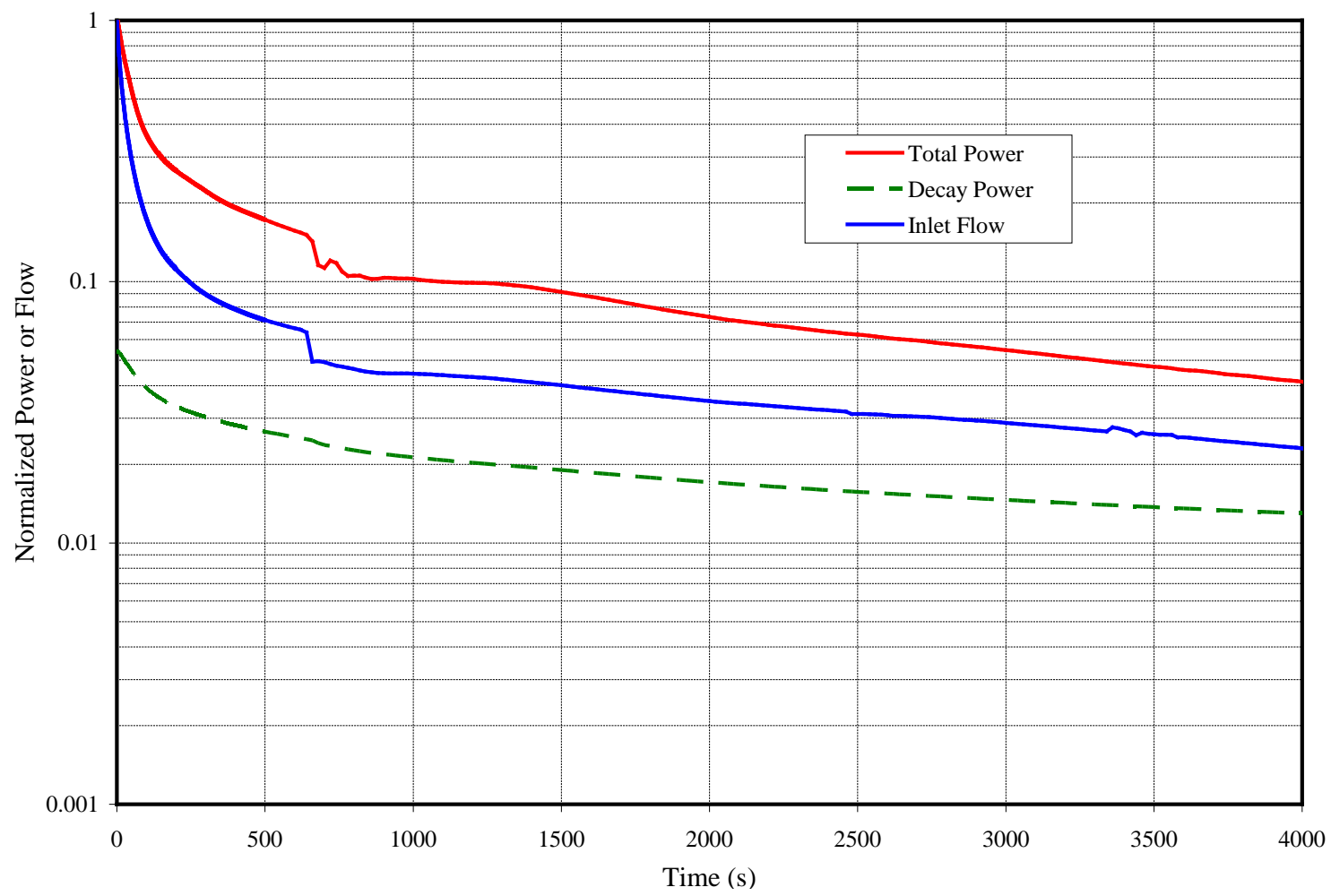

Figure III.7-27 Oxide Fuel ULOF Transient Power and Channel 4 Flow (50\% CRDL Feedback)

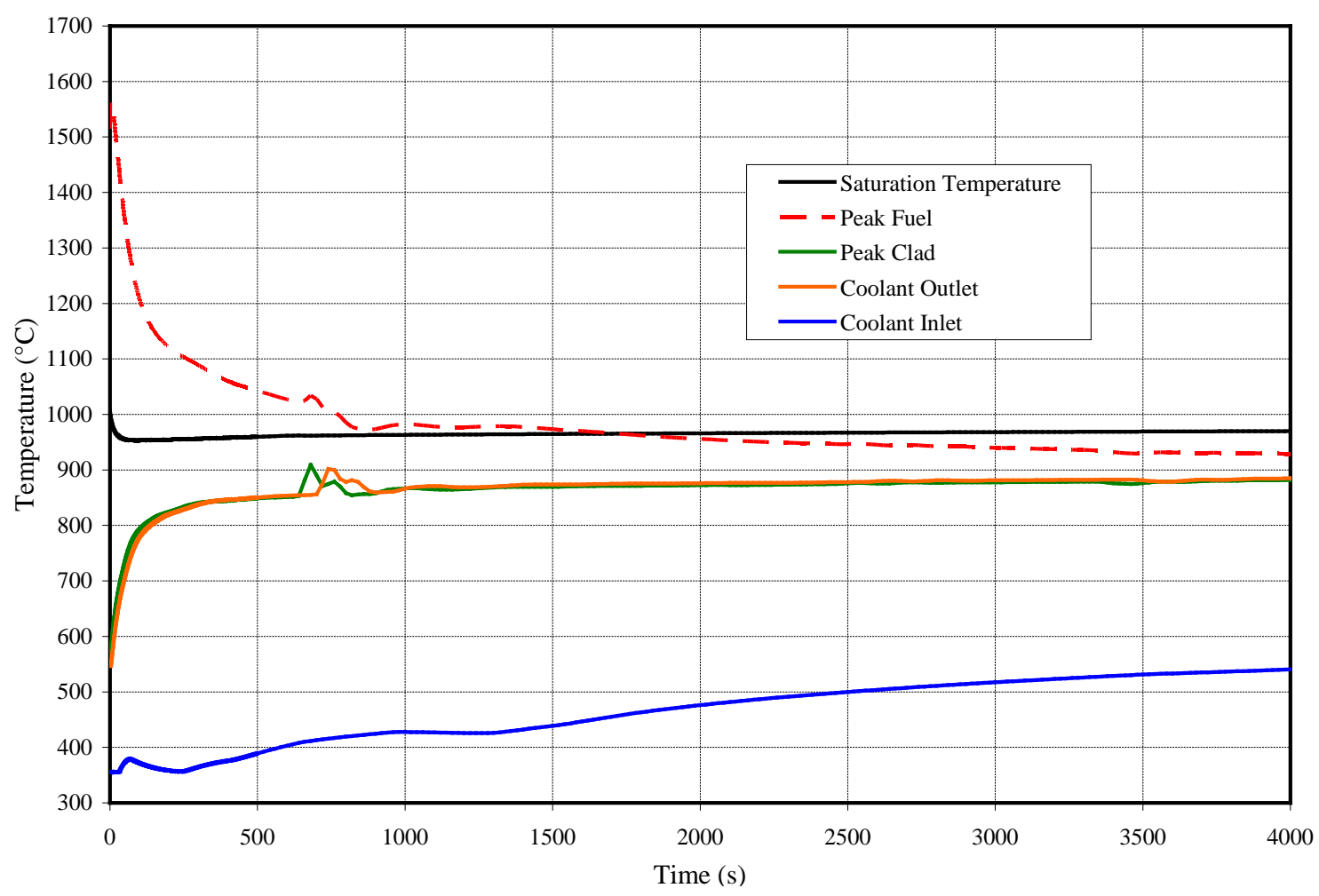

Figure III.7-28 Oxide Fuel ULOF Transient Temperatures for Channel 4 (50\% CRDL Feedback) 


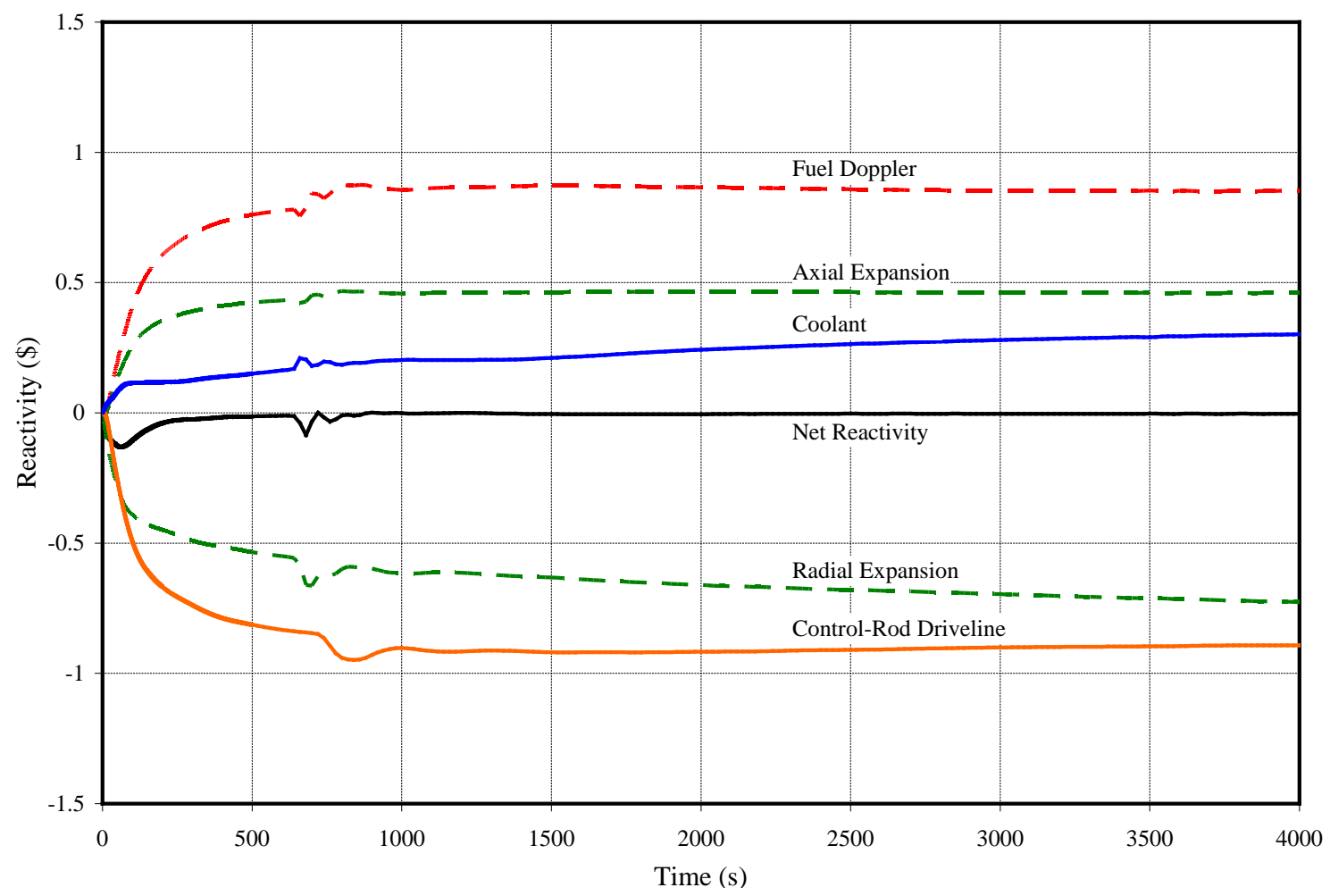

Figure III.7-29 Oxide Fuel ULOF Transient Reactivity Feedback (50\% CRDL Feedback)

As with case (a), control-rod driveline and radial expansion are the main contributors to the initial negative reactivity feedback but the contribution is reduced by about $30 \%$ for the controlrod driveline feed back. The power-flow ratio reaches a quasi-asymptotic value towards the end of this period, so the channel temperatures which are initially driven by this, reach quasiequilibrium and follow the core inlet temperature. As in the base case, the net reactivity has reestablished a quasi-equilibrium around critical but at higher power and higher core temperatures.

As in the base case, the flow coast-down provided by the inertia of the primary pumps ends at approximately 650 seconds when the shafts stop turning. Similarly at this point, natural circulation has not yet been fully established, so with the abrupt drop in flow, the cladding and local coolant temperatures begin to rise to form a second temperature peak around $900^{\circ} \mathrm{C}$ at approximately 700 seconds. There is less of a rise in the fuel temperature. As in case (a), the second temperature peak also causes additional thermal expansion and negative reactivity feedback and this once again starts a period of damped oscillations between the fission power and the thermal hydraulic feedback effects. But the oscillations are damped out much sooner than in the base case and the conditions soon reach a quasi-equilibrium at about 1000 seconds. Beyond this point the fission power is noticeably a larger part of the total power than in case (a). As in case (a), in the long term beyond 20000 seconds, the total power still remains above the heat rejection capability of the DRACS and overall system temperatures are still rising. At 20,000 seconds, the development of quasi-equilibrium natural circulation conditions continues to hold peak coolant and cladding temperatures to around $850^{\circ} \mathrm{C}$ which is about $50^{\circ} \mathrm{C}$ higher than the base case. 
The significance of this ULOF CRDL feedback parametric is best illustrated in Figure III.732. As shown for channel 4, fuel temperatures are well below the melting point. In contrast, the peak cladding temperatures approach $900^{\circ} \mathrm{C}$. Moreover, the major significance of this ULOF accident parametric analysis is that for channel 4, during the transient, the minimum margin to coolant boiling is now only about $50^{\circ} \mathrm{C}$. The analysis further suggests that examination of an enhanced scram shut down capability would be advisable. Results from these parametrics are presented below.

\section{c) SASS Case with Reduced CRDL Feedback}

Reference should be made to the discussion above for the base case (a) and the reduced CRDL feedback case (b). Since case (b) led to the results with the minimum margin to coolant boiling in the core, this reduced CRDL feedback case should provide the more challenging case for examining the quantitative benefits of introducing a SASS device. In this parametric case, the total SASS scram worth is $\$ 8.70$. The insertion is initiated by the coolant outlet temperature of channel 1 (inner driver subassembly) reaching $727^{\circ} \mathrm{C}(1000 \mathrm{~K})$. In line with case (b), the control rod driveline feedback has been reduced from the base case by $50 \%$, and additional details are available in the discussion of that case. This ULOF transient is initiated by the same set of failures as for the base case (loss of forced flow and loss of normal heat rejection) and the reactor protection system also fails to scram the reactor; so the accident proceeds from full power. All heat rejection is through the DRACS, with a design heat rejection of $0.5 \%$ of full power at nominal conditions. Results from the analysis of this ULOF accident sequence are shown in Figure III.7-30, III.7-31, and III.7-32. As can be expected, the results are a combination of those from the PLOF accident and the ULOF case (b).

Figure III.7-30 shows the histories for the total reactor power, the decay heat production, and the coolant flow in channel 4 (the peak inner core assembly). It can be seen that the fission power decreases as the transient proceeds along the lines of case (b). SASS scram occurs at 80 seconds after initiation of the transient when the total power has been reduced to $40 \%$. Core outlet fuel, peak clad, and coolant outlet temperatures for channel 4 are shown in Figure III.7-31. Coolant and cladding temperatures increase to approximately $760^{\circ} \mathrm{C}$ and $780^{\circ} \mathrm{C}$ respectively within the first 80 seconds at which point scram occurs. In contrast, the fuel temperature drops to about $1250^{\circ} \mathrm{C}$ at the scram point as the heating causes the reactivity feedbacks shown in Figure III.7-32. The net reactivity is at about 12 cents sub-critical at scram. The minimum channel 4 safety margins occur at this point and are higher than those reported in case (b) since the scram occurs at an early time in the heat up transient. The margin to boiling in channel 4 is about $170^{\circ} \mathrm{C}$. Post scram in the transient period beyond 80 seconds, the system response is similar to that of the PLOF sequence.

As in the PLOF sequence, the flow coast-down provided by the inertia of the primary pumps ends at approximately 650 seconds when the shafts stop turning. At this point, natural circulation has not yet been fully established, so with the abrupt drop in flow, the cladding and local coolant temperatures begin to rise to form a second broad temperature peak or plateau around $500^{\circ} \mathrm{C}$ at approximately 700 seconds. Reference should be made to the discussion on the PLOF sequence. The transient is now all decay heat driven and the total power eventually decreases to below the heat rejection capability of the DRACS and overall system temperatures decrease. The margins during this phase of the accident are larger than the margins at normal operation. 


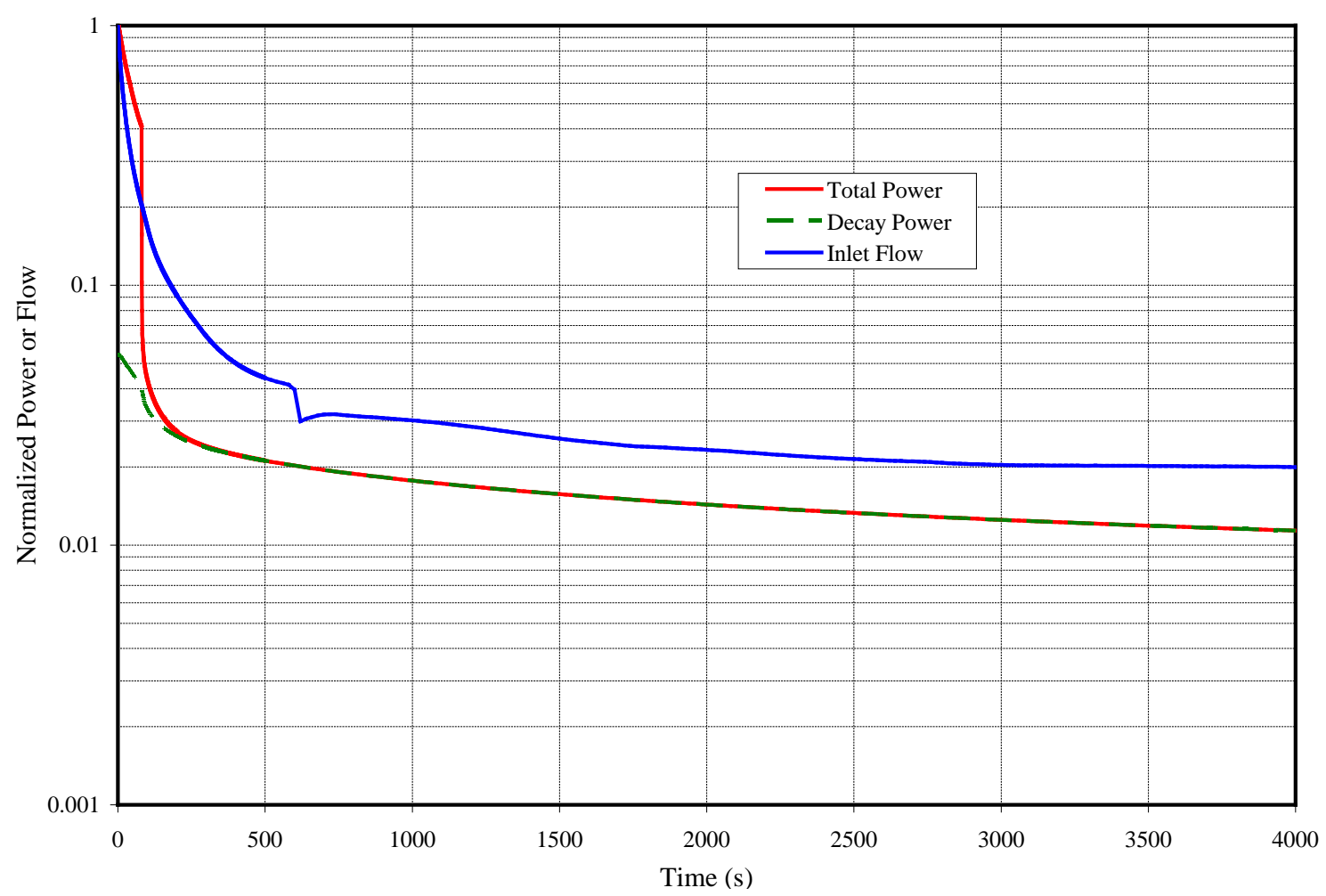

Figure III.7-30 Oxide Fuel ULOF Transient Power and Channel 4 Flow (50\% CRDL Feedback, Secondary Control System Trip at 1000 K)

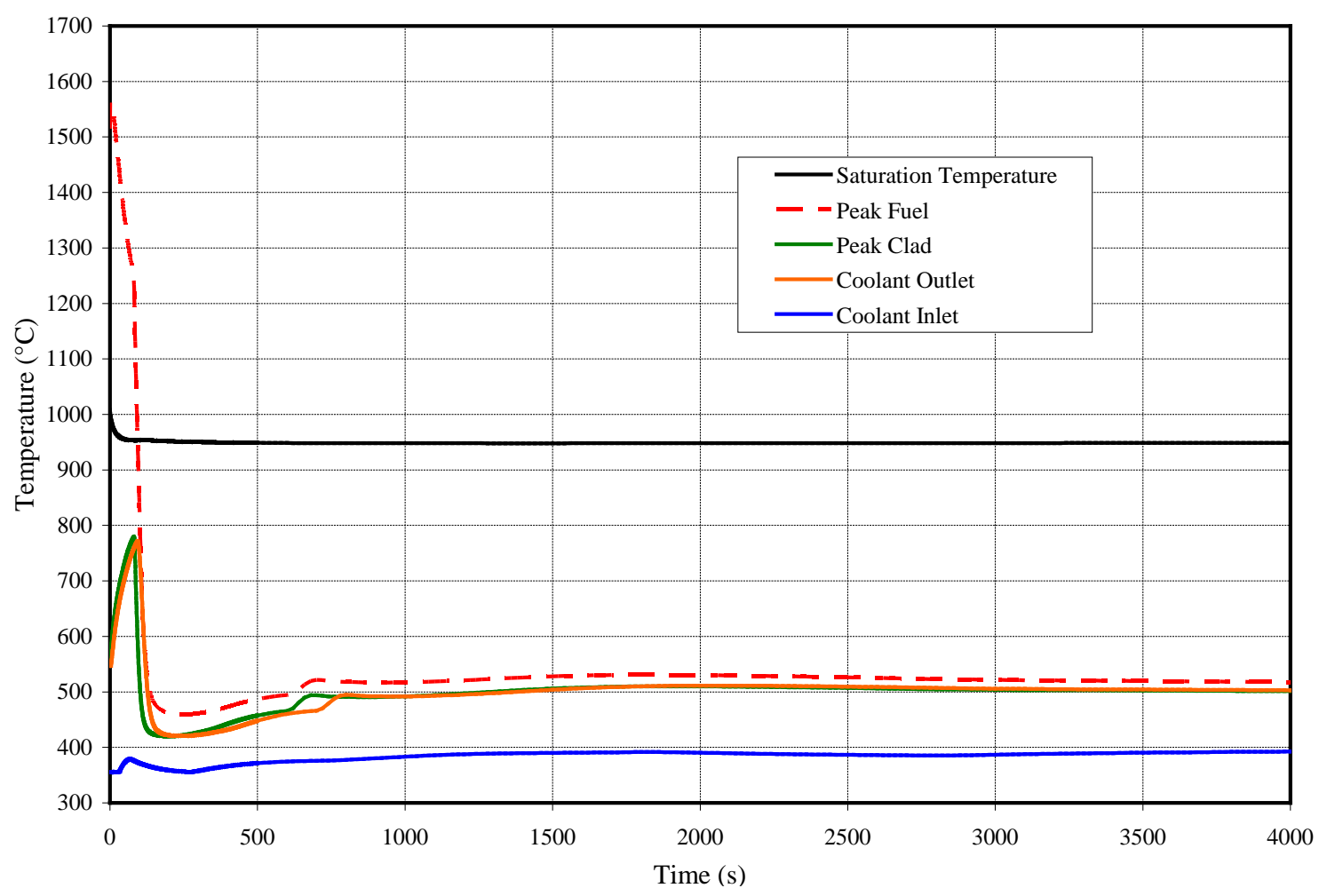

Figure III.7-31Oxide Fuel ULOF Transient Temperatures for Channel 4 ( $50 \%$ CRDL Feedback, Secondary Control System Trip at 1000 K) 


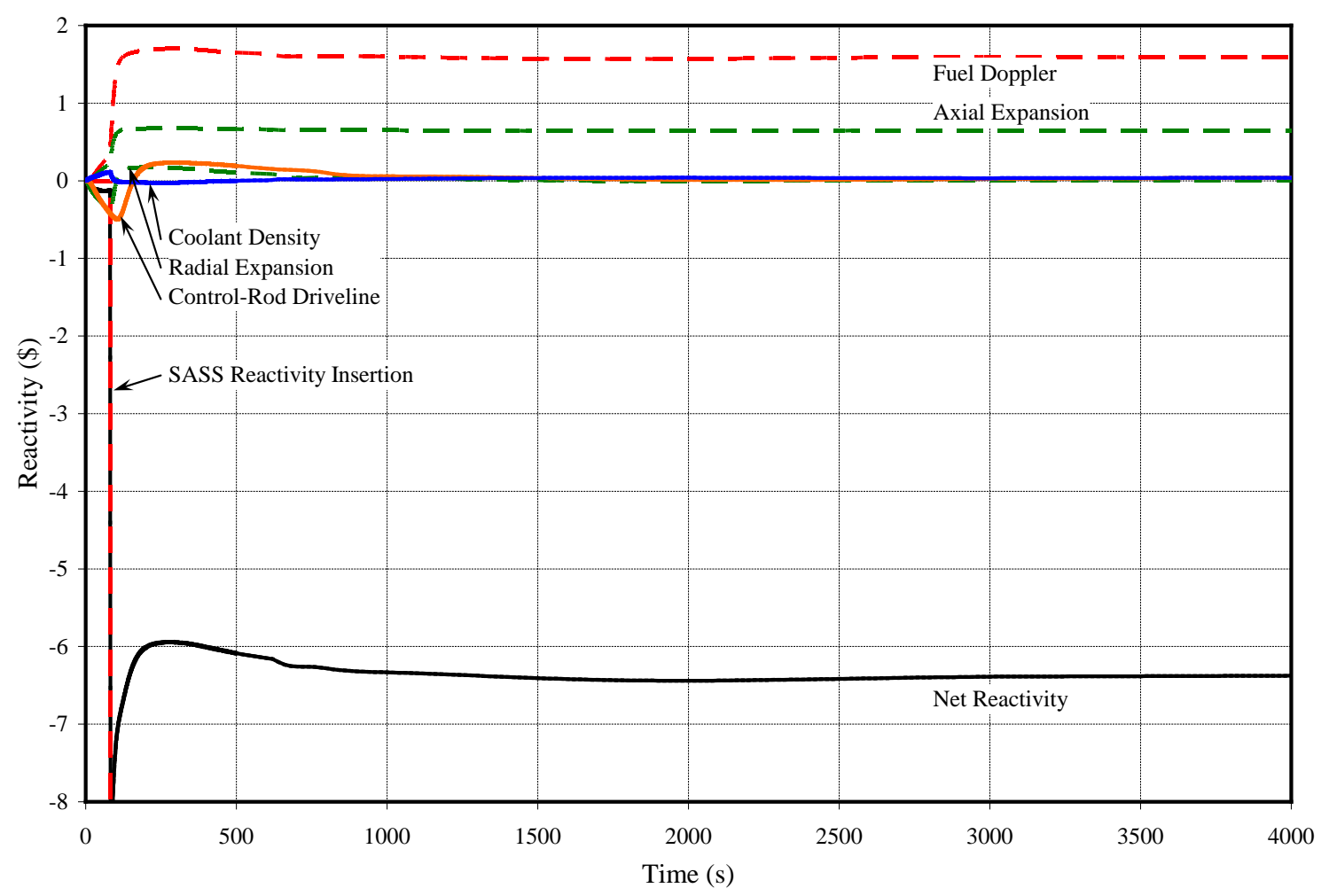

Figure III.7-32 Oxide Fuel ULOF Transient Reactivity Feedback (50\% CRDL Feedback, Secondary Control System Trip at $1000 \mathrm{~K}$ )

The significance of the ULOF accident analysis results for the SASS case is confirmed in Figure III.7-31. As shown for channel 4, the inclusion of the SASS has significantly increased the minimum core coolant boiling margin to about $170^{\circ} \mathrm{C}$ from the $50^{\circ} \mathrm{C}$ obtained in case (b). Peak cladding temperatures approach $780^{\circ} \mathrm{C}$ as opposed to $900^{\circ} \mathrm{C}$ without the SASS in case (b). Fuel temperatures are well below the melting point.

\section{III.7.5 References}

1. American National Standard Decay Heat Power in Light Water Reactors, ANSI/ANS-5.1-2005, American Nuclear Society, 2005.

2. J. E. Cahalan, A. M. Tentner, and E. E. Morris, "Advanced LMR Safety Analysis Capabilities in the SASSYS-1 and SAS4A Computer Codes," Proceedings of the International Topical Meeting on Advanced Reactors Safety, American Nuclear Society, Vol. 2, pp. 1038-1045, Pittsburgh, PA, April 17-21, 1994.

3. L. Leibowitz and R. A. Blomquist, "Thermal Conductivity and Thermal Expansion of Stainless Steels D9 and HT9,” International Journal of Thermophysics, Vol. 9, No. 5, pp. 873-883, September, 1988. 


\section{III.7.6 Evaluation Of Safety Design Criteria}

This section provides a survey of safety design criteria applicable to sodium cooled fast reactors. Criteria selected for consideration include 1) generalized design criteria specified by Title 10, Part 50, Appendix A of the Code of Federal Regulations for application to light water nuclear power reactors, 2) American National Standard general safety design criteria for a liquid metal reactor nuclear power plant, and 3) U.S. Department of Energy nuclear reactor safety design criteria. Considerations of design criteria by U.S. Nuclear Regulatory Commission in licensing and safety evaluation of proposed liquid metal cooled nuclear power reactor designs are reviewed. Applicability of the selected set of design criteria to sodium cooled fast reactor design features and operational characteristics are discussed. Recommendations are also made for modifications to existing safety design criteria for relevance to sodium cooled fast reactor designs.

The safety philosophy guiding the design, construction, and operation of nuclear facilities in the U.S. is based on the principle of "defense in depth" [1]. The objectives of "defense in depth' are first to protect the health and safety of the public and plant operating personnel, and second to preserve the facility investment by assuring its operational readiness. In terms of physical elements, “defense in depth" is exemplified by multiple, successive barriers to guard against the escape of radioactivity from nuclear facilities. However, in the evolution of U.S. nuclear safety philosophy, the "defense in depth" principle has been extended and applied to all aspects of nuclear facility design, construction, and operation, so that all safety critical functions are achieved by multiple systems/procedures/processes that are diverse and independent.

In the nuclear facility design process, the "defense in depth" principle has fostered the development of guidelines for identifying those engineered systems that are important for safety. Safety class systems are designed to be very reliable. They are constructed using specifications and materials that will assure functionality. In addition, multiple systems of diverse design are provided so that failure of any single safety system will not put people or equipment at risk.

The system configurations and functional requirements for nuclear facilities are routinely documented early in the design process as a set of safety design criteria. Commercial water cooled nuclear power plants licensed by the U.S. Nuclear Regulatory Commission must comply with general design criteria documented in the Code of Federal Regulations [2]. Suggestions for modifications of these criteria for application to liquid metal cooled reactor designs have been supplied by representatives of the nuclear power industry [3]. Nuclear research reactors built by the U.S. Department of Energy must comply with safety design criteria documented in a DOE Order [4].

The purpose of this Appendix is to provide an evaluation of documented nuclear reactor safety design criteria, with the aim of identifying changes necessary for application to a sodiumcooled fast reactor design. Existing general design criteria developed by the USNRC for light water reactors [2], by industry for liquid metal reactors [3], and by USDOE for research reactors [4] are examined for relevance. Recommendations are made for modifications to the existing safety design criteria for application to sodium-cooled system designs. 


\section{III.7.6.1 Safety Design Criteria Comparison}

In the methodological framework built on the defense-in-depth foundation, the role of safety design criteria is to set requirements for design performance. During the formative stages of design development, safety design criteria specify the configuration and functional performance characteristics the design must have for it to receive construction and operation approvals from the regulatory agency. Once the design is developed, safety analyses are performed and documented to quantify the margins between the safety requirements and expected performance. After the design is constructed, safety tests are performed to verify design safety performance. Therefore, it is necessary to establish safety design criteria early in the design process, and the requirements set by the safety design criteria largely determine the plant configuration, equipment inventory, and equipment arrangement.

The U.S. Nuclear Regulatory Commission has developed a set of general safety design criteria for commercial light water-cooled nuclear power reactors [2]. These criteria are the base-line requirements for nuclear power reactors in the U.S. However, the 10CFR50 Appendix A requirements are intended for application to light water reactors, and so are only partly applicable to the sodium cooled reactor designs.

During the years of the U.S. sodium cooled fast reactor development program, industry representatives developed an American National Standards Institute standard [3] for safety design criteria applicable to liquid metal cooled reactors. This set of criteria followed the organization and intent of 10CFR50 Appendix A, but modified certain criteria details for applicability to the low pressure, chemically reactive liquid metal coolant.

The U.S. Department of Energy has developed a set of safety design criteria [4] to apply to USDOE reactors that are exempt from USNRC regulation. Such reactors include one-of-a-kind designs built for research, and other special purpose reactors. The USDOE criteria are similar in organization and intent to the 10CFR50 Appendix A criteria, with some variations to address generically the design variations of the USDOE reactors.

Table III.7-5 contains a listing of the design criteria from 10CFR50 Appendix A, and a cross reference to the criteria proposed in Refs. 3 and 4. These three sets of safety design criteria have been reviewed for applicability to the sodium cooled fast reactor design. The "Comments" column in Table III.7-5 references the list of comment statements that follow the Table and record the results of the review. The review takes into account the technical safety evaluations performed by the USNRC for the PRISM [5] and SAFR [6] sodium cooled fast reactor designs. 
Table III.7-5 Safety Design Criteria Cross Comparison

\begin{tabular}{|c|c|c|c|c|}
\hline Criterion/Requirement & $\begin{array}{c}\text { 10CFR50, } \\
\text { App. A }\end{array}$ & $\begin{array}{c}\text { ANSI/ANS } \\
54.1\end{array}$ & $\begin{array}{c}\text { DOE } \\
5480.30\end{array}$ & Comments \\
\hline \multicolumn{5}{|c|}{ I. Overall Requirements } \\
\hline $\begin{array}{c}\text { Single Failure } \\
\text { Sing }\end{array}$ & & & 8.c.1 & 1,2 \\
\hline Quality Standards and Records & GDC 1 & 3.1 .1 & 8.c. 2 & 1 \\
\hline $\begin{array}{l}\text { Design Bases for Protection Against } \\
\text { Natural Phenomena }\end{array}$ & GDC 2 & 3.1.2 & 8.c.3 & 1 \\
\hline Fire Protection & GDC 3 & 3.1 .3 & 8.c. 4 & 1 \\
\hline $\begin{array}{l}\text { Protection Against Sodium and NaK } \\
\text { Reactions }\end{array}$ & & 3.1 .4 & & 3 \\
\hline $\begin{array}{l}\text { Environmental and Dynamics Effects } \\
\text { Design Bases }\end{array}$ & GDC 4 & 3.1.5 & $\begin{array}{l}\text { 8.c.5, } \\
\text { 8.c.10 }\end{array}$ & 4 \\
\hline $\begin{array}{l}\text { Sharing of Structures, Systems, and } \\
\text { Components }\end{array}$ & GDC 5 & 3.1.6 & 8.c. 6 & 1 \\
\hline Sodium Heating Systems & & 3.1 .7 & & 3 \\
\hline Siting & & & 8.c.7 & 5 \\
\hline Human Factors Engineering & & & 8.c.9 & 6 \\
\hline Safeguards and Security & & & 8.c.11 & 6 \\
\hline $\begin{array}{l}\text { Reactor Decontamination and } \\
\text { Decommissioning }\end{array}$ & & & 8.c.13 & 6 \\
\hline Support Systems & & & 8.c.15 & 6 \\
\hline $\begin{array}{l}\text { Non-Safety Class Structures, Systems, } \\
\text { and Components }\end{array}$ & & & 8.c.16 & 6 \\
\hline \multicolumn{5}{|c|}{ II. Protection by Multiple Fission Product Barriers } \\
\hline Reactor Design & GDC 10 & 3.2 .1 & 8.d.3.a & 1 \\
\hline Reactor Inherent Protection & GDC 11 & 3.2 .2 & 8.d.3.b & 7 \\
\hline $\begin{array}{l}\text { Suppression of Reactor Power } \\
\text { Oscillations }\end{array}$ & GDC 12 & 3.2.3 & 8.d.3.c & 1 \\
\hline Instrumentation and Control & GDC 13 & 3.2 .4 & 8.d.5.a & 1 \\
\hline Reactor Coolant Pressure Boundary & GDC 14 & 3.2 .5 & 8.d.1.a & 1 \\
\hline Reactor Coolant System Design & GDC 15 & 3.2 .6 & 8.d.6.a & 8 \\
\hline Containment Design & GDC 16 & 3.2.7 & 8.c.8 & 1 \\
\hline Electric Power Systems & GDC 17 & 3.2 .8 & 8.d.2.a & 1 \\
\hline $\begin{array}{l}\text { Inspection and Testing of Electric } \\
\text { Power Systems }\end{array}$ & GDC 18 & 3.2 .9 & 8.d.2.b & 1 \\
\hline Control Room & GDC 19 & 3.2 .10 & 8.d.5.e & 1 \\
\hline Remote Shutdown & & & 8.d.5.f & 9 \\
\hline $\begin{array}{l}\text { Heating, Ventilation, and Air } \\
\text { Conditioning (HVAC) Systems }\end{array}$ & & & 8.d.7.a,b,c & 10 \\
\hline
\end{tabular}


Table III.7-5 Safety Design Criteria Cross Comparison (cont.)

\begin{tabular}{|c|c|c|c|c|}
\hline Criterion/Requirement & $\begin{array}{l}\text { 10CFR50, } \\
\text { App. A }\end{array}$ & $\begin{array}{c}\text { ANSI/ANS } \\
54.1\end{array}$ & $\begin{array}{c}\text { DOE } \\
5480.30\end{array}$ & $\begin{array}{l}\text { Comm- } \\
\text { ents }\end{array}$ \\
\hline \multicolumn{5}{|c|}{ III. Protection and Reactivity Control Systems } \\
\hline $\begin{array}{l}\text { Protection System Functions } \\
\end{array}$ & GDC 20 & 3.3 .1 & 8.d.4.a & 1 \\
\hline $\begin{array}{l}\text { Protection System Reliability and } \\
\text { Testing }\end{array}$ & GDC 21 & 3.3.2 & 8.d.4.a & 1 \\
\hline $\begin{array}{l}\text { Protection System Independence } \\
\end{array}$ & GDC 22 & 3.3 .3 & 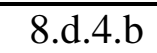 & 1 \\
\hline Protection System Failure Modes & GDC 23 & 3.3.4 & 8.d.4.c & 1 \\
\hline $\begin{array}{l}\text { Separation of Protection and Control } \\
\text { Systems }\end{array}$ & GDC 24 & 3.3 .5 & 8.d.4.d & 1 \\
\hline $\begin{array}{l}\text { Protection System Requirements for } \\
\text { Reactivity Control Malfunctions }\end{array}$ & GDC 25 & 3.3.6 & 8.d.4.e & 1 \\
\hline $\begin{array}{l}\text { Reactivity Control System Redundancy } \\
\text { and Capability }\end{array}$ & GDC 26 & 3.3.7 & 8.d.5.b & 11 \\
\hline $\begin{array}{l}\text { Combined Reactivity Control Systems } \\
\text { Capability }\end{array}$ & GDC 27 & 3.3.8 & 8.d.5.c & 11 \\
\hline Reactivity Limits & GDC 28 & 3.3 .9 & 8.d.5.d & 11 \\
\hline $\begin{array}{l}\text { Protection Against Anticipated } \\
\text { Operational Occurrences }\end{array}$ & GDC 29 & 3.3.10 & 8.d.4.f & 1 \\
\hline \multicolumn{5}{|c|}{ IV. Fluid Systems } \\
\hline $\begin{array}{l}\text { Assurance of Adequate Reactor Coolant } \\
\text { Inventory }\end{array}$ & & 3.4.1 & & 12 \\
\hline $\begin{array}{l}\text { Quality of Reactor Coolant Pressure } \\
\text { Boundary }\end{array}$ & GDC 30 & 3.4.2 & 8.d.1.b & 1 \\
\hline $\begin{array}{l}\text { Fracture Prevention of Reactor Coolant } \\
\text { Pressure Boundary }\end{array}$ & GDC 31 & 3.4.13 & 8.d.1.c & 4 \\
\hline $\begin{array}{l}\text { Inspection of Reactor Coolant Pressure } \\
\text { Boundary }\end{array}$ & GDC 32 & 3.4.3 & 8.d.1.d & 1 \\
\hline Reactor Coolant Makeup & GDC 33 & & 8.d.6.c & 13 \\
\hline $\begin{array}{l}\text { Reactor and Intermediate Coolant and } \\
\text { Cover Gas Purity Control }\end{array}$ & & 3.4.4 & & 14 \\
\hline Intermediate Coolant System & & 3.4 .5 & & 14 \\
\hline $\begin{array}{l}\text { Inspection and Surveillance of } \\
\text { Intermediate Coolant Boundary }\end{array}$ & & 3.4.6 & & 14 \\
\hline Residual Heat Removal & GDC 34 & 3.4.7 & 8.d.6.b & 1 \\
\hline Emergency Core Cooling & GDC 35 & & 8.d.6.d & 13 \\
\hline $\begin{array}{l}\text { Inspection of Emergency Core Cooling } \\
\text { System }\end{array}$ & GDC 36 & 3.4.8 & 8.d.6.d & 15 \\
\hline $\begin{array}{l}\text { Testing of Emergency Core Cooling } \\
\text { System }\end{array}$ & GDC 37 & 3.4 .9 & 8.d.6.d & 15 \\
\hline Containment Heat Removal & GDC 38 & & 8.c. 8 & 16 \\
\hline $\begin{array}{l}\text { Inspection of Containment Heat } \\
\text { Removal System }\end{array}$ & GDC 39 & & 8.c. 8 & 16 \\
\hline
\end{tabular}


Table III.7-5 Safety Design Criteria Cross Comparison (cont.)

\begin{tabular}{|c|c|c|c|c|}
\hline Criterion/Requirement & $\begin{array}{l}\text { 10CFR50, } \\
\text { App. A } \\
\end{array}$ & $\begin{array}{c}\text { ANSI/ANS } \\
54.1 \\
\end{array}$ & $\begin{array}{c}\text { DOE } \\
5480.30 \\
\end{array}$ & Comments \\
\hline $\begin{array}{l}\text { Testing of Containment Heat } \\
\text { Removal System }\end{array}$ & GDC 40 & & 8.c.8 & 16 \\
\hline Containment Atmosphere Cleanup & GDC 41 & 3.5.11 & 8.c.8 & 16 \\
\hline $\begin{array}{l}\text { Inspection of Containment } \\
\text { Atmosphere Cleanup Systems }\end{array}$ & GDC 42 & 3.5.12 & 8.c.8 & 16 \\
\hline $\begin{array}{l}\text { Testing of Containment } \\
\text { Atmosphere Cleanup Systems }\end{array}$ & GDC 43 & 3.5 .13 & 8.c. 8 & 16 \\
\hline Cooling Water & GDC 44 & 3.4 .10 & 8.d.6.e & 1 \\
\hline $\begin{array}{l}\text { Inspection of Cooling Water } \\
\text { System }\end{array}$ & GDC 45 & 3.4.11 & 8.d.6.e & 1 \\
\hline Testing of Cooling Water System & GDC 46 & 3.4 .12 & 8.d.6.e & 1 \\
\hline \multicolumn{5}{|c|}{ V. Reactor Containment } \\
\hline Containment Design Basis & GDC 50 & $\begin{array}{c}3.5 .1 \\
\text { 3.5.2, 3.5.3 }\end{array}$ & 8.c.8 & 17 \\
\hline $\begin{array}{l}\text { Fracture Prevention of Containment } \\
\text { Pressure Boundary }\end{array}$ & GDC 51 & 3.5.4 & 8.c.8 & 1 \\
\hline $\begin{array}{l}\text { Capability for Containment } \\
\text { Leakage Rate Testing }\end{array}$ & GDC 52 & 3.5 .5 & 8.c.8 & 1 \\
\hline $\begin{array}{l}\text { Provisions for Containment Testing } \\
\text { and Inspection }\end{array}$ & GDC 53 & 3.5.6 & 8.c.8 & 1 \\
\hline $\begin{array}{l}\text { Piping Systems Penetrating } \\
\text { Containment }\end{array}$ & GDC 54 & 3.5.7 & 8.c.8 & 1 \\
\hline $\begin{array}{l}\text { Reactor Coolant Pressure Boundary } \\
\text { Penetrating Containment }\end{array}$ & GDC 55 & 3.5 .8 & 8.c.8 & 1 \\
\hline Primary Containment Isolation & GDC 56 & 3.5 .9 & 8.c.8 & 1 \\
\hline Closed System Isolation Valves & GDC 57 & 3.5 .10 & 8.c.8 & 1 \\
\hline \multicolumn{5}{|l|}{ VI. Fuel and Radioactivity Control } \\
\hline $\begin{array}{l}\text { Control of Releases of Radioactive } \\
\text { Materials to the Environment }\end{array}$ & GDC 60 & 3.6.1 & $\begin{array}{l}\text { 8.c.12.a, } \\
\text { 8.c.14 }\end{array}$ & 1 \\
\hline $\begin{array}{l}\text { Fuel Storage and Handling and } \\
\text { Radioactivity Control }\end{array}$ & GDC 61 & 3.6.2 & $\begin{array}{l}\text { 8.d.8.a, } \\
\text { 8.d.8.d }\end{array}$ & 1 \\
\hline $\begin{array}{l}\text { Prevention of Criticality in Fuel } \\
\text { Storage and Handling }\end{array}$ & GDC 62 & 3.6.3 & 8.d.8.b & 1 \\
\hline Monitoring Fuel and Waste Storage & GDC 63 & 3.6 .4 & 8.d.8.c & 1 \\
\hline Monitoring Radioactivity Releases & GDC 64 & 3.6 .5 & $\begin{array}{l}\text { 8.c.12.b, } \\
\text { 8.c.14 }\end{array}$ & 1 \\
\hline
\end{tabular}


Comment 1 . The relevant criteria from Refs. 2, 3, and 4 are essentially congruent in intent and are applicable to the liquid metal-cooled reactor design.

Comment 2. The single failure criterion for safety class structures, systems, and components is listed as a definition applied to specified criteria in Refs. 2 and 3. The single failure criterion is explicit in Ref. 4.

Comment 3. Explicit requirement for liquid metal coolant that considers the impact of chemical reactivity or thermophysical properties.

Comment 4. The 10CFR50 criterion addresses phenomena for a high pressure water system. The criteria should be revised to address phenomena relevant to low pressure, chemically reactive liquid metal coolant.

Comment 5. Siting criteria are considered in 10CFR100.

Comment 6. Explicit USDOE requirement beyond the scope of 10CFR50 Appendix A.

Comment 7. In LWR designs, the requirement for a prompt, negative power coefficient is met by the combination of the negative fuel Doppler coefficient and the negative moderator density coefficient. If the sodium cooled fast reactor coolant void coefficient is positive, the regulator may require additional design features to compensate, as noted in the PRISM [5] and SAFR [6] safety evaluation reports.

Comment 8. Systems cited should include the liquid metal heating system.

Comment 9. The USDOE separate requirement for a remote shutdown capability is included in GDC 19.

Comment 10. USDOE criterion for confinement superseded by containment criterion, GDC 16.

Comment 11. 10CFR50 Appendix A criteria contain references to LWR specific reactivity mechanisms (Xe, cold shutdown, ECCS boron injection, rod dropout, cold coolant shock) that are either irrelevant or require re-interpretation for fast spectrum LMR.

Comment 12. Liquid metal reactor criterion for maintaining core submersion in coolant is the equivalent of ECCS requirement for light water reactor (GDC 33, 35).

Comment 13. 10CFR50 Appendix A criterion is not applicable for liquid metal coolant.

Comment 14. Intermediate loop criteria for liquid metal cooled design.

Comment 15. The liquid metal reactor design does not include an ECCS, but inspection and testing requirements are applied to the residual heat removal system.

Comment 16. In the light water reactor design, the containment heat removal system is intended to reduce temperature and pressure following a loss-of-coolant accident. Because such an accident sequence is not a design basis for the liquid metal cooled reactor, this criterion may not be relevant.

Comment 17. In the light water reactor design the containment design is based on loss of coolant accident consequences. For the low pressure liquid metal cooled reactor, in which a pipe break event is much less severe (leak before break), an alternative design basis accident must be specified. 


\section{III.7.6.2 Evaluation of Safety Design Criteria}

The cross comparison of safety design criteria in Section III.7-1 shows that the sets of design criteria defined by the USNRC [2], the ANSI [3], and the USDOE [4] are generally and specifically convergent with regard to scope and content. The NRC design criteria are intended for application to light water cooled power reactors, and the ANSI criteria were proposed for sodium cooled reactors.

In its safety evaluation reports for the PRISM [5] and SAFR [6] designs, the USNRC has provided analyses of the applicability of the criteria in Ref. 2 to specific sodium cooled fast reactor designs, taking Ref. 3 into account. These analyses have been reviewed with a perspective of the general characteristics of a sodium cooled fast reactor system. From this review, the following general statements can be made with regard to the applicability of the design criteria in Ref. 2 to sodium cooled systems:

1. Many of the 10CFR50 Appendix A general design criteria are directly applicable to the sodium cooled fast reactor systems, without wording changes or modifications. The intent of the design criterion is clear, and the design implication for application to a sodium cooled system is apparent. Criteria included in this class are GDCs 1, 2, 3, $5,10,12,13,14,16,17,18,19,20,21,22,23,24,25,29,30,32,34,44,45,46,51$, 52, 53, 56, 60, 61, 62, 63, 64.

2. Some of the 10CFR50 Appendix A general design criteria are worded with reference to specific light water reactor design features, performance characteristics, or regulatory requirements. These criteria must be reworded to preserve the original intent for sodium cooling. A listing of the criteria in this class and suggested changes are given in Table III.7-6. 
Table III.7-6 Suggested Changes to 10CFR50 Appendix A General Design Criteria for Application to Sodium Cooled Systems

\begin{tabular}{|c|c|}
\hline GDC No. & Suggested Change \\
\hline 4 & $\begin{array}{l}\text { This criterion is written in reference to a high pressure, water coolant, and includes } \\
\text { explicit references to loss-of-coolant accidents (LOCAs) and pipe ruptures. The } \\
\text { words should be changed 1) to include dynamic and environmental phenomena } \\
\text { relevant to low pressure sodium, e.g. the environmental effects of aerosols and } \\
\text { oxidation products, 2) to delete references to dynamic and environmental accident } \\
\text { phenomena specific to water coolant, and 3) to include references to generic design } \\
\text { basis events, i.e. "anticipated operational occurrences." }\end{array}$ \\
\hline 11 & $\begin{array}{l}\text { This criterion requires a prompt inherent nuclear feedback effect to compensate a } \\
\text { rapid reactivity increase in the power operating range. NRC reviews of the PRISM } \\
\text { and SAFR designs highlighted the positive coolant void reactivity worth as an area } \\
\text { of significant concern for a beyond-design-basis loss-of-flow-without-scram } \\
\text { accident sequence. The criterion should be modified to specifically exclude or } \\
\text { include coolant voiding effects, depending on the safety strategy dealing with } \\
\text { severe accident. }\end{array}$ \\
\hline 15 & $\begin{array}{l}\text { This criterion is written to require that the reactor coolant pressure boundary is built } \\
\text { to withstand design basis conditions. Reference } 5 \text { specifically requires inclusion of } \\
\text { the sodium heating system in the listing of coolant systems subject to this } \\
\text { requirement. }\end{array}$ \\
\hline 26 & $\begin{array}{l}\text { This criterion requires two independent reactivity control systems, one of which } \\
\text { shall use control rods. Specific control reactivity requirements are listed in terms of } \\
\text { LWR performance characteristics. The criterion should be rewritten in terms of } \\
\text { equivalent sodium cooled fast reactor characteristics. }\end{array}$ \\
\hline 27 & $\begin{array}{l}\text { This criterion specifies combined control reactivity requirements including liquid } \\
\text { poison injection. The criterion should be rewritten to eliminate the explicit mention } \\
\text { of liquid poison addition. }\end{array}$ \\
\hline 28 & $\begin{array}{l}\text { This criterion requires that the reactivity control system be designed to limit the } \\
\text { possible rate of reactivity addition to avoid damage to the reactor and its associated } \\
\text { structures, systems, and components. Specific control reactivity requirements are } \\
\text { listed in terms of LWR performance characteristics. The criterion should be } \\
\text { rewritten in terms of equivalent sodium cooled fast reactor characteristics. }\end{array}$ \\
\hline 31 & $\begin{array}{l}\text { This criterion specifies conditions and phenomena to be considered in the design of } \\
\text { the reactor coolant pressure boundary. For sodium coolant, Ref. } 5 \text { specifies } \\
\text { addition of coolant chemistry and mechanical properties degradation to the list of } \\
\text { considered phenomena, in recognition of the chemically active nature of sodium. }\end{array}$ \\
\hline
\end{tabular}


Table III.7-6 Suggested Changes to 10CFR50 Appendix A General Design Criteria for Application to Sodium Cooled Systems (cont.)

\begin{tabular}{|c|c|}
\hline GDC No. & Suggested Change \\
\hline 33 & $\begin{array}{l}\text { This criterion provides for a coolant supply system to assure reactor coolant } \\
\text { inventory in the event of a small break in the coolant pressure boundary. As } \\
\text { formulated, the criterion is not relevant to low pressure sodium coolant. } \\
\text { Reference } 3 \text { specifies a replacement criterion dealing with assurance of adequate } \\
\text { coolant inventory to maintain core cover and operation of the residual heat } \\
\text { removal system in all cases. }\end{array}$ \\
\hline 35 & $\begin{array}{l}\text { This criterion provides for emergency core cooling in the event of a loss of } \\
\text { coolant accident. Such an event is outside the design basis for the low pressure } \\
\text { sodium coolant system. Coolant inventory, core covering, and residual heat } \\
\text { removal are assured by GDC } 33 \text { and } 34 \text {. }\end{array}$ \\
\hline 36 & $\begin{array}{l}\text { This criterion provides for inspection of the emergency core cooling system. } \\
\text { Reference } 5 \text { recommends rewriting this criterion to provide for inspection of the } \\
\text { residual heat removal system (GDC 34). }\end{array}$ \\
\hline 37 & $\begin{array}{l}\text { This criterion provides for testing of the emergency core cooling system. } \\
\text { Reference } 5 \text { recommends rewriting this criterion to provide for testing of the } \\
\text { residual heat removal system (GDC 34). }\end{array}$ \\
\hline 38 & $\begin{array}{l}\text { This criterion provides for the design of a containment heat removal system with } \\
\text { the capability of rapidly reducing the temperature and pressure within the } \\
\text { containment following a loss-of-coolant accident. The LOCA sequence is not } \\
\text { relevant to the low pressure sodium coolant system. The criterion should be } \\
\text { rewritten with wording to replace the LOCA reference with the appropriate } \\
\text { design basis accident reference. }\end{array}$ \\
\hline 39 & $\begin{array}{l}\text { This criterion provides for inspection of the containment heat removal system, } \\
\text { and includes specific reference to design features relevant to water cooled } \\
\text { systems. The criterion should be rewritten to include design characteristics } \\
\text { relevant to sodium cooled systems. }\end{array}$ \\
\hline 40 & $\begin{array}{l}\text { This criterion provides for testing of the containment heat removal system, and } \\
\text { includes specific reference to design features relevant to water cooled systems. } \\
\text { The criterion should be rewritten to include design characteristics relevant to } \\
\text { sodium cooled systems. }\end{array}$ \\
\hline 41 & $\begin{array}{l}\text { This criterion provides for the design of a system to control fission gases and } \\
\text { combustible gases in containment following a postulated accident. Reference } 3 \\
\text { recommends modification of this criterion to include sodium aerosols and } \\
\text { combustion products, and to identify sodium leakage and interaction with } \\
\text { concrete as events in the accident sequence. The criterion should be rewritten to } \\
\text { include design characteristics relevant to sodium cooled systems. }\end{array}$ \\
\hline 42 & $\begin{array}{l}\text { This criterion provides for inspection of the containment atmosphere cleanup } \\
\text { system provided by GDC } 41 \text {. The criterion should be rewritten to include design } \\
\text { characteristics relevant to sodium cooled systems. }\end{array}$ \\
\hline
\end{tabular}


Table III.7-6 Suggested Changes to 10CFR50 Appendix A General Design Criteria for Application to Sodium Cooled Systems (cont.)

\begin{tabular}{|c|l|}
\hline GDC No. & \multicolumn{1}{c|}{ Suggested Change } \\
\hline 43 & $\begin{array}{l}\text { This criterion provides for testing of the containment atmosphere cleanup system } \\
\text { provided by GDC 41. The criterion should be rewritten to include design } \\
\text { characteristics relevant to sodium cooled systems. }\end{array}$ \\
\hline 50 & $\begin{array}{l}\text { This criterion stipulates design basis conditions and phenomena for the } \\
\text { containment, and makes specific mention of loss-of-cooling accidents and metal- } \\
\text { water interactions. The criterion should be rewritten to include design } \\
\text { characteristics relevant to sodium cooled systems. Reference } 5 \text { recommends } \\
\text { replacement of the LOCA sequence with the appropriate postulated accident, and } \\
\text { replacement of metal-water interactions with phenomena relevant to sodium } \\
\text { cooling. }\end{array}$ \\
\hline 55 & $\begin{array}{l}\text { This criterion provides for isolation design requirements for lines connected to the } \\
\text { reactor coolant pressure boundary that penetrate the containment. In sodium } \\
\text { cooled systems, this criterion also applies to lines connected to the reactor cover } \\
\text { gas space. }\end{array}$ \\
\hline
\end{tabular}

Reference 5 identifies nine additional design criteria relevant to sodium cooled fast reactor designs that are not explicitly stated in 10CFR50 Appendix A. Six of these additional nine criteria are also cited in Ref. 3. These nine criteria are as follows:

1. Protection Against Sodium Reactions. (Cited in Ref. 3 as Criterion 3.1.4). This criterion explicitly provides for measures to protect against the consequences of chemical reactions resulting from sodium leaks. It calls for prevention, detection, and consequence mitigation design features, as well as measures to protect personnel and equipment from corrosive and potentially radioactive oxidation products.

2. Sodium Heating Systems. (Cited in Ref. 3 as Criterion 3.1.7). This criterion provides safety and performance requirements for systems intended to maintain as a liquid. Such systems are required to perform assuming a single failure.

3. Heat Transport System Design. (No corresponding Criterion in Ref. 3). This requirement ensures sufficient reactor cooling for normal operation and anticipated operational occurrences by providing two independent coolant flow paths between the reactor and the heat sinks, and stipulates that the integrity of the reactor coolant pressure boundary shall be maintained for postulated accidents. This criterion covers the same requirements as GDCs 34 (Residual Heat Removal), 35 (Emergency Core Cooling), and 44 (Cooling Water) in the original 10CFR50 Appendix A.

4. Assurance of Adequate Reactor Coolant Inventory. (Cited as Criterion 3.4.1 in Ref. 3). This criterion provides for sufficient coolant inventory to assure residual heat removal for normal operation, anticipated operational occurrences, and postulated accidents assuming a single failure. The criterion has the same intent as the original GDC 33 (Reactor Coolant Makeup).

5. Design of the Intermediate Coolant System. (Cited as Criteria 3.4.5 and 3.4.6 in Ref. 3). These criteria provide for the design, inspection, testing, and surveillance of the 
intermediate coolant system, and cover the intent of the original GDCs 44, 45, and 46 (Cooling Water).

6. Reactor and Intermediate Coolant and Cover Gas Purity Control. (Cited as Criterion 3.4.4 in Ref. 3). This criterion requires systems to monitor and maintain the purity of reactor and intermediate coolants and cover gases within specified design limits.

7. Inspection and Testing of Residual Heat Removal Systems. (Cited as Criteria 3.4.8 and 3.4.9 in Ref. 3). This criterion provides for inspection, testing, and surveillance of the residual heat removal system. The intent of these criteria is the same as that of the original GSCs 36 and 37, which provide for inspection and testing of the emergency core cooling system for water cooled reactor designs.

8. Protection Against Fuel Rod Failure Propagation. (No corresponding Criterion in Ref. 3). This criterion was proposed for early sodium cooled fast reactor designs for which fuel irradiation experience was limited. As fuel irradiation experience was gained, confidence in fuel performance was assured, and this criterion was explicitly excluded in Ref. 5, which notes that fuel design limits and failure performance are included by GDCs 10, 27, and 35.

9. Protection Against Coolant Flow Blockage. (No corresponding Criterion in Ref. 3). This criterion requires the fuel assembly design to include specific features to prevent and minimize the likelihood of coolant flow blockages, so that such events can be eliminated from the design basis. This consideration arises due to use of ducted fuel assemblies, for which inlet flow blockages or restrictions could lead to fuel damage or failure.

\section{References}

1. U.S. Atomic Energy Commission, The Safety of Nuclear Power Reactors (Light Water-Cooled) and Related Facilities, WASH-1250, July, 1973.

2. U.S. Nuclear Regulatory Commission, Code of Federal Regulations, Title 10, “Energy,” Part 50, "Domestic Licensing of Production and Utilization Facilities," Appendix A, "General Design Criteria for Nuclear Power Plants.”

3. American National Standards Institute/American Nuclear Society, General Safety Design Criteria for a Liquid Metal Reactor Nuclear Power Plant, ANSI/ANS-54.11989, American Nuclear Society, La Grange Park, Illinois.

4. U.S. Department of Energy, Nuclear Reactor Safety Design Criteria, Order DOE 5480.30, Chg. 1: 3-14-01.

5. U.S. Nuclear Regulatory Commission, Preapplication Safety Evaluation Report for the Power Reactor Innovative Small Module (PRISM) Liquid Metal Reactor, Final Report, NUREG-1368, February, 1994.

6. U.S. Nuclear Regulatory Commission, Preapplication Safety Evaluation Report for the Sodium Advanced Fast Reactor (SAFR) Liquid-Metal Reactor, NUREG1369,December,1991. 


\section{III.8 Supporting Analyses for Heat Transport Systems}

The purpose of this section is to document analyses that have been carried out in support of the development of the various heat transport systems for ABR. These supporting analyses include parametric calculations for sizing of the Intermediate Heat Exchanger (IHX) and Direct Reactor Auxiliary Cooling System (DRACS) heat exchangers, including models for calculating pressure drop on the primary (shell) and secondary (tube) sides. The various modeling activities that have been carried out in this area are summarized below.

As noted in Sections II.3 and II.6, thermal hydraulic analyses were performed in order to form the technical basis for the sizing of both the IHX and DRACS in-vessel heat exchangers. In terms of the thermodynamic analysis required to estimate the overall unit size needed to achieve the specified thermal rating, the forced convection heat transfer coefficients on shell and tube sides are calculated using the well-known Lockhart-Martinelli correlation which was developed on the basis of forced convection heat transfer from liquid metals:

$$
N u=\frac{h D_{e}}{k}=5.0+0.025(R e \cdot P r)^{0.8}
$$

where:

$$
\begin{array}{lll}
\mathrm{Nu} & = & \text { Nusselt number, } \\
h & = & \text { forced convection heat transfer coefficient, } \\
\mathrm{Pr} & = & \text { Prandlt number }=\mu \mathrm{c} / \mathrm{k}, \\
k & = & \text { sodium thermal conductivity } \sim 69 \mathrm{~W} / \mathrm{m}-\mathrm{K}, \\
\mathrm{C} & = & \text { specific heat } \sim 1283 \mathrm{~J} / \mathrm{kg}-\mathrm{K}, \\
\mathrm{Re} & = & \text { Reynolds number }=\rho \mathrm{Du} / \mu \\
\mathrm{D} & = & \text { equivalent diameter of flow channel }=\text { tube ID for tube-side flow, } \\
& = & (4 \cdot \text { channel flow area }) / \text { wetted perimeter for shell side. }
\end{array}
$$

For pitch-to-diameter (P/D) ratios of $>1.1$, the above correlation results in errors of no more than $10 \%$ on the shell side of the IHX.

Aside from the thermodynamic calculations, the pressure drop on the tube side of the heat exchangers is calculated to facilitate pump selection and design considerations. In particular, the pressure drop is calculated using the standard Bernoulli equation modification to account for frictional drag as well as tube bank entrance and exit form losses; i.e.,

$$
\Delta P_{t}=\left(K_{e n t r}+K_{e x i t}+f L / D_{i}\right) \frac{1}{2} \rho u^{2}
$$

where:

$$
\begin{array}{lll}
u & = & \text { flow velocity, } \\
K_{\text {entr }} & = & \text { tube entrance loss coefficient (typically taken as } \sim 0.5), \\
K_{\text {entr }} & = & \text { tube exit loss coefficient (typically taken as } \sim 1.0), \\
f & = & \text { friction factor given by Blasius correlation }=0.3164 / R^{1 / 4},
\end{array}
$$




$$
\begin{array}{lll}
R e & = & \text { Reynolds number }=\rho D_{i} u / \mu \\
D_{i} & = & \text { tube inner diameter, } \\
L & = & \text { tube bundle length, } \\
\mu & = & \text { sodium viscosity } \sim 2.76 \cdot 10^{-4} \mathrm{~kg} / \mathrm{m} \text {-sec, and } \\
\rho & = & \text { sodium density } \sim 850 \mathrm{~kg} / \mathrm{m}^{3} .
\end{array}
$$

The pressure drop analysis on the shell side is complicated by the fact that an orificed baffle plate design has been adopted in order to minimize pressure drop. Although well-established correlations have been developed for evaluating pressure drop for the case of impervious plates (e.g., see Kern [1]), suitable correlations for the case of orificed plates could not be identified in the literature. On this basis, a correlation was developed and validated against other IHX designs that feature orificed baffle plates. The specific correlation is presented below; complete modeling details can be found in reference [2].

For the case of orificed baffle plates, the flow pressure drop is evaluated through the following correlation:

$$
\Delta P_{s}=\frac{C}{2 \rho}\left(N_{b}+1\right)\left(\frac{\mu N_{T}}{D_{o}}\right)^{0.135}\left(F G_{c}\right)^{1.73}
$$

where:

$$
\begin{aligned}
& D_{o}=\quad \text { tube outside diameter, } \\
& G_{c}=\quad \text { mass flux through bundle in cross flow }=\frac{\dot{m}}{B\left(P-D_{o}\right) N_{T}} \text {, } \\
& N_{T}=\quad \text { number of tubes in cross-flow, perpendicular to flow direction, } \\
& N_{b}=\quad \text { number of baffle plates, } \\
& P=\text { tube pitch, } \\
& B=\text { baffle plate pitch (i.e., distance between plates), } \\
& \dot{m}=\quad \text { shell-side mass flowrate, } \\
& C=\left\{\begin{array}{l}
3.59+8.34\left(1.44-P / D_{o}\right), \quad P / D_{o}<1.44, \\
3.59, \quad P / D_{o} \geq 1.44
\end{array}\right.
\end{aligned}
$$

and $F$ is the fraction of the total shell side mass flowrate that remains in a cross-flow configuration during flow past an individual baffle plate. This fraction is evaluated through the following transcendental equation:

$$
1-F=\omega F^{0.865}
$$

where:

$$
\omega=\frac{\pi}{2} N_{x}^{1.5}\left(\frac{C}{K_{h}}\right)^{0.5} \frac{D_{h}^{2}}{\left[\left(P-D_{o}\right) B\right]^{0.865}}\left(\frac{\mu N_{T}}{D_{o} \dot{m}}\right)^{0.135}
$$


and:

$$
\begin{array}{lll}
D_{h} & = & \text { orifice hole diameter, } \\
K_{h} & = & \text { orifice hole form loss coefficient } \sim 1.5, \text { and } \\
N_{x} & = & \text { number of tube rows in cross flow, parallel with flow direction. }
\end{array}
$$

In terms of the approach for carrying out the parametric calculations, the number of heat exchanger tubes is treated as the independent variable. The required tube length is then calculated as the key model output, and the minimum heat exchanger cross-sectional area is given by tube unit cell cross-sectional area multiplied by the total number of tubes. This approach provides the overall heat exchanger size to be selected on the basis of the available cross sectional area, or the available elevation space, whichever is desired.

In terms of the design calculations for the IHX, tube diameter was selected to be identical to the PRISM IHX. For completeness, the calculations were carried for a variety of P/D ratios with the temperature differentials on the primary and secondary sides fixed at the design levels shown in Table II.3.3.1. The thermal conductivity of the 9Cr-1Mo tubes was taken as $28 \mathrm{~W} / \mathrm{m}-{ }^{\circ} \mathrm{C}$.

The results of the calculations for the IHX are shown in Figures III.8-1 through III.8-4, which provide the tube sheet planar area, tube length, and pressure drop across the shell and tube sides vs. number of $\mathrm{HX}$ tubes for a range of P/D ratios from 1.2 to 1.8. To compromise between the planar area of the reactor vessel occupied by the IHXs and the primary side pressure drop, an intermediate value of the P/D ratio of 1.4 was selected for the current design. Given the equipment distribution within the reactor vessel, $\sim 2.3 \mathrm{~m}^{2}$ of cross sectional area is available to accommodate each IHX. Factoring in the shell thickness of $1.9 \mathrm{~cm}$, as well as the downcomer pipe planar area, then the available area for the tube sheet is reduced to $\sim 2.25 \mathrm{~m}^{2}$. Thus, from Figure III.8-15, an IHX with a total of $\sim 4500$ tubes is selected to fit into the available space. From Figure III.8-16, for the case of $\mathrm{P} / \mathrm{D}=1.4$, the active tube length must be $4.78 \mathrm{~m}$ to achieve the target thermal rating of $250 \mathrm{MW}$ per IHX. With the tube length specified, the minimum required elevation space to accommodate the IHXs in the vessel plan view is determined. From Figures III.8-17 and III.8-18, the pressure drop across the primary and secondary sides of the tube bank are found to be 18.2 and $14.8 \mathrm{kPa}$, respectively, for the case of $\mathrm{P} / \mathrm{D}=1.4$. The primary side pressure drop thus sets the pressure head required to drive the $1256 \mathrm{~kg} / \mathrm{sec}$ sodium flowrate through the IHX at $\sim 2.2 \mathrm{~m}$ at full power conditions.

Figures III.8-5 through III.8-8 provide the analogous plots of planar area, tube length, and pressure drop across shell and tube sides vs. number of heat exchanger tubes for both 2.5 and 5.0 MW DRACS heat exchanger designs that were evaluated as part of the shutdown heat removal system design evaluation. As described in Section II.6, the final design adopted the $2.5 \mathrm{MW}$ units, which corresponds to $0.25 \%$ long term decay heat removal capacity. Key design parameters for these units are summarized in Table II.6.1.1.

\section{References}

1. D. Q. Kern, Process Heat Transfer, McGraw-Hill Book Co., New York, NY, 1950.

2. M. T. Farmer, Intra-Laboratory memo to C. Grandy, ANL, May 18, 2006. 


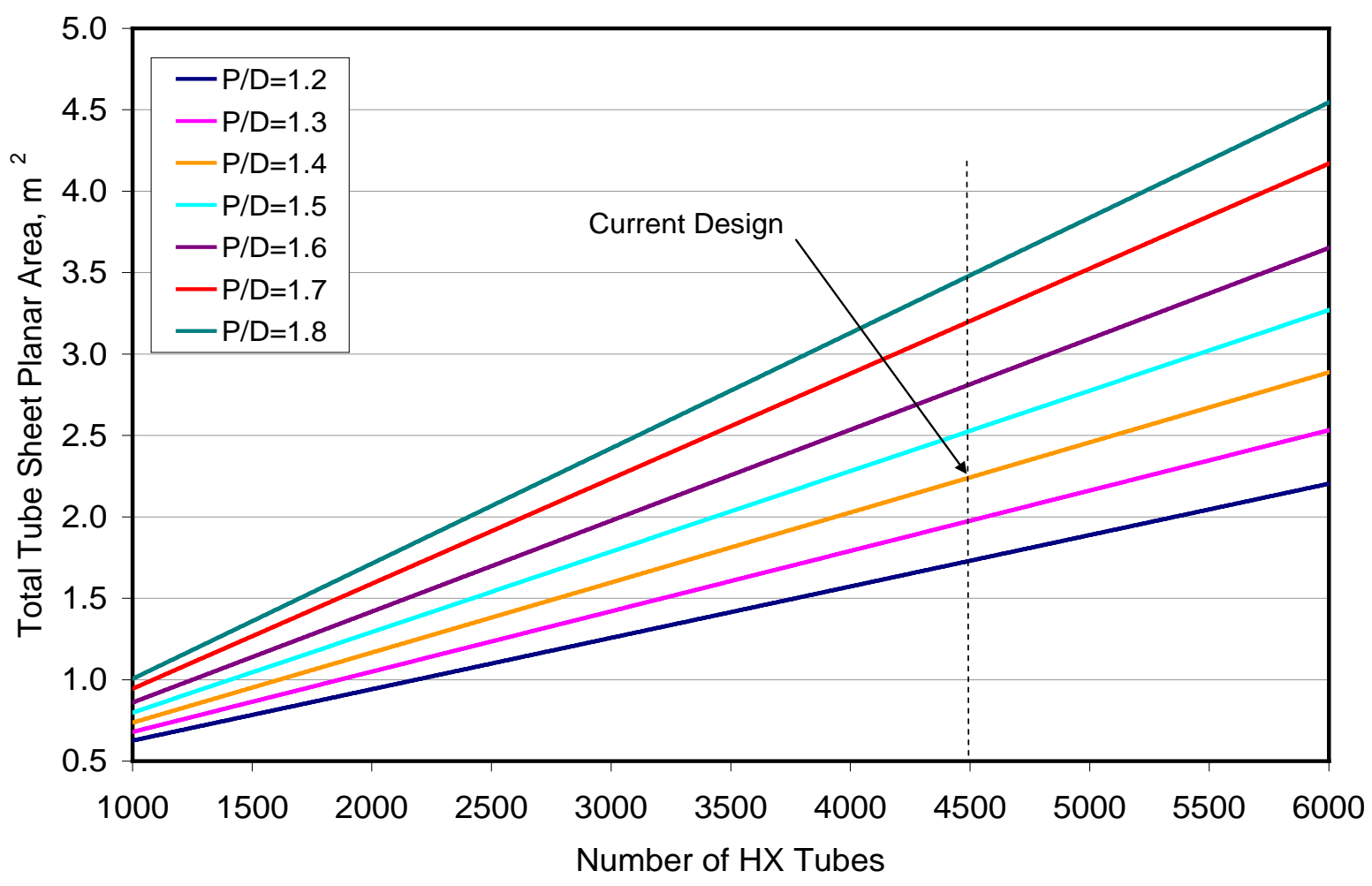

Figure III.8-1 IHX Tube Sheet Planar Area vs. No. of Tubes for Various P/D Ratios

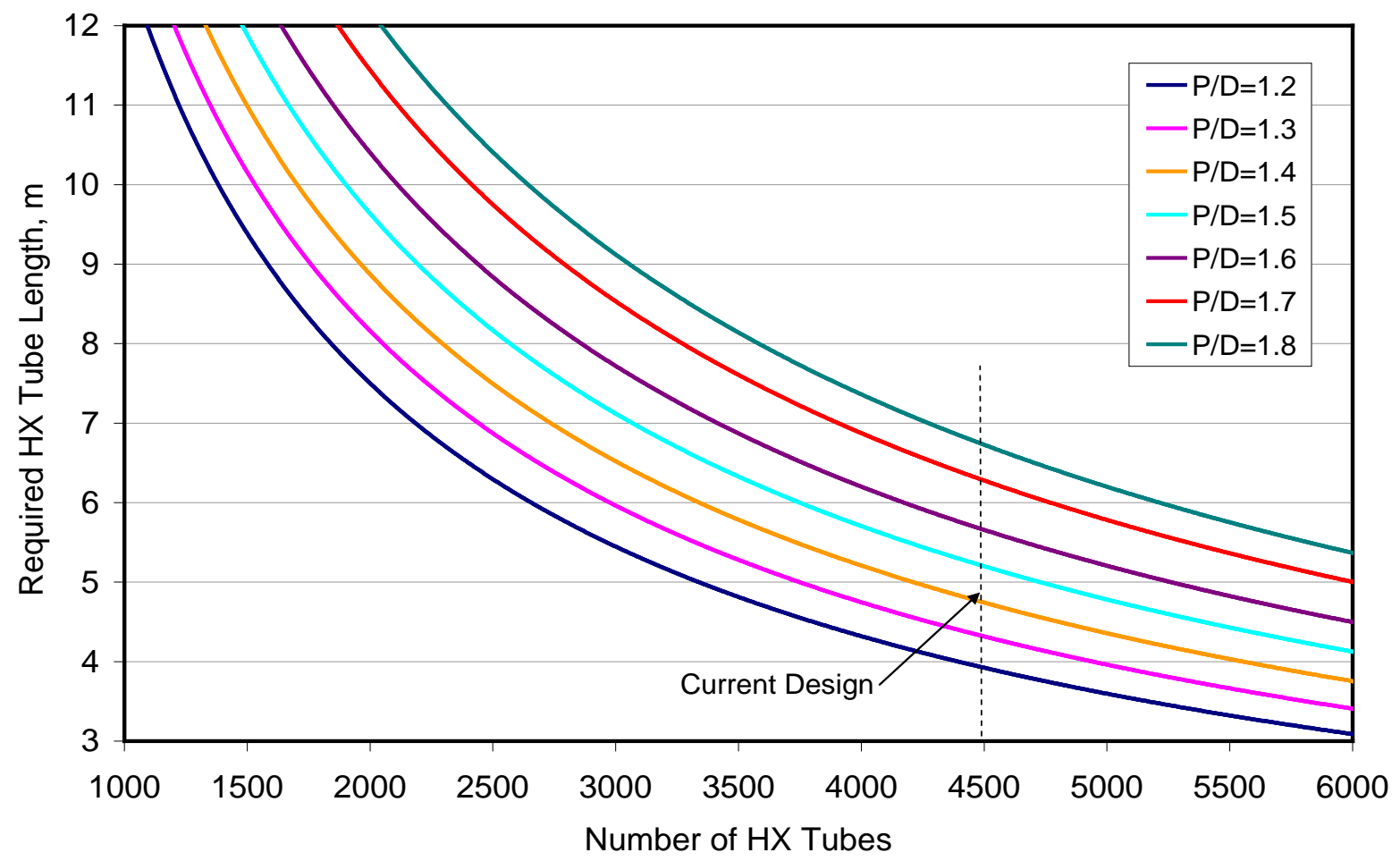

Figure III.8-2 IHX Tube Length vs. No. of Tubes for Various P/D Ratios 


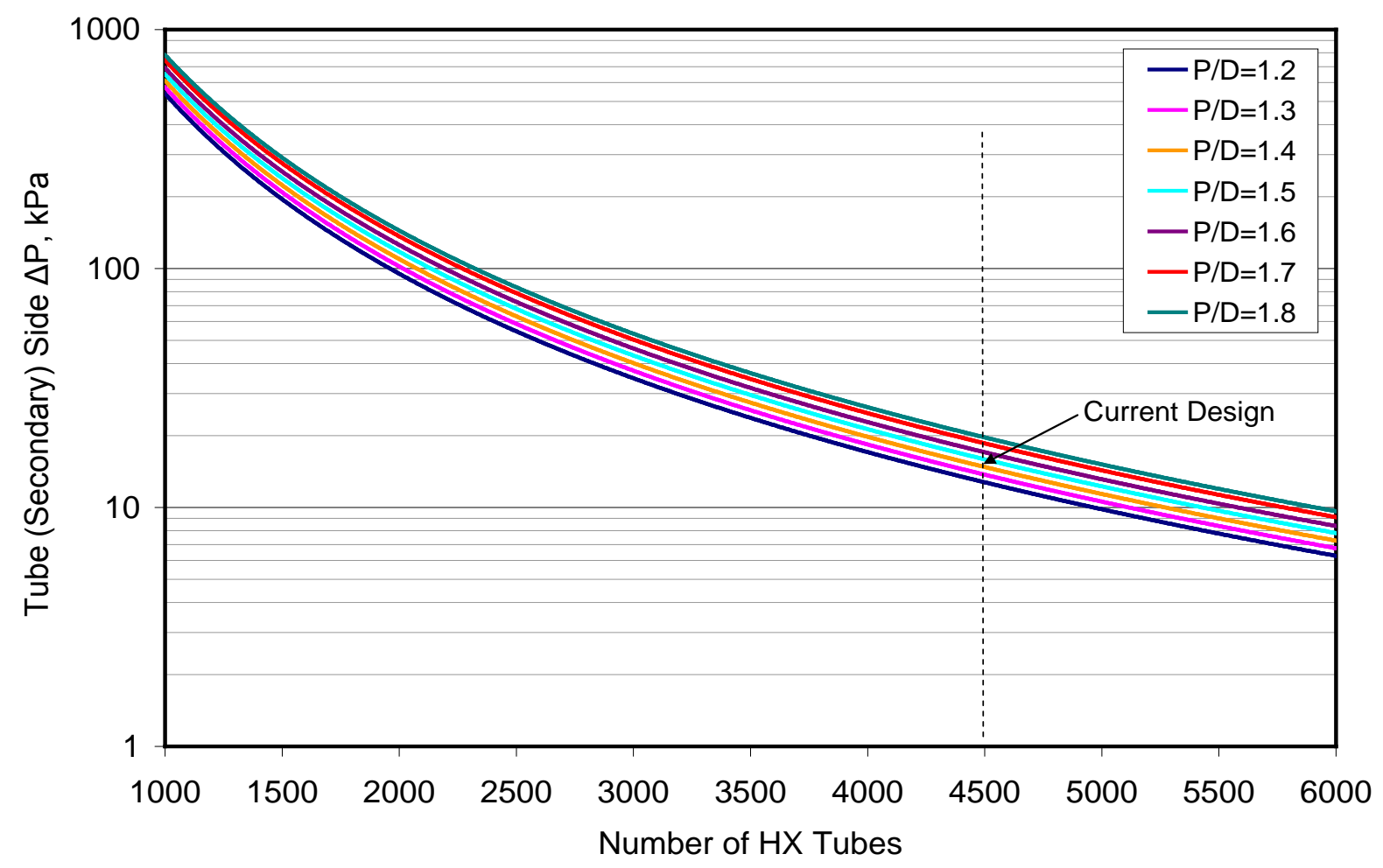

Figure III.8-3 IHX Secondary (Tube) Side $\Delta \mathrm{P}$ vs. No. of Tubes for Various P/D Ratios

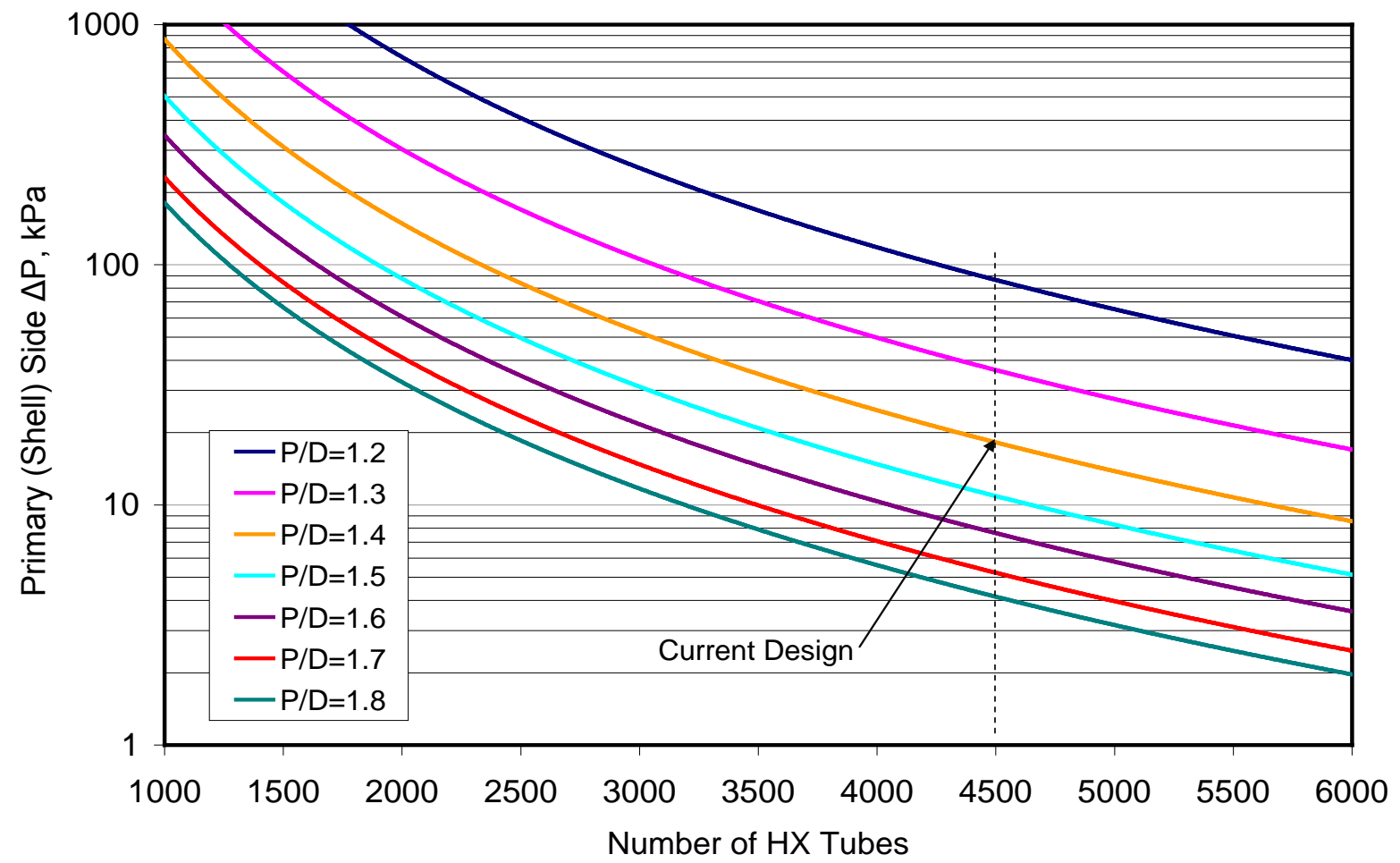

Figure III.8-4 IHX Primary (Shell) Side $\Delta P$ vs. No. of Tubes for Various P/D Ratios 


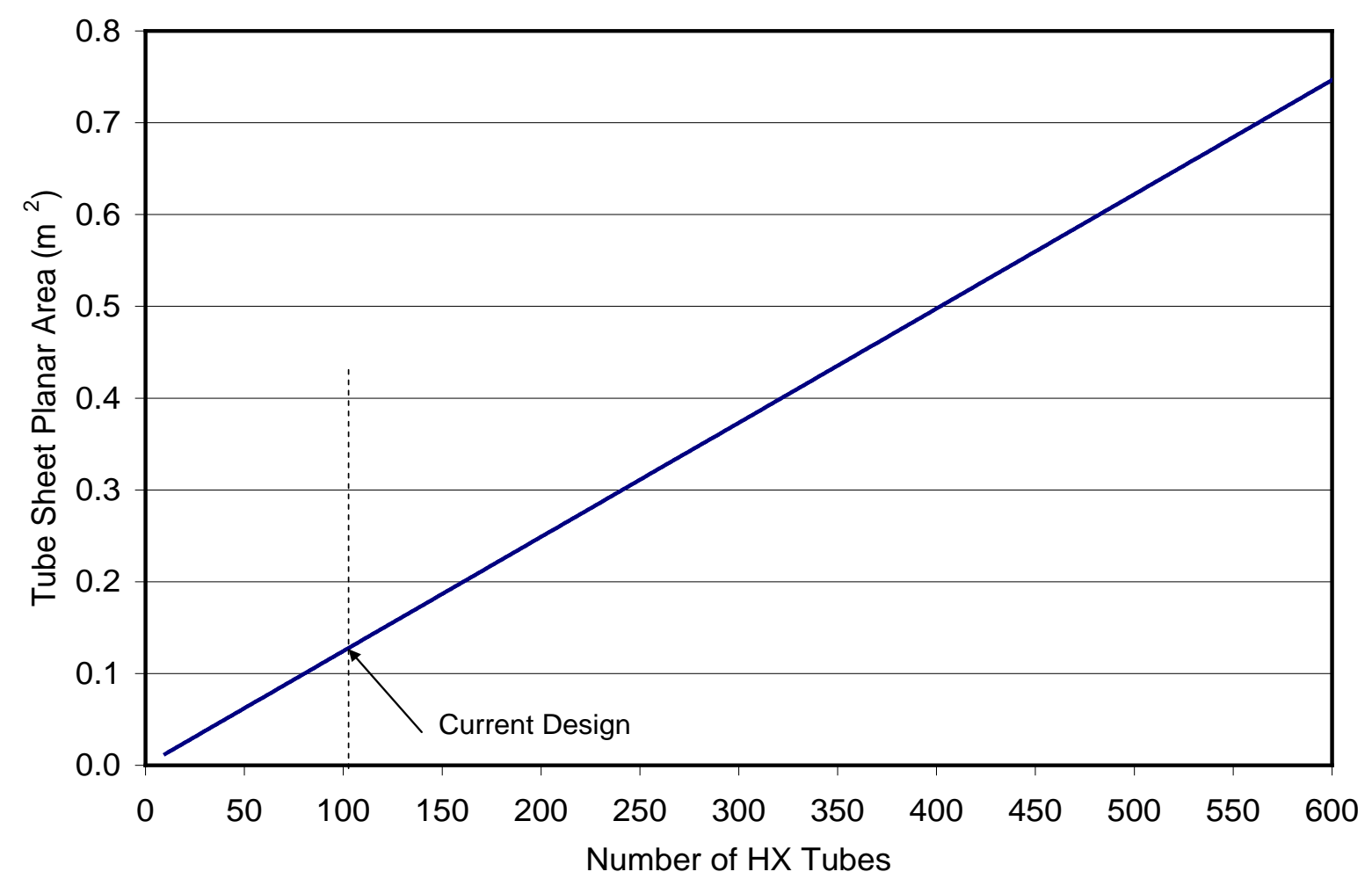

Figure III.8-5 DRACS HX Tube Sheet Planar Area vs. Number of Tubes

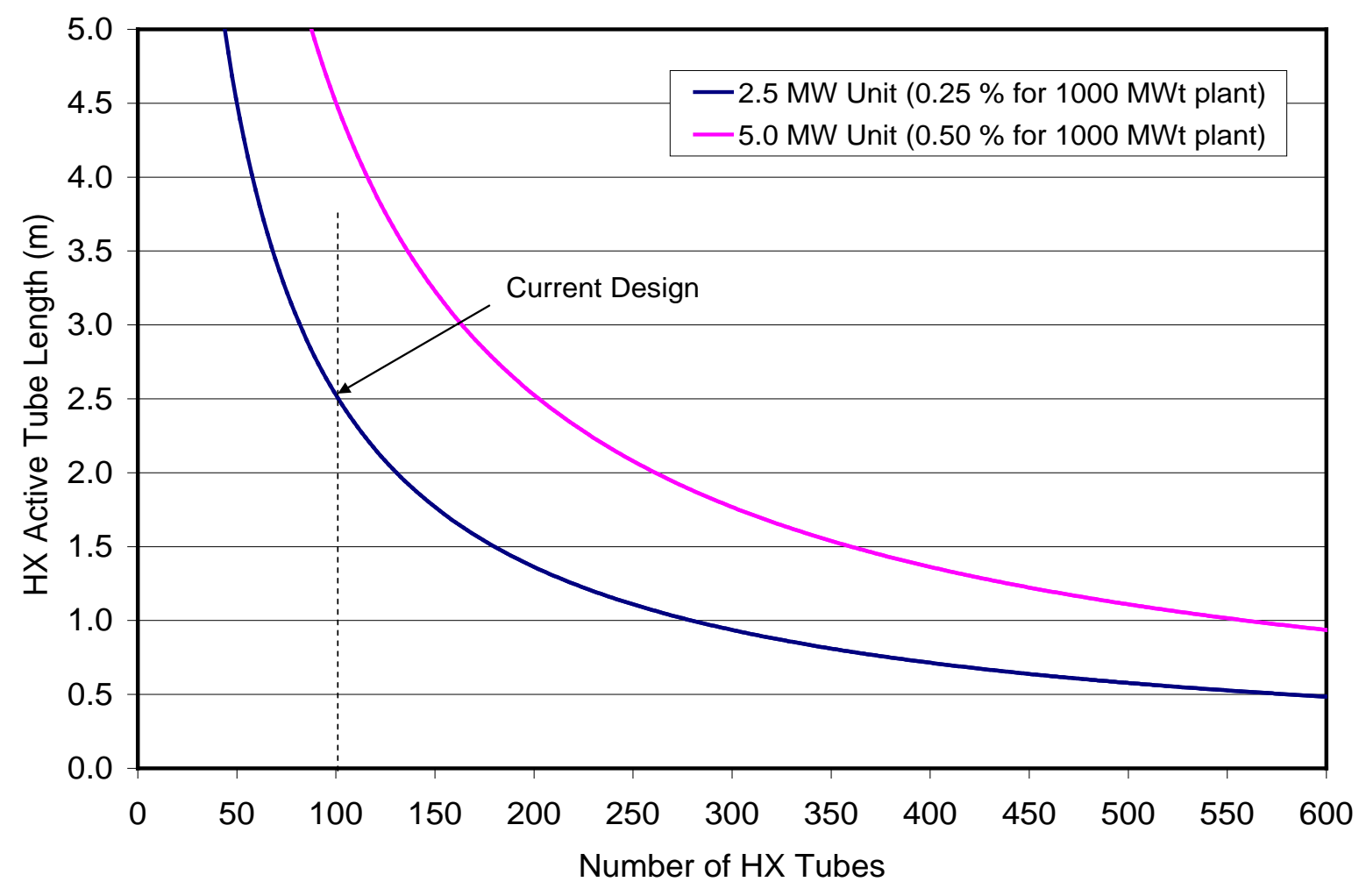

Figure III.8-6 DRACS HX Tube Length vs. Number of Tubes 


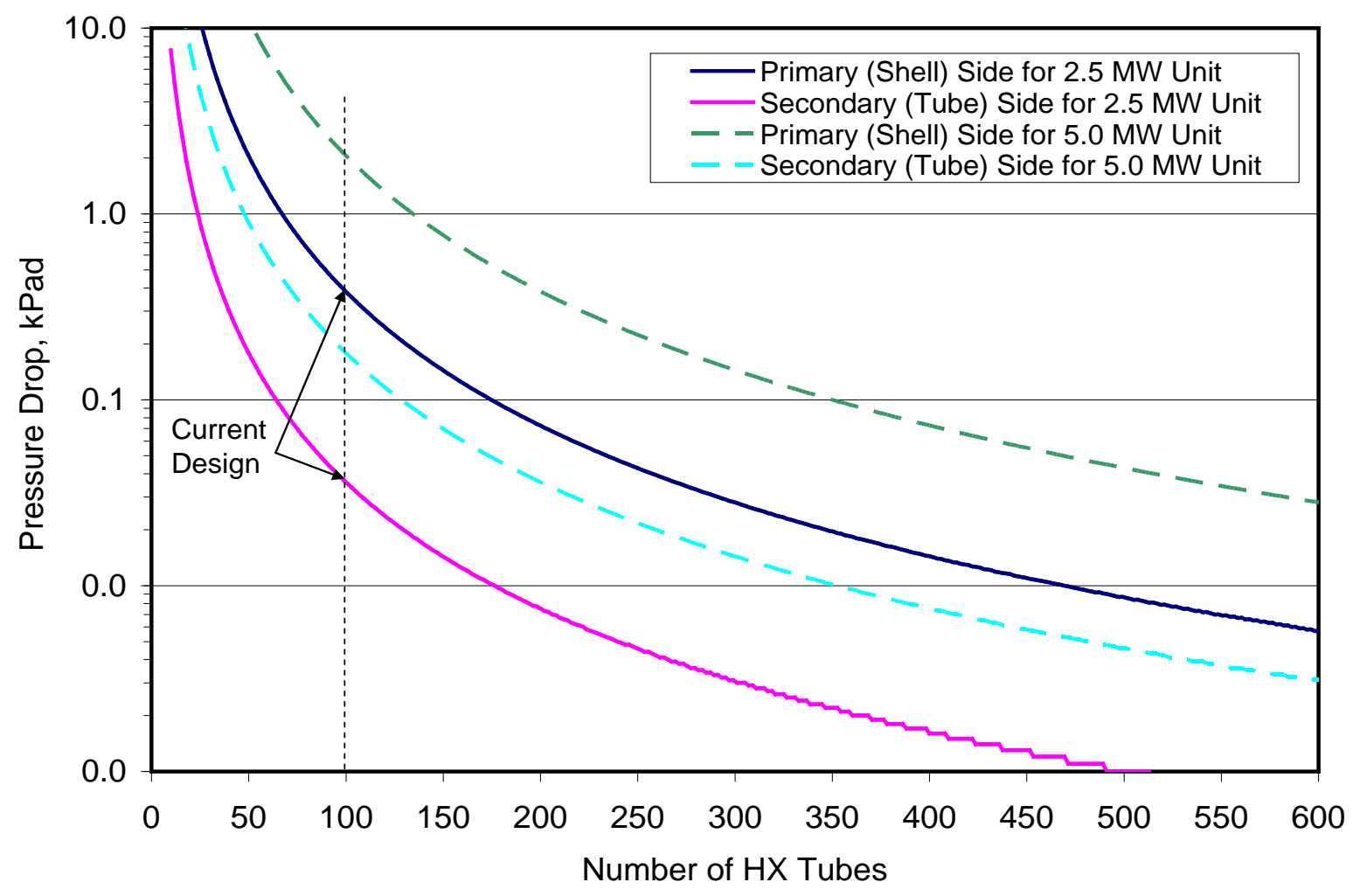

Figure III.8-7 DRACS HX Primary (Shell) - Secondary (Tube) Side $\Delta P$ vs. No. of Tubes 


\section{III.9 Fueling and Refueling Operations}

\section{III.9.1 Estimated Refueling Time}

In the previous ABTR study, refueling time was estimated using information of refueling experience in EBR-II [8].

In EBR-II, the spent and new fuels were transported between the core and the in-vessel basket during the normal refueling operation with reactor shutdown (unrestricted fuel handling). The spent assemblies were then unloaded from the reactor vessel to the fuel examination facility by the inter-building coffin (IBC) while the reactor was operating (restricted fuel handling). The durations of unrestricted and restricted fuel handling procedure in EBR-II are shown in Tables III.9-1 and Table III.9-2, respectively [9]. The unrestricted and restricted fuel handling operations took approximately one hour and ten hours per subassembly, respectively.

Table III.9-1 Restricted fuel handling in EBR-II

\begin{tabular}{|l|l|r|}
\hline \multicolumn{2}{|l|}{ Item } & Time(min) \\
\hline Preparing & & 150 \\
\hline Spent Fuel handling & Core to FHM gripper & 13 \\
& FHM gripper to TA & 6 \\
& TA to basket & 11 \\
\hline New Fuel Handling & Basket to TA & 10 \\
& TA to FHM gripper & 6 \\
& FHM gripper to core & 17 \\
\hline Concluding & & 85 \\
\hline Total & & 298 \\
\hline
\end{tabular}

ABTR and ABR store the new and spent fuels at the positions around the core barrel to reduce the diameter of the reactor vessel since the basket storage method used in EBR-II requires a large space in the reactor vessel. Thus ABTR and ABR must rotate the plug to load and unload fuels to and from the positions around the core barrel with reactor shutdown. Therefore ABTR and ABR refueling might take as long as the restricted fuel handling of ten hours per subassembly.

The number of refueling items depends on the number of core components and their number of batches. ABTR has 24 inner and 30 outer core assemblies with the batch numbers of 12 and 15 respectively. Thus ABTR changes two inner and two outer core subassemblies during every refueling. Including other items such as test fuel assemblies and control rods, the number of exchanging items in a refueling is expected to be seven. The maximum refueling time is estimated to be 70 hours [8].

In the case of the ABR, the refueling time seems to become much longer since the number of exchanged core components is relatively large. The metal and oxide ABR core has 180 driver fuel assemblies with the batch numbers of four and five respectively. Thus the metal and oxide ABR cores change 45 and 36 driver fuel subassemblies respectively per refueling. Additional ABR has 15 primary and four secondary control rods. Assuming the life time of the primary 
control rods are the same of the fuel subassemblies, the number of exchanged core component per refueling is 49 and 40 for metal and oxide fuel cores respectively. Therefore the refueling times are estimated to be 490 and 400 hours respectively (23 and 19 days respectively with 24hours duty and $90 \%$ efficiency).

Table III.9-2 Unrestricted fuel handling in EBR-II

\begin{tabular}{|l|l|r|}
\hline \multicolumn{2}{|l|}{ Item } & Time(min) \\
\hline New Fuel Handling & Load new assembly in temporary coffin & 30 \\
& Transport FUM to temporary coffin & 5 \\
& Load new assembly in FUM and Transport & \\
& FUM to fuel transfer port & 130 \\
& Prepare assembly insertion & 3 \\
& Assembly insertion & 33 \\
& FUM gripper to transfer arm & 7 \\
& Transfer arm to basket & 12 \\
\hline Spent Fuel Handling & Basket to Transfer arm & 10 \\
& Transfer arm to FUM & 35 \\
& Conclude unloading & 5 \\
& Transport FUM to coffin pit & 10 \\
& FUM to IBC & 15 \\
\hline IBC Handling & Transport IBC to FEF & 165 \\
& Return IBC to coffin pit & 135 \\
\hline Total & & 595 \\
\hline
\end{tabular}

The refueling time can be reduced by installing extra IBC's, or additional new fuel handling facilities. An extra IBC can reduce the refueling time dramatically since it allows that a parallel operations of IBC transportation and fuel transportation between the reactor vessel and IBC. The critical path with two IBC's is the IBC transportation time of 300 minutes, while the spent fuel transportation time from the FUM to IBC is 15 minutes. Therefore, the refueling time per subassembly is estimated to be 315 minutes (5 hours 15minutes).

Three or more IBC's can only reduce the refueling time from 315 minutes to 295 minutes, since the critical path with three IBC's becomes the fuel transportation time between the reactor vessel and the IBC, as shown in Table 7.4. Further reduction of refueling time with three IBC's can be achieved by adding a fuel preheating step before the temporary coffin (TC) operation. In the EBR-II refueling scheme, the new fuel was preheated at the FUM, and it took 90 minutes. Thus, preheating an IBC can shorten the refueling time by 90 minutes. In this case, the refueling time per assembly is estimated to be 205 minutes, as shown in Table 7.5. Instead of three IBC's, a combination of two IBC's and a new fuel buffer zone in the reactor building yields the shortest 
refueling time of 205 minutes. This value seems to be relatively longer than that in the conventional sodium cooled reactor with EVS (for example, refueling time in Monju is 90 minutes) [10]. The whole core refueling times are estimated to be 168 and 137 hours respectively (8 and 6 days respectively with 24-hours duty and 90\% efficiency). The whole core refueling times with EVS are roughly estimated to be 74 and 60 hours (4 and 3 days respectively with 24-hours duty and 90\% efficiency). Since the ABR operation cycle is one year, these refueling time differences roughly showed that the availability of ABR could be increased by $1 \%$ with additional EVS. 
Table III.9-3 Refueling Chart with two IBCs

\begin{tabular}{|l|l|r|r|}
\hline Fuel Type & Operation & time(min) & Chart \\
\hline \multirow{5}{*}{ New Fuel } & To TC & 35 \\
& TC to FUM and FUM to FTP & 130 \\
\cline { 2 - 2 } & Insertion to RV & 36 \\
\cline { 2 - 3 } & FUM to Basket & 19 \\
\hline \multirow{3}{*}{ Spent Fuel } & Basket to TA & 10 \\
\cline { 2 - 3 } & TA to FUM & 35 \\
\cline { 2 - 3 } & FUM handling & 15 \\
\cline { 2 - 3 } & FUM to IBC & 15 \\
\hline IBC & IBC transportation & 300 & $280 \mathrm{~min}$ \\
\hline
\end{tabular}

Table III.9-4 Refueling Chart with three IBCs and new fuel preheating before TC

\begin{tabular}{|c|c|c|c|}
\hline Fuel Type & Operation & time(min) & Chart \\
\hline \multirow[t]{4}{*}{ New Fuel } & $\mathrm{TC}$ & 35 & \\
\hline & TC to FUM and FUM to FTP & 40 & \\
\hline & Insertion to RV & 36 & \\
\hline & FUM to Basket & 19 & \\
\hline \multirow[t]{4}{*}{ Spent Fuel } & Basket to TA & 10 & \\
\hline & TA to FUM & 35 & \\
\hline & FUM operation & 15 & \\
\hline & FUM to IBC & 15 & \\
\hline \multicolumn{4}{|c|}{$\begin{array}{l}\text { TC: Temporary Coffin } \\
\text { FHM: Fuel handling Machine } \\
\text { TA: Transfer Arm } \\
\text { FTP: Fuel Transfer Port } \\
\text { IBC: Inter Building Coffin }\end{array}$} \\
\hline
\end{tabular}




\section{III.9.1.1 Handling Outside Reactor Building}

\section{III.9.1.1.1 Spent Fuel Handling}

Since ABR pursuing cost reduction, ABR is not planed to equip any ex-vessel spent fuel storage in the rector building at this design stage. However ABR might need interim spent fuel storage or/and a shipping cask handling facility outside reactor building. Interim spent fuel storage provides long term and lager capacity storage for spent fuels before they are shipped to fuel recycle facilities. If $A B R$ do not equip any interim storage, $A B R$ at least need a shipping cask handling facility with buffer storage, a spent fuel cleaning facility and shipping cask handling facility like the fuel handling cell (FHC) in the CRBR (See Figure III.9-1).

If $\mathrm{ABR}$ do not plan to have interim storage outside reactor building, the buffer storage at the shipping cask must have enough positions for spent fuels from one refueling at least: 45 and 36 for the metal and oxide fuel core respectively.

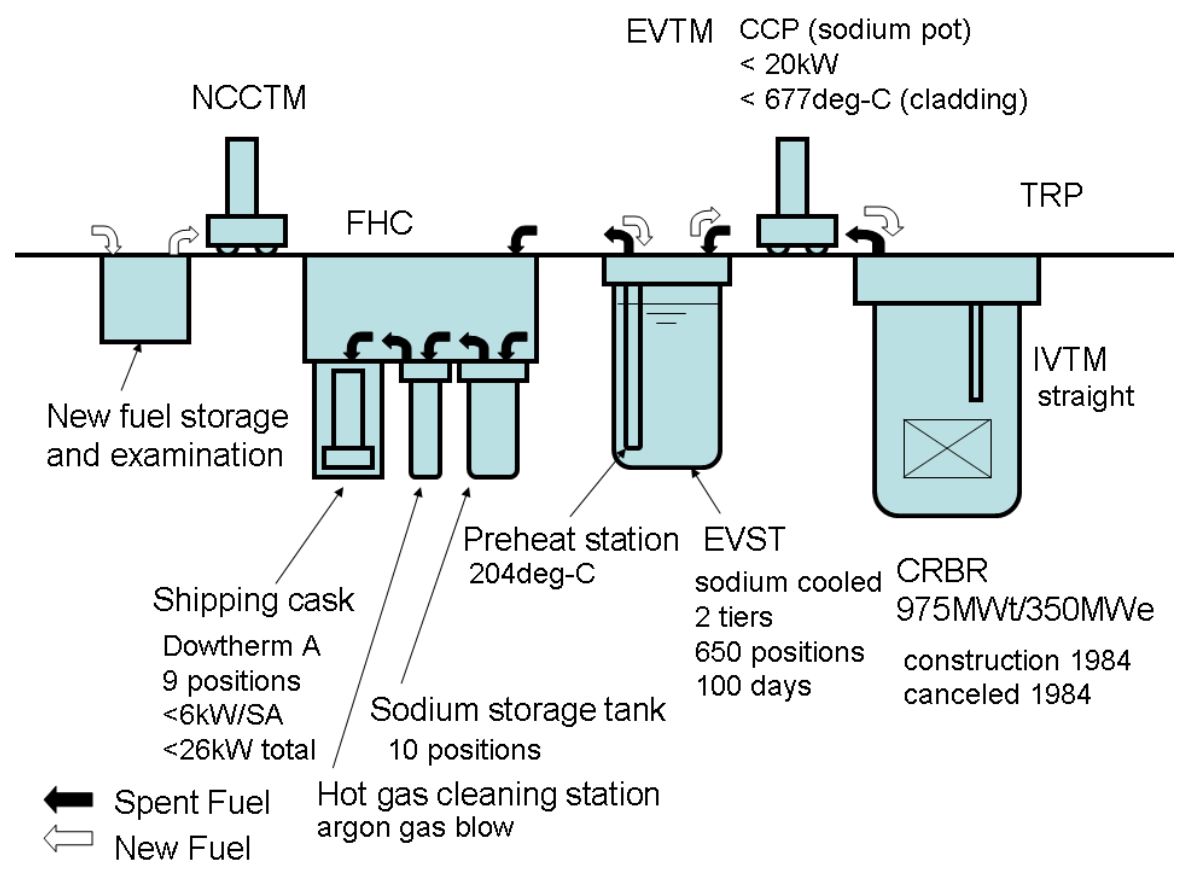

Figure III.9-1 Fuel Handling Sequence in CRBR

Besides it is strongly recommended the commercial fast reactors have large spent fuel storage capacity, since reactor operations must cease when this capacity is met. From the viewpoint of economics, water or dry storage options appear to be the simplest and easiest to implement.

The major concern about interim storage is the integrity of the cladding during long term storage. A considerable amount of experience has been gathered with stainless steel clad fuels stored in water pools. However, long term storage experience with new cladding materials such as HT-9 has not yet been accumulated. Since cladding integrity strongly depends on the cladding material and storage circumstances, new materials require fundamental experiments on corrosion under storage conditions, as well as long term storage demonstration at a prototype 
reactor. In addition, for sodium-bonded metal fuels, water pool storage requires the consideration of sodium-water reactions in the event of cladding failure during interim storage.

Active gas cooling storage could be an option. SNR-300 had an active gas cooling storage behind an EVS. This system had three independent cooling circuits to provide redundancy. The active gas cooling storage has disadvantages at safety and cost. Since loss of cooling accidents could result a large amount of spent fuel failures, redundancy of the cooling system is important. If fact, the SNR-300 gas cooled store had three independent cooling system considering redundancy. The active gas cooling also requires a certain amount of emergency power supply against station blackout. These redundant cooling system and emergency power supply is thought to raise the storage cost.

Passive dry storage could be more preferable than active gas cooling storage when it provides enough cooling capacity. HNPF adopt helium gas thimbles under water pool. Since helium provides good thermal conductivity, the HNPF helium gas thimble was designed to accommodate a $21 \mathrm{~kW}$ decay heat spent fuel.

Passive dry storage techniques developed for LWRs or gas cooled reactors could be also an option. Modular Vault Dry Storage (MVDS) is a one of passive dry storage design and already operated at the Fort Saint Brain gas reactor plant in the US and at a VVER-440 plant in Hungary. The MVDS accommodates spent fuels in thimbles which are cooled with natural circulated air $[11,12]$. A feasibility study is required since fast reactor spent fuels have higher decay heat densities compared to LWRs or gas cooled reactors. Preliminary feasibility of the Modular Vault Dry Storage was shown by thermal analyses on Advanced Burner Test Reactor (ABTR) spent fuels. The analyses showed that the MVDS could accommodate ABTR spent fuels with about $1 \mathrm{~kW}$ decay heat [13]. Fundamental experiments would be beneficial to reveal thermal radiation effects which play an important role in passive dry cooling, since radiation heat transfer is a strong function of the cladding surface condition (or emissivity).

At this design stage, ABR is planed to have in-vessel storage capacity just for one year. Therefore decay heat from a spent fuel is rather high: $5 \mathrm{~kW}$ per subassembly. Available storages for those high decay heat spent fuels are a sodium pool, water pool, active gas cooling storage and helium gas thimbles in a water pool from the view point of cooling.

In the metal fuel core case, water pool storage is not suitable because of sodium-bonding. And helium gas thimbles might cause impact on the reactor vessel caver gas system, since the reactor vessel cover gas must be helium when adopting helium gas thimble storage. Thus active gas cooling storage seems the most likely choice as for the ABR buffer storage.

Another issue on the metal fuel is coolant for shipping casks. In the case of light water reactors, spent fuels are shipped by water filled shipping casks which have a lot of experiences. However water filled casks are not suitable to metal fuels due to sodium bonding. Therefore shipping casks for metal fuels are limited to sodium, dry or synthetic. Sodium or synthetic casks have not accumulated enough experiences. Sodium casks might be involved in safety or license issues. CRBR tried to utilize synthetic filled casks for spent fuel shipping but the project was terminated before construction. Dry cask is supposed to have experiences but the major disadvantage is limitation of cooling capacity. The decay heat limit for a dry cask is roughly 2 $\mathrm{kW}$ at maximum as shown in the previous study [14]. In this case, ABR needs interim storage to reduce decay heat. And shipping cost might increase because the dry cask can accommodate only one or two spent fuels. Anyway, shipping cask decision for the metal fuel design is important because it could cause impact on the fuel handling system. 
In the case of oxide fuel core, water storage and shipping cask are available. Water pool can accommodate high decay heat spent fuels and spent fuels can be cooled by natural circulation in the pool. Beside water pool storage can provide large capacity storage with relatively less impact on construction cost.

\section{III.9.1.1.2 Fresh Fuel Handling}

ABR needs fresh fuel buffer in the reactor building or outside the reactor building. And the refueling time estimation showed that preheating at the fresh fuel handling facility could contribute significant reduction of refueling time. In past and existing sodium cooled reactors, various fresh fuel handling facilities accumulated experiences. For example, Fast Flux Test Facility (FFTF) was equipped with a core component conditioning station (CCCS). In FFTF, new fuels were transported from shipping casks to the CCCS and preheated in the CCCS prior to entry into the sodium system [15]. (See Figure III.9-2)

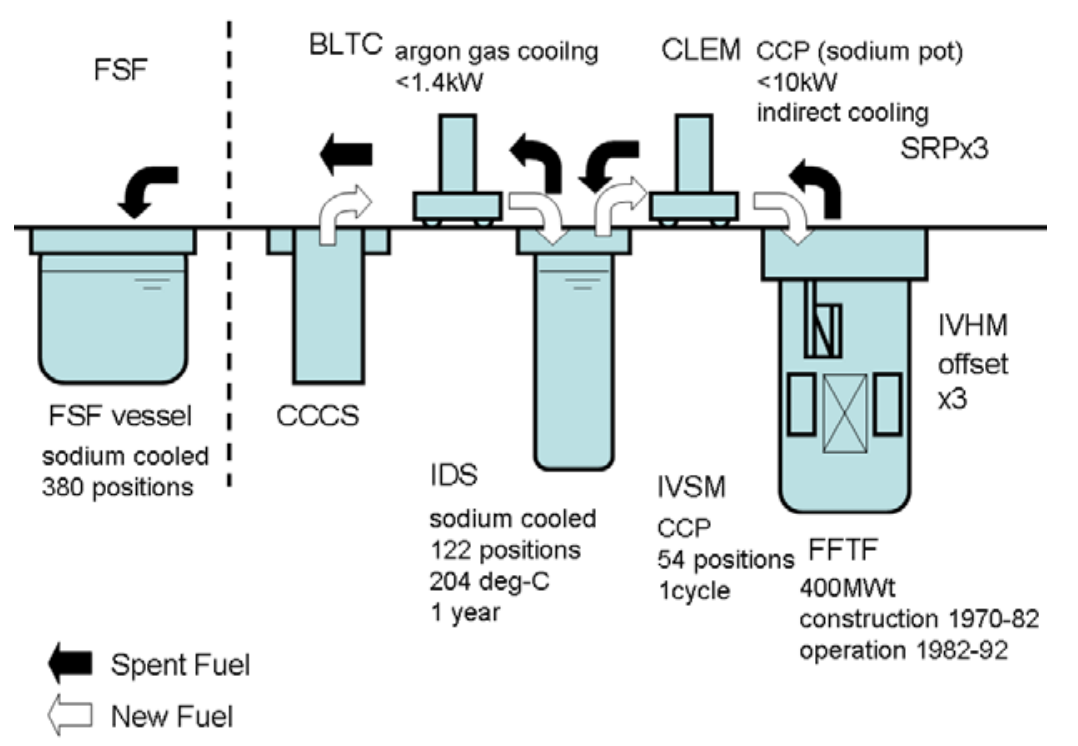

Figure III.9-2 Fuel Handling Sequence in CRBR

In the CRBR case, fresh fuel was received at the new fuel storage and examination location. The fresh core component transfer machine transferred a fresh assembly to the preheating station in the EVST. The fresh fuel was preheated in argon gas to $204{ }^{\circ} \mathrm{C}$ before sodium immersion to prevent thermal stress due to sodium immersion. After the preheating it was put into one of the CCP positions in the EVST prior to transfer to the reactor vessel.

Because ABR is going to utilize transuranic-bearing fuels, it will require special consideration on the fresh fuel storage and shipping. Because of the minor actinides, fresh transuranic-bearing fuels will have much higher decay heat and radiation levels than those of conventional fast reactor fuels containing mixtures of only uranium and plutonium. The decay heat from a fresh fuel subassembly depends on fuel inventory and minor actinide contents. For example, in the cases of Small Modular Fast Reactor (a sodium cooled reactor design with TRUbearing fuel), decay heat level of the TRU-bearing fresh fuel is estimated to be $1.2 \mathrm{~kW}$ for a subassembly with metal fuel of $2.1 \%$ minor actinide per heavy metal [14]. This decay heat level is more than ten times comparing with the conventional uranium-plutonium mixed fuel. The 
decay heat from a fresh fuel subassembly could increase with a larger subassembly and a higher minor actinide containing fuel. Because of these higher decay heat and radiation levels, TRUbearing fresh fuel will most likely require special shipping casks and storage that can accommodate these features without degrading the fuel characterisitics. The TRU shipping cask and storage facilities are expected to be of the dry type to avoid any damage from the interaction of residual moisture on the fuel with sodium coolant. The cladding temperature during shipping and storage must be kept below the cladding creep temperature to avoid creep damage before core loading. In this case, passive decay heat removal from the shipping cask is critical, since any active cooling device is not suitable for an economical shipping cask. Since the only experience with fresh transuranic-bearing fast reactor fuel handling has been at the experimental level, a detailed feasibility study that most likely includes fundamental experiments will be required.

\section{III.9.2 System Design Options}

\section{III.9.2.1 Historical Overview of Fuel Handling System}

In the previous technology gap analysis, fuel handling systems in past and existing sodium cooled reactors were investigated. The gap analysis concluded that a standardized fuel handling system for a commercial fast reactor is yet to be established. Major design goals for the fuel handling system of a commercial plant are low construction cost and short outages due to refueling operations. One of most expensive components in the fuel handling system is the sodium-cooled ex-vessel storage (EVS) system. ABR do not plan to have any EVS in the reactor vessel enhancing cost reduction. However removing EVS, ABR might be required to have interim or buffer storage outside the reactor building. And refueling without EVS generally seems to result longer reactor outage. Thus the system design survey study on the ABR fuel handling system is recommended. From EVS this point of view, fuel handling systems for liquid metal cooled reactors can be categorized by the primary sodium storage and ex-vessel transportation methods, as shown in Table III.9-5.

Table III.9-5 Four Categories of Sodium Cooled Reactor Fuel Handling Systems

\begin{tabular}{|c|c|c|c|}
\hline Category & $\begin{array}{c}\text { Primary } \\
\text { Sodium Storage }\end{array}$ & $\begin{array}{c}\text { Ex-Vessel } \\
\text { Transportation }\end{array}$ & Experience \\
\hline A & no sodium & gas & EBR-I, SRE, HNPF, DFR, Rapsodie \\
\cline { 3 - 4 } & storage & pot & EBR-II \\
\hline B & IVS & gas & Joyo \\
\cline { 3 - 4 } & & pot & - \\
\hline C & IVS+EVS & gas & Fermi, FFTF, PFR, Phenix \\
\cline { 3 - 4 } & & pot & SEFOR, KNK \\
\hline D & EVS & gas & CRBR, Superphenix, SNR-300, Monju \\
\cline { 3 - 4 } & & pot &
\end{tabular}

In early small experimental reactors, sodium-cooled storage was not required since the fuel element decay heat levels were not high (e.g, DFR and Rapsodie fuels elements had maximum decay heat levels of 2.5 and $2 \mathrm{~kW}$, respectively). For these reactors, spent fuel was transferred to interim storage with gas cooling. The EBR-I, SRE, HNPF, DFR and Rapsodie fuel handling 
systems belong to this category (Category A). The major advantage of this category is the low construction cost by omitting the EVS system. On the other hand, the major disadvantage is the long reactor outage due to refueling operations, and the high decay heat levels during ex-vessel transportation. In Category A, the fuel handling system must conduct fresh fuel preheating, fresh fuel loading, spent fuel unloading, spent fuel cleaning, and spent fuel transfer to interim storage during reactor shutdown. Since early experimental reactors were generally not concerned with outage time, they could adopt this type of fuel handling system. However, for commercial reactors, this approach is not viable due to the long outage time associated with refueling operations. Moreover, the subassembly decay heat level will be as much as an order of magnitude higher (e.g., peak subassembly heat levels for CRBR and Superphenix at refueling are 20 and $35 \mathrm{~kW}$, respectively). Forced gas cooling for these types of assemblies would be very difficult due to the sodium vapor generation issues. Finally, redundancy and a high level of reliability would be needed for a gas cooled system, since loss of cooling would rapidly lead to unacceptably high fuel cladding temperatures and possible cladding breach for these high power subassemblies.

EBR-II and Joyo both adopted in-vessel storage (IVS) without EVS (Category B). The major advantage of this approach is the same as Category A; i.e., reduced construction cost when EVS is omitted. The principal difference from Category A is the reduced decay heat of the subassemblies during ex-vessel transfers. The EBR-II IVS could receive or send fuel subassemblies during reactor operation, because the storage rack was placed outside the core barrel, and had its own rotation mechanism. This system significantly reduced the duration of refueling outages, since only in-vessel fuel transfers between the core and IVS had to be conducted when the reactor was shutdown. There two major disadvantage of this type of IVS: the enlarged diameter of the reactor vessel due to the space for the storage rack and maintenance problems introduced by active parts inside the reactor vessel.

The Joyo IVS is placed around the core barrel and can not be accessed by the ex-vessel transfer machine during reactor operations, because access to a target subassembly requires motion of the rotatable plug. However, the design of the Joyo IVS has less impact on the reactor vessel structure. Joyo is the only reactor that uses the sodium pot ex-vessel transfer technique without EVS. Joyo has an argon atmosphere transfer rotor for pot handling operations, and utilizes forced gas cooling for fuel movements between the transfer rotor and the cleaning machine.

Although IVS is an effective means of allowing the decay heat level of spent fuel to decline prior to transfer, space for this storage may be limited. In addition, spent fuel storage requirements for prototype or commercial reactors are generally greater than that of small test reactors, since there are more assemblies with higher burnup that require a longer decay time prior to transfer. Fermi, FFTF, PFR and Phenix utilized both IVS and EVS (Category C). Conversely, CRBR, Superphenix, SNR-300 and Monju utilize only EVS (Category D). Aside from the normal task of removing decay heat, EVS offers several operational advantages; namely, EVS effectively provides a buffer stage which allows fresh fuel preheating and spent fuel cleaning operations to be performed while the reactor is operating. Most designs elect to use the sodium transfer pot technique between the reactor vessel and EVS. EVS also functions as a sodium pot handling station. Finally, sodium pots make passive cooling possible during exvessel transfers since the interstitial sodium provides a highly effective heat transfer medium, as well as heat sink. Finally, the elimination of forced gas circulation reduces maintenance problems caused by sodium vapor condensation and buildup. 
To select a suitable fuel handling system for ABR, a tradeoff study is recommended. The competition should consider construction costs and outage duration as two of the key criteria for judging the relative merits of the above four categories. Additional design and experimental technology development may be required to fully decide the issue.

\section{III.9.2.2 ABR Fuel Handling Design Options}

Category A systems of course can achieve low construction cost without EVS neither IVS. However in the ABR case, decay heat of spent fuels still remains in a level of $15 \mathrm{~kW}$ per subassembly on 30 days after reactor shutdown. Gas cooling transportation of such a high decay heat subassembly must consider about safety problem, since loss of cooling could cause cladding failure in a several minutes. Especially in the case of metal fuel, water pool storage which can provide high cooling capacity is not recommended for interim or buffer storage because there might be sodium-water reaction and failure propagation.

Category B to which the present ABR fuel handling system design belongs can also eliminate EVS while allowing the decay heat to diminish with IVS. The reduction of decay heat depends on storage duration in IVS. Capacity of IVS is relatively limited comparing with EVS, since increment of IVS capacity causes impacts on reactor vessel designs. The ABR IVS was estimated to provide one year storage and spent fuels seem to still have approximately $5 \mathrm{~kW}$ decay heat after IVS. This level of decay heat requires forced gas circulation cooling during exvessel transportation. Since the decay heat level is relatively higher than those experienced in gas cooling ex-vessel transportation at past sodium cooled reactors, additional technology development is required on reliable forced gas cooling systems.

Besides ABR is recommended to have interim or buffer storage outside the reactor building, since it requires a long time to prepare spent fuel shipping and spent fuels need accommodation during the shipping preparation. In the case of the metal fuel core, additional feasibility study and technology development is required on reliable forced gas cooling storage. Forced gas cooling storage is required to have at least three independent cooling system considering redundancy. The active gas cooling also requires a certain amount of emergency power supply against station blackout. These redundant cooling system and emergency power supply is thought to cause impact on the storage cost. On the other hand, the case of the oxide fuel core, water pool storage which were widely used as interim storage in past sodium cooled reactors is available.

Categories A and B also has an issue on refueling time. In this study, ABR refueling time was estimated 205 minutes at least. This value is relatively longer than those of Categories $\mathrm{C}$ and D (for example, Monju refueling time is estimated 90 minutes). Since long refueling time causes impact on reactor outage time and plant availability, shortening of refueling time is important in commercial reactors from the economical point of view.

Categories C and D have already accumulated a satisfactory experience base for high decay heat spent fuels. Adopting large capacity EVS, under sodium storage duration could be increased and decay heat could be smaller than Category A and B. For example, if ABR has IVS and EVS with spent fuel positions for five batches (IVS: 70 positions and EVS: 155 positions), duration of under sodium storage becomes five years. In the metal fuel core case, decay heat is estimated about $1.2 \mathrm{~kW}$ per subassembly after five years storage. The previous ABTR study showed that this level of decay heat might be accommodated in passive gas cooling storage. Thus there is a possibility that ABR could adopt passive gas cooling storage. 
Available design options for the metal fuel ABR fuel handling system are summarized at Table III.9-6 and III.9-7 for the metal fuel core and oxide fuel core respectively. The major differences between metal and oxide fuel systems are cooling methods for buffer/interim storage and shipping casks. Neither water pool storage nor water filled shipping cask could be available for metal fuels, since metal fuels have sodium bonding. If one dared to choice water pool storage or water filled shipping casks for metal fuels, safety experiments would be required to confirm consequences of sodium-water reactions in cases of metal fuel cladding failures. Especially, the shipping cask will require enough toughness against inside sodium-water reaction.

As previously mentioned, power reactors like ABR are recommended to have interim storage which has large capacity. Without interim storage, troubles on spent fuel shipping or fuel recycling could cause significant reactor outage. However, at this design stage, ABR does not plant to have interim storage considering low construction cost. The reference design has 70 positions at the IVS and would be required at least 45 positions at the buffer storage outside reactor building. Fuel handling time is longer (205 minutes) than that of a system with EVS even if the ABR fuel handling system utilizes three inter-building casks. Since decay heat is still at the level of $5 \mathrm{~kW}$ per subassembly on shipping, dry cask is not available. Development of a shipping cask which can accommodate high decay heat spent fuels is required.

Option M1 is another design without interim storage. Disadvantage of this system is EVS construction cost. Major advantages of the system with EVS are reduced refueling time and elimination of other buffer storage. The previous refueling time estimation showed that fuel handling system with EVS could increase reactor availability by $1 \%$. The rough cost competition between the reference and option M1 would be as follows:

\begin{tabular}{|l|l|}
\hline reference & option M1 \\
\hline IVS (70 positions) & EVS (45 positions) \\
\hline active gas cooling storage (45 positions) & sodium pot cask + active gas cooling cask \\
\hline active gas cooling cask x 3 & - \\
\hline $\begin{array}{l}\text { low availability penalty } \\
\text { (1\% of a whole plant construction) }\end{array}$ & - \\
\hline
\end{tabular}

Option M2 and M3 show fuel handling concepts which have interim storage. The spent fuels are accommodated in IVS and EVS while their decay heat goes down to a level which can be acceptable to passive gas cooling storage. The major advantage of these design options is that the reactor plant could be independently operated and would not be suffered from reactor outage caused by shipping or recycling problems. After interim storage, dry shipping casks are available since decay heat from spent fuels is in a level of $1 \mathrm{~kW}$.

In the case of the oxide fuel system, the reference design can adopt water pool buffer storage and water filled shipping casks. Since the water pool storage can provide high cooling capacity, more aggressive concept without IVS or EVS is possible (option O1). This aggressive design would require development of an active gas cooling cask for a $21 \mathrm{~kW}$ decay heat spent fuel. 


\begin{tabular}{|l|l|l|}
\hline reference & option O1 & option O2 \\
\hline IVS (70 positions) & - & EVS (45 positions) \\
\hline water pool (45 positions) & water pool (45 positions) & sodium pot cask \\
\hline active gas cooling cask x 3 & active gas cooling cask x 3 & active gas cooling cask \\
\hline $\begin{array}{l}\text { low availability penalty } \\
\text { (1\% of a whole plant) }\end{array}$ & $\begin{array}{l}\text { low availability penalty } \\
\text { (1\% of a whole plant) }\end{array}$ & - \\
\hline
\end{tabular}

Options O3 to O5 are concepts with interim storage. Since water pool storage can accommodate relatively higher decay heat spent fuels, IVS and EVS capacity is not restricted like metal fuel cases. 
Table III.9-6 Design options for the metal fuel ABR fuel handling system

\begin{tabular}{|c|c|c|c|c|c|c|c|}
\hline & IVS & transportation 1 & EVS & transportation 2 & $\begin{array}{l}\text { buffer/interim } \\
\text { storage }\end{array}$ & \begin{tabular}{|l} 
shipping \\
options
\end{tabular} & \begin{tabular}{|l}
$\begin{array}{l}\text { refueling } \\
\text { time }\end{array}$ \\
\end{tabular} \\
\hline Reference & $\begin{array}{l}45 \text { positions } \\
\text { (present: } 70 \\
\text { positions) }\end{array}$ & $\begin{array}{l}\text { active gas cooling } \\
\text { cask x } 3 \\
(5 \mathrm{~kW} / \mathrm{SA})\end{array}$ & N/A & N/A & $\begin{array}{l}\text { active gas cooling } \\
45 \text { positions } \\
(5 \mathrm{~kW} / \mathrm{SA})\end{array}$ & \begin{tabular}{|l} 
sodium \\
synthetic \\
(5kW/SA) \\
\end{tabular} & 205 minutes \\
\hline Option M1 & N/A & $\begin{array}{l}\text { sodium pot } \\
(20 \mathrm{~kW} / \mathrm{SA})\end{array}$ & 45 positions & $\begin{array}{l}\text { active gas cooling } \\
\text { (5 kW/SA) }\end{array}$ & N/A & \begin{tabular}{|l} 
sodium \\
synthetic \\
(5kW/SA) \\
\end{tabular} & 90 minutes \\
\hline Option M2 & 70 positions & $\begin{array}{l}\text { sodium pot } \\
(5 \mathrm{~kW} / \mathrm{SA})\end{array}$ & 155 positions & $\begin{array}{l}\text { active gas cooling } \\
(1.2 \mathrm{~kW} / \mathrm{SA})\end{array}$ & $\begin{array}{l}\text { passive gas } \\
\text { cooling } \\
\text { interim storage } \\
(1.2 \mathrm{~kW} / \mathrm{SA})\end{array}$ & $\begin{array}{l}\text { sodium } \\
\text { synthetic } \\
\text { dry } \\
(1 \mathrm{~kW} / \mathrm{SA})\end{array}$ & 90 minutes \\
\hline Option M3 & N/A & $\begin{array}{l}\text { sodium pot } \\
(20 \mathrm{~kW} / \mathrm{SA})\end{array}$ & 225 positions & $\begin{array}{l}\text { active gas cooling } \\
(1.2 \mathrm{~kW} / \mathrm{SA})\end{array}$ & $\begin{array}{l}\text { passive gas } \\
\text { cooling } \\
1.2 \mathrm{~kW} / \mathrm{SA} \\
\text { interim storage }\end{array}$ & $\begin{array}{l}\text { sodium } \\
\text { synthetic } \\
\text { dry } \\
(1 \mathrm{~kW} / \mathrm{SA}) \\
\end{array}$ & 90 minutes \\
\hline
\end{tabular}


Table III.9-7 Design options for the oxide fuel ABR fuel handling system

\begin{tabular}{|c|c|c|c|c|c|c|c|}
\hline & IVS & transportation 1 & EVS & transportation 2 & $\begin{array}{l}\text { buffer/interim } \\
\text { storage }\end{array}$ & $\begin{array}{l}\text { shipping } \\
\text { options }\end{array}$ & $\begin{array}{l}\text { refueling } \\
\text { time }\end{array}$ \\
\hline Reference & $\begin{array}{l}36 \text { positions } \\
\text { (present: } 70 \\
\text { positions) }\end{array}$ & $\begin{array}{l}\text { active gas cooling } \\
\text { cask x } 3 \\
(5 \mathrm{~kW} / \mathrm{SA})\end{array}$ & N/A & N/A & $\begin{array}{l}\text { water pool } \\
36 \text { positions } \\
(5 \mathrm{~kW})\end{array}$ & $\begin{array}{l}\text { water } \\
(5 \mathrm{~kW} / \mathrm{SA})\end{array}$ & 205 minutes \\
\hline Option O1 & N/A & $\begin{array}{l}\text { active gas cooling } \\
\text { cask x } 3 \\
(21 \mathrm{~kW} / \mathrm{SA})\end{array}$ & NA & N/A & $\begin{array}{l}\text { water pool } \\
36 \text { positions } \\
(21 \mathrm{~kW})\end{array}$ & $\begin{array}{l}\text { water } \\
(5 \mathrm{~kW} / \mathrm{SA})\end{array}$ & 205 minutes \\
\hline Option O2 & NA & $\begin{array}{l}\text { sodium pot } \\
(21 \mathrm{~kW} / \mathrm{SA})\end{array}$ & 36 positions & $\begin{array}{l}\text { active gas cooling } \\
\text { (5 kW/SA) }\end{array}$ & N/A & $\begin{array}{l}\text { water } \\
(5 \mathrm{~kW} / \mathrm{SA})\end{array}$ & 90 minutes \\
\hline Option O3 & $\begin{array}{l}36 \text { positions } \\
\text { (present: } 70 \\
\text { positions) }\end{array}$ & $\begin{array}{l}\text { active gas cooling } \\
\text { cask x } 3 \\
(5 \mathrm{~kW} / \mathrm{SA})\end{array}$ & N/A & N/A & $\begin{array}{l}\text { water pool } \\
\text { interim storage } \\
(5 \mathrm{~kW})\end{array}$ & $\begin{array}{l}\text { water } \\
(1 \mathrm{~kW} / \mathrm{SA})\end{array}$ & 205 minutes \\
\hline Option O4 & NA & $\begin{array}{l}\text { sodium pot } \\
(21 \mathrm{~kW} / \mathrm{SA})\end{array}$ & 36 positions & $\begin{array}{l}\text { active gas cooling } \\
(5 \mathrm{~kW} / \mathrm{SA})\end{array}$ & $\begin{array}{l}\text { water pool } \\
\text { interim storage } \\
(5 \mathrm{~kW})\end{array}$ & $\begin{array}{l}\text { water } \\
(1 \mathrm{~kW} / \mathrm{SA})\end{array}$ & 90 minutes \\
\hline Option O5 & N/A & $\begin{array}{l}\text { sodium pot } \\
(21 \mathrm{~kW} / \mathrm{SA})\end{array}$ & 180 positions & $\begin{array}{l}\text { active gas cooling } \\
(1.7 \mathrm{~kW} / \mathrm{SA})\end{array}$ & $\begin{array}{l}\text { water pool } \\
\text { interim storage } \\
(1.7 \mathrm{~kW})\end{array}$ & $\begin{array}{l}\text { water } \\
(1 \mathrm{~kW} / \mathrm{SA})\end{array}$ & 90 minutes \\
\hline
\end{tabular}




\section{References}

1. K.P. Johnson, "Experience with Fermi Fuel Handling”, Proceedings of 23rd Conference on Remote Systems Technology, p. 390, November, 1975.

2. L.E. Glasgow, "Sodium Reactor Experiment Operating Experience”, Transactions of ANS, vol. 4, No. 2, p202, 1961.

3. D.M. Romrell, D.M. Art, R.D. Redekopp and J.B. Waldo, J.L. Marshall, "Fast Flux Test Facility Fuel Handling Experience November 1979 to August 1988”, Nuclear Technology, Vol. 86, p.264, 1989.

4. E. Hutter, "Experience with EBR-II Fuel Handling”, Proceedings of $23^{\text {rd }}$ Conf. on Remote System Technology, p379, 1975.

5. R.C. Marison, M.R. Neuman, V.J. Retkauskas, F.M. Shoemaker, L.R. Tollbom and L.A. Trego, "Fast Flux Test Facility Major Component Testing Experience and Plant Construction Experience”, Proceedings of 1976 ASME-ANS International Conference -Advanced Nuclear Energy Systems-, Pittsburg, PA, USA, March, 1976.

6. D.W.M. Hornberger and C.L. Boyd, "FFTF Fuel Handling System Features and Experience”, Proceedings of 23rd Conf. on Remote Systems Technology, p. 397, San Francisco, CA, USA, Nov, 1975.

7. P.H. Delvis, W. Rodwel, "Mechanisms in Sodium”, Proceedings of a Symposium on Progress in Sodium-Cooled Fast Reactor Engineering, Monaco, March, 1970.

8. Y.I. Chang, P.J. Finck and C. Grandy, “Advanced Burner Test Reactor Preconceptual Design Report”, ANL-ABR-1 (ANL-AFCI-173), 2006.

9. EBR-II design descriptions, Volume II, Chapter 5, 1971.

10. Y. Chikazawa, S. Usui, M. Konomura, H. Ikeda, "Design Study on a Fuel Handling System in a Sodium Cooled Reactor”, JAEA-Research 2006-032, 2006.

11. A.J. Holt, “A modular vault dry storage facility for Fort St Vrain”, Nuclear Engineering International, vol. 35, p105, 1990.

12. D. Deacon, “The Developed Technology of Modular Vault Dry Storage”, Nuclear Engineering International, vol. 29, p. 24, 1984.

13. Y. Chikazawa and C. Grandy, "Thermal Analysis on Passive Dry Fuel Storage for ABTR”, Transaction of ANS, No. vol. 96, p. 167, July, 2007

14. Y. Chikazawa and C. Grandy, "Thermal Analysis on Shipping Cask for LMFR Fresh Trans-Uranium Fuel Transportation”, Proceeding of ICAPP 2007, No. 7132, Nice, France, June, 2007.

15. R.O. Haroldsen, F.D. McGinnis and G.K. Whitham, "Operational and Maintenance of the EBR-I Liquid Metal-Cooled Reactor”, Transactions of ANS, vol. 6, supplement, p. 2, 1963. 


\section{III.10 Primary Pump Design Analyses}

\section{III.10.1 Mechanical Pump Design}

A schematic of a mechanical pump, indicating major essential components is shown in Figure III.10-1. Since the design called "Fermi" type was considered superior to that called "Hallam" (see Figure III.10-2), a Fermi type design was used in this study. The mechanical pump shown in Figure III.10-1 is a Fermi type. To estimate the size of this type of mechanical pump, Byron Jackson has developed a series of nomographs [1]. A size estimation of a mechanical pump for ABR was done using the nomographs shown in Figures III.10-4 through III.10-9. In these nomographs, sodium flow rate, head pressure, and operating temperature are needed as inputs (see Figure III.10-3).

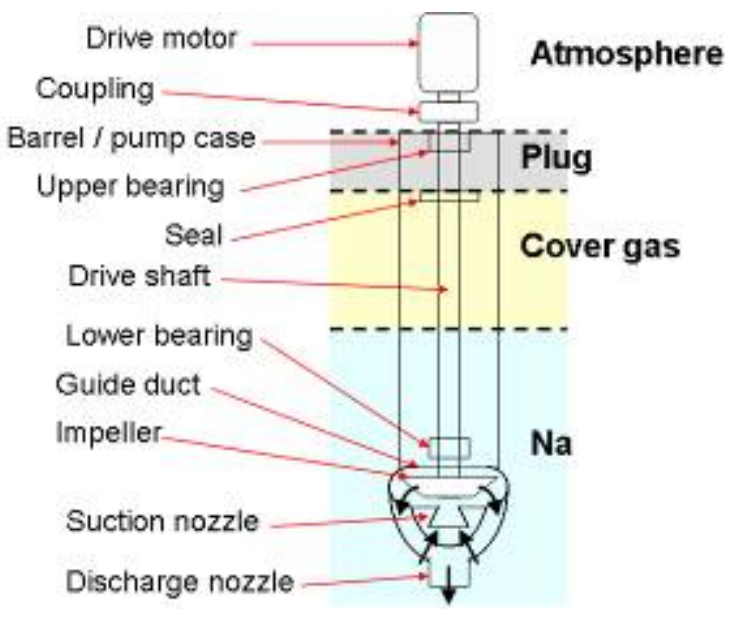

\section{Figure III.10-1 Schematic of mechanical pump}

In the first nomograph, the specific speed and rotation speed are determined. In the second nomograph, the required power is determined. Then, in the third nomograph, the shaft diameter is determined. In the fourth nomograph, the pump case diameter, which is related to the impeller diameter, is determined. In the fifth nomograph the size of the pump outer barrel is determined, however, for the pool type configuration, the outer barrel is not used and the pump casing is suspended directly in the pool of Na. Finally in the sixth nomograph, the suction and discharge nozzle diameters are determined. A summary of those parameters obtained as well as input values are presented in Figure III.10-10. This procedure estimates only the diameter of the pump. The length of the pump can be estimated from other considerations, which are that the submergence depth needs to be greater than 0.9-1.2 $\mathrm{m}$ to prevent vortexing [2]. 


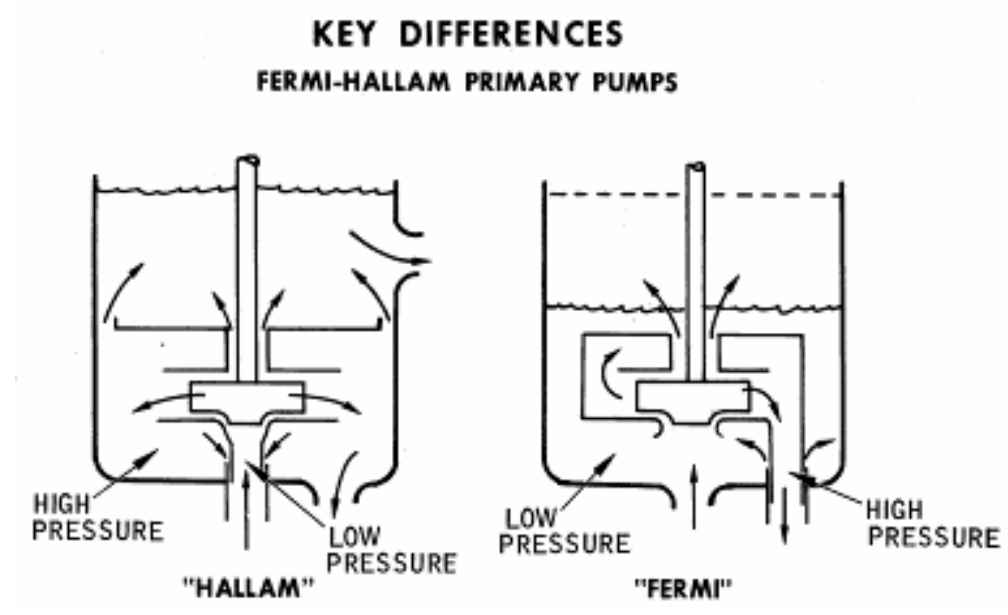

Figure III.10-2 Key differences between Fermi and Hallam designs

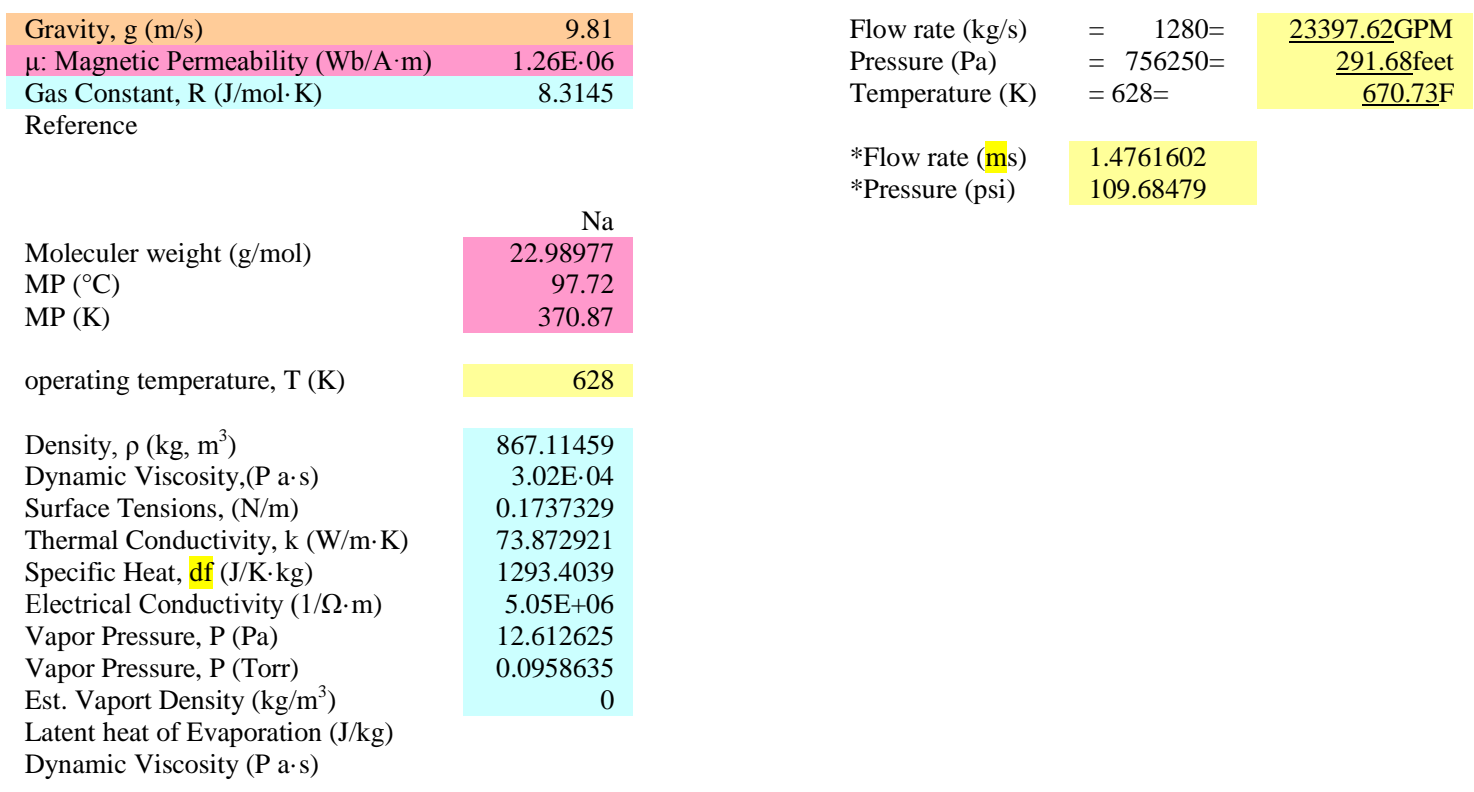

Figure III.10-3 Input data for mechanical pump design nomograph 


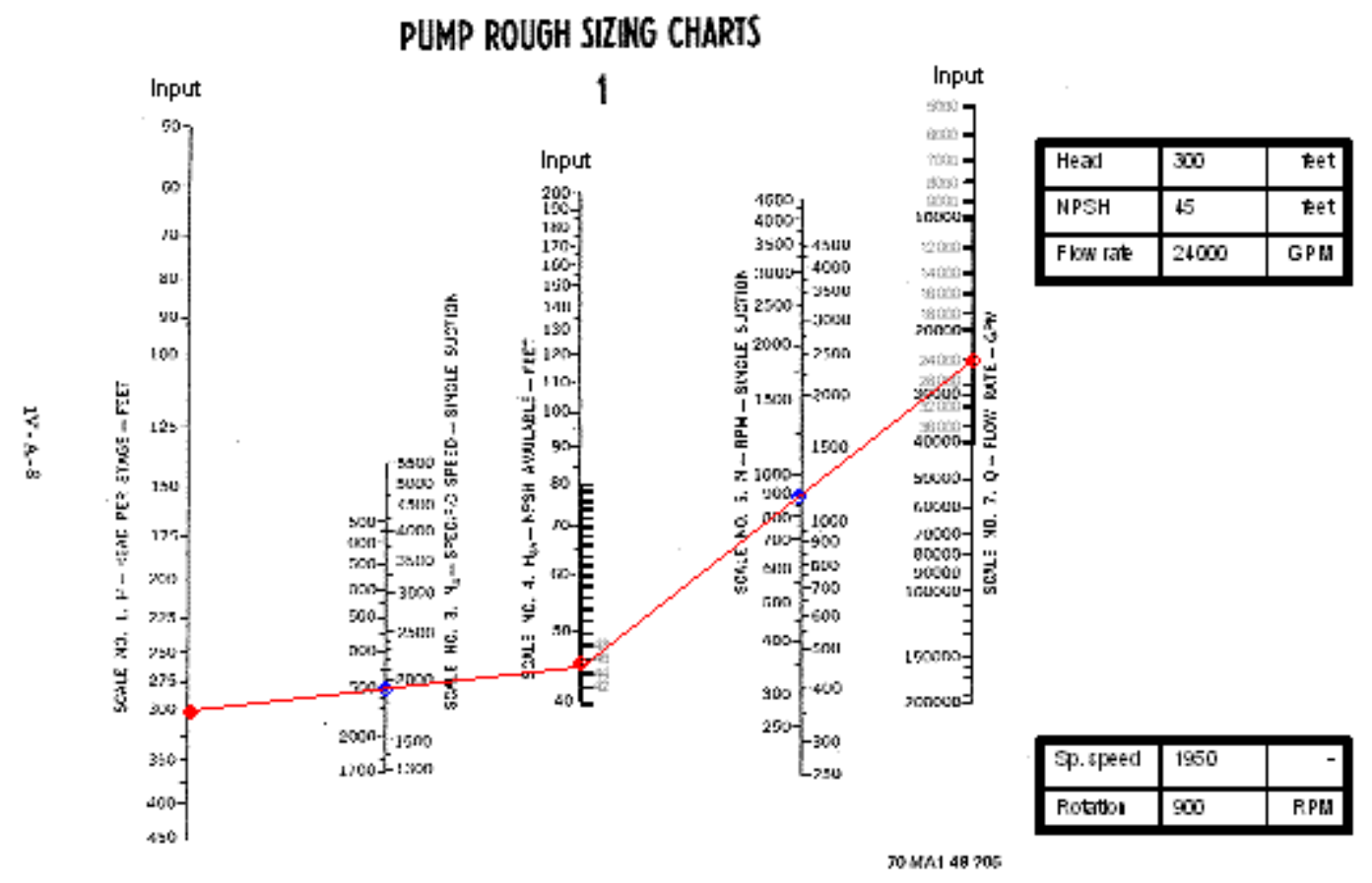

Figure III.10-4 Mechanical pump design nomograph-1

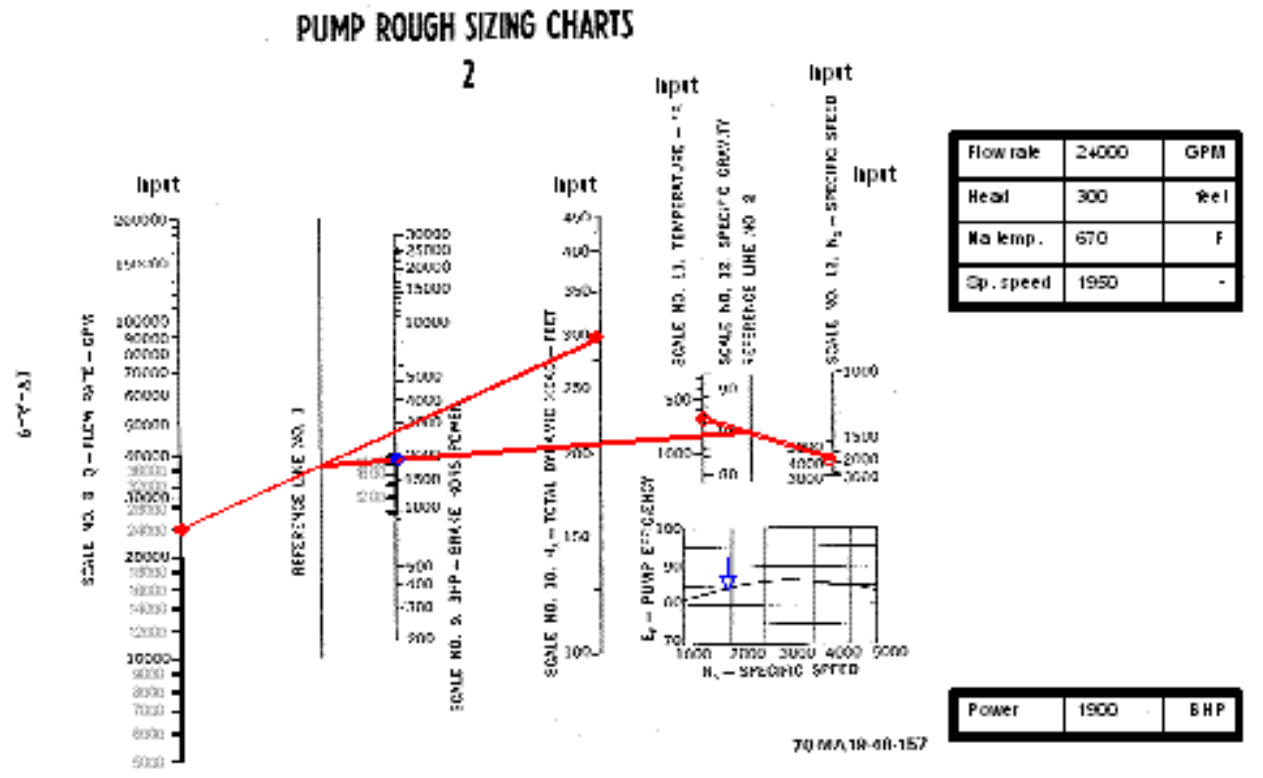

Figure III.10-5 Mechanical pump design nomograph-2 


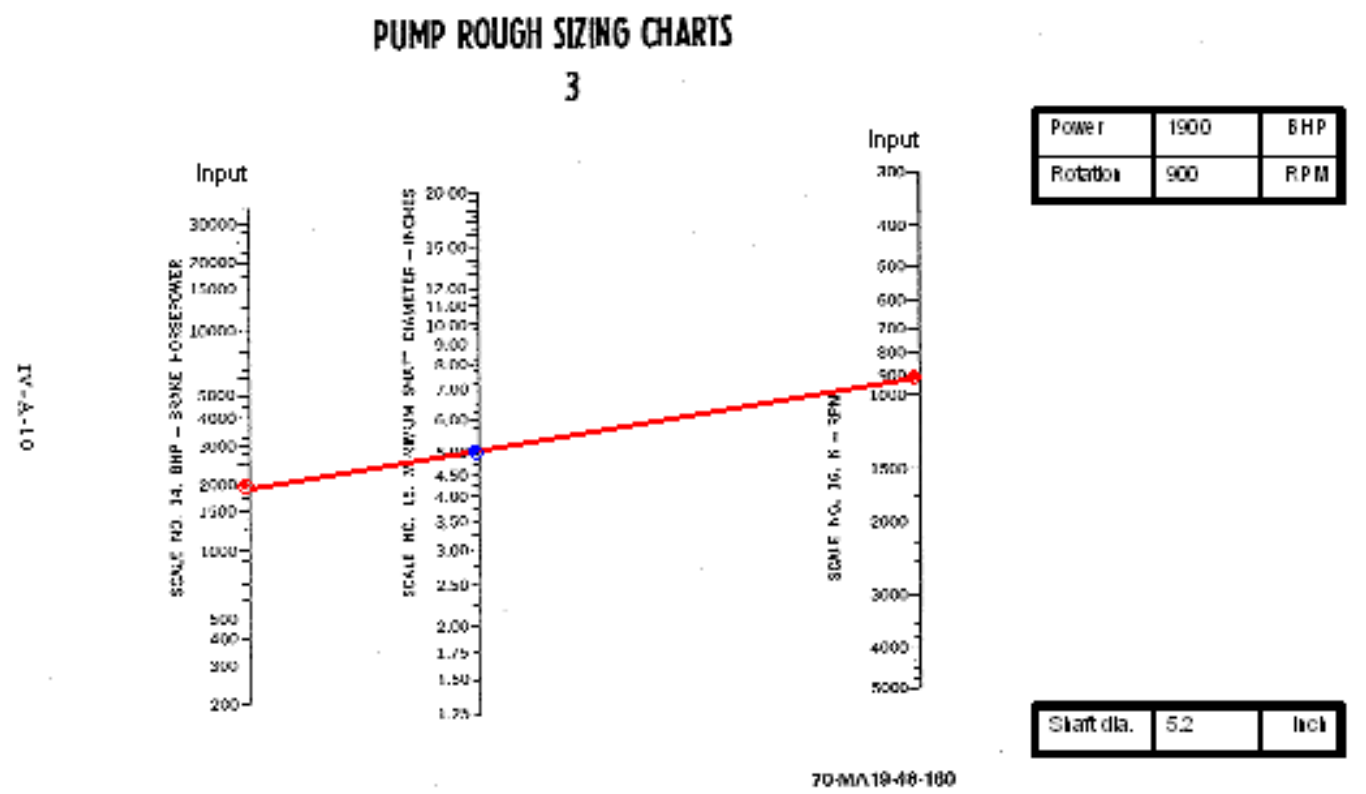

Figure III.10-6 Mechanical pump design nomograph-3

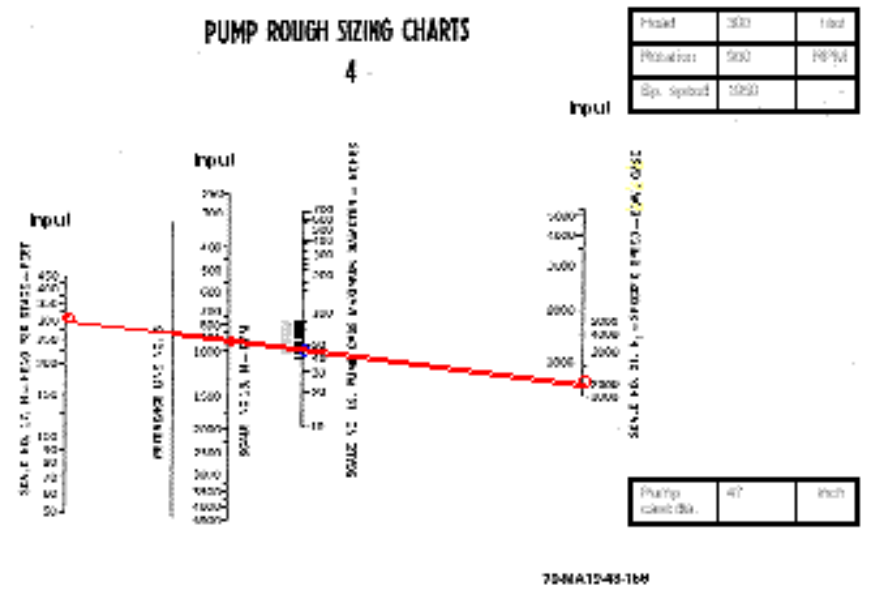

Figure III.10-7 Mechanical pump design nomograph-4 


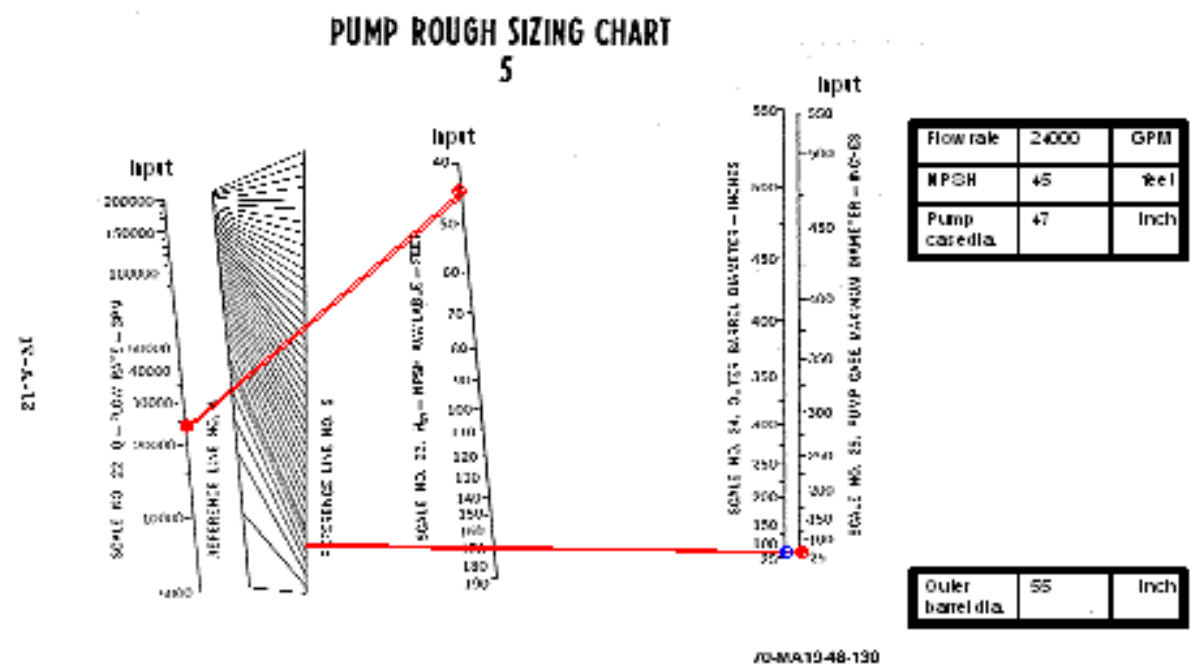

Figure III.10-8 Mechanical pump design nomograph-5

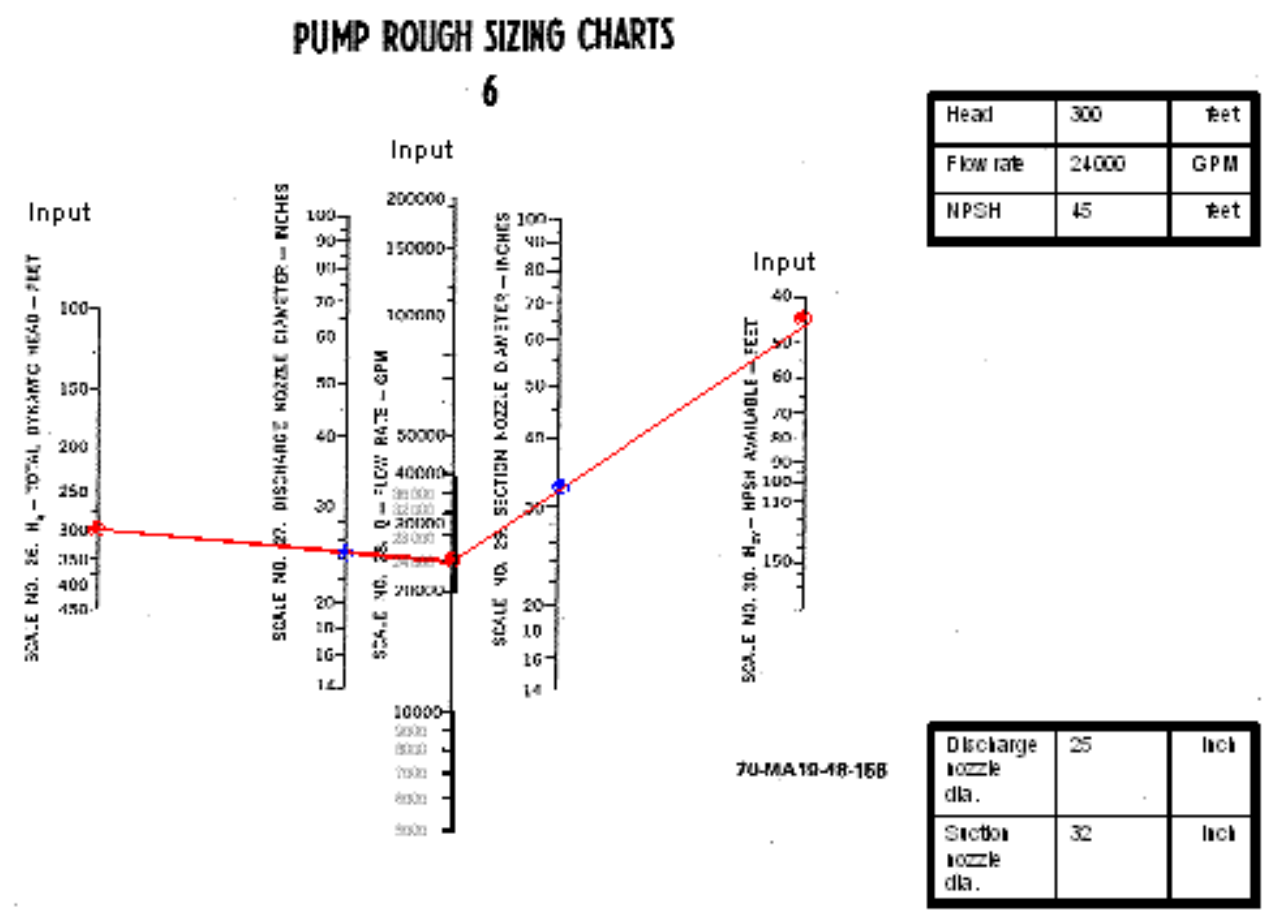

Figure III.10-9 Mechanical pump design nomograph-6 


\begin{tabular}{|l|l|r|}
\multicolumn{1}{|c}{ Input } \\
\hline Head & 300 & feet \\
\hline NPSH & 45 & feet \\
\hline Flow rate & 24000 & GPM \\
\hline Na temp & 670 & F \\
\hline
\end{tabular}

Estimated Parameters

\begin{tabular}{|l|c|c|c|c|}
\hline Specific speed & 1950 & - & 1950 & - \\
\hline Rotation & 900 & RPM & 900 & RPM \\
\hline Power & 1900 & BHP & 1416830 & W \\
\hline Shaft dia. & 5.2 & inch & 0.13 & $\mathrm{~m}$ \\
\hline Pump case dia. & 47 & inch & 1.19 & $\mathrm{~m}$ \\
\hline Outer barrel dia. & 55 & inch & 1.40 & $\mathrm{~m}$ \\
\hline Discharge nozzle dia. & 25 & inch & 0.64 & $\mathrm{~m}$ \\
\hline Suction nozzle dia. & 32 & inch & 0.81 & $\mathrm{~m}$ \\
\hline
\end{tabular}

Figure III.10-10 Estimated design parameters for ABR mechanical pump

\section{III.10.2 Electromagnetic Pump}

The EM pump design optimization studies were carried out using an ANL developed code based on the calculational model developed to estimate the performance of various ALIPs. At the design operating condition (nominal operating condition for the ABR design) and a given pump length, the code estimates the diameter of the pump without return duct, mass, and efficiency of the ALIP. To optimize some parameters for the pump, the code repeatedly calculates the performance of the pump at a fixed operating condition and pump length while varying the following parameters:

1. Diameter of the pump duct,

2. Number of coils,

3. Number of coils per pole per phase,

4. Drive frequency.

In this analysis, the diameter of the pump is limited to within $0.6 \mathrm{~m}$, such that the ALIP can be installed in the opening for the mechanical pump design presented in Section II.3.2.1. An example design for a primary pump is shown in Table III.10-1. Performance curves are shown in Figure III.10-11 and III.10-12. 
Table III.10-1 EM Pump design parameters

\begin{tabular}{|c|c|c|c|c|c|}
\hline Items & $\begin{array}{l}\text { Spec. } \\
\text { (SI) }\end{array}$ & Unit & $\begin{array}{l}\text { Spec. (Eng } \\
\text { Units) }\end{array}$ & & Remarks \\
\hline Type & ALP & & & & return single stator \\
\hline Quantity & 4 & & 4 & & \\
\hline Mass Flow rate & 1280 & $\mathrm{~kg} / \mathrm{s}$ & - & - & $\begin{array}{l}\text { Reference number (volumetric flow } \\
\text { rate is used for pump sizing) }\end{array}$ \\
\hline Volumetric Flow rate & 1.48 & $\mathrm{~m}^{3} / \mathrm{s}$ & 23458 & gpn & \\
\hline Pump head (psig) & 0.76 & $\mathrm{MPa}$ & 110 & psig & $\begin{array}{l}\text { It is currently estimated that the } \\
\text { core pressure drop is } \sim 60 \text { psig and } \\
\text { the IHX primary side pressure drop } \\
\text { is 5-10psig, so the pump specificed } \\
\text { here mayb be oversized. }\end{array}$ \\
\hline Temperature & 355 & ${ }^{\circ} \mathrm{C}$ & 671 & ${ }^{\circ} \mathrm{F}$ & cold pool temperature \\
\hline Power & 2315 & KW & & & \\
\hline Efficiency & 48.59 & $\%$ & 49 & $\%$ & \\
\hline Drive voltage & 480 & $\mathrm{~V}$ & 480 & $\mathrm{~V}$ & \\
\hline Drive current & 4564 & A & 4564 & $\mathrm{~A}$ & \\
\hline Drive Frequency & 40 & $\mathrm{~Hz}$ & 40 & $\mathrm{~Hz}$ & \\
\hline Pole count & 8 & & 8 & & Usually $3 \mathrm{x}$ pole count $=$ coil count \\
\hline Coil count & 24 & & 24 & & \\
\hline $\begin{array}{l}\text { Net positive suction } \\
\text { head available }\end{array}$ & & $\begin{array}{l}\mathrm{m} \text { of } \\
\text { sodium }\end{array}$ & 0 & $\begin{array}{l}\text { feet of } \\
\text { sodium }\end{array}$ & $\begin{array}{l}\text { Roughly, the expected cover gas } \\
\text { pressure will be } \sim 1 \text { atm which is } \\
\text { equivalent to } \sim 40 \text { feet of sodium } \\
\text { head plus } 4-5 \text { feet of sodium to the } \\
\text { inlet of ht e sodium pump - see } \\
\text { drawing. }\end{array}$ \\
\hline Mass & 6176 & $\mathrm{~kg}$ & 13587 & lbm & \\
\hline Pump height (length) & 1.6 & $\mathrm{~m}$ & 5.2 & $\mathrm{ft}$ & \\
\hline Pump diameter & 1.18058 & $\mathrm{~m}$ & 3.9 & $\mathrm{ft}$ & \\
\hline $\begin{array}{l}\text { Inner core - inner } \\
\text { diameter }\end{array}$ & 0.141 & $\mathrm{~m}$ & 0.5 & $\mathrm{ft}$ & \\
\hline $\begin{array}{l}\text { Inner core - outer } \\
\text { diameter }\end{array}$ & 0.222 & $\mathrm{~m}$ & 0.7 & $\mathrm{ft}$ & \\
\hline $\begin{array}{l}\text { Inner core -containment } \\
\text { thickness }\end{array}$ & 1 & $\mathrm{~mm}$ & 0.0 & inches & \\
\hline Duct - inner diameter & 0.42542 & $\mathrm{~m}$ & 1.4 & $\mathrm{ft}$ & \\
\hline Duct - outer diameter & 0.43 & $\mathrm{~m}$ & 1.4 & $\mathrm{ft}$ & \\
\hline Duct - wall thickness & 2.29 & $\mathrm{~mm}$ & 0.1 & inches & \\
\hline $\begin{array}{l}\text { Pump housing - inner } \\
\text { diameter }\end{array}$ & 0.956 & $\mathrm{~m}$ & 3.1 & $\mathrm{ft}$ & \\
\hline $\begin{array}{l}\text { Pump housing - outer } \\
\text { diameter }\end{array}$ & 0.976 & $\mathrm{~m}$ & 3.2 & $\mathrm{ft}$ & \\
\hline $\begin{array}{l}\text { Pump housing - wall } \\
\text { thickness }\end{array}$ & 10 & $\mathrm{~mm}$ & 0.4 & inches & \\
\hline Return path - thickness & 0.1 & $\mathrm{~m}$ & 0.3 & $\mathrm{ft}$ & \\
\hline Casing - inner diameter & 1.176 & $\mathrm{~m}$ & 3.9 & $\mathrm{ft}$ & \\
\hline Casing - outer diameter & 1.18058 & $\mathrm{~m}$ & 3.9 & $\mathrm{ft}$ & \\
\hline Casing - wall thickness & 2.29 & $\mathrm{~mm}$ & 0.1 & inches & \\
\hline Material & 304 SS & & & & \\
\hline
\end{tabular}




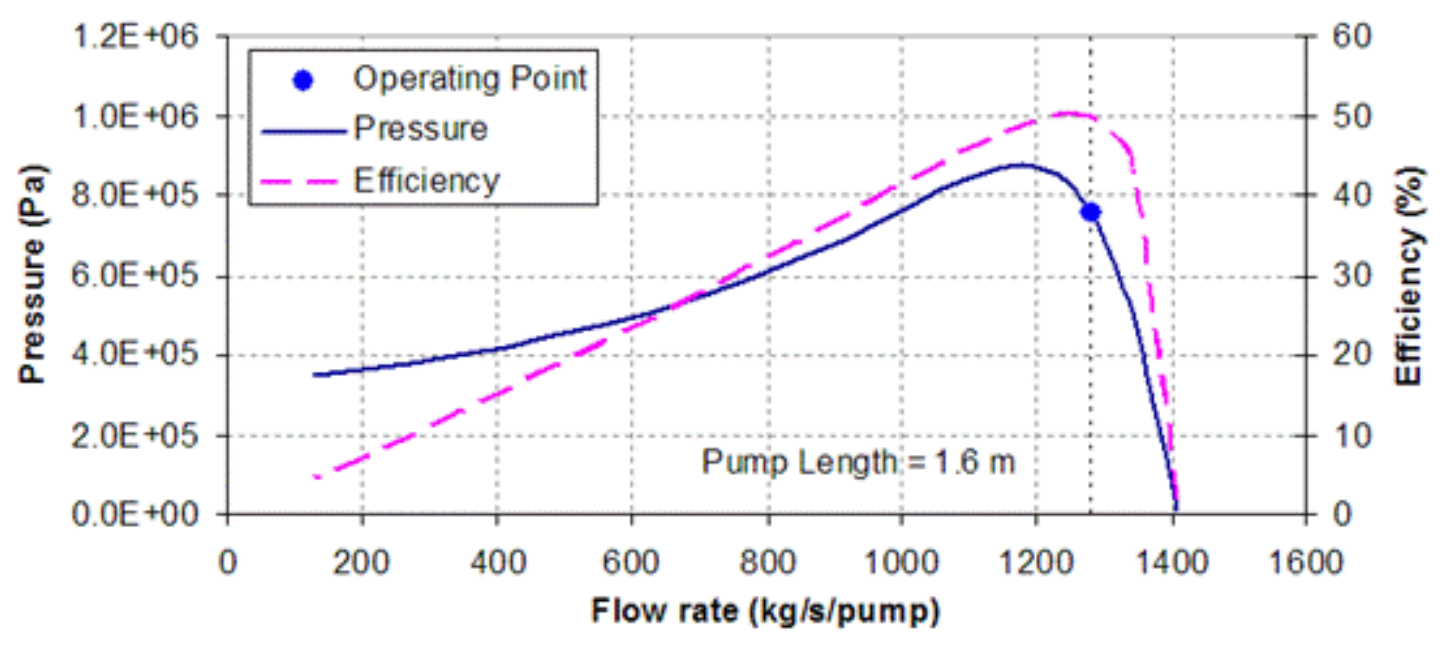

Figure III.10-11 Performance Curve of 1.6 m ALIP

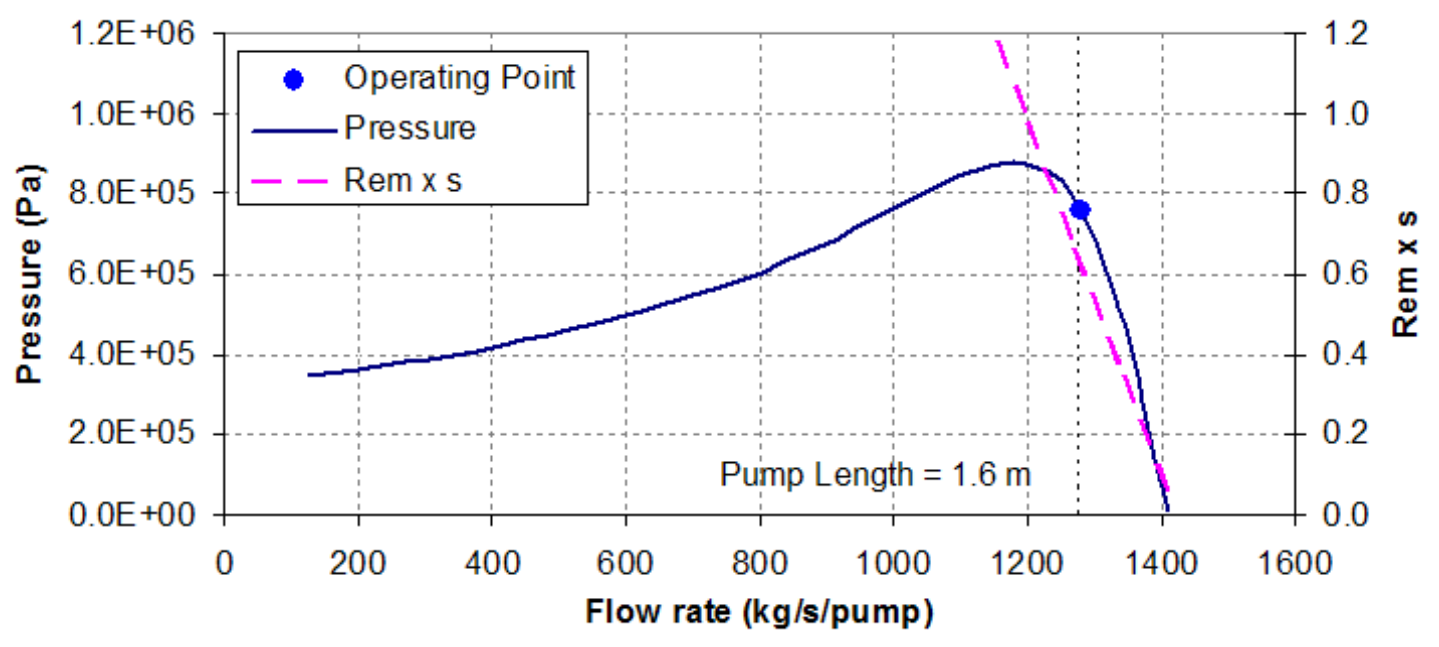

Figure III.10-12 Stable Range of Operation for 1.6 m ALIP

The operating point (primary coolant flow rate of $1280 \mathrm{~kg} / \mathrm{s}$ and pressure head of $760 \mathrm{kPa}$ ) is shown as a blue dot in these figures. It must be noted that stable operation of the pump requires that $\operatorname{Rem} \times s<\sim 1.5$ where Rem is the magnetic Reynolds number and $s$ is the slip defined below [3-4]:

$$
\operatorname{Rem}=2 \pi f \frac{\mu}{\rho}\left(\frac{L_{p}}{\pi}\right)^{2}\left(\frac{\tau}{\tau_{a}}\right), \text { and } s=\frac{v_{s}-v_{f}}{v_{s}},
$$

where $f$ is the drive frequency, $\mu$ is the magnetic permeability and $\mu \cong \mu_{0}$ that is the magnetic permeability of vacuum, $\rho$ is the resistivity of the working fluid, $L_{p}$ is the pole pitch, $\tau$ is the flow channel height, $\tau_{a}$ is the total non-magnetic gap height, $v_{s}$ is the synchronous velocity defined as $v_{s}=2 L_{p} f$, and $v_{f}$ is the fluid velocity. 
Once all dimensions and design parameters are calculated, performance curves of the pump as a function of the drive voltage and frequency are generated and compared with the system curve (flow rate vs pressure drop curve) for the ABR design to check the stability of the pump at various off-normal conditions. However, the system curve for the ABR has not yet been developed. Similar stability analyses were conducted for Small Modular Fast Reactor (SMFR) [5]. In the following, results for the SMFR were used to explain the method of stability analysis and significance of it.

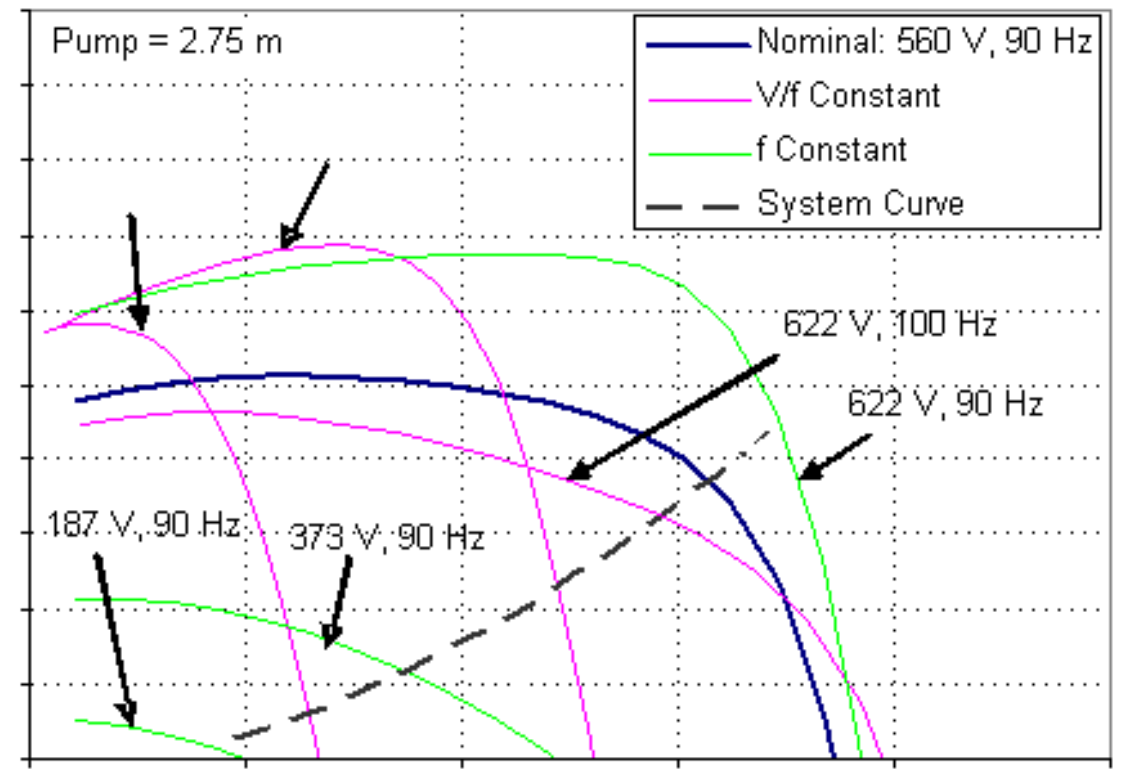

Figure III.10-13 Comparison of Different Control Methods

One typical method to control an induction motor is constant $V / f$ control. In this method, the ratio of the drive voltage, $V$ to drive frequency, $f$ is kept constant while the drive voltage is reduced from the rated value. This method is used to provide maximum torque at reduced motor speed for an induction motor. Another method is constant frequency control in which the drive frequency is kept constant while changing drive voltage. For hydrodynamic pump applications, since pressure head required at reduced flow rate is generally smaller than that at nominal operating conditions, large pressure head is not required while the pump operates at the reduced flow rates and thus constant frequency method may be suitable. An example comparison of $V / f$ constant control and $f$ constant control is shown in Figure III.10-14. This figure shows that when a flow rate greater than the nominal value is required, the $V / f$ constant control cannot meet the required pumping characteristics. For example, the ALIP performance line for the $622 \mathrm{~V}$ and $100 \mathrm{~Hz}$ operation crosses the system curve at a flow rate smaller than that at the nominal condition. On the other hand, the $f$ constant control provides adequate pumping characteristics at a flow rate greater than the nominal value (see the ALIP performance line for the $622 \mathrm{~V}$ and 90 $\mathrm{Hz}$ operation). However, it is shown that both methods can provide satisfactory pumping characteristics at reduced flow rates. It is also confirmed that the coil temperatures are well below the maximum demonstrated temperature of $1023 \mathrm{~K}$ at any operating conditions. The highest coil temperature occurs when the pump is driven at increased drive voltage using the $f$ constant control method ( $<\sim 900 \mathrm{~K}$ at $622 \mathrm{~V}$ and $90 \mathrm{~Hz}$ operation and $<\sim 940 \mathrm{~K}$ at $650 \mathrm{~V}$ and 90 Hz operation). 


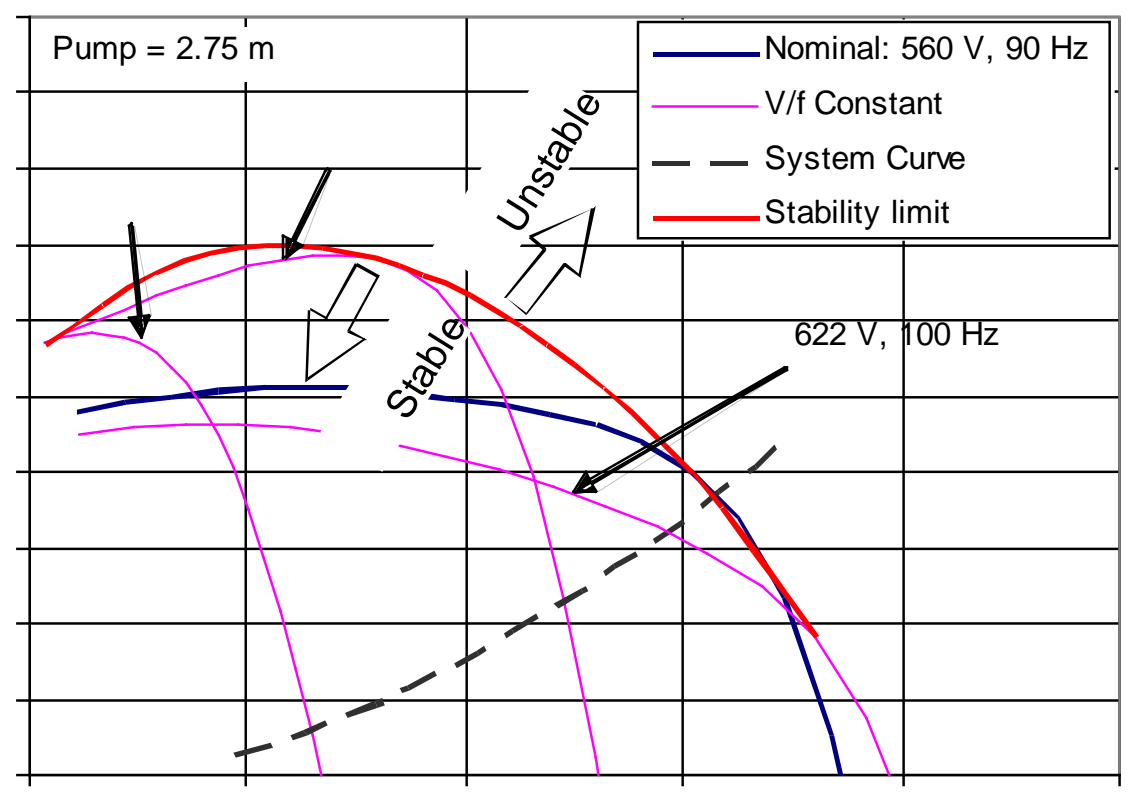

Figure III.10-14 Stability Diagram for V/f Constant Control

The next figures show the region in which the ALIP operation is stable ( $\operatorname{Rem} \times s<\sim 1.5$ ) for V/f constant control (Figure III.10-14) and f constant control (Figure III.10-15). Figure III.10-14 shows that when a flow rate greater than the nominal value is required, an ALIP controlled using the V/f constant control method becomes unstable. On the other hand, an ALIP controlled using the f constant control method becomes unstable, when a flow rate smaller than the nominal value is required, as shown in Figure III.10-15. Table III.10-2 summarizes the applicability of different control methods.

Table III.10-2 Applicability of Different Control Methods

\begin{tabular}{|c|c|c|c|c|}
\hline & \multicolumn{2}{|c|}{ Reduced Flow Rate } & \multicolumn{2}{c|}{ Increased Flow Rate } \\
\cline { 2 - 5 } & Pumping & Stability & Pumping & Stability \\
\hline V/f Constant & Good & Good & No Good & No Good \\
\hline f Constant & Good & No Good & Good & Good \\
\hline
\end{tabular}




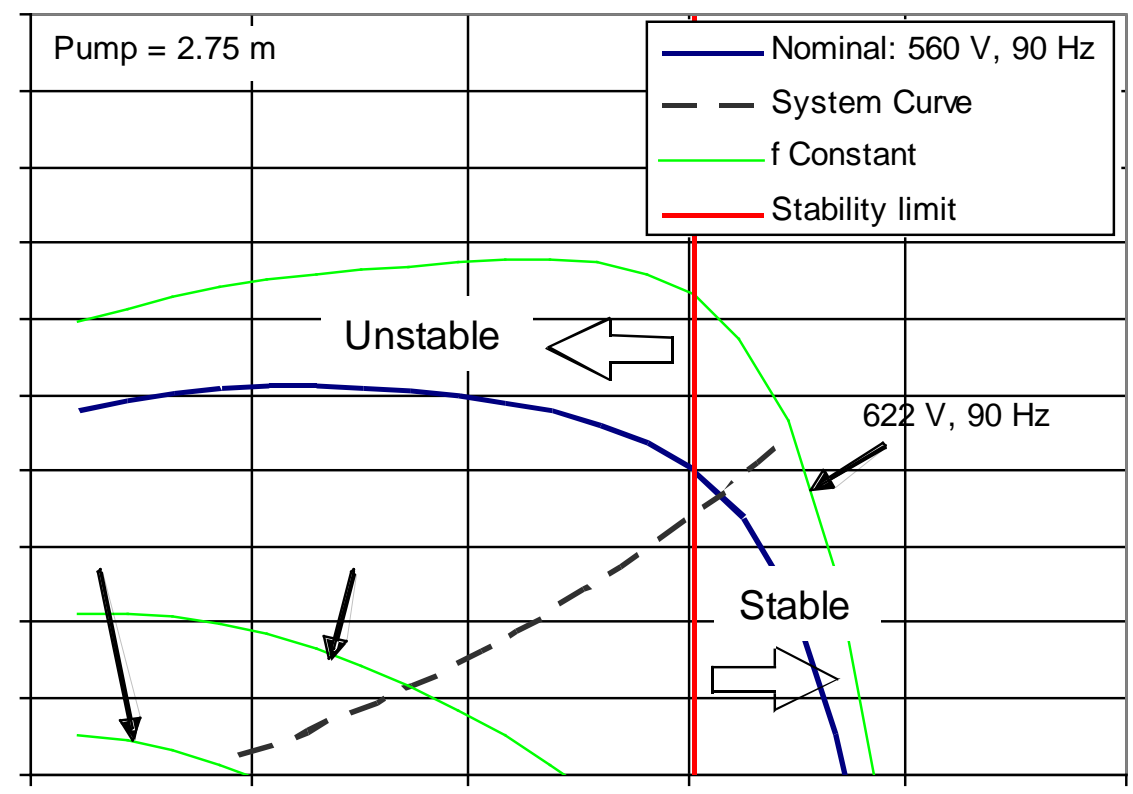

Figure III.10-15 Stability Diagram for f Constant Control

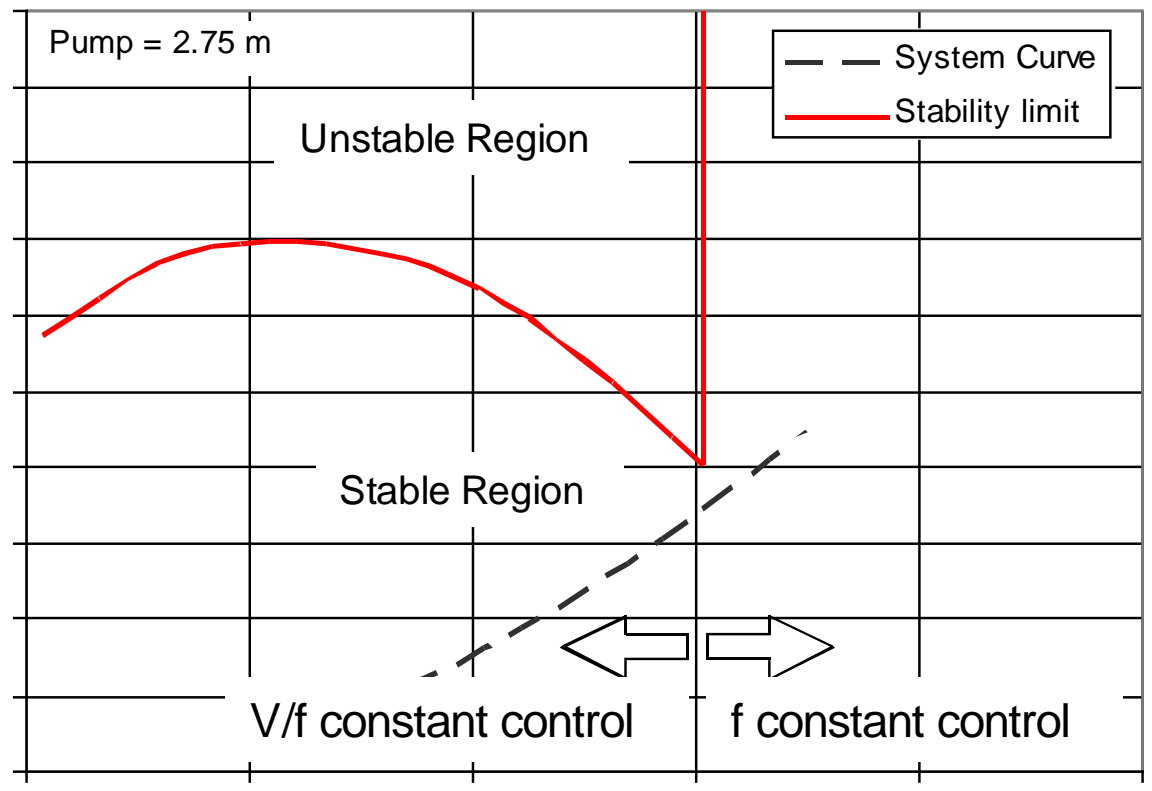

Figure III.10-16 Overall Stability Diagram 
These results indicate that the $V / f$ constant control method is appropriate for ALIP operation at reduced flow rates below the nominal operating condition and the $f$ constant control method is suitable for ALIP operating at increased flow rates above the nominal operating condition (see Figure III.10-16). Detailed pump operation curves for a $2.75 \mathrm{~m}$ ALIP using this mixed control method are shown in Figure III.10-17.

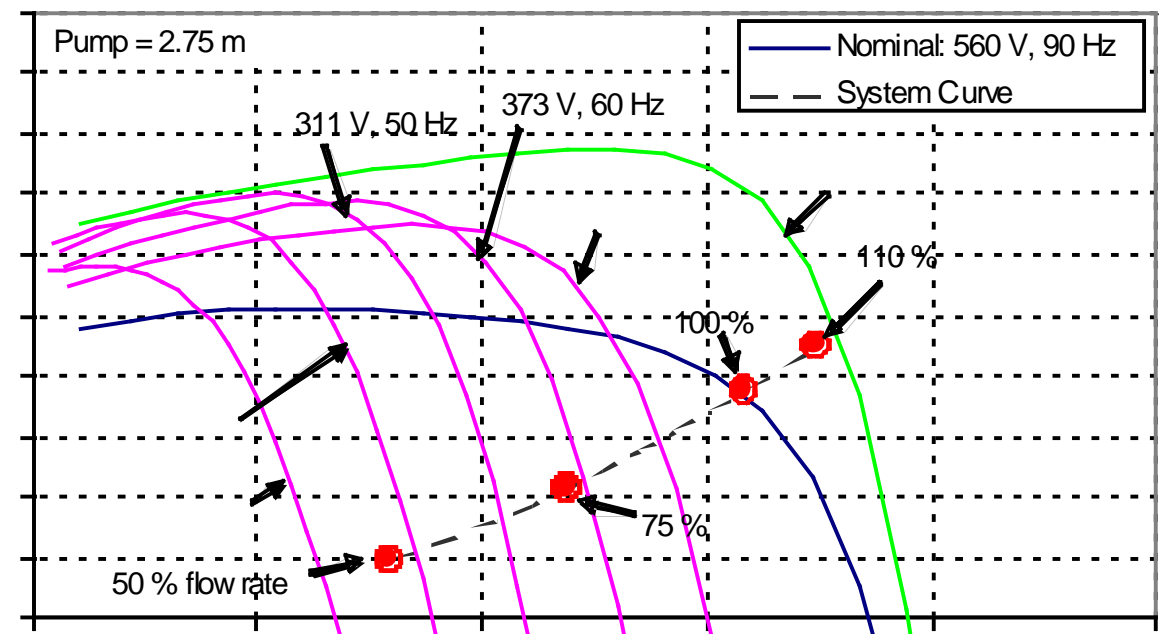

Figure III.10-17 Pump Operation Curves and System Curve for 2.75 m ALIP

It has been shown that all discussions above are also applicable to other pumps with similar size and performances as well.

These results show that using the $V / f$ constant control at reduced flow rates below the nominal operating condition and the $f$ constant control at increased flow rates above the nominal operating condition, the designed ALIPs for various pump lengths are all capable of meeting the flow rate-pressure requirements for the SMFR design. To employ this mixed control method, the power supply for the ALIP must be able to vary both voltage and frequency simultaneously. It must be noted that the ALIP may be designed such that the nominal operating condition of the pump is set at $110 \%$ flow rate point and the ALIP is controlled using only the V/f constant control in the entire operating range to simplify the control scheme. Once the system curve for the ABR is developed, a similar analysis needs to be performed to confirm that the pump designed for the ABR can be stably operated at all conditions; however, since the size and performance of the ABR pumps are very similar to the SMFR pumps, the analysis is expected to show that the ABR pumps can be stably operated using similar control methods described here. 


\section{References}

1. Charnock, H. O. and Holz, H. B., "Sodium Systems Components," Sodium Technology, Session IV, Atomic International, North American Rockwell, 1970.

2. Babcock, W., "State of Technology Study - Pumps Experience with High Temperature Sodium Pumps in Nuclear Reactor Service and Their Application to FFTF,” BNWL-1049 UC-80, Reactor Technology, Battelle Memorial Institute Pacific Northwest Laboratories, Richland, Washington, 1969.

3. H. Ota et al., "Development of $160 \mathrm{~m}^{3} / \mathrm{min}$ Large Capacity Sodium-Immersed SelfCooled Electromagnetic Pump,” J. of Nucl. Sci. and Tech. v41, No4, pp511-523, 2004.

4. R. Stieglitz, "MHD-features of the Main Service and Bypass Pump in the MEGAPIE Design,” ISSN 0947-8620, Forschungszentrum Karlsruhe GmbH, Karlsruhe, 2003.

5. ANL-SMFR-1, Small Modular Fast Reactor Design Description, 2005. 


\section{Appendix A}




\section{LIST OF ACRONYMS}

$\begin{array}{ll}\text { ABR } & \text { Advanced Burner Reactor } \\ \text { ANL } & \text { Argonne National Laboratory } \\ \text { ANS } & \text { American Nuclear Society } \\ \text { ANSI } & \text { American National Standards Institute } \\ \text { ASME } & \text { American Society of Mechanical Engineers } \\ \text { ATWS } & \text { Anticipated Transient Without Scram } \\ \text { BOC } & \text { Beginning of Cycle } \\ \text { B\&PV } & \text { Boiler and Pressure Vessel } \\ \text { CEA } & \text { Commissariat a l'Energie Atomique } \\ \text { CFD } & \text { Computational Fluid Dynamics } \\ \text { DBE } & \text { Design Basis Events } \\ \text { DOE } & \text { Department of Energy } \\ \text { DRACS } & \text { Direct Reactor Auxiliary Cooling System } \\ \text { EBR-II } & \text { Experimental Breeder Reactor II } \\ \text { EM } & \text { Electromagnetic } \\ \text { EMAT } & \text { Electromagnetic Acoustic Transducer } \\ \text { EOC } & \text { End of Cycle } \\ \text { EPA } & \text { Environment Protection Agency } \\ \text { FFTF } & \text { Fast Flux Test Facility } \\ \text { HEPA } & \text { High Efficiency Particulate Air } \\ \text { HTR } & \text { High Temperature Recuperator } \\ \text { HVAC } & \text { Heating, Ventilation and Air Conditioning } \\ \text { HX } & \text { Heat Exchanger } \\ \text { IBC } & \text { International Building Code } \\ \text { IEEE } & \text { Institute of Electrical and Electronics Engineers } \\ \text { IHTS } & \text { Intermediate Heat Transport System } \\ \text { IHX } & \text { Intermediate Heat Exchanger } \\ \text { ISI } & \text { In-Service Inspection } \\ \text { IVIM } & \text { In-Vessel Inspection Machine } \\ \text { JNC } & \text { Japan Nuclear Cycle Development Institute } \\ \text { LMR } & \text { Liquid Metal Reactor } \\ \text { LRB } & \text { Laminated Rubber Bearing } \\ \text { LTR } & \text { Low Temperature Recuperator } \\ \text { MOC } & \text { Middle of Cycle } \\ \text { MOX } & \text { Mixed Oxide } \\ \text { MWe } & \text { Megawatt Electric } \\ \text { NDE } & \text { Non-Destructive Examination } \\ \text { NDHX } & \text { Natural Draft Heat Exchanger } \\ \text { NPSH } & \text { Net Positive Suction Head } \\ \text { NRC } & \text { Nuclear Regulatory Commission } \\ \text { OBE } & \text { Operating Basis Earthquake } \\ \text { OSHA } & \text { Occupational Safety and Health Administration } \\ \text { PHTS } & \text { Printed Circuit Heat Exchanger } \\ & \end{array}$




$\begin{array}{ll}\text { PLOF } & \text { Protected Loss of Flow } \\ \text { PLOHS } & \text { Protected Loss of Heat Sink } \\ \text { PRISM } & \text { Power Reactor Inherently Safe Module } \\ \text { RBCB } & \text { Run Beyond Cladding Breach } \\ \text { RCV } & \text { Remote Controlled Vehicle } \\ \text { SHRS } & \text { Shutdown Heat Removal System } \\ \text { SS } & \text { Stainless Steel } \\ \text { SSE } & \text { Safe Shutdown Earthquake } \\ \text { TRU } & \text { Transuranic } \\ \text { ULOF } & \text { Unprotected Loss of Flow } \\ \text { ULOHS } & \text { Unprotected Loss of Heat Sink } \\ \text { UTOP } & \text { Unprotected Transient Overpower }\end{array}$




\section{APPENDIX A}

\section{A.1 Requirements for a Standard Commercial Advanced Burner Reactor $^{9}$}

\section{Introduction}

1. The Advanced Burner Reactor (ABR) is a key component of the Global Nuclear Energy Partnership (GNEP) initiative for recovering transuranic elements (TRU) from spent nuclear fuel and recycling these elements as reactor fuel. By recycling the TRU as fuel, the longterm radiation hazard resulting from the spent fuel is significantly reduced, the demand on a geologic repository for spent fuel is also significantly reduced and useful energy is recovered by fissioning the TRU in the ABR.

2. This document is intended to convey the expectations of the Department of Energy (DOE) for the principal attributes of an ABR design. It is to be used along with the regulations and guidance of the US Nuclear Regulatory Commission (NRC), the US Environmental Protection Agency (EPA) and other appropriate requirements documents and standards to formulate an overall set of requirements for an ABR design. The design team is expected to use creativity and ingenuity in developing a design that satisfies these expectations. The design team may propose an exception or alternative to one or more of the provisions of this document in formulating its overall design requirements, but shall justify the proposed change to DOE's satisfaction.

3. The requirements included in this document are intended to apply to a design for a standard commercial nuclear power plant based on an Advanced Burner Reactor. Since these nuclear power plants would be operated in the commercial sector, they will be licensed by the US Nuclear Regulatory Commission (NRC), and this requirements document reflects that expectation.

4. Current plans are that a prototype ABR would be designed, built and operated as part of a development and demonstration program, having the goals of demonstrating the transmutation of TRU in a fast reactor, demonstrating operation of the ABR as part of the system for recovering and recycling TRU as fuel, qualifying the transmutation fuel, testing new materials and components, and supporting design certification of the standard commercial ABR power plant by the NRC under 10CFR52. The prototype ABR must be sufficiently close in design to the commercial ABR to effectively perform these functions. Therefore, the requirements set out in this document should apply to the prototype ABR as well, with a few additional requirements to reflect the purposes of the prototype. These additional requirements are clearly indicated in the document.

\footnotetext{
${ }^{9}$ The document also provides additional requirements for a prototype ABR. See the Introduction, paragraph 4.
} 
5. As a prototype of a fast reactor intended to be used in a commercial nuclear power plant, it is assumed herein that the prototype ABR will be licensed by the US NRC as specified by the Energy Reorganization Act of 1974. However, if a decision is made that DOE will license the prototype ABR, rather than NRC, it will be necessary to make appropriate changes to references to NRC regulations to refer instead to the corresponding DOE regulations, primarily those found in 10CFR830 and 835, and related Orders and other documents.

6. Both the commercial ABR and a prototype ABR will be subject to International Atomic Energy Agency (IAEA) safeguards. If operated in the US, it is an open question as to whether the IAEA will actually choose to safeguard the facility. Should an ABR be constructed in another country, it would be subject to IAEA safeguards. Accordingly, design of the ABR shall incorporate the ability to meet international safeguards criteria.

\section{Requirements}

\section{High-level Requirements}

\subsection{Functional Objectives}

1.1.1. Electricity production in a commercial environment: The ABR shall be designed for safe and efficient production of electricity. Also, the ABR shall be designed to operate in a commercial environment (i.e., on an electrical grid). Accordingly, an efficient energy transport and conversion system is required, and the ABR shall be designed with sufficient operational control, flexibility, and availability to ensure compatibility with the demands of such an environment.

1.1.1.1. Capacity Factor: For the ABR power production mission, the commercial ABR design shall be such that a capacity factor greater than $90 \%$ can be achieved.

1.1.2. Power plant size: The reference power rating for the prototype $\mathbf{A B R}$ is expected to be in the range of 250 to $2000 \mathrm{MWt}$; in any case, it shall be large enough to fulfill the objectives of the prototype ABR set out in paragraph 4, above. It is expected that the commercial ABR will be larger, in the 1500 MWe class. For the commercial ABR, either a single unit or a modular, multiple power unit approach may be proposed by the design team.

1.1.3. Burning of plutonium and minor actinides: The principal purpose of the Advanced Burner Reactor (ABR) is to transmute transuranic elements (TRU) by fissioning. Therefore, the ABR shall be designed to operate safely and efficiently using 'transmutation fuel' containing plutonium and minor actinides. In the prototype $\mathbf{A B R}$, it may be necessary to allow for flexibility in operation with various fuel types, considering both fuel 
composition (TRU, U/Pu, HEU, etc.) and fuel form (oxide, metal, etc.), so the prototype ABR shall have the capability to operate safely and efficiently for a range and/or mixture of fuel types.

1.1.3.1. Transuranic consumption targets: The $A B R$ shall be designed for a net consumption ("burning") of transuranics (TRU) at a rate of 0.25 to 0.50 grams TRU/MWt-day when optimized for cost and other performance characteristics. The design team shall propose the core design options, such as absence of blankets, needed to achieve this goal. The prototype ABR shall include the capability for testing advanced fuel forms and alternate core configurations designed for a wider range of TRU management options (i.e., significant TRU enrichment variations, fuels containing no fertile material, etc.).

1.1.4. Cost objective: To be successful, the ABR must function as part of a commercially viable system, which includes LWRs, ABRs, reprocessing and fuel-fabrication facilities and waste disposition. A design objective shall be that the capital and operating cost of the future commercial ABR will be such that, when considered in the context of the overall benefits of the ABR transmutation system, the cost of the electricity produced is competitive in the marketplace. The design of the prototype ABR shall minimize cost consistent with achieving the principal goals of the ABR development and demonstration program

1.1.5. Non-proliferation and nuclear security: The ABR design shall include proliferation resistance as a design objective. The design of the standard commercial ABR shall facilitate domestic and international safeguards activities and include provisions to protect against theft or diversion of nuclear materials and to protect against security-related threats. The design of the prototype ABR shall enable testing of advanced equipment and systems that can enhance proliferation resistance and nuclear security.

\subsection{Supporting Technical Requirements}

1.2.1. Fast neutron spectrum: To ensure that transmutation of plutonium and minor actinides can be accomplished efficiently without excessive buildup of higher actinides, the ABR shall provide a fast neutron spectrum.

1.2.2. Sodium coolant: The ABR design shall take advantage of wellestablished fast reactor coolant and component technology and operating experience to the maximum extent possible to minimize technical risk and expedite design and construction. To this end, the reactor shall use liquid sodium coolant. 
1.2.3. Design of a prototype reactor: The prototype $\mathbf{A B R}$ shall be designed to satisfy the functions of a prototype reactor, including but not limited to demonstration of reactor based transmutation of TRU isotopes, qualification of the transmutation fuel, testing of advanced structural materials and developing an understanding of the cost drivers of this portion of the overall system. Accommodation of testing of advanced fuels, components and systems shall be a design goal for the prototype ABR.

1.2.4. Irradiation environment in the prototype ABR: The prototype ABR shall be sized to provide an irradiation environment that is prototypic of the conditions to be expected in commercial ABRs. A single module of a modular plant design or a reduced size version of a large, single unit design would be acceptable if a prototypic environment is achieved.

\section{General Requirements}

2.1. Compliance with regulations, codes and standards: The ABR design shall comply with NRC regulations and other applicable codes and standards, including but not limited to those published by the American Society of Mechanical Engineers (ASME), the American Society of Civil Engineers (ASCE), the American Nuclear Society (ANS), and the Institute of Electrical and Electronic Engineers (IEEE). In case of conflict between NRC's requirements and those of other codes and standards, the NRC requirements shall take precedence.

2.1.1. Proven design methods and design features shall be used to the extent possible. When new or innovative features are introduced, they shall be proven by analysis, test or both, followed by demonstration in the prototype reactor.

2.2. Detailed design requirements and documentation: The design team shall divide the reactor and plant design into appropriate systems. For each system, a System Design Description (SDD) shall be prepared to document the detailed functions and requirements for that system, along with the details of the design. The design team shall show that the detailed functions and requirements in the SDD are traceable to and support the requirements in this document and/or any other higher level requirements agreed with DOE, and that the design satisfies the detailed functions and requirements.

2.3. Quality assurance: All safety-significant ${ }^{10}$ structures, systems and components (SSCs) of the ABR shall be designed, built and operated in accordance with a quality

\footnotetext{
${ }^{10}$ The term 'safety significant' SSCs includes those designated as 'safety related,' 'safety significant' and 'important to safety' in current NRC terminology. The functionality of such SSCs plays a role in meeting the acceptance criteria for licensing basis events (see section 3.3),
} 
assurance program that meets the requirements of 10CFR50, Appendix B, or an alternative standard (such as ANSI N45.2.11, "Quality Assurance Requirements for the Design of Nuclear Power Plants") as agreed with the NRC. Non-safety significant SSCs may be designed, built and operated under an appropriate alternative quality assurance program that will ensure that high industrial and commercial quality standards are observed.

2.4. Licensing by the NRC: The ABR shall be designed and operated to ensure that the public, the site workers and the environment are protected against undue exposure to radiation. The design, development and demonstration program shall be conducted with the long term goal of obtaining design certification of a standard design under 10CFR52. Appropriate interactions with the NRC shall take place prior to submittal of a formal license application. In line with the NRC's philosophy of using riskinformed, performance-based approaches, the ABR's design shall be based on that philosophy from the outset. Interactions with the NRC shall be a major vehicle for assuring that the philosophy is being appropriately followed.

2.5. Meeting NRC's safety goals: The ABR safety design requirements shall meet the Commission's Quantitative Health Objectives as expressed in the Safety Goal Policy Statement $^{11}$, as well as the Commission's expectations for safety of new and advanced design reactors $^{12}$.

2.5.1. To facilitate future licensing outside the USA, accepted international safety standards (such as the IAEA Safety Standards) should be considered in formulating the safety design requirements for the ABR.

\section{Safety and Security Requirements}

3.1. Safety assessment and PRA: As required by the NRC, a comprehensive safety assessment shall be performed and documented in a Safety Analysis Report. This safety assessment shall include a probabilistic risk assessment (PRA), which shall be performed as an integral contributor to informing the design process. The PRA shall be used to ensure that an acceptable risk profile is achieved, and to help to select the licensing basis (or design basis) events and to ensure that defense-in-depth is achieved.

3.1.1. The PRA shall be of sufficient quality (i.e., scope, level of detail and technical adequacy) to support risk-informed licensing decisions. Appropriate PRA quality standards shall meet NRC’s expectations,

so they require special treatment, the nature of which depends on the function and importance of the SSC.

${ }^{11}$ Safety Goal Policy Statement, "Safety Goals for the Operation of Nuclear Power Plants," 51 FR 30028, August 21, 1986

12 "Commission Policy Statement on the Regulation of Advanced Nuclear Power Plants," 51 FR 24643, July 8, 1986; 59 FR 45461, July 12, 1994 
specifically those expressed in the Commission's Phased Approach to PRA Quality.

3.1.2. It shall be assumed that the PRA will be maintained as a 'living PRA' to support operations, inspection and maintenance activities throughout the life of the plant. Appropriate quality standards will be established and observed.

3.2. Frequency-consequence curve: An acceptable risk profile shall be derived based on the existing regulations in 10CFR20, 50 and 100, and EPA Protective Action Guidelines and expressed in a frequency-consequence curve (F-C curve) relating frequencies of undesired event sequences to the corresponding acceptable consequences. ${ }^{13}$ In its draft "Framework for Development of a Risk-Informed, Performance-Based Alternative to 10 CFR Part 50," the NRC has developed the relationship shown in the Appendix to this requirements document. Use of this F-C curve or an alternative shall be agreed with the NRC.

3.3. Protection against off-normal and accident events: The ABR shall be designed to provide protection against off-normal and accident events. Licensing basis events (or off-normal, design-basis and beyond-design basis accidents) shall be defined. The performance of the ABR in providing protection against these events shall satisfy the F-C curve and the following additional deterministic criteria (demonstrated by calculations at the $95 \%$ probability value):

3.3.1. For 'frequent' events expected to occur at least once in the lifetime of a plant (frequency greater than $10^{-2} /$ reactor-year), there shall be no impact on safety analysis assumptions and no barrier failure. Redundant means of reactor shutdown and decay heat removal shall remain functional.

3.3.2. For 'infrequent' events expected to occur at least once in the lifetime of a population of plants (frequency less than $10^{-2}$ /reactor-year and greater than $10^{-5}$ /reactor-yr), a coolable geometry shall be maintained, at least one physical barrier shall remain, and at least one means of reactor shutdown and one means of decay heat removal shall remain functional.

3.3.3. For 'rare' events not expected to occur in the lifetime of a population of plants (frequency less than $10^{-5} /$ reactor-yr), no additional deterministic criteria apply.

${ }^{13}$ In the absence of a specific site for the prototype ABR, site related calculations shall be based on the most limiting of the characteristics of the 11 sites identified for detailed siting studies for integrated spent fuel recycling facilities under the GNEP initiative. 
3.3.4. In addition to the above requirements, the design team shall establish as goals a core damage frequency of $10^{-6} /$ reactor-yr and a large release ${ }^{14}$ frequency not exceeding $10^{-6} /$ site-yr for multi-reactor sites.

3.4. Defense-in-depth: The ABR design shall provide for defense-in-depth against uncontrolled release of radioactive material. The design shall provide suitable features to prevent accidents, limit accident progression, maintain containment integrity and mitigate radiological consequences of a release. Defense-in-depth measures shall also be provided for physical protection and protection against security-related threats. Safety margins shall be provided to account for uncertainties in performance of humans and SSCs, and uncertainties in the analyses. The design team may develop additional deterministic criteria to evaluate the design features for defense-in-depth, in addition to meeting the criteria of Section 3.3.

3.4.1. Principles of redundancy, diversity and independence shall be observed to ensure that no single failure or removal from service of an active or passive component can result in loss of a safety or security function or loss of required redundancy in performance of a safety or security function. Performance of safety and security functions shall not depend on a single element of design, construction, maintenance or operation. At least two redundant, diverse and independent means for reactor shutdown and decay heat removal shall be provided, and there shall be at least two barriers to fission product release.

3.5. Risk-informed classification: Classification of structures, systems and components shall be risk-informed, and safety significant SSCs shall be identified and given special attention throughout their life cycle to ensure that they can perform their functions in accordance with the assumptions made in the PRA and SAR.

3.6. Passive safety features: In addition to the engineered systems described in Section 3.4 to provide defense-in-depth, the ABR design shall incorporate passive means of performing the fundamental safety functions of reactivity control, heat removal and containment of radioactive materials to the extent possible consistent with design goals for operability, reliability and cost. Examples of passive means include inherent negative reactivity feedbacks, thermal expansion of fuel and structures, low excess reactivity and mechanical stops to limit the magnitude of potential positive reactivity insertion from control rod withdrawal, natural circulation heat removal systems, guard vessels to limit sodium leakage, and a low leakage containment building.

3.6.1. The ABR design shall include features that reduce to as low a level as practical the likelihood and consequences of a sodium fire, sodium-water or sodium-concrete interaction or chemical interaction of sodium with

\footnotetext{
${ }^{14} \mathrm{~A}$ large release is a release of volatile radionuclides into the environment that could result in a prompt fatality to a member of the general public.
} 
other materials with which it is not compatible. Sodium leak detection systems shall be provided. Use of inerted cells for sodium equipment, capability for rapid draining of the secondary sodium system and similar measures may be considered.

3.7. Protection against security-related events: As a minimum, the ABR shall have the same level of protection against security-related threats as established by 10CFR73 and the NRC's post-9/11 orders ${ }^{15}$, and shall satisfy the NRC's expectations for security design of new reactors ${ }^{16}$. A security vulnerability assessment shall be performed during the design phase of the facility, as well as prior to licensing and operation. Such an assessment shall consider, as a minimum, the areas mandated by the NRC. Security shall be accomplished by design to the extent practical. Defensein-depth shall be provided to compensate for uncertainties. Details of the application of probabilistic performance standards shall be agreed to with the NRC.

3.7.1. The ABR design shall provide for detection and surveillance sufficient to detect theft or diversion of material that could result in an Extraordinary Nuclear Occurrence, as defined in 10CFR140.

3.8. Safety and security preparedness: Specific design features to provide for safety and security preparedness shall be incorporated into the ABR design. Examples of such features may include provisions for protecting the control room(s), emergency operations center and vital areas of the plant in case of an accident or attack. At a minimum, the expectations of the NRC shall be met in the design.

3.9. Emergency preparedness: Appropriate design features for emergency preparedness shall be included. Examples may include an auxiliary control room, emergency operations center, emergency communications, accident management measures and emergency procedures.

3.10. Integrated design: Safety, security and emergency preparedness shall be considered as an integrated whole in the design of the ABR.

\section{Design Requirements}

4.1. Design life: The design life of the ABR shall be determined based on the expected lifetime of materials used in structures, systems and components (SSCs). Aging and degradation mechanisms shall be considered in the design. It is expected that the ABR will have a design life similar to that of advanced light water reactors.

\footnotetext{
${ }^{15}$ All Operating Reactor Licensees, Order Modifying License EA-02-26, 67 FR 9792, March 4, 2002, and EA-03-086, 68 FR 24517, May 7, 2003

${ }^{16}$ SECY 05-0120, "Security Design Expectations for New Reactor Licensing Activities," September 9, 2005
} 
4.2. Design for simplicity, low cost and high availability: The ABR design shall emphasize simplicity, ease of fabrication, installation, operation, inspection and maintenance to promote reliability of SSCs, minimize capital and operating cost and maximize availability of the plant.

4.3. Design for reliability: Structures, systems and components shall be designed for a high level of reliability to increase plant availability, decrease costs and reduce risk. Principles of redundancy, diversity, physical separation and independence shall be applied to eliminate the likelihood of common cause failures.

4.3.1. Auxiliary services and equipment that support a safety significant function shall be considered to be part of that function and classified accordingly. Failure of a non-safety significant SSC shall not affect safety by causing failure of a safety significant SSC.

4.4. Design for flexibility in siting: The ABR shall be suitable for deployment on the widest possible variety of sites within the United States. To this end, the design shall reduce vulnerability to external events, such as earthquakes, floods, wind storms, human-caused external events, etc.

4.4.1. In the absence of identification of a specific site, the prototype ABR shall be suitable for deployment on any of the sites that have been identified for detailed siting studies for integrated spent fuel recycling facilities under the GNEP initiative.

4.5. Seismic design: The ABR's safety-significant structures, systems and components shall be designed for earthquake resistance using the current NRC regulatory scheme, or (if it is adopted) the performance-based scheme that the NRC has recently proposed for design certification of new light-water reactors (LWRs). The objective of the approach taken for the ABR (like that taken in design of the LWRs that have received design certification) shall be that the ABR could be sited in most of the United States without modifying the seismic design basis. (It is recognized that in areas of very high seismicity, such as coastal California, a special design approach may be necessary.)

4.6. Design for external events: The ABR design shall include generic provisions to accommodate hazards other than earthquakes, high winds and external flooding that originate from outside the plant site, including but not limited to external explosion, toxic gas release, rare meteorological events, floods, etc. Specific provisions depending on the site characteristics shall be incorporated as necessary when a site is identified.

4.7. Protection against internal fires: The ABR design shall provide comprehensive systems for protection against internal fires, considering both normal fires and sodium fires. The impact of smoke and toxic fumes from fires shall be considered in the 
design of the control room and safety significant SSCs. Use of a risk-informed approach to design for fire protection shall be agreed with the NRC.

4.8. Normal heat transport: The normal system for heat transport from the reactor to the power conversion system shall be such that radioactive sodium does not circulate outside of the containment boundary. The system may consist of conventional primary and secondary sodium loops separated by an intermediate heat exchanger or an innovative design may be proposed by the designers. In any case, the reactor core must be protected against potential damage from energetic events such as sodiumwater reactions in the heat transport system. This heat transport system shall have sufficient capacity to prevent exceeding acceptable fuel design limits and to maintain conditions of normal operation within the design limits of the ASME Boiler and Pressure Vessel Code (B\&PV Code), Section III, Levels A and B. The heat transport system shall be configured to ensure natural circulation capability and to limit sodium inventory loss in the event of a rupture or leak such that the core is not uncovered at any time.

4.9. Shutdown heat removal: A separate and redundant shutdown heat removal system shall be provided. It shall provide for passive, continuous operation and shall be considered safety-significant. This system shall have sufficient capacity to prevent structures from exceeding ASME B\&PV Code Section III Level B limits and fuel design limits under normal conditions if the normal heat transport system is not available. It shall provide sufficient capacity to prevent structures from exceeding ASME B\&PV Code Section III Level C limits and fuel safety limits under accident conditions within the design basis.

4.10. Instrumentation and control systems: A highly reliable instrumentation and control system capable of maintaining plant conditions within prescribed operating ranges and providing reliable information on plant processes shall be provided. It is expected that the control room(s) and the I\&C systems will use digital technology, proven hardware and verified and validated software, and have growth capability to accommodate future hardware and software changes and enhancements. The system shall incorporate self-diagnostic and on-line maintenance features.

4.11. Data communication and handling: State-of-the-art systems for communicating and handling plant data shall be provided.

4.12. Reactivity control: Two independent and diverse reactivity control systems shall be provided. Each system shall have the capability to ensure that the reactor can be brought to and maintained in a safe shutdown state under all operating and accident conditions within the design basis assuming failure of other systems.

4.13. Reactor protection system: An IEEE Class $1 \mathrm{E}$ reactor protection system, considered safety-significant, separate and independent from the plant control system, shall be provided to ensure reactor shutdown, prevent fuel damage and prevent structures from exceeding ASME B\&PV Code Section III Level C limits under 
accident conditions within the design basis. The design shall be such as to minimize the likelihood that an operator action can prevent the reactor protection system from performing its safety functions or inhibit the functioning of any passive safety feature.

4.14. Diagnostics: Diagnostic systems shall be provided to detect off-normal conditions. Systems for detecting sodium leakage and cladding failures during operation and shutdown shall be provided.

4.15. Reduce challenges to safety systems: The plant design shall include reliable equipment for all non-safety functions. If failure of an item of equipment performing a non-safety function can challenge safety-significant systems, the design shall reduce the frequency of such challenges to an acceptable level.

4.16. Post-accident monitoring: A system for monitoring safe shutdown conditions shall be provided. It shall be designed to monitor selected system parameters in their anticipated ranges under conditions of normal operation, frequent and infrequent events. The parameters to be monitored shall include those related to the fission process and the integrity of the fuel, reactor coolant system boundary and the containment. This system shall be highly reliable and fault tolerant, and shall be considered safety significant.

4.17. Equipment qualification: Safety-significant equipment shall be designed with due consideration of the environment expected to be present when it is called upon to perform its safety function. Possible effects of external events and equipment aging shall be considered.

4.18. Radiation protection for plant personnel: The ABR design shall include sufficient shielding and other measures for radiation protection to ensure that radiation levels within and outside the plant are acceptable and that dose to plant personnel is as low as reasonably achievable. The integrated whole-body exposure for workers in routine operations, maintenance and inspection shall meet NRC's requirements. Radiation protection shall be provided to limit individual exposure to within those requirements.

4.19. Containment: The ABR design shall include a containment system that provides a low-leakage, safety-significant structure as the final physical barrier against radiological release. The containment system shall also provide sufficient shielding to satisfy radiation protection requirements and serve as a barrier against damage to the reactor from outside attack. The design team shall specify the appropriate codes and standards for design and construction of the containment.

4.19.1. The containment system, including all access openings and penetrations, internal compartments and internal structures shall be designed to accommodate the calculated pressure and temperature conditions associated with normal operation and accident conditions within the design basis without exceeding the design leakage rate. The design shall 
account for thermal energy sources, such as decay heat, fires and potential exothermic chemical reactions, as well as any mechanical challenges.

4.19.2. The design containment leak rate shall meet NRC's leak rate requirements. The design shall permit periodic leak rate testing and surveillance of the containment boundary in accordance with NRC requirements.

4.19.3. The containment shall satisfy regulatory requirements for physical protection of vital SSCs located within the containment.

4.19.4. In the commercial and prototype $\mathbf{A B R}$, the containment shall include hatches and internal spaces that are large enough to allow for removal and replacement of major components such as intermediate heat exchangers and primary pumps.

4.20. Fuel handling: The ABR design shall provide for receipt, handling, storage, security, international verification and accountability of fresh fuel entering the facility. Adequate cooling for the fresh fuel shall be provided. Similarly, the design shall provide for handling, storage, security, verification and accountability, cooling, preparation for shipment and dispatch of spent fuel. Suitable equipment for fuel handling shall be provided.

4.20.1. The design shall facilitate control and accountancy of nuclear material throughout its presence in the facility, including receipt and storage of fresh fuel, loading fresh fuel into the reactor, unloading and interim storage of spent fuel, and preparation for shipment and removal from the facility of spent fuel. Appropriate viewing equipment or other means of identifying fuel assemblies in storage and in the reactor shall be provided.

4.21. Inspection and maintenance: The design shall facilitate inspection and maintenance. To the extent possible, systems and components shall be designed so that routine inspection and maintenance can be performed during operation.

4.21.1. The design shall allow for access, viewing, inspection and testing of systems and components, working and lay-down space for component repair or replacement, and to facilitate good housekeeping and contamination control.

4.21.2. An in-service inspection (ISI) program shall be developed that meets the intent of the ASME B\&PV Code Section XI, Division 3. The ISI program shall be applied to safety-significant SSCs and shall ensure that the safetysignificant SSCs maintain their ability to perform their safety functions. For other SSCs, the design shall include inspection provisions consistent with maintaining safe, operable conditions throughout the plant. 
4.21.3. State-of-the-art techniques for inspection and maintenance of SSCs that are under sodium shall be considered.

4.22. Human Factors: The ABR design shall include considerations of human factors and human-machine interface. Automatic control and safety functions shall be provided to reduce the demands on operators. Plant operating procedures and diagnostics shall be automated to the extent needed to facilitate operator actions. There shall be a clear distinction of functions between operating personnel and the automatic systems provided.

4.22.1. The design shall promote the success of operator actions with due regard for the complexity of operator actions required, the time available for action, the physical environment to be expected and the psychological demands to be made on the operator. The need for intervention by the operator involving complex actions on a short time scale shall be avoided to the extent feasible.

4.22.2. The control room design shall incorporate human factors considerations, and shall provide for protection of the operating staff during accidents and external events.

4.23. Waste Minimization and Management: Design of the ABR shall consider minimization of the volume and activity of radioactive waste produced during operation. Suitable facilities shall be included for management of radioactive waste in accordance with applicable regulations. These facilities shall be considered in the safety, reliability and availability assessments of the plant.

4.24. Decommissioning: The ABR shall be designed to facilitate decommissioning, and to minimize the waste produced and the radiation hazards associated with decommissioning.

\section{Acknowledgment}

The author is grateful to his colleagues at Argonne, the other participating National Laboratories and the Department of Energy for the many constructive and useful comments and suggestions that they provided during development of this document.

Rev 11a

25 July 2007 


\section{Appendix}

\section{A Frequency-Consequence Curve}

The frequency-consequence curve shown here was developed by the US NRC and presented in the draft "Framework for Development of a Risk-Informed, Performance-Based Alternative to 10CFR Part 50,” NUREG-1860 Working Draft, July 2006.

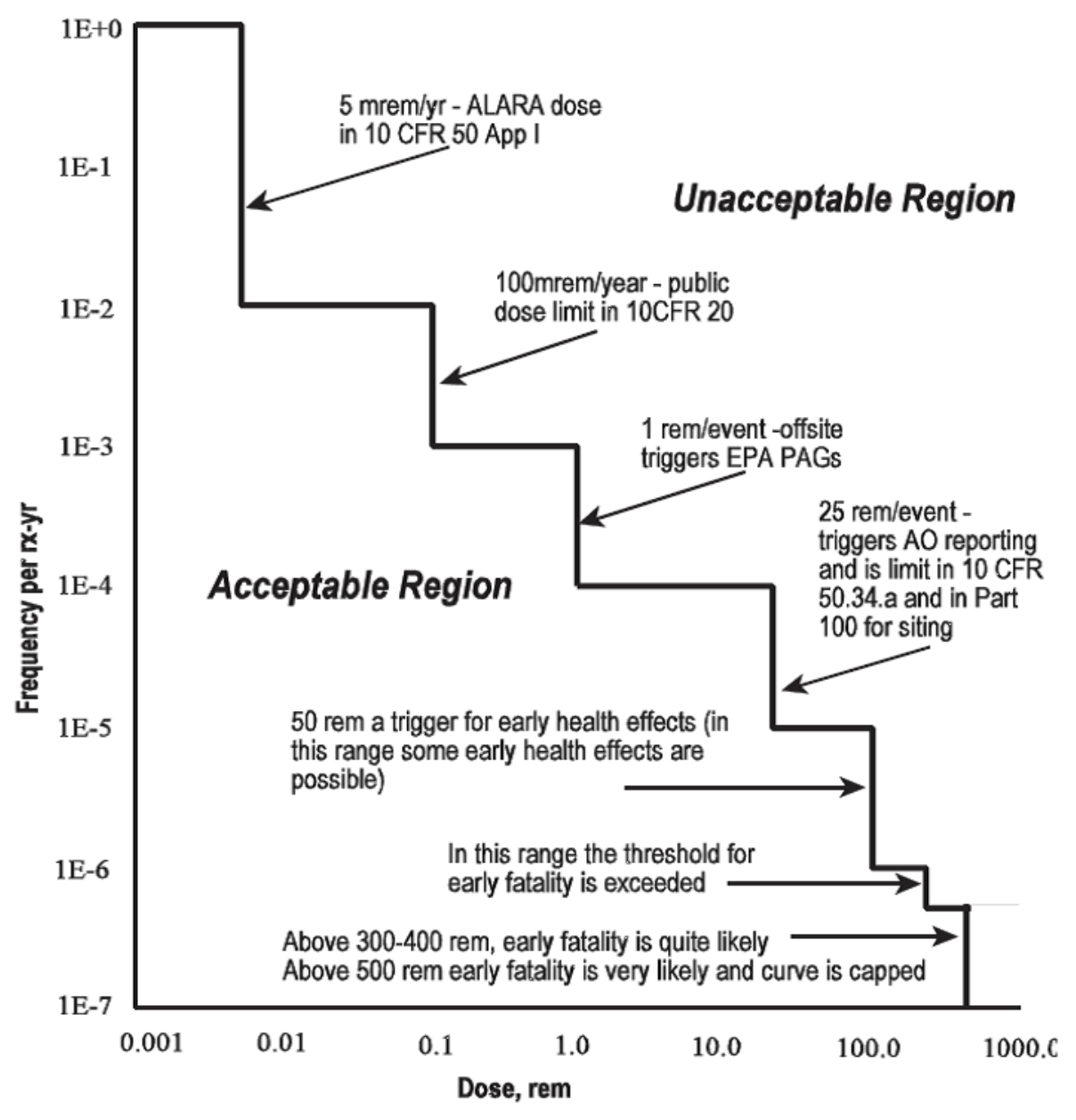

Figure A-1 


\section{Argonne}

\section{Division}

Argonne National Laboratory

9700 South Cass Avenue, Bldg. \#208

Argonne, IL 60439

www.anl.gov 